\title{
Investigations into the Mesophase Behaviour and Equilibrium Dynamics of a Family of Aerosol OT-related Surfactants
}

\author{
by \\ Graham Scott Fairweather
}

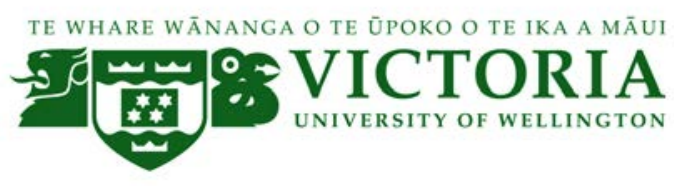

\author{
A thesis \\ submitted to Victoria University of Wellington \\ in fulfilment of the \\ requirements for the degree of \\ Doctor of Philosophy \\ in Chemistry
}

Victoria University of Wellington

2017 



\section{Preface}

This dissertation is an account of experimental work carried out between January 2010 and December 2015 in the School of Chemical and Physical Sciences at Victoria University of Wellington. All of this work was carried out solely by myself and the schematics and figures that are included are of my creation unless specifically stated otherwise.

The results and discussion chapters of this thesis are presented in two parts to distinguish between the different topics. Part I discusses the self-assembly of surfactant systems containing Group 1 alkali metal counterions, while in Part II ammonium cations were used. In addition, Appendix E reports some rheological properties of the systems outlined in the main body of the thesis.

Much of the experimental work involved the use of microscopes to study fluid and dynamic systems. Important observations made during the course of this work are described to the best of my ability and are supplemented with a range of captured images that are representative of these observations. To fully explain and document what occurs in these systems on a wide range of lengthscales, many pictures have been provided. To avoid disrupting the written account, pictures have been kept to a logical size. However, several key figures are reproduced in larger size in the appendices, along with a number of additional images. 


\section{Abstract}

Surfactants are ubiquitous, with many important applications in physical and biological sciences. Increasingly, they are being utilised for their liquid crystalline properties rather than in their traditional roles as detergents, emulsifiers or wetting agents. This is because these liquid crystalline mesophases offer a large range of different architectures with varying degrees of structural order and functionality. Presently, surfactants used in these applications are those with behaviours that are already understood due to their routine use in industry. However, the concept of producing surfactants by rational design would be a significant step forward and could potentially revolutionise the field. Such a step is not trivial due to the hierarchical and complex nature of self-assembly. In order to realise this goal, we have to understand how the various contributions to the free energy of the system can be manipulated to affect the building blocks of the system on different lengthscales. How do changes made on the sub-nanometre lengthscale alter the physical dynamics of a system as a whole?

In order to answer this question, we must gather experimental evidence for different systems using a range of complementary techniques to probe the system dynamics on different lengthscales. Here we report the characterisation of phase behaviour and the evolution of microstructure for a family of surfactants. We investigated this using a combination of smallangle X-ray spectroscopy, polarising optical microscopy and cryogenic scanning electron microscopy, producing seven new phase diagrams. We also report the flow properties of these systems, which were studied using static and dynamic rheology.

The starting point of this study is sodium bis(2-ethylhexyl)sulfosuccinate (Na-AOT), which is an industrially important surfactant that forms lyotropic liquid crystalline phases in water. This behaviour has been extensively studied previously and presents over large concentration and temperature ranges. The system has also been a source of debate and confusion in regards to a possible transition within the lamellar phase. We present a review of the literature and, with the addition of our own investigation, show that system does not undergo any of the previously proposed transitions within this region. We show that the anomalies reported in the literature can be explained by a change from a swollen and highly connected lamellar phase to a more classical ordered lamellar phase with increasing surfactant concentration. This change is a result of different intermolecular forces governing the system as the lamellar bilayer repeat distance decreases. The impressive stability of the lamellar phase is mediated through formation of different types of topological defects, which change from positive to negative 
Gaussian curvature as the bilayer elasticity varies. We then use Na-AOT as a base system for comparison with a family of related surfactants to detail how molecular changes alter the self-assembly of each new system.

The first comparison system discussed is the an analogue of Na-AOT with reduced branching, sodium bis(1,3-dimethylbutyl) sulfosuccinate (Na-butylAMA). The use of this system allowed us to investigate the role of the surfactant's branching ethyl groups in the self-assembly of the $\mathrm{Na}$-AOT system. We show that the less bulky tail region and reduced conformational freedom of Na-butyIAMA dramatically reduces the stabilisation of the lamellar phase, with liquid crystalline phases only presenting at surfactant concentrations above $50 \mathrm{wt} \%$. We also report that the phase progression of the Na-AOT system can be closely reproduced by modification of the Na-butylAMA headgroup via ion exchange. Replacing the sodium counterion with potassium restores the balance between headgroup and tail volumes and allows the stabilisation of the lamellar phase at low concentrations.

Alterations were made to the steric bulk and electronic properties of the AOT headgroup without changing the chemical functionality by replacing the sodium cation with two other Group 1 alkali metals: lithium and potassium. Cryo-SEM images show how these modifications dramatically alter the elasticity in the system. In the case of Li-AOT, this results in a significant shift of the phase boundaries to lower concentrations, with extended stability of the ordered inverted bicontinuous cubic and inverted hexagonal phases. The K-AOT system shows increased elasticity due to more negative Gaussian curvature. This drives the system to form a more disordered sponge phase, which can reversibly transition to a lamellar phase upon heating. These changes are explained in terms of the different hydration and headgroup affinities of the cations, which both alter the elastic moduli of the surfactant bilayer.

The AOT system was also studied with three biologically relevant ammonium-based counterions: ammonium $\left(\mathrm{NH}_{4}^{+}\right)$, choline $\left(\mathrm{Ch}^{+}\right)$and acetylcholine $\left(\mathrm{AcCh}^{+}\right)$. With the first being of comparable size to $\mathrm{K}^{+}$, the effects of increased polarisability and hydrogen bonding on phase behaviour were investigated. The different microstructures observed in the AOT system when using $\mathrm{Na}^{+}, \mathrm{K}^{+}$and $\mathrm{NH}_{4}^{+}$are discussed in terms of the different contributions to the Gaussian curvature, including ion pair formation, hydrogen bonding, hydration and steric constraints on molecular packing. In comparison to the other counterions investigated, choline and acetylcholine are more weakly hydrated, have greater affinity for the sulfonate anion, and provide additional steric bulk to the AOT headgroup. This removes some degrees of freedom in these systems, and hence increases bilayer rigidity, causing significant stabilisation of their lamellar phases. As a result, lamellar phases dominate both systems over nearly their entire concentration ranges.

The bilayer elasticity was further investigated by the addition of electrolyte to the lamellar phases of the $\mathrm{NH}_{4}$-AOT and Ch-AOT systems. Both are shown to exhibit salt-induced phase transitions, forming sponge and cubic phases depending on the starting surfactant concentration. These transitions were achieved with small amounts of $\mathrm{NaCl}$ in the case of $\mathrm{NH}_{4}$-AOT, due to its highly disordered microstructure. By contrast, as a result of the increased bilayer rigidity, 
the Ch-AOT system is shown to have a highly stable lamellar phase that can accommodate large amounts of salt.

Having elucidated the phase behaviour and microstructure of each surfactant system, the viscoelastic properties of a number of the systems were investigated. The flow properties of the lamellar and sponge phases were studied by static and dynamic rheology. The shear behaviour of the sponge phases were observed to be dependent upon concentration, with both Newtonian and shear thinning behaviours displayed. The lamellar phases of several systems demonstrated shear-dependent phase transitions. These transitions were also monitored by cryo-SEM, which showed the formation of multilamellar vesicles (onions) at a critical shear rate. Upon increasing shear, the onions decreased in size and assembled in a close-packed arrangement.

In summary, this study addresses the confusion surrounding the widely disputed lamellar phase of the Na-AOT system. The observed changes in physical properties are explained by the evolution of defect behaviours on multiple lengthscales. Through a series of molecular modifications, a range of different systems have been produced and their liquid crystalline phase behaviours and microstructures characterised. The relative stabilisation of lamellar and sponge phases are rationalised in terms of the various competing contributions to the total free energy. These phases are then manipulated through changes in temperature, electrolyte addition and shear, with the induction of several different phase transitions observed. By systematically changing the AOT cation, we have demonstrated the role of the counterion in mesophase stability and system elasticity. We have shown that through careful counterion selection, we can enhance or reduce the stabilisation of curved interfaces. This has allowed us to compile a set of considerations, which can be applied to other surfactant systems in order to better predict the potential outcomes of alterations in these systems, and is a step towards rational design in the field. 


\section{Acknowledgements}

It gives me great pleasure to acknowledge the help and support offered by an array of people, who each in their own way made this thesis possible.

Firstly, to Professor Kathryn McGrath, without whom this work would never have been completed. I cannot thank you enough for your support, understanding and enthusiasm. I was so grateful when you took a gamble on me (a forensic and analytical chemist) to undertake a PhD in physical chemistry and I could never have imagined how much that gratitude would grow. At no stage was this journey easy, but without you it would have been insurmountable. Thank you for being both a brilliant and caring supervisor.

My thanks also go to my co-supervisor Associate Professor Justin Hodgkiss, as well as David Flynn (for assistance with cryo-SEM), Dr Kathryn Allan and Dr Melanie Nelson (for their NMR expertise), and the support staff at SCPS, in particular the chemistry laboratory technicians, administrative staff, workshop guys and the glassblower Grant Franklin.

I also thank Patricia Stein, who completely underestimates just how good at her job she actually is. When in crisis mode and feeling like everything is falling apart around you, there is something very calming about entering a room and being greeted by a woman on a giant bouncy ball who offers a beaming smile, chocolate and the words "we will fix this".

Many friends were made during the process of completing this thesis, and I appreciate the support and encouragement offered on both sides of the globe. I thank the following people in particular for making this (at times a truly horrible) experience actually quite enjoyable. Natasha Evans, Melanie Nelson, Jaime-Anne Elliott, Helen Rowley, Sarah Hoyte and Teresa Vaughan (nothing quite beats cups of tea and understanding). Ash Dangerfield (I will never not smile upon hearing The Cure), Jacqui Barber (keep bouncing Zebedee), Joe Gallaher (for the company on the all-nighters) and the CMFC (for the exquisite drams). I also thank my lab group (both past and present), and Judy Vaughan (for being a caring person with friends in high places).

I would also like to thank The MacDiarmid Institute for Advanced Materials and Nanotechnology, Victoria University of Wellington and The Curtis-Gordon Fund for various scholarships/financial contributions. 
My gratitude goes to the Allan family for their help and encouragement, warm meals, sausages, and a roof over my head.

I thank my family - Mum, Dad and Dave - for their encouragement, for believing in me and for funding this adventure (even when it dragged on).

And lastly, to Kathryn Allan, much like the Kathryn listed above you've been amazing throughout. I thank you deeply from the bottom of my heart and look forward to the good times to come. 


\section{Contents}

Preface

$\begin{array}{ll}\text { Abstract } & \text { iii }\end{array}$

Acknowledgements $\quad$ vii

Table of Contents $\quad$ ix

List of Figures $\quad$ xi

List of Tables $\quad$ xvi

Glossary $\quad$ xvii

1 Introduction 1

Phase Behaviour and Curvature . . . . . . . . . . . . . . . 3

Relevant Mesophase Structures . . . . . . . . . . . . . . . . . 9

Intermolecular Forces . . . . . . . . . . . . . . . . . . . . . . . . 12

Defect Structures . . . . . . . . . . . . . . . . . . . . . . . . . . . . . . . . . . . . 14

The Role of Counterions . . . . . . . . . . . . . . . . . . . . . . 17

2 Experimental $\quad 19$

Surfactant Synthesis and Purification . . . . . . . . . . . . . . . . . . . 19

Preparation of Surfactant Samples and Phase Diagrams . . . . . . . . . . . . . . . 22

Small-Angle X-ray Scattering . . . . . . . . . . . . . . . . . . . . . . 28

Optical Microscopy . . . . . . . . . . . . . . . . . . . . . . . 32

Cryogenic Scanning Electron Microscopy $\ldots \ldots$. . . . . . . . . . . 36

I Alkali Metal Counterions $\quad 43$

3 Sodium Aerosol OT $\quad 45$

Sodium AOT: Introduction . . . . . . . . . . . . . . . . . . . . . . . . . . . 45

Sodium AOT: Phase Behaviour . . . . . . . . . . . . . . . . . . . . . 48

Sodium AOT: Lamellar Phase Literature Review . . . . . . . . . . . . . . . . . . . 50

Sodium AOT: Experimental Results . . . . . . . . . . . . . . . . . . . 67

Sodium AOT: Discussion $\ldots \ldots \ldots$. . . . . . . . . . . . . . . 97 
4 Sodium and Potassium Aerosol MA 103

Sodium AMA: Introduction . . . . . . . . . . . . . . . . . . . . . . . . 103

Sodium butylAMA: Experimental Results . . . . . . . . . . . . . . . . . . . . 107

Sodium butylAMA: Discussion . . . . . . . . . . . . . . . . . . . . . 123

Potassium butylAMA: Experimental Results . . . . . . . . . . . . . . . . . 127

Potassium butylAMA: Discussion . . . . . . . . . . . . . . . . . . . . . . . . . 139

5 Potassium and Lithium Aerosol OT 141

Potassium and Lithium AOT: Introduction . . . . . . . . . . . . . . . . . . . . . . 141

Potassium AOT: Experimental Results . . . . . . . . . . . . . . . . . . . . . . 143

Lithium AOT: Experimental Results . . . . . . . . . . . . . . . . . . . . . . . . . 158

Potassium and Lithium AOT: Summary f . . . . . . . . . . . . . . . . . . . . 171

$\begin{array}{ll}\text { II Ammonium Counterions } & 177\end{array}$

6 Ammonium Aerosol OT 181

Ammonium AOT: Introduction . . . . . . . . . . . . . . . . . . . . . . . . . . . . . . 181

Ammonium AOT: Experimental Results . . . . . . . . . . . . . . . . . . . . . . . . 182

Ammonium AOT: Discussion . . . . . . . . . . . . . . . . . . . . . . . . . 193

Addition of Salt to the Ammonium AOT System: Experimental Results . . . . . . . . 196

Addition of Salt to the Ammonium AOT System: Discussion . . . . . . . . . . . . . . . 201

7 Choline and Acetylcholine Aerosol OT 205

Choline and Acetylcholine AOT: Introduction . . . . . . . . . . . . . . . . 205

Choline AOT: Experimental Results . . . . . . . . . . . . . . . . . . . . 209

Choline AOT: Summary . . . . . . . . . . . . . . . . . . . . . . . . . . . . 223

Addition of Salt to the Choline AOT System: Experimental Results . . . . . . . . . . . 224

Addition of Salt to the Choline AOT System: Summary . . . . . . . . . . . . . . . . . . 234

Acetylcholine AOT: Experimental Results . . . . . . . . . . . . . . . . . 235

Acetylcholine AOT: Summary . . . . . . . . . . . . . . . . . . . . . . . . . . . . 251

Choline and Acetylcholine AOT: Discussion . . . . . . . . . . . . . . . . . . . 252

8 Concluding Remarks $\quad 257$

$\begin{array}{ll}\text { Bibliography } & 263\end{array}$

Appendix A: NMR Spectra of Na-butylAMA 285

Appendix B: Concentration Gradient POM Images 293

Appendix C: Additional POM Images 299

Appendix D: Enlargements of Selected Cryo-SEM Images 311

Appendix E: Rheology 329 


\section{List of Figures}

1.1 Schematic illustrations of surfactant packing. $\ldots \ldots \ldots \ldots \ldots \ldots \ldots$

1.2 Commonly observed lyotropic mesophases. . . . . . . . . . . . . 4

1.3 The relationship between the principal curvatures of a surface and its mean and Gaussian curvatures. . . . . . . . . . . . . . . . 6

1.4 An illustration of the curvature frustration associated with monolayers adopting a

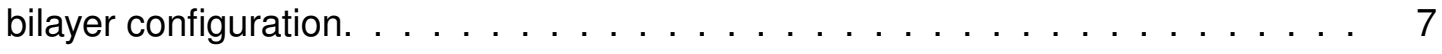

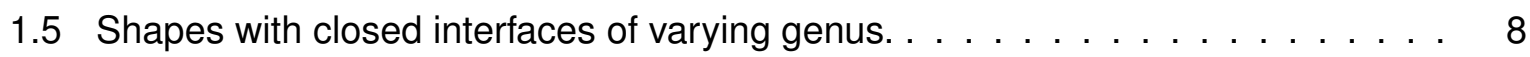

1.6 Theorised variation in self-assembly as a function of packing parameter and

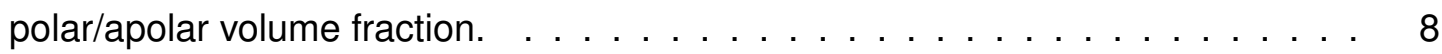

1.7 Schematic representations of lamellar and inverse hexagonal phases. . . . . . 9

1.8 Mathematical models of the space groups of bicontinuous cubic phases. . . . . 11

1.9 A saddle-splay minimum surface, a schematic representation of an $L_{3}$ phase, and a mathematical $L_{3}$ phase model. . . . . . . . . . . . . . . 11

1.10 The relationship between lamellar, sponge and bicontinuous cubic phases, and their dependency on the elastic constants $\kappa$ and $\bar{\kappa}$. . . . . . . . . 13

1.11 Selected examples showing the topology around line defect singularities. . . . 15

1.12 Schematic examples of lamellar phase defects. . . . . . . . . . . 16

1.13 A schematic illustration of Collins' law of matching water affinities. . . . . . . 18

1.14 The relative kosmotropic/chaotropic natures of the cations used in this research. 18

$2.1{ }^{1} \mathrm{H}$ NMR spectra of Li-AOT, Na-AOT and K-AOT in $\mathrm{CDCl}_{3} \ldots \ldots \ldots \ldots$

$2.2{ }^{1} \mathrm{H}$ NMR spectra of AcCh-AOT, Ch-AOT, $\mathrm{NH}_{4}$-AOT and Na-AOT in $\mathrm{CDCl}_{3} \ldots \ldots .24$

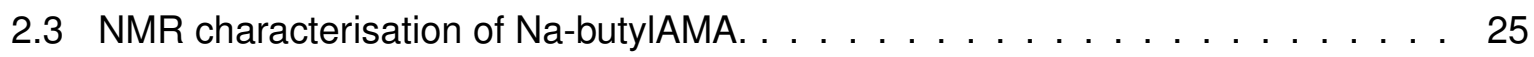

$2.4{ }^{1} \mathrm{H}$ NMR spectra of K-butylAMA, Na-butylAMA and $\mathrm{Na}-\mathrm{AOT}$ in $\mathrm{CDCl}_{3} \ldots \ldots .26$

2.5 An example plot of phase observations at different concentrations and temperatures used as the basis for creating a phase diagram. . . . . . . . 28

2.6 Schematic representations of two different SAXS instrumentation set-ups. . . . . 29

2.7 Examples of SAXS data for lamellar and hexagonal phases. . . . . . . . 30

2.8 An example of SAXS/WAXS data with 2D and 1D representations shown. . . . 31

2.9 An anisotropic material between crossed polarisers displaying birefringence. . . 32

2.10 Examples of FCD-II (left) and FCD-I (right) defects commonly encountered in this research, observed using POM. . . . . . . . . . . . . . . 33

2.11 Examples of POM textures encountered in this research. . . . . . . . . 34

2.12 Cryo-SEM images of samples frozen at an insufficiently rapid rate. . . . . . . . 38

2.13 Cryo-SEM images obtained via the sample preparation method used in this work. 39 
2.14 Cryo-SEM images for a single sample obtained with different sample preparation methods. . . . . . . . . . . . . . . . . . . . . . . . . . . 40

3.1 Molecular structure of Na-AOT. . . . . . . . . . . . . . . . . . . . 45

3.2 Plot showing the increasing number of Na-AOT publications between 1900 and $2015 \ldots \ldots \ldots \ldots \ldots \ldots$. . . . . . . . . . . . . . . . . . . . . . . . . .

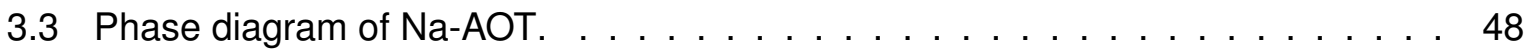

3.4 Two stabilised conformations of the Na-AOT succinate skeleton. . . . . . . . . . 56

3.5 Schematic representation of the structural model for the Na-AOT lamellar phase proposed by Callaghan et al. . . . . . . . . . . . . . . . . . . . . . . . . . . 61

3.6 Schematic representation of the structural model for the Na-AOT lamellar phase proposed by Chidichimo et al. . . . . . . . . . . . . . . . . . . . . . . . . . . . 62

3.7 Simulated models of the Na-AOT lamellar phase between 30 and 70 wt \% surfactant, produced by Yang et al. . . . . . . . . . . . . . . . . . . . . . . . . . . 64

3.8 SAXS profiles of the Na-AOT lamellar phase with increasing surfactant concentration. . . . . . . . . . . . . . . . . . . . . . . 68

3.9 Changes in $d$ spacing with increasing concentration for the Na-AOT system. . . . 70

3.10 Evolution of the first-order Bragg peak with increasing temperature for $\mathrm{Na}$-AOT samples of 46 and 53 wt $\%$ surfactant. . . . . . . . . . . . . . . . . . . . 72

3.11 The effect of temperature on $d$ spacing in the Na-AOT system. . . . . . . . . . 73

3.12 Evolution of the first-order Bragg peak with increasing temperature for $\mathrm{Na}-\mathrm{AOT}$ samples of 20 and $38 \mathrm{wt} \%$ surfactant. . . . . . . . . . . . . . . . . . . . . . 74

$3.133 \mathrm{D}$ and 2D plots showing the evolution of SAXS profiles of $34 \mathrm{wt} \%$ and $40 \mathrm{wt} \%$ Na-AOT upon increasing temperature. . . . . . . . . . . . . . . . . . . 76

3.14 Schematic representation of trans-gauche isomerism in the Na-AOT tail region upon heating. . . . . . . . . . . . . . . . . . . . . . 77

3.15 POM concentration gradient of the Na-AOT system. $\ldots \ldots \ldots$. . . . . 78

3.16 POM images of Na-AOT between 10 and 45 wt $\%$ surfactant. . . . . . . . . . . . 80

3.17 POM images of Na-AOT between 50 and 85 wt \% surfactant. . . . . . . . . . . . 81

3.18 Schematic representation of the defect types formed in the Na-AOT lamellar phase with increasing concentration. . . . . . . . . . . . . . . . . 85

3.19 Cryo-SEM images of Na-AOT samples of 25 and 38 wt \% surfactant. . . . . . . . 87

3.20 Cryo-SEM images of Na-AOT samples of 45 and 60 wt \% surfactant. . . . . . . . 89

3.21 Cryo-SEM images of Na-AOT samples of 70 and $75 \mathrm{wt} \%$ surfactant. . . . . . . . 91

3.22 Schematic representation of the "crumpling transition" observed in the Na-AOT system at $\sim 43$ wt $\%$ surfactant. . . . . . . . . . . . . . . . . . . . . 93

3.23 Schematic representations of point defects occurring on the lengthscale of surfactant bilayers. . . . . . . . . . . . . . . . . . . . . . . . . 93

3.24 Schematic representation of line dislocations observed in the concentrated region of the Na-AOT lamellar phase.

3.25 Cryo-SEM images showing the changes in the connectivity of the lamellar phase with increasing Na-AOT concentration. . . . . . . . . . . . . . . . . . . . 95

4.1 Molecular structures and CAS numbers of Na-butyIAMA, Na-pentylAMA and Na-hexyIAMA. 
4.2 Phase diagrams of Na-butylAMA and Na-AOT.

4.3 SAXS profiles of the Na-butyIAMA lamellar phase with increasing surfactant concentration. . . . . . . . . . . . . . . . . . . . . . . . . 109

4.4 SAXS profiles of Na-butylAMA $\mathrm{V}_{/ /}$and $\mathrm{H}_{/ /}$phases. . . . . . . . . . . . . 110

4.5 Plots showing the reciprocal d spacings $v s . \sqrt{ }\left(h^{2}+k^{2}+l^{2}\right)$ for the cubic phases of the Na-butylAMA and Na-AOT systems. . . . . . . . . . . . . . . . . . . . . 111

4.6 Changes in $d$ spacing with increasing concentration for the Na-butylAMA and Na-AOT systems.

4.7 Evolution of the first-order Bragg peak with increasing temperature for Na-butylAMA samples of 58,70 and 81 wt $\%$ surfactant. . . . . . . . . . . . . 113

4.8 POM concentration gradient of the Na-butylAMA system. . . . . . . . . . . . 114

4.9 POM images of Na-butylAMA with increasing concentration. . . . . . . . . . . . 115

4.10 Cryo-SEM images of a sample of $45 \mathrm{wt} \%$ Na-butylAMA. . . . . . . . . . . . . 117

4.11 Cryo-SEM images of a sample of $55 \mathrm{wt} \%$ Na-butylAMA. . . . . . . . . . . . . 118

4.12 Comparison of the lamellar phase undulations at 55 wt \% Na-butylAMA and 38 wt \% Na-AOT. . . . . . . . . . . . . . . . . . . . . . . . . . . . . . . . . . . 119

4.13 Cryo-SEM images of a sample of $60 \mathrm{wt} \%$ Na-butylAMA. . . . . . . . . . . . . 120

4.14 Cryo-SEM images of a sample of $70 \mathrm{wt} \%$ Na-butylAMA. . . . . . . . . . . . . . 122

4.15 Cryo-SEM images of a sample of 78 wt \% Na-butylAMA. . . . . . . . . . . . . . 123

4.16 Phase diagram of K-butylAMA . . . . . . . . . . . . . . . . . . 128

4.17 SAXS profiles of the K-butylAMA system with increasing surfactant concentration. 130

4.18 Plot showing the reciprocal d spacings vs. $\sqrt{ }\left(h^{2}+k^{2}+l^{2}\right)$ for the cubic phase of the K-butylAMA system . . . . . . . . . . . . . . . . . . . . . 131

4.19 POM images of K-butylAMA with increasing concentration. . . . . . . . . . . . 133

4.20 Cryo-SEM images of a sample of $30 \mathrm{wt} \%$ K-butyIAMA. . . . . . . . . . . . . 135

4.21 Cryo-SEM images of a sample of $50 \mathrm{wt} \%$ K-butylAMA . . . . . . . . . . . . . 137

4.22 Cryo-SEM images of a sample of $70 \mathrm{wt} \% \mathrm{~K}$-butylAMA . . . . . . . . . . . . . 138

4.23 Comparative cryo-SEM images of $\sim 70$ wt $\%$ surfactant samples of Na-butylAMA, K-butylAMA and Na-AOT. . . . . . . . . . . . . . . . . . . . 139

5.1 Phase diagram of the K-AOT system. . . . . . . . . . . . . . . . . . . 144

5.2 SAXS profiles of the K-AOT system, showing the $L_{\alpha}-L_{3}$ transition with increasing surfactant concentration. . . . . . . . . . . . . . . . . . . . . . 145

5.3 SAXS profiles of the K-AOT system, showing the formation of $V_{/ /}$and $H_{/ /}$phases. 146

5.4 Plots showing the reciprocal d spacings $v s . \sqrt{ }\left(h^{2}+k^{2}+l^{2}\right)$ for the cubic phases of the K-AOT system. . . . . . . . . . . . . . . . . . . . . . . . . . . . . . . . . . 147

5.5 SAXS profiles of the K-AOT system with increasing temperature at $\sim 30 \mathrm{wt} \%$ K-AOT and $\sim 35$ wt $\%$ K-AOT. . . . . . . . . . . . . . . . . . . . . . . . . . . . 148

5.6 SAXS profiles of the K-AOT system with increasing temperature at $\sim 50$ wt \% K-AOT and $\sim 55$ wt \% K-AOT. . . . . . . . . . . . . . . . . . . . . . . . . . . 149

5.7 SAXS profiles for a sample of $55 \mathrm{wt} \% \mathrm{~K}$-AOT at various temperatures. . . . . . . 150

5.8 Cryo-SEM images of $40 \mathrm{wt} \% \mathrm{~K}$-AOT obtained by freezing a sample that had been held at $80^{\circ} \mathrm{C}$ for several days. . . . . . . . . . . . . . . . . 151

5.9 POM concentration gradient of the K-AOT system. . . . . . . . . . . . . . . 153

5.10 POM images of K-AOT with increasing concentration. . . . . . . . . . . . . . 154 
5.11 POM images of the $\mathrm{L}_{3}-\mathrm{L}_{\alpha}$ phase transition in a $35 \mathrm{wt} \% \mathrm{~K}$-AOT sample with increasing temperature. . . . . . . . . . . . . . . . . . . . 155

5.12 Cryo-SEM images of the K-AOT samples of 17 and $25 \mathrm{wt} \%$ surfactant. . . . . 157

5.13 Cryo-SEM images of the K-AOT sponge phase. . . . . . . . . . . . . . . 158

5.14 Phase diagram of the Li-AOT system. . . . . . . . . . . . . . . . . . 159

5.15 SAXS profiles of the Li-AOT lamellar phase with increasing surfactant concentration. . . . . . . . . . . . . . . . . . . . . . . . 160

5.16 SAXS profiles of the Li-AOT system, showing the formation of $\mathrm{V}_{/ /}$and $\mathrm{H}_{/ /}$phases. 161

5.17 Plots showing the reciprocal $d$ spacings $v s . \sqrt{ }\left(h^{2}+k^{2}+l^{2}\right)$ for the cubic phases of the Li-AOT system. . . . . . . . . . . . . . . . . . . . . . . . 162

5.18 The theoretical stability of different $V_{/ /}$space groups as a function of chain volume and shape parameter. . . . . . . . . . . . . . . . . . . . . . . . . . 164

5.19 POM concentration gradient of the Li-AOT system. . . . . . . . . . . . . . 165

5.20 POM images of Li-AOT with increasing concentration. . . . . . . . . . . . . . 166

5.21 Cryo-SEM images of a sample of $30 \mathrm{wt} \%$ Li-AOT. . . . . . . . . . . . . . . . 168

5.22 Cryo-SEM images of a sample of 50 wt \% Li-AOT. . . . . . . . . . . . . . . . . 169

5.23 Schematic representation of the evolution of counterion binding and hydration in the K-AOT system with increasing concentration. . . . . . . . . . . . . . . . 174

5.24 Schematic representation of the evolution of counterion binding and hydration in the Li-AOT system with increasing concentration. . . . . . . . . . . . . . . . 175

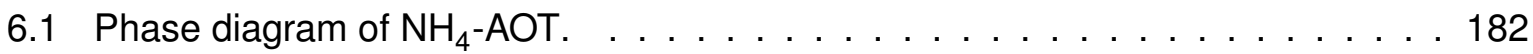

6.2 Phase diagrams of K-AOT, Na-AOT and $\mathrm{NH}_{4}$-AOT . . . . . . . . . . . . 183

6.3 SAXS profiles of the $\mathrm{NH}_{4}$-AOT system with increasing surfactant concentration. . 185

6.4 Plots showing the reciprocal $d$ spacings $v s . \sqrt{ }\left(h^{2}+k^{2}+l^{2}\right)$ for the cubic phases of the $\mathrm{NH}_{4}$-AOT system . . . . . . . . . . . . . . . . . . . . 186

6.5 POM images of $\mathrm{NH}_{4}$-AOT with increasing concentration. . . . . . . . . . . . . 188

6.6 Comparison of the POM textures of $\mathrm{NH}_{4}$-AOT at 43 wt $\%$ and $\mathrm{Na}-\mathrm{AOT}$ at 50 wt $\% .189$

6.7 Cryo-SEM images of a sample of $15 \mathrm{wt} \% \mathrm{NH}_{4}$-AOT. . . . . . . . . . . . . . . 190

6.8 Cryo-SEM images of a sample of 25 wt $\% \mathrm{NH}_{4}$-AOT. . . . . . . . . . . . . . . . 191

6.9 Cryo-SEM images of a sample of $50 \mathrm{wt} \% \mathrm{NH}_{4}$-AOT. . . . . . . . . . . . . . . . 192

6.10 Cryo-SEM images of a sample of 60 wt $\% \mathrm{NH}_{4}$-AOT . . . . . . . . . . . . . . . . 192

6.11 Cryo-SEM images of a sample of $70 \mathrm{wt} \% \mathrm{NH}_{4}$-AOT. . . . . . . . . . . . . . . 193

6.12 Phase diagram of the system AOT-NaCl-water at low salt content. . . . . . . . . 197

6.13 Cryo-SEM images of a sample of 25 wt $\% \mathrm{NH}_{4}$-AOT and 1.6 wt $\% \mathrm{NaCl}$ supporting the classification of the $\mathrm{L}_{3}$ phase. . . . . . . . . . . . . . 198

6.14 Stacked SAXS plots showing the effect on phase behaviour of the addition of $\mathrm{NaCl}$ to specified concentrations of $\mathrm{NH}_{4}$-AOT. . . . . . . . . . . . . . . . . 198

6.15 Stacked SAXS plots showing the effect on phase behaviour of increasing temperature for specified concentrations of $\mathrm{NH}_{4}$-AOT and $\mathrm{NaCl}$. . . . . . . . . . . 200

6.16 POM showing the temperature-induced sponge to lamellar transition for a sample of 48 wt $\% \mathrm{NH}_{4}-\mathrm{AOT}$ and $1 \mathrm{wt} \% \mathrm{NaCl}$ at various temperatures. . . . . . . . . 201

7.1 Molecular structure of the choline counterion. . . . . . . . . . . . . . . . 205

7.2 Molecular structures of phosphatidylcholine and sphingomyelin. . . . . . . . . 206 
7.3 Molecular structure of the acetylcholine counterion. . . . . . . . . . . . . . . 208

7.4 Phase diagram of the Ch-AOT system. . . . . . . . . . . . . . . . . . . . 210

7.5 Bulk samples of Ch-AOT viewed under crossed polarised light. . . . . . . . . . 211

7.6 POM concentration gradient for Ch-AOT. . . . . . . . . . . . . . . . . . 211

7.7 SAXS profiles of the Ch-AOT system with increasing surfactant concentration. . 213

7.8 Changes in d spacing with increasing concentration for Ch-AOT and Na-AOT systems. . . . . . . . . . . . . . . . . . . . . . . . . . 214

7.9 POM images of Ch-AOT with increasing concentration. . . . . . . . . . . . . 215

7.10 POM texture of pure Ch-AOT at $25^{\circ} \mathrm{C}$, obtained by slow cooling from an isotropic melt. . . . . . . . . . . . . . . . . . . . . . . . . . 217

7.11 Cryo-SEM images of a sample of $15 \mathrm{wt} \%$ Ch-AOT. . . . . . . . . . . . . . 218

7.12 Cryo-SEM images of a sample of $25 \mathrm{wt} \%$ Ch-AOT. . . . . . . . . . . . . . . . 219

7.13 Cryo-SEM images of a sample of $50 \mathrm{wt} \%$ Ch-AOT. . . . . . . . . . . . . . . 221

7.14 Cryo-SEM images of a sample of $75 \mathrm{wt} \%$ Ch-AOT. . . . . . . . . . . . . . . . 222

7.15 Cryo-SEM images of a sample of $95 \mathrm{wt} \%$ Ch-AOT. . . . . . . . . . . . . . . . 223

7.16 Bulk samples of $\sim 40$ wt \% Ch-AOT with increasing $\mathrm{NaCl}$ concentrations viewed under crossed polarised light. . . . . . . . . . . . . . . . . . . . . . . 225

7.17 Stacked SAXS plots showing the effect of increasing $\mathrm{NaCl}$ concentration at various Ch-AOT surfactant concentrations. . . . . . . . . . . . . . . . . . 228

7.18 Plots showing the observed reciprocal d spacings vs. $\sqrt{ }\left(h^{2}+k^{2}+l^{2}\right)$ for cubic phases of the Ch-AOT system. . . . . . . . . . . . . . . . . . . . . . 229

7.19 Cryo-SEM images showing the variety of phases formed in samples of varying Ch-AOT/NaCl/water composition. . . . . . . . . . . . . . . . . . . . 231

7.20 POM images showing the change of defect behaviour in the lamellar phase at $\sim 60$ wt $\%$ Ch-AOT upon increasing salt concentration. . . . . . . . . . . . . 233

7.21 A plot of viscosity vs. time for samples of $\sim 60 \mathrm{wt} \%$ Ch-AOT with various $\mathrm{NaCl}$ concentrations. . . . . . . . . . . . . . . . . . . . . . 234

7.22 Phase diagrams of AcCh-AOT and Ch-AOT. . . . . . . . . . . . . . . . 236

7.23 SAXS profiles of the AcCh-AOT system with increasing surfactant concentration. 239

7.24 Plots of $q^{*}$ intensity vs. azimuth angle for concentrations of 90,95 and 100 wt $\%$ AcCh-AOT, and the corresponding 2D SAXS profiles. . . . . . . . . . . . . . 240

7.25 WAXS profiles for AcCh-AOT and Ch-AOT at concentrations of 90 and $100 \mathrm{wt} \% .241$ 7.26 Changes in d spacing with increasing concentration for the AcCh-AOT, Ch-AOT and Na-AOT systems. . . . . . . . . . . . . . . . . . . . . . . 241

7.27 POM images of AcCh-AOT with increasing concentration. . . . . . . . . . . . . 245

7.28 POM image of a $20 \mathrm{wt} \%$ AcCh-AOT sample, showing the interface between the two phases. . . . . . . . . . . . . . . . . . . . . . 246

7.29 Cryo-SEM images of a sample of 15 wt \% AcCh-AOT. . . . . . . . . . . . . . . . 247

7.30 Cryo-SEM images of a sample of $25 \mathrm{wt} \%$ AcCh-AOT. . . . . . . . . . . . . . . 248

7.31 Cryo-SEM images of a sample of $50 \mathrm{wt} \%$ AcCh-AOT. . . . . . . . . . . . . . . . 250

7.32 Cryo-SEM images of a sample of 75 wt \% AcCh-AOT. . . . . . . . . . . . . . . . 251 


\section{List of Tables}

1.1 Types of lyotropic mesophases and associated structures. . . . . . . . . . . 5

3.1 Values for the $q$ spacing of the first-order Bragg peak $\left(\mathrm{q}^{\star}\right)$ and the corresponding bilayer repeat distance $(d)$ at selected Na-AOT concentrations and temperatures. 73

4.1 Values for the $q$ spacing of the first-order Bragg peak $\left(q^{*}\right)$ and the corresponding bilayer repeat distance $(\mathrm{d})$ at selected Na-butylAMA concentrations and temperatures. . . . . . . . . . . . . . . . . . . . . . . . . 112

5.1 Structural parameters for the $V_{/ /}$phases formed in the AOT and butyIAMA systems.163

6.1 Specific ion parameters for the ammonium ion and alkali metal ions. . . . . . . 194

6.2 Values of $\mathrm{q}^{*}$ and $\mathrm{d}$ for selected concentrations of $\mathrm{NH}_{4}-\mathrm{AOT}$ and $\mathrm{NaCl}$. . . . . 202

7.1 Structural parameters for various compositions of $\mathrm{Ch}-\mathrm{AOT}$ and $\mathrm{NaCl}$. . . . . . . 226 


\section{Glossary}

\begin{tabular}{|c|c|}
\hline $2 P$ & two-phase region \\
\hline $\mathrm{AcCh}^{+}$ & acetylcholine cation \\
\hline AcCh-AOT & acetylcholine bis(2-ethylhexyl)sulfosuccinate \\
\hline$\overline{\mathbf{B}}$ & compressibility modulus \\
\hline CCD & charge-coupled device \\
\hline $\mathrm{Ch}^{+}$ & choline cation \\
\hline Ch-AOT & choline bis(2-ethylhexyl)sulfosuccinate \\
\hline CMC & critical micelle concentration \\
\hline cosy & homonuclear correlation spectroscopy \\
\hline cryo-SEM & cryogenic scanning electron microscopy \\
\hline d & bilayer repeat distance \\
\hline $\mathbf{d}_{\alpha}$ & lamellar phase bilayer repeat distance \\
\hline $\mathbf{d}_{3}$ & sponge phase characteristic distance \\
\hline DDCOSY & diffusion-diffusion correlation spectroscopy \\
\hline DEXSY & diffusion exchange spectroscopy \\
\hline DSC & differential scanning calorimetry \\
\hline FCD-I & Type I focal conic domain \\
\hline FCD-II & Type II focal conic domain \\
\hline $\mathbf{H}$ & mean curvature \\
\hline HCR & high concentration region \\
\hline $\mathbf{H}_{\text {II }}$ & inverse hexagonal phase \\
\hline hkl & Miller indices \\
\hline HMBC & heteronuclear multiple-bond spectroscopy \\
\hline HSQC & heteronuclear single-quantum correlation spectroscopy \\
\hline ICR & intermediate concentration region \\
\hline IR & infrared \\
\hline$\kappa$ & bending modulus \\
\hline $\bar{\kappa}$ & Gaussian curvature modulus \\
\hline $\mathrm{K}$ & Gaussian curvature \\
\hline K-AOT & potassium bis(2-ethylhexyl)sulfosuccinate \\
\hline$k_{\mathrm{B}} T$ & thermal energy \\
\hline K-butylAMA & potassium bis(1,3-dimethylbutyl) sulfosuccinate \\
\hline $\mathrm{L}_{\alpha}$ & lamellar phase \\
\hline $\mathrm{L}_{1}$ & micellar phase \\
\hline $\mathrm{L}_{3}$ & sponge phase \\
\hline
\end{tabular}




$\begin{array}{ll}\text { LCR } & \text { low concentration region } \\ \text { Li-AOT } & \text { lithium bis(2-ethylhexyl)sulfosuccinate } \\ \text { MLV } & \text { multilamellar vesicle } \\ \text { Na-AOT } & \text { sodium bis(2-ethylhexyl)sulfosuccinate } \\ \text { Na-butyIAMA } & \text { sodium bis(1,3-dimethylbutyl) sulfosuccinate } \\ \text { Na-hexyIAMA } & \text { sodium bis( } n \text {-hexyl) sulfosuccinate } \\ \text { Na-pentyIAMA } & \text { sodium bis(1-methylpentyl) sulfosuccinate } \\ \text { NH }_{\mathbf{4}} \text {-AOT } & \text { ammonium bis(2-ethylhexyl)sulfosuccinate } \\ \text { NMR } & \text { nuclear magnetic resonance } \\ \text { PFG } & \text { pulsed field gradient } \\ \text { POM } & \text { polarising optical microscopy } \\ \text { q } & \text { reciprocal spacing of an X-ray reflection } \\ \text { q } & \text { reciprocal spacing of the first-order Bragg peak } \\ \text { qmax } & \text { reciprocal spacing of the highest intensity X-ray reflection } \\ \text { S } & \text { disclination strength } \\ \text { SANS } & \text { small-angle neutron scattering } \\ \text { SAXS } & \text { small-angle X-ray scattering } \\ \text { SDS } & \text { sodium dodecyl sulfate } \\ \text { SEM } & \text { scanning electron microscopy } \\ \text { TEM } & \text { transmission electron microscopy } \\ \text { V II } & \text { inverse bicontinuous cubic phase } \\ \text { WAXS } & \text { wide-angle X-ray scattering } \\ & \end{array}$




\section{Chapter 1}

\section{Introduction}

Liquid crystalline structures (mesophases) exist between the ordered and disordered states of solids and liquids. They have some degree of orientational order but not the long-range positional ordering of crystalline materials. The term is broad, encompassing a wide range of compounds with different chemical functionalities, and hence a large array of structures and properties. Pure materials that form temperature-dependent mesophases are classified as thermotropic liquid crystals, while lyotropic liquid crystals are multicomponent systems in which mesophase formation requires the additional degrees of freedom provided by a solvent. Nonamphiphilic examples of lyotropic systems include DNA ${ }^{1}$ and $\mathrm{Kevlar}^{\circledR},{ }^{2}$ but the term generally refers to structures that form via the self-assembly of amphiphilic molecules, including diblock copolymers, lipids and surfactants.

This work details the lyotropic mesophases formed by several surfactant systems, and except when stated otherwise, refers to binary systems of surfactant and water. As amphiphilic molecules, surfactants contain both hydrophobic and hydrophilic components. Their selfassembly is driven by the hydrophobic effect, which causes molecules to aggregate in a way that shields the non-polar parts of the molecule from interacting with water molecules. This limits the entropy cost that arises from water forming clathrate-like solvation shells around the hydrophobic region. ${ }^{3}$ Aggregated structures are formed above the critical micelle concentration $(\mathrm{CMC})$ and Krafft temperature (once aggregation has the lower entropy cost). As illustrated in Figure 1.1, molecules of different shapes, or exposed to different forces, will pack in different ways with varying interfacial curvature. As such, self-assembly can produce normal (Type I) or inverse (Type II) structures, with varying degrees of 1D, 2D or 3D order.

The formation of aggregate structures with a diverse range of architectures, varying chemical functionalities, and synergic physical properties (in terms of both order and flow) mean that lyotropic systems are used in a variety of applications and fields. Their use is wide-ranging and growing rapidly, so a comprehensive review is outside the scope of this introduction. However, we direct the interested reader to the pioneering nanotemplating work of Attard, ${ }^{4}$ the hybrid systems produced by Constantin et al., ${ }^{5}$ and the potential role of surfactant/DNA complexes in 

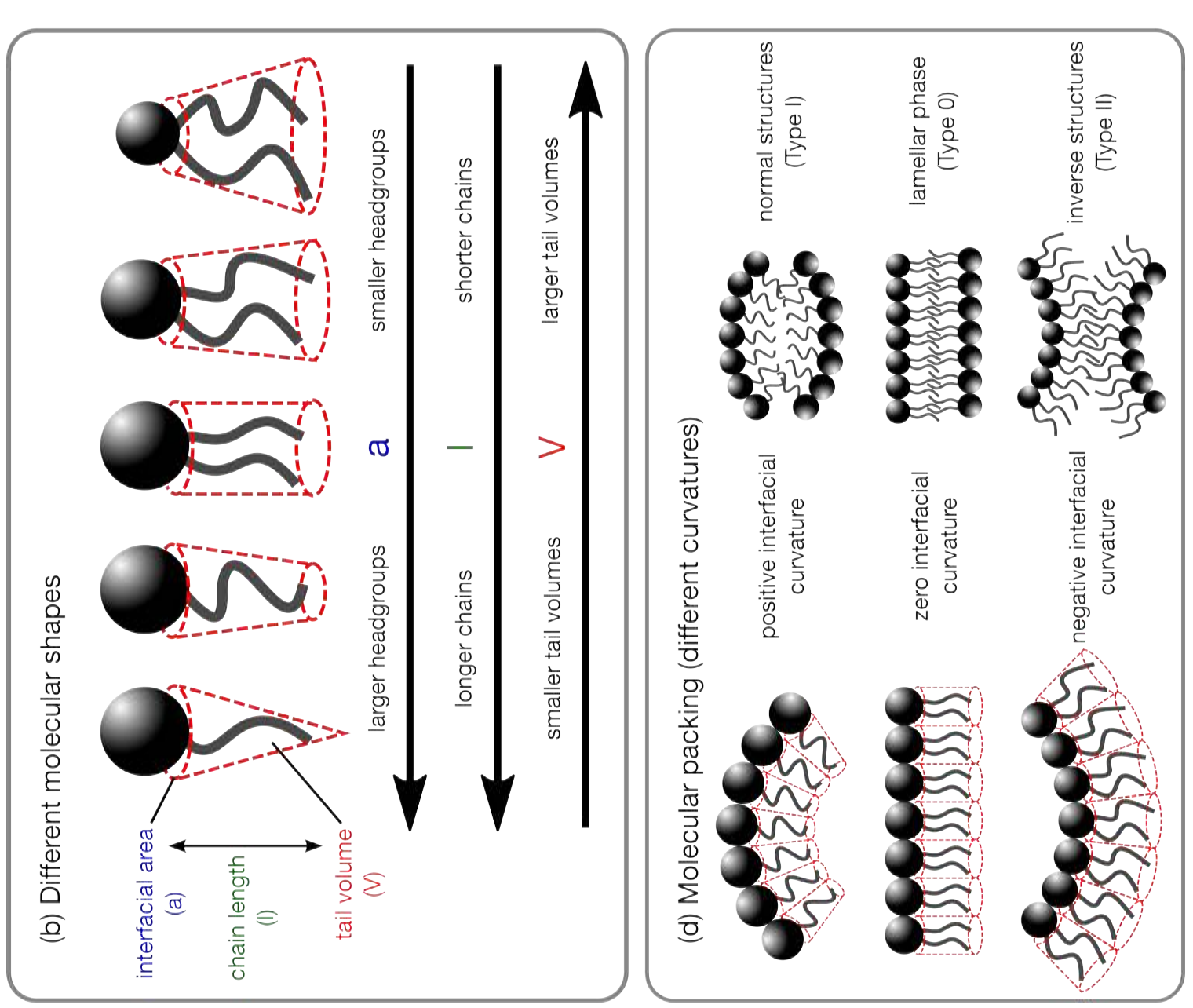

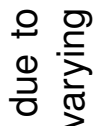

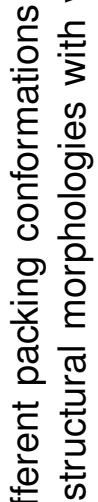
告 声蓄 Ð 을 을 क ํำ 흠 오 $\subseteq$ 올 댕
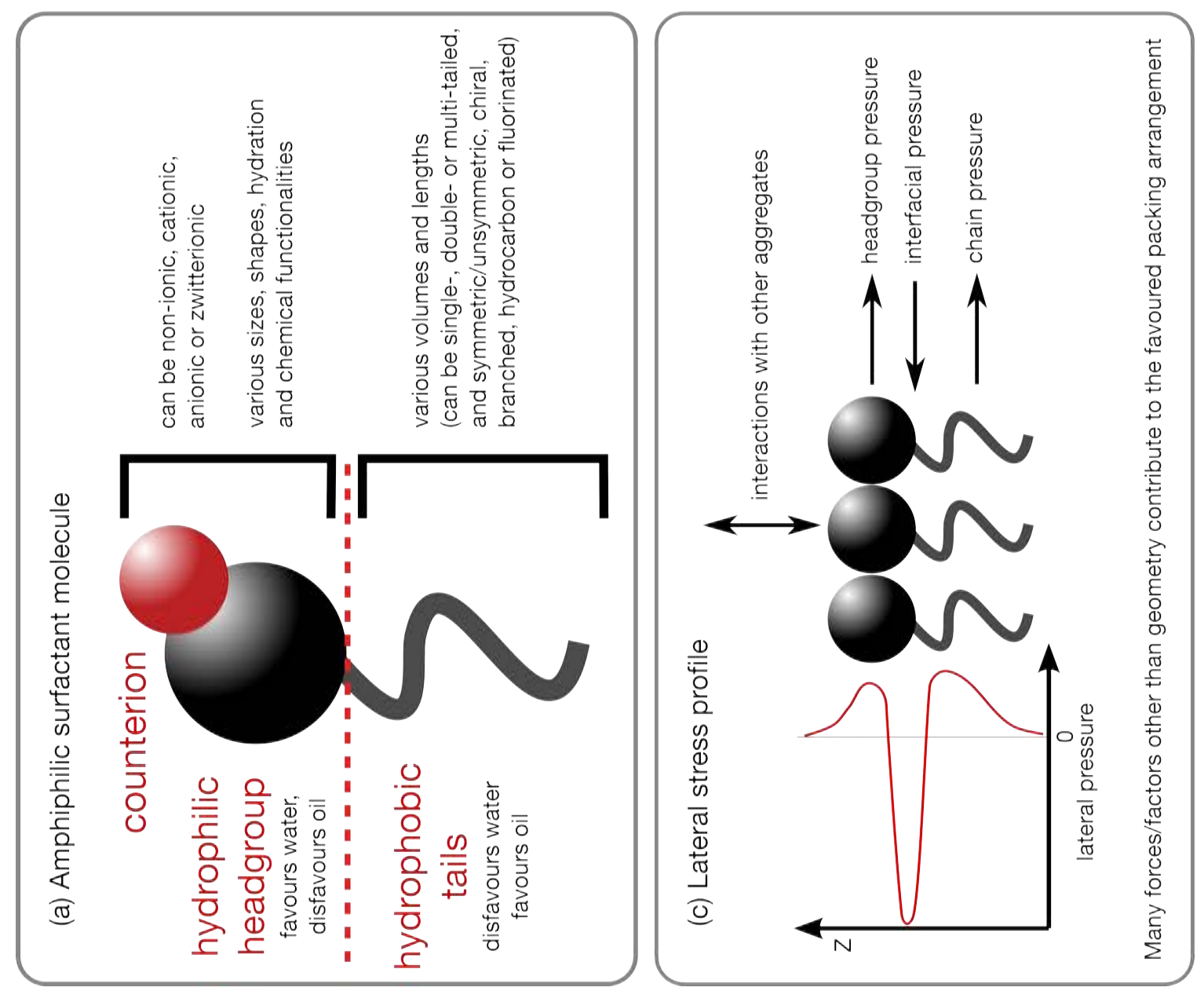

弯 $\frac{\infty}{5}$ సే 온흐 을 웜 을 은 苋 등 음

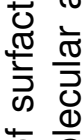
능응 थ 든 쥴 옹 음 일

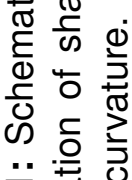
둥 즘 일 융 司它 
gene therapy. ${ }^{6,7}$ The most important role of lyotropic structures is in living systems where they are ubiquitous. Cellular membranes are known to adopt complex geometries during various processes and phospholipids form important structures such as the myelin sheath. Lyotropic phases are already utilised in drug delivery systems, ${ }^{8}$ but a better understanding of how they are regulated and controlled within the chaotic environments of biological systems could lead to rational design, biomimicry, and improved understanding and manipulation of biological processes.

\section{Phase Behaviour and Curvature}

Figure 1.2 illustrates commonly observed lyotropic mesophases, while additional structures and nomenclatures are listed in Table 1.1. Indications of molecular shape and interfacial curvature are also shown in Figure 1.2, which are important factors in surfactant self-assembly and in determination of the free energy structures. It is not uncommon for surfactant self-assembly to be described solely in terms of the dimensionless packing parameter $\mathrm{P}$, an estimation of the molecular shape based on interactions at the hydrophobic/hydrophilic interface $(P=V / a l) .9,10$ However, while this is a convenient "rule of thumb" to predict general behaviour, it is a poor measure of interfacial curvature as it neglects the contribution of many forces that act upon the system. Purely steric models do not account for the bending energy that arises from the fluid nature of mesophases, nor the fact that they are free energy structures and so a function of thermodynamic parameters, which are not constant. The packing parameter on its own is only able to predict the formation of spherical, cylindrical and flat structures and cannot rationalise the more complex three-dimensional bicontinuous structures that are observed in many systems. The shape of the molecule is clearly important in determining the aggregate structure, but geometry alone provides no indication of the rigidity/elasticity of the structures formed. The shape must be considered in relation to the thermodynamics of the system and the various contributions to the free energy. Unfortunately, it is still somewhat common within the literature for a single value of $P$ for a surfactant (based on Tanford's equations ${ }^{11}$ ) to be quoted as if a constant.

A better understanding of aggregation behaviour is gained from considering the elasticity of surfactant monolayers and the energy involved in inducing curvature. To do this we must consider both Gaussian and mean curvature, which are defined in terms of vector quantities that characterise the rate and tangent direction of maximum and minimum bending at a surface, i.e. the principal curvatures $c_{1}$ and $c_{2}$ (see Figure 1.3). Gaussian curvature $(K)$ and mean curvature $(\mathrm{H})$ are intrinsic and extrinsic measurements, respectively, defined as $\mathrm{K}=\mathrm{C}_{1} \mathrm{C}_{2}$ and $H=1 / 2\left(c_{1}+c_{2}\right)$. $H$ is the average interfacial curvature, while $K$ reveals the surface characteristics. Figure 1.3 presents shapes with different signs of Gaussian curvature and shows that $\mathrm{K}$ cannot be altered through bending alone (parabolic surfaces with $\mathrm{K}=0$ form if either $c_{1}$ or $c_{2}$ is zero). When $c_{1}$ and $c_{2}$ have the same sign, $K$ is positive, resulting in elliptic surfaces, while hyperbolic surfaces form when $c_{1}$ and $c_{2}$ are of opposite sign. Of particular importance is the case where $c_{1}$ and $c_{2}$ have opposing signs but equal magnitudes, which 


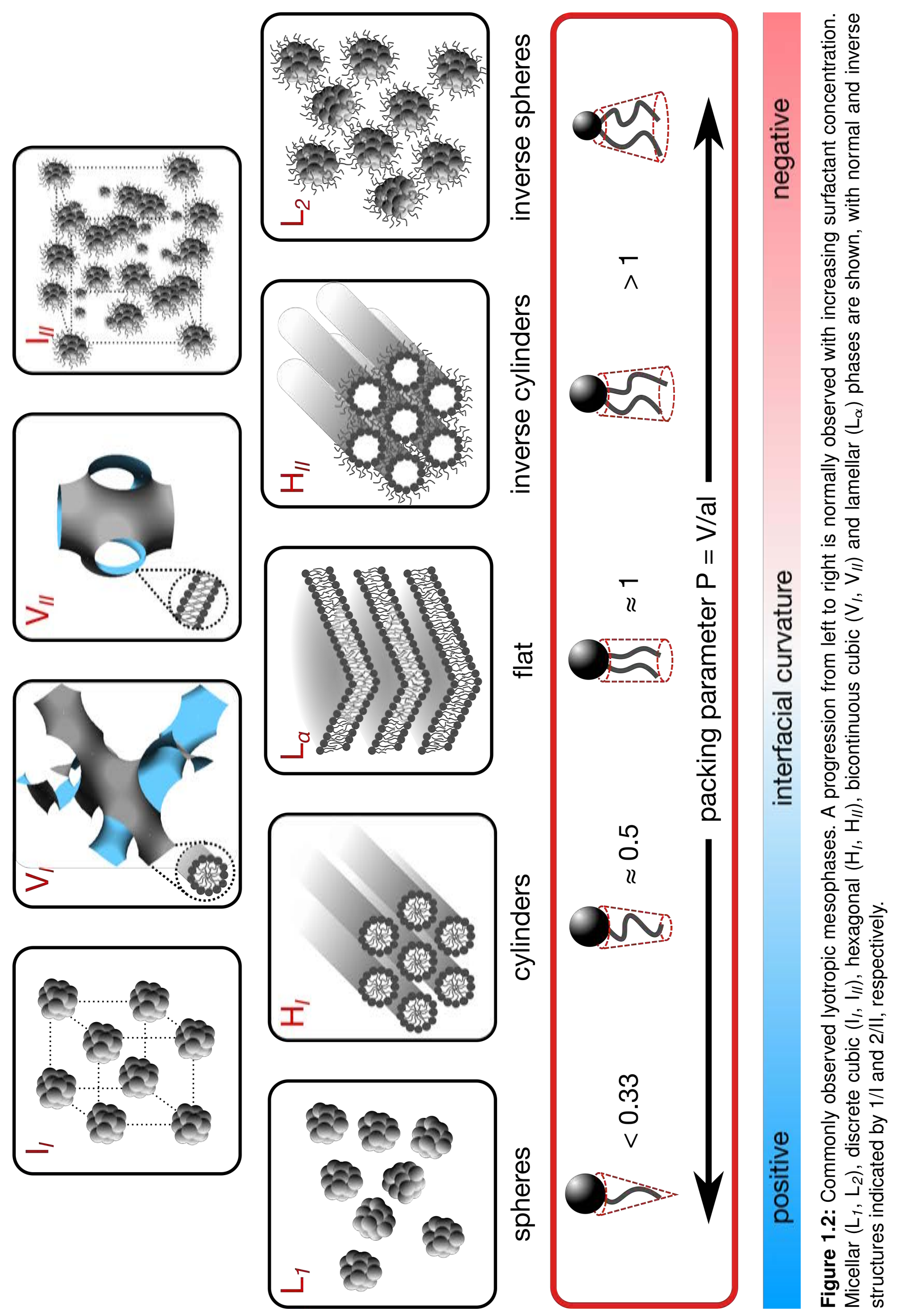


Table 1.1: Types of lyotropic mesophases and associated structures.

\begin{tabular}{|c|c|c|c|}
\hline Type & Symbol & Symmetry & Alternative symbol \\
\hline \multicolumn{4}{|l|}{ Disordered } \\
\hline Micellar & $\mathrm{L}_{1}$ & - & \\
\hline Inverse micellar & $\mathrm{L}_{2}$ & - & \\
\hline Sponge & $\mathrm{L}_{3}$ & - & \\
\hline Vesicular & $\mathrm{L}_{4}$ & - & \\
\hline \multicolumn{4}{|l|}{ Layered } \\
\hline Lamellar (fluid) & $\mathrm{L}_{\alpha}$ & $1 \mathrm{D}$ & \\
\hline Lamellar (gel, untilted) & $\mathrm{L}_{\beta}$ & $1 \mathrm{D}$ & \\
\hline Lamellar (gel, tilted) & $\mathrm{L}_{\beta}^{\prime}$ & $1 \mathrm{D}$ & \\
\hline Lamellar (gel, interdigitated) & $\mathrm{L}_{\beta 1}$ & $1 \mathrm{D}$ & \\
\hline Lamellar (rippled) & $\mathrm{P}_{\beta}$ & $1 \mathrm{D}$ & \\
\hline Lamellar (helically coiled chains) & $\mathrm{L}_{\delta}$ & $1 \mathrm{D}$ & \\
\hline Lamellar (crystalline - 2D) & $\mathrm{L}_{c r}^{2 \mathrm{D}}$ & $2 \mathrm{D}$ & $\mathrm{L}_{\mathrm{c}}^{2 \mathrm{D}}$ \\
\hline Lamellar (crystalline - 3D) & $\mathrm{L}_{c r}$ & $3 D$ & $\mathrm{~L}_{\mathrm{c}}^{3 \mathrm{D}}$ \\
\hline Cubic & & $3 \mathrm{D}$ & $Q, C$ \\
\hline Discrete cubic & $\mathrm{l}_{1}$ & $\mathrm{Pm} 3 \mathrm{n}, \mathrm{Fm} 3 \mathrm{~m}, \mathrm{Im} 3 \mathrm{~m}, \mathrm{P} 4_{3} 32$ & \\
\hline Discrete cubic (inverse) & $\mathrm{I}_{\|}$ & $\mathrm{Fd} 3 \mathrm{~m}$ & \\
\hline Bicontinuous cubic & $\mathrm{V}_{l}$ & Im3m, la3d, Pn3m & \\
\hline Bicontinuous cubic (inverse) & $\mathrm{V}_{\|}$ & Im3m, la3d, Pn3m & \\
\hline Columnar & & $2 \mathrm{D}$ & \\
\hline Hexagonal & $\mathrm{H}_{l}$ & P6m & \\
\hline Hexagonal (inverse) & $\mathrm{H}_{\|}$ & P6m & \\
\hline Hexagonal (complex) & $\mathrm{H}_{l c}$ & P6m & \\
\hline Hexagonal (inverse complex) & $\mathrm{H}_{/ / c}$ & P6m & \\
\hline Ribbon (rectangular) & $\mathrm{Rb}_{\text {। }}$ & Cmm, Pgg, Pmm & \\
\hline Ribbon (inverse rectangular) & $\mathrm{Rb}_{\|}$ & Cmm, Pgg, Pmm & \\
\hline Ribbon (oblique) & $\mathrm{M}_{l}$ & $\mathrm{P} 1, \mathrm{P} 2$ & \\
\hline Ribbon (inverse oblique) & $\mathrm{M}_{\|}$ & $\mathrm{P} 1, \mathrm{P} 2$ & \\
\hline Mesh & & $3 \mathrm{D}$ & $\mathrm{Mh}$ \\
\hline Rhombohedral & $\mathrm{R}_{l}$ & $\mathrm{R} 3 \mathrm{~m}$ & \\
\hline Rhombohedral (inverse) & $\mathrm{R}_{\| \prime}$ & & \\
\hline Tetragonal & $\mathrm{T}_{1}$ & $14 \mathrm{~mm}$ & \\
\hline Tetragonal (inverse) & $\mathrm{T}_{/ /}$ & & \\
\hline Disordered & $\mathrm{Mh}_{0}$ & & \\
\hline Other & & $3 D$ & \\
\hline Hexagonal micellar & - & $\mathrm{P}_{3} / \mathrm{mmc}$ & \\
\hline Polycontinuous & 3etc & $\mathrm{P}_{3} / \mathrm{mcm}$ (and others proposed) & \\
\hline Bicontinuous (non-cubic) & $\mathrm{Bc}_{l}, \mathrm{Bc}_{/ l}$ & R3c (not confirmed) & \\
\hline
\end{tabular}

Note on nomenclature: Some researchers use Arabic numerals rather than Roman numerals to designate normal and inverse structures. Others use a superscript " $\mathrm{i}$ " for inverse structures so they can also use a subscript " $\alpha$ " to denote the fluid nature of the tails. For example, an inverse hexagonal phase may be written as $\mathrm{H}_{\|}, \mathrm{H}_{2}$ or $\mathrm{H}_{\alpha}^{\mathrm{i}}$. 
results in hyperbolic surfaces (such as saddle-splay surfaces) in which the magnitude of $\mathrm{K}$ varies along the surface but $\mathrm{H}=0$. This curvature is observed in the bicontinuous cubic and sponge phases, which are described as minimal surfaces. As the Gaussian curvature is not altered by bending alone, structures with non-zero Gaussian curvatures require stretching or constriction of the bilayer and so have an associated elastic energy cost.
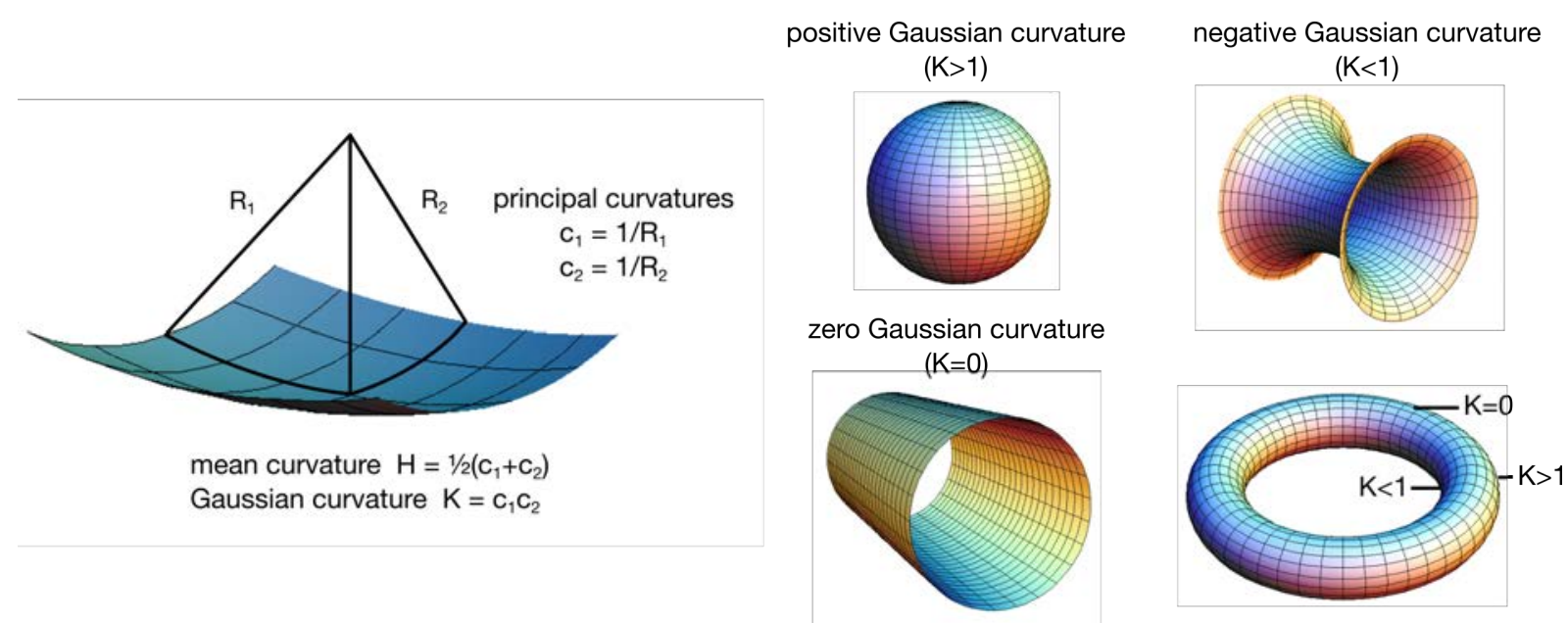

Figure 1.3: The relationship between the principal curvatures of a surface and its mean and Gaussian curvatures. Several examples of different Gaussian curvatures are shown.

The elasticity of a bilayer can be related to the Gaussian and mean curvatures, using the expression of membrane free energy derived by Helfrich, ${ }^{12}$ and the elastic energy per unit area can thus be written as:

$$
\mathrm{F}_{\text {elastic }}=2 \kappa\left(\mathrm{H}-\mathrm{C}_{0}\right)^{2}+\bar{\kappa} \mathrm{K}
$$

where $c_{0}$ is the spontaneous curvature and $\kappa$ and $\bar{\kappa}$ are the elastic moduli. These three components are incredibly important and require further explanation.

The spontaneous curvature $\mathrm{c}_{0}$ is a measure of the inherent tendency of each monolayer comprising a bilayer to curve. Mathematically it is twice the value of $\mathrm{H}$ for one monolayer when $\mathrm{K}=0$, and so essentially represents the equilibrium mean curvature that the monolayers would adopt in the absence of any constraints (i.e. if it was governed by packing/lateral steric forces alone) and so it is $\mathrm{c}_{0}$ that the previously discussed packing parameter actually describes. Monolayers will not favour a flat morphology at all concentrations or temperatures and so adopting a bilayer structure has an associated energy cost, as illustrated in Figure 1.4. This must be met by the adoption of unfavourable bilayer conformations or through deformation. If existing as this deformed structure is less energetically favourable than existing as a different structure, then a phase transition is induced. If retaining a bilayer has the lower cost, then deformation will result in deviation from the idealised structure through the formation of defects. 


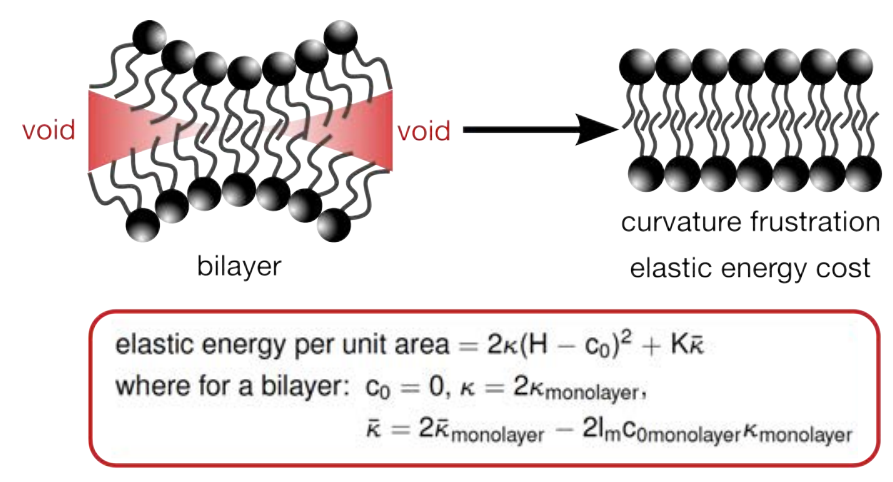

Figure 1.4: An illustration of the curvature frustration associated with monolayers adopting a bilayer configuration.

The first of the elastic moduli, $\kappa$, is the modulus of the mean curvature, and is a measure of the energy cost associated with deforming a bilayer from its ideal spontaneous mean curvature through bending, and thus is termed the bending rigidity modulus (note that $\kappa=2 \kappa$ monolayer). This value is always positive in stable mesophases, but its relative magnitude in comparison to the thermal energy $k_{\mathrm{B}} T$ is important in determining whether thermal energy can destabilise the structure. $\bar{k}$ is the Gaussian curvature modulus (sometimes called the saddle-splay modulus) and represents the energy required to form surfaces of non-zero Gaussian curvature. The value of $\bar{\kappa}$ can be negative or positive, resulting in positive or negative Gaussian curvature, respectively, thus affecting the relative stability of normal and inverse phases. In contrast to bending deformations, the energy associated with changing the Gaussian curvature of a bilayer is not twice that of deforming the monolayer. As such, $\bar{\kappa}=2 \bar{\kappa}_{\text {monolayer }}-2 \mathrm{l}_{\mathrm{m}} \mathrm{C}_{\text {omonolayer }} \kappa_{\text {monolayer }}$, and so $\bar{\kappa}$ is closely related to the spontaneous curvature and monolayer thickness $\left(\mathrm{I}_{\mathrm{m}}\right)$, as well as the value of $\kappa .^{13}$

A significant step in understanding lyotropic mesophases was provided by Hyde, who related the mean and Gaussian curvatures of the bilayer to Israelachvili's packing parameter through the equation $\mathrm{P}=\mathrm{V} / \mathrm{al}=1+\mathrm{HI}_{\mathrm{m}}+\left(\mathrm{KI}_{\mathrm{m}}{ }^{2}\right) / 3 .{ }^{14,15}$ This enabled the hydrophobic/hydrophilic interface to be modelled as a thin film, the total curvature of which is governed by both the local geometric packing constraints of the surfactant molecules and the macroscopic constraints of spontaneous and non-spontaneous curvatures. A second assumption that the lowest energy structures will have homogeneous interfaces then allowed the topology of the surface to be related to the Gauss-Bonnet theorem so that:

$$
\oint \mathrm{KdA}=4 \pi(1-\mathrm{g})
$$

where the genus $(\mathrm{g})$ is the topology of a closed interface (i.e. the number of handles/holes through the structure: see Figure 1.5). As $\bar{\kappa}$, which had previously (incorrectly) been assumed to be negligible, is a measure of the cost of changing $\mathrm{K}$ it follows that it is this modulus that controls the topology of the surface. This allows the rationalisation of bicontinuous surfaces of different genus and thus periodic pores. Hyde compiled a "phase diagram" relating theorised structural types to the shape and polar volume of surfactants (shown in Figure 1.6). Importantly, 


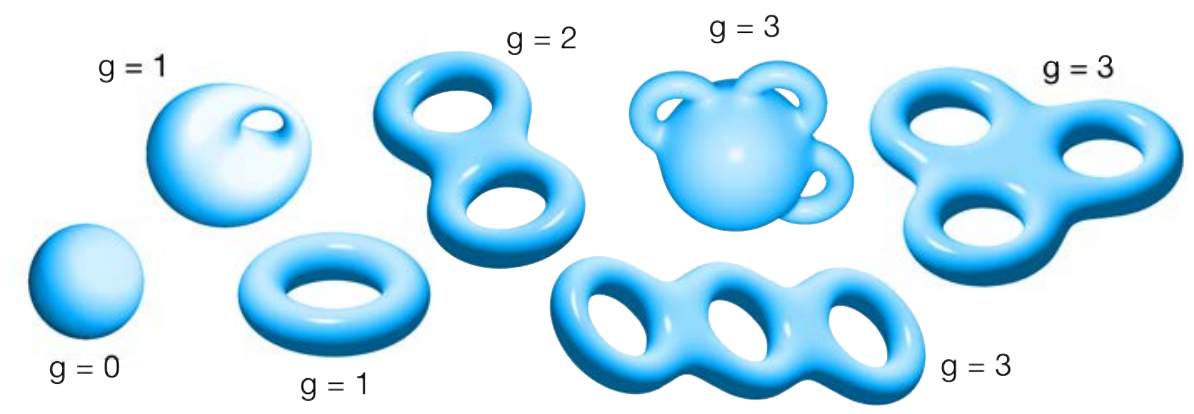

Figure 1.5: Shapes with closed interfaces of varying genus (different numbers of handles/holes).

this methodology shows that for any given shape parameter, a number of different mesophase types are available.

The work of Hyde was important in finally rationalising the formation of $V_{\| l}$ phases and in advancing the field of other bicontinuous phases with minimal surfaces or near-zero curvature. Many intermediate mesh structures were subsequently theorised by those with a mathematical interest in minimal surfaces, and some have since been definitively characterised through experiment and found to be of biological relevance. However, their characterisation is problematic, as it relies on the assignment of $\mathrm{X}$-ray reflections based on assigned lattice parameters taken from those reflections. These can be few in number, weak and/or absent. However, interesting

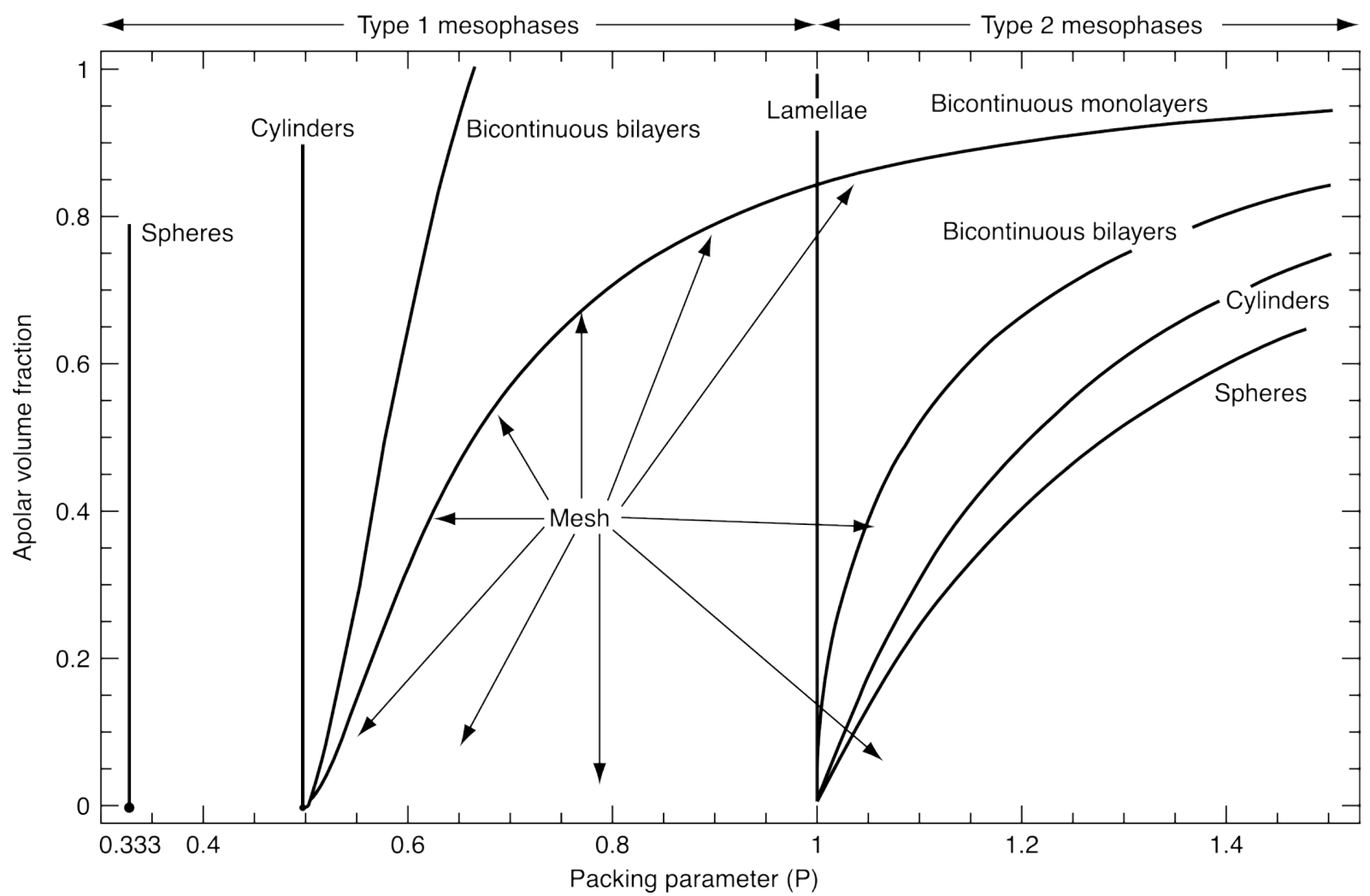

Figure 1.6: Theorised variation in self-assembly as a function of packing parameter and polar/apolar volume fraction, duplicated from the work of Hyde. ${ }^{14}$ (c) 2001 John Wiley \& Sons, Ltd. 
architectures have been observed including the new examples of polycontinuous phases, in which three or more distinct domains are separated by a single continuous surfactant layer. ${ }^{16,17}$

Like the packing parameter, Hyde's work did not account for the role of competing intermolecular interactions between surfactant aggregates, and was unable to predict specific phase formation in advance for any given surfactant. Despite significant expansion of the field since this work, there remains no suitable model (or even universally accepted and unambiguous design rules) that can predict the phase behaviour of a surfactant with any confidence based upon its molecular structure. In fact, the answer to this problem is probably becoming harder to conceive. Novel phases, and new variants of those that already exist, are still being characterised. Thus, an accurate model must be able to predict the formation of increasingly complex topologies. Additionally, there is a rapidly growing diversity in surfactant archetypes that are being produced and investigated specifically because they exhibit different surface tension and self-assembly properties. The current interest in hyperbranched, bola, gemini and star-like surfactants introduces further complexities, ${ }^{18-21}$ which are certainly poorly rationalised by the previous methods that neglect the significant role of chain frustrations and packing. The incorporation of different chemical functionalities then stretches the definition of a surfactant and introduces supramolecular self-assembly. ${ }^{22}$

\section{Relevant Mesophase Structures}

The idealised lamellar $\left(L_{\alpha}\right)$ phase, depicted in Figure 1.7, is the simplest structure to describe. Bilayers with zero interfacial curvature form indefinitely flat layers, which alternate with water layers, producing a regular repeat distance and 1D order. This results in anisotropic properties, good thermodynamic stability, and relatively low viscosity as unrestricted bilayers can move independently along two axes under shear. Ideally, zero curvature is retained upon dilution and the periodicity increases to accommodate thicker water layers. One-dimensional ordering pro-

(a) lamellar $\left(\mathrm{L}_{a}\right)$

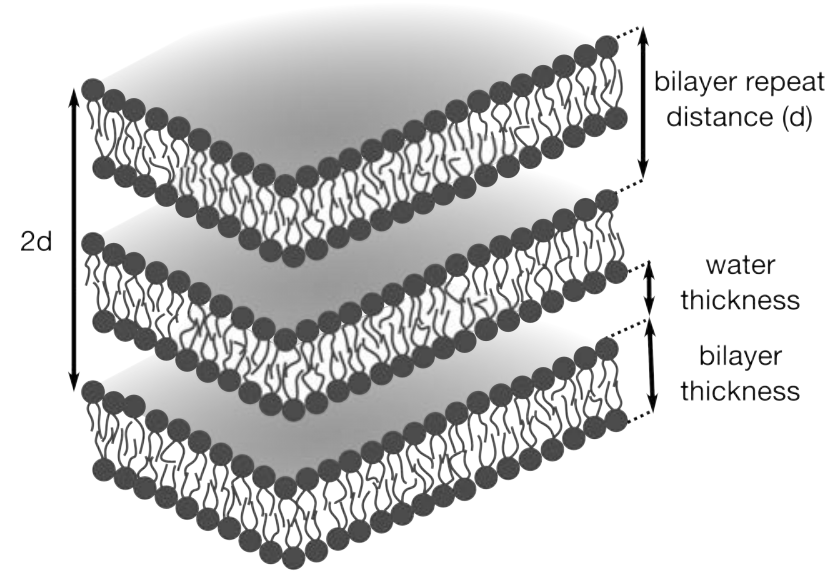

(b) inverse hexagonal $\left(\mathrm{H}_{\|}\right)$

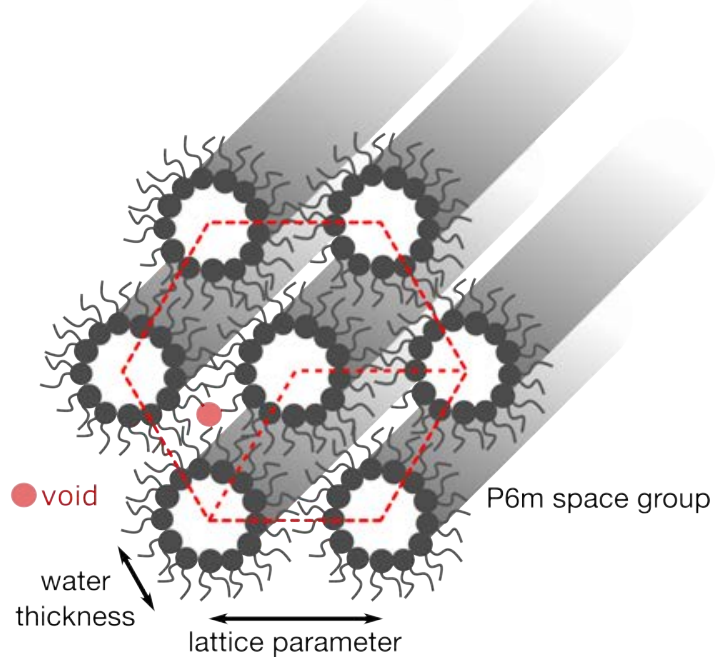

Figure 1.7: Schematic representations of (a) lamellar and (b) inverse hexagonal phases. 
duces simple $X$-ray diffraction patterns in the ratio $1: 2: 3 \ldots$ allowing for simple identification. The subscript " $\alpha$ " indicates a fluid hydrocarbon chain environment, although various ordered/frozen arrangements can exist at lower temperatures, producing gel phases with tilted, coiled or interlocked tail regions (see Table 1.1). However, as described above, bilayer formation incurs a curvature frustration energy cost, and no system will have truly zero interfacial curvature over large concentration or temperature ranges. The energy cost is met through elastic deformations (bending, twisting, splay), which produce lamellar structures of varying topology and defective microstructures, dependent upon the values of $\kappa$ and $\bar{\kappa}$.

The inverse hexagonal $\left(\mathrm{H}_{\| l}\right)$ phase has negative interfacial curvature and forms at high surfactant concentrations. The structure comprises inverse micellar aggregates/columns of indefinite length, which pack together in a $2 \mathrm{D}$ hexagonal array with a $\mathrm{P} 6 \mathrm{~m}$ space group, giving rise to $X$-ray reflections in the ratio of $1: \sqrt{ } 3: \sqrt{ } 4: \sqrt{ } 7 \ldots$ Other columnar phases (listed in Table 1.1) are variants of the $\mathrm{H}_{/ /}$phase, in which the symmetry of the columnar shape and/or packing array is reduced (except the $\mathrm{H}_{/ l c}$ phase, which retains both but is formed of cylinders resembling elongated vesicles rather than inverse micelles). The $\mathrm{H}_{/ /}$phase is limited to high surfactant concentrations as swelling is inhibited by a chain-stretching energy cost, which arises due to voids within the hydrocarbon region (see Figure 1.7) that are filled by tails adopting unfavourable stretched conformations. This energy cost increases with the internal diameter of the cylinders and as the interfacial curvature decreases. $\mathrm{L}_{\alpha}-\mathrm{H}_{/ /}$transitions occur when the tail length of the surfactant is long enough to reduce the size of the void. When this is not the case, the two phases are separated by additional phases, which normally adopt bicontinuous cubic $\left(\mathrm{V}_{\text {II }}\right)$ structures.

Bicontinuous cubic structures comprise single, highly curved indefinite bilayers interwoven with water, producing continuous three-dimensional crystallographic networks. They are formed by an ordered arrangement of hyperbolic/saddle surfaces, meaning that $V_{\text {/I }}$ structures have zero mean curvature and varying (between zero and negative) Gaussian curvature. They are best described as triply periodic minimum surfaces and can be visualised as a bilayer folded around the mathematical minimum structures shown in Figure 1.8, which shows the three $V_{\text {II }}$ space groups observed experimentally in lyotropic systems. The term bicontinuous describes the result of the minimal surface rather than its physical structure and the phases are actually composed of three interpenetrating domains, with the continuous surfactant structure dividing the water into two separate, uninterrupted congruent domains. The structures shown in Figure 1.8 are closely related in terms of energy, but the mechanism of $\mathrm{V}_{I I}-\mathrm{V}_{\text {II }}$ transitions is unknown, despite the structures being interrelated mathematically by the (physically unlikely) Bonnet transformation, which maintains zero mean curvature. ${ }^{23}$

The 3D cubic ordering results in highly viscous materials with equivalent refractive indices that do not give rise to characteristic optical textures under crossed polarised light (in contrast to the anisotropic $\mathrm{L}_{\alpha}$ and $\mathrm{H}_{/ /}$phases). This isotropy means that optical microscopy cannot distinguish between different cubic space groups (but it can rule out other intermediate phases - generally low symmetry rhombic or tetragonal variants). X-ray diffraction is used instead, with cubic space groups having a large number of allowed reflections. However, there are at least three possible 
(a)
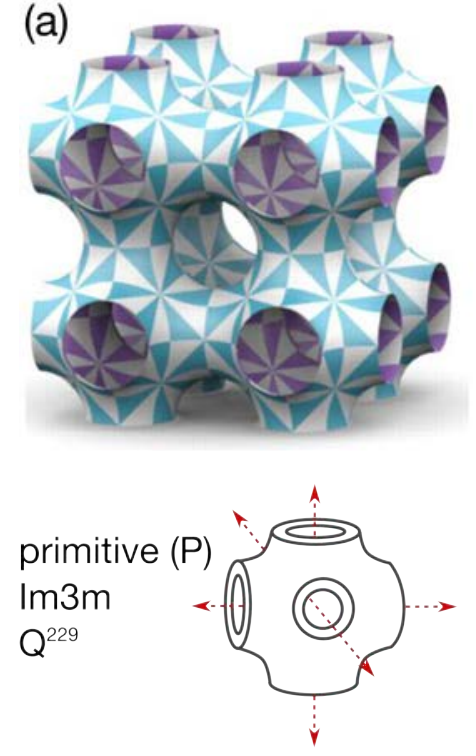

(b)
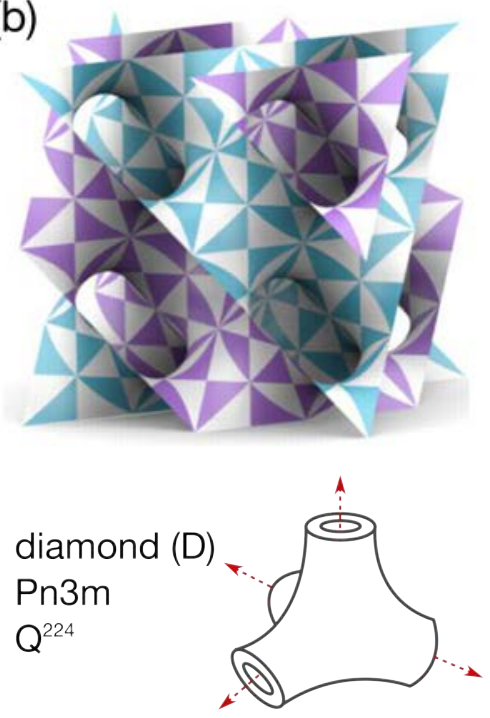

(c)
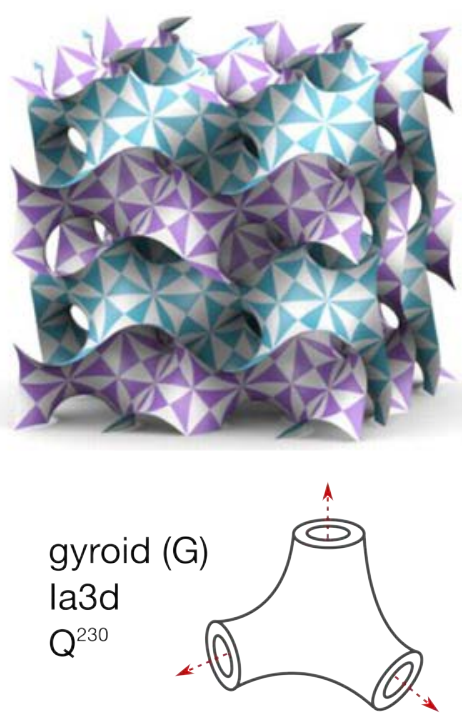

Triply periodic minimal surfaces, $\mathrm{H}=0, \mathrm{~K}<0$

Figure 1.8: Mathematical models of the space groups of bicontinuous cubic phases. Red arrows indicate water channels in the structures. Models are duplicated from the work of Castle et al., ${ }^{24}$ by permission of the Royal Society.

structures and they share many of these reflections. The phases must be assigned based on systematic absences, and if the number of peaks observed (or their intensity) is low then assignments are often tentative (and have been proved incorrect in the past).

Several low-symmetry (2D ordered) variants of the $V_{/ /}$phase exist, perhaps the most interesting of which is the sponge $\left(L_{3}\right)$ phase. The structure of the $L_{3}$ phase (see Figure 1.9) can be described as a translationally disordered (or melted) $V_{/ I}$ phase. It is bicontinuous and formed of saddle-splay/hyperbolic interfaces (strictly $\mathrm{K}<0$ ), but they are randomly arranged and no longer conform to a lattice. The phase can exist between $L_{\alpha}$ and $V_{\| l}$ phases or upon promotion of disorder in a $L_{\alpha}$ phase, and can coexist with both $L_{\alpha}$ and $V_{/ /}$phases. Thus, the $L_{3}$ structure can also be related to the $L_{\alpha}$ phase, as both phases are formed from the same local bilayer structure. The $L_{3}$ phase can be considered as a $L_{\alpha}$ phase that loses translational order through the combination of thermal fluctuations and a high genus defect formation producing

(a) saddle-splay surface

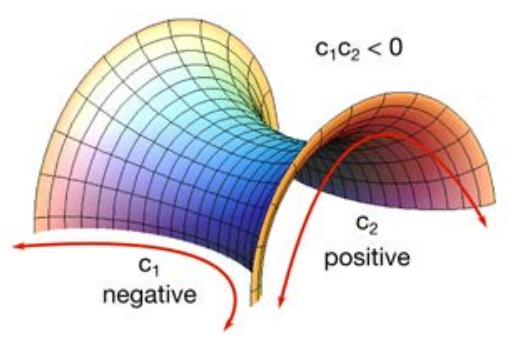

(b) sponge phase $\left(L_{3}\right)$

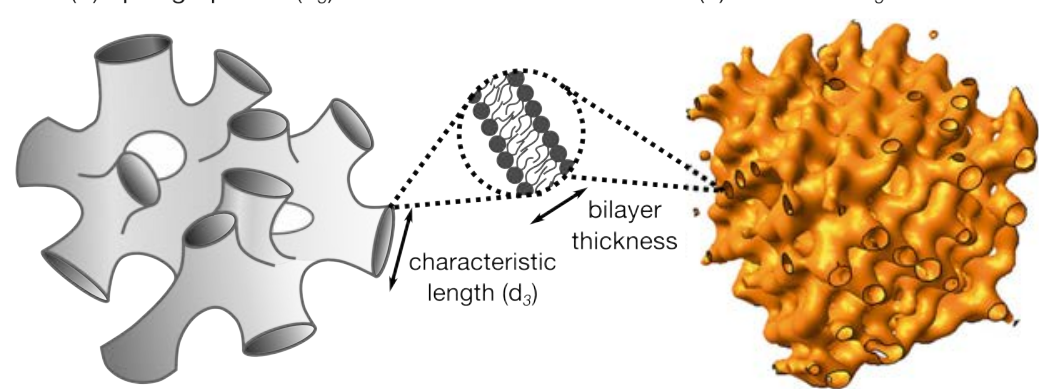

Figure 1.9: (a) A saddle-splay minimum surface, (b) a schematic representation of an $L_{3}$ phase, and (c) a mathematical $\mathrm{L}_{3}$ phase model. Image (c) is duplicated from the work of Angelova et al. ("Sponge phase organization with a nonperiodic lipid bilayer"). ${ }^{25}$ With permission of Springer, (c) Springer-Verlag Berlin Heidelberg 2011. 
connections between bilayers. The characteristic length/pore size $\left(d_{3}\right)$ of a $L_{3}$ phase is generally $\sim 1.2-1.5$ times the size of the corresponding $\mathrm{L}_{\alpha}$ repeat distance $\left(\mathrm{d}_{\alpha}\right)$ and $\sim 2-3$ times the size of corresponding passages of $V_{/ /}$phases, although exceptions to both have been reported and $L_{3}$ and $\mathrm{L}_{\alpha}$ structures with comparable $d$ spacings have been observed in some ionic systems. ${ }^{25-30}$

The disordered $L_{3}$ phases are isotropic and do not give rise to regular diffraction peaks. They are normally assigned based on their remarkably low viscosities (given their relatively high surfactant concentration) and by electron microscopy. They have poor spatial correlations but an average characteristic length and are likely stabilised by some localised short-range entropic interactions. They normally exhibit Newtonian flow when exposed to low shear and flow birefringence at high shear. As continuous domains allow for relatively unrestricted ion movement, they normally have high conductivity values. Despite the "random" arrangement of the $L_{3}$ phase, different variations appear to exist. Both symmetric and asymmetric forms (with respect to the bilayer) have been reported, with the two water domains separated in the former and connected by additional defective pores in the latter. ${ }^{31}$ Beck et al. have also reported "novel" thermodynamically stable $L_{3}$ phases in ionic systems, with $d_{3}$ spacings and properties such as low flow birefringence that do not strictly conform to the accepted $L_{3}$ character. ${ }^{29,30}$

Sponge phases provide an excellent example of the reasons purely geometric explanations of surfactant systems (that persist in some fields) are inadequate. To rationalise the relative stabilities of $L_{\alpha}, L_{3}$ and $V_{/ /}$phases, the elasticity of the bilayer must be considered. Whether originating from a classical $L_{\alpha}$ or $V_{/ /}$phase, the formation of the $L_{3}$ phase requires increased flexibility (decreased bending rigidity $\kappa$ ), which is provided through thermal fluctuations such that the value of $\kappa$ is comparable to the thermal energy $\left(\kappa \approx k_{\mathrm{B}} T\right)$. As such, the formation of the $L_{3}$ phase can be described as an entropy-driven order-disorder transition. However, the $\mathrm{L}_{\alpha}-\mathrm{L}_{3}$ transition also requires increased topological complexity, which is driven by Gaussian curvature (more positive $\bar{\kappa}$ ). The relative stability of these phases is dependent upon both $\kappa$ and $\bar{\kappa}$, as illustrated in Figure 1.10. If we consider the previously discussed equations $\kappa=2 \kappa_{\text {monolayer }}$ and $\bar{\kappa}=2 \bar{\kappa}_{\text {monolayer }}-2 \mathrm{l}_{\mathrm{m}} \mathrm{C}_{\text {omonolayer }} \kappa_{\text {monolayer }}$, then for fluid bilayers with low values of $\bar{\kappa}_{\text {monolayer }}$, the magnitude of $\bar{k}$ is controlled by the second term and its sign is governed by that of the spontaneous curvature. Sponge phases are observed for positive values of $\bar{\kappa}$ (negative Gaussian curvature) and so the $L_{\alpha}-L_{3}$ transition requires the molecular shape to invert (larger tail volume than headgroup area). $\mathrm{L}_{\alpha}-\mathrm{L}_{3}$ transitions can be induced using salt, temperature, alcohols (cosurfactants) or shear. Each of these factors alters the elasticity of the bilayer by changing the intermolecular forces that act upon it. So, while mesophase stability can be explained in terms of curvature, these are free energy systems and we must consider the intermolecular contributions that control them.

\section{Intermolecular Forces}

Self-assembly can be described very simply as purely being the result of competing attractive and repulsive forces. Complexities within the assembled structure are a result, on some 


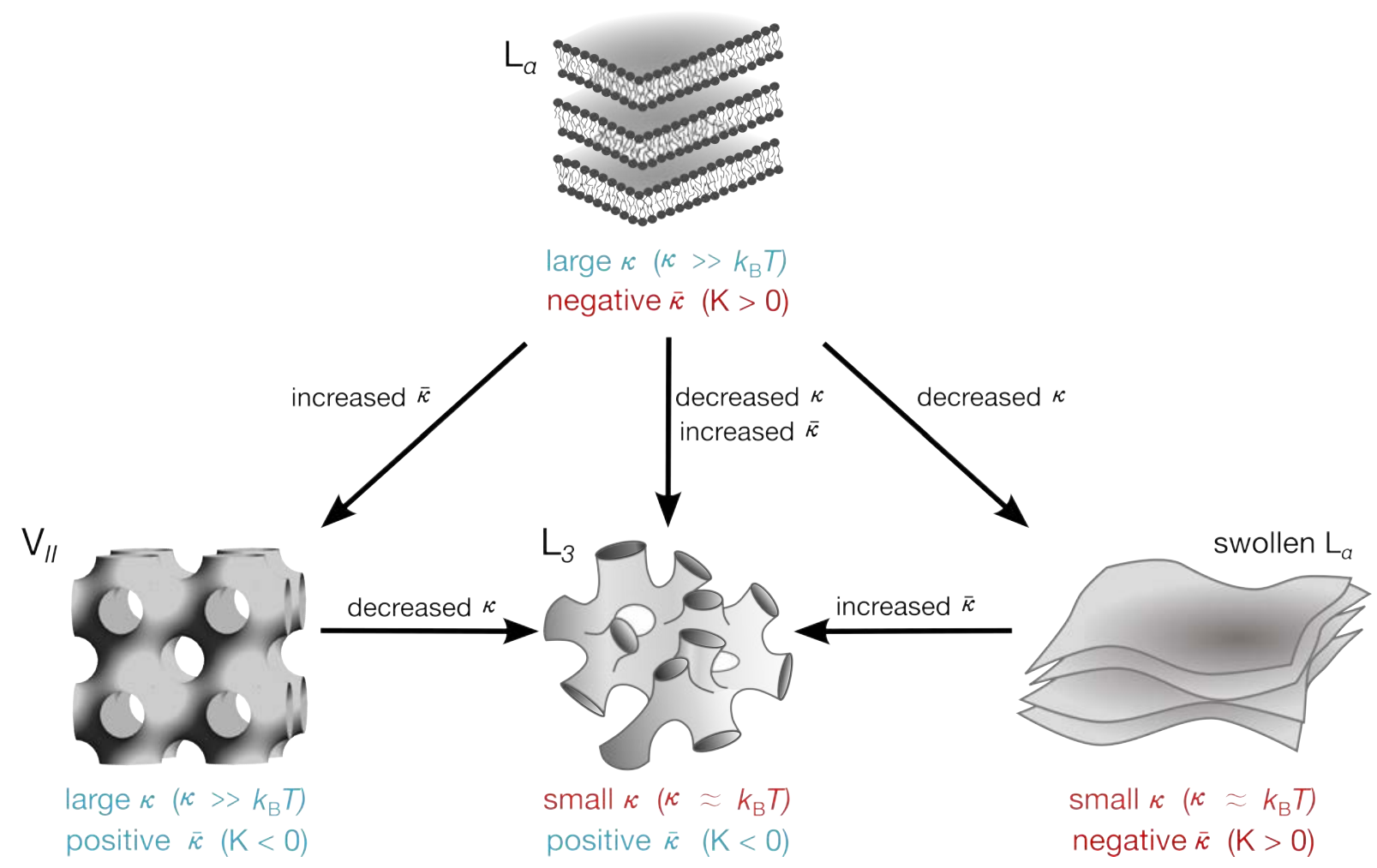

Figure 1.10: The relationship between lamellar, sponge and bicontinuous cubic phases, and their dependency on the elastic constants $\kappa$ and $\bar{\kappa}$.

level, of these interactions. However, the reality of surfactant self-assembly is complicated because these forces are interrelated in non-trivial ways and change not only as a function of environmental conditions, but also indirectly as a result of changes occurring in the opposing forces. Furthermore, not all of these forces contribute on all lengthscales or in all systems. As with any self-assembled system, the assembly of the building blocks is governed by the forces that act upon them, but the nature of those forces depends upon the structure building blocks. The variety in molecular structure and chemical functionality of surfactants results in a range of different behaviours. Despite this, the overriding factor in all lyotropic systems is the hydrophobic effect.

The hydrophobic effect promotes the aggregation of molecules in a way that separates their polar and apolar components from the solvent. It is rational that explanations of surfactant selfassembly focus on the hydrophobic/hydrophilic interface, and hence the lateral forces along a bilayer that affect the molecular packing. The lateral forces are not limited to the hydrophobic forces and tail-tail/headgroup-headgroup steric interactions. Electrostatic repulsion and van der Waals attractive forces compete within the headgroup of ionic surfactants, and it is changes in these forces (in particular in the electrostatic component) that can significantly alter the elastic properties of the bilayer, and thus its curvature.

The forces in surfactant systems are not limited to operating laterally. They also operate between aggregates and are important in rationalising the relative stabilities of different phases (shown in Figure 1.10 in regards to the $L_{\alpha}, L_{3}$ and $V_{/ /}$phases). The magnitudes of these interlayer forces also contribute to, or destabilise, the bilayer elasticity (in particular the magnitude 
of $\kappa$ ). The main two forces are again electrostatic and van der Waals forces, and as before the result of their competition will vary greatly with concentration and temperature. This is because their magnitudes scale differently as a function of interaggregate spacing, and their shielding is affected by the hydration of the surfactant and the degree of binding of the counterion (particularly the Coulombic forces between adjacent headgroup ions).

Additional forces acting between bilayers are steric hydration forces and Helfrich undulation forces. The first of these is a strong short-range $(\sim 20-30 \AA)$ repulsive force that prevents the close approach of aggregates. The exact nature of these forces is still debated, but they appear to be at least partially entropic in nature and may be a combined result of headgroups protruding from the bilayer and the need to dehydrate or overlap hydration shells to allow bilayers to approach. Helfrich undulation forces are long-range entropic forces that are dependent upon the relative value of the thermal energy $k_{\mathrm{B}} T$ compared to the bilayer rigidity. They were first theorised by Helfrich, and arise from thermally induced out-of-plane fluctuations. ${ }^{32}$ They occur in systems with flexible bilayers (with low values of $\kappa$ ) and the repulsive force arises from the entropic confinement resulting from the undulating bilayers being restricted by those on either side. Helfrich undulation forces are common in ionic systems and produce a steric repulsion interaction that competes with the van der Waals attractive forces and results in the stabilisation of swollen lamellar phases at large bilayer repeat distances. ${ }^{33}$

\section{Defect Structures}

So far, we have shown that lyotropic mesophases must satisfy the free energy requirements of the system and that the structures formed can be rationalised in terms of curvature and elasticity. When the elastic energy cost of a structure becomes untenable, a phase transition occurs. However, that is not to say that when a particular phase is formed it adopts the idealised thermodynamic structure or incurs trivial energy costs. The wide range of competing intermolecular forces acting upon the system means that at no point will all parts of each surfactant molecule be truly chemically and physically satisfied, especially over the large concentration ranges at which some phases exist. As such, the elastic energy cost and curvature demands of the system must be satisfied. This is achieved by deformation of the structure through a combination of bending, splaying and twisting (i.e. through the formation of defect structures).

It is important to remember that mesophases are ordered fluids, hence the molecules can move and the structure is in a constant state of rearrangement. The formation of defects in such systems should be expected, but despite their name we stress that they should not be considered as faults. As well as dissipating energy to stabilise the mesophase structures, defects are fundamental to their physical properties and play key roles in facilitating phase transitions. Such structures have important roles within biological systems and if we consider the properties of membrane-based structures, topological "defects" are essential in controlling/facilitating their permeability and fusion, and the incorporation of proteins. As such, defects 
are interesting phenomena and investigating the evolution of mesophase microstructure as a function of concentration and temperature is important in understanding surfactant selfassembly and determining possible transition mechanisms.

Many defects can be classified as line defects, which disrupt either the rotational symmetry of the structure (disclinations), the translational symmetry (dislocations) or (most commonly) a combination of the two. In some cases, these are comparable to defects formed in solids by the Volterra process, where a medium is cut along one surface and the newly formed faces are displaced or removed. ${ }^{34}$ Schematic representations showing the topology of a system around several singularity examples are shown in Figure 1.11 along with the values of the parameter $\mathrm{s}$, which is the strength of a disclination where the angle at which the director turns on a closed curve around the centre is $2 \pi \mathrm{s}$. Defects of different strengths can be determined using polarising microscopy as they interact with light differently producing characteristic patterns of extinction.

disclination $s=-1 / 2$
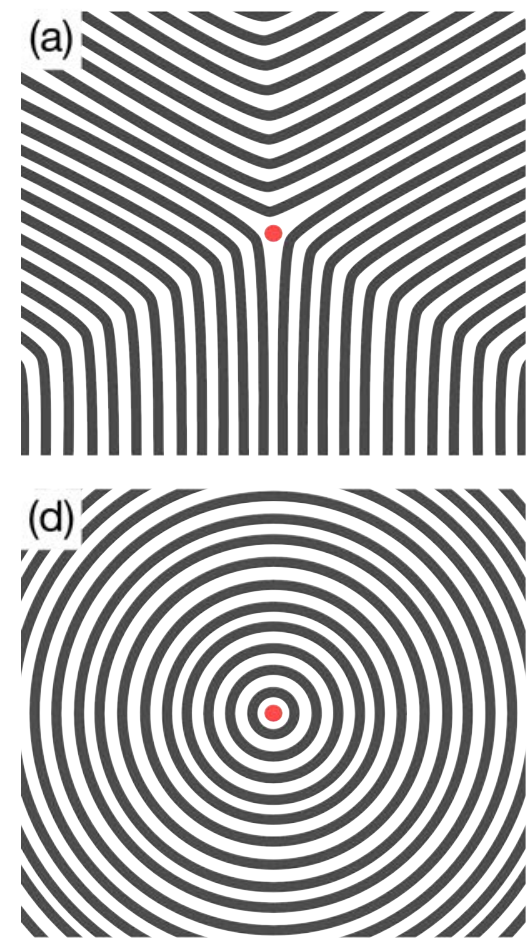

disclination $\mathrm{s}=+1$ disclination $s=+1 / 2$
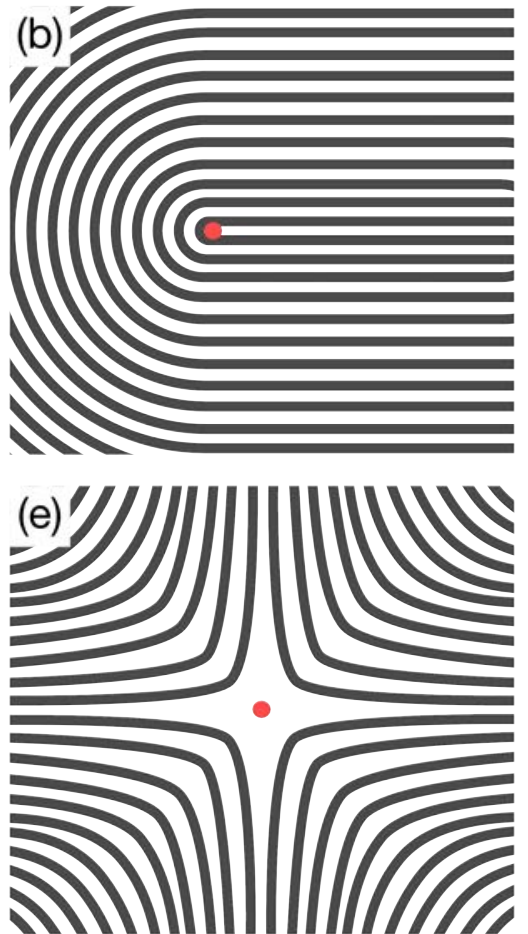

disclination $s=-1$ edge dislocation
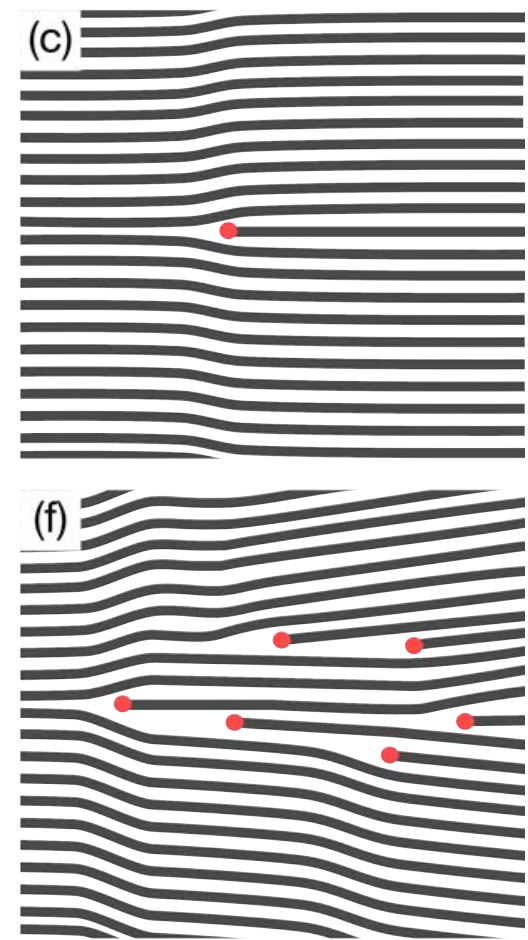

series of dislocations

Figure 1.11: Selected examples showing the topology around line defect singularities. Note that (f) shows an imperfect series of edge dislocations that may reorientate the surrounding regions and act as a domain boundary.

Figure 1.12 shows various types of defects that are observed in $L_{\alpha}$ phases on different lengthscales. On a global scale, and unlike the continuous $V_{\| /}$and $L_{3}$ phases, $L_{\alpha}$ bilayers deviate from the idealised description of indefinite bilayers by forming domains with different director alignments. These defects are separated by boundaries that can take various forms, but are comprised of groups of further (smaller lengthscale) defects. Domain boundaries can be sharp (e.g. a series of edge dislocations, sometimes considered a single wedge disclination: see Figure 1.11) or appear highly disordered (e.g. separated by a space iteratively filled by focal 
conic domains of varying size). ${ }^{35,36}$ In focal conic domains (FCDs), layers are folded around two conjugated focal lines of an ellipse and a hyperbola, such that they are perpendicular to the straight lines joining any part of the ellipse to the hyperbola. They can have negative or positive Gaussian curvature, termed FCD-I and FCD-II focal conic domains ${ }^{\ddagger}$ where bilayers curve away from or around the defect singularity, respectively. FCD-I defects, with negative Gaussian curvature, can assemble into chains (joined by edge dislocations) termed "oily streaks", which are visible using polarising microscopy and reduce the elastic energy by allowing curvature without compression of the bilayers. FCD-I defects also exist individually and are observed when oily streaks break down as a result of a longitudinal instability. FCD-Ils do not assemble into further structures but do anneal into spherulite structures. ${ }^{37-39}$

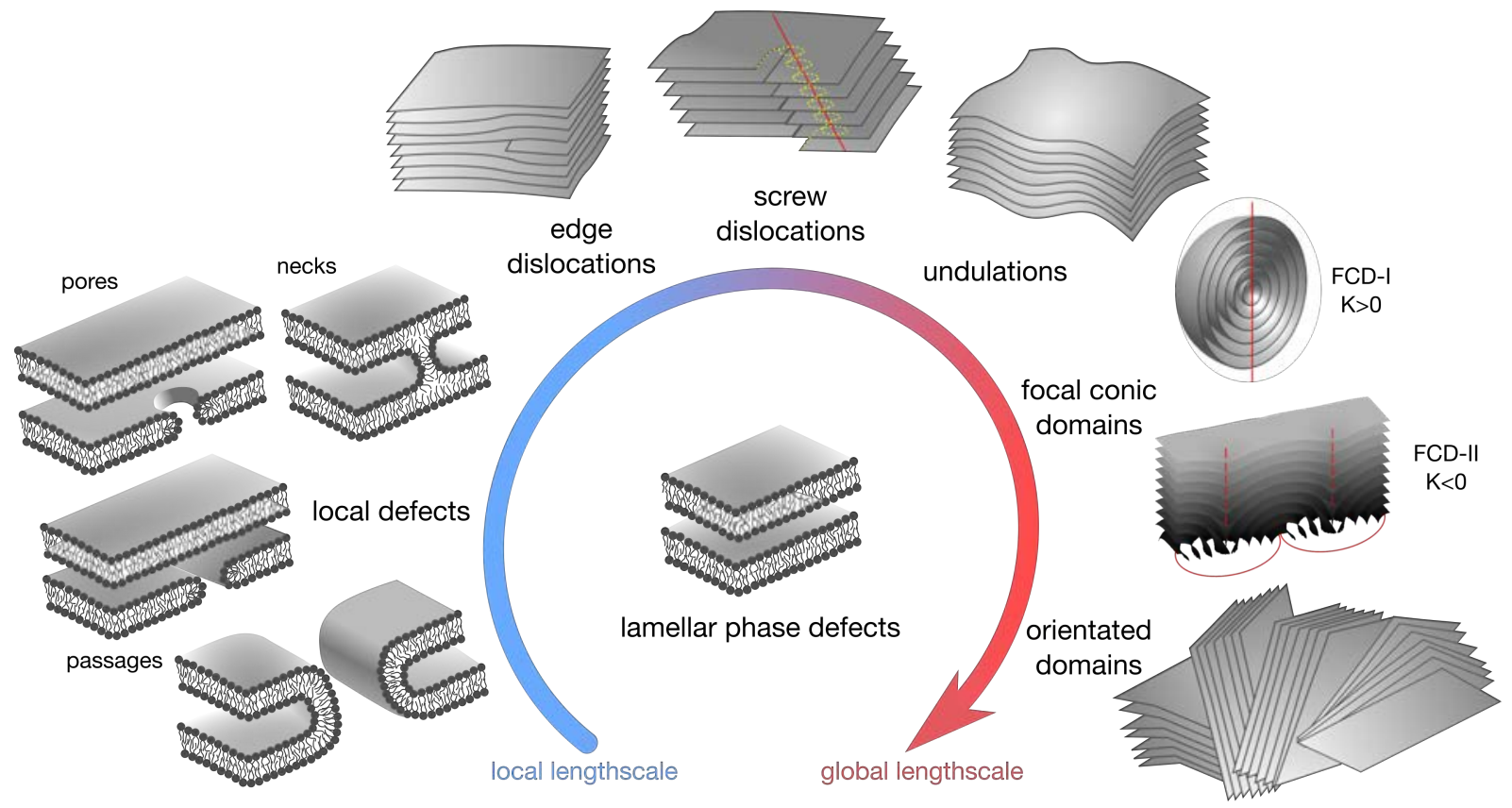

Figure 1.12: Schematic examples of lamellar phase defects.

Two specific types of dislocation defect are also illustrated in Figure 1.12, both of which increase connectivity between bilayers. Edge dislocations arise from the termination of one bilayer plane, while screw defects are low in energy and provide connectivity through multiple bilayers. ${ }^{40}$ Connectivity is also affected by the formation of localised point defects, which take the form of "pores", "necks" and "passages". ${ }^{41}$ These defects can either enhance or break the connectivity between the surfactant and water layers, and they are likely a key factor in the previously discussed $L_{\alpha}-L_{3}$ phase transition. For a further in-depth analysis of soft matter defects and their physical stabilities see the work of Kléman. ${ }^{42,43}$

FNote that FCD-I and FCD-II denote "Type I" and "Type II" defects; however, this is not consistent with the Type I and Type II assigned to normal and inverse mesophases. Type I FCD defects have negative Gaussian curvature, whereas inverse phases, which generally have negative Gaussian curvature, are termed Type II mesophases. 


\section{The Role of Counterions}

The range of different surfactant behaviours is vast. As we are unable to sufficiently predict the phase behaviour of any molecule, it is difficult to judge whether the observed behaviour of any system is particularly remarkable, and thus indicative of specific packing requirements resulting from an interesting molecular structure. Observations of phase transitions that progress in the opposite order from the normal trend (such as those occurring in the monoolein-based systems ${ }^{44}$ ) are an exception, as is when structurally similar surfactants are observed to display drastically different behaviours (such as the difference in didodecyldimethylammonium systems with chloride and bromide counterions ${ }^{45,46}$ ), or in specific relation to this thesis, when discontinuous behaviour is reported within a single phase (e.g. the Aerosol OT system ${ }^{47}$ ). This thesis reports on our investigation into a family of Aerosol OT-related surfactants, with particular focus on role of the branched tails (which are often neglected, but have been shown to be important in the formation of microemulsions ${ }^{48}$ ) and the counterion, another often neglected but potentially very important - factor in self-assembly.

The presence of the counterion in ionic surfactants adds an additional degree of freedom that can impact upon each contributor to the system self-assembly. It provides steric and electronic contributions to the system, as when bound it is considered as part of the headgroup and partially shields electrostatic forces. As well as providing steric bulk, the degree of counterion binding can alter the structures of the headgroup, tails and water at the interface. How the counterion affects each parameter is not constant for any system as the extent of binding and hydration change with both concentration and temperature. This complexity, combined with the "specific ion effects" observed within some systems, means that the role of the counterion within any given system is not easily predicted. However, the empirical "matching water affinities" rule proposed by Collins ${ }^{49}$ does allow some generalisations to be made, and although this law is simple and has exceptions, it has been shown to be remarkably reliable (at least for spherical point charges) and has been used to rationalise many specific ion effects in biological systems. ${ }^{50}$

Collins' law can be summarised as "like seeks like", or more specifically oppositely charged ions in solution will form close (inner sphere) ion pairs when their water affinities (free energy of hydration) are equally matched. This is shown schematically in Figure 1.13, and means that chaotropic and kosmotropic ions (which disfavour and favour interactions with water, respectively) will not bind strongly when paired together. The rule can be rationalised as domination of the strongest available intermolecular interaction. Despite tightly bound hydration shells, the electrostatic attraction between small kosmotropic ions results in the expulsion of water and close ion pair formation. The attraction between large chaotropic ions is not as great, but they readily shed their weakly bound hydration shells as water favours interactions with itself rather than the ion. In the case of mismatched ions, the strongest interaction is between the kosmotropic ion and the water, and so the ions retain their hydration water and form weakly solvated ion pairs or dissociate. 


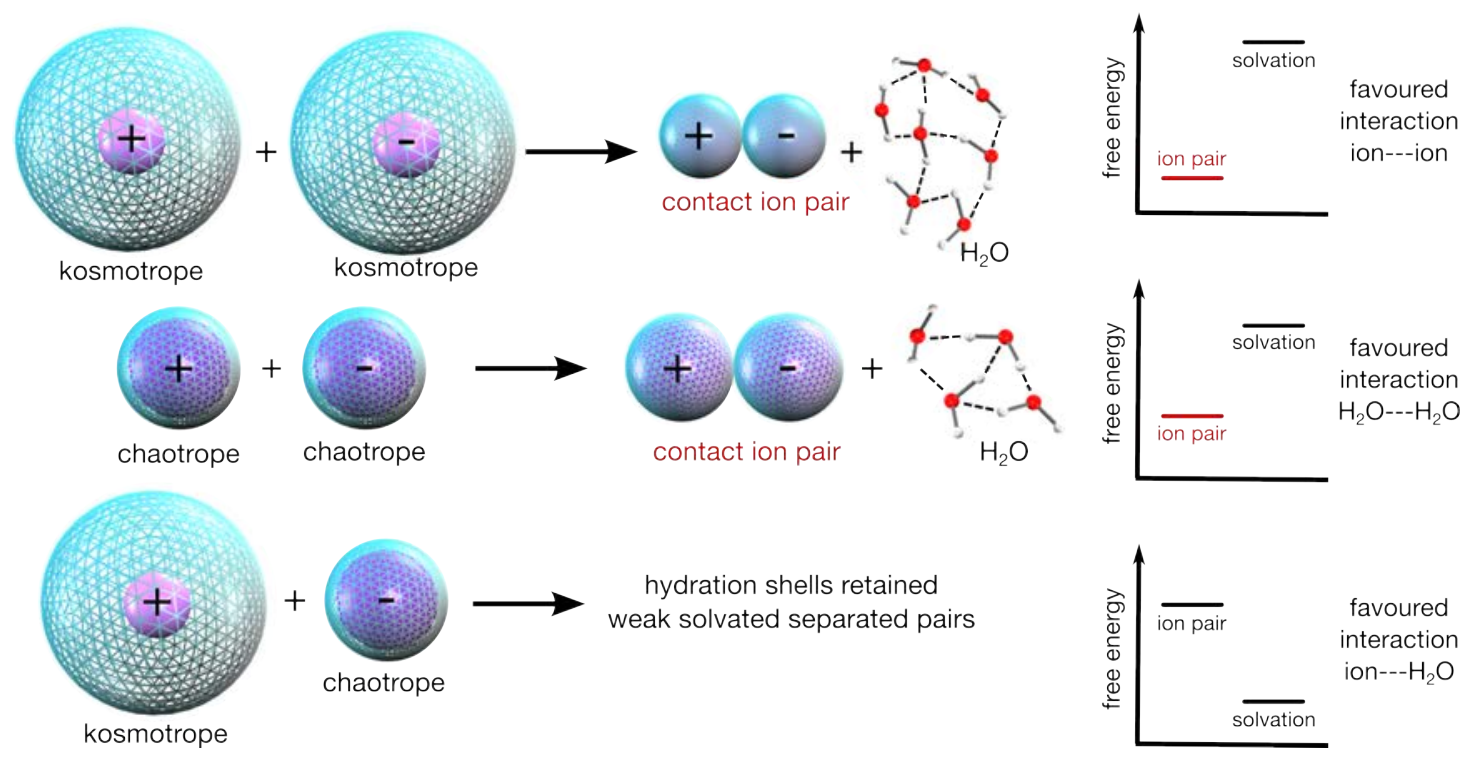

Figure 1.13: A schematic illustration of Collins' law of matching water affinities.

This thesis reports on a family of Aerosol OT-related surfactants, elucidating and comparing their phase and defect behaviours upon self-assembly in water. All of the surfactants employed are anionic, containing a sulfosuccinate ion within their headgroups that is described as "soft" and chaotropic. Using Collins' law, we can state that the kosmotropic counterions used within this work should form the weakest surfactant-counterion pairs, while the chaotropic ions used should bind more strongly (see Figure 1.14). The role of the counterion in surfactant selfassembly, with respect to effects on the evolution of both the macro and microstructure, is the main focuses of this thesis. Two types of counterion were used in experimental work, simple point charges (group 1 alkali metals) and non-spherical ammonium ions, which are presented separately in Part I and Part II of this thesis, respectively. Before this work is discussed the selfassembly of $\mathrm{Na}$-AOT is reviewed in an attempt to rationalise previously described anomalies in the system, and the role of tail branching is discussed. This introduction has covered the key points of lyotropic liquid crystals required to understand the work presented here, with the roles of elasticity and defect behaviours of particular importance in the following chapters.

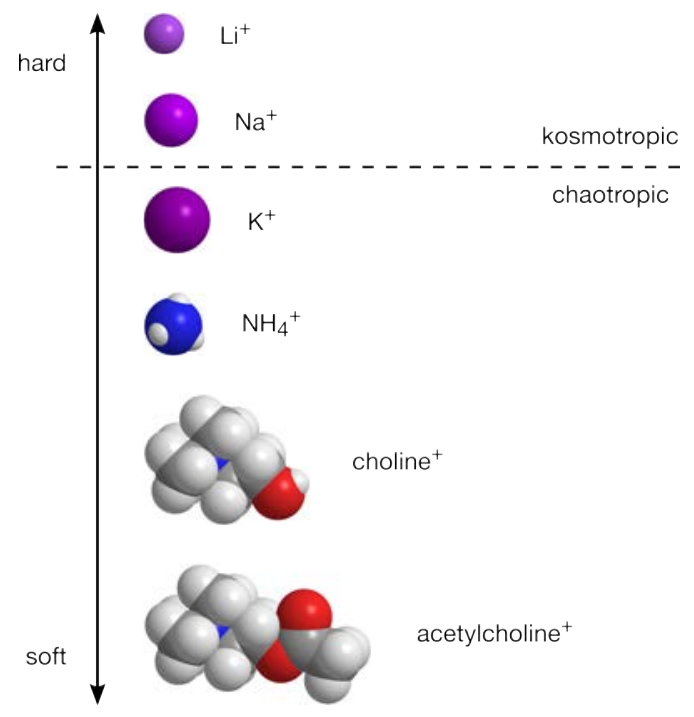

Figure 1.14: The relative kosmotropic/chaotropic natures of the cations used in this research. 


\section{Chapter 2}

\section{Experimental}

This thesis reports on the investigation of surfactant systems on a range of lengthscales, both in- and out-of-equilibrium, monitoring the structural and dynamic changes that occur. Such an investigation cannot be completed using a single experimental technique. Instead, a range of complementary techniques must be used, in order to probe the different physical properties of the system on various lengthscales. This chapter describes the synthesis, purification and sample preparation of the surfactants investigated in this research. The experimental approach to each of the investigative techniques and methods employed is then explained, along with the theory, limitations and rationale behind their use in the study of surfactant systems.

\section{Surfactant Synthesis and Purification}

Lyotropic phase behaviour can be significantly altered by the presence of impurities. ${ }^{51}$ In particular, the presence of salts can change the electrostatic interactions of the systems and weakly amphiphilic molecules (such as long chain alcohols) can act as cosurfactants. Such changes can modify the position of phase boundaries, and phases existing over narrow surfactant concentrations can be removed completely. In more extreme cases, contaminants can induce phase separation and surfactant degradation, as observed in the sodium dodecyl sulfate (SDS)/water system with the introduction of dodecanol. ${ }^{52}$ To ensure high purity in the systems studied here, ultrapure water (double-distilled and Millipore filtered to a resistivity of 18.2 M $\Omega . c m$ at $25^{\circ} \mathrm{C}$ ) was used and all surfactants were purified before use. Starting materials were purchased from Sigma-Aldrich, Inc. Acetylcholine chloride was synthesised using a literature method. ${ }^{53}$ Analytical grade solvents were used as received, other solvents were double-distilled before use. Cation exchange resin beads were purchased from Merck Millipore. Nuclear magnetic resonance (NMR) spectroscopy was used to characterise all products. NMR spectra were recorded using a Varian Unity Inova spectrometer operating at $500 \mathrm{MHz}$ for ${ }^{1} \mathrm{H}$ spectra and $125 \mathrm{MHz}$ for ${ }^{13} \mathrm{C}$ spectra, and all chemical shifts, $\delta(\mathrm{ppm})$, were referenced to the residual solvent peak of the $\mathrm{CDCl}_{3}$ solvent. ${ }^{54}$ 


\section{Purification of Na-AOT}

Crude Na-AOT was dissolved in methanol to give a $1.0 \mathrm{M}$ surfactant solution. This solution was vacuum filtered through a $0.22 \mu \mathrm{m}$ pore size hydrophobic micropore filter. Ultrapure water (10\% by volume) was added, and the resulting solution washed twice with petroleum ether $(500 \mathrm{~mL}$ petroleum ether per $300 \mathrm{~mL}$ surfactant solution for each wash). The lower aqueous layer was retained, and the solvent removed by rotary evaporation under reduced pressure. The purified Na-AOT was then freeze-dried for 48 hours.

\section{Synthesis of Li-AOT, K-AOT, $\mathrm{NH}_{4}$-AOT and AcCh-AOT}

The synthesis of $\mathrm{Li}$-AOT, $\mathrm{K}-\mathrm{AOT}, \mathrm{NH}_{4}$-AOT and AcCh-AOT was adapted from a literature method. ${ }^{55}$ Crude Na-AOT was dissolved in hexane or heptane to give a $0.1 \mathrm{M}$ surfactant solution. The appropriate metal nitrate, ammonium nitrate or acetylcholine chloride was dissolved in ultrapure water to give a $1.0 \mathrm{M}$ salt solution. Equal volumes of the two solutions were shaken and allowed to separate overnight. The upper organic layer was retained, and shaken with two further equal volume portions of salt solution. The solvent was removed from the resulting surfactant solution by rotary evaporation under reduced pressure, and the AOT salt product purified using the method for Na-AOT above. This method of ion-exchange is well-established in the literature and has been shown previously via atomic absorption experiments to be sufficient for near-quantitative counterion exchange. ${ }^{56-59}$

\section{Synthesis of Ch-AOT}

The synthesis of Ch-AOT was adapted from the only reported method for sodium/choline ionexchange in any surfactant at the time this work was carried out. ${ }^{60}$ An ion-exchange column was loaded with $500 \mathrm{~g}$ of strong acidic cation exchange resin beads (Type 1, with a resin capacity of 1.7 milliequivalents) as a slurry in ultrapure water. The column was washed with $1 \mathrm{M} \mathrm{HCl}$ to ensure full protonation of the resin material. Ultrapure water was washed through the column until a neutral $\mathrm{pH}$ was achieved. The resin was loaded with choline ions by passing $3.65 \mathrm{~L}$ of $1 \mathrm{M}$ choline chloride solution through the column (four times the maximum loading capacity of the resin) resulting in an effluent $\mathrm{pH}$ of $\sim 3$, and complete loading was ensured by passing $1 \mathrm{~L}$ of $0.1 \mathrm{M}$ choline hydroxide solution through the column resulting in a $\mathrm{pH}$ of $10-11$. The column was neutralised by passing $1 \mathrm{~L}$ of ultrapure water through the column, giving a final $\mathrm{pH}$ of 7. A $0.05 \mathrm{M}$ solution containing $125 \mathrm{~g}$ of purified Na-AOT (less than one third of the maximum resin capacity) was passed through the column. The resulting Ch-AOT solution was collected from the column after discarding the forerunnings. Ethanol was added to the solution to form an azeotropic mixture, and the solvent was removed by rotary evaporation under 
reduced pressure followed by freeze-drying for 72 hours. The resulting extremely hygroscopic Ch-AOT was then dried at $\sim 0.1 \mathrm{mmHg}$ for 24 hours and transferred to a glove box for storage..$^{\ddagger}$

\section{NMR Characterisation of AOT Salts}

Proton NMR spectra of the AOT salts are shown in Figures 2.1 and 2.2. The cation present in the AOT salt primarily affects the NMR chemical shifts and/or coupling constants relating to the protons in the AOT headgroup. The relevant ${ }^{1} \mathrm{H}$ NMR data for the headgroups (including any protons present in the cations) are listed below:

Na-AOT Selected ${ }^{1} \mathrm{H}$ NMR $\delta\left(500 \mathrm{MHz}, \mathrm{CDCl}_{3}\right): 3.17\left(\mathrm{~m}, 2 \mathrm{H}, \mathrm{CH}_{2} \mathrm{C}=\mathrm{O}\right), 4.35(\mathrm{br}, 1 \mathrm{H}$, $\mathrm{CHSO}_{3}{ }^{-}$).

Li-AOT Selected ${ }^{1} \mathrm{H}$ NMR $\delta\left(500 \mathrm{MHz}, \mathrm{CDCl}_{3}\right): 3.15\left(\mathrm{~m}, 2 \mathrm{H}, \mathrm{CH}_{2} \mathrm{C}=\mathrm{O}\right), 4.35(\mathrm{dd}, \mathrm{J}=9.0$, $5.4 \mathrm{~Hz}, 1 \mathrm{H}, \mathrm{CHSO}_{3}{ }^{-}$).

K-AOT Selected ${ }^{1} \mathrm{H}$ NMR $\delta\left(500 \mathrm{MHz}, \mathrm{CDCl}_{3}\right): 3.09\left(\mathrm{dd}, J=17.4,3.8 \mathrm{~Hz}, 1 \mathrm{H}, \mathrm{CH}_{2} \mathrm{C}=\mathrm{O}\right), 3.16$ (dd, $\left.J=17.4,10.4 \mathrm{~Hz}, 1 \mathrm{H}, \mathrm{CH}_{2} \mathrm{C}=\mathrm{O}\right), 4.22\left(\mathrm{br}, 1 \mathrm{H}, \mathrm{CHSO}_{3}{ }^{-}\right.$).

$\mathrm{NH}_{4}$-AOT Selected ${ }^{1} \mathrm{H}$ NMR $\delta\left(500 \mathrm{MHz}, \mathrm{CDCl}_{3}\right): 3.06$ (dd, $J=17.4,3.9 \mathrm{~Hz}, 1 \mathrm{H}, \mathrm{CH}_{2} \mathrm{C}=\mathrm{O}$ ), 3.15 (dd, $J=17.4,11.0 \mathrm{~Hz}, 1 \mathrm{H}, \mathrm{CH}_{2} \mathrm{C}=\mathrm{O}$ ), $4.18\left(\mathrm{dd}, \mathrm{J}=10.8,4.0 \mathrm{~Hz}, 1 \mathrm{H}, \mathrm{CHSO}_{3}{ }^{-}\right.$), $6.68(\mathrm{br}$, $4 \mathrm{H}, \mathrm{NH}_{4}^{+}$).

Ch-AOT Selected ${ }^{1} \mathrm{H}$ NMR $\delta\left(500 \mathrm{MHz}, \mathrm{CDCl}_{3}\right): 3.04$ (dd, $\left.J=17.3,3.5 \mathrm{~Hz}, 1 \mathrm{H}, \mathrm{CH}_{2} \mathrm{C}=\mathrm{O}\right)$, 3.17 (dd, $\left.J=17.2,11.5 \mathrm{~Hz}, 1 \mathrm{H}, \mathrm{CH}_{2} \mathrm{C}=\mathrm{O}\right), 3.29\left(\mathrm{~s}, 9 \mathrm{H}, \mathrm{NCH}_{3}\right), 3.64\left(\mathrm{~m}, 2 \mathrm{H}, \mathrm{CH}_{2} \mathrm{~N}\right), 4.03(\mathrm{~m}$, $2 \mathrm{H}, \mathrm{CH}_{2} \mathrm{OH}$ ), 4.08 (dd, $J=11.4,3.6 \mathrm{~Hz}, 1 \mathrm{H}, \mathrm{CHSO}_{3}{ }^{-}$).

AcCh-AOT Selected ${ }^{1} \mathrm{H}$ NMR $\delta\left(500 \mathrm{MHz}, \mathrm{CDCl}_{3}\right): 2.11\left(\mathrm{~s}, 3 \mathrm{H}, \mathrm{C}(=\mathrm{O}) \mathrm{CH}_{3}\right), 3.07(\mathrm{dd}, J=17.4$, $3.2 \mathrm{~Hz}, 1 \mathrm{H}, \mathrm{CH}_{2} \mathrm{C}=\mathrm{O}$ ), 3.19 (dd, $\left.J=17.4,11.4 \mathrm{~Hz}, 1 \mathrm{H}, \mathrm{CH}_{2} \mathrm{C}=\mathrm{O}\right), 3.35\left(\mathrm{~s}, 9 \mathrm{H}, \mathrm{NCH}_{3}\right), 3.91$ (m, $\left.2 \mathrm{H}, \mathrm{CH}_{2} \mathrm{~N}\right), 4.09$ (dd, $\left.J=11.4,3.2 \mathrm{~Hz}, 1 \mathrm{H}, \mathrm{CHSO}_{3}{ }^{-}\right), 4.52\left(\mathrm{~m}, 2 \mathrm{H}, \mathrm{CH}_{2} \mathrm{OC}(=\mathrm{O}) \mathrm{Me}\right)$.

\section{Purification and NMR Characterisation of Na-butylAMA}

As noted in Chapter 4, it was intended that sodium bis( $n$-hexyl) sulfosuccinate (Na-hexylAMA) would be studied as part of this research. On this basis, dihexyl sulfosuccinate sodium salt solution (product number 86146, lot number BCBG9784V, CAS number and product image corresponding to Na-hexylAMA) was purchased from Sigma-Aldrich Inc. ${ }^{61}$ and purified using the method for Na-AOT above. However, upon characterisation of the purified material by ${ }^{1} \mathrm{H}$ NMR spectroscopy, it was found that the spectrum of the material did not match the expected integration pattern or correspond to a previously reported ${ }^{1} \mathrm{H} N M R$ spectrum of Na-hexylAMA. ${ }^{62}$ Full characterisation of this material was undertaken using ${ }^{1} \mathrm{H}$ and ${ }^{13} \mathrm{C}$ NMR spectroscopy, homonuclear correlation spectroscopy (COSY), heteronuclear singlequantum correlation spectroscopy (HSQC), and heteronuclear multiple-bond spectroscopy

\footnotetext{
FThis method was found to be problematic due to foaming at the rotary evaporation stage. In future, this synthesis may be improved by using methanol rather than ultrapure water for the column exchange process, or the synthetic method for the other AOT salts described above may be employed.
} 
$(\mathrm{HMBC}),{ }^{\ddagger}$ and the material was determined to be sodium bis(1,3-dimethylbutyl) sulfosuccinate (Na-butylAMA). Notably, the phased HSQC spectrum confirms the presence of only two distinguishable $\mathrm{CH}_{2}$ environments in the molecule, consistent with Na-butylAMA, whereas based on the ${ }^{13} \mathrm{C}$ NMR data of similar molecules, Na-hexylAMA would contain six distinguishable $\mathrm{CH}_{2}$ environments. Details of the NMR characterisation of Na-butylAMA are shown in Figure 2.3, and the ${ }^{1} \mathrm{H}$ and ${ }^{13} \mathrm{C}$ NMR data are listed below:

Na-butylAMA ${ }^{1} \mathrm{H}$ NMR $\delta\left(500 \mathrm{MHz}, \mathrm{CDCl}_{3}\right): 0.86\left(\mathrm{br}, 12 \mathrm{H}, \mathrm{OCH}\left(\mathrm{CH}_{3}\right) \mathrm{CH}_{2} \mathrm{CH}\left(\mathrm{CH}_{3}\right)_{2}\right)$, $1.21\left(\mathrm{~m}, 8 \mathrm{H}, \quad \mathrm{OCH}\left(\mathrm{CH}_{3}\right) \mathrm{CH}_{2} \mathrm{CH}\left(\mathrm{CH}_{3}\right)_{2}\right), 1.59\left(\mathrm{~m}, 4 \mathrm{H}, \quad \mathrm{OCH}\left(\mathrm{CH}_{3}\right) \mathrm{CH}_{2} \mathrm{CH}\left(\mathrm{CH}_{3}\right)_{2}\right), \quad 3.09$ $\left(\mathrm{m}, 2 \mathrm{H}, \mathrm{CH}_{2} \mathrm{C}=\mathrm{O}\right), 4.23\left(\mathrm{~m}, 1 \mathrm{H}, \mathrm{CHSO}_{3}{ }^{-}\right), 4.98\left(\mathrm{~m}, 2 \mathrm{H}, \mathrm{OCH}\left(\mathrm{CH}_{3}\right) \mathrm{CH}_{2} \mathrm{CH}\left(\mathrm{CH}_{3}\right)_{2}\right) ;{ }^{13} \mathrm{C}$ NMR $\delta\left(125 \mathrm{MHz}, \mathrm{CDCl}_{3}\right):$ 19.9-20.4 (8s, $\left.\mathrm{OCH}\left(\mathrm{CH}_{3}\right) \mathrm{CH}_{2} \mathrm{CH}\left(\mathrm{CH}_{3}\right)_{2}\right)$, 22.4-23.0 (12s, $\left.\mathrm{OCH}\left(\mathrm{CH}_{3}\right) \mathrm{CH}_{2} \mathrm{CH}\left(\mathrm{CH}_{3}\right)_{2}\right)$, 24.4-24.8 (6s, OCH $\left.\left(\mathrm{CH}_{3}\right) \mathrm{CH}_{2} \mathrm{CH}\left(\mathrm{CH}_{3}\right)_{2}\right)$, 33.3-33.7 (4s, $\mathrm{CH}_{2}-$ $\mathrm{C}=\mathrm{O}), 44.8-45.2\left(8 \mathrm{~s}, \mathrm{OCH}\left(\mathrm{CH}_{3}\right) \mathrm{CH}_{2} \mathrm{CH}\left(\mathrm{CH}_{3}\right)_{2}\right), 61.4-61.6\left(4 \mathrm{~s}, \mathrm{CHSO}_{3}{ }^{-}\right), 70.2-72.0$ (5s, $\left.\mathrm{OCH}\left(\mathrm{CH}_{3}\right) \mathrm{CH}_{2} \mathrm{CH}\left(\mathrm{CH}_{3}\right)_{2}\right), 169.2-171.1(6 \mathrm{~s}, \mathrm{C}=\mathrm{O})$.

\section{Synthesis and NMR Characterisation of K-butyIAMA}

K-butylAMA was synthesised from purified Na-butylAMA and potassium nitrate using the method described for Li-AOT, K-AOT, $\mathrm{NH}_{4}$-AOT and AcCh-AOT above. ${ }^{1} \mathrm{H}$ NMR spectra of Na-butylAMA and K-butylAMA (with Na-AOT for comparison) are shown in Figure 2.4. The cation present in the butylAMA salt primarily affects the NMR chemical shifts relating to the protons in the butylAMA headgroup. The relevant ${ }^{1} \mathrm{H}$ NMR data for the headgroups of Na-butylAMA and K-butyIAMA are listed below:

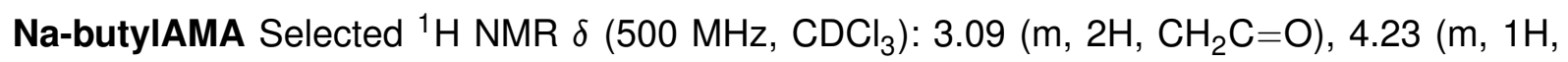
$\mathrm{CHSO}_{3}{ }^{-}$).

K-butyIAMA Selected ${ }^{1} \mathrm{H}$ NMR $\delta\left(500 \mathrm{MHz}_{\mathrm{CDCl}}\right): 3.07\left(\mathrm{~m}, 2 \mathrm{H}, \mathrm{CH}_{2} \mathrm{C}=\mathrm{O}\right), 4.15(\mathrm{~m}, 1 \mathrm{H}$, $\mathrm{CHSO}_{3}{ }^{-}$).

\section{Preparation of Surfactant Samples and Phase Diagrams}

\section{Surfactant Sample Preparation}

Samples of a wide range of surfactant concentrations were prepared by accurately weighing known amounts of dried surfactant and ultrapure water (double-distilled and Millipore filtered to a resistivity of $18.2 \mathrm{M} \Omega . \mathrm{cm}$ at $25^{\circ} \mathrm{C}$ ) into sample vials, and the masses recorded using an analytical balance accurate to five decimal places. The sample vials were then sealed by either flame sealing or crimping, dependent upon the type of sample vial used. For analysis of phase

\footnotetext{
¥These NMR spectra can be found in Appendix A.
} 


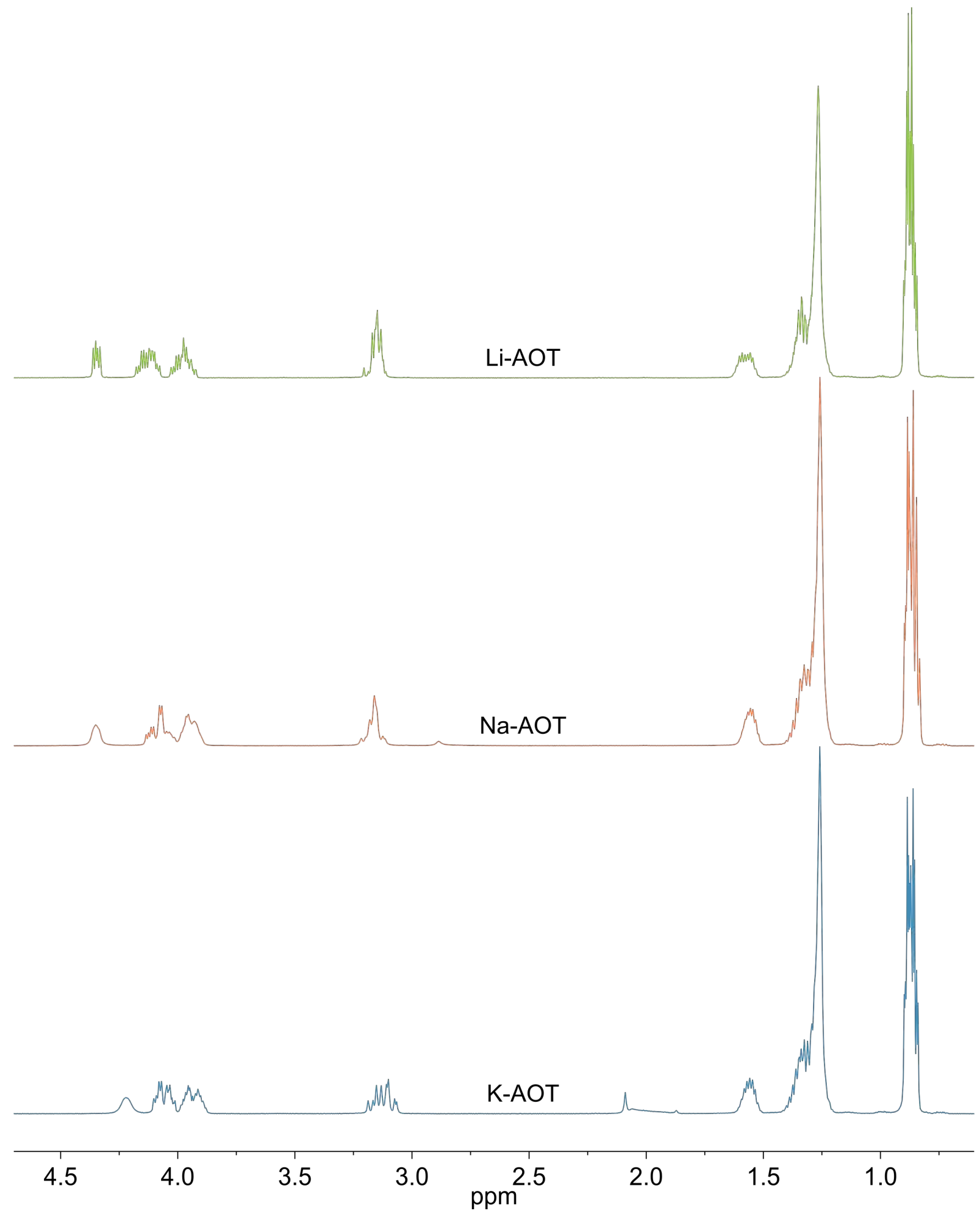

Figure 2.1: ${ }^{1} \mathrm{H}$ NMR spectra of Li-AOT, Na-AOT and K-AOT in $\mathrm{CDCl}_{3}$. 


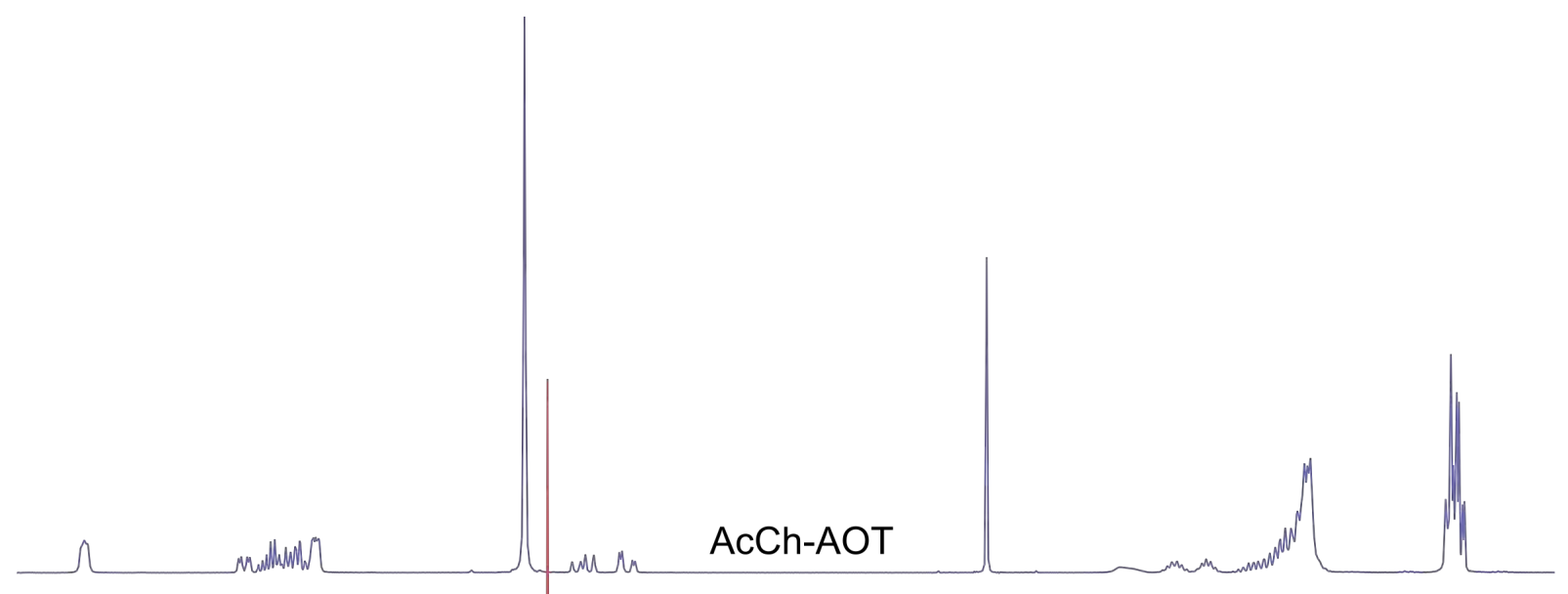
Whathrour Ch-AOT

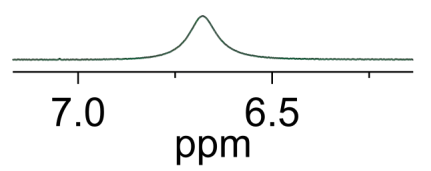

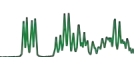
whln $\mathrm{NH}_{4}$-AOT

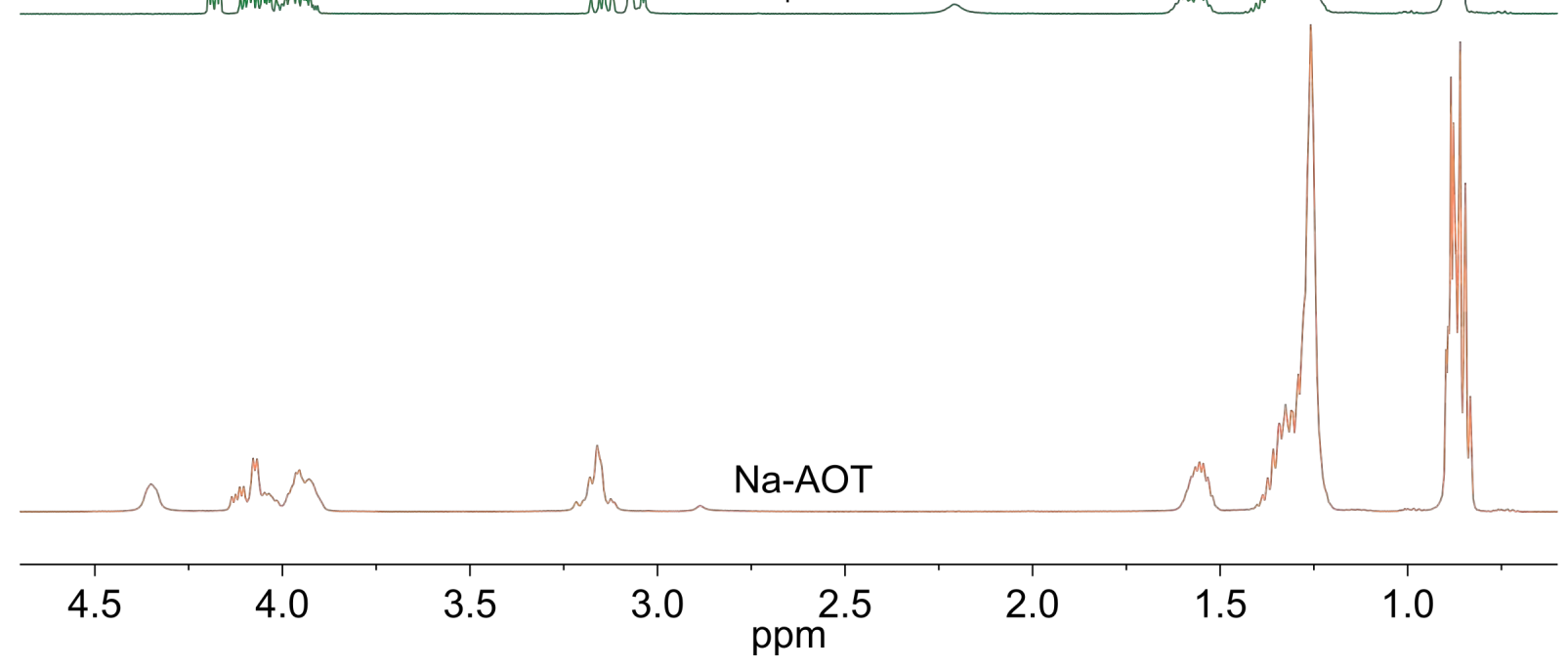

Figure 2.2: ${ }^{1} \mathrm{H}$ NMR spectra of AcCh-AOT, Ch-AOT, $\mathrm{NH}_{4}$-AOT and $\mathrm{Na}-\mathrm{AOT}$ in $\mathrm{CDCl}_{3}$. 


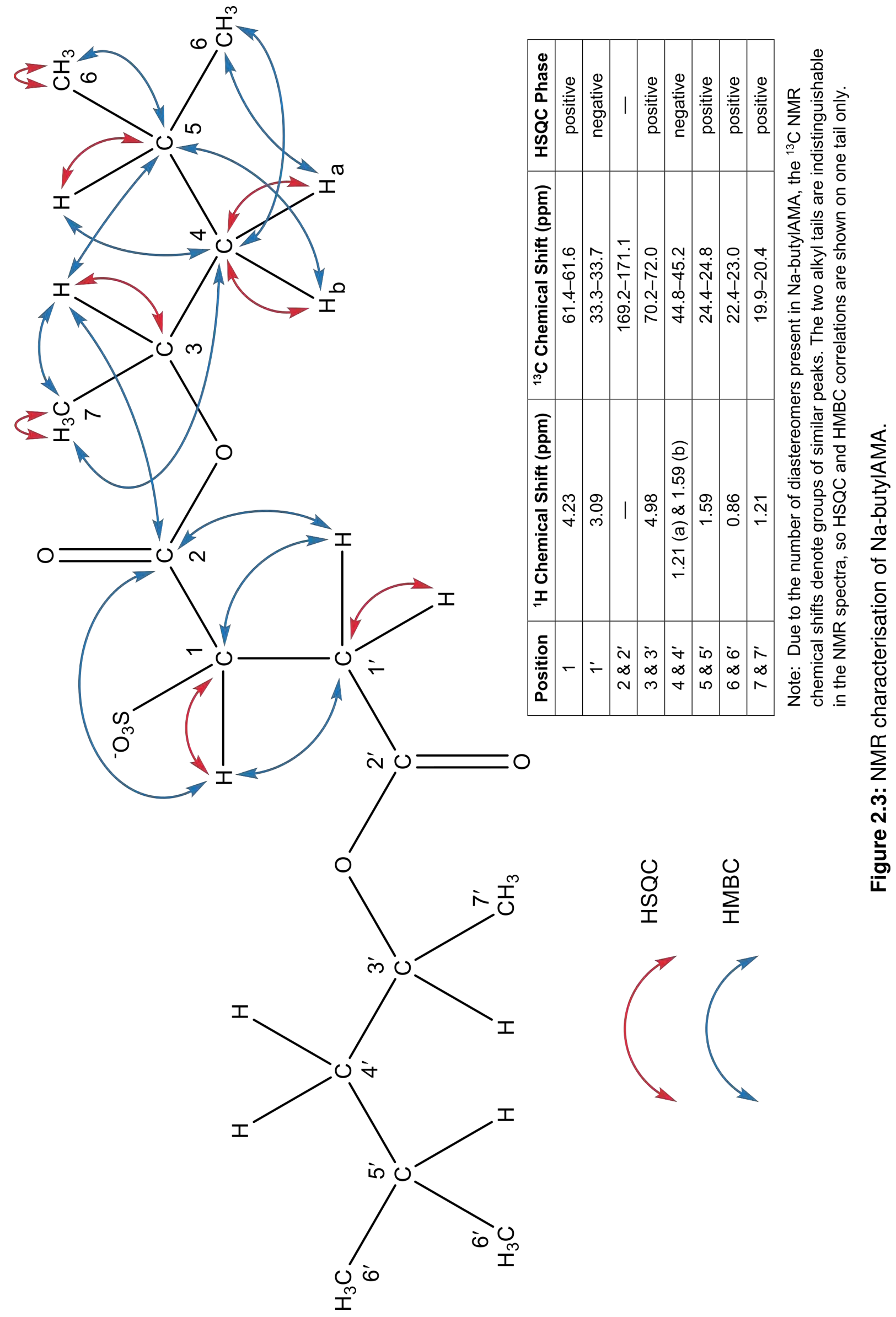




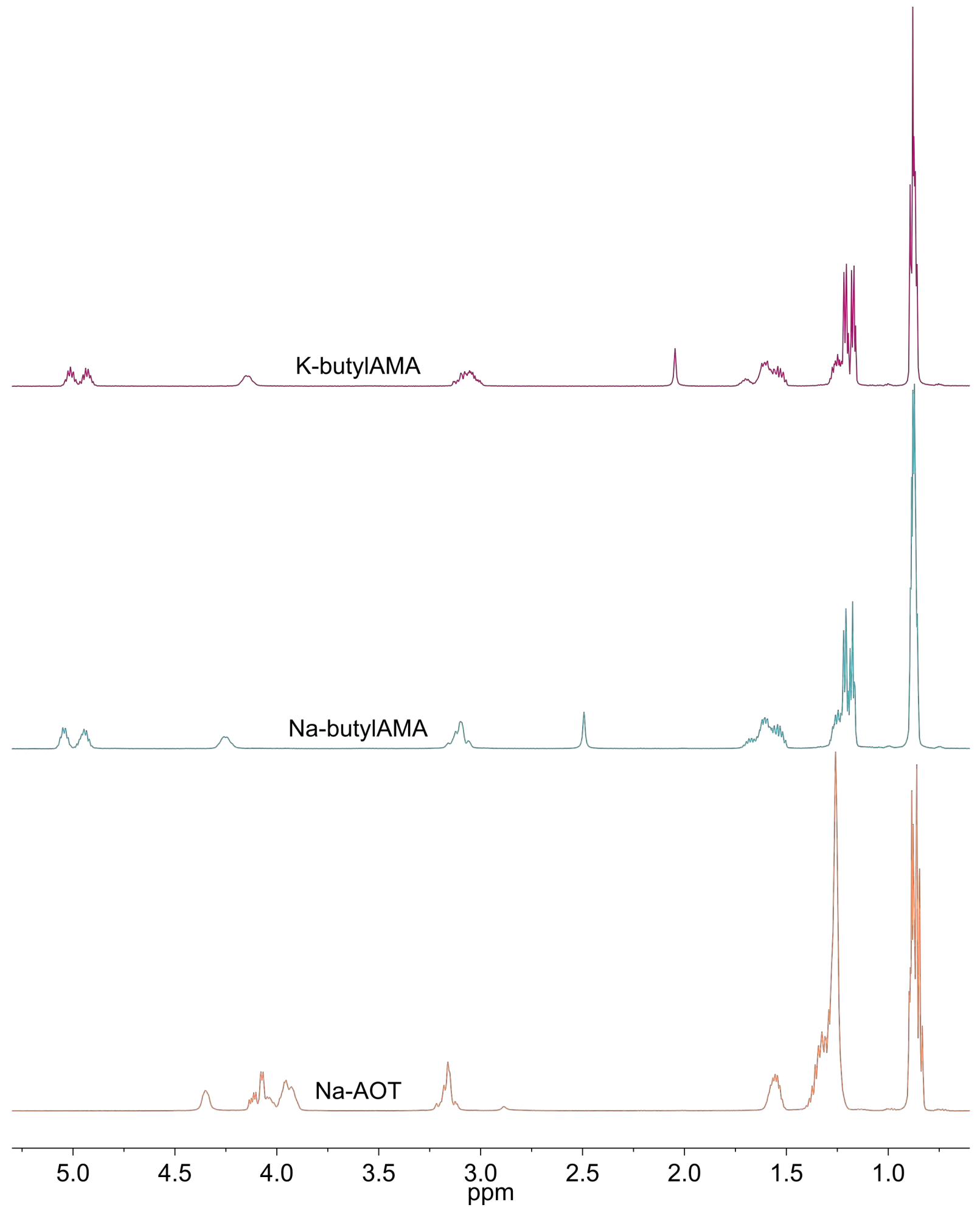

Figure 2.4: ${ }^{1} \mathrm{H}$ NMR spectra of K-butylAMA, Na-butylAMA and $\mathrm{Na}-\mathrm{AOT}$ in $\mathrm{CDCl}_{3}$. 
and microstructural behaviours, the samples produced were no larger than $2 \mathrm{~mL}$ in volume, and samples prepared for rheological measurements were limited to a volume of $5 \mathrm{~mL}$. The samples were equilibrated prior to any experimental use through regular equilibration cycles, which consisted of periods of heating (to approximately $60{ }^{\circ} \mathrm{C}$ in an oven), agitation using a vortex mixer, and centrifugation. The equilibration of samples was monitored regularly by viewing through crossed polarised filters and checking for homogeneity. Samples generally reached their equilibration state after several months, although the length of time varied for different surfactants and with concentration. The equilibration of samples was confirmed via POM before use in other experiments.

\section{Phase Diagram Preparation}

Each new phase diagram presented in this thesis was constructed based on several hundred observations, using several of the techniques outlined in this chapter. For each surfactant, multiple samples were prepared at concentration intervals of approximately $2.5 \mathrm{wt} \%$, at concentrations of 2.5-100 wt \%. Once equilibrated, sealed samples were placed in a Boekel Scientific multi-deck dry block heater and heated at a rate of $\sim 1{ }^{\circ} \mathrm{C} \mathrm{min}^{-1}$. Samples were continually observed between crossed polarisers to ascertain the approximate positions of phase boundaries, with observations documented at temperature intervals of $2.5^{\circ} \mathrm{C}$. Once the approximate positions of the phase boundaries were established, samples were reheated and observations made at more frequent temperature intervals. The characterisation of the phase behaviour was confirmed by POM and SAXS. The transition temperatures of phase boundaries were then confirmed by $\mathrm{POM}$, using a heating rate of $0.25^{\circ} \mathrm{C} \mathrm{min}^{-1}$ in close proximity to the phase boundary. POM observations were generally in excellent agreement with the bulk sample observations. For all systems, additional samples of equivalent concentration were prepared and no discrepancies in transition temperature were observed. Observations from bulk samples were compiled in the form of a graph (for an example see Figure 2.5), which were then used as the basis to create phase diagrams, with the transitional areas fine-tuned using SAXS and POM measurements. In the case of Na-AOT, samples were analysed as described here and the observed phase behaviour is in excellent agreement with Na-AOT phase diagrams reported elsewhere. ${ }^{63,64}$ 


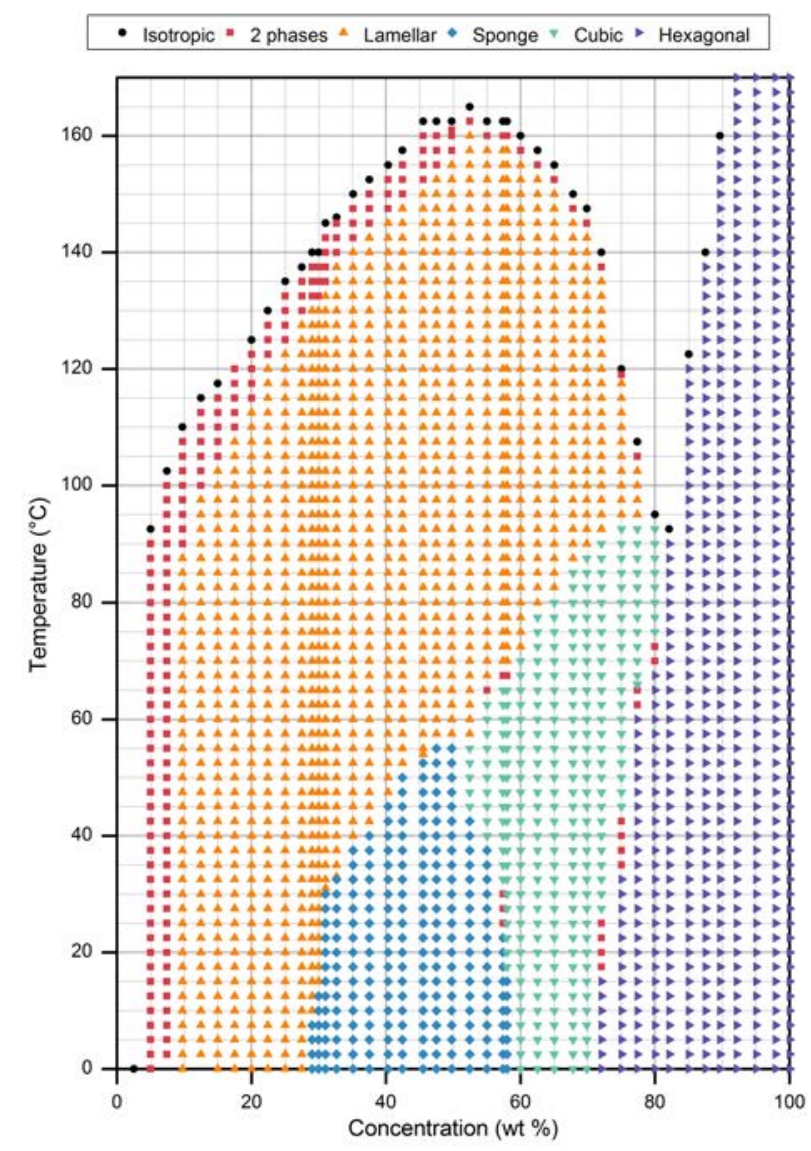

Figure 2.5: An example plot of phase observations at different concentrations and temperatures used as the basis for creating a phase diagram. The example shown is for K-AOT.

\section{Small-Angle X-ray Scattering}

Schematic representations of a small-angle X-ray scattering (SAXS) instrument are shown in Figure 2.6, in which a monochromatic beam of X-rays interacts with a sample, causing elastic scattering of $X$-rays due to differences in electron density. A beam stop prevents unscattered light from reaching the detection system and causing damage or swamping the signal. The detection system is often in the form of a CCD camera or imaging plates, placed at a small angle $\left(0.1-10^{\circ}\right)$ to the incident $\mathrm{X}$-ray direction achieved by increasing the distance between the sample and the detector, which detects the emitted X-rays, or the so-called scattering pattern. The intensities observed are functions of the scattering angle and provide averaged, representative structural information and characteristic distances of the molecular packing symmetry in the sample. From Bragg's law $(n \lambda=2 d \sin \theta)$, the larger the diffraction angle, the smaller the lengthscale probed, hence the use of SAXS provides detail on lengthscales larger than powder or single crystal X-ray crystallography. However, due to the wavelength of the radiation source used, the lengthscales are much smaller than those in static light scattering, and so SAXS is used to provide information on the size, shape and order in the nanometre range, while wide-angle X-ray scattering (WAXS) can be used to gain information on the atomic scale of a few Ångströms. 


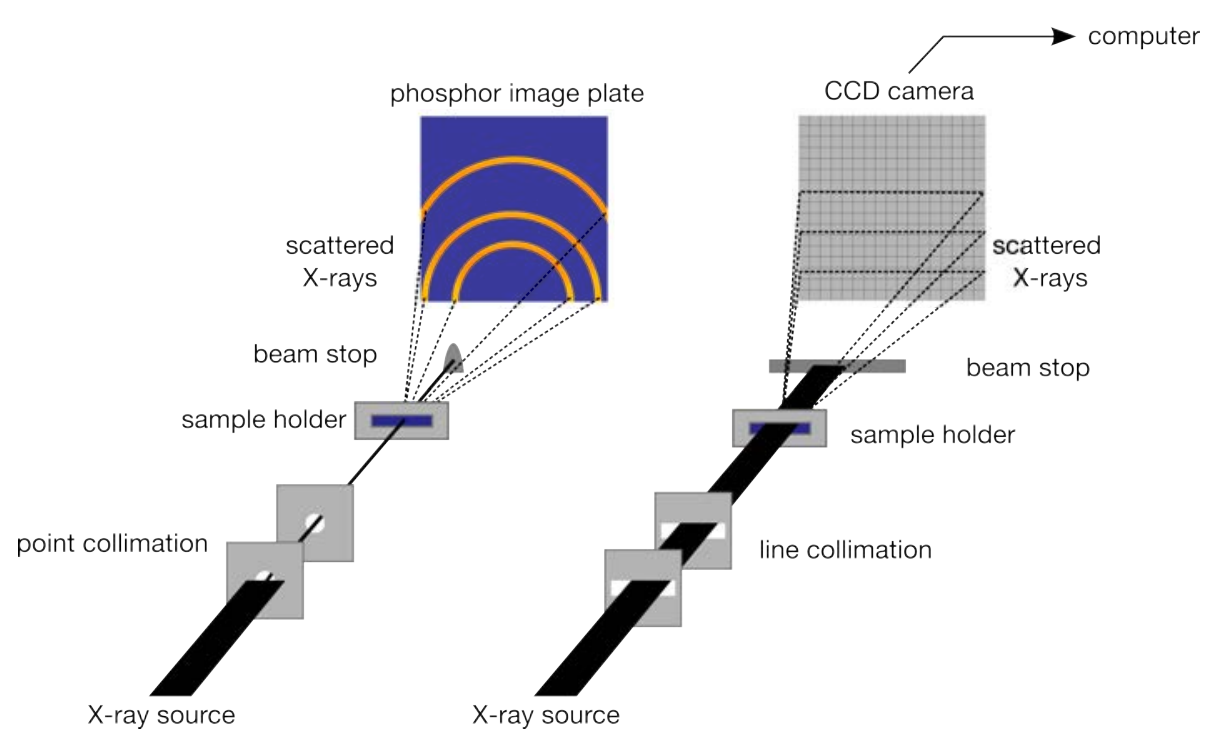

Figure 2.6: Schematic representations of the two different SAXS instrumentation set-ups used in this work, with point and line collimated X-ray beams detected by phosphor imaging plates and a CCD camera, respectively.

Upon interaction with a sample, a fraction of the $\mathrm{X}$-rays pass through the sample, a fraction are absorbed and transmitted into other forms of energy such as heat or fluorescence, and a fraction will be scattered in other directions. In SAXS the sample thickness is kept small to avoid absorption, allowing the scattered X-rays to be viewed. Neighbouring molecules/structures produce synchronous, coherent waves, which interact with others and produce interference patterns, containing structural information when detected. Interference patterns can be in-phase (constructive), out-of-phase (destructive) or in-between, depending on the observation angle, the orientation, and the distance between the neighbouring molecules or repeating structures. The intensity at the detector is dependent upon the interference, and thus an interference pattern is obtained characteristic of the distances between the molecules/structures. Each distance is measured relative to the wavelength of the applied radiation and is reported as a function of the scattering vector $q$ (the dimension of $q$ is the reciprocal of the lengthscale, $\mathrm{nm}^{-1}$ or $\AA^{-1}$, and so it is a measure of the structure in reciprocal space), where $\lambda$ is the wavelength of the incident $\mathrm{X}$-rays and $\theta$ is half the angle of reflection, such that:

$$
q=\frac{4 \pi}{\lambda} \sin \theta
$$

Each sample has an inherent form factor, with characteristic oscillations governed by the particle's shape, in the form of an interference pattern (essentially a Fourier transform of the shape of the electron cloud/distribution). When structures are packed more closely, such as in liquid crystalline systems, a structure factor (interparticle/lattice interference) multiplies with the form factor due to neighbouring particle interactions, and in ordered structures this gives rise to Bragg peaks, which detail the distances between ordered structures. These Bragg peaks form characteristic patterns dependent upon the symmetry of the ordered structure. 
As observed scattering patterns are due to inhomogeneities in the distribution of electrons in the sample and these differences can be between types of atoms, individual molecules or molecular assemblies, the ability of SAXS to probe distances in the nanometre domain makes it suitable for the analysis of the order within the liquid crystal microstructure, such as bilayer repeat distances in lamellar phases. SAXS has been used to characterise lyotropic liquid crystals for many years, with characteristic patterns of Bragg peaks observed for different mesophases. The lamellar phase in its ideal $L_{\alpha}$ form gives the most simple of these patterns, with q values in the ratio of $1: 2: 3: 4: 5: 6 \ldots$, whilst hexagonal phases give $q$ value ratios of $1: \sqrt{ } 3: \sqrt{ } 4: \sqrt{ } 7: \sqrt{ } 9: \sqrt{ } 12 \ldots$ and cubic phases give different ratios dependent upon their structural arrangement. In addition to the peak ratios, q spacings, the peak intensities and the sharpness of peaks, as well as the presence of any smaller and broader peaks, provide further information of the mesophase structure. Example patterns are shown in Figure 2.7.
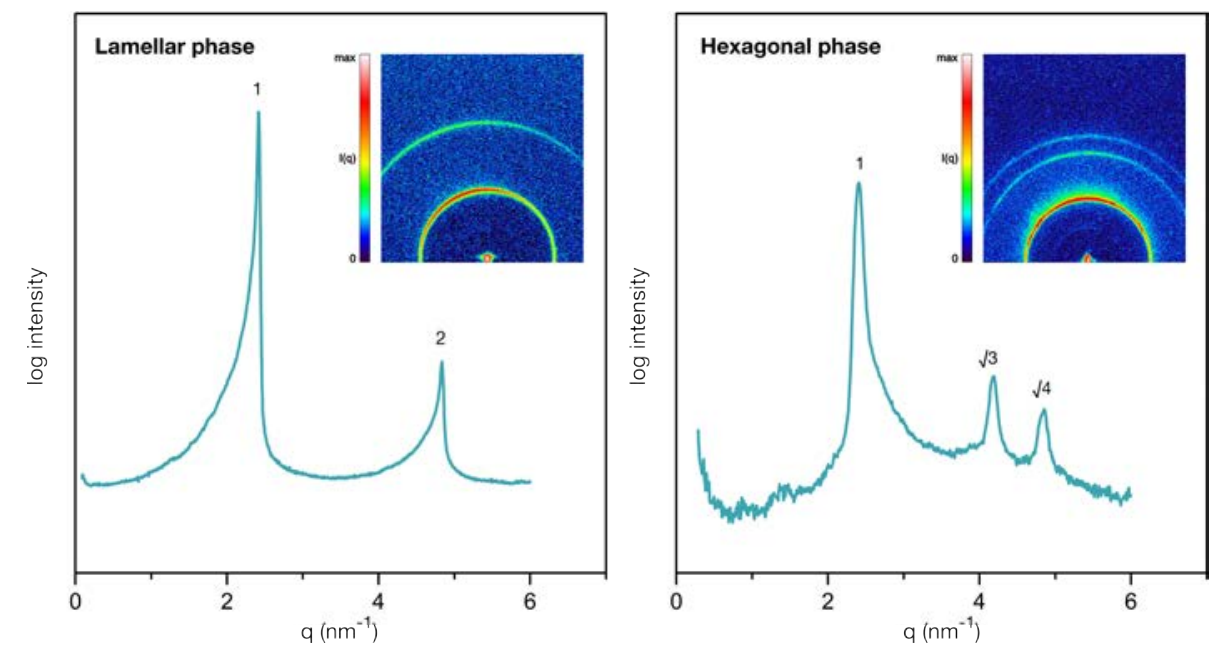

Figure 2.7: Examples of SAXS data for lamellar and hexagonal phases.

While SAXS provides information about the order and interaction between lamellar bilayers, the use of WAXS to measure the scattering at wide angles provides information at the atomic lengthscale. WAXS can therefore provide information about the internal bilayer structure and thickness, displaying patterns characteristic of different hydrocarbon arrangements and interactions. The $L_{\alpha}$ phase with disordered "fluid" hydrocarbon environments give rise to one broad signal, while more ordered phases give rise to series of sharp signals (for an example see Figure 2.8).

Although SAXS analysis can provide information that allows us to infer structural information, SAXS patterns themselves do not give morphological information directly. It is the intensity of the Fourier transform of the electron density, which is then interpreted to give structural information. Limitations of the technique arise in the interpretation of the scattering pattern, as two different morphologies can potentially give rise to very similar scattering patterns, from which conclusions are drawn. As such it is essential that SAXS is carried out in conjunction with other techniques or that other structural information is already available. In the case of the systems investigated here, SAXS analysis is a complementary technique to the optical and electron microscopy techniques detailed in this section. SAXS gives averaged results that are 

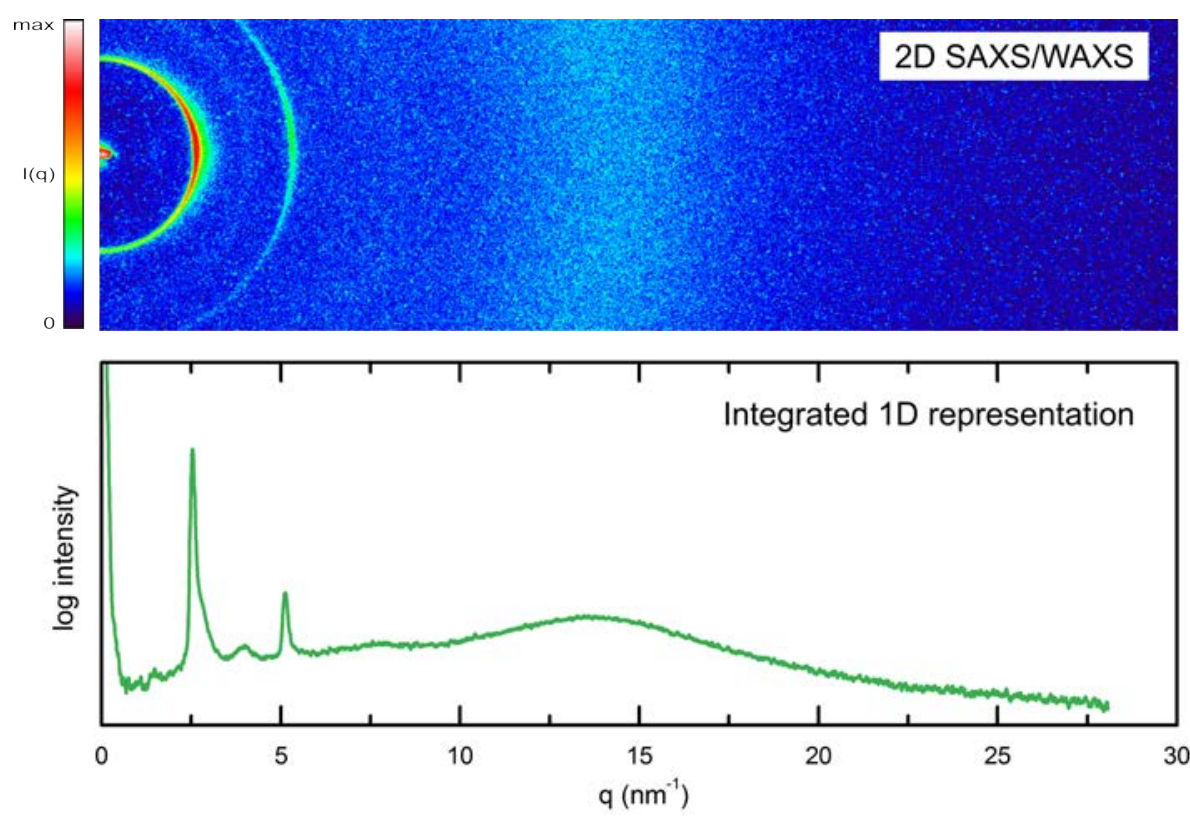

Figure 2.8: An example of SAXS/WAXS data with 2D and 1D representations shown.

representative of the sample, but cannot provide the small, localised structure information that microscopy techniques provide.

Small angle X-ray scattering experiments were performed using a SAXSess system (AntonPaar $\mathrm{GmbH}$ ), with a PANalytical PW3830 X-ray generator operating at $40 \mathrm{kV}$ and $50 \mathrm{~mA}$, and producing Ni-filtered $\mathrm{Cu} \mathrm{K} \alpha$ radiation with a wavelength of $0.1542 \mathrm{~nm}$. Samples were loaded in quartz flow cells and the temperature was controlled using a TCS 300-c sample stage. The maximum temperature was limited to $90{ }^{\circ} \mathrm{C}$ in accordance with the manufacturer's recommended guidelines for the flow cell. Where samples were too viscous for loading in a flow cell, a paste cell was used; however, this was found not to be air-tight on heating and the samples were sheared significantly during the loading process.

The 1D scattering curves were obtained using a line collimation system and captured using a Princeton Instruments-SCX CCD camera, with a sample-to-detector distance of $309.0 \mathrm{~mm}$ (exposure time $360 \times 10 \mathrm{sec}$ ). The 2D SAXS and SAXS/WAXS images used to investigate the orientational behaviour of samples were obtained using a point focus collimation and imaged on two-dimensional phosphor storage plates (exposure time $90 \mathrm{~min}$ ). Images were obtained using a Perkin Elmer Cyclone Plus imaging plate reader operated using OptiQuant software. A sample-to-detector distance of $261.2 \mathrm{~mm}$ was used. Unfortunately, equipment malfunction meant that the CCD camera was not available throughout the experimental work, so the investigation of some systems was limited to 2D studies, with 1D profiles then obtained via Azimuthal integration of the $2 \mathrm{D}$ data. 


\section{Optical Microscopy}

Polarising optical microscopy (POM) has long been a standard technique in the study of liquid crystals. This is because the anisotropy of different phases gives rise to optical textures with characteristic patterns, allowing the phases to be distinguished from one another. This provides a quick and convenient way to directly view a sample and obtain characteristic macroscopic structural information. The use of a heating stage allows temperature-based transitions to be observed and so aids in the construction of a phase diagram. However, not all phases can be distinguished using this technique and information inferred about surfactant microstructure is limited.

POM utilises the birefringence property that some liquid crystalline phases exhibit. Upon interaction with an anisotropic material, light is doubly refracted into two waves of different velocities and orthogonal polarisations (shown in Figure 2.9). The two waves have different refractive indices $(n)$, where $n=c / v$ ( $c$ is the velocity of light in a vacuum and $v$ is the velocity of light in the material). The refractive indices change with changes in polarisation and direction of propagation of light through the material.

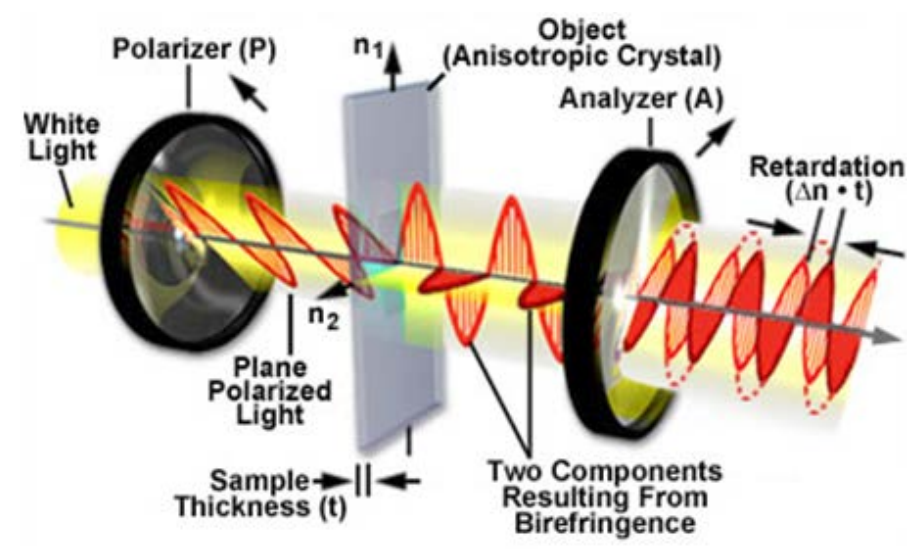

Figure 2.9: An anisotropic material between crossed polarisers displaying birefringence. Image property of Olympus America Inc. ${ }^{\odot 65}$

Any birefringence displayed by a mesophase is governed by its structural morphology. The three-dimensional order in cubic phases gives rise to equivalent refractive indices, such that $\mathrm{n}_{1}=\mathrm{n}_{2}=\mathrm{n}_{3}$, and so cubic phases do not exhibit birefringence and are optically isotropic. This is also the case for completely disordered phases, such as the sponge phase. In cases where two indices are identical, and so differ in only one direction, such that $n_{1}=n_{2} \neq n_{3}$, structures exhibit birefringence and are said to be uniaxial. This is the case for lamellar and hexagonal mesophases, with the birefringence given as $\Delta n=n_{3}-n_{1}$. Note that $n_{1}$ is often termed $n_{0}$ or $n_{\|}$, representing the ordinary index, and $n_{3}$ is often termed $n_{e}$ or $n_{\perp}$, describing the extraordinary index. The optical anisotropy in uniaxial systems can display positive or negative birefringence, dependent upon whether $n_{1}$ or $n_{3}$ is larger. When $n_{1}$ is larger, meaning that the refractive index perpendicular to the longest (or director) axis is larger (such as in hexagonal phases), the birefringence is considered to be negative. Conversely, when $n_{3}$ is larger than $n_{1}$, 
due to the refractive index parallel to the director axis being larger (as is generally the case in the lamellar phase), the birefringence is positive. In cases where all three refractive indices are distinct, the materials are termed biaxial, and the birefringence is defined as the difference between the largest and smallest indices. This is the case for rarer phases such as rectangular and distorted variations of hexagonal phases.

In a typical polarising optical microscope, light travels from the source and through a polariser before interacting with the sample, which is mounted on a rotatable heating stage to control the orientation and temperature of the sample. After passing through the sample, light passes through a second polariser placed at a $90^{\circ}$ angle to the first. This crossed polarisation means that the only light observed has undergone a change in polarisation, due to optical retardation with the two perpendicular components growing out of phase. A camera mounted above the microscope allows the recording of textures as high definition images.

The orientation of a liquid crystalline phase will normally vary throughout a sample under a microscope. This is particularly true for a phase with many differently orientated domains or defect points, such as a lamellar phase, where the director axis drastically changes. Where the director is orientated parallel or perpendicular to the polarisers, the area will appear dark as the incident light propagates according to a single index of refraction, and no light passes through the second of the crossed polarisers. When the director is orientated at an angle between 0 and $90^{\circ}$, a phase difference between two indices of refraction is observed. The light is then elliptically polarised and will pass through both crossed polarisers, resulting in the area appearing bright. As the orientational order changes upon mesophase evolution (via changes in temperature, concentration or shear), the indices of refraction and thus optical textures observed will vary. As the amount of optical retardation is a function of the wavelength of the light, a variety of colour intensities can be observed. Additionally, microscopes can be fitted with quarter-wave plates, which convert linearly polarised light to circularly polarised light. These plates can enhance the visibility of samples and can be used to determine the sign of birefringence of materials. This is particularly useful in identifying different phases within a single sample.

Examples of POM textures that will be regularly encountered in this thesis are shown in Figures 2.10 and 2.11. Figure 2.10 shows two examples of lamellar defects, while Figure 2.11 shows images characteristic of lamellar and inverse hexagonal phases with various defect structures.
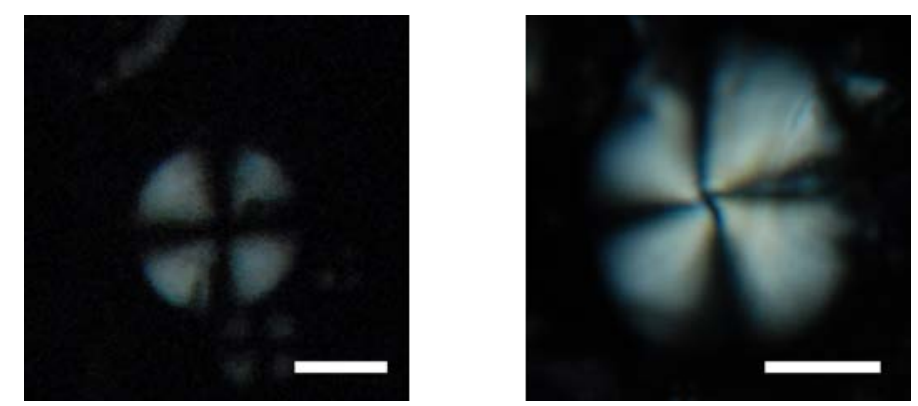

Figure 2.10: Examples of FCD-II (left) and FCD-I (right) defects commonly encountered in this research, observed using POM. Scale bars are $40 \mu \mathrm{m}$. 

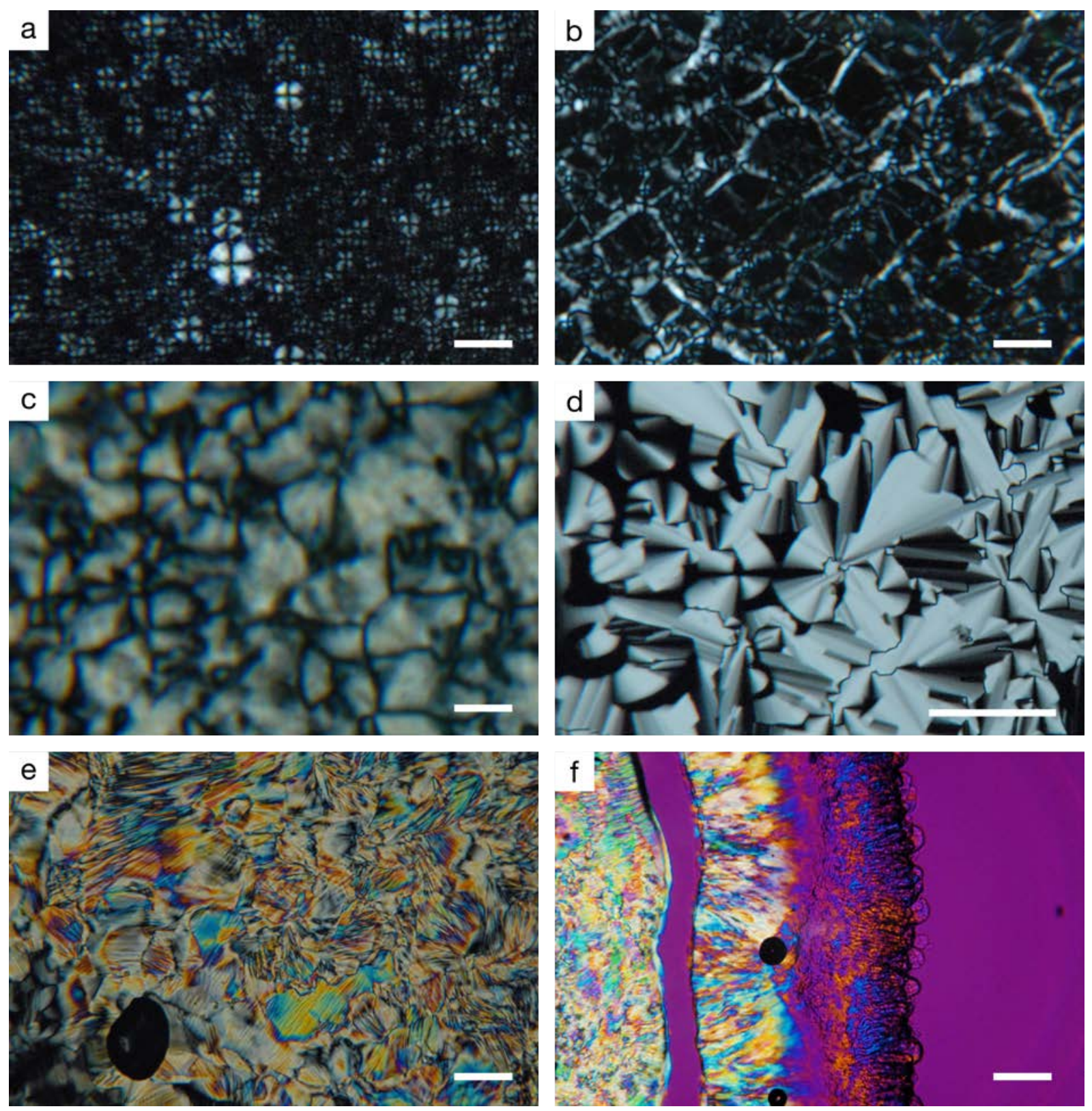

Figure 2.11: Examples of POM textures encountered in this research. Scale bars are $50 \mu \mathrm{m}$. (a) $A L_{\alpha}$ phase with a number of Maltese crosses of various sizes that identify the primary defect type as that of FCD-II defects (spherulite/onion-like structures of positive Gaussian curvature and disclination strength $s=+1$ ).

(b) $A L_{\alpha}$ phase with defects of predominantly negative Gaussian curvature, of the type FCD-I, that aggregate to form chains, termed oily streaks. Individual defects may be observed, or defects may form striated bands in which individual FCD-Is cannot be distinguished.

(c) $A L_{\alpha}$ phase at high concentration in which the various defect structures are packed into a characteristic mosaic-like pattern.

(d) An inverse hexagonal phase exhibiting a characteristic fan texture. Focal conic surfaces form to minimise the local strain energy and distinct lines or brushes separating domains are due to the changing curvature around line disclinations of $s= \pm 1 / 2$.

(e) A second representation of an inverse hexagonal phase, where a fan-like pattern is still observed, but the phase appears brightly coloured due to the thickness of the sample.

(f) A concentration gradient image captured using a quarter-wave plate. Water penetrates towards the pure surfactant creating a gradient of the phases found in the system. 
Despite being one of the standard techniques used to characterise phase behaviour in the broad field of liquid crystal research, POM does have several drawbacks and introduces some experimental difficulties, particularly when studying lyotropic systems. These problems arise from difficulties associated with preserving the structure of the sample upon preparation and keeping the concentration of the sample constant over the time period of heating and/or study. Sandwiching a sample between a glass slide and a cover slip inevitably introduces the possibility of applying significant shear to a sample and therefore changing its morphology. It is also a challenge to ensure that samples of different viscosities are examined at comparable thicknesses. To minimise these problems in this thesis, all microscope slides were prepared in triplicate and images were only captured when considered to be representative of the texture presented by the sample on all slides. Cover slips were placed onto the sample with care directly under the microscope to ensure no deviation from the texture observed in the bulk sample droplet. In addition, flattened capillaries with defined $100 \mu \mathrm{m}$ path lengths (termed "microslides") were used to compare different concentrations at the same thickness. Samples were not studied solely using microslides due to loading shear often resulting in monodirectional alignment of the sample.

As all the systems studied contain water as the solvent, unsealed samples can lose or gain water over time resulting in a concentration change. However, such changes occur gradually (starting at the edge of the slide) and are generally clearly evident. To prevent this issue, all slides were prepared directly before analysis and any slides that were kept for a longer period of time were sealed with a quick-drying epoxy resin that was not miscible with the sample. Potential concentration changes are more problematic in temperature investigations and sample confinement is necessary as sample flow is promoted by the decrease in viscosity and increase in thermal energy associated with heating. In these cases, samples were sealed with an epoxy putty (KnetFlux ${ }^{\circledR}$ ) with a melting temperature of $240{ }^{\circ} \mathrm{C}$, or in a microslide sealed with polytetrafluoroethylene $\left(\right.$ Teflon $\left.^{\circledR}\right)$ tape, which has a melting point of $327^{\circ} \mathrm{C}$.

All microscope slides were examined using a Nikon OPTIPHOT2-POL optical microscope equipped with crossed polarisers, a quarter-wave plate, and a rotating sample stage, at 40, 100 and $200 \times$ magnification. The temperature was controlled using a Mettler Toledo heating stage accurate to $\pm 0.4^{\circ} \mathrm{C}$. Images were acquired using a Nikon D200 digital single-lens reflex camera fitted to the microscope. Concentration gradients were prepared by the standard isothermal concentration gradient method, in which a small amount of pure surfactant is placed between a microscope slide and cover slip (either as a thin layer of the pure wax or as melted crystals) and a droplet of water added at one side of the cover slip. As the water gradually penetrates the surfactant, a gradient is formed and the phase progression of the system is revealed through the appearance of bands of characteristic patterns. The water penetration was observed under the microscope, and images were captured under crossed polarised light with and without a quarter-wave plate. Where possible, microslides were loaded by capillary action, but many samples were loaded via syringe due to their high viscosities. All POM images were collected at room temperature unless stated otherwise. 


\section{Cryogenic Scanning Electron Microscopy}

In contrast to SAXS, which provides structural information on a macroscopic scale, scanning electron microscopy (SEM) can be used to probe defect architectures and local structure in real space. The sample surface is imaged by a high-energy electron beam, focused to a spot less than a nanometre in diameter, and scanned in a raster pattern over a rectangular area of the specimen surface. The resulting signals arising from the electron beam-specimen interactions produce high-resolution images revealing the surface topography with structural detail as low as 1 to $5 \mathrm{~nm}$ in size. The narrow electron beam results in SEM micrographs having a large depth of field, producing images that detail the three-dimensional structure at the sample surface. Reducing the size of the raster scan increases the magnification, and a typical SEM has a wide range of control over magnification, normally from $\times 10$ to $\times 500,000$ (however, the resolution capabilities are reduced when SEM is adapted for cryogenic use).

Various signals are produced when the primary electron beam interacts with the sample surface in SEM analysis, including secondary electrons, back-scattered electrons, characteristic X-rays, cathodoluminescence, Auger electrons, transmitted electrons and specimen current. Secondary electron detectors are generally found in all SEMs, and this detector type was used to image lyotropic liquid crystalline samples in this work. Secondary electrons are produced when an electron from the primary beam collides with an electron from a specimen atom, causing an ionisation event and the emission of an electron. At the detector, the signals representing these electrons are electronically amplified and displayed as variations in brightness on a cathode ray tube, before being captured digitally.

The specimen chamber in an SEM instrument is kept under high vacuum and so conventional SEM samples must be dry. This is obviously not feasible for water-based samples such as the surfactant/water systems in this work. Instead, a replica of the sample must be made or cryogenic scanning electron microscopy (cryo-SEM) used, in which a droplet of sample is frozen and viewed at low temperature (the microscope's specimen chamber is maintained at $-140{ }^{\circ} \mathrm{C}$ ). These techniques allow the samples to be etched and fractured, exposing the internal structure and allowing visualisation of internal order. As such, cryo-SEM can be used to provide insight into the degree and nature of defects in lyotropic systems. As well as visualising domain sizes and point defects, information about sample topology can be inferred through visualisation of the curvature and connectivity of surfaces revealed by sublimation.

While various freeze-fracture electron microscopy techniques have been used in previous studies of lyotropic liquid crystalline systems, instrumentation advances have resulted in a change in methodology. Compared to the replica-based procedures used previously, modern techniques allow samples to be viewed under the microscope directly. Both methods involve similar freezing and fracturing, with a thin layer of sample sandwiched between two plates being rapidly vitrified for maximum preservation of the structural morphology. Previously, the fracture face was replicated with carbon and platinum vapour deposition creating a thin film, which was then studied under the microscope. While this was a time-consuming process producing fragile 
films, the films were resistant to electron-beam damage and the resolution of the microscope was not limited by the need to operate at low temperatures. This allowed the capture of highresolution transmission electron microscopy (TEM) images, and samples could be stored for later reference.

With modern instruments, samples can be frozen, fractured and then viewed directly under the microscope. This is a faster process, and facilitates the preparation of much larger numbers of samples. The greater number of samples, and so increased diversity of fracture planes, provides a better overview of the features present in samples and greater confidence in the validity/reproducibility of results. However, samples are more susceptible to electron-beam damage, cannot be stored and re-imaged, and do not allow for the resolution required to capture high magnification images of single defect centres or bilayers. The advantages of direct imaging have been heralded; ${ }^{66}$ however, the decline of replica-based methods has also been lamented. ${ }^{67}$ Samples in this work would ideally have been prepared using both methodologies and analysed via SEM and TEM; however, the capability to prepare replicas (which may have allowed for improved resolution, especially of highly viscous samples) was not available.

As SEM rather than TEM was used in this work, the achievable resolution was limited and individual bilayers and connections could not be resolved. However, a broad array of data were available at low magnifications, and the direct imaging of samples provided a superior depth of field, revealing global structural information. In particular, defect structures from the macroscale to the sub-microscale were observed, and the cryo-SEM images obtained corroborate the POM investigation and further reveal the nature of domains, domain boundaries, point defects, and series of line defects. Despite the achieved resolution being insufficient to observe local structural defects, the topological nature of the sample can still be cautiously inferred as some local features manifest on larger lengthscales, and the variety and depth of fracture planes reveals the 3D surface, thus providing information regarding curvature and degree of connectivity/porosity. Before cryo-SEM can be confidently used as a characterisation technique, reliable freezing and sublimation procedures must be established, and the reproducibility and representativeness of each sample confirmed. Additionally, it should be used as a complementary technique rather than in isolation, as the unpredictable nature of the sample fracture can result in sample features being misidentified. In this work, the cryo-SEM images produced are in excellent agreement with those obtained by POM, which (alongside the study of multiple samples of equivalent concentrations) indicates that the sampled materials are representative of the bulk. A large range of images are provided in this work to reveal structures on different lengthscales, and low magnification images are provided in addition to those at higher magnifications to demonstrate that the structural features highlighted at high magnification are representative of the whole sample. A non-destructive sublimation procedure was established after trialling a range of sublimation times and temperatures, and samples were imaged before and after sublimation to ensure the preservation of structural features (it should be noted that sublimation can cause an apparent swelling of structural features but this was not evident upon use of the established procedure). 
A common method of sample preparation for cryo-SEM is a plunge-freeze method in which a small amount/droplet of sample is mounted on a stub attached to a sample holder at one end of a long transfer rod. The sample is rapidly frozen in a liquid nitrogen slush $\left(\sim-210^{\circ} \mathrm{C}\right)$ before being transferred under vacuum to the preparation chamber. The sample is then fractured with a knife to expose the internal microstructure, sublimed to remove superficial water, and coated with a thin layer of platinum to make it conductive. The sample is transferred to a cold stage in the SEM chamber and observed at low temperature. Although this method was followed initially in this work, it was found to be inadequate as much of each sample was not frozen/vitrified at a sufficiently rapid rate, and samples were riddled with artefacts due to the formation of ice crystals upon quench cooling. Examples of poor freezing are shown in Figure 2.12, in which only a small region at the edge has negligible ice damage. Similar images are frequently found in the literature, despite the obvious damage to the sample microstructure. ${ }^{68}$ Cryo-protective agents such as glycerol and DMSO are available, but were not used in this study as additives can dramatically affect the phase behaviour of liquid crystalline systems.
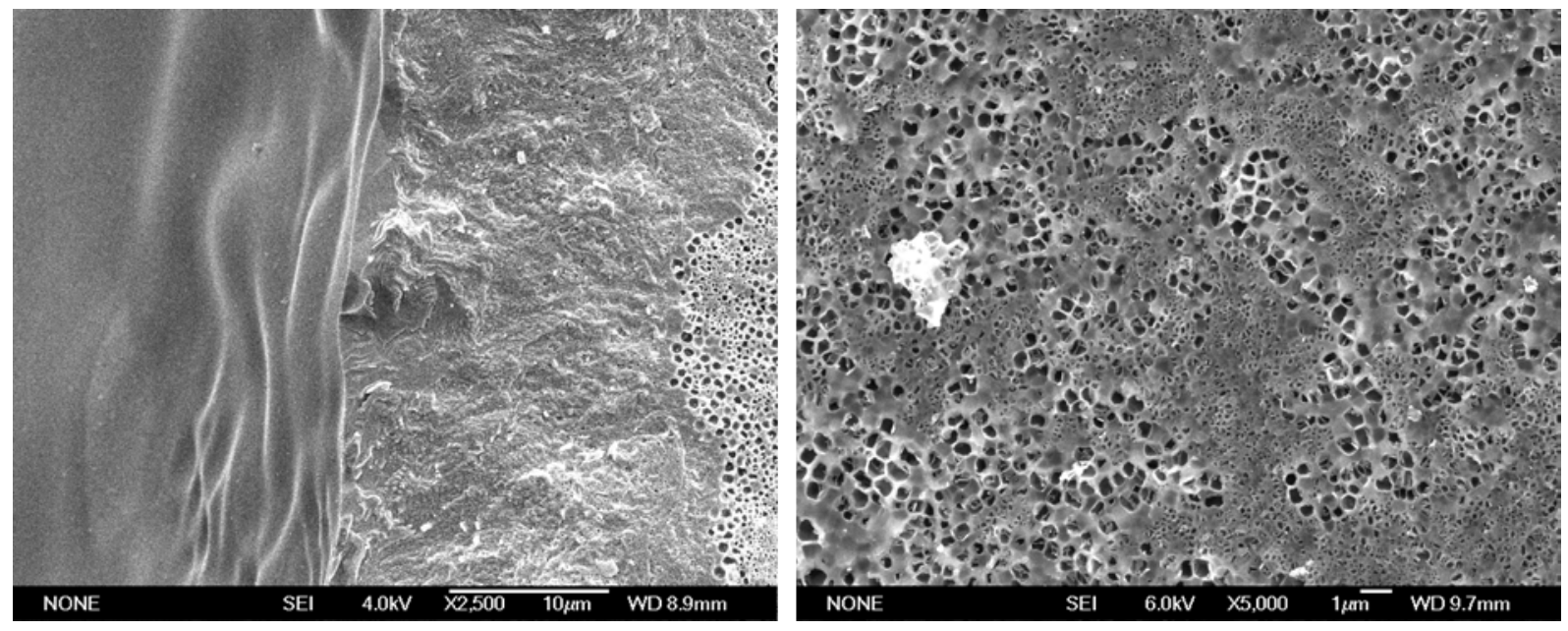

Figure 2.12: Cryo-SEM images of samples frozen at an insufficiently rapid rate. The three areas of (a) show the outside of the droplet, the edge of the droplet providing "real" information, and the damaged internal microstructure (also shown in (b), note the non-uniform size of the pores).

To improve sample vitrification, the above method was altered to maximise the surface area-tovolume ratio of the sample. A pair of tweezers was modified to enable clamping of two copper rivets together, and small sample volumes were sandwiched between the rivets creating a thin layer of sample. The tweezers were then plunged into a nitrogen slush and the rivets mounted on a sample stage and transferred to the SEM preparation chamber. This sandwiching method is based on a report by Issman et al. ${ }^{69}$ and produced consistent results and predominantly uniform samples (see Figure 2.13).

Due to the additional complications involved with frozen samples, we were unable to use the SEM to its full resolution capability for two reasons. Firstly, the cooling system used to keep the microscope chamber at $-140{ }^{\circ} \mathrm{C}$ resulted in significant vibration and hence image distortion. This problem was most severe at high magnifications, when images were captured at slow scanning rates in a small raster pattern. Secondly, the surfactant samples were prone 

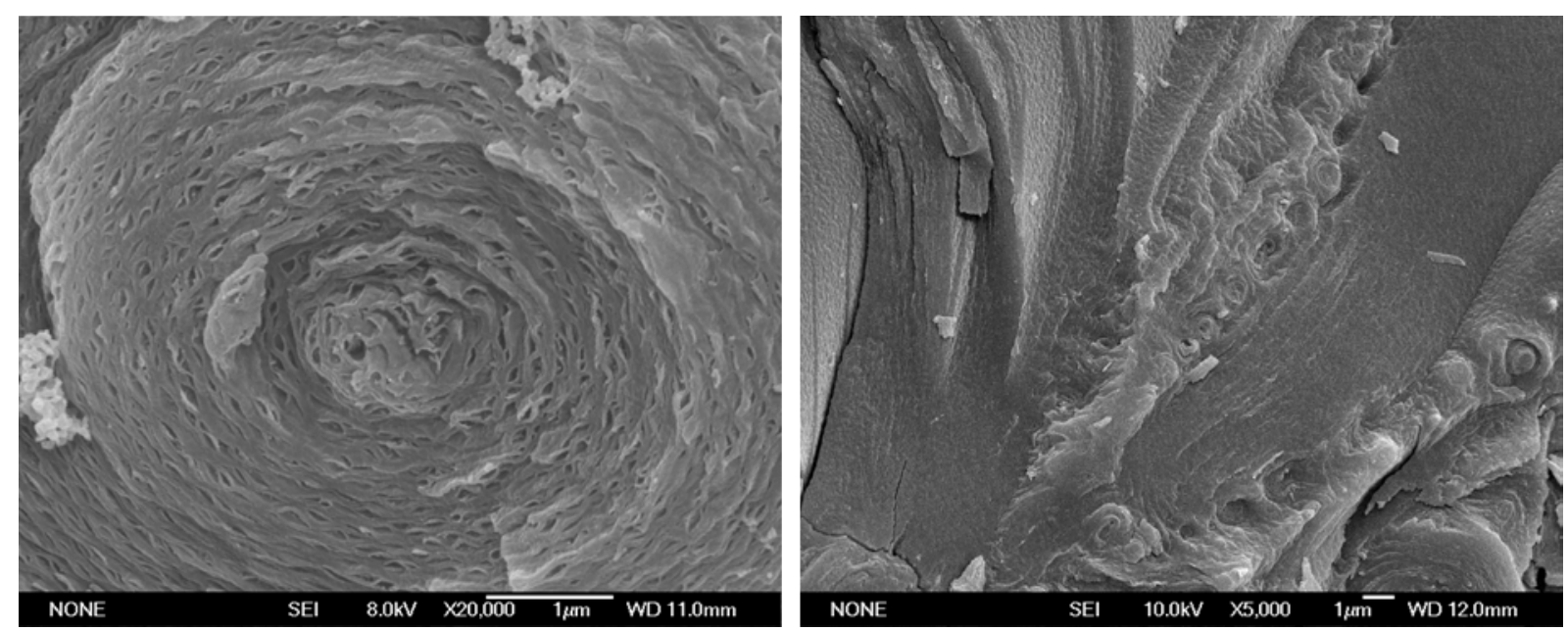

Figure 2.13: Cryo-SEM images obtained via the sample preparation method used in this work. Image (a) shows an individual defect, while (b) shows series of aligned defects and regions of lamellar bilayers orientated in different directions.

to significant damage, in relatively short periods of time, when exposed to the electron beam. This was observed in all samples at magnifications of $\times 40,000$, and at magnifications as low as $\times 5,000$ in highly concentrated/viscous samples. The fragility of the samples also meant that backscattered electron analysis was not feasible, which is unfortunate as the combination of this technique and high resolution imaging may provide important information about the nature of the surfactant counterion.

During the later stages of this work, a liquid propane jet-freezer (generally considered second only to a high-pressure freezer for vitrification of thick or viscous samples ${ }^{\ddagger}$ ) became available for use. This technique involves sandwiching a droplet of sample between two copper plates, creating a thin layer of sample to allow efficient freezing, and is comparable to the sandwiching of material between two copper rivets discussed above. The images obtained using this method were comparable with those obtained via the previously described method, providing similar levels of structural detail (see Figure 2.14).

The experimental work was conducted using a Jeol JSM-6500F microscope fitted with a Gatan Alto 2500 cryo attachment, operated at accelerating voltages of 3-10 kV. The cryo-preparation chamber was operated at $-140{ }^{\circ} \mathrm{C}$ and samples were sublimed at a maximum of $-95^{\circ} \mathrm{C}$ for $5 \mathrm{~min}$ to enhance the topological contrast, and coated with up to three $120 \mathrm{sec}$ sputter coatings of Pt. Upon cooling to $-140^{\circ} \mathrm{C}$, samples were transferred to the microscope chamber via an interlocked airlock and the microscope was maintained at $-140^{\circ} \mathrm{C}$.

\footnotetext{
${ }^{\ddagger}$ High-pressure freezing efficiently vitrifies samples as the pressure $(2,100 \mathrm{bar})$ is too high for water to crystallise. However, alcohol is used as a pressure-transducing liquid, potentially making it unsuitable for lyotropic systems. ${ }^{69}$
} 

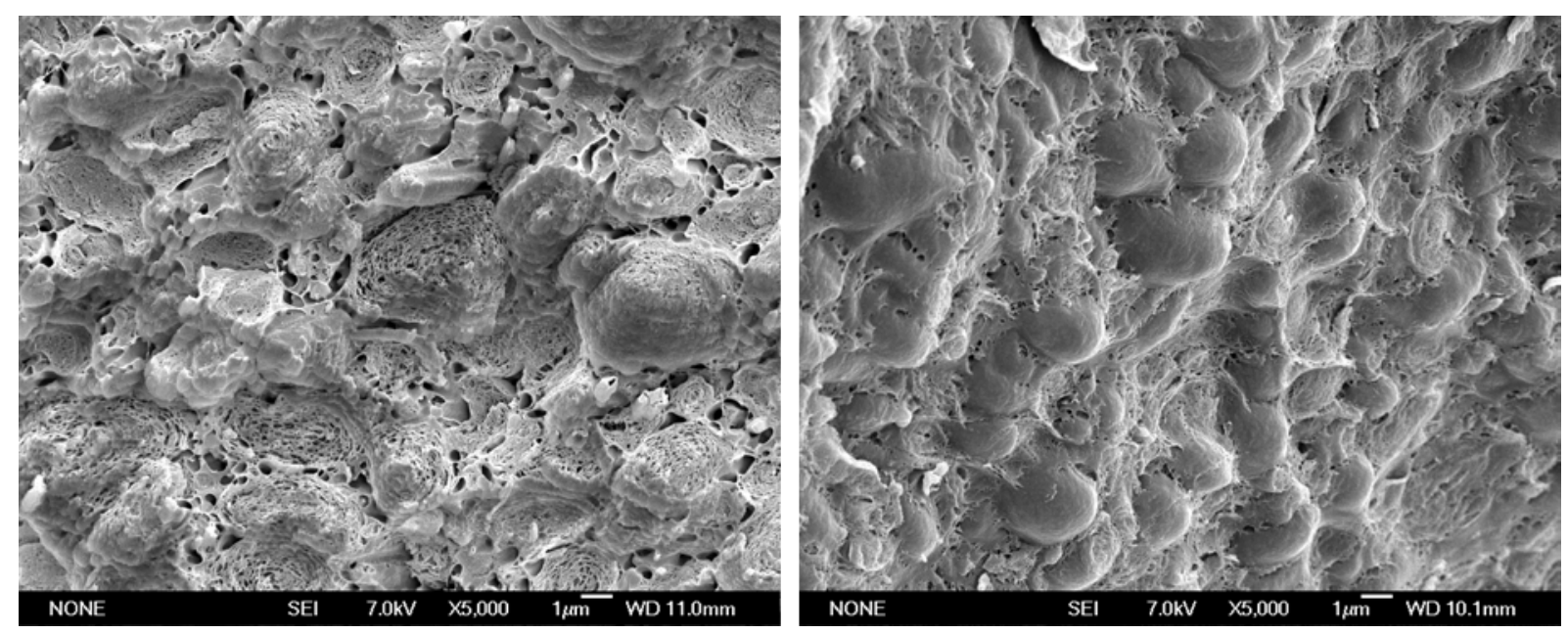

Figure 2.14: Cryo-SEM images for a single sample obtained with different sample preparation methods. The results of the method employed in this work (left) are comparable with those obtained using a jet-freezer (right). 


\section{Part I}

\section{Alkali Metal Counterions}




\section{Chapter 3}

\section{Sodium Aerosol OT}

\section{Sodium AOT: Introduction}

Sodium bis(2-ethylhexyl) sulfosuccinate is an anionic surfactant, commonly referred to by the commercial names Aerosol OT, AOT, docusate and DOSS (dioctyl sulfosuccinate). In this thesis it is referred to as Na-AOT, to allow distinction between the systems discussed in subsequent chapters, where different counterions are used. It is a two-tailed surfactant, with each tail further branched with an additional short alkyl chain (see Figure 3.1). Although the two hydrophobic (2-ethylhexyl) chains are identical, the molecule is asymmetric due to the structure of the polar sulfosuccinate headgroup. One ester group is separated from the sulfonate group by an additional methylene unit, giving rise to non-equivalent acyl groups. Thus, the branching point of the non-identical chains $(\mathrm{C} 1)$ is a chiral centre. Each tail has a further chiral centre within the hydrophobic region ( $\mathrm{C} 4$ and $\left.\mathrm{C}^{\prime}\right)$ due to the tail branching. The presence of three chiral centres results in eight possible stereoisomers that exist as four enantiomeric pairs. The hydrophilic headgroup is anionic upon dissociation of the sodium cation in solution. The linear length of the molecule has been reported as $\sim 12 \AA$, and $\sim 15 \AA$ when associated with a water molecule. ${ }^{70}$<smiles>[B]CCC[C@H](C[18OH])COC(=O)[Te][C](C(=O)OC[C@H](C[18OH])CCC[13CH3])S(=O)(=O)O[NH3+]</smiles>

Figure 3.1: Molecular structure of Na-AOT. 
Given the widespread importance of surfactants in a huge array of practical applications (the estimated annual global surfactant production exceeds 16 million tons, and is forecasted to reach 24 million tons with a market worth of US $\$ 46$ billion by $2022^{71}$ ) it is unsurprising that $\mathrm{Na}-\mathrm{AOT}$, a surfactant with a relatively low cost and ease of manufacture, has undergone extensive study. A structure search for $\mathrm{Na}$-AOT provides in excess of 14,500 references and highlights its increasing industrial importance (see Figure 3.2). ${ }^{\ddagger}$ It is used in a wide range of applications, including as an emulsifier, dispersant, wetting agent, food additive, pesticide adjuvant, and as a non-active component of various medications. Additionally, Na-AOT is on the World Health Organization's List of Essential Medicines, due to its use in treating constipation. ${ }^{72}$ It is also the most widely used surfactant in studies and applications involving reverse micelle encapsulation. ${ }^{73}$

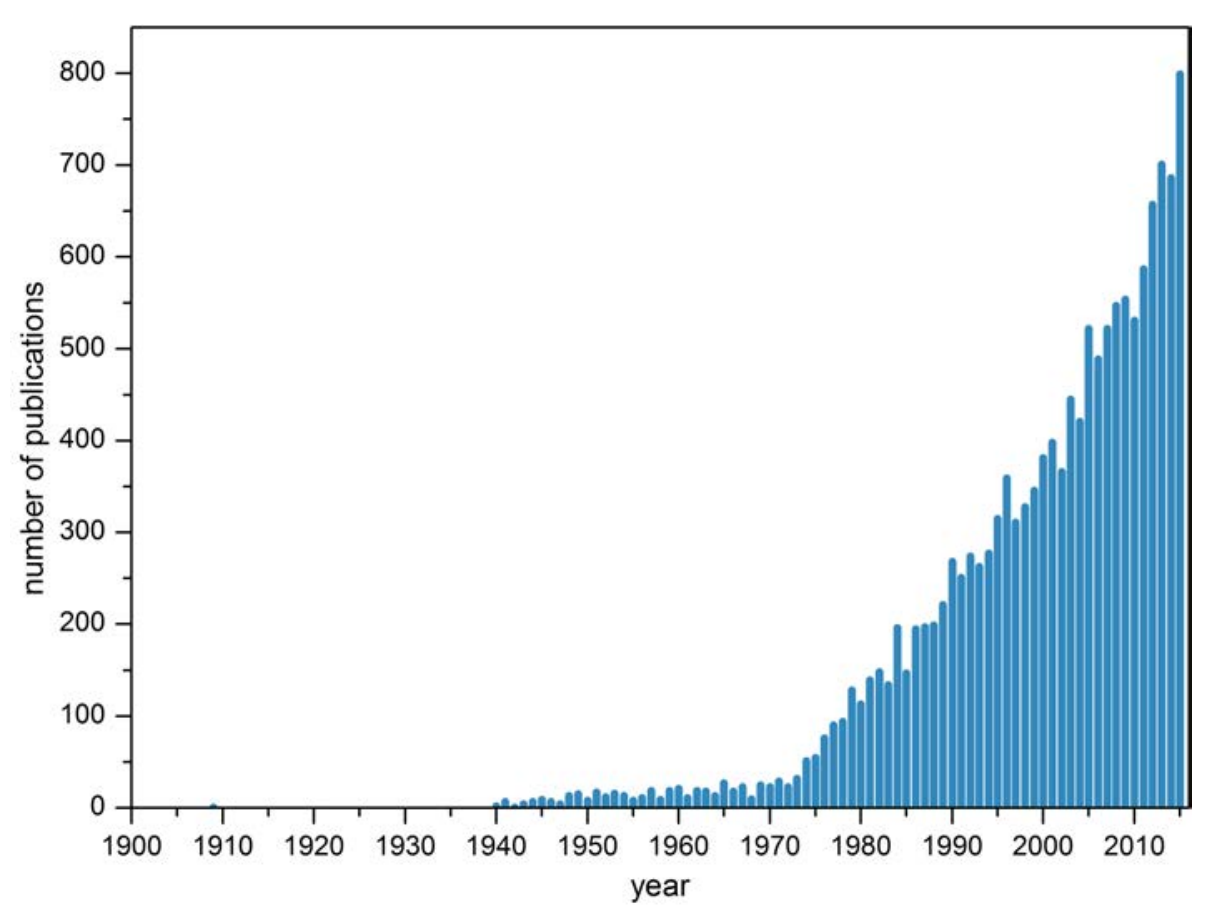

Figure 3.2: Plot showing the increasing number of Na-AOT publications between 1900 and 2015.

Aside from the low cost, Na-AOT is a popular choice for a diverse range of applications due to its numerous useful properties, which make it an efficient and versatile surfactant. It has a low CMC (0.12 wt \% in water), excellent solubility in both polar and non-polar solvents, and good thermal stability. ${ }^{48,74-76}$ Also, its ability to dramatically decrease surface tension makes it an excellent wetting agent. ${ }^{77}$ One of the most useful, and uncommon, properties of Na-AOT is the ability to form reverse micelles in oil/water mixtures in the absence of a cosurfactant. It was the first surfactant reported to have this property and has since been estimated to be utilised in over $90 \%$ of research using reverse micelles. ${ }^{73,78}$ As a cosurfactant is not needed, Na-AOT can form both discrete droplet and bicontinuous microemulsions in ternary systems that are more simple than the quaternary systems required by other surfactants to produce the same behaviour. As such, Na-AOT has often been studied as a model system for microemulsions due to its remarkable properties.

\footnotetext{
‡Literature search conducted November 2016.
} 
The versatility of Na-AOT is highlighted by the work of Nave et al., who produced a fourpart series of papers titled "What is so special about AOT?". ${ }^{48,75,79,80}$ They investigated the underlying reasons for the efficiency of $\mathrm{Na}$-AOT in a variety of roles (which often outperforms other surfactants), and whether or not the cause is unique to its molecular structure. They produced a series of analogous molecules with structural variations in the degree of chain branching, headgroup structure and tail region polarity, and revealed several interesting findings. All of the branched surfactants studied form ternary systems with microemulsion phases. In contrast, none of the equivalent linear surfactants are able to stabilise microemulsions without the addition of a short-chain alcohol to act as a cosurfactant.

Calculations of the effective headgroup area of Na-AOT at the hydrophobic/hydrophilic interface showed that the addition of branching to the surfactant tails results in an area up to $20 \AA^{2}$ larger than in the linear variants (an increase of $\sim 40 \%$ ) at the CMC. ${ }^{48}$ The increase in the effective interfacial area was also shown to be greater if an additional $\mathrm{CH}_{2}$ group was added to the tail region rather than the headgroup. This highlights the importance of the surfactant tails within these systems in determining the molecular packing. ${ }^{79}$ While Nave et al. concluded that $\mathrm{Na}$-AOT is not unique, in that it fits within the logical progression of series of analogues with varying tail structures, they also showed that formation of microemulsions requires a certain degree of disorder within the hydrophobic domain so that negatively curved interfaces can be stabilised. In most systems, this disorder has to be provided through the inclusion of an additional component, whereas in Na-AOT, this disorder is provided through the alkyl branches. Nave et al. showed that the sulfosuccinate motif can be used to produce a range of versatile surfactants with properties that can be significantly altered by modification of the alkyl chains. In particular, such changes alter the solubility of the surfactants with respect to temperature. In the case of $\mathrm{Na}-\mathrm{AOT}$, the combination of the relatively short tails and the positioning of the ethyl branches at $\mathrm{C} 4$ and $\mathrm{C}^{\prime}$ ', in close proximity to the headgroup, result in a hydrophobic/hydrophilic interface that allows the beneficial properties of Na-AOT (including its excellent solubilisation properties) to prevail at room temperature, another major factor increasing its practical use.

The surfactants synthesised by Nave et al. were not investigated in terms of liquid crystalline behaviour, as their study primarily focused on the solubility properties of the surfactants at high dilutions. However, in the case of Na-AOT, the full aqueous phase behaviour has received considerable attention in the literature, and the system has been shown to form an array of lyotropic phases. The inherent molecular shape of Na-AOT, which can in general terms be described as having a slightly bulkier hydrophobic region than hydrophilic headgroup, results in the preferential formation of lamellar and Type II structures with little or negative interfacial curvature. The fact that these structures persist over a wide range of surfactant concentrations and temperatures, along with the commercial importance and accessibility of Na-AOT, led to the system being used as a model in the early study of bilayered systems. Currently, the liquid crystalline phases of Na-AOT are used in a variety of applications, such as templating of functional materials ${ }^{81,82}$ and drug delivery systems. ${ }^{83,84}$

Despite being extensively studied, with investigations spanning over five decades, the lyotropic behaviour of $\mathrm{Na}$-AOT is still not completely resolved. The evolution of the microstructure of the 
phases upon increasing concentration is still a subject of debate. Simplistic rationalisations of surfactant self-assembly, such as the surfactant packing parameter, have been shown to neglect the role of the hydrophobic tails on the interfacial packing. ${ }^{85}$ The work by Nave et al. shows that the bulky and branched tails in Na-AOT are clearly important in facilitating the formation of cosurfactant-free microemulsions. It is likely that the tails are also of fundamental importance in governing the aqueous phase behaviour at higher concentrations to a greater extent than in "normal", unbranched surfactants.

\section{Sodium AOT: Phase Behaviour}

The binary $\mathrm{Na}-\mathrm{AOT} /$ water phase diagram is shown in Figure 3.3. At surfactant concentrations above the CMC (0.12 wt \% Na-AOT), a micellar phase extends to $2.5 \mathrm{wt} \% \mathrm{Na}$-AOT (at $25^{\circ} \mathrm{C}$ ). A relatively wide two-phase region exists between this aqueous solution and a lamellar phase formed at higher concentrations. This two-phase region consists of micelles and dispersed lamellar bilayers, and has been reported to exist up to $\sim 10 \mathrm{wt} \% \mathrm{Na}$-AOT. ${ }^{63,86}$ However, it has also been noted that for Na-AOT concentrations up to $17.5 \mathrm{wt} \%$, the lamellar phase formed is unstable, and samples are liable to phase separate under the high gravity fields of centrifugation generally used in achieving sample equilibration. As such, some researchers have chosen to assign the two-phase to lamellar phase boundary to a Na-AOT concentration of $17.5 \mathrm{wt} \%{ }^{87}$

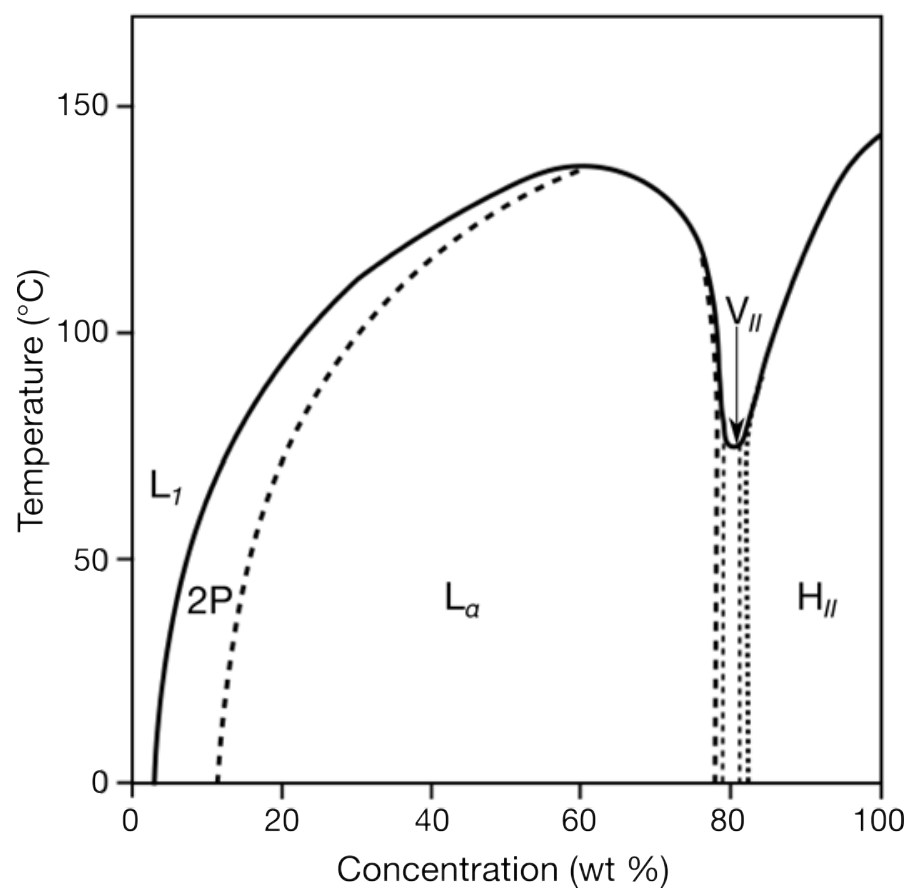

Figure 3.3: Phase diagram of Na-AOT, showing a two-phase region (2P) and lamellar $\left(\mathrm{L}_{\alpha}\right)$, inverse bicontinuous cubic $\left(\mathrm{V}_{I I}\right)$ and inverse hexagonal $\left(\mathrm{H}_{\| I}\right)$ phases, reproduced from Rogers and Winsor. ${ }^{64}$

A lamellar phase dominates the phase diagram of the system, extending up to $77 \mathrm{wt} \%$ $\mathrm{Na}-\mathrm{AOT}$ at $25^{\circ} \mathrm{C}$. This phase has, however, been reported to show anomalous or discontinuous 
behaviour at concentrations of approximately 33-45 wt \%. Within this range the lamellar phase has been reported to show unusual electrical conductivity, birefringence, water diffusion and swelling behaviour upon changes in concentration and temperature. ${ }^{47,63,86}$ This behaviour indicates that a structural rearrangement may occur within this region, incongruous with classical lamellar swelling behaviour. Due to its discontinuous nature, the lamellar phase has previously been described in terms of three concentration regions - termed low, intermediate and high - which will be referred to as LCR (10-33 wt \% Na-AOT), ICR (33-45 wt \% Na-AOT) and HCR (45-76 wt \% Na-AOT), respectively. The lamellar phase of the system is discussed in greater detail in the next section.

At Na-AOT concentrations above 77 wt \%, the system forms Type II structures, with an inverse bicontinuous cubic phase and an inverse hexagonal columnar phase observed. The viscous bicontinuous cubic phase exists over a narrow concentration range between 77 and 82 wt \% Na-AOT. The three equivalent refractive indices of the cubic phase mean this phase is isotropic and shows no birefringence under a crossed polarised microscope. The phase has been confirmed by X-ray diffraction studies and presents up to eight Bragg reflections of varying intensities, in the ratio $\sqrt{ } 3: \sqrt{ } 4: \sqrt{ } 7: \sqrt{ } 8: \sqrt{ } 10: \sqrt{ } 11: \sqrt{ } 12: \sqrt{ } 13 .{ }^{88}$ Thus, the phase has been characterised as a body-centred cubic structure with the space group la3d and the unit cell parameter is reported as $69 \AA$. Such structures exist as short identical amphiphilic rods, wherein the surfactant headgroups are facing inwards, and the rods are assembled in equivalent, interpenetrating, but unconnected, three-dimensional networks. Above $82 \mathrm{wt} \% \mathrm{Na}-\mathrm{AOT}$, the system forms an inverse hexagonal phase, wherein water is confined within amphiphilic rods that are arranged in a two-dimensional lattice. This phase presents characteristic fan-like textures under crossed polarised microscopy and produces Bragg reflections in the ratio $1: \sqrt{ } 3: \sqrt{ } 4$ by $X$-ray diffraction, ${ }^{88}$ indicative of a $\mathrm{P} 6 \mathrm{~m}$ space group. ${ }^{\ddagger}$ Molecular modelling studies have further indicated that whilst the configuration of pure Na-AOT is hexagonal, it is trigonal on a local scale, with the cross-section of each rod containing three Na-AOT molecules with polar headgroups facing inwards and the protruding tails efficiently packed, with tessellating ethyl branches. ${ }^{89}$

Aside from changes with surfactant concentration, the weak interactions that govern the selfassembly of the Na-AOT system are dependent on changes in temperature, ionic strength, $\mathrm{pH}$ and additives. The effect of temperature is shown in the phase diagram, which shows that the temperature at which the transition to an isotropic solution occurs changes significantly with concentration. This transition temperature increases with concentration up to approximately $60 \mathrm{wt} \%$, decreases in the vicinity of the cubic phase, and increases again in the hexagonal region.

Of the ternary systems that have been produced with $\mathrm{Na}-\mathrm{AOT}$, the inclusion of $\mathrm{NaCl}$ is most relevant to this work, as it shows that the phase behaviour of the system can be manipulated through changing the electrostatic contribution to the free energy. In particular, it implies that

\footnotetext{
${ }^{\ddagger}$ Note that it has also previously been reported that the $\sqrt{ } 4$ reflection is not observed in the Na-AOT system, but that the $\sqrt{ } 7$ is observed. ${ }^{89}$ The SAXS experiments carried out for this thesis are in agreement with the data published by Ekwall et al., ${ }^{88}$ with peaks in the ratio $1: \sqrt{ } 3: \sqrt{ } 4$ observed. However, the intensities of the $\sqrt{ } 4$ and $\sqrt{ } 7$ reflections may be dependent on concentration, equilibration time or shear effects upon sample loading.
} 
the bilayer elastic constants $\kappa$ and $\bar{\kappa}$ are susceptible to these changes. It has been reported that the increase in ionic strength upon addition of $1.3-1.7 \mathrm{wt} \% \mathrm{NaCl}$ to the system results in the stabilisation of the lamellar region to concentrations as low as $5 \mathrm{wt} \% .{ }^{90}$ The addition of $\mathrm{NaCl}$ in excess of $2 \mathrm{wt} \%$ promotes the formation of sponge and bicontinuous cubic phases dependent upon the Na-AOT concentration, and the coexistence of sponge and lamellar phases in the water-rich region of this system.

In the $\mathrm{Na}-\mathrm{AOT} / \mathrm{H}_{2} \mathrm{O} / \mathrm{NaCl}$ system, the stabilisation of the lamellar phase to low $\mathrm{Na}-\mathrm{AOT}$ concentrations indicates that the bending rigidity of the Na-AOT bilayer $(\kappa)$ is in the range of the thermal energy $k_{\mathrm{B}} T$, such that thermal undulations may also be a governing factor in the phase stabilisation. The formation of a sponge phase, upon the addition of relatively small amounts of electrolyte, indicates that the lamellar phase is already subjected to a high degree of curvature and that it likely contains connective defects between bilayers. The topology of the sponge phase is induced due to changes to $\bar{\kappa}$. If both $\kappa$ and $\bar{\kappa}$ are susceptible to change, and these changes induce phase transitions, upon shielding of the electrostatic contribution of the counterion, then it is likely that altering the counterion itself will also bring about different phase behaviours, a matter further discussed in subsequent chapters.

\section{Sodium AOT: Lamellar Phase Literature Review}

As stated previously, although the phase diagram of the Na-AOT/water system was reported in 1967, the evolution of the lamellar phase microstructure over its full concentration range has not been satisfactorily characterised. As the primary focus of this thesis is the formation and manipulation of the lamellar phases produced by a family of AOT-related systems, the anomalies reported for the Na-AOT lamellar phase must be addressed before the system can subsequently be used as a base system for comparison with related surfactants. To do this, we must first review the work reported in the literature for the Na-AOT system and consider the validity of the conclusions. The liquid crystalline behaviour of the system has received much attention, with reports spanning over five decades, yet the system still provokes debate. Reports in the literature provide conflicting and sometimes paradoxical results, and are rationalised in different ways. Various structural models have been proposed to account for the anomalies in the system, but as yet, no single model has adequately explained all the experimental observations.

One reason for the confusion surrounding the Na-AOT system may stem from the fact that it was among the first systems to have its liquid crystalline phase behaviour fully characterised. Binary and tertiary Na-AOT systems underwent a period of great interest in the late 1960s and early 1970s, as the field of liquid crystals became popular. These initial investigations provided insight into the chemistry of concentrated surfactant systems and laid the foundations of later research. However, much of this work pre-dates many modern experimental techniques and instrumentation advances, and as such, it should not be surprising that some of the conclusions drawn have since been shown to be erroneous by subsequent advances in the understanding 
of the self-assembly of lyotropic liquid crystals. However, this early work is still frequently cited in the literature and some of the proposed models used to explain the behaviour of the lamellar phase incorporate these outdated ideas. In particular, the SAXS data reported by Fontell have misled later researchers and will be discussed in more detail below. ${ }^{63}$

Generally, research fields display a cumulative increase in the knowledge base, with newer studies building upon those that preceded them. However, the literature relating to the liquid crystalline behaviour of $\mathrm{Na}$-AOT does not always follow this logical progression. This may in part be due to the surfactant having been referred to by a variety of names in different fields. Additionally, much of this research pre-dates the modern-day convenience of electronic resources such as online database searches and supplementary data files, meaning that much of the research was completed in comparative isolation, with little knowledge of concurrent or previous studies. One significant example is the introductory statement in a paper by Sheu et al. in 1987 (more than 20 years after the phase diagram of the Na-AOT system was produced) that the system forms "a mesomorphous phase consisting of parallel rod-like aggregates of AOT molecules in hexagonal array" below $29 \mathrm{wt} \% \mathrm{Na}-\mathrm{AOT}$, and a lamellar phase up to a Na-AOT concentration of $82 \mathrm{wt} \% .{ }^{91}$ Like others before us, ${ }^{92}$ we can find no published evidence to support this claim, and our own investigation shows it to be erroneous. The fact that a cubic phase was not reported at 78-82 wt \% Na-AOT implies that this assertion may have been based upon outdated observations. Regardless, these claims clearly contradict the accepted behaviour of the system, yet the aggregation behaviour at concentrations below 29 wt \% $\mathrm{Na}-\mathrm{AOT}$ has been cited as if accurate as recently as $2015 .^{93}$

A further possible complication is the report from Hubbard et al. that Na-AOT samples of some concentrations can take over three months to fully equilibrate. ${ }^{94,95}$ This raises the question of whether or not different research groups have reported upon the system at different states of equilibration, rather than the truly energetically preferred state. If studies of the system have been carried out on samples that are actually in different metastable states or have significantly different defect structures, then it may explain some of the erroneous data and conflicting reports that can be found in the published literature.

The above issues make it difficult to discuss the history of Na-AOT research in a chronological fashion, and the sheer quantity means we are also unable to detail all work to date. As such, the literature discussed below is that which is most relevant to the present study (i.e. possible structural changes within the Na-AOT lamellar phase region, from molecular to micrometre lengthscales) and will be addressed in terms of the experimental technique used.

\section{Early Work}

Aside from early communications in which Na-AOT was incorrectly reported to exist as a lamellar phase at all concentrations, ${ }^{96}$ the first full characterisation of the liquid crystalline phase behaviour of the system was carried out by Rogers and Winsor. ${ }^{64,86}$ They primarily used polarising optical microscopy and reported the effects of concentration, temperature and 
several additives on the system, showing the phase behaviour to be dependent upon all three. They also reported an anomaly in the lamellar phase region, having observed the birefringence to undergo a change in optic sign, switching from negative to positive with increasing surfactant concentration. This was deemed to be counterintuitive, as birefringence is normally expected to decrease with reduced solubility. Additionally, they observed the change in optic sign upon increasing temperature and rationalised it in terms of a significant change occurring within the polar headgroup of Na-AOT. However, this was later discounted when it was shown that the headgroup does not appreciably change in size within the lamellar region. ${ }^{63}$

Rogers and Winsor undoubtedly completed a comprehensive microscopy investigation of the $\mathrm{Na}$-AOT system in order to compile the phase diagram. Unfortunately however, they did not publish the microscopy images they obtained and the work pre-dated any interest in the defect behaviour of lyotropic systems. As such, we are unable to infer anything beyond the reported phase progression and change in birefringence. POM is of course a standard experimental technique in the study of lyotropic liquid crystals and so other groups have routinely used it when investigating the Na-AOT system. However, this has generally been to confirm the equilibration of the samples, or to illustrate the effect of shear on samples. A full range of microscopy images showing the whole lamellar region is not available and a definitive characterisation of the defect behaviour of this system has not been published.

Another common tool in the elucidation of lyotropic phase diagrams is X-ray diffraction. In the case of Na-AOT, several groups published X-ray results of the system. Philippoff et al. ${ }^{96}$ and Park et al. ${ }^{97}$ elucidated the basic phase transitions, but provided an insufficient range of samples to fully explore the lamellar phase. Fontell provided a comprehensive X-ray investigation, and defined three concentration regions based on different behaviours (LCR, ICR and HCR). ${ }^{63}$ Fontell's results show that the thickness of lamellar bilayers and the area of the polar headgroup remain approximately constant throughout the lamellar region. He also showed that the swelling behaviour of the bilayers upon dilution is one-dimensional in the LCR and HCR, ranging from $\sim 180-20 \AA$ at each extreme. In the ICR, Fontell observed only one weak reflection (rather than the two seen for the other regions), which occurred at a $q$ value double that expected for one-dimensional swelling. He concluded that a break in one-dimensional swelling and an associated structural rearrangement take place, involving a drastic change in the bilayer repeat distance.

It has since been generally accepted that the assignment of the single observed peak in the ICR as the first-order lamellar Bragg peak was erroneous, and the peak is actually the second-order lamellar peak (this will be discussed in greater detail later in the chapter). As such, the implied (but highly unlikely) drastic compression and expansion of bilayers within the lamellar region does not in fact occur. It should be noted that Fontell's peak assignment was stated to be an "assumption" and not definitive. However, these data and the subsequent conclusions were not questioned for many years. Unfortunately, this error has resulted in Fontell's paper becoming arguably the most important within the Na-AOT literature. Many subsequent researchers have attempted to rationalise the results of their own work in a way that is consistent with Fontell's data/conclusions. As such, it is now evident that many of the conclusions and proposed models 
for the Na-AOT lamellar phase are based on fallacious arguments. Although the concentration regions defined by Fontell are based on an incorrect interpretation of his data, the terms LCR, ICR and HCR will be used within this literature review for consistency with other publications. The reduction in intensity of the first-order Bragg peak with the ICR is still relevant as it is not characteristic of a classical lamellar phase, and several other researchers have reported anomalous behaviour within this region. It is important to note, however, that these terms are somewhat misleading, as they assume that all the changes occurring within the system are due to a single structural change, where in fact the system undergoes many changes and they do not all occur within the ICR.

Fontell's group provided much of the early literature on the Na-AOT system, investigating how electrical conductivity, density, vapour pressure and Raman spectroscopy intensities vary with concentration. ${ }^{88,98,99}$ They also investigated the phase behaviour of tertiary systems and the effect of replacing the sodium cation with divalent counterions, namely magnesium and calcium. ${ }^{88,100}$ The results of the electrical conductivity and Raman spectroscopy investigations showed dramatic variations within the ICR. The electrical conductivity was shown to begin to increase within this region, and to continue to increase, until a sharp decrease was observed within the HCR (above 60 wt \% Na-AOT). The electrical conductivity was also shown to be temperature dependent. Fontell proposed that the changes in conductivity could be due to the extent of binding or movement of the sodium cations, either within the water layer or along the polar surface of the bilayer. This is a convincing rationalisation of the data, although another possible contributing factor could be dehydration of the counterion, particularly when considering the reported decrease at high surfactant concentrations. Due to the large change in bilayer spacing within the lamellar region of Na-AOT, changes occurring in the cation environment are likely to be more evident than in surfactant systems with lamellar phases existing over smaller concentration ranges. Fontell noted that if such changes were to take place, it is feasible that the arrangement of the polar groups would also be altered and could then influence the chain configuration and surfactant packing. Conversely, we suggest that conformational changes in the AOT molecule could equally be the driving factor, changing the surfactant packing and resulting in bilayer defects that may then alter the electrical conductivity.

With increasing Na-AOT concentration, Faiman, Lundström and Fontell observed significant changes in only one Raman spectral band. ${ }^{99}$ Interestingly, this band is not due to the sulfate in the surfactant headgroup, but was assigned to the antisymmetric methylene rocking/deformation modes. The reported change concerns the band centred at $1460 \mathrm{~cm}^{-1}$ and is a change in relative intensity rather than peak position or shape. The change is also non-linear, in general the peak intensity increases with concentration, but was observed to fall in the ICR. Such a change would indicate that in the ICR a subtle conformational change occurs within the hydrocarbon tails. Unfortunately, the error associated with the data is significant, and while it was also stated that similar changes in peak intensity were observed with temperature variations, the recorded data were deemed not to be of publishable quality. 


\section{Vibrational Spectroscopy and Spectrophotometry}

Given the high degree of error and lack of any further communications regarding the observed temperature variations, the Raman spectroscopy investigation by Faiman et al. should be regarded as preliminary. As such, it is unfortunate that further Raman spectroscopy investigations of the lamellar phase, building on this group's observations, are lacking. Moran et al. assigned the stretching modes of the sulfonate group and investigated the changes induced by exchange of the sodium cation, ${ }^{101}$ while Nagasoe et al. improved the assignment of the peaks relating to the hydrocarbon tail environment by studying straight-chain analogues of Na-AOT, which correlate with different rotational isomers dependent upon tail length. ${ }^{102}$ These investigations significantly improve upon the spectral assignments of Faiman et al., but do not provide any further insight into any concentration-induced structural changes. The only Raman spectroscopy publication since the work of Faiman et al. to sample within the lamellar region of Na-AOT is by Arunagirinathan et al., who used micro-Raman primarily to investigate myelin formation or "surfactant outgrowth" upon dilution with water. ${ }^{103}$

Although the focus of this study was myelin formation in the Na-AOT system, which occurs at the lower concentrations of the lamellar region, Arunagirinathan et al. also sampled within the more concentrated regions for comparison. Their experimental procedure focused the Raman laser upon the desired area of a microscope slide bearing a standard concentration gradient of Na-AOT (produced by promoting water penetration of the pure surfactant). This technique means that samples of known concentration were not studied. However, by comparing the sample areas marked on the published image with a concentration gradient of our own, we were able to deduce that three of the spectra produced relate to an unknown concentration within the LCR, ICR or HCR. This is not ideal for investigating the exact concentrations at which any structural changes may occur, and the sampled region relating to the LCR was in the form of a myelin, which may not truly represent a sample prepared at an equivalent concentration. However, the study still provides useful information regarding changes to the molecular configuration of $\mathrm{Na}$-AOT at different concentrations within the lamellar region.

The study identified four regions of interest: CC-CS stretching $\left(740-790 \mathrm{~cm}^{-1}\right), \mathrm{C}-\mathrm{C}$ skeletal stretching $\left(1050-1085 \mathrm{~cm}^{-1}\right), \mathrm{C}-\mathrm{H}$ bending $\left(1450-1460 \mathrm{~cm}^{-1}\right)$ and $\mathrm{C}-\mathrm{H}$ stretching $\left(2850-2950 \mathrm{~cm}^{-1}\right)$. The latter three relate to changes in the hydrocarbon tail environment, while the CC-CS stretching highlights the only observed changes within the headgroup, with peaks at 785 and $745 \mathrm{~cm}^{-1}$ relating to trans and gauche conformations of the CC-CS bond, respectively. They showed that within the LCR both trans and gauche conformations are present, but only the trans conformation is observed in the ICR and HCR. The spectroscopic regions relating to the hydrocarbon tail environment are significantly more complex, a result of the large number of carbon and hydrogen atoms in similar chemical environments (exacerbated by the presence of multiple diastereomers). However, a general trend towards rotational isomers with more ordered tail orientations that pack more efficiently is evident at higher concentrations. The peaks in the LCR reveal a mixture of tail orientations, comprised of both ordered trans configurations and bent or kinked gauche configurations. This implies that the hydrocarbon environment in the LCR is a mixture of splayed and non-splayed tails, which will promote 
disorder and allow flexibility in the surfactant packing. This may be a factor in any non-classical lamellar behaviour within the LCR. Interestingly, the $\mathrm{C}-\mathrm{C}$ skeletal stretching showed that in the ICR the relative peak intensity relating to gauche configurations within the succinate skeleton dramatically increases, and is a further indication that the hydrocarbon tails alter the surfactant packing within this region either due to, or facilitating, a structural transition.

Although they highlight changes in the $1450-1460 \mathrm{~cm}^{-1}$ spectral region, Arunagirinathan et al. did not observe the intensity anomalies described by Faiman et al., but have reported the observation of two peaks in this area rather than a singlet. These peaks were assigned as trans and gauche contributions of the hydrocarbon chains. The authors suggested that these differences could be due to increased hydration within their samples, which (in contrast to those of Fontell) were not sealed. However, it is more likely that the observation of two peaks is due to the improved resolution available with modern instrumentation. The intensity anomaly may not have been observed by Arunagirinathan et al. as the ICR was sampled more extensively by Fontell et al. than Arunagirinathan et al., who only reported a single Raman spectrum for each of the lamellar regions.

As in the Raman spectroscopy, the infrared spectrum of Na-AOT is complicated by the presence of multiple diastereomers, giving rise to a complex series of bands. Again, much of the characterisation of these peaks was completed by Nagasoe et al. The authors initially studied the simpler straight-chained analogues of Na-AOT, for which crystal structures were available, and used their findings to assist with assigning the peaks in the Na-AOT spectrum. ${ }^{104,105}$ However, it was not until the topic was revisited by the same research group that significant information regarding possible concentration-dependent structural changes was reported. Okabayashi et al. investigated the conformations of the succinate skeletons of Na-AOT and various straight-chained dialkyl sulfosuccinate analogues, with a particular focus on the $\mathrm{CH}$ and $\mathrm{CH}_{2}$ deformation vibrational modes $\left(1300-1450 \mathrm{~cm}^{-1}\right)$. They assigned IR signals to two stabilised conformations of the Na-AOT succinate skeleton. The two conformations display drastically different torsion angles of the ${ }^{1} \mathrm{C}-{ }^{1} \mathrm{C}-{ }^{2} \mathrm{C}-{ }^{3} \mathrm{O}$ segment within the headgroup, which arise through rotation of the ${ }^{1} \mathrm{C}-{ }^{2} \mathrm{C}$ bond, resulting in the presence of splayed or parallel alkyl chains (see Figure 3.4).

Although the stacking of parallel chains may be considered to be the more efficient configuration in a lamellar phase, the straight-chained surfactants showed an increased stabilisation of splayed tails with increasing concentration. It was reasoned that the hydrocarbon tails may be too short to preferentially stabilise the stacking of parallel chains, and so a "finger-joint" model of interdigitated surfactant molecules was proposed for the bilayer. This is supported for the $n$-hexyl variant by a reduced bilayer thickness of $30 \AA$, compared with the theoretical value of $35.5 \AA$ obtained using Tanford's equation. ${ }^{106}$ In comparison, Na-AOT (which has a bilayer thickness significantly less than its straight-chained analogue) displays peaks in the IR spectrum corresponding to both splayed and parallel tail conformations in the lamellar phase. Okabayashi et al. suggested a mixed contribution to the bilayer from the bending of the branched hydrocarbon chains and a finger-joint arrangement of molecules. It is clear from the published spectra that the relative contribution of the splayed and parallel conformations 

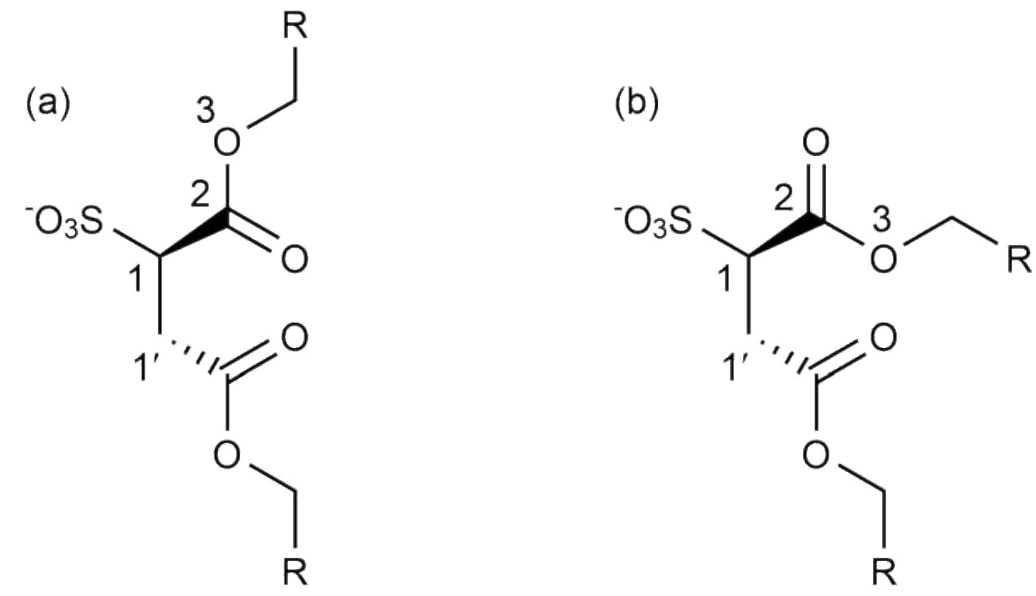

$$
\mathrm{R}=\mathrm{CH}\left(\mathrm{CH}_{2} \mathrm{CH}_{3}\right) \mathrm{CH}_{2} \mathrm{CH}_{2} \mathrm{CH}_{2} \mathrm{CH}_{3}
$$

Figure 3.4: Two stabilised conformations of the Na-AOT succinate skeleton, resulting in (a) splayed or (b) parallel alkyl chains.

changes with concentration; however, an insufficient number of concentrations were sampled to fully determine the extent of the structural changes and at exactly which concentrations they occur.

Given that at high surfactant concentrations the X-ray repeat distances published by Fontell (including the water layer) are significantly less than twice the length of an extended Na-AOT molecule, ${ }^{63}$ it was already evident that the bilayer must be composed of interdigitated molecules. Likewise, an increased splaying of the tails at high concentrations may also be inferred from the transition to Type II structures above $78 \mathrm{wt} \%$ Na-AOT. However, the evidence that this splaying is facilitated through rotational isomerism within the Na-AOT molecule (and is likely further enhanced by the tail branching), and results in a drastic change in the effective surface area at the hydrophobic/hydrophilic interface, may be significant in rationalising the observed properties of the surfactant. Indeed, when these results are combined with the Raman spectroscopic data, it is evident that the hydrocarbon tail region of the Na-AOT molecule can adopt a wide range of different packing modes, potentially producing an extremely high degree of flexibility in the lamellar bilayer.

A further study of the Na-AOT system using IR spectroscopy was completed by Boissiere et al. who were interested in the behaviour of the water molecules rather than the Na-AOT molecules. As such, they probed the intermolecular water interactions by monitoring changes in the $\mathrm{O}-\mathrm{H}$ stretching mode over a range of concentrations. ${ }^{107}$ They modelled this spectroscopic region using Gaussian functions to fit contributions from three distinct spectroscopic components, which they termed network $\left(3320 \mathrm{~cm}^{-1}\right)$, intermediate $\left(3465 \mathrm{~cm}^{-1}\right)$ and multimer $\left(3585 \mathrm{~cm}^{-1}\right)$ water. These components differ in their degree of connectivity, with on average hydrogen bonding to four, three and two other molecules, respectively. At concentrations up to $35 \mathrm{wt} \% \mathrm{Na}$-AOT, the relative proportions of each component show little variation. Above this concentration, the multimer contribution shows little change, but there is a significant drop in the contribution from network water coupled with a rise in that of intermediate water. These changes were attributed 
to an increasing proportion of "hydration water molecules" forming a buffer between the outer layer of surfactant and bulk-like water. The position of the $S=0$ stretch was reported to be constant throughout the lamellar region, suggesting that the hydration of the AOT headgroup itself does not change, remaining fully hydrated ( 2.6 water molecules per ionic headgroup).

Despite the change in water behaviour reported by Boissiere et al. coinciding with the ICR, the fitting of three functions to such a broad peak could surely be achieved in a number of ways and is in the authors' own words "ambiguous". Indeed, the three spectroscopic components of water discussed are all observed within bulk water itself, and so their presence alone does not confirm the assignment of different water populations. It is also not obvious what the change in water environments implies about the overall lamellar structure. A decrease in the relative amount of bulk water is surely consistent with normal constriction of lamellar bilayers upon increasing concentration. Although, alterations in the water structure could also be due to changes in the hydration of the counterion, or due to a rearrangement of the bilayered structure. However, the modelling of one broad $\mathrm{O}-\mathrm{H}$ band in an IR spectrum is not enough evidence for either.

Further evidence has more recently been provided by the same research group through a study that combined IR spectroscopy with differential scanning calorimetry (DSC). ${ }^{108}$ This work significantly improved upon earlier studies using DSC, which included incorrectly assigned peaks ${ }^{109}$ or only sampled at the lower end of the lamellar region. ${ }^{110}$ Prouzet et al. observed three endothermic peaks in the DSC profiles, each representing a distinct thermally homogeneous water population, at $-12,-10$ and $-2{ }^{\circ} \mathrm{C}$. These populations were assigned as bulk water existing between lamellar bilayers; bound water, which interacts strongly with the surfactant headgroup; and intermediate water, a thin layer of approximately two water molecules in thickness, located between the previous layers and in close enough proximity to the surfactant bilayer to be significantly disturbed by its presence. Each of the three populations was shown to present a different distribution of the three spectroscopic components of water identified by IR spectroscopy. Bulk water is predominantly network water, whilst the intermediate and bound water were shown to present a lower degree of hydrogen bonding. Although this further study provides greater evidence for a continuous change in the water structure above $35 \mathrm{wt} \% \mathrm{Na}-\mathrm{AOT}$, it does not provide any more detail regarding the cause of the change or the implications for the mesophase structure.

Another publication using a spectroscopic technique is a study by Nallet and Barois, who further investigated the reported change in birefringence within the lamellar region of the Na-AOT system. ${ }^{111}$ This report is primarily composed of theoretical work that does not relate to one lamellar phase of a specific system; however, the assertions made within the text were then confirmed using UV-visible spectrophotometry. The experimental work within the paper was carried out on the SDS/hexanol/dodecane/water system rather than the Na-AOT system, but the findings are applicable to all lamellar systems. It was shown that when a change from negative to positive birefringence occurs in such systems, it can be reasoned by considering the overall birefringence as the sum of two different contributions, one of which is heavily dependent upon the dilution. The first component is the intrinsic birefringence of each lamellar bilayer. This arises because each bilayer is composed of an approximately parallel arrangement of Na-AOT 
molecules. The value of this contribution is typically positive and normally governs the overall birefringence of a lamellar phase. The second component is the "form birefringence", which is the birefringence arising from the stack of lamellar bilayers.

Nallet and Barois showed that at low dilutions the intrinsic birefringence of the bilayers dominates over the form birefringence. This is generally the observed behaviour and results in positive birefringence. However, at higher dilutions the negative contribution of the form birefringence can prevail. This occurs when the bilayers are no longer flat parallel sheets, but deviate from the idealised structure due to undulating, or crumpling, of the bilayers. This is most often the case in highly swollen lamellar phases stabilised by Helfrich's entropic forces rather than electrostatic interactions. At the dilution at which the negative contribution of the form birefringence matches the positive contribution of the bilayer, the overall birefringence is zero, and thus samples appear dark between crossed polarisers. The form birefringence is sensitive to thermal fluctuations and so the optical properties of samples can be altered by small temperature adjustments. As such, samples with compositions within the vicinity that will produce a structure with zero birefringence can appear blue or yellow when viewed through crossed polarisers. This property may provide a simple distinction between a non-birefringent lamellar sample and a truly isotropic phase. The study by Nallet and Barois shows that one of the previously (and often) cited anomalies of the Na-AOT lamellar phase is neither unique to the system or indicative of strange behaviour. However, the paper also provides an indication that the lamellar structure of the LCR may be a crumpled or undulating structure that is sensitive to thermal fluctuations. This is a key point to note, as it implies that within the LCR, the bending rigidity of the bilayer $(\kappa)$ has a similar value to the thermal energy, $k_{\mathrm{B}} T$, and that both must be taken into account when considering the intermolecular forces that stabilise the structure formed.

\section{Small-Angle X-ray/Neutron Scattering}

Since the early X-ray data reported by Fontell, ${ }^{63}$ several studies of the Na-AOT system have utilised small-angle X-ray or neutron scattering techniques (SAXS and SANS, respectively). However, the role of the SAXS or SANS measurements has generally been confined to confirming the phase assignment or the bilayer repeat distance of samples, rather than a continued investigation of the intensity anomalies reported by Fontell. No further full and comprehensive experimental investigations into the region have been reported to date. However, the phenomenon has been reported in the $\mathrm{Na}-\mathrm{AOT} /$ water $/ \mathrm{NaCl}$ system, where it was proposed that Bragg peaks occurring at a wave vector that coincides with the minimum value of the form factor would be significantly reduced in intensity and possibly disappear. ${ }^{90}$ This concept was investigated further by Nallet et al., ${ }^{112}$ who studied the intensity anomaly of the Na-AOT system using both SAXS and SANS. Crucially, they showed that the reduced intensity of the first-order scattering peak is only observed in the SAXS spectra, with the SANS profiles presenting no anomalous intensity variations. The $X$-ray profiles also reveal significant diffuse scattering at large wave vectors, present in the spectra as a broad hump in the $q$ range of $\sim 1$ to $5 \mathrm{~nm}^{-1}$, which is also not present in the SANS profiles. The fact that the broad scattering and intensity 
variations are only present in the X-ray studies indicate that they arise from the contribution to the scattered intensity of the form factor of the bilayer. This is due to the Fourier transform of the electron density distribution and so not a component in neutron scattering experiments. The shape of the diffuse scattering peak was reported to be independent of concentration and the intensity was stated to scale linearly. The scattering was also reported to be sensitive to the scattering length density profile of the amphiphilic bilayer.

Nallet et al. proposed models to quantitatively describe and explain the experimental observations. By combining a simple model for the electron density profile of the Na-AOT bilayer with the Caille model for thermal fluctuations, ${ }^{113}$ they proposed models for the small angle scattering that incorporate both geometric and thermodynamic contributions. The theoretical data are in good agreement with the experimental data, including the intensity variations that occur with surfactant concentration in the SAXS spectra. The thermal fluctuations are incorporated into the model through the term $\eta$, a unitless line shape component termed the Caille exponent, where $q_{0}$ is the $q$ value at the Bragg peak maximum, $k_{\mathrm{B}} T$ is the thermal energy, $\mathrm{K}$ is the curvature modulus $(\mathrm{K}=\kappa / \mathrm{d})$ and $\overline{\mathrm{B}}$ is the compressibility modulus, such that:

$$
\eta=\frac{q_{0}^{2} k_{B} T}{8 \pi \sqrt{K \bar{B}}}
$$

The model of Nallet et al. allows the effect of individual parameters upon the scattering profile to be probed. They showed the structure factor to be heavily dependent upon the selected value for $\eta$, demonstrating that it quickly reaches its asymptotic value of 1 when $\eta$ is not zero. The form factor was shown to tend towards zero at $q$ values much smaller than those corresponding to the thickness of the bilayer. This offers a convincing explanation for the decrease in intensity of the first-order Bragg peak, as the intensity of this peak would diminish for sample compositions that give rise to a Bragg peak that coincides with the zero value of the form factor. This is due to the electron density profile of the bilayer adopting a two-square profile, with the electronic density of the headgroup estranged from that of the tails or solvent. This work highlights the effect of elastic fluctuations, arising from the Landau-Peierls instability. It shows how the magnitude of the elastic constants of the bending modulus, $\kappa$, Gaussian curvature modulus, $\bar{\kappa}$, and compressibility modulus, $\bar{B}$, have a large effect on the small angle scattering of the system and must be considered alongside the form and structure factors when analysing the shape of the Bragg peaks.

In a later study, Zhuang et al. reported (and offered rationalisations for) the sharpening and shift of Bragg peaks to higher q spacings, with increases in concentration or temperature, and also upon addition of a cosurfactant, to the Na-AOT system. ${ }^{114}$ They stated that this is synonymous with a move to a more ordered structure and attributed it to a switch from flexible to planar bilayers. Their presented scheme suggests that planar bilayers are induced by a simultaneous reduction in the effective surfactant tail length and an increase in headgroup area. Thus, they explained the changes in the system in terms of the simplified surfactant packing parameter. In 
calculating the reported changes to the headgroup area and tail volume, it is assumed that the thickness of the water layer remains unchanged upon variations in temperature, and also that changes to the Bragg peaks with concentration or temperature are due to a compression of the bilayer (i.e. a reduced bilayer thickness, presumably as a result of increased interdigitating of the $\mathrm{Na}-\mathrm{AOT}$ tails). This reasoning is not in agreement with the reports discussed previously, which indicate that both the size of the surfactant headgroup and bilayer thickness remain effectively unchanged throughout the lamellar region. Zhuang et al. also offered a rationalisation for the intensity variations of the first-order Bragg peak, proposing that they occur due to the scattering being sensitive to small structural fluctuations and neighbouring bilayers. They also stated that an increase in bridging or micellar defects could cause a reduction in peak intensity. While the first of these explanations, that of small structural fluctuations, may be consistent with the work of Nallet et al., the work of Nallet et al. was not addressed within this publication, and the role of thermal fluctuations was ignored. While the concept of a transition from flexible to planar bilayers may have some merit, the simplistic explanations that do not consider the elastic components of the Na-AOT bilayer and are not consistent with the findings of others are less convincing.

\section{Nuclear Magnetic Resonance Spectroscopy}

The Na-AOT system has been extensively studied with various forms of NMR spectroscopy. The first report of NMR characterisation of the liquid crystalline regions of Na-AOT was completed by Franses and Hart, ${ }^{115}$ who used ${ }^{1} \mathrm{H}$ and ${ }^{13} \mathrm{C}$ NMR to determine the location of phase boundaries of the system, and also interpreted the molecular motion at various concentrations from the peak linewidths. It should be noted that the results include an error margin of up to $\pm 5 \mathrm{wt} \%$, and so the values quoted were tentative and not all are in good agreement with values stated elsewhere. Unsurprisingly, they reported a decrease in molecular motion moving from the isotropic to the two-phase region, and a significant broadening of peaks upon entering the lamellar phase, due to significantly reduced molecular motion. Indeed, due to slow molecular motion in the lamellar phase, the ${ }^{1} \mathrm{H}$ NMR peaks of the headgroup are not observed from 5-70 wt \% Na-AOT. Similar behaviour was also reported for the ${ }^{13} \mathrm{C}$ NMR spectra with peaks due to carbon atoms in the headgroup becoming broader, while those of the tail region remain sharp, highlighting the different environments experienced by the hydrophobic and hydrophilic parts of the molecule. In the more concentrated region of the phase diagram, three substantial changes were reported in the ${ }^{1} \mathrm{H}$ NMR water signal. Two of these changes are representative of phase transitions, forming bicontinuous cubic and hexagonal phases, whilst the third occurs at $50 \mathrm{wt} \%( \pm 5 \mathrm{wt} \%)$ roughly coinciding with the change from the ICR to the HCR. A change in the thermal properties of the water was also observed in this region, with no bulk-like water freezing above this surfactant concentration.

Pulsed field gradient (PFG) NMR was used by Callaghan and Söderman to monitor the diffusion of water molecules in Na-AOT samples of various concentrations. ${ }^{47}$ This technique is sensitive to the diffusion of water on the lengthscale of approximately $1000 \AA$ (over five times that of the longest reported interbilayer spacing in the system), meaning the technique is primarily 
suited to measuring lateral or 2D diffusion. In the LCR between 16 and $31 \mathrm{wt} \% \mathrm{Na}-\mathrm{AOT}$, the water diffusion fits well with a model for 2D diffusion; however, the values for the self-diffusion coefficient in this region are very low, up to a factor of four below that of free water. Upon entering the ICR, the $2 \mathrm{D}$ diffusion is maintained, although a significant increase was observed in the diffusion coefficient values. In the HCR (above 45 wt \% Na-AOT), a 2D diffusion model no longer fits the experimental data, and the results are more akin (although not a perfect fit) to the 3D diffusion of Brownian motion. At concentrations above $60 \mathrm{wt} \%$, the samples show a progressive decline in their self-diffusion coefficient and a return to 2D diffusion. This decrease in water diffusion coincides with the decrease in electrical conductivity observed by Fontell.

Callaghan and Söderman proposed a tentative structural model to rationalise the changes in diffusion (see Figure 3.5). They proposed that the low rates of diffusion observed in the LCR are due to a high number of bridging defects between bilayers, which restrict the movement of water molecules. They suggested that upon increasing surfactant concentration, the bilayer repeat distance falls, resulting in the packing curvature needed for such bridges becoming unfavourable. The destruction of such defects could result in localised regions containing high concentrations of "free" amphiphile molecules, which they proposed then form bilayered micellar discs between the lamellar layers. With the further compression of bilayers resulting from increasing surfactant concentration (moving to the HCR), such discs would then begin to break down, returning to classical lamellar bilayers. The pseudo-3D diffusion within this region was attributed to the constant rearrangement of lamellar domain boundaries, which could allow water to diffuse into domains aligned along different directional axes. It was proposed that such behaviour would gradually decrease as bilayers are compressed further, creating a more closely packed, ordered and rigid state which would exhibit 2D diffusion.

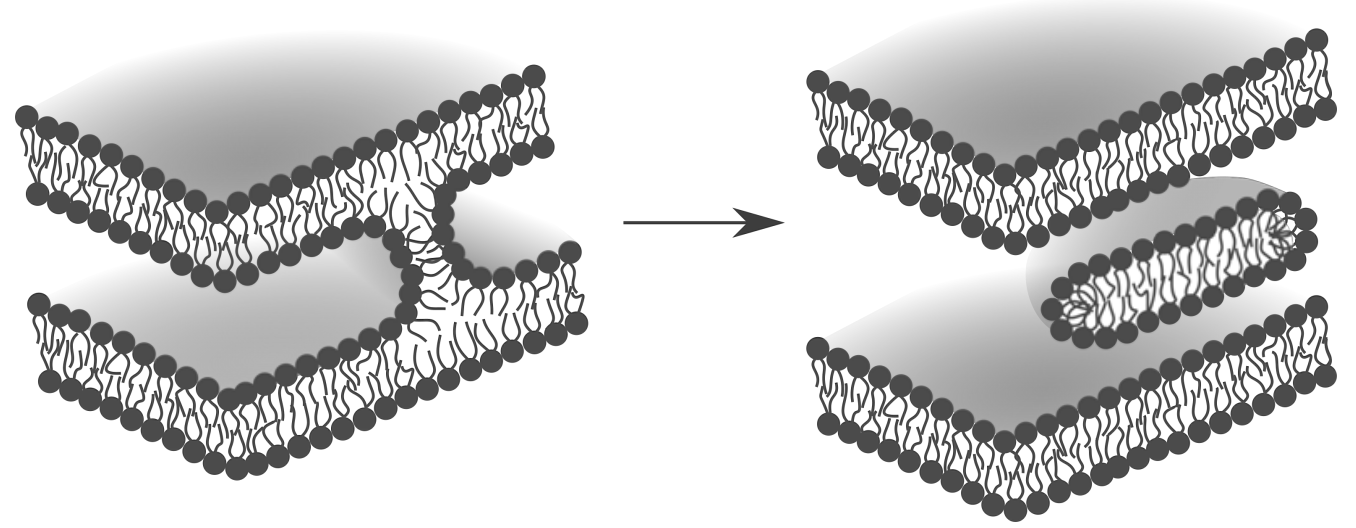

Figure 3.5: Schematic representation of the structural model for the Na-AOT lamellar phase proposed by Callaghan et al.

Despite achieving very similar results, Chidichimo et al. disputed the conclusions of Callaghan et al., suggesting that a structure of highly connected bilayers proposed in the LCR would leave the aqueous domains in a structural form similar to that of inverted ribbons. ${ }^{116}$ They stated that the high viscosity associated with ribbon structures is not present in the LCR, and that such a phase would only be viable in highly concentrated samples. Using POM in conjunction with 
deuterium NMR and PFG-NMR experiments, they suggested an alternative model based on a rippled lamellar phase (see Figure 3.6), previously observed in some phospholipid systems. They suggested a system with ripple angles between $50^{\circ}$ and $65^{\circ}$ forms below 20 wt \% $\mathrm{Na}-\mathrm{AOT}$, which then coexists with non-rippled bilayers at 20-28 wt \%, and is replaced by a "defectless" or normal $L_{\alpha}$ phase above this concentration. Comparison of deuterium NMR lineshapes for relaxed and perturbed samples reveals different behaviours, with the former being less broad and displaying less biaxial character. Chidichimo et al. stated that the system is prone to mechanically-induced deformations and suggested that this is the cause for the anomalies in the X-ray diffraction data of Fontell and thus the identification of the ICR region.

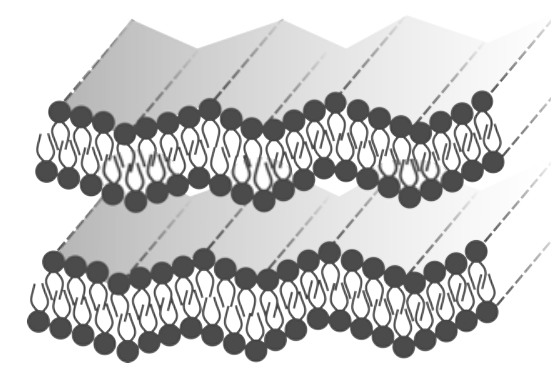

Figure 3.6: Schematic representation of the structural model for the Na-AOT lamellar phase proposed by Chidichimo et al.

The discrepancy between the Callaghan et al. and Chidichimo et al. led to a third investigation using PFG-NMR, with Coppola et al. also investigating the water diffusion in relaxed and perturbed samples. ${ }^{117}$ Although they agreed that an observable change is evident in the line shapes of deuterium NMR spectra of relaxed and perturbed samples, they also concluded that mechanical stresses affect the macroscopic properties of the lamellar domains rather than the microscopic properties. They observed a reduction in the size of lamellar domains, but stated that the geometry of the microstructure is not affected. While the publications offer different rationalisations for the microstructure of the Na-AOT lamellar phase, PFG-NMR spectroscopy unquestionably shows that the water diffusion in the Na-AOT lamellar phase undergoes concentration-dependent changes, with the motion restricted at lower concentrations and increased above $33 \mathrm{wt} \% \mathrm{Na}-\mathrm{AOT}$.

Further diffusion NMR experiments have been performed on the Na-AOT lamellar phase in the form of diffusion exchange spectroscopy (DEXSY) and diffusion-diffusion correlation spectroscopy (DDCOSY), in order to monitor the diffusion of water and hence investigate the size and orientation of the lamellar domains, respectively. ${ }^{94,95}$ DEXSY NMR maps the exchange of water molecules between domains, with the timescale of this exchange correlating to the lengthscale of the domain. Average domain sizes for samples of 25 and 50 wt $\%$ Na-AOT were quoted as 5 and $12 \mu \mathrm{m}$, respectively. These values are lower than those observed using other experimental techniques such as electron microscopy. This is possibly indicative of a dense network of local defects in the microstructure, which inhibit the movement of water and so change the water diffusion coefficient. DDCOSY was shown to be more sensitive to partial alignment of lamellae than the deuterium NMR experiments used in previous studies. The DDCOSY investigation revealed an interesting trend in the samples analysed, in that the more 
concentrated samples show a greater tendency to align. Samples of 33 and $50 \mathrm{wt} \% \mathrm{Na}-\mathrm{AOT}$ display anisotropic alignment of lamellar bilayers, whereas samples of $25 \mathrm{wt} \% \mathrm{Na-AOT}$ show no significant bulk alignment and were reported to be globally isotropic. It was also reported that barriers to diffusion are more prevalent along one director plane and that this observation is consistent with the model of interbilayer micellar discs proposed by Callaghan et al. ${ }^{47}$ Although only three Na-AOT concentrations were sampled within these two studies (one from each of the lamellar concentration regions), the combination of techniques provided much detail regarding the scale of defects within the microstructure of the lamellar phase and the effect upon water diffusion. The NMR investigations were also supported by several electron microscopy images as reported below.

\section{Electron Microscopy}

Hubbard et al. supplemented their NMR investigations with several SEM images of cryogenically frozen Na-AOT samples of 25,33 and 50 wt \% surfactant. ${ }^{94}$ They reported a defect-rich microstructure at $25 \mathrm{wt} \%$ and described it as being more akin to a metastable sponge phase than a typical $\mathrm{L}_{\alpha}$ phase, due to the high number of bridging and hole defects. At 50 wt \%, a more typical lamellar morphology was observed with a reduced number of defects. The $33 \mathrm{wt} \%$ sample was reported to show characteristics found in each of the other samples. Domain boundaries were estimated from low magnification images and the domain sizes were reported to range between 10 and $100 \mu \mathrm{m}$ for the $25 \mathrm{wt} \%$ sample and between 10 and $50 \mu \mathrm{m}$ at $50 \mathrm{wt} \% \mathrm{Na}$-AOT. The ability of the cryo-SEM to allow details to be obtained at both higher and lower magnifications meant that the technique was preferred over cryo-TEM as it was judged to reveal a greater depth of structural information. In recent years, technological advancements in electron microscopy have led to the freeze-fracture replica technique employed in the study by Hubbard et al. being replaced with the direct imaging of fractured samples. This change in methodology results in different structural information being revealed by the technique as shown in the results of this thesis. The Na-AOT system has not previously been studied this way, although a related lecithin-containing system (Na-AOT/L- $\alpha$ phosphatidylcholine/isooctane/water) has been studied, and the lamellar to inverse hexagonal phase transition imaged. Spherical vesicles ranging in size from $100 \mathrm{~nm}$ to $10 \mu \mathrm{m}$ could clearly be viewed within the lamellar phase and the progression to hexagonal tubules was also observed. ${ }^{68}$

Although only a proof of concept study, one further investigation used freeze fracture atomic force microscopy to view two lyotropic systems. The binary water systems of Na-AOT and pentaethylene glycol monododecyl ether (C12E5) were both viewed using this technique, although only one sample of each system was investigated. ${ }^{118}$ While the technique showed some promise, with interbilayer distances calculated from the observed images in agreement with those obtained from SAXS investigations, the quality of the visual observations was poor. Thus, the technique reveals very little information pertaining to the microstructure of the system and falls some way short of the capability of cryo-SEM, which appears to be the experimental technique most suited to the study of the structure of lyotropic systems at this time. 


\section{Computational Simulation}

The self-assembly of the Na-AOT system has also been simulated, on a mesoscopic lengthscale, using dissipative particle dynamics. ${ }^{119}$ Modelling the Na-AOT molecule as a simplified structure of four spherical particles representing the surfactant headgroup, two tails and water, Yang et al. modelled the system at concentration intervals of $10 \mathrm{wt} \%$. The models produced, shown in Figure 3.7, are in general agreement with the phase diagram of the system, with structures consistent with the lamellar, cubic and hexagonal phases modelled within the correct concentration ranges. At $40 \mathrm{wt} \% \mathrm{Na}$-AOT (the only simulation to coincide with the ICR) the modelled data indicate a significant shift from normal swelling lamellar behaviour. This seemingly matches much of the previously discussed experimental data for the Na-AOT system, as it is within the region in which various anomalies have been reported for the system, and Yang et al. proposed a structural transition within this region based upon their simulations. The authors interpreted their modelled structure as being a "pseudo-reversed hexagonal phase" composed of rod-like micelles.

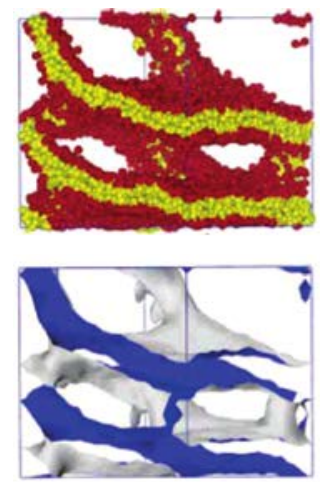

30 wt \%
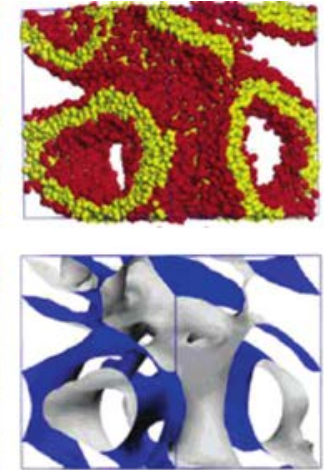

40 wt \%
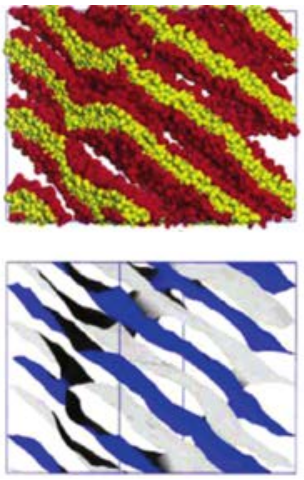

50 wt \%
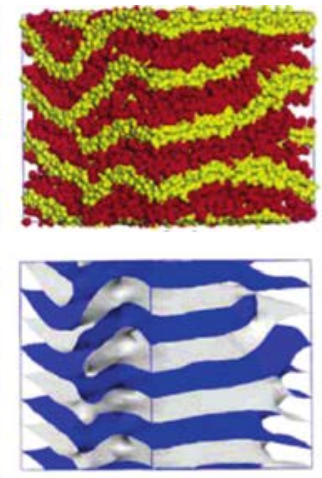

60 wt \%

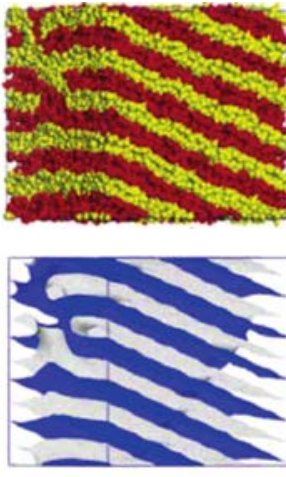

70 wt \%

Figure 3.7: Simulated models of the Na-AOT lamellar phase between 30 and 70 wt \% surfactant, produced by Yang et al. Top: Ball-and-stick representations with the Na-AOT headgroup and tail regions depicted in red and yellow, respectively. Bottom: Representations of the electron density distribution of water particles (blue) in the form of isodensity surfaces. Reprinted with permission from J. Phys. Chem. B, 2006, 110, 21735-21740. Copyright (C) 2006 American Chemical Society.

While the simulation of Yang et al. clearly predicts a deviation from classical lamellar behaviour within the ICR, the argument for a hexagonal structure is not convincing. Although it was claimed that the model fits well with the various anomalies described in the literature for the $\mathrm{Na}$-AOT lamellar phase, we argue that the diffusion data calculated in the paper are not in agreement with the various NMR diffusion studies that have been completed. Additionally, while the proposed pseudo-reversed hexagonal structure may be consistent with the initial conclusions of Fontell, it does not correlate with the SAXS models or SANS data of Nallet et al., ${ }^{112}$ which indicate the retention of a lamellar structure consistent with 1D swelling. Although in many fields computational chemistry models can provide accurate structural predictions, the complex subtleties of the forces governing lyotropic self-assembly are yet to be fully understood, and the simplification of the Na-AOT structure in this example likely ignores one the fundamental factors behind the energetically favoured mesostructure. The superior 
microemulsion properties of Na-AOT, along with the IR and Raman spectroscopic studies described previously, indicate that the remarkable properties of Na-AOT are at least partially a result of the branched alkyl tails and the ability of the molecule to adopt drastically different structural conformations through rotational isomerism. By modelling the Na-AOT tails as two spherical particles, the contribution of the tails to the flexibility of the Na-AOT molecule and bilayer is neglected, which is not ideal as it is likely this flexibility that sets the Na-AOT apart from other surfactants.

\section{Summary}

This review has detailed publications concerned with the structure of the Na-AOT lamellar phase at different compositions. The Na-AOT system has clearly been investigated extensively, with a wide range of experimental techniques that have probed the system on a variety of lengthscales. However, this substantial amount of work has yet to be sufficiently collated and used to definitively characterise the Na-AOT lamellar phase, and thus resolve the debate regarding the nature of a possible structural transition within the region. The characterisation of the lamellar phase structure has undoubtedly been complicated by inaccuracies in the literature, many of which stem from the concept of a deviation from 1D swelling within the lamellar phase. ${ }^{63}$ This concept has since been shown to be incorrect, and we propose that the division of the Na-AOT lamellar phase into the LCR, ICR and HCR (which were based upon incorrectly interpreted data) is similarly misleading. As the various intermolecular forces that govern the free energy (and thus self-assembly) of the system change, it should be expected that the mesophase structure will adapt to compensate. As the magnitude of each of these forces does not vary linearly with the bilayer repeat distance, it follows that a lamellar phase existing over a large concentration range will similarly not display continuous behaviour throughout the region. A number of parameters are observed to change within the Na-AOT lamellar system; however, many do not correlate with the defined LCR, ICR and HCR boundaries, but are in fact continuous changes that occur over large concentration ranges of the lamellar region.

Of the various properties well documented to change within the lamellar region of Na-AOT, those that have been continuously cited as being anomalous (and hence proposed to be indicative of a structural transition) are the electrical conductivity, water diffusion, bilayer spacing and birefringence. Two of these four anomalous properties have now been shown to actually be consistent with lamellar behaviour. The bilayer spacing has been demonstrated to be consistent with 1D swelling, and the change in birefringence can be rationalised as a geometrical effect which promotes a negative contribution to the form birefringence. ${ }^{111,112}$ Given this evidence, it becomes highly unlikely that the Na-AOT system presents a unique structural transition, such as those proposed in the literature. ${ }^{47,116,119}$

While the birefringence and X-ray intensities of the Na-AOT system are now rationalised, and are consistent with a lamellar phase, the other reports of discontinuous behaviour within the system still need to be explained. The changes in water diffusion are mirrored by changes in 
electrical conductivity and so the phenomena likely arise from the same source. In addition, the Na-AOT molecules themselves are reported to undergo several changes within the lamellar region, as evidenced by various IR and Raman spectroscopic investigations. The molecules adopt different conformations depending on the sample composition and will promote different packing arrangements within the bilayer, resulting in changes to the bilayer elasticity. ${ }^{102-105,108}$

The X-ray intensities and change in the sign of the birefringence should no longer be considered as anomalous; however, these observations are still not consistent with a classical lamellar phase, and the rationalisation of the results may be significant in finally resolving the Na-AOT microstructure. In each case the observations are explained as being consistent with a lamellar phase sensitive to thermal undulations. This implies that for some surfactant concentrations, the lamellar phase is not composed of a flat stack of parallel bilayers (that would give rise to positive birefringence and intense $X$-ray reflections) but has a large degree of flexibility. As such, the elastic moduli and bilayer compressibility of the bilayer must have a key role in determining the energetically favoured structural morphology. It is also implied that the lamellar phase is at least partially stabilised by entropic forces at low surfactant concentrations. If this is the case, the structure formed at low concentrations is likely that of a highly swollen and defective lamellar phase.

Surprisingly, the defect behaviour of the Na-AOT system has not previously been fully characterised, although the reports of Hubbard et al. are consistent with the system presenting a variety of different defect behaviours, dependent upon the surfactant concentration. ${ }^{94,95}$ The nature of the defects in the lamellar microstructure and the extent of bilayer undulations within a lamellar phase will change with concentration. Such changes in the defect behaviour are the likely cause of the observed variations in electrical conductivity and water diffusion as they can significantly alter the connectivity between the water and surfactant domains. As such, the defect behaviour may also offer an explanation for the reported changes that occur within the water network within the Na-AOT lamellar phase. ${ }^{107,108}$ The potentially significant role of defects in the self-assembly of the Na-AOT system has previously been overlooked and forms a significant part of the experimental work of this chapter.

We concluded from this review of the literature that in order to gain further understanding of the self-assembly behaviour of the Na-AOT system, our study should focus on the defect structures formed by the system. As such, the following section details our attempt to finally resolve the debate surrounding possible structural transitions in the Na-AOT system by fully characterising the defect behaviour of the lamellar phase, and investigating the apparent sensitivity to thermal fluctuations. We have used a combination of POM, cryo-SEM and SAXS to characterise the system on a variety of lengthscales. These three techniques have been used to study the $\mathrm{Na-AOT}$ system previously (as discussed in this review); however, a comprehensive microscopy study, including a sufficient range of sample concentrations to fully detail the behaviour of the system, has not previously been completed. 


\section{Sodium AOT: Experimental Results}

\section{Sodium AOT SAXS}

Although the SAXS scattering for the Na-AOT system has previously been studied (including an explanation of Fontell's anomalous results by Nallet et al. ${ }^{63,112}$ ), we have repeated the experiments and briefly explain our own results here for several reasons. Firstly, the Na-AOT system will be used as a base system with which to compare related systems throughout this thesis, and hence it is important to have a reliable, fully characterised set of SAXS data for comparison in the following chapters. Given the evidence of Hubbard et al. that Na-AOT samples require long equilibration times, ${ }^{94,95}$ it was deemed important that new experimental data were obtained using samples that were fully equilibrated over a period of several months, rather than those of a metastable intermediate. Additionally, our new experimental data allow us to definitively show that Fontell's concept of discontinuous swelling was based on a flawed assumption, and to further investigate the role of thermal fluctuations in the Na-AOT system. While the explanation by Nallet et al. indicated that thermal fluctuations must be considered to have a role in the Na-AOT lamellar phase bilayer structure, the implications of this on the evolution of the system's microstructure was not discussed.

The Na-AOT system was investigated over the full concentration range; however, the following discussion focuses on samples within the lamellar phase region of the system, in particular those that show the progression from non-classical to classical lamellar behaviour. Samples at other concentrations, such as those within the cubic and hexagonal regions, did not provide any information not already covered in the literature review above. Figure 3.8 shows selected $1 \mathrm{D}$ and 2D scattering profiles for the Na-AOT lamellar phase between 20 and $55 \mathrm{wt} \%$ $\mathrm{Na}$-AOT. These scattering profiles clearly illustrate the variations in intensity and strong diffuse scattering described previously and attributed to a combined scattering contribution from thermal fluctuations and the form factor of the bilayer. ${ }^{112}$

The 1D scattering profiles in Figure 3.8 generally show a simple Bragg pattern in the $q$ spacing ratio of $1: 2$, although at 35 and $40 \mathrm{wt} \% \mathrm{Na}-\mathrm{AOT}$ the first-order peak is noticeably absent. From 20 to $30 \mathrm{wt} \% \mathrm{Na}-\mathrm{AOT}$, the first-order Bragg peak diminishes in intensity with increasing concentration. At higher concentrations, the first-order peak reappears, and the intensity of the peak increases significantly with concentration. In contrast, the second-order Bragg peak shows a continuous increase in intensity with surfactant concentration over the entire lamellar phase. The second-order peak does not coincide with the $q$ space region in which the form factor contribution of the bilayer is zero, and as such the second-order peak is a better indicator than the first-order peak for the lamellar swelling behaviour in the Na-AOT system. Further weak scattering, existing either as a shoulder or as a broad isolated peak, is observed at some concentrations and can be attributed to the third order reflection. The third order Bragg peak was not observed as a sharp peak in any Na-AOT sample at any concentration; however, it should be noted that the intensity of the peak was sometimes more prominent in samples that had not been fully equilibrated. 


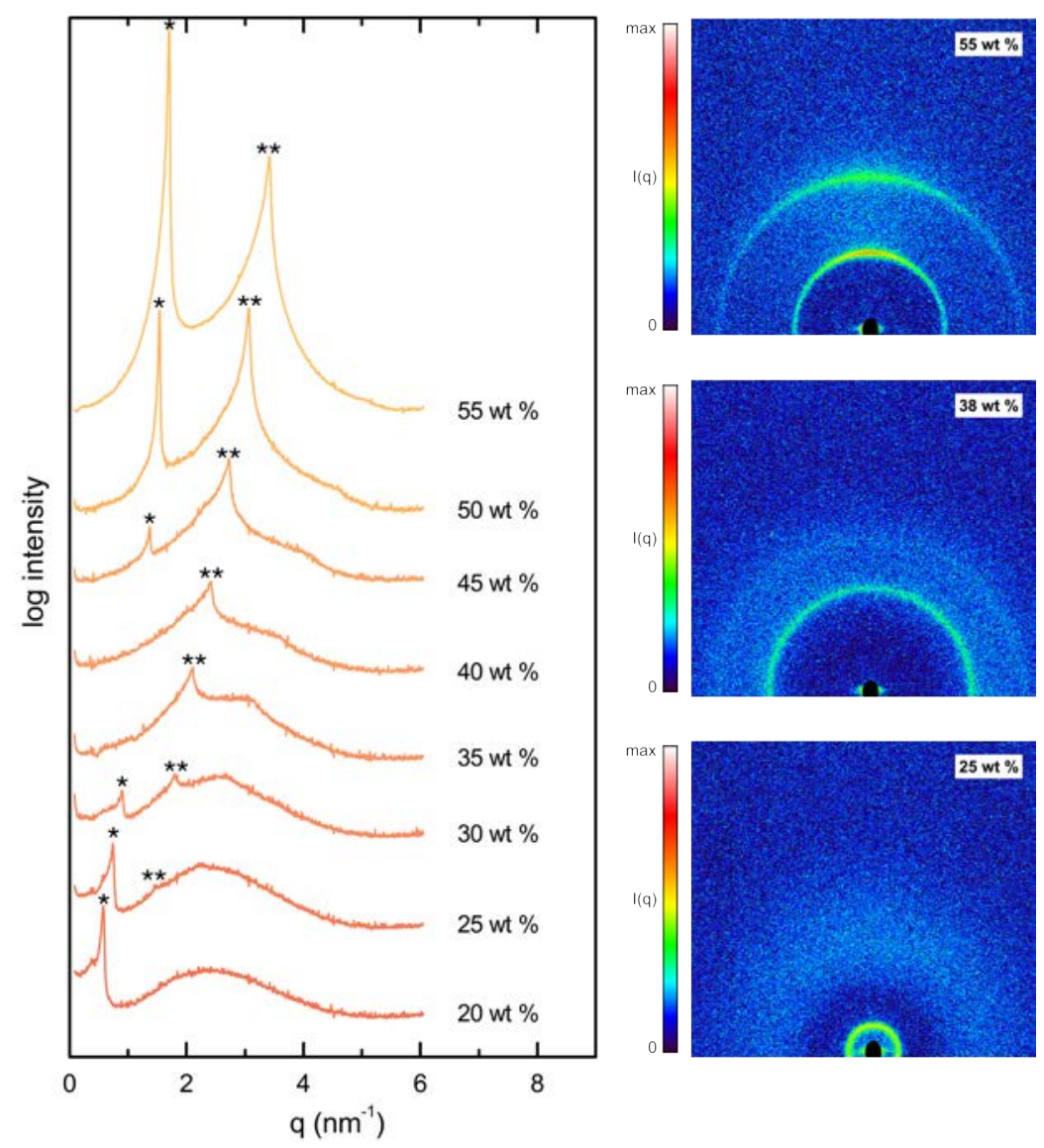

Figure 3.8: SAXS profiles of the Na-AOT lamellar phase with increasing surfactant concentration. Both 1D (left) and 2D (right) profiles are shown, obtained using line and point collimation, respectively. Bragg peaks are shown in the 1D image with single and double asterisks, indicating first- and second-order peaks, respectively. Both data sets show the loss in intensity of the first-order reflection and the presence of diffuse scattering.

The diffuse scattering due to the thermal fluctuations in the bilayer is present at all concentrations as a broad hump in the q space region of $\sim 1.0-4.5 \mathrm{~nm}^{-1}$. This scattering is an indication of the contribution of entropic Helfrich forces to the stabilisation of the system. At the lower $\mathrm{Na}$-AOT concentrations the diffuse scatter masks the second and third order lamellar peaks, indicating that these peaks are weak and hence the long-range order in the system is also weak, consistent with a highly defective structure. Such broad scattering is a common feature in the SAXS spectra of $L_{3}$ phases, disordered variants of the lamellar phase, which are spongelike in appearance and riddled with pores and passages. In the present case, it is likely that the structure of the Na-AOT lamellar phase is also highly defective and the surfactant and/or water regions are highly connected. At concentrations above 45 wt \% Na-AOT, the Bragg peaks significantly increase in intensity and the diffuse scattering, while not entirely absent, is no longer a dominant feature of the lamellar phase. This is a result of the progression towards more normal lamellar behaviour, with increased long-range ordering between bilayers and a reduction in local defects and fluctuations along the bilayers.

Selected 2D SAXS profiles for concentrations of 25, 38 and $55 \mathrm{wt} \% \mathrm{Na}$-AOT are also shown in Figure 3.8. These images are consistent with the $1 \mathrm{D}$ data, clearly illustrating the initial loss and subsequent return of the first-order lamellar scattering. Of these three concentrations, 
the loss in first-order scattering intensity is only observed at $38 \mathrm{wt} \% \mathrm{Na}-\mathrm{AOT}$, with firstorder Debye-Scherrer rings prominent in the images relating to 25 and $55 \mathrm{wt} \%$. At $38 \mathrm{wt} \%$ $\mathrm{Na}$-AOT, the scattering region in which the first-order ring should be present appears dark and is indistinguishable from background scattering, a result of this region coinciding with the form factor minimum, where the scattering contribution is zero. At $25 \mathrm{wt} \% \mathrm{Na}-\mathrm{AOT}$, this scattering region (i.e. the same distance from the primary beam) also appears dark, indicating that the form factor of the bilayer at $25 \mathrm{wt} \%$ may not be significantly different to that of the bilayer at 38 wt \% Na-AOT. However, as the Debye-Scherrer ring at 25 wt \% Na-AOT does not coincide with the scattering region of the form factor minimum, samples at these concentrations were historically considered to display different behaviours.

The 2D SAXS profiles also prominently show the diffuse scattering that occurs at higher $\mathrm{q}$ values. At 25 wt \% Na-AOT, this diffuse scattering masks the second-order Bragg peak due to the weak ordering within these samples. At $55 \mathrm{wt} \% \mathrm{Na}-\mathrm{AOT}$, the intensity of this diffuse scattering appears to have decreased slightly, with the intense Bragg peaks dominating the image. The presence of diffuse scattering at the higher concentration implies that, although the system adopts a structure more typical of classical lamellar behaviour and the role of Helfrich forces is reduced or negligible at high concentrations, the bilayers likely retain some elasticity. Thus, the local structure is still defective and bilayers (to a certain extent) continue to form connective defects to neighbouring layers. One further feature of note in the $2 \mathrm{D}$ profiles is the increase in monodirectional ordering of the scattering at higher surfactant concentrations. This is also likely a result of the Na-AOT system adopting a more classical lamellar structure with greater ordering at higher concentrations. However, these samples were loaded into the SAXS instrument using a flow cell and the possibility that the increased order is due to a shear effect cannot be ruled out.

A range of Na-AOT samples were also analysed by SAXS/WAXS. The only observed feature of the wide-angle region was a single broad peak, which was present in all samples within the lamellar region of the Na-AOT system, indicative of the fluidity of the hydrocarbon tail region. The absence of any additional peaks within this region shows that the phase does not undergo a formal transition to a structure with an ordered tail arrangement. This is evidence against the formation of a rippled lamellar phase (similar to those observed in several phospholipid systems ${ }^{120}$ ) proposed by Chidichimo et al. ${ }^{116}$ Rippled phases give rise to characteristic scattering within the wide-angle region that presents as a significantly sharper peak than any observed within the Na-AOT lamellar phase. ${ }^{121}$ However, it should be noted that the lack of a formal rippled phase does not contradict the evidence presented above that the bilayer contains significant undulations at low concentrations, as the alkyl chain environment is not significantly ordered within the disordered swollen arrangement of highly elastic bilayers.

Figure 3.9 shows plots of the bilayer repeat distance, $d$, in the Na-AOT system as a function of surfactant concentration. Our own experimental results are presented in Figure 3.9 (a), showing that discontinuous swelling does not occur. In Figure 3.9 (b), the same data set is used to show that Fontell's results can be recreated by assuming that the dominant Bragg peak corresponds to the first-order lamellar reflection at all concentrations. When the $q$ spacing 
of the second-order Bragg peak is attributed to the first-order peak within Fontell's "ICR", the calculated bilayer spacings of the system imply a drastic structural reorganisation has taken place involving a constriction of bilayers. However, when this peak is correctly assigned as the second-order Bragg peak, the system presents no abnormal swelling behaviour. Figure 3.9 (b) is consistent with Fontell's published results, which were subsequently quoted and reproduced elsewhere, ${ }^{87,114,122,123}$ even after an explanation of the intensity anomaly was published. ${ }^{112}$ To the best of our knowledge, Figure 3.9 is the first example of the Na-AOT bilayer spacings presented in a way that definitively shows that no drastic deviation from 1D swelling occurs within the Na-AOT lamellar phase. This figure also shows that Fontell's data can be reproduced with the incorporation of a single incorrect assumption. Aside from the incorrect assignment of the "ICR", the results presented here are in good agreement with those obtained by Fontell, indicating that differing equilibration times do not significantly affect the bilayer spacing of the system.
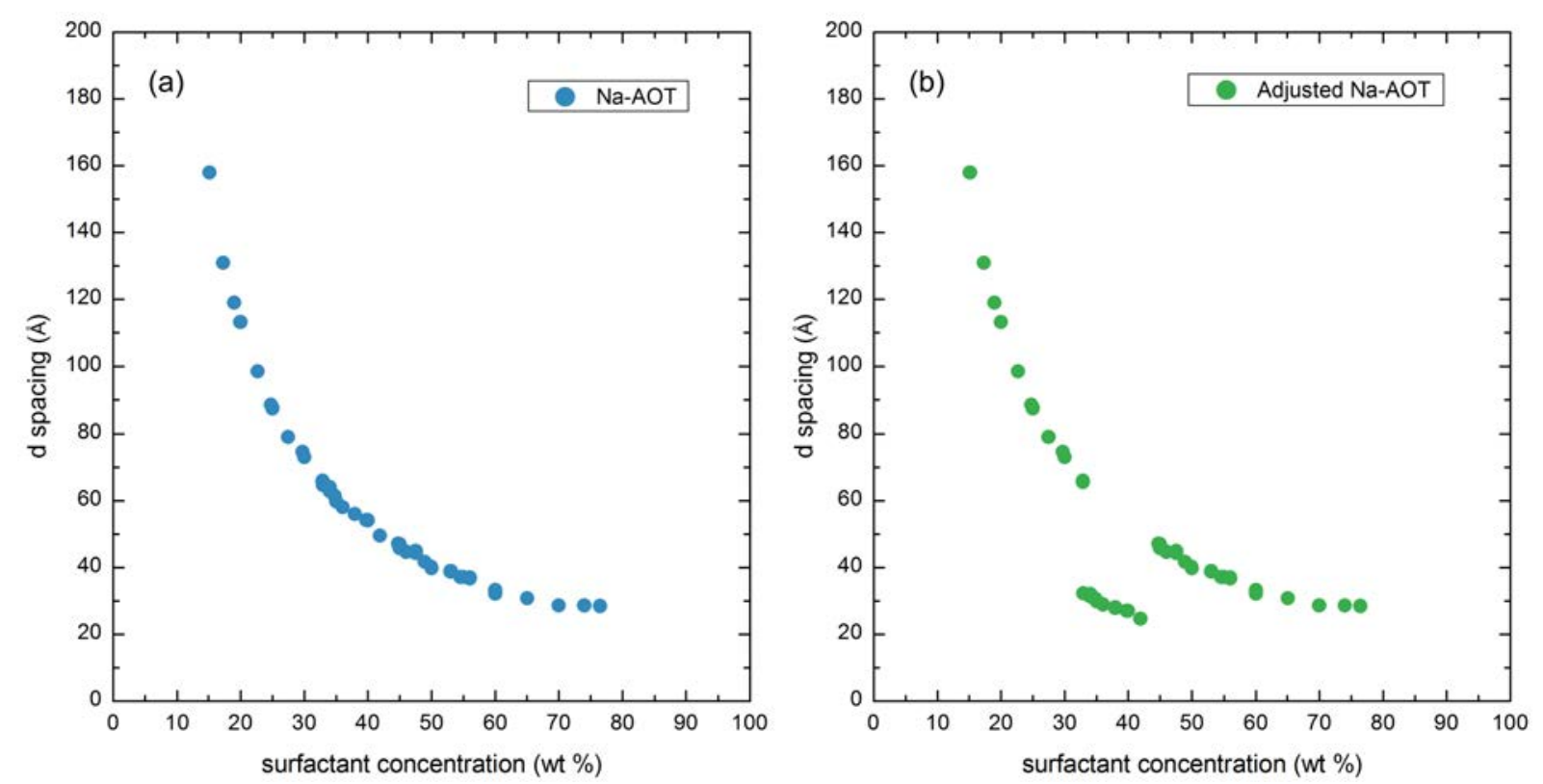

Figure 3.9: Changes in d spacing with increasing concentration for the Na-AOT system. Both plots show the same experimental data, with (a) demonstrating that the swelling behaviour of the system is not anomalous and (b) demonstrating that Fontell's results can be reproduced if the first-order Bragg peak is misidentified.

The dominance of diffuse scattering in the SAXS spectra of the Na-AOT system at $25{ }^{\circ} \mathrm{C}$ (see Figure 3.8) illustrates that thermal fluctuations are important in this system, with the resulting repulsive Helfrich forces playing a key role in the stabilisation of the lamellar phase, particularly at surfactant concentrations below $45 \mathrm{wt} \%$. In contrast to those at high concentrations, the second-order Bragg peaks produced by samples below $45 \mathrm{wt} \%$ are weak and broad. When combined with the evidence that Helfrich forces are a significant energy component at these concentrations, this implies that the lamellar phase has very weak long-range order and hence is likely to have a highly disordered, defective and undulating structure. As the bilayer correlations in the system are governed by forces that are particularly sensitive to variations in temperature, the $\mathrm{L}_{\alpha}$ phase of $\mathrm{Na}$-AOT was investigated for its temperature-dependent behaviour. 
The thermal behaviour of lyotropic lamellar phases is difficult to predict as different systems display significantly different behaviours upon heating, dependent on the composition of the surfactant and the intermolecular forces acting upon it. In the case of Na-AOT, the phase diagram indicates that the lamellar phase does not transition to any other liquid crystalline phases with increasing temperature and is stable to temperatures in excess of $100{ }^{\circ} \mathrm{C}$ at all but low concentrations. However, the Na-AOT $\mathrm{L}_{\alpha}$ phase is governed by many factors that do change with temperature, and can alter the system in opposing ways. Subtle differences can change the outcome of this competition and there are examples of other surfactant systems in which lamellar phases form more ordered or more disordered structures upon heating, ${ }^{124-127}$ and other examples where the phase either swells or contracts. ${ }^{128,129}$ The forces that govern selfassembly change with concentration and so different trends can also be observed in a single system. Na-AOT is an anionic surfactant and so we must consider the role of the counterion and changes in electrostatic shielding (intrabilayer and interbilayer repulsion), alongside changes in the entropic forces and steric effects on the Na-AOT molecules, which occur upon increased thermal energy and decreased hydration. We briefly consider the temperature dependence of the following parameters:

- van der Waals forces: The magnitude of van der Waals forces change with temperature because they depend upon a single temperature-dependent parameter, the Hamaker constant. However, in comparison to the relative changes in the other forces with changing temperature, the van der Waals forces can be considered to remain approximately constant. ${ }^{130}$

- Steric hydration forces: Given their name, it would be rational to assume that short-range steric hydration forces would weaken upon dehydration, and this has been reported by (and is the accepted theory of) some researchers. ${ }^{131}$ However, these forces have been shown to be at least partially entropic in nature, rather than a factor of the water structure, and so others have reported the forces to strengthen with increasing temperature. ${ }^{132}$ These forces are not a factor when the water thickness is above $\sim 20-30 \AA$.

- Helfrich forces: Helfrich forces are proportional to $T^{2}$ and so are expected to increase with temperature. ${ }^{32}$ This increase is normally strengthened by a reduction in $\kappa$ upon increasing temperature, as a result of a decrease in the bilayer thickness and thus increase in bilayer flexibility. ${ }^{125}$

- Electrostatic forces: An increase in temperature will result in dehydration of the headgroups within the bilayer, and a reduction in the shielding of electrostatic forces. This will result in an increase in the electrostatic repulsion between headgroups and between bilayers. The increased repulsion between headgroups will result in an increased contribution to the value of $\kappa$.

- Counterion dissociation: It has been reported for Na-AOT microemulsions that an increase in temperature results in an increased dissociation of the $\mathrm{Na}^{+}$counterion. ${ }^{133}$ This would remove any steric contribution of the counterion from the headgroup area, possibly altering the conformation of the surfactant. However, the primary effect would likely be a further decrease in electrostatic shielding.

- Molecular shape: The molecular shape of surfactant molecules can become either more or less inverted with temperature depending upon the exact molecular structure. However, 
the effective headgroup area of anionic surfactants generally increases with temperature as a result of increased steric repulsion, while the length of the tails normally decreases due to increased molecular motion, regardless of the type of surfactant. ${ }^{134}$ In the case of $\mathrm{Na}-\mathrm{AOT}$, the large number of possible rotational isomers may facilitate a greater change in the molecular shape than would normally be expected.

For the Na-AOT lamellar phase, the thermal behaviour can be simplified to a dependence upon the interplay between the entropic forces (increased due to the additional thermal energy), the electrostatic contribution (increased by dehydration and counterion dissociation), and any steric changes in the Na-AOT molecule (due to rearrangement in the tail region or change in the effective headgroup size). It is logical to assume that an increase in thermal energy would result in an increase in the undulations, and this has been reported in non-ionic systems. ${ }^{125,130}$ An increase in $k_{\mathrm{B}} T$ could then drive the lamellar phase formed by samples of $\sim 45-55 \mathrm{wt} \%$ Na-AOT (whose SAXS spectra were shown in Figure 3.8 to be dominated by Bragg peaks but retain some diffuse scattering) towards more undulating structures. This loss in order would be evident in the SAXS spectra by a broadening of peaks and a decrease in intensity. However, the results of this work show that the opposite is true. The Bragg peaks of the Na-AOT samples at concentrations above $45 \mathrm{wt} \%$ are generally observed to sharpen slightly, increase in intensity, and shift to higher q spacings with increasing temperature (see examples in Figure 3.10). The higher q spacing, caused by a decrease in bilayer repeat distance, can be explained by the expected decrease in tail volume and resulting decrease in the bilayer thickness. However, the sharpening of the Bragg peaks and increase in intensity reveals an increase in ordering, not the decrease expected due to additional bilayer fluctuations. This must be the result of an increase rather than decrease in the value of $\kappa$ and implies that the increase in electrostatic repulsion (and possible reorganisation of the Na-AOT tails) sufficiently negates the increased value of $k_{\mathrm{B}} T$ at high Na-AOT concentrations.
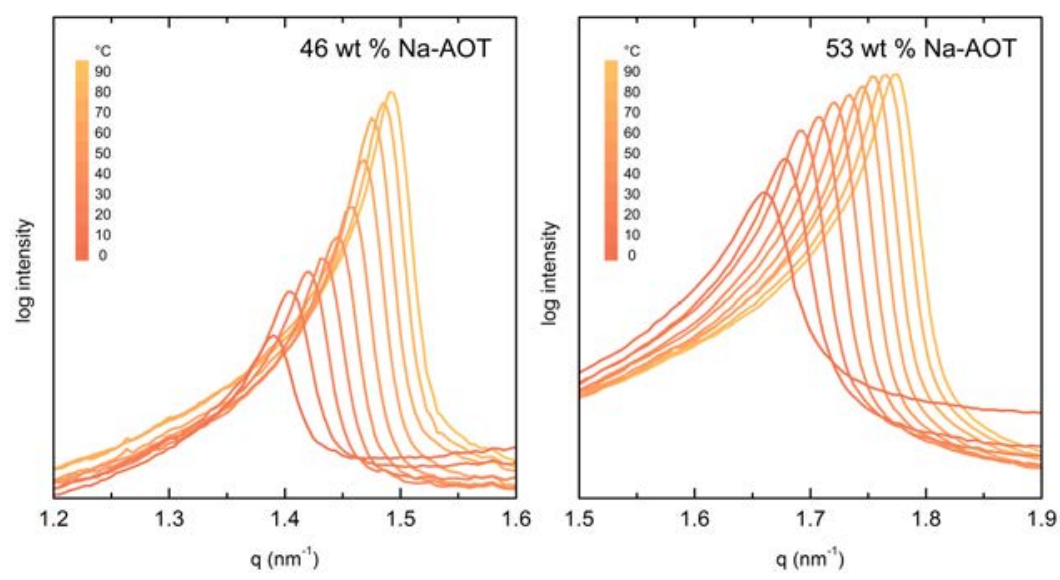

Figure 3.10: Evolution of the first-order Bragg peak with increasing temperature for Na-AOT samples of 46 and $53 \mathrm{wt} \%$ surfactant. Note an increase in q, slight sharpening of the peaks, and increase in intensity at higher temperatures.

The decrease in d spacing with temperature is not only observed at high surfactant concentrations, but is a trend observed throughout the lamellar region, with selected values shown in Table 3.1 and Figure 3.11. At many concentrations, the shift is not large and may be 
Table 3.1: Values for the $q$ spacing of the first-order Bragg peak $\left(\mathrm{q}^{*}\right)$ and the corresponding bilayer repeat distance (d) at selected Na-AOT concentrations and temperatures.

\begin{tabular}{cccccccc}
\hline \multirow{2}{*}{ Concentration (wt \%) } & \multicolumn{3}{c}{$\mathrm{q}^{*}\left(\mathrm{~nm}^{-1}\right)^{\S}$} & & \multicolumn{3}{c}{$\mathrm{d}$ spacing $(\AA)$} \\
\cline { 2 - 5 } \cline { 7 - 9 } & $25^{\circ} \mathrm{C}$ & $50^{\circ} \mathrm{C}$ & $90^{\circ} \mathrm{C}$ & & $25^{\circ} \mathrm{C}$ & $50{ }^{\circ} \mathrm{C}$ & $90^{\circ} \mathrm{C}$ \\
\hline 20.0 & 0.576 & 0.588 & 0.650 & & 109.14 & 106.80 & 96.65 \\
24.9 & 0.723 & 0.744 & 0.757 & & 86.88 & 84.44 & 82.98 \\
29.7 & 0.877 & 0.887 & 0.918 & & 71.63 & 70.86 & 68.43 \\
33.0 & 0.973 & 0.992 & 1.013 & & 64.58 & 63.34 & 62.03 \\
35.0 & 1.053 & 1.073 & 1.103 & & 59.67 & 58.56 & 56.96 \\
38.0 & 1.147 & 1.169 & 1.206 & & 54.78 & 53.77 & 52.10 \\
40.0 & 1.204 & 1.230 & 1.262 & & 52.21 & 51.08 & 49.81 \\
42.5 & 1.295 & 1.336 & 1.366 & & 48.52 & 47.03 & 46.00 \\
44.8 & 1.357 & 1.388 & 1.412 & & 46.30 & 45.27 & 44.50 \\
47.5 & 1.457 & 1.517 & 1.556 & & 43.12 & 41.42 & 40.38 \\
50.0 & 1.562 & 1.632 & 1.682 & & 40.23 & 38.50 & 37.36 \\
54.9 & 1.753 & 1.780 & 1.824 & & 35.84 & 35.30 & 34.45 \\
60.0 & 1.901 & 1.938 & 1.984 & & 33.05 & 32.42 & 31.67 \\
\hline
\end{tabular}

§ For Na-AOT concentrations between 35.0 and $42.5 \mathrm{wt} \%$, the $\mathrm{q}^{*}$ value is taken as half of the second-order q value.

explained by a change in the bilayer thickness (and thus its reduced contribution to the value of d). However, the magnitude of the change is not consistent, and generally decreases with increasing concentration, with the exception of erratic variations at intermediate concentrations. At the lower concentration end of the lamellar region, the change in $d$ spacing is significant and is an indication of another contributing factor that promotes bilayer constriction within this region. It should be noted that hysteresis was observed in SAXS experiments obtained when cycling the temperature; however, with sufficient relaxation time at $25^{\circ} \mathrm{C}$, the $\mathrm{q}$ spacings

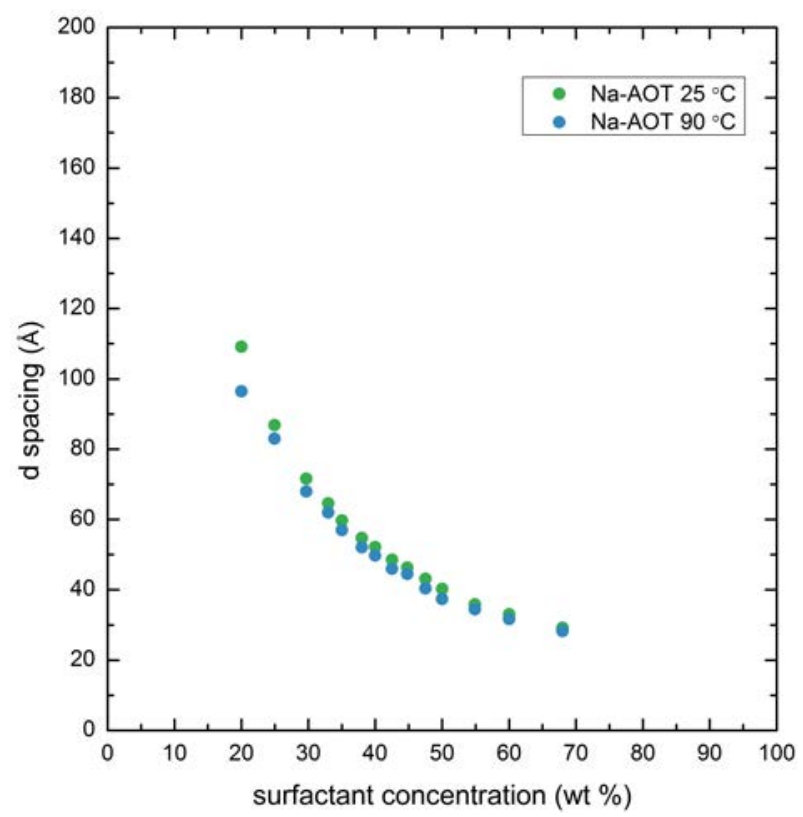

Figure 3.11: The effect of temperature on d spacing in the Na-AOT system. A larger change is seen at low surfactant concentrations, but $1 \mathrm{D}$ swelling is essentially retained. 
eventually returned to their initial values and so changes in repeat distance are not due to changes in the sample composition through loss of water.

The combined decrease in $d$ spacing and increase in peak intensity, as shown in Figure 3.10 for samples of 46 and $53 \mathrm{wt} \% \mathrm{Na}-\mathrm{AOT}$, results in the SAXS spectra at increased temperatures for a fixed $\mathrm{Na}$-AOT concentration mimicking those obtained by increasing the surfactant concentration at a fixed temperature. An increase in temperature producing the same trends observed with increasing surfactant concentration also holds true for samples at lower concentrations; however, the differences in the scattering patterns at these concentrations appear more significant, as this mimicking of the concentration-dependent behaviour includes the intensity variations of the Bragg peaks.

The SAXS spectra of low surfactant concentration samples that display first-order Bragg peaks at $25{ }^{\circ} \mathrm{C}$ were observed to lose this peak upon heating (a $20 \mathrm{wt} \% \mathrm{Na}$-AOT example is shown in Figure 3.12). This loss in intensity occurs due to the reduction in the bilayer repeat distance, shifting the peaks towards the region of $q$ space that coincides with the form factor minimum, and hence is the same trend observed with increasing concentration. Compared with samples at high concentrations, the changes in the SAXS patterns at lower concentrations are more significant, and alongside the intensity variation of the first-order Bragg peak, changes in the magnitude of the diffuse scattering is also evident. The more drastic changes in appearance may be due the scattering being weaker to begin with (and thus changes appear larger in magnitude). However, the more likely reason is that the highly disordered structures formed at low concentrations are more sensitive to changes in thermal energy than those at higher concentrations, which are limited in their degree of thermal fluctuations through a combination of stronger intermolecular interactions and smaller repeat distances that limit the capacity of the bilayer to undulate (as a general rule, the water thickness has to be greater than the bilayer thickness for out-of-plane fluctuations to be allowed). ${ }^{135}$
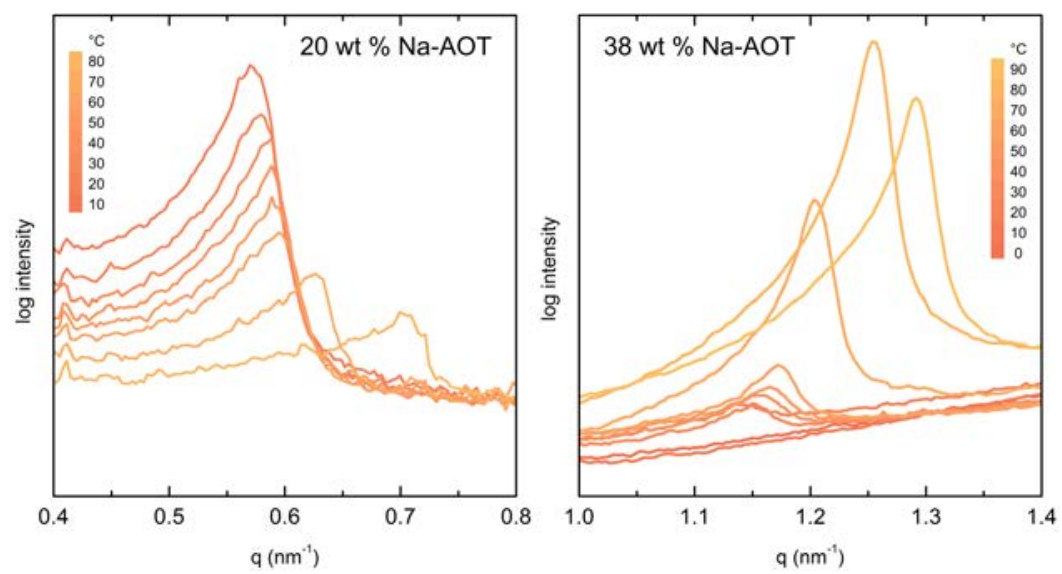

Figure 3.12: Evolution of the first-order Bragg peak with increasing temperature for Na-AOT samples of 20 and $38 \mathrm{wt} \%$ surfactant. At $20 \mathrm{wt} \%$, the peak decreases in intensity and shifts to higher q spacings. At $38 \mathrm{wt} \%$, the peak is absent at low temperatures, but becomes prominent at higher temperature, with increases in q spacing and intensity. However, the trends at $38 \mathrm{wt} \%$ are erratic, with the variation in q spacing not consistent, and a drop in intensity observed at $90^{\circ} \mathrm{C}$. 
Surfactant concentrations where the first-order Bragg peak is absent at $25^{\circ} \mathrm{C}$ were also shown to be sensitive to thermal fluctuations, while still following the general trend of producing SAXS spectra similar to those observed with increasing concentration. As the temperature is increased, the first-order Bragg peak reappears and then increases in intensity as the SAXS patterns gradually begin to resemble those of classical lamellar phases with well-defined Bragg peaks. This can be seen in Figure 3.12 for a 38 wt \% Na-AOT sample, although it is noticeable that the changes are considerably more erratic than those shown at other concentrations (there are sharp changes in the $\mathrm{q}$ spacing and the intensity of the peak decreases again at $90{ }^{\circ} \mathrm{C}$ ).

The overall trend observed throughout the lamellar phase region is that increases in temperature produce increases in ordering similar to those observed with increasing concentration. This implies that the increase in electrostatic repulsion between headgroups more than compensates for the increase in thermal energy that would be expected to result in increased undulation. While the trend described above was observed throughout the lamellar region, we note that samples between 34 and $40 \mathrm{wt} \%$, however, often display significant variations in thermal behaviour with very minor changes in concentration. These variations concern the second-order Bragg peaks and diffuse scattering. The second-order Bragg peaks are observed to increase in some samples but decrease in others, with no obvious trend. This can be seen in Figure 3.13, which also illustrates the large decrease in diffuse scattering, suggesting a significant change in the form factor (and thus structure) of the bilayer, which likely arises due to the increased motion within the alkyl tail region and possible conformational changes. The lack of an obvious trend in the second-order peak intensity may be because the changes are a factor of metastable defects. Samples within this concentration region were extremely fluid, which may be an indication of a defective local structure. If different samples have different types/compositions of local point defects, then the structures may produce different metastable structures upon heating. Curiously, samples at several concentrations displayed the behaviour seen at $34 \mathrm{wt} \% \mathrm{Na}$-AOT (shown in the 3D image of Figure 3.13), where the first-order Bragg peak disappeared and then reappeared. This implies a large increase in order, comparable to a significant increase in surfactant concentration of $\sim 8 \mathrm{wt} \%$. As the sample concentration was confirmed upon cooling not to have changed, the various forces that govern the behaviour must change significantly, which again may be due to the starting state of the microstructure providing a favourable energetic pathway upon heating.

It is somewhat paradoxical that an increase in thermal energy does not result in an increase in thermal undulations. The result is, however, consistent with the changes in the birefringence of the lamellar phase, where samples between $\sim 30$ and 45 wt \% Na-AOT are observed to change from negative to positive birefringence upon a sufficient increase in temperature. ${ }^{64}$ This is now known to be due to a decrease in undulations. ${ }^{111}$ Broadly speaking, in most lamellar phases Helfrich forces are either dominant due to lack of charged species, or are not observed at all because they are negated by electrostatic forces. So, the key question in the Na-AOT system is not why are the undulations suppressed by electrostatic forces at high temperatures, but why are they are not suppressed at low temperatures? 

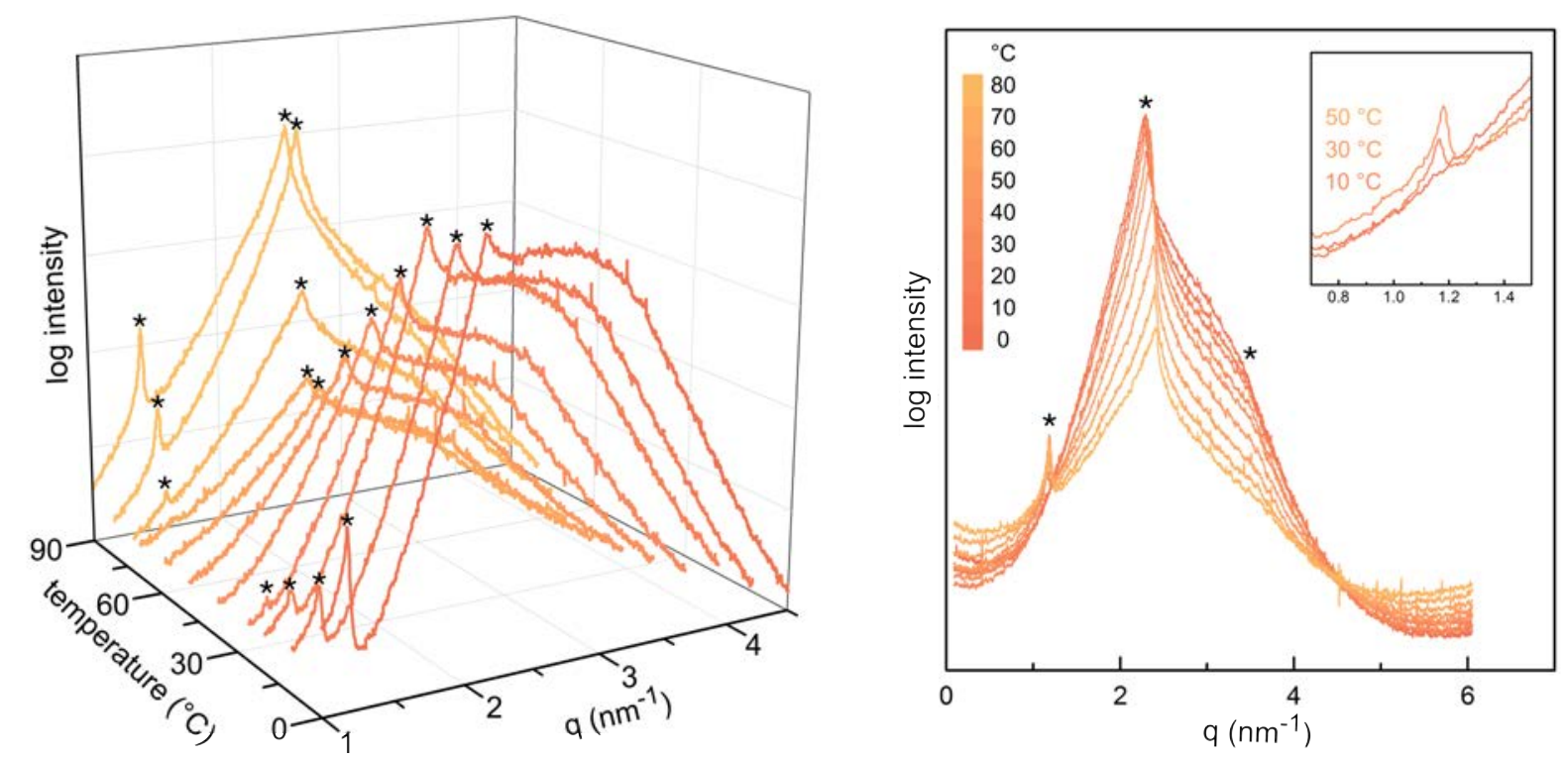

Figure 3.13: $3 \mathrm{D}$ and $2 \mathrm{D}$ plots showing the evolution of SAXS profiles of $34 \mathrm{wt} \%$ (left) and 40 wt \% Na-AOT (right) upon increasing temperature. The data sets of the two samples are presented in different ways to best illustrate the different behaviours that are exhibited within this region of the lamellar phase. Bragg peaks are marked by asterisks in both plots. The 3D plot at $34 \mathrm{wt} \% \mathrm{Na}$-AOT shows that the intensity of the first-order Bragg peak initially decreases with temperature, and the peak is not observed within a temperature range of $\sim 40-70{ }^{\circ} \mathrm{C}$, before it returns and subsequently increases in intensity again. The second-order Bragg peak shows similar intensity variations with temperature. The diffuse scattering, most evident at $\sim 3-4 \mathrm{~nm}^{-1}$, significantly decreases with temperature and does not reappear at high temperatures. The 2D plot at $40 \mathrm{wt} \% \mathrm{Na}$-AOT does not as adequately illustrate the variation in the first-order Bragg peak (although this is shown in the inset to increase in intensity with temperature), but more adequately displays the small shifts to higher q spacings. The second-order Bragg peak, diffuse scattering and possible third order contribution all decrease in intensity with increasing temperature.

The answer to this question is undoubtedly a combination of many factors. We can offer two explanations that are likely both significant contributing factors. Firstly, the counterion binding at low temperatures is sufficiently strong to significantly shield the headgroup repulsion. Evidence for this is provided by the temperature dependence of Na-AOT-based microemulsions, which show the opposite behaviour to non-ionic systems in that the spontaneous curvature decreases at high temperatures, a property that has been attributed to headgroup repulsion upon counterion dissociation. Secondly, the branched surfactant tails may promote undulations in the bilayer at low temperatures through the adoption of various rotational conformations that are then not stabilised at higher temperatures. Tentative evidence for this can be found in the reported intensity variations observed in Raman spectra by Faiman et al., ${ }^{99}$ the likely cause of which is the same molecular changes observed with increasing concentration. ${ }^{102-104}$ Further support is again given by Na-AOT microemulsions that display similar behaviour. ${ }^{136,137}$ This implies that upon increasing temperature, the Na-AOT tails adopt more kinked conformations through rotation of $\mathrm{C}-\mathrm{C}$ bonds, which would explain the decrease in bilayer thickness and also result in an increase in interfacial area at high temperatures (illustrated schematically in Figure 3.14). There are many examples in phospholipid systems of trans-gauche isomerisation upon heating, and this is sometimes accompanied by a pretransition involving an undulating or rippled structure. ${ }^{138} \mathrm{~A}$ conformational change in the 
Na-AOT tail region explains the more drastic changes in the SAXS patterns observed for lower concentration samples, as those of higher concentration have been reported to be comprised of predominantly one splayed conformation rather than a mixture and so will be unlikely to undergo a similar isomerisation. ${ }^{103,104}$ Hence, the electron density distribution, and thus form factor, will not be as significantly altered at higher surfactant concentrations.
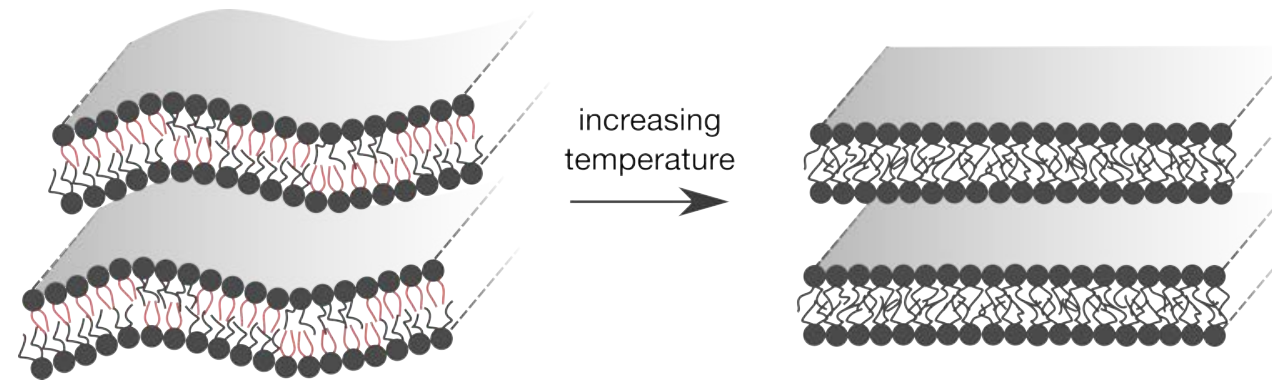

Figure 3.14: Schematic representation of trans-gauche isomerism in the Na-AOT tail region upon heating. Increased molecular motion within this region at higher temperatures causes the region to become more fluid and disordered, and results in a reduction in the bilayer thickness. However, the undulations in the Na-AOT bilayer are suppressed due to a reduction in the trans isomers (shown in red), which at low temperatures provide the variation in packing modes that promote an undulating structure. Despite the fluid tail region and reduced thickness, the high temperature bilayer may be stabilised through the increased lateral pressure provided by the tails at the hydrophobic/hydrophilic interface and an increase in electrostatic repulsion (that arises due to counterion dissociation and dehydration).

The SAXS spectra showing the thermal behaviour of the Na-AOT system reveal two important points: the sensitivity of the elastic properties of the Na-AOT bilayer to temperature, and a possibly important role of the Na-AOT tails. Despite the lamellar phase persisting at higher temperatures, the structure is clearly sensitive to small changes in thermal energy, and so the elastic properties of the bilayer can be manipulated by small variations in temperature. This confirms that the values of $k_{\mathrm{B}} T$ and $\kappa$ are (at some concentrations and temperatures) approximately equal, and means that the stability of the Na-AOT lamellar phase depends on the interplay between the resulting Helfrich forces and the electrostatic and van der Waals forces. However, this competition is then further complicated by the conformational isomerism of the Na-AOT molecule and any possible metastable defects that may result from structural changes.

\section{Sodium AOT Optical Microscopy}

Concentration gradient POM images (described in Chapter 2) provide limited insight into the defect behaviour in surfactant systems. This is because they are generally captured at low magnifications, and for any point of interest the exact surfactant/water composition is unknown. However, the technique is a useful first step when characterising the phase behaviour of a binary surfactant/water system. In the case of the Na-AOT system, the phase behaviour has been characterised previously, and the above discussion clearly shows that the system retains a lamellar structure rather than undergoing any of the previously suggested structural transitions. 
However, the concentration gradient POM image of the system, shown in Figure 3.15, illustrates how these ideas would arise. While the inverse hexagonal and inverse bicontinuous cubic phases are easily identified based on their characteristic textures (fan-like and isotropic, respectively), the regions of positive and negative birefringence within the lamellar phase are remarkably different in appearance.

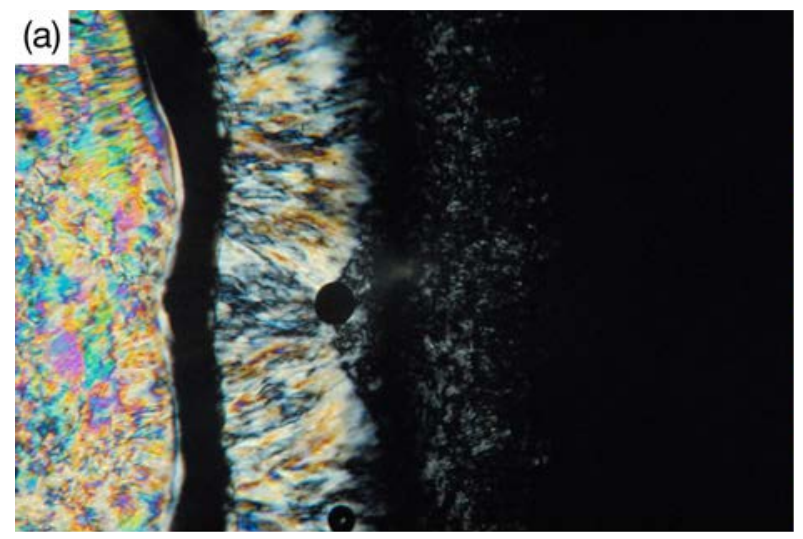

$\mathbf{H}_{l l} \quad \mathbf{V}_{l l} \quad \mathbf{L}_{a}$

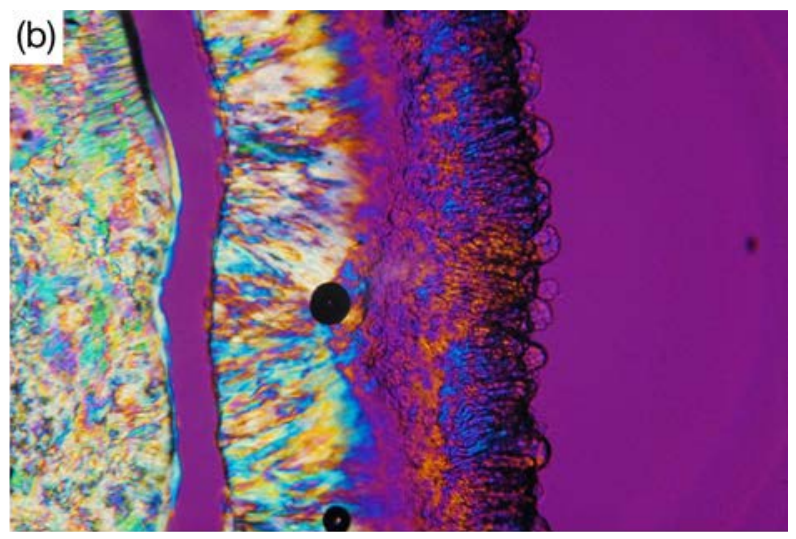

$\begin{array}{llll}\mathbf{H}_{\|} & \mathbf{V}_{\|} & \mathrm{L}_{a} & \text { myelin }\end{array}$

Figure 3.15: Concentration gradient of the Na-AOT system at $\times 40$ magnification and $\sim 25^{\circ} \mathrm{C}$ viewed under (a) crossed polarised light and (b) with the addition of a quarter-wave plate.

Although the change from positive to negative birefringence with dilution in the Na-AOT system has been shown to be consistent with the retention of lamellar bilayers, the Na-AOT system clearly exhibits different behaviours either side of this divide. Changes in the SAXS patterns and birefringence upon dilution are explained in terms of entropic forces providing an increasing contribution to the total energy of the system. It follows that the differences observed between the positively and negatively birefringent lamellar regions in the concentration gradient are likely due to the decrease in the long-range order upon the swelling of the lamellar phase. The swollen nature of the phase at low concentrations is evidenced by the formation of myelin structures at the surfactant/water boundary, clearly visible when the concentration gradient is captured using a quarter-wave plate: see Figure 3.15 (b). In contrast to the concentrated region of the lamellar phase, which was observed to be highly birefringent and viscous, the dilute region was weakly birefringent and a quarter-wave plate was required to capture the two regions clearly within a single image. The dilute region was also found to be less viscous (discussed in Appendix E), and the texture appears uneven (or "bumpy") within this region rather than aligning flush against the confining cover slip. This apparent unevenness originates within the region in which the optic sign of the birefringence changes, and entropic forces likely have an important role in governing the self-assembly. Based upon the previous SAXS measurements and the work of Nallet et al., we can infer that the uneven appearance of the texture is likely due to the presence of a highly swollen lamellar phase. This phase is formed of bilayers that are only stabilised via weak intermolecular interactions, meaning that it will be likely to undergo constant rearrangement and begin to flow with continued penetration of water towards the pure material at the centre of the slide. 
The defect behaviour of the Na-AOT system was characterised using a combination of POM and cryo-SEM, which were used to examine samples of known composition. Neither technique used individually can provide enough detail to fully characterise the system with confidence. POM reveals the nature of larger defects (greater than $1 \mu \mathrm{m}$ ) and is suitable for larger sample volumes. It is a well-established technique and can be used to confirm that samples are correctly equilibrated and the observed defect behaviours are representative of the entire sample. POM also allows in situ observations to be made as the experimental conditions are changed, and thus samples can be monitored over time or upon changes in temperature. Cryo-SEM can provide much more information about the local defect behaviour and the domain boundaries within the system; however, the area of sample viewed is relatively small. As the sample is cryogenically frozen, the samples are not viewed in their dynamic state and observations cannot be made in situ with changes to the energy of the system. A cryo-SEM methodology has not yet been produced that allows the reliable freezing of samples from elevated temperatures and so cryo-SEM observations are generally of samples frozen from their room temperature state. Through a combination of the two techniques, which in our investigations were shown to be in good agreement, we are able to characterise the Na-AOT system in more detail.

The POM images of Na-AOT samples in Figures 3.16 and 3.17 show how the defect behaviour of the $L_{\alpha}$ phase evolves as a function of concentration. Samples at the low concentration end of the two-phase $\left(L_{1} / L_{\alpha}\right)$ region (not shown) give predominantly isotropic POM textures due to the disordered nature of the micellar phase. As the Na-AOT concentration increases, the relative increase in the amount of lamellar phase results in anisotropy, and large Maltese crosses become the prominent feature of the textures, as observed at $10 \mathrm{wt} \% \mathrm{Na}$-AOT (see Figure 3.16). Maltese crosses indicate the presence of spherulite structures composed of lamellar bilayers. Within the two-phase region these structures are isolated within an isotropic background and so can be considered as multilamellar vesicles (MLVs) existing within a bulk water/micellar medium. As the Na-AOT concentration increases, the number of Maltese crosses increases, and the spherulites become incorporated within a global lamellar structure. As such, we define them as focal conic domains of positive Gaussian curvature (FCD-II), i.e. as defects within the lamellar structure rather than as MLVs.

The terms FCD-II, MLV and onion are often used interchangeably as they relate to the same spherical structural arrangement composed of stacked bilayers. However, the terms are not truly synonymous, as they can be distinguished based on how they interact with the surrounding medium. In this discussion, FCD-Ils are considered as defects embedded within a global lamellar structure, while MLVs (and onions) are considered as singular, disconnected structures. MLVs can exist as isolated structures within a biphasic solution (as observed in the two-phase region of the Na-AOT system) or can be induced by the shearing of some lamellar samples. In the case of shearing, a concentrated array of MLVs is formed and the sample is composed almost entirely of densely packed spherulite structures (sometimes called an onion phase and discussed for the Na-AOT system in Appendix E). In the case of FCD-II defects, the boundary with the surrounding medium can be sharp or gradual. The bilayers immediately surrounding the point of the singularity are highly curved, and the curvature demands decrease 

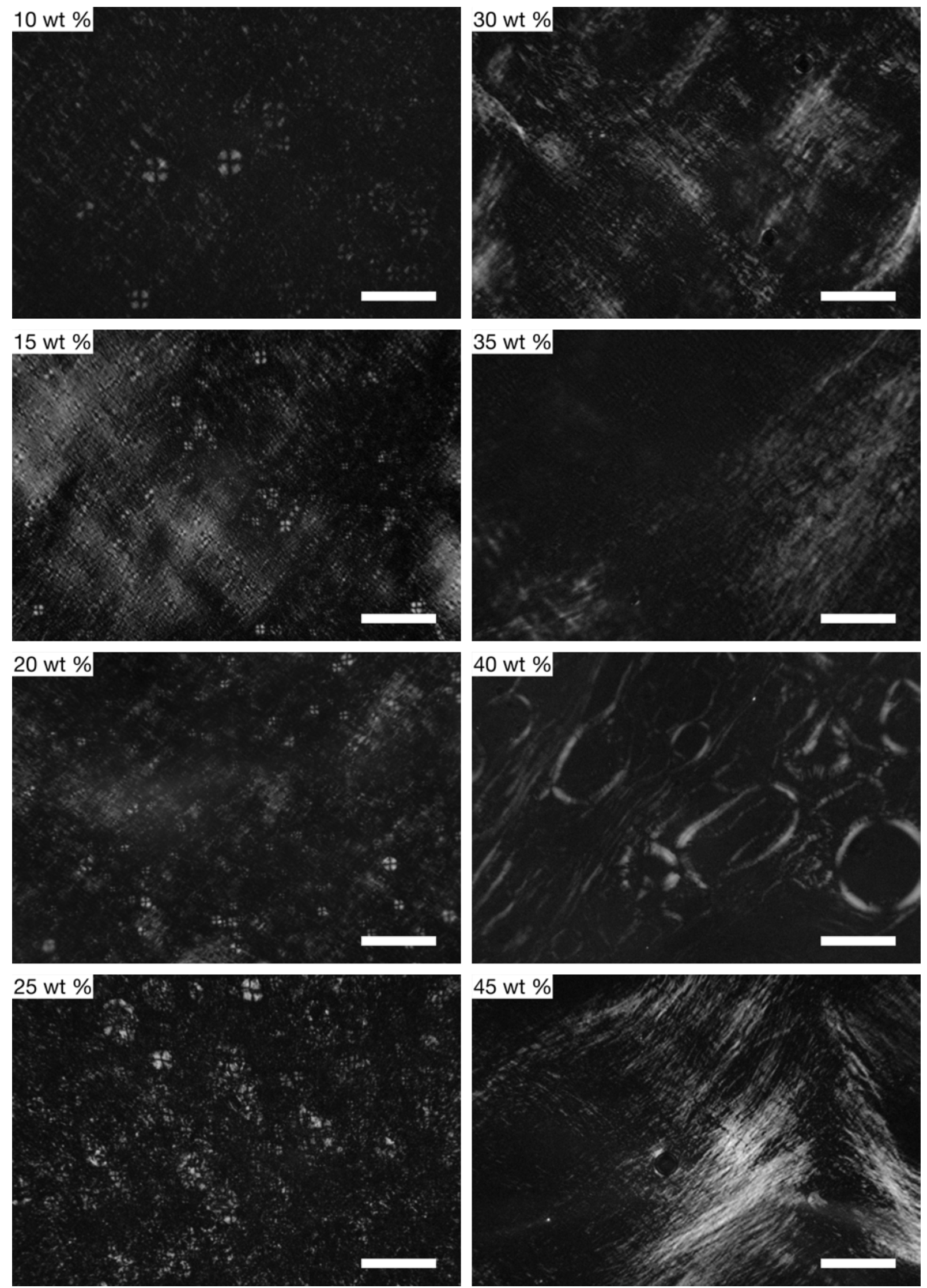

Figure 3.16: POM images of Na-AOT between 10 and 45 wt \% surfactant at $\sim 25{ }^{\circ} \mathrm{C}$. FCD-II defects are observed at low concentrations, which are replaced by grainy and streaky textures upon increasing concentration. A change from negative to positive birefringence occurs around 35-40 wt \% Na-AOT. Scale bars are $100 \mu \mathrm{m}$. 

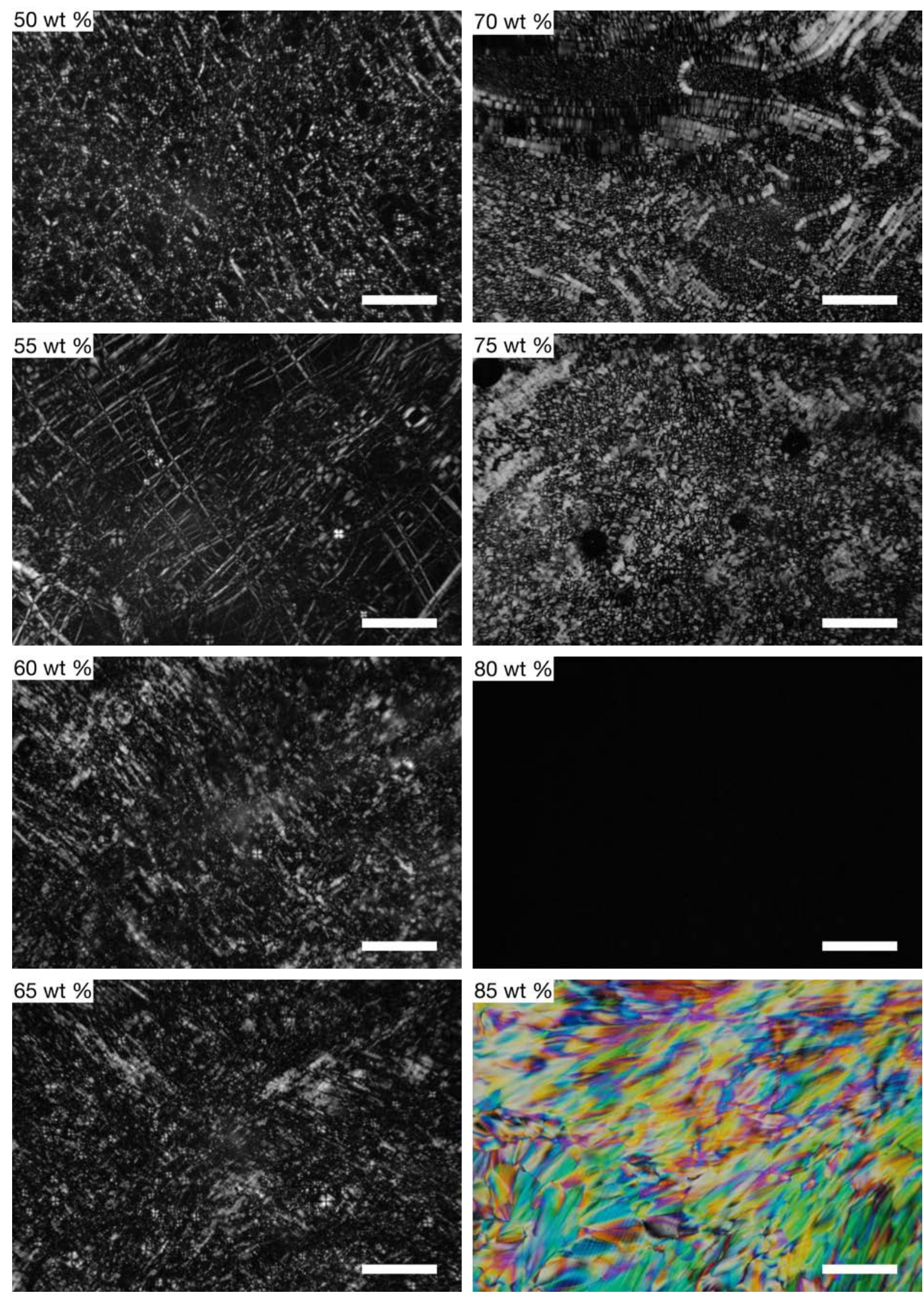

Figure 3.17: POM images of Na-AOT between 50 and 85 wt $\%$ surfactant at $\sim 25{ }^{\circ} \mathrm{C}$. A progression from oily streaks composed of FCD-I defects to mosaic patterns is observed in the lamellar phase with increasing concentration. Isotropic and fan-like textures reveal the presence of $\mathrm{V}_{/ /}$and $\mathrm{H}_{/ /}$phases, respectively. Scale bars are $100 \mu \mathrm{m}$. 
significantly for the bilayers further removed from the defect. The bilayers may gradually relax into the global lamellar phase, such that the focal conic domain is embedded within a lamellar phase of the same average repeat spacing, with no distinct boundary defining the end of the defect and the beginning of another domain. Equally, the FCD-lls may form sharp boundaries with other lamellar domains, such as bilayers orientated in other directions or with other FCD-Ils. As the boundary conditions (and thus packing constraints) of the FCD-Ils change, the defects can compensate by deviating from the ideal spherulite shape, which is clearly observed within the Na-AOT system as the number of FCD-IIs increases.

Images showing sample concentrations of 15, 20 and 25 wt \% illustrate the gradual change in the nature of the FCD-II defects. When compared with the biphasic region, there is a significant increase in the number of defects and the majority are smaller in size, although some large and easily identifiable defects remain at all three concentrations. The backgrounds of the images have regions that appear to be isotropic and birefringent regions that appear "grainy". When the sample stage was rotated, the grainy regions gradually became dark, while the previously dark regions appeared anisotropic and grainy. This implies that the structure contains an averaged ordering within these regions, possibly through the formation of large domains of directionally ordered bilayers. However, this pattern is also consistent with the formation of many FCD-II defects too small to be observed individually, due to the resolution capabilities of the microscope. As the Na-AOT concentration increases, the Maltese crosses become significantly distorted (shown clearly at $25 \mathrm{wt} \%$ ), indicating that the bilayers adopt non-spherical and disordered arrangements around the point of the FCD singularity. It should be noted that FCD-Ils can be elongated by small amounts of shear during sample preparation; however, such structures are generally observed to regain a spherical shape over time, while the defects in the Na-AOT system were observed to be very stable and hence the distortions result from the packing constraints imposed by their surroundings (such as interactions with other defects) and/or changes in the elasticity (and thus curvature requirements) of the bilayer.

At Na-AOT concentrations of 30 and 35 wt \%, few undistorted FCD-II defects are observed and the dominant feature of the textures are the grainy domains, which at $30 \mathrm{wt} \%$ are smaller and randomly orientated. For samples between 35 and $40 \mathrm{wt} \% \mathrm{Na}$-AOT, individual defect points were rarely observed and the textures appeared predominantly isotropic, although some grainy domains and a small number of oily streaks were also observed. In contrast to samples at both lower and higher Na-AOT concentrations, there is an apparent significant decrease in the number of defects present in the lamellar phase within this concentration range. However, as previously noted, the optic sign of the birefringence changes within this region, meaning that features are less easily identified. Additionally, these samples were observed to be of low viscosity and the streaky features in the $40 \mathrm{wt} \% \mathrm{Na}$-AOT image are characteristic of a sample under flow.

Based on the SAXS data, this is the region in which the contributions of Helfrich and electrostatic forces to the energy of the system are delicately balanced. Thus, it is an area of significant interest as changes in the defect structure and connectivity of the lamellar phase likely take place in this region. As such, this area was sampled extensively in an attempt to 
gain additional information. However, despite the use of different light sources (the change in birefringence is dependent upon the wavelength of light passing through the sample), very little information was garnered, as the number of observable defects remained very low. However, some regions of birefringence were observed in which defects similar to those observed either side of the loss of birefringence coexisted. To rule out the possibility that the apparent reduction in defect concentration was due to the low viscosity resulting in thinner samples (and thus a reduction in the number of defects present), the samples were also viewed in microslides with a defined thickness of $100 \mu \mathrm{m}$, producing textures not remarkably different from those shown in Figure 3.16. The problems associated with the weakly birefringent material mean that POM is not sufficient to confidently characterise the defect behaviour of the system in this region. As such, cryo-SEM was used to gain further insight and is discussed below.

At 50 wt \% Na-AOT, the lamellar phase is clearly birefringent and the defects are once again clearly discernible. Figure 3.17 shows that the defects formed at $50 \mathrm{wt} \% \mathrm{Na}$-AOT are significantly different to those at lower concentrations. There is a large number of defects, which are predominantly FCD-Is, and can be seen both individually and conjoined in the form of oily streaks (although some distorted FCD-II defects also remain). FCD-I and FCD-II defects have the same disclination "strength" but the opposite sign ( $s=-1$ and $s=+1$, respectively). Although they can be distinguished in POM images as the brushes of the crosses are characteristically different, in this research the identification was confirmed by observing the defects upon rotation of the sample stage. The pattern formed by FCD-I defects is observed to rotate in the opposite direction to the sample, whereas the pattern of FCD-II defects rotates in the same direction. The domination of the texture by FCD-I defects shows an overall move towards more negative Gaussian curvature and thus indicates a change in the elasticity of the bilayer.

At $55 \mathrm{wt} \% \mathrm{Na}-\mathrm{AOT}$, the oily streaks are longer than those observed at $50 \mathrm{wt} \%$ and the decrease in both the number of individually discernible FCD-I defects and the size of those that are observed indicates that the defects composing the streaks are smaller. Upon further increases in concentration (60 and 65 wt \% Na-AOT in Figure 3.17), the streaks begin to align and form small orientated streaky domains. The POM image for $70 \mathrm{wt} \% \mathrm{Na}$-AOT shows the continued presence of oily streaks, some of which appear obviously striated, but between the streaky regions the dominant feature is now the "marbled" or mosaic-like pattern that reveals the structure to be highly curved with a complex packed array of defects, which are clearly distorted by those surrounding them.

The textures for samples at 80 and $85 \mathrm{wt} \% \mathrm{Na}$-AOT are characteristic of $\mathrm{V}_{/ /}$and $\mathrm{H}_{/ /}$phases. The $3 D$ ordering of the $V_{/ /}$phase results in isotropic textures, while the $\mathrm{H}_{/ /}$phase produces brightly coloured textures with fan-like domains, formed by the rapid growth of the phase in different directions from many nucleation points. The domains are separated by line disclinations that minimise the local strain, and the size, orientation and colour of the fan-like domains are determined by the shear involved in preparing microscope slides of the extremely viscous material. As the features in textures of the $\mathrm{H}_{/ /}$phase are a factor of the slide preparation, they provide no relevant information other than the initial classification of the phase formed. 
Given that the SAXS profiles of the Na-AOT lamellar phase could be changed significantly by altering the temperature, this effect was also investigated by POM. The birefringence of samples between 33 and $42 \mathrm{wt} \% \mathrm{Na}$-AOT could be easily manipulated by varying the temperature, and is consistent with the changes in the SAXS spectra reported above. However, somewhat surprisingly, the defects within textures in sealed samples were not observed to change significantly with increases in temperature, except in the vicinity of the phase boundary between the lamellar phase and isotropic fluid. Elevations in temperature that promoted the rise or fall of the first-order Bragg peak of low concentration lamellar samples in SAXS experiments, did not result in significant changes to the defect behaviour when observed by POM. This implies that once formed, the FCD defects are stable and do not change form, despite the thermally induced changes to the bilayer elasticity. An exception to this was observed when unsealed samples were heated. The lack of confining walls meant that samples were not restricted from flowing. As such, samples were observed to significantly change under the microscope as the temperature increased. FCD-II defects were observed to move through the sample, and in some cases, deform and eventually break up. FCD-I defects were observed to elongate and become obviously striated as the samples flowed. These effects are likely in part due to flow resulting in a thinning of the sample, but could also be due to changes in concentration due to dehydration of the sample.

The general trend revealed by POM observations can be described simply as the progression towards defects of increasingly negative curvature with increasing surfactant concentration, illustrated in Figure 3.18. A gradual transition from FCD-Ils of positive Gaussian curvature to FCD-Is of negative Gaussian curvature is observed with increasing concentration. This progression is logical, as while the phase behaviour of the system is dominated by a wideranging $L_{\alpha}$ phase, it forms phases of positive and negative Gaussian curvature at lower and higher concentrations, respectively. The focal conic defects are formed to satisfy the curvature frustration of the Na-AOT bilayer, which arises from the inherent desire of each of its monolayer components to curve. As the forces governing the self-assembly change with concentration, so do both the elasticity of the lamellar bilayers and the subsequent energy costs that need to be dissipated through the formation of defects. The Na-AOT lamellar phase exists over a large concentration range and so the curvature requirements at each extreme are different. Defects of positive curvature are formed at the dilute extreme, where it borders a micellar phase. Defects of negative curvature are required at high concentrations with oily streaks formed, which then give way to highly curved mosaic-like arrangements in the proximity of the bicontinuous cubic phase. The lamellar phases of most binary systems do not exist over such large concentration ranges, and so do not contain the range of defects (and inversion of curvature) observed in the Na-AOT system. However, the transition from FCD-II to FCD-I upon increasing concentration has been shown in non-binary systems, such as in the cetylpyridinium chloride/hexanol/brine system studied by Kléman. ${ }^{139}$ The observation of this behaviour in the binary Na-AOT system suggests that the Na-AOT bilayer has an inherent flexibility (likely due to the branched tails) that is only matched in most other systems through the inclusion of a cosurfactant.

The switch in defect behaviour from positive to negative Gaussian curvature on increasing concentration coincides with the change in birefringence, and thus may be partially due to the 


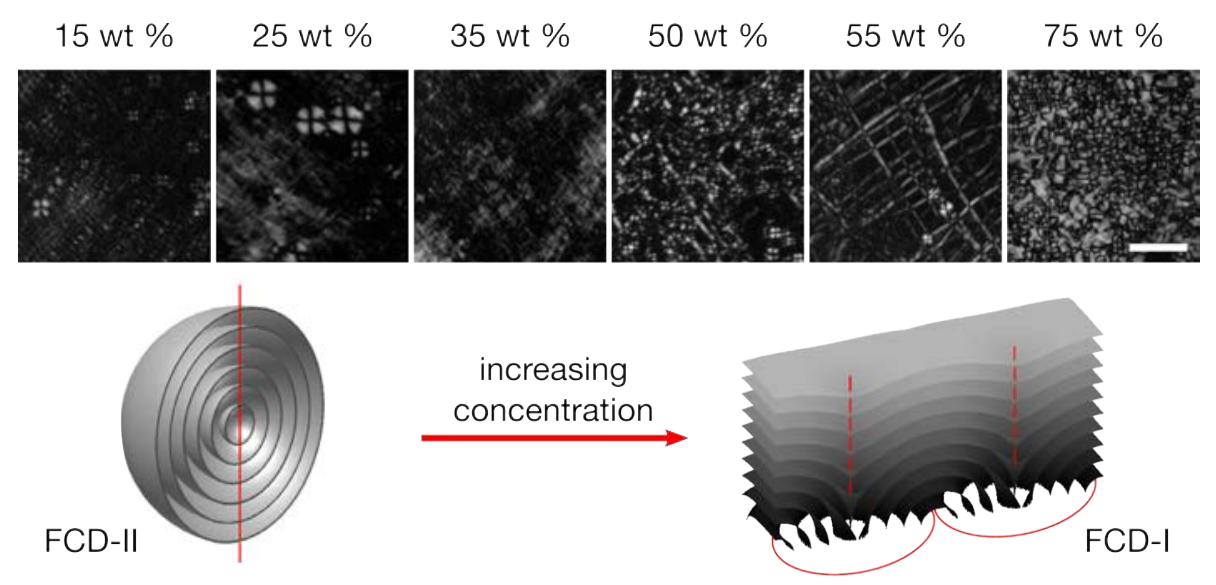

Figure 3.18: Schematic representation of the defect types formed in the Na-AOT lamellar phase with increasing concentration. The system undergoes an inversion in curvature resulting in a change from defects of positive Gaussian curvature to defects of negative Gaussian curvature, which exist as spherulite structures, oily streaks or mosaic patterns. This illustration shows idealised defect forms, when in reality, the defects distort upon changes in concentration, as shown in the POM images. Scale bars are $50 \mu \mathrm{m}$.

changes in the elasticity of the bilayer and governing forces within this region. However, it should also be stressed that although reduced in number, FCD-II and FCD-I defects were observed to coexist within this region, and that observations of their structure over the full lamellar phase concentration range showed that the defects change gradually in order to satisfy the evolving energy demands of the system. They were observed to change in size, shape, orientation and degrees of interaction. These defects clearly have an important role in stabilising the lamellar phase, and the ability of the defects to adapt in this way should not be underestimated. As the simplistic indefinite flat bilayer description of the lamellar phase is outdated and clearly untrue for real systems, the idealised schematic representations of the defects themselves (such as those shown in Figure 3.18) may in time come to be considered as an unhelpful oversimplification.

\section{Sodium AOT Cryo-SEM}

The POM images presented above illustrate the important role of large FCDs in the stabilisation of the Na-AOT lamellar phase. The role of these defects is to dissipate the curvature energy costs of the system that arise from the packing of monolayers (with a non-zero value of mean curvature) into a stacked arrangement of bilayers. The overall change from positive to negative Gaussian curvature reveals how the curvature frustration of the bilayer changes with concentration. However, the POM does not reveal the nature of defects that exist on smaller lengthscales. As such, cryo-SEM was used in this research to investigate the local structure of the lamellar phase, revealing how the connectivity between bilayers, and the degree of deviation from the classical description of flat indefinite bilayers, changes with concentration. The cryo-SEM images also provide a clearer illustration of the distortion of large FCD defects observed via POM, and the boundaries between lamellar domains. 
Cryo-SEM images obtained at Na-AOT concentrations of 25 and 38 wt \% Na-AOT are shown in Figure 3.19. The dominant structural feature in the images of both concentrations, not revealed in the POM investigation, is the obvious disorder within the microstructure. There is a high degree of connectivity and the lamellar phase clearly deviates from flat bilayers, with bilayer undulations and flexibility evident. At low magnifications, the sample of $25 \mathrm{wt} \%$ Na-AOT - shown in Figure 3.19 (a) - displays behaviour that is consistent with the POM observations, with several large spherical FCD-II defects of over $10 \mu \mathrm{m}$ observed alongside many smaller and distorted FCD-II defects. Several examples are marked in red, although we are unable to determine the exact boundaries between the defects and the surrounding phase. Similarly, while different regions of the sample appear to have an average direction of orientation, no distinct domain boundaries are visible. The reason for this is revealed in the higher magnification images, which show the phase to be very disordered on a local scale. Figure 3.19 (b) shows a focal conic defect that merges with neighbouring regions despite them aligning in different directions, while Figure 3.19 (c) is an enlargement of the area indicated in Figure 3.19 (a) and partially shows the two large FCD-Ils and the area between them. Both images reveal the large number of microstructural defects present, in the form of connective passages between surfactant bilayers, pores that provide water pathways through layers, and closed defects that restrict water movement. On local lengthscales, the sample displays characteristics that resemble those of the $L_{3}$ phase, with many connected and randomly orientated passages forming continuous surfactant and water networks. However, the closed defects disrupt the continuity of both domains and imply that the positive Gaussian curvature restricts the formation of a true $L_{3}$ phase. The phase is obviously less disordered than $L_{3}$ phase, and has an average bilayer repeat distance and regions with overall average directional order. However, the bilayers are clearly very flexible and so can facilitate changes in directional order without the formation of harsh curvature environments, which are unfavourable and usually result in distinct boundaries between domains. As such, the low concentration lamellar samples do not exhibit defects that are commonly associated with the lamellar phase, such as screw defects and clusters of small FCD defects in the form of grain boundaries.

At $38 \mathrm{wt} \% \mathrm{Na}$-AOT, the degree of disorder within the microstructure is significantly reduced and directional order is clearly evident. However, there remains a large amount of connectivity between bilayers, and the undulation is obvious. Whether viewed from an angle parallel (sideon) or perpendicular (top-down) to the bilayers - Figure 3.19 (f) shows both views - the deviation from the idealised flat stack of layers is easy to perceive, despite the overall structure being instantly recognisable as a $L_{\alpha}$ phase. This sample concentration is within the region previously described as anomalous, and the sample showed no first-order Bragg peak in SAXS experiments and very little birefringence under a polarising optical microscope. It is clear from the images that the only deviation from lamellar ordering is through bilayer undulation. In fact, compared to samples of either higher or lower surfactant concentrations, there are less large defects present. This was stated tentatively in the POM investigation as the observation could have been a factor of the weak birefringence. The cryo-SEM shows that some FCD-II defects remain, but they are significantly smaller than many of those observed at $25 \mathrm{wt} \% \mathrm{Na}$-AOT (generally between 0.5 and $2 \mu \mathrm{m}$ ) and are significantly reduced in number. One FCD-II defect is shown in Figure 3.19 (e), and although the sample has cracked due to damage from the electron 

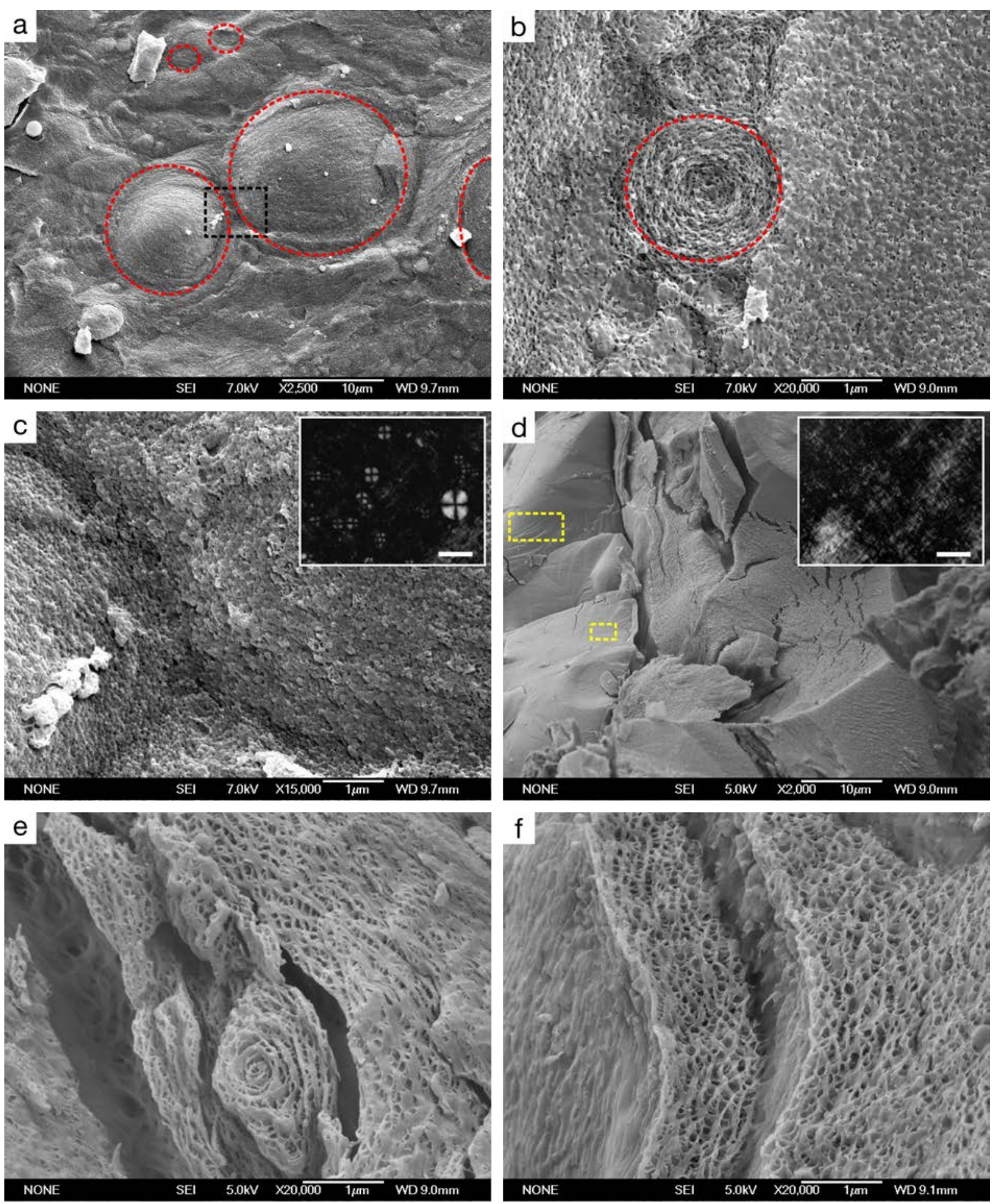

Figure 3.19: Cryo-SEM images of Na-AOT samples of $(a-c) 25$ and (d-f) 38 wt \% surfactant. Red circles highlight examples of FCD-II defects. Black rectangle in (a) is enlarged in (c). Yellow boxes in (d) highlight features consistent with screw dislocations. The insets show the corresponding POM images, with scale bars of $50 \mu \mathrm{m}$.

beam, it is noteworthy that the defect is almost seamlessly integrated into the surrounding orientated phase, despite its small size. This is a further indication that the Na-AOT bilayers retain flexibility at this concentration. This flexibility also facilitates the gradual redirection of the bilayer orientation resulting in continuous regions of moderate curvature. In contrast to the images at 25 wt \% Na-AOT, there is evidence of lamellar domains along different directional axes, and features akin to those identified as screw defects elsewhere (highlighted in yellow). ${ }^{140}$ 
Figure 3.20 shows cryo-SEM images for samples of 45 and 60 wt \% Na-AOT, both concentrations at which the properties of the Na-AOT system have been reported to change. The lowest concentration at which typical lamellar behaviour is reported (in that the first-order SAXS peak and positive birefringence are observed) is approximately $45 \mathrm{wt} \% \mathrm{Na}-\mathrm{AOT}$. It is also above this concentration that Callaghan et al. reported that the water diffusion changed from $2 D$ to $\sim 3 D$, while at $60 \mathrm{wt} \%$ they observed a change back to 2D diffusion, which also coincides with the drop in electrical conductivity. ${ }^{47}$

Comparison of Figure 3.20 with the cryo-SEM images for lower Na-AOT concentrations reveals a drastic loss in the degree of undulation along the bilayers when the Na-AOT concentration is increased from 38 to $45 \mathrm{wt} \%$. Although some fluctuation is still evident - most obvious in the higher magnification image shown in Figure 3.20 (c) - the phase is considerably more ordered. Connectivity between the surfactant bilayers remains, but there is a significant reduction in the number of connections, and those that are present are grouped together between small regions of compacted bilayers. Despite these obvious differences between the structures at 38 and $45 \mathrm{wt} \%$, there are also some similarities. The number of large defects remains low and the structure is arranged into several domains with different directional axes. Although not many large defects are observed, some FCD defects are present in the form of FCD-Is that align to form isolated oily streaks. There are also areas in Figure 3.20 (c) in which the ordering of the bilayers is clearly distorted, which is likely due to the formation of edge dislocations, but could be caused by defect points below the plane of view.

From the previously described SAXS results, samples of $60 \mathrm{wt} \% \mathrm{Na}$-AOT are expected to show classical lamellar behaviour, as this concentration produced strong Bragg reflections far greater in intensity than any diffuse scattering. As such, thermal undulations would not be expected to have a significant role in the structure of the system. The POM images at this concentration showed short clusters of oily streaks in a texture typical of lamellar systems. The low magnification cryo-SEM image for 60 wt \% Na-AOT - shown in Figure 3.20 (d) correlates well with the POM and SAXS results, as it shows a large domain of ordered lamellar bilayers, with several other domains visible in the background. Partially bisecting this region is an oily streak, indicated by the red arrow. This image also shows a feature not observed at the previously discussed concentrations, in the form of distinct grain boundaries separating domains of different orientations. The most obvious of these boundaries is highlighted in yellow and is composed of a cluster of defects. The nature of these defects is not obvious but may be a collection of oily streaks composed of small FCD-Is, or the result of screw dislocations. The presence of a periodic line of pitted defects in the same region indicates that there are streaks of FCD-I defects below the region of observable structure, consistent with the pits resulting from the hyperbolas of the FCD-Is when viewed from above. Despite the obvious presence of a typical ordered $\mathrm{L}_{\alpha}$ phase at $60 \mathrm{wt} \% \mathrm{Na}$-AOT, the two images at higher magnifications show that the structure still contains connective defects and that the bilayers are not planar. The microstructure in Figure 3.20 (e) is similar to that shown in Figure 3.20 (c) for the sample at $45 \mathrm{wt} \% \mathrm{Na}$-AOT. Figure 3.20 (f) also shows the presence of many edge dislocations at $60 \mathrm{wt} \%$ $\mathrm{Na}-\mathrm{AOT}$, which are primarily $\mathrm{s}=+1 / 2$. The formation of such defects is consistent with a typical lamellar phase and provides further connectivity between the bilayers. 

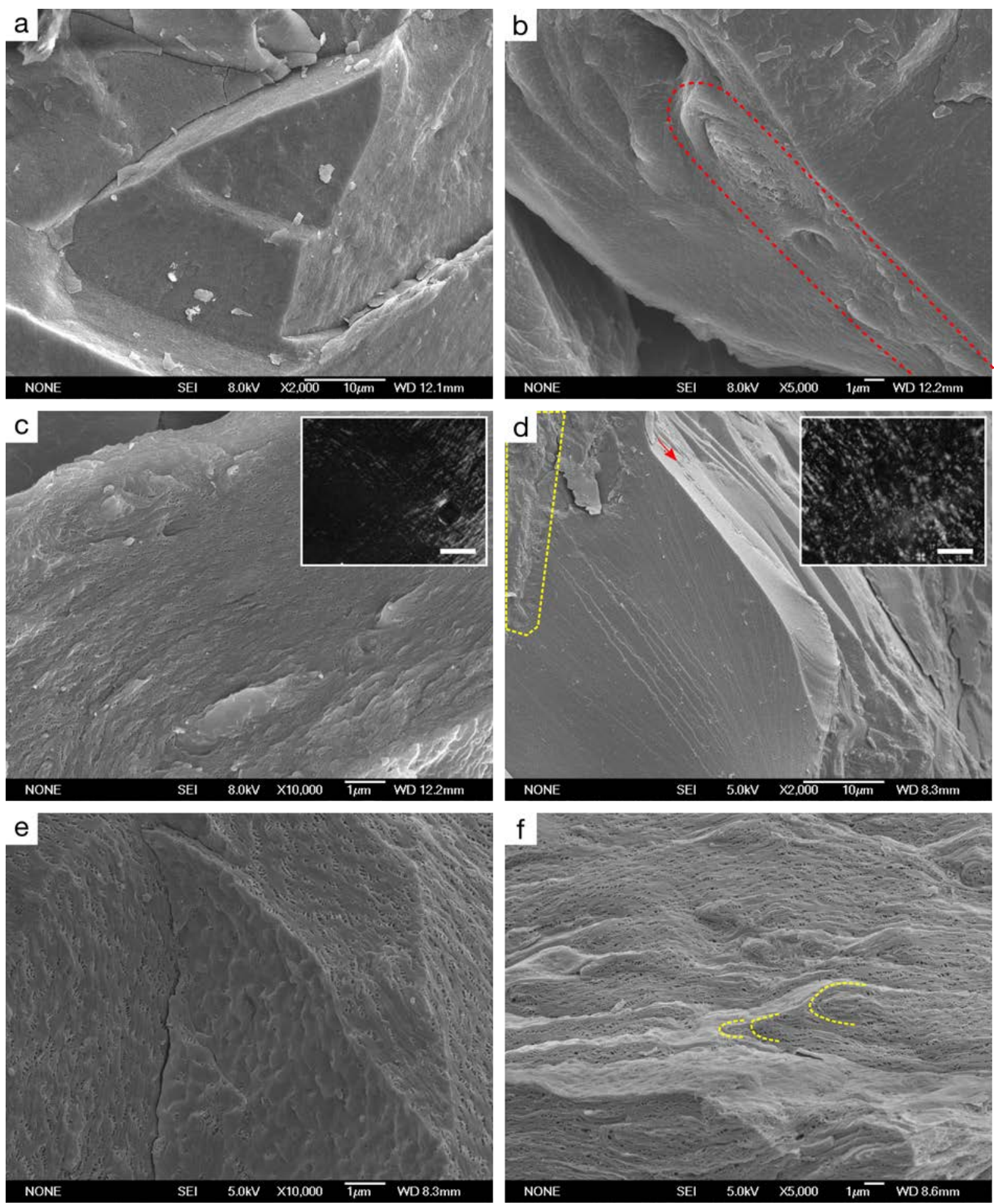

Figure 3.20: Cryo-SEM images of Na-AOT samples of (a-c) 45 and (d-f) 60 wt \% surfactant. Red dashed line in (b) shows a oily streak. Red arrow in (d) highlights an oily streak within a lamellar domain. Yellow dashed line in (d) indicates a domain boundary composed of a cluster of defects. Yellow dashed lines in ( $f$ ) illustrate an example of a series of edge defects. These can be identified as $s=+1 / 2$, although they are likely paired with either complementary $s=-1 / 2$ defects or (given the gradual tilt of the structure towards a plane parallel to the imaging angle) screw dislocations. The insets show the corresponding POM images, with scale bars of $50 \mu \mathrm{m}$. 
At 70 and 75 wt \% Na-AOT, POM images showed that with increasing surfactant concentration, a continuing trend towards negative curvature results in a change in the structure from many short oily streaks to mosaic-like patterns. The cryo-SEM images for samples of the same concentrations are once again in excellent agreement with the POM images. Cryo-SEM images at $70 \mathrm{wt} \% \mathrm{Na-AOT}$ (shown in Figure 3.21) display a more ordered local structure in comparison with images at lower concentrations, with a reduction in connective defects. However, the macrostructure reveals a large increase in FCD-I defects, due to the continued progression towards negative curvature. Many oily streaks are observed in Figure 3.21 (c), along with saddle-splay surfaces and packed defects that deviate from the idealised shape. The large number of defects and increase in curvature results in few ordered classical lamellar domains such as those observed at $60 \mathrm{wt} \%$.

At a concentration of 75 wt \% surfactant, the $L_{\alpha}$ phase of the Na-AOT system is in close proximity to the $L_{\alpha}-V_{/ /}$phase boundary. Given that the $V_{/ /}$phase is a bicontinuous structure that conforms to an infinite periodic minimal surface, it is not surprising that the lamellar phase is highly defective and exhibits large amounts of curvature. In particular, the images in Figure 3.21 show the formation of many saddle-splay surfaces that likely facilitate this transition. The POM texture shows a mosaic pattern formed by the close packing of many different defects. The cryo-SEM image is consistent with this description, showing a packed array of small domains, most of which are formed around distorted oily streaks. These streaks range between $\sim 5$ and $15 \mu \mathrm{m}$ in length and are composed of adjoined FCD-I defects with a maximum individual size of $\sim 2 \mu \mathrm{m}$. The streaks in excess of $10 \mu \mathrm{m}$ are observed to be highly curved.

Samples with high concentrations of surfactant (above approximately $65 \mathrm{wt} \% \mathrm{Na}-\mathrm{AOT}$ ) were prone to damage from the electron beam, and exhibited cracking during attempts to obtain high magnification images. An example of this is shown in Figure 3.21 (f), where the dark lines around the two prominent streaks are an artefact of damage rather than a structural feature. Despite being restricted to low magnifications, it is clear that the bilayers no longer undulate and, despite the macroscopic curvature, the local bilayer structure appears to be highly ordered. Such ordering is expected in the high concentration lamellar phase, as small bilayer repeat distances result in strong intermolecular forces and the curvature involved in forming connective neck defects becomes energetically unfavourable. However, at small repeat distances the steric hydration forces result in strong repulsion between bilayers and likely contribute to the $L_{\alpha}-V_{\text {II }}$ transition, which requires the bilayers to curve into tunnels on local lengthscales. This likely occurs at slightly higher surfactant concentrations than those illustrated; however, the fragile nature of the material under the electron beam means we were unable to observe this.

Great care must be taken when interpreting electron microscopy images of lyotropic liquid crystals for several reasons: there is the possibility that an inadequate freezing rate will result in alterations to the structure caused by the slow crystallisation of water, or is insufficiently rapid to capture short-lived defects; the small volumes able to be viewed may not truly represent the whole sample; the sublimation process can result in slight swelling of the structure at points at which water is removed, and as such these features may appear slightly larger than they are in their true state, a factor that is often neglected; and the structural features such as fractured 

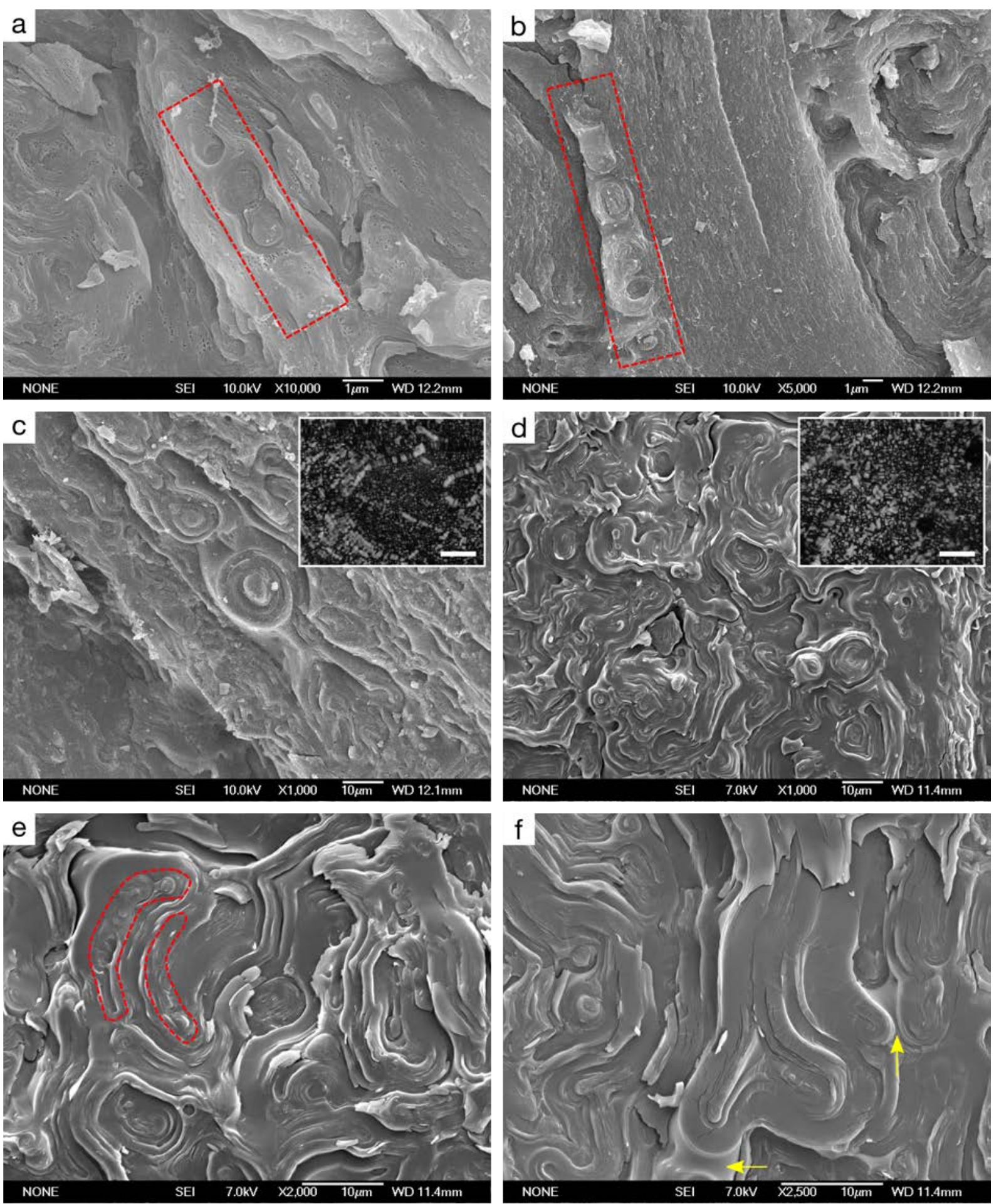

Figure 3.21: Cryo-SEM images of Na-AOT samples of $(a-c) 70$ and $(d-f) 75$ wt \% surfactant. Red boxes in (a) and (b) highlight examples of oily streaks. Red dashed lines in (e) highlight the non-linearity of streaks within small curved lamellar domains. Yellow arrows in (f) highlight obvious saddle-splay surfaces formed by the packing of domains. The insets show the corresponding POM images, with scale bars of $50 \mu \mathrm{m}$.

FCDs are only viewed from one angle and could easily be misidentified and hence assigned the wrong eccentricity. Despite these potential issues, we are confident in the cryo-SEM images used in this thesis. Efforts were undertaken to ensure that the freezing process was reliable (see Chapter 2), and the samples produced cryo-SEM images that were impressively uniform. These images were not studied in isolation, and showed excellent agreement with 
POM images of the same samples. The large FCD defects and trends observed in the POM investigation are mirrored in the cryo-SEM images. This indicates that the cryo-SEM images are representative of the sample, while the POM textures aid in the assignment of defect structures in the cryo-SEM images. We can then use high magnification cryo-SEM images to provide additional information about structural features on smaller lengthscales. Despite the resolution being insufficient to observe individual molecules or bilayers, the images allow the visualisation of 3D surfaces, and localised features such as undulations and connectivity are manifested on the lengthscales investigated. Additionally, domain boundaries and regions of saddle-like and dome-like surfaces can also be visualised.

The cryo-SEM images discussed above (and in particular those of $38 \mathrm{wt} \% \mathrm{Na}$-AOT) do not provide any evidence to support the theories of structural transitions previously proposed. ${ }^{47,116,119}$ The structure is clearly a lamellar phase, and there is no indication of the formation of intermicellar discs or a pseudo-hexagonal arrangement of bilayers. However, the proposal by Callaghan et al. that the structure contains many connective neck defects at low surfactant concentrations holds true, and while the system does not form a ripple phase, the undulating bilayer structure does somewhat resemble the phase proposed by Chidichimo et al. (although this occurs within a different range of sample concentrations than was suggested for the ripple phase).

The cryo-SEM images presented above support our assertions from the SAXS data that at concentrations below $\sim 43$ wt \% Na-AOT entropic repulsive forces in the form of Helfrich's undulation interactions become a relevant contributor the free energy of the system. The system is likely delicately balanced with the values of the bending modulus $\kappa$ and the thermal energy $k_{\mathrm{B}} T$ approximately equal (due to the flexible bilayer of Na-AOT having a low value for $\kappa$ ). Small alterations in the energy of the system, arising from changes in the concentration or temperature, can then promote either the van der Waals or Helfrich forces overcoming the other. When Helfrich forces are dominant, the result is out-of-plane fluctuations, and when the van der Waals forces dominate, more ordered bilayers are observed. The images at $38 \mathrm{wt} \%$ $\mathrm{Na}$-AOT clearly show that at this concentration the system forms a swollen undulating $\mathrm{L}_{\alpha}$ phase. While this change is not a phase transition, the relative strength of the forces that govern this structural modification change abruptly with small variations in the bilayer repeat distance. As such, the change between ordered and fluctuating bilayers may occur over a relatively small concentration range, and so there is a pseudo-"crumpling transition" between a swollen $L_{\alpha}$ phase of disordered fluctuating bilayers and more ordered bilayers (represented schematically in Figure 3.22). The fact that this change occurs abruptly explains many of the previously reported discontinuous physical properties of the lamellar phase within this region.

The schematic shown in Figure 3.22 is obviously an oversimplification, and it is evident from cryo-SEM images that the $L_{\alpha}$ phase cannot be described accurately solely in terms of undulations. Point defects, such as those illustrated in Figure 3.23, are shown to provide connectivity between the surfactant and water domains to varying degrees dependent upon the $\mathrm{Na}$-AOT concentration. This connectivity is particularly evident at low concentrations, which are highly defective and contain a large number of neck defects between surfactant domains, which 


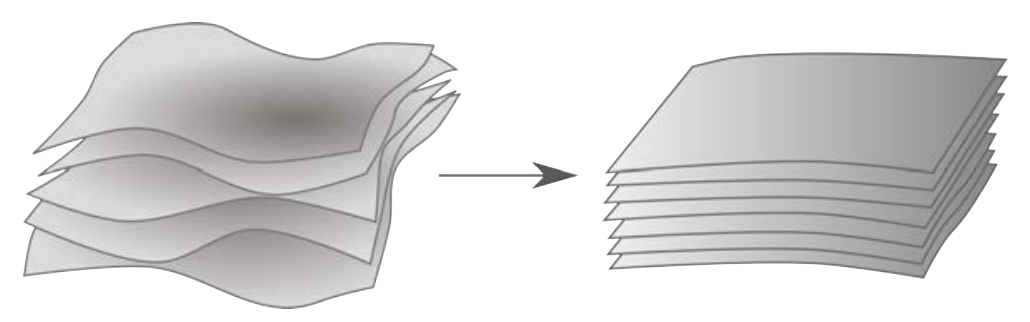

Figure 3.22: Schematic representation of the "crumpling transition" observed in the Na-AOT system at $\sim 43 \mathrm{wt} \%$ surfactant. Relatively ordered bilayers at high concentrations give way to undulating bilayers at low concentrations due to thermal fluctuations.

likely contribute to the stabilisation of the swollen lamellar structure, by introducing an extra entropic attractive interaction. ${ }^{141}$ The undulation of the $L_{\alpha}$ phase and the formation of neck defects are energetically favourable as a result of the thin and flexible Na-AOT bilayer, with a low value of $\kappa$. This flexibility allows the bilayer to adopt different local curvatures (both positive and negative), and likely arises due to the freedom of the AOT tails, which can adopt different configurations due to their branched structure. The formation of such defects is dependent upon the surfactant concentration, as both $\kappa$ and $\bar{\kappa}$ undergo significant changes as the bilayers adapt to the changing intermolecular forces, and the number of topological defects falls significantly above the "crumpling transition".

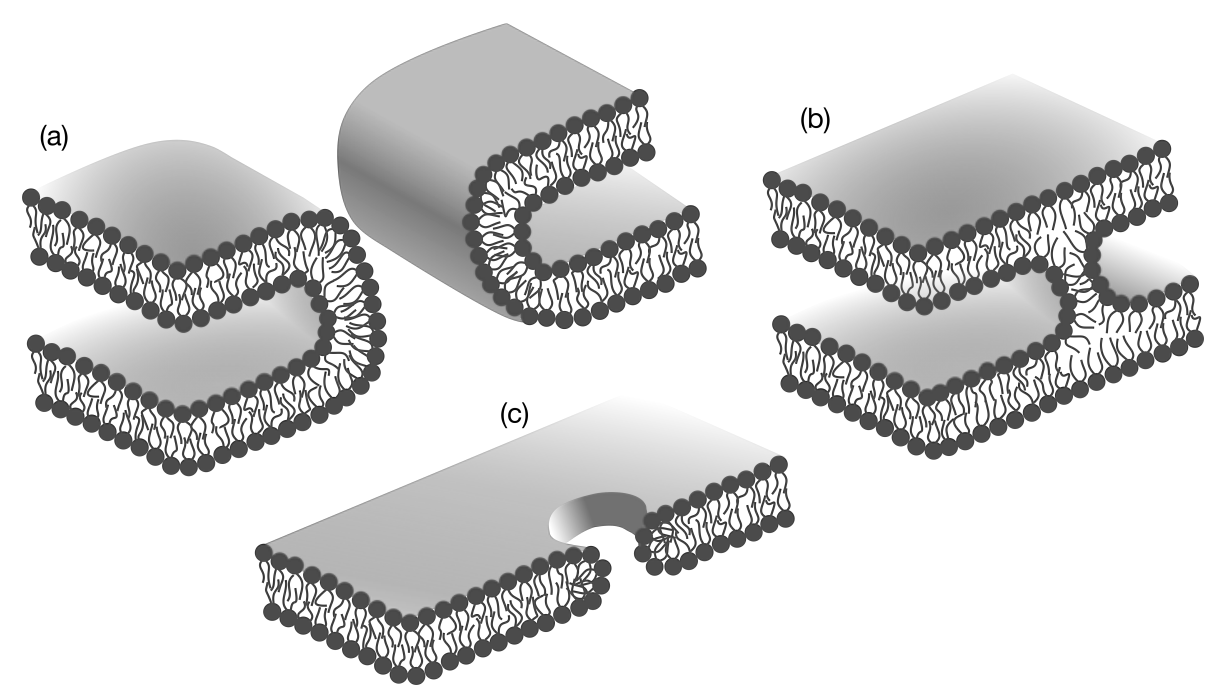

Figure 3.23: Schematic representations of point defects occurring on the lengthscale of surfactant bilayers. These defects, termed (a) channels, (b) necks or passages, and (c) pores, ${ }^{41}$ introduce connectivity in the system between the water and surfactant domains, the surfactant domain, or the water domain, respectively. These simple representations are shown individually for clarity; however, they can all be observed at the same sample concentration and even within a single bilayer, despite requiring different curvature energies.

At higher concentrations, the thermal fluctuations do not have a significant role in the structure of the $L_{\alpha}$ phase and cryo-SEM reveals the formation of different defects. The change from FCD-I to FCD-II defects has already been described, but the cryo-SEM reveals the nature of domain boundaries and the formation of edge and screw dislocations that provide connectivity between the domains and reduce the strain energy at boundaries. Depictions of edge and screw defects are shown individually in Figure 3.24, although they can also form mixed disclinations, 
which is likely the case in Figure 3.20 (f). Figure 3.24 also shows how screw defects may facilitate the reorientation of bilayers into domains along different directional axes. The largest ordered domains were observed at $60 \mathrm{wt} \% \mathrm{Na}$-AOT and it is apparent that the domain size initially increases at concentrations above the "crumpling transition", but then decreases again as the negative curvature dictates the structure of the system upon approaching the $V_{\text {/l }}$ phase.
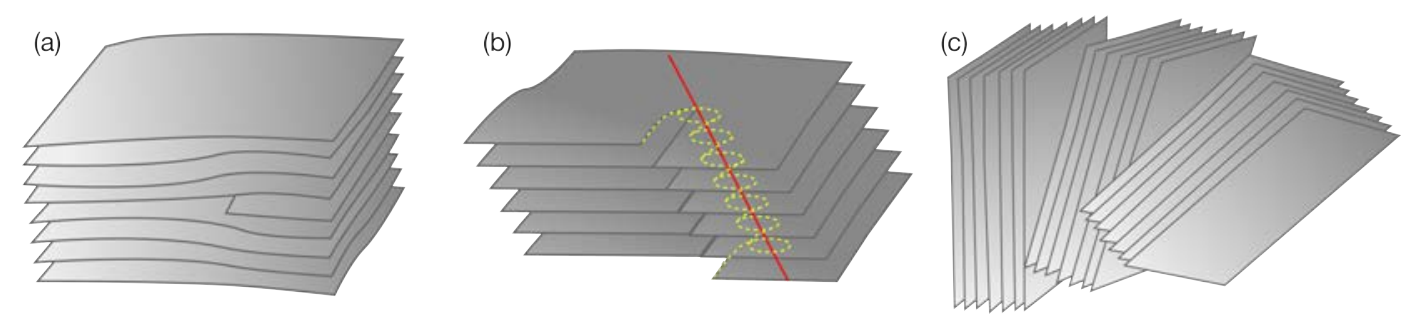

Figure 3.24: Schematic representation of line dislocations observed in the concentrated region of the Na-AOT lamellar phase. Although shown individually, the (a) edge and (b) screw dislocations can also pair to form mixed dislocations. Screw dislocations can result in the reorientation of the lamellar phase, forming domains along different directional axes, depicted in (c).

The significant changes to the elastic constants $\kappa$ and $\bar{\kappa}$ with concentration are key to the self-assembly of the Na-AOT $L_{\alpha}$ phase. They result in a flexible bilayer at low surfactant concentrations that is highly connected due to many point defects. These connections are lost at high concentrations as the bilayer becomes more rigid and connectivity is provided through the formation of different defect structures, such as line defects. These changes in connectivity, and in particular the formation of many point defects at low surfactant concentrations, will impact the physical properties of the system that require movement of water or ions through the structure. As such, our cryo-SEM investigation can provide a rationalisation for the changes that have been reported to occur in the structure and diffusion of water, and the electrical conductivity. ${ }^{47,63,107,108,116,117}$ These properties show the same trends with increasing surfactant concentration, and thus occur due to the same changes in connectivity. The reasoning will be described in terms of water diffusion with the use of Figure 3.25, which shows cryo-SEM images at different sample concentrations acquired from an angle perpendicular to the orientation of the bilayers. This provides a "top-down" view of the lamellar stacks and so shows the bilayer surface, thus illustrating any pores connecting the water layers and any deviation from flat bilayers.

Despite the 20 wt \% Na-AOT sample shown in Figure 3.25 being fractured in a way that exposes several opposing planes from different angles, assigning one face as the top of a stack of lamellae is not possible. This is because the long-range ordering to produce such as stack is not present, and all the exposed surfaces appear equivalent. There is clearly a large amount of disorder in the phase, and the surfactant structure appears continuous and highly connected. However, it is not obvious to what extent there is connectivity and freedom of movement within the water domain. The nature of the defects, many of which are of positive curvature and produce a globular appearance, is consistent with the $L_{\alpha}^{H}$ phase - a lamellar phase containing water-filled defects and no correlation between bilayers, reported by Funari et al. to form via first-order phase transitions in C16EO6 and C22EO6 systems ${ }^{142,143}$ - implying pockets of 

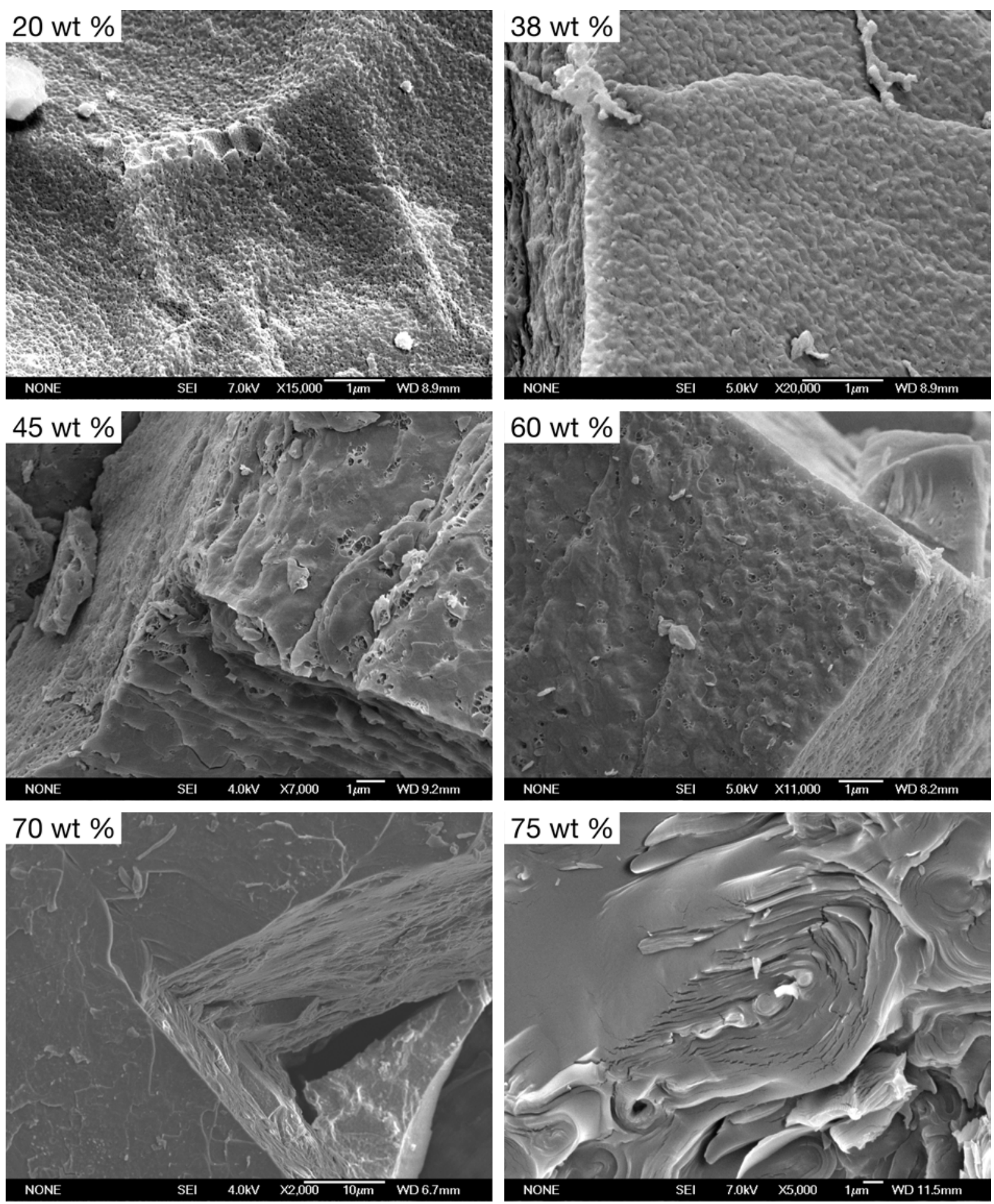

Figure 3.25: Cryo-SEM images showing the changes in the connectivity of the lamellar phase with increasing Na-AOT concentration. The images show lamellar stacks from an angle perpendicular to the directional order of the bilayer, providing a "top-down" view of a bilayer surface, revealing the extent of deviation from flat structures, and the size and frequency of pore defects.

trapped water, likely variations of the channel defects shown in Figure 3.23 (a). The implication of this is that the diffusion of water molecules in any direction will be severely disrupted by the surfactant phase, offering a convincing explanation for the water diffusion coefficients reported previously, which were four times lower than that of bulk water. ${ }^{47}$ 
The image at 20 wt \% Na-AOT is in good agreement with that shown by Hubbard et al. for a sample of $25 \mathrm{wt} \% \mathrm{Na}$-AOT. ${ }^{94}$ However, having also obtained images at low magnifications, our investigation does not support their statement that domain sizes are $\sim 100 \mu \mathrm{m}$ within this concentration region. We did not observe distinct phase boundaries at low concentrations, but a disordered and continuous phase in which the movement of water will be restricted by the large number of defects. This conclusion is in much closer agreement with the DEXSY NMR results also reported by Hubbard et al., that indicated water movement was restricted to $1-5 \mu \mathrm{m}$ over timescales suitable for identifying domain sizes.

At $38 \mathrm{wt} \% \mathrm{Na}$-AOT some very small pores through the bilayer are observed, but it is the uneven and knobbled surface resulting from the undulating structure that is the main structural feature. If we consider the lateral diffusion of water molecules between two such surfaces, it is clear that the random protrusions due to the out-of-plane fluctuations will obstruct the movement of water. This obstruction is also evident from the side-on view of the phase shown in Figure 3.19, due to the large number of neck defects. While the diffusion of water is significantly impeded at this concentration, it is to a lesser extent than in the highly defective structure at $25 \mathrm{wt} \% \mathrm{Na}$-AOT. This observation is consistent with the diffusion NMR experiments of Callaghan et al., which showed an increase in the measured diffusion coefficients of water above $~ 32 \mathrm{wt} \% \mathrm{Na}$-AOT. ${ }^{47}$ The POM investigation presented above (see Figure 3.16) indicated that the orientated lamellar domains (rather than a continuous disordered $\mathrm{L}_{3^{-}}$or $\mathrm{L}_{\alpha}^{\mathrm{H}}$-like structure) are first formed within this region.

The surfaces of samples at 45 and $60 \mathrm{wt} \% \mathrm{Na}$-AOT are significantly flatter than those at lower concentrations, and there is an apparent increase in the size and number of pores through the bilayers. Between 45 and $60 \mathrm{wt} \% \mathrm{Na}-\mathrm{AOT}$, the water diffusion measured via NMR methods was reported to be more akin (although not a perfect fit) to unrestricted 3D diffusion, rather than 2D diffusion. If we consider the diffusion of water along these surfaces shown in Figure 3.25, the movement is certainly less restricted than at $38 \mathrm{wt} \% \mathrm{Na}-\mathrm{AOT}$. The pores within the bilayers may allow for some vertical water movement; however, the pseudo-3D diffusion is more likely a result of the line disclinations/dislocations that introduce connectivity and/or grain boundaries, which can reorientate in a fluid system on the timescale of the NMR measurements.

Callaghan et al. reported that water diffusion reverts to $2 \mathrm{D}$ at concentrations above $60 \mathrm{wt} \%$ $\mathrm{Na}-\mathrm{AOT}$. This can be explained by the domains decreasing in size as the structure adopts increasingly negative curvature. The image at $70 \mathrm{wt} \% \mathrm{Na}$-AOT does show an ordered stack of bilayers, but connectivity between the layers is not evident, and no screw or tilt defects were observed at this concentration. At 75 wt \% Na-AOT, acquiring an image with a top-down view of a stack of bilayers was not possible as the phase is highly curved and most of the areas between defects are saddle-splay surfaces rather than flat regions. The mosaic-like structures at these high surfactant concentrations prohibit the movement of water as they are composed of small packed domains. Additionally, the small bilayer thickness means that the water layer is very thin $(\sim 5-10 \AA)$, which also prohibits unrestricted diffusion. 


\section{Sodium AOT: Discussion}

To satisfactorily describe the self-assembly of the Na-AOT system, the role of each factor governing the system at any given concentration must be considered and explained in terms of its effects on the different components of the system, from macroscopic to molecular lengthscales. This can be summarised by the following points:

- The intermolecular forces that dictate the structure of the Na-AOT system change significantly and discontinuously with concentration, resulting in the lamellar phase of the system being governed by different forces at each concentration extreme.

- The system adapts to changes in intermolecular forces through structural variations on all lengthscales, adopting non-classical lamellar structures and a variety of defect types that change and distort with concentration to efficiently dissipate the energy costs associated with bilayered structures.

- The structural variations that allow the retention of a lamellar phase over a large concentration range are facilitated by the elastic constants of the bilayer, which both change significantly with concentration. The magnitude of $\kappa$ increases with concentration, resulting in an extremely flexible bilayer at low concentrations and a rigid bilayer at high concentrations, while the value of $\bar{\kappa}$ changes from negative to positive, resulting in an inversion of bilayer curvature and topological changes, allowing the formation of different defect structures.

- The formation of the adaptable Na-AOT bilayer is facilitated by the structure of Na-AOT itself. The short tails of the Na-AOT molecule result in a thin bilayer, a factor that increases the bilayer's flexibility, while the tail branching means that the molecule can adapt to changes in the system energy by adopting different structural configurations, thus altering the hydrophobic/hydrophilic interfacial region of the bilayer and modifying the bilayer elasticity.

The self-assembly of surfactants is further complicated by these factors being interrelated. Changes in the relative contribution of the intermolecular forces results in the system adapting on the molecular lengthscale, which then alters the overall structure. Conversely, changes to the molecule, brought about by different packing environments, dehydration, or counterion dissociation (resulting from changes in concentration or temperature) can alter the van der Waals and electrostatic contributions.

The literature review presented earlier in this chapter shows that the Na-AOT system has not previously been explained adequately in terms of all the factors listed above, with in particular the characterisation of the defect behaviour lacking. The Na-AOT system is often unsatisfactorily explained as having a packing parameter close to one, with no further explanation of how the packing changes with concentration. There has also been a general assumption that the discontinuous physical properties in the lamellar region is anomalous behaviour, rather than the logical adaptation of the system to the discontinuous changes in the governing forces. The bilayer spacing in the Na-AOT lamellar phase varies from $\sim 10$ to $\sim 180 \AA$. In lamellar systems that exist on larger lengthscales, a difference of $170 \AA$ may not result in significant changes 
in the relative contribution of the intermolecular forces. However, for the Na-AOT system this equates to an 18-fold increase and encompasses different regions in which short-range or long-range forces stabilise the phase.

As in any lamellar phase formed by an anionic surfactant, both van der Waals attractive forces and electrostatic (Coulombic repulsion) forces act upon the system. These forces are well understood in terms of the DLVO theory, and have opposing effects on the system. Firstly, both forces act between adjacent bilayers promoting or disrupting their stabilisation. Secondly, both forces act along bilayers through the interactions of adjacent headgroups. There is obviously greater variation in the magnitude of these forces between different bilayers, as this spacing changes significantly with concentration. However, the role of these forces along the bilayer should not be assumed to be constant, as factors such as hydration and the counterion binding can alter their strength. Electrostatic forces decay exponentially with increasing bilayer spacing, while van der Waals forces decay to a lesser extent. The electrostatic forces are also more easily affected by changes in the system, such as those that alter hydration or additional shielding of the charged groups. These two forces are common to all anionic lamellar phases; however, the Na-AOT system is also stabilised by two entropic forces: steric hydration and Helfrich forces.

Steric hydration forces are short-range repulsion forces that normally act over distances of $\sim 20$ to $30 \AA$ (decreasing in value exponentially over this region) and prevent the close approach of the lamellar bilayers. ${ }^{132}$ Many lamellar systems do not have bilayer repeat distances that equate to a water thickness this small and so these forces do not need to be considered. However, the Na-AOT bilayers approach within $10 \AA$ of each other and so strong repulsion forces are likely a factor in the curved domains and subsequent cubic phase formed at high surfactant concentrations. In contrast, Helfrich forces are long-range forces normally associated with extremely large bilayer repeat distances and have been reported to stabilise lamellar phases in non-ionic systems with repeat distances of over $3000 \AA .{ }^{33}$ However, they can also be important at significantly lower distances, providing that the distance between the bilayers is larger than the bilayer thickness, as reported for the non-ionic system C10E4, in which the undulation forces dominate at repeat distances similar to those observed in the Na-AOT system. ${ }^{135}$ In fact, the lamellar phase of the dimiristoylphosphatidylcholine $/ D_{2} \mathrm{O}$ system has been reported to be governed by Helfrich forces at an interbilayer distance of just $20.5 \AA$. ${ }^{125}$

The bilayer thickness in the Na-AOT system remains approximately constant at $\sim 18-20 \AA$ within the lamellar region. This means that the points at which the short-range steric hydration forces are expected to diminish and the Helfrich forces can feasibly begin to contribute to the energy of the system are within the same region. The different natures of these forces mean that the regions in which these forces contribute to the system will not overlap. It is, however, clear that both forces operate at concentration ranges within the lamellar phase. Given that the Na-AOT lamellar phase is susceptible to changes in thermal energy and shows evidence of substantial undulations below $43 \mathrm{wt} \%$ surfactant, it is reasonable to state that the Helfrich forces provide a significant contribution below this point ( $50 \AA \mathrm{d}$ spacing). The Na-AOT lamellar phase is also observed to form significantly curved domains and restrict water diffusion at concentrations 
above $\sim 60 \mathrm{wt} \%$. This can in part be attributed to the contribution of steric forces, and implies that they are the dominant force in the system once the water thickness is below $\sim 13 \AA(\sim 33 \AA$ d spacing). At concentrations between 43 and $60 \mathrm{wt} \% \mathrm{Na}$-AOT, the persistence of some diffuse scattering in the SAXS spectra and evidence from the cryo-SEM images shows that some degree of undulation is still present in the bilayer. However, it is only at concentrations below $43 \mathrm{wt} \%$ that Helfrich forces appear to be the dominant factor. This could be due to a conformational change in the surfactant at this concentration and/or the steric hydration forces becoming negligible at concentrations below this point. It should be noted that the interbilayer distances obtained via SAXS measurements at $43 \mathrm{wt} \% \mathrm{Na}$-AOT are in the region of the $30 \AA$ limit at which steric hydration forces become negligible, and those at $60 \mathrm{wt} \% \mathrm{Na}-\mathrm{AOT}$ are approximately double the bilayer thickness.

The role of Helfrich forces in the Na-AOT system do not appear to have previously been considered. This is presumably because it is a binary system comprised of an anionic surfactant and water, leading to the assumption that the electrostatic contribution of the bilayer is sufficient to restrict the role of out-of-plane fluctuations and formation of disordered $L_{3}$ structures. Such an assumption is generally (but not always ${ }^{144}$ ) valid, as the electrostatic repulsion contributes to the magnitude of $\kappa$, resulting in bilayers too rigid for such structures to be energetically favoured without the further addition of a salt or cosurfactant (to screen the interactions or provide extra fluidity). ${ }^{145}$ However, our experimental results demonstrate that the Na-AOT system forms a bilayer that is extremely flexible, such that in the dilute region $\kappa \approx k_{\mathrm{B}} T$ and thus the contribution of undulations, and resulting entropic repulsion forces, should be considered in the system. The presence of an extra stabilising force is likely the reason that the lamellar phase can exist over such a large concentration range, and implies that the long-range electrostatic contribution is weak. However, the role of electrostatic forces should not be underestimated, as clearly demonstrated by the SAXS data at high temperatures. The Na-AOT system can be considered as intermediate to systems in which Helfrich forces are negated by electrostatic forces and nonionic systems in which Helfrich forces dominate. As such, the Na-AOT lamellar phase stability at low surfactant concentrations is a delicate balance between significant contributions of Helfrich, van der Waals and electrostatic interactions. The importance of electrostatic forces is evidenced by the addition of $\mathrm{NaCl}$ to the system, which shields and hence reduces their contribution. ${ }^{90}$ Depending on the amount of $\mathrm{NaCl}$ added, the concentration range of the lamellar phase can be extended, or the system can be forced to form a sponge phase.

The lamellar phase of $\mathrm{Na}-\mathrm{AOT}$ is stabilised at low concentrations upon the addition of $\mathrm{NaCl}$ because the result of increased shielding of electrostatic forces is a reduction in the headgroup repulsion, and so an increase in the bilayer flexibility (lower $\kappa$ ). The lower the value of $\kappa$, the greater the magnitude of Helfrich repulsive forces (as they are inversely related), and so bilayers have an additional stabilising force. ${ }^{146}$ This suggests that when the $\mathrm{NaCl}$ is not added to the system, there is a point of dilution (the $L_{1}-L_{\alpha}$ phase boundary) at which the magnitude of the long-range Helfrich forces is no longer sufficient for the forces to compete with and balance the attractive van der Waals forces, and so the lamellar phase is no longer stabilised. It follows that the magnitude of the van der Waals and Helfrich forces are sufficiently competitive, and thus both are important in stabilising the lamellar phase, at concentrations between $\sim 10$ 
and $\sim 43 \mathrm{wt} \% \mathrm{Na}$-AOT. However, the relative contribution of the two forces gradually changes with concentration, which results in the different structural features observed in the cryo-SEM images shown previously in Figure 3.19 and the resulting differences in water diffusion. At the higher surfactant concentrations within this region, the lamellar phase is composed of undulating bilayers, with additional stabilisation provided by connecting neck defects. Upon moving to higher $d$ spacings, the van der Waals contribution gradually begins to determine the structural features, producing a more disordered and highly defective structure with many of the neck defects replaced by features of increasingly positive Gaussian curvature.

The crossover between van der Waals and Helfrich forces in the dilute region can be explained by the electrostatic repulsion. As the bilayer repeat distance increases, both the van der Waals and Helfrich forces decrease in strength, which in non-ionic systems occurs at a rate of $\sim 1 / \mathrm{d}^{3}$ for both forces. ${ }^{144}$ However, in the Na-AOT system the decay of the van der Waals forces is clearly slower. This is a result of the dependency of the Helfrich forces on the bilayer rigidity. In contrast to non-ionic systems, as the bilayer repeat distance increases in the Na-AOT system, short-range electrostatic forces still contribute to the value of $\kappa$. The Na-AOT bilayer will retain some rigidity and thus the degree of undulation and magnitude of the Helfrich forces will decay at a faster rate. When the van der Waals forces truly dominate the Helfrich forces, the result is the formation of MLVs, which are observed in the two-phase region via POM. In the region in which the relative contributions of the Helfrich and van der Waals forces change gradually, the continued relative increase in the attractive component upon dilution results in the larger FCD-II structures observed via POM and cryo-SEM. However, the local structure is a mixture determined by the relative contribution of the two forces which either drive the bilayers to undulate or aggregate, resulting in a disordered and defective structure.

The evidence of Helfrich forces within the Na-AOT system is significant as they are not normally relevant in binary "counterion only" systems. However, the contribution of Helfrich forces is consistent with the non-liquid crystalline properties that have made Na-AOT commercially important. The emulsifying capabilities of Na-AOT mean that it is one of only a few reported surfactants that can promote the formation of reverse micelles or microemulsions without the addition of a cosurfactant. The defect behaviour reported in this chapter, with focal conic defects of both signs observed, is also usually associated with ternary systems. The Na-AOT system does not display any extraordinary behaviour that is not known to exist in liquid crystalline phases; however, the system is remarkable in that it clearly exhibits behaviour normally associated with the presence of a cosurfactant. This implies that the Na-AOT molecule provides a fundamental flexibility to the system that is normally only achievable through the inclusion of an additive. In line with the work of Nave et al. on the emulsifying behaviour of the system, this flexibility is unlikely to be a property unique to Na-AOT, but a factor of the branching within the tail region. ${ }^{48}$ This is a factor in surfactant self-assembly that has not undergone extensive research, but is a growing topic of interest. ${ }^{147}$

The flexibility of the Na-AOT bilayer is clearly evident, with both elastic constants changing significantly within the lamellar region. The magnitude of $\kappa$ increases with concentration, while the value of $\bar{\kappa}$ is indicated by the defect structures formed, which change from predominantly 
positive to predominantly negative Gaussian curvature. When the value of $\kappa$ is low (but above the limit of micelle formation), surfactant systems form $L_{\alpha}$ or $L_{3}$ phases if the value of $\bar{\kappa}$ is negative or positive, respectively. The fact that at low surfactant concentrations the Na-AOT system forms a lamellar phase and FCD-II defects (and that it borders a micellar phase) shows that $\bar{\kappa}$ is negative, and hence the overall Gaussian curvature is positive. However, the lamellar phase at low Na-AOT concentrations is highly defective, and on local lengthscales forms connective defects of both positive and negative Gaussian curvature, giving this phase significant $\mathrm{L}_{3}$-like characteristics. The curvature at low $\mathrm{Na}$-AOT concentrations is clearly easily manipulated, as it has been shown previously that the system can produce $a L_{3}$ phase (negative Gaussian curvature) upon the addition of salt, ${ }^{90,148}$ and then onion phases (positive Gaussian curvature) on shear. ${ }^{149}$ For low concentration samples to be composed of lamellar bilayers and exhibit macroscopic properties of the lamellar phase, while simultaneously retaining some $\mathrm{L}_{3}$-like character on the micrometre lengthscale, is an indication that the Na-AOT bilayer is not only flexible, but also adaptable and able to facilitate localised curvature. This is another trait that could be easily rationalised if the system contained a cosurfactant, but must instead be a result of the ethyl branches of the Na-AOT molecule.

The above observations suggest that the branching of the Na-AOT molecule enables properties that are not characteristic of binary systems. The molecule results in an extremely flexible bilayer, capable of forming a variety of structures and allowing drastic changes in the elasticity. This is partially a result of the short alkyl chains in Na-AOT interdigitating and thus forming a thin bilayer (which reduces the value of $\kappa$ ), but must primarily be attributed to the branched tails of the surfactant. The branches in Na-AOT are in close proximity to the surfactant headgroup and so can introduce flexibility at the hydrophobic/hydrophilic interface through rotational isomerism, forming isomers that either increase or decrease the tail contribution. The Na-AOT system is often inadequately explained in terms of its packing parameter, which is often quoted as a single somewhat arbitrary value, neglecting the fact that the molecular shape of any surfactant molecule will change depending upon concentration and the energy of the system. The branching of the Na-AOT tails means that the effect of such changes is amplified, and the surfactant can efficiently regulate its tail volume to adapt to changes in the system's energy.

The Raman and IR spectroscopy work discussed in the literature review shows that the $\mathrm{Na}$-AOT tails adopt a mixture of trans and gauche rotational isomers at low concentrations, but only gauche isomers at high concentrations. It also describes the concentration-dependent stabilisation of two different conformations of the sulfosuccinate skeleton, which adjusts the molecule at the interfacial region and promotes either splayed/kinked or parallel tails. At low concentrations (and below the "crumpling transition") a mixture of splayed/kinked and parallel chains will introduce a significant amount of flexibility and curvature into the bilayer. This has the effect of reducing $\kappa$ and thus allowing out-of-plane fluctuations. It also allows the Na-AOT molecules to adapt within the fluid system and form short-lived regions of either planar or curved bilayers, which explains the $L_{3}$-like character observed in low concentration samples. Above the "crumpling transition" and thus approaching the formation of Type II structures, the tails are reported to primarily adopt splayed conformations, which accounts for the increase in 
the magnitude of $\kappa$ and the switch in $\bar{\kappa}$, resulting in more classical lamellar bilayers and FCD-I defects.

Another important factor is that the ethyl branches result in the molecule having three chiral centres and so eight distinct stereoisomers. As such, the self-assembly of Na-AOT in water does not form a true binary system, but a racemic mixture of Na-AOT in water. If we were to consider each of the eight isomers as distinct, then the system would be a nine-component system and thus incredibly complex. In practical terms, overcomplicating the system in this way would be unhelpful as the isomers are likely very similar. However, we can assume that $\mathrm{Na}-\mathrm{AOT}$ is a mixture of stereoisomers, and that some will have configurations with higher degrees of enforced splay and thus larger tail volumes than others. This essentially means that $\mathrm{Na}-\mathrm{AOT}$ is a mixed surfactant system, and the observed properties that are more akin to systems containing cosurfactants than standard binary systems are explained in terms of $\mathrm{Na}$-AOT acting as its own cosurfactant. As with the different rotational isomers discussed above, curvature in the system (such as the large number of connecting neck defects or channels that result in the defective $L_{3}$-like character in low concentration $L_{\alpha}$ samples) may be explained by the preferential assembly of stereoisomers of different geometries at sites of localised curvature. Considering the system as a mixed surfactant system also provides an explanation for the long equilibration times of Na-AOT samples. However, given that the isomers are still closely related, and most cosurfactants are specifically selected in order to introduce different behaviours and hence are significantly different in molecular structure to the main component, a better description of the system would be either a pseudo-binary or pseudo-mixed surfactant system.

In conclusion, this chapter combines a comprehensive review of the literature with new experimental data, which has enabled the rationalisation of the lamellar behaviour of the $\mathrm{Na}$-AOT system. As such, we are now able to highlight inaccuracies in the previous literature and definitively show that the region is composed of a continuous lamellar phase exhibiting normal swelling behaviour. The phase is facilitated in spanning a large concentration range by the adaptability of the chiral Na-AOT molecule, which forms different rotational isomers and therefore introduces flexibility in the bilayer. This flexibility promotes the formation of lamellar structures and defects with varying degrees of order.

This research provides two interesting topics that are further described in subsequent chapters. Firstly, the role of tail branching is a key property of the system and so the following chapter details an investigation into the effect on the phase behaviour if the tails are altered. Secondly, the existence of an extremely flexible bilayer, but formation of a sponge phase upon the addition of $\mathrm{NaCl}$, suggests that the long-range electrostatic forces are weak and so comparable in magnitude to opposing forces. The role of the electrostatic forces has been investigated by changing the counterion of the Na-AOT system. 


\section{Chapter 4}

\section{Sodium and Potassium Aerosol MA}

\section{Sodium AMA: Introduction}

In the previous chapter, the stability of the extended lamellar phase in the Na-AOT system was explained in terms of the bilayer being the preferred packing motif of the AOT molecules over a large concentration range. The associated packing costs are met through the formation of different defect structures and through conformational changes in the molecule, primarily flexibility in the tail packing. As most systems do not provide a continuous change from a swollen to classical lamellar phases, the continuous change observed in the Na-AOT system is likely aided by the branched tails, an unusual feature in these molecules. In order to investigate the role of the ethyl branches present in Na-AOT, the initial plan for this work was to study the liquid crystalline properties of the straight-chained linear analogue, sodium bis( $n$-hexyl) sulfosuccinate. However, towards the end of this research, it was discovered that the product purchased as the straight-chain analogue was in fact a different structural isomer, namely sodium bis(1,3-dimethylbutyl) sulfosuccinate. The details of the structural characterisation of this material can be found in Chapter 2. Although this was not the intended structure for investigation, it still provides a suitable comparison to Na-AOT, as any changes in phase behaviour can be attributed to the significantly reduced tail volume of the surfactant.

Sodium bis(1,3-dimethylbutyl) sulfosuccinate is more commonly known by the commercial name Aerosol ${ }^{\circledR}$ MA, a USA registered trademark belonging to CYTEC Industries Inc. (formerly American Cyanamid Company). It is normally sold as an $80 \%$ solution in a water/alcohol mixture with $\sim 5 \%$ ethanol or isopropanol, termed Aerosol MA-80 and Aerosol MA-80I, respectively. ${ }^{150}$ Like Na-AOT, it is used commercially as an anionic surfactant, and displays a similar range of useful properties at ambient temperatures. It is a highly efficient emulsifying, wetting and dispersion agent, with a high tolerance to salt. It promotes low interfacial tension, particularly between water and chlorinated solvents. Applications include the production of paper, paints, inks, adhesives and batteries, and use in emulsion polymerisation. It is also US 
Food and Drug Administration (FDA) approved and used in pharmaceutical coatings, and is US Environmental Protection Agency (EPA) exempt allowing its use in pesticide formulations. ${ }^{151}$

Despite being a commercially used surfactant, the literature review undertaken during the process of this work found many examples of "mistaken identity" between this surfactant and two of its structural isomers. The structure of sodium bis(1,3-dimethylbutyl) sulfosuccinate is shown in Figure 4.1 alongside these isomers, sodium bis(1-methylpentyl) sulfosuccinate and sodium bis(n-hexyl) sulfosuccinate, which differ in terms of tail branching. Each of the three structures presented were found to have been referred to within the published literature as sodium dihexyl sulfosuccinate, Aerosol MA, Aerosol MA-80, Aerosol MA-80I or AMA. In the absence of any established nomenclature to adequately differentiate between the structures, the names Na-butyIAMA, Na-pentylAMA and Na-hexylAMA will be used in this work, distinguishing them by their butyl, pentyl and hexyl chains, respectively.

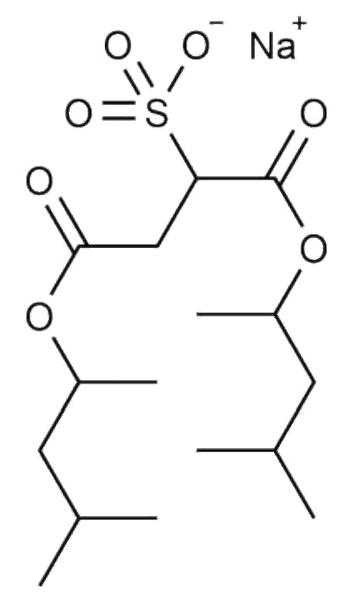

Na-butyIAMA

sodium bis(1,3-dimethylbutyl) sulfosuccinate

CAS number 2373-38-8

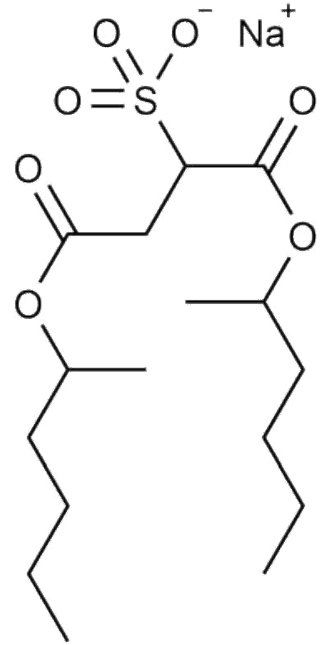

Na-pentyIAMA

sodium bis(1-methylpentyl) sulfosuccinate

CAS number 6001-97-4

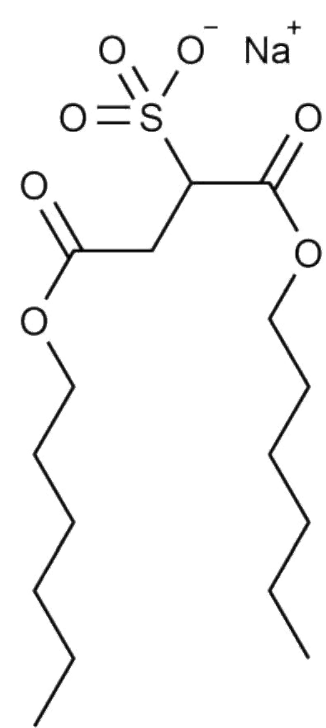

Na-hexyIAMA

sodium bis( $n$-hexyl) sulfosuccinate

CAS number 3006-15-3

Figure 4.1: Molecular structures and CAS numbers of Na-butyIAMA, Na-pentylAMA and Na-hexylAMA.

These three isomers sharing the same generic name is not an insignificant problem. Literature searches based on molecular structure or CAS number conducted using SciFinder ${ }^{\circledR}$, revealed many literature articles in which the isomer used in the research is classified under the wrong structure in the database. The lack of a rational and coherent naming convention, and the use of the non-descriptive terms Aerosol MA or dihexyl sulfosuccinate (which do not differentiate between isomeric forms), both within the literature and by chemical suppliers, has resulted in inaccuracies in publications. Many examples were found in which researchers have unknowingly reported on a different surfactant than that stated, have referred to data or findings 
from other researchers that have not used the same molecule, or have not given any indication of the structure of the isomer used. In cases where the surfactant is used for properties that all three isomers likely possess, this issue may well have no impact on the research. However, in other examples there may be a significant impact upon the findings, especially in research such as that presented here, where the structure of the surfactant is of fundamental importance.

This issue is not a recent phenomenon, with the term Aerosol MA having been used by different researchers to refer to different isomers as far back as the 1940s, but to the best of our knowledge the confusion has not been addressed in any published work to date. The earliest example we have found of this term being used to describe any isomer other than Na-butyIAMA was the work by Vetter in 1946 that used Na-pentylAMA. ${ }^{152}$ This work was later cited by Stamm et al., although they were in fact studying Na-butylAMA. ${ }^{153}$ The only studies relating to the lyotropic phase behaviour of any of these isomers is also confusing. Winsor ${ }^{154}$ referred to the work of Luzzati et al. ${ }^{155,156}$ and reported the observation of several lyotropic phases in the Aerosol MA system, similar to the Na-AOT system. However, Winsor showed the structure of the surfactant as a branched isomer, while the original work by Luzzati et al. showed the structure as the linear Na-hexyIAMA.

In recent years Na-hexylAMA has received considerable attention, particularly in the study of microemulsions. Much of this research has centred around the modelling of the hydrophilic-lipophilic deviation or characteristic curvatures of surfactants. Na-hexylAMA has featured prominently in this work, extensively studied itself and subsequently used as the reference system for many other surfactant mixtures. ${ }^{157-164}$ These characteristics are dependent upon the structure of the surfactant, and one calculation parameter is the length of the hydrocarbon tail region, which in this case is calculated based on an unbranched hexyl chain. As such, it is obviously important that the correct structural isomer is used in these studies. This research was carried out with Na-hexylAMA sourced from a number of different suppliers, including Sigma-Aldrich, which was the supplier used in our research. Given our experience with this product and the issues in the literature, it is reasonable to question whether these studies actually used the correct isomer. Unfortunately, the purchased surfactant was used as received in all cases, and no structural characterisation was reported.

Additionally, the authors of a number of the papers procured the surfactant from CYTEC Industries Inc. who advertise the product under the general name Aerosol MA with the chemical description "sodium dihexyl sulfosuccinate". ${ }^{150}$ Without further information, this would logically suggest the product is Na-hexyIAMA; however, the CYTEC Material Safety Data Sheets for this product (sold either as a water/ethanol or water/isopropanol solution) give the molecular structure and CAS number for Na-butylAMA. ${ }^{165,166}$ We can find no evidence that CYTEC (or American Cyanamid previously) have ever sold either Na-hexylAMA or Na-pentylAMA, even though throughout the literature there are examples of authors reporting that their Na-hexyIAMA, ${ }^{160,161,167}$ Na-pentyIAMA ${ }^{152,168,169}$ or Na-butylAMA ${ }^{170-172}$ was purchased from this company under the name Aerosol MA, while many have not specified which isomer was used. ${ }^{173-175}$ It should be noted that CYTEC may have provided these groups with the stated isomers; however, without any supporting structural characterisation data, it would be 
imprudent to assume that this is the case. One indication this is not the case is the assertion made in several examples that the Na-hexylAMA isomer was selected due to its ability to facilitate cosurfactant-free microemulsions. ${ }^{157,158}$ It has previously been stated by Nave et al. (who synthesised and characterised the surfactant) that unbranched sulfosuccinates, including Na-hexyIAMA, do not have this ability. ${ }^{75}$

It is very likely that all three structural isomers of this surfactant have been used correctly in various literature reports; however, without synthetic or characterisation data (one or both of these are given only in the minority of papers) there is no clear way of determining which are accurate and which are not. The most widespread error would appear to be cases in which researchers (including ourselves) have attempted to purchase Na-hexylAMA as an $80 \%$ solution, but instead have received Na-butyIAMA. Without obtaining comprehensive historical records from all the suppliers, it is impossible to state with any certainty which isomer may have been sold under the generic names Aerosol MA and sodium dihexyl sulfosuccinate. Indeed, we have been unable to ascertain whether our case is that of an isolated "bad batch" or whether the product is consistently misrepresented. ${ }^{\ddagger}$ However, in the only example in the literature in which Na-hexylAMA sold by Sigma-Aldrich was used and NMR data given, there is evidence that the chemical supplied was actually Na-butylAMA. Wilfred et al. used the chemical with no further purification to synthesise several ionic liquids. ${ }^{176}{ }^{1} \mathrm{H}$ NMR data of the ionic liquids were collated, although no peak assignments were attempted. Upon comparison of the NMR data with those obtained in this work (see NMR characterisation in Chapter 2), it is evident that it is in good agreement and the reported ionic liquids contained butyIAMA anions.

It is clear that the combination of the use of non-specific terminology and the indications that other research groups have also received the wrong structural isomer mean that the validity of much of the published literature is questionable. It is unfortunate that it has not been standard procedure to purify and characterise the product prior to use. However, beyond the discovery that the surfactant investigated within this chapter is not that which it was originally believed to be, this work is not affected by any inaccuracies in the literature. This is due to the limited research carried out into the liquid crystalline behaviour of any of the three isomers. However, any references or comparisons to the previous literature that are made beyond this point are limited to reports that included characterisation of the surfactant used, which is primarily those authors that synthesised the molecules themselves.

\footnotetext{
${ }^{\ddagger}$ As of February 2017, the Sigma-Aldrich quality assurance team have corroborated our assignment of the material we received as Na-butyIAMA and have temporarily ceased selling the product until the issue is resolved with their supplier.
} 


\section{Sodium butyIAMA: Experimental Results}

\section{Sodium butyIAMA Phase Behaviour}

The phase diagram of Na-butylAMA is presented alongside that of Na-AOT in Figure 4.2, and was elucidated based on a combination of observations of sealed bulk samples in a controlled heating block, POM and SAXS measurements. The obvious difference between the phase diagrams of Na-butylAMA and Na-AOT is that the Na-butylAMA lamellar phase does not exist over such a large concentration range, as it is no longer the favoured state at low surfactant concentrations. The Na-butylAMA system forms a pure lamellar phase between $\sim 50$ and 81 wt \% surfactant. An inverse bicontinuous cubic phase exists over a narrow concentration range of $\sim 82-85 \mathrm{wt} \%$, and an inverse hexagonal phase is formed at higher surfactant concentrations, which includes the pure surfactant.
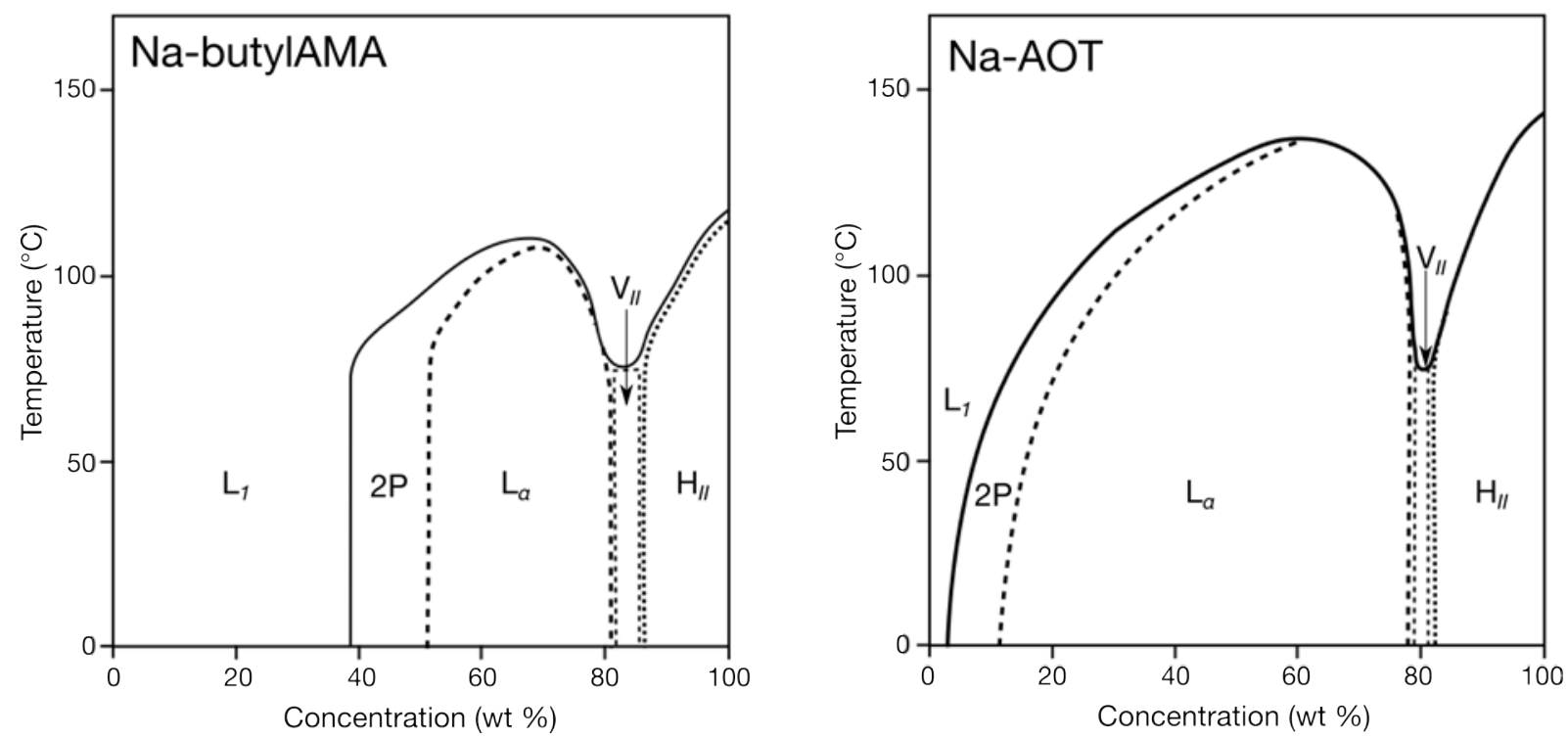

Figure 4.2: Phase diagrams of Na-butylAMA and Na-AOT, showing the relative positions of the two-phase regions $(2 \mathrm{P})$, and the lamellar $\left(\mathrm{L}_{\alpha}\right)$, inverse bicontinuous cubic $\left(\mathrm{V}_{I I}\right)$ and inverse hexagonal $\left(\mathrm{H}_{I I}\right)$ phases.

Comparison of the two phase diagrams shows that the result of the reduced tail branching in the Na-butyIAMA system is a reduction in the stability of the lamellar phase. This difference in molecular structure between the two surfactants clearly has a greater influence at low concentrations than at high concentrations. The phase progression at high concentrations remains the same, with $\mathrm{V}_{\| /}$and $\mathrm{H}_{\| /}$phases formed and the $\mathrm{V}_{\| /}$phase occupying a similar concentration range of $\sim 4 \mathrm{wt} \%$. The locations of the phase boundaries in the two systems are only slightly shifted, with the $\mathrm{L}_{\alpha}-\mathrm{V}_{\| /}$and $\mathrm{V}_{\text {II }}-\mathrm{H}_{\|}$phase boundaries occurring at $\sim 82$ and $\sim 86$ wt $\%$ surfactant in the Na-butylAMA system and $\sim 78$ and $\sim 82 \mathrm{wt} \%$ surfactant in the Na-AOT system, respectively. 
The region in which the Na-AOT system presents a lamellar phase but the Na-butyIAMA system does not is the dilute region (below $\sim 50 \mathrm{wt} \%$ surfactant). In the Na-AOT system, this is the region in which the lamellar phase is highly defective, exhibits on average positive Gaussian curvature, and is stabilised by an additional intermolecular force due to out-of-plane fluctuations. The concentrations at which the Na-butylAMA system does present a lamellar phase correlate with the region in which the lamellar phase of the Na-AOT system shows positive birefringence, predominantly negative Gaussian curvature, and more classical lamellar behaviour with significantly reduced bilayer fluctuation. This implies that the Na-butyIAMA molecule is not able to provide a significantly bulky tail volume at the hydrophobic/hydrophilic interface. As such, the curvature of the bilayer cannot stabilise a dilute lamellar phase such as that observed with Na-AOT, as the spontaneous curvature of the bilayer is too high and the value of $\kappa$ is not in the region of $k_{\mathrm{B}} T$, and hence additional intermolecular forces (i.e. Helfrich forces) are unable to act upon the system. The increased spontaneous curvature is also the likely cause of the reduced stability of the Na-butylAMA system with increasing temperature, with the melting of the lamellar phase occurring at temperatures approximately $20{ }^{\circ} \mathrm{C}$ lower than in the Na-AOT system.

\section{Sodium butyIAMA SAXS}

The SAXS results of the Na-butyIAMA lamellar phase are presented in Figure 4.3, with selected $1 \mathrm{D}$ and $2 \mathrm{D}$ profiles shown. In stark contrast to the scattering described for the Na-AOT system, the lamellar phase of Na-butyIAMA produces spectra characteristic of classical lamellar behaviour throughout its concentration region. Sharp Bragg peaks in the ratio of $1: 2$ are observed at all concentrations within the $L_{\alpha}$ phase. These peaks do not show any intensity anomalies, nor are they masked by diffuse scattering at any point. The intensities of both the first and second-order Bragg peaks increase with concentration, before decreasing again in proximity to the $L_{\alpha}-V_{/ /}$phase boundary. The peaks also shift to higher q spacings as a result of the reduction in bilayer repeat distance with increased concentration.

The 2D profiles shown in Figure 4.3 are consistent with the $1 \mathrm{D}$ spectra, and indicate the Na-butylAMA system forms a classical and ordered lamellar phase, as the scattering is clearly aligned in one direction for all sample concentrations. It is notable that the $2 \mathrm{D}$ profiles do not show the broad diffuse scattering ring observed in the Na-AOT spectra. Even at the lowest concentration shown (52.5 wt \%) there is no complete ring of weak intensity scatter. There is, however, some weak scattering in the region of the Bragg peaks in samples of 52.5 and $62.5 \mathrm{wt} \% \mathrm{Na}$-butylAMA, with the same alignment. This feature is also observed in the 1D spectra of 58 and 60 wt \% surfactant, as broadness at the base of the Bragg peaks. This scattering is not likely to be due to the thermal undulations that were observed in the Na-AOT system, as the scattering is monodirectional in the $2 \mathrm{D}$ profiles and was not observed to be sensitive to changes in temperature. Instead, this scattering implies some level of variation in the bilayer repeat distance of these samples, and thus suggests the possibility of defects in the lamellar system that result in localised deviations from this distance. 


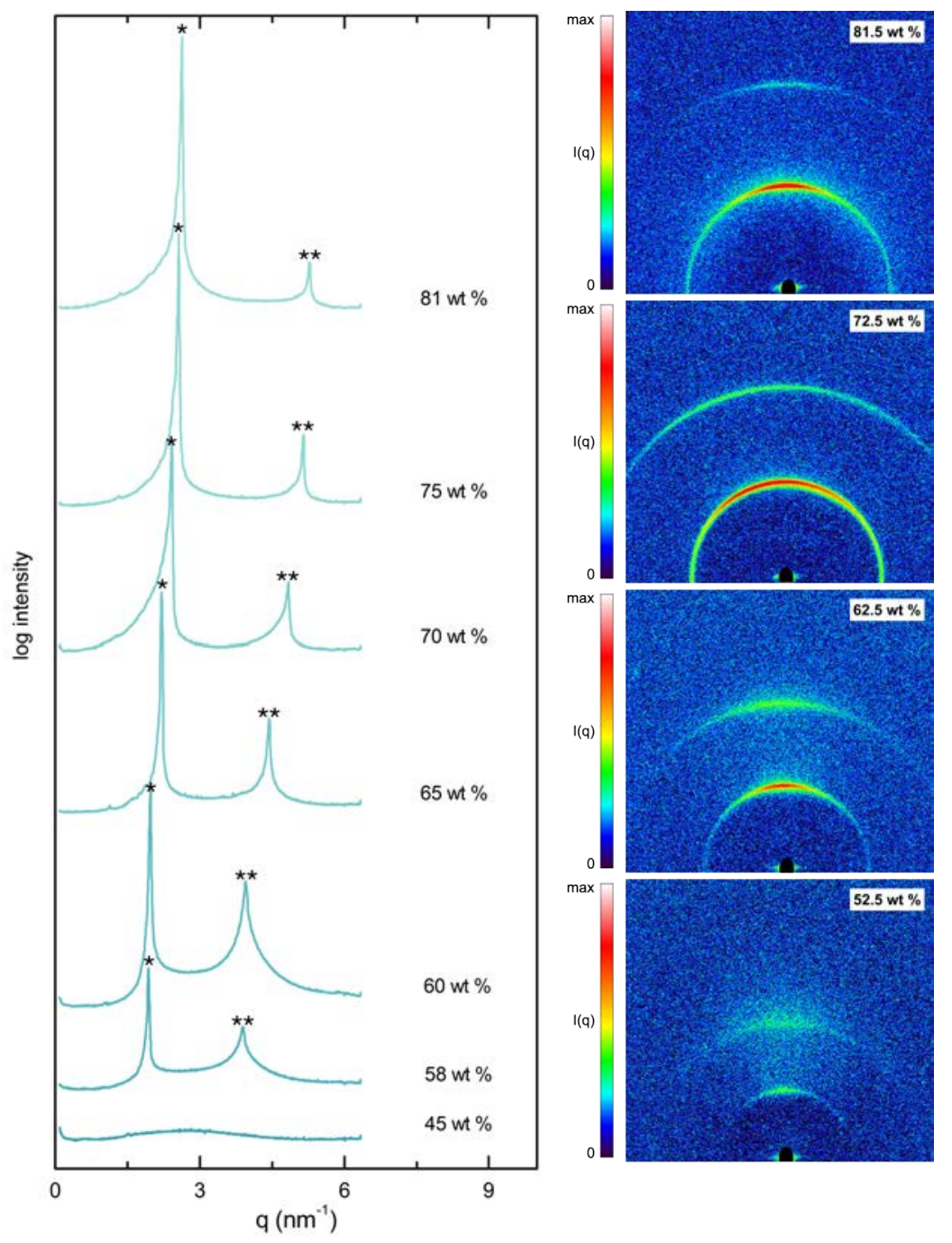

Figure 4.3: SAXS profiles of the Na-butyIAMA lamellar phase with increasing surfactant concentration. Both 1D (left) and 2D (right) profiles are shown, obtained using line and point collimation, respectively. Bragg peaks are shown in the 1D image with single and double asterisks, indicating first- and second-order peaks, respectively. Both data sets show the characteristic scattering of a classical lamellar phase; however, the second-order reflections broaden with decreasing concentration.

The formation of $\mathrm{V}_{/ /}$and $\mathrm{H}_{/ /}$phases was also confirmed by SAXS, as illustrated by the example shown in Figure 4.4, which presents the 1D and 2D scattering profiles for a sample of $85.5 \mathrm{wt} \%$ $\mathrm{Na}$-butyIAMA. This concentration is within the small two-phase region between the pure $\mathrm{V}_{\text {II }}$ and $H_{/ /}$phases. Bragg peaks in the ratio of $1: \sqrt{ } 3: \sqrt{ } 4$ arise due to the high concentration $H_{/ /}$ phase, while seven Bragg peaks in the spacing ratio $\sqrt{ } 6: \sqrt{ } 8: \sqrt{ } 14: \sqrt{ } 16: \sqrt{ } 20: \sqrt{ } 22: \sqrt{ } 24$ confirm the formation of the bicontinuous cubic phase, and allow the assignment of the cubic symmetry as the la3d space group, based on a gyroid structure (see Figure 4.5). This is the same space group observed in the $\mathrm{V}_{\text {/I }}$ phase of the Na-AOT system. The $\mathrm{V}_{\text {/I }}$ and $\mathrm{H}_{\text {/I }}$ phases share the same peak as their first-order Bragg peaks, representing the repeat distance along the [211] and [100] planes of each phase, respectively. This is due to the epitaxial relationship between the two phases in this region of the phase diagram, with the growth of the $\mathrm{H}_{/ /}$phase from the $V_{\text {II }}$ phase resulting in the hexagonal columns being orientated by the cubic phase along one anchoring plane. ${ }^{177}$ 

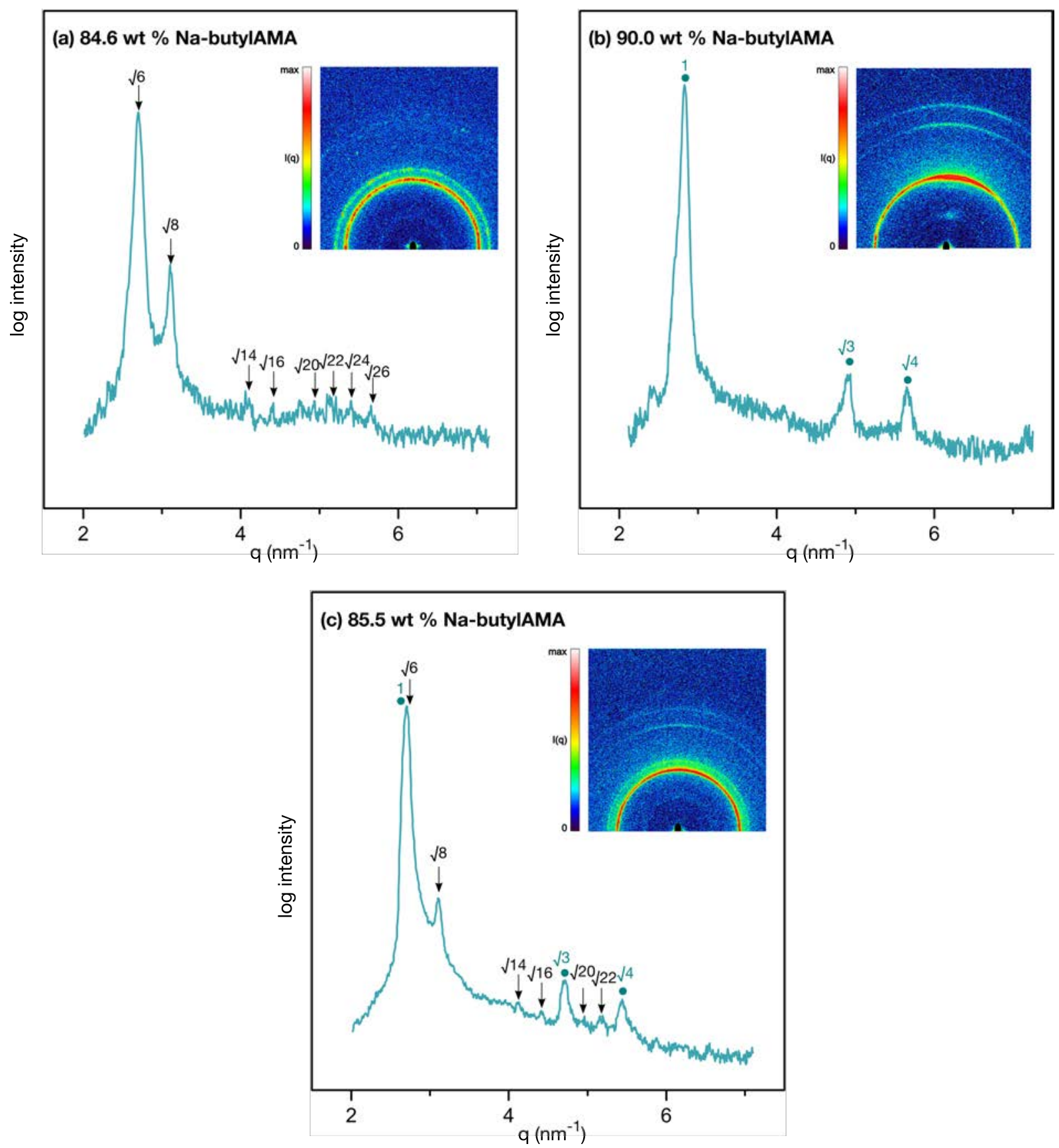

Figure 4.4: SAXS profiles of Na-butylAMA showing (a) a $\mathrm{V}_{/ /}$phase, (b) a $\mathrm{H}_{/ /}$phase, and (c) a mixture of the two. The 1D spectra show peaks due to $V_{/ /}$and $H_{/ /}$phases, denoted by arrows and circles, respectively. The weak $V_{\| /}$reflections are not obvious from the $2 \mathrm{D}$ profiles alone, and many are not a continuous scattering rings, but are composed of several small intense diffraction spots, indicative of a reflection arising from cubic ordering.

The calculated d spacings of the Na-butylAMA lamellar phase are plotted in Figure 4.6, along with comparative values for the Na-AOT system. In the region in which both systems form a lamellar phase, the $d$ spacings are comparable, indicating that the structures are likely similar. The values for the Na-butylAMA system are slightly lower than those of the Na-AOT system, but the difference is less than $4 \AA$. The $L_{\alpha}-V_{\text {/l }}$ phase boundaries of the two systems occur at slightly different concentrations and so a small difference in $\mathrm{d}$ spacing at equivalent concentrations is expected. However, the primary reason for the difference in $d$ spacing is likely to be that the bilayer thicknesses in the two systems are not equivalent. The $d$ spacing 

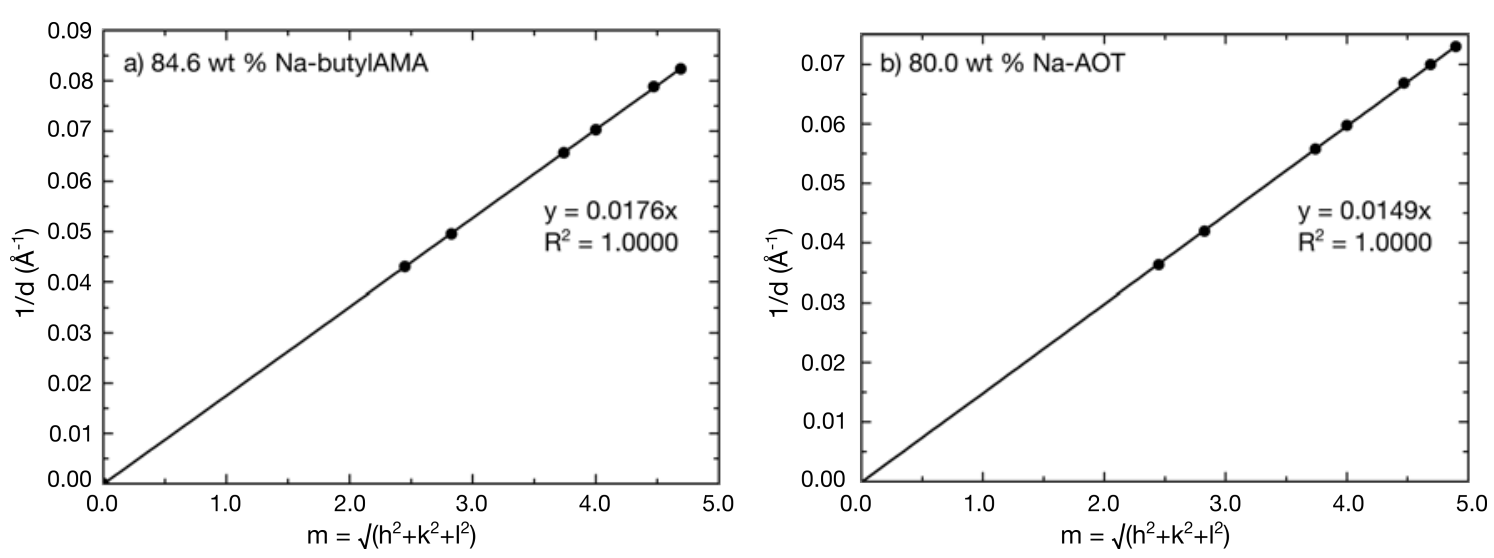

Figure 4.5: Plots showing the reciprocal d spacings vs. $\sqrt{ }\left(h^{2}+k^{2}+l^{2}\right)$ for the cubic phases of (a) the Na-butyIAMA system and (b) the Na-AOT system for comparison, where hkl are the assigned Miller indices. Correct assignments of the lattice types and space groups are confirmed by the linearity of the plots and the intercepts of the fitted lines at the origins. Lattice parameters are calculated as the reciprocal of the gradient of the fitted lines and are (a) $56.8 \AA$ and (b) $67.1 \AA$ for the sample concentrations shown.

incorporates the thickness of two monolayers alongside that of the water layer. A difference in d spacing of $\sim 4 \AA$ equates to a difference in each monolayer of only $\sim 2 \AA$. This difference can be rationalised by the shorter alkyl chains in the Na-butylAMA molecule. Given that the effective tail length contribution (apparent zigzag distance) of each additional carbon atom in alkyl chains is $1.26 \AA$, ${ }^{178}$ the two fewer $\mathrm{C}-\mathrm{C}$ bonds in Na-butylAMA would be expected to result in alkyl tails around $2.52 \AA$ shorter than those of Na-AOT. As the tails of Na-butylAMA have a combined total of four terminal $\mathrm{CH}_{3}$ groups rather than the two of $\mathrm{Na}-\mathrm{AOT}$ and these extra methyl groups provide additional bulk at the end of the tail region, it may be expected that this added bulk would disrupt the interdigitating of the surfactant tails in Na-butylAMA. However, the results obtained suggest this is not the case, and the Na-butylAMA molecules are able to pack in a way that produces a thin bilayer, in the region of $16 \AA$.

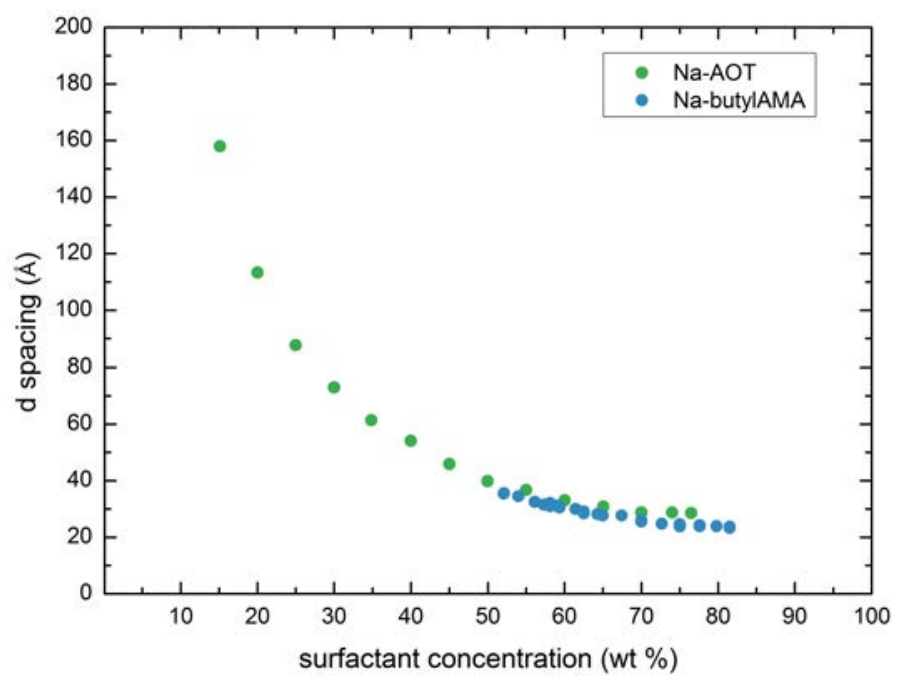

Figure 4.6: Changes in d spacing with increasing concentration for the Na-butylAMA and $\mathrm{Na}$-AOT systems. Where the form factor interference results in the disappearance of the firstorder Bragg peak in the Na-AOT system, the $d$ spacing was calculated using the position of the second-order Bragg peak. 
The limiting values of $d$ spacing in the Na-butylAMA system are $\sim 23$ and $\sim 38 \AA$, meaning that the water layer of the lamellar spacing is limited to a thickness of $\sim 7-22 \AA$. It follows that alongside the van der Waals and electrostatic forces, the steric hydration repulsive forces will provide a significant contribution to the self-assembly of the lamellar phase over most of this region. If we account for the differences in the $d$ spacings being predominantly due to the bilayer thicknesses, then the $\mathrm{L}_{\alpha}-\mathrm{H}_{\text {/I }}$ transition in both systems occurs at a limiting water thickness of $\sim 7 \AA$ and it is logical that the two surfactants both form $V_{\text {/I }}$ phases with the same space group.

The Bragg peaks of the Na-butyIAMA lamellar phase shift to slightly higher values of $q$ upon increasing temperature, meaning a reduction in the bilayer repeat distance. This can be attributed to changes in the relative contributions of each intermolecular force, likely as a result of dehydration of both the headgroup and counterion. Altering the energy of the system affects the surfactant geometry and disrupts the delicate balance of intermolecular forces. This can result in an increase or decrease in the bilayer repeat distance, with examples of both types of behaviour known. ${ }^{128,129}$ In the case of Na-butyIAMA, a decrease in d spacing means a relative increase in the contribution of attractive forces, and is consistent with the behaviour of the Na-AOT system at equivalent concentrations. However, the change in d spacing is not large and is less significant at higher concentrations, as shown in Table 4.1. The change in $\mathrm{d}$ spacing is not accompanied by any significant variation in the intensity of the Bragg peaks, which only significantly change in proximity to the two-phase boundary (see Figure 4.7).

Table 4.1: Values for the $q$ spacing of the first-order Bragg peak $\left(q^{*}\right)$ and the corresponding bilayer repeat distance $(d)$ at selected Na-butyIAMA concentrations and temperatures.

\begin{tabular}{cccccccc}
\hline \multirow{2}{*}{ Concentration (wt \%) } & \multicolumn{3}{c}{$\mathrm{q}^{*}\left(\mathrm{~nm}^{-1}\right)$} & & \multicolumn{3}{c}{$\mathrm{d}$ spacing $(\AA)$} \\
\cline { 2 - 4 } \cline { 6 - 8 } & $25^{\circ} \mathrm{C}$ & $50^{\circ} \mathrm{C}$ & $90^{\circ} \mathrm{C}$ & & $25^{\circ} \mathrm{C}$ & $50{ }^{\circ} \mathrm{C}$ & $90^{\circ} \mathrm{C}$ \\
\hline 52.1 & 1.793 & 1.835 & 1.895 & & 35.04 & 34.24 & 33.16 \\
58.1 & 1.959 & 1.982 & 2.062 & & 32.07 & 31.70 & 30.47 \\
60.0 & 2.005 & 2.051 & 2.119 & & 31.34 & 30.63 & 29.65 \\
62.5 & 2.158 & 2.185 & 2.242 & & 29.16 & 28.76 & 28.02 \\
64.3 & 2.231 & 2.249 & 2.258 & & 28.16 & 27.94 & 27.82 \\
70.0 & 2.416 & 2.433 & 2.456 & 26.01 & 25.82 & 25.58 \\
75.0 & 2.535 & 2.548 & 2.563 & & 24.79 & 24.66 & 24.51 \\
77.6 & 2.590 & 2.598 & 2.610 & 24.26 & 24.18 & 24.07 \\
81.5 & 2.649 & 2.662 & - & 23.72 & 23.60 & - \\
\hline
\end{tabular}

The change in $d$ spacing may be solely the result of a decrease in bilayer thickness upon increasing temperature (due to increased molecular motion within the tail region). This would mean that the water thickness does not change with temperature, which may explain why the Bragg peak intensity is largely independent of temperature for most sample concentrations. However, the magnitude of change in d spacing is not consistent, but decreases at higher concentrations, which suggests other contributing factors. If a change in bilayer thickness is not responsible for the change in $d$ spacing, then the relative increase in attractive forces may be due to either an increase in $\kappa$ and/or $\bar{\kappa}$ resulting from counterion dissociation and an increase in intrabilayer electrostatic repulsion (these effects would have to also overcome the increase in electrostatic contribution to the repulsion between bilayers). Alternatively, given 

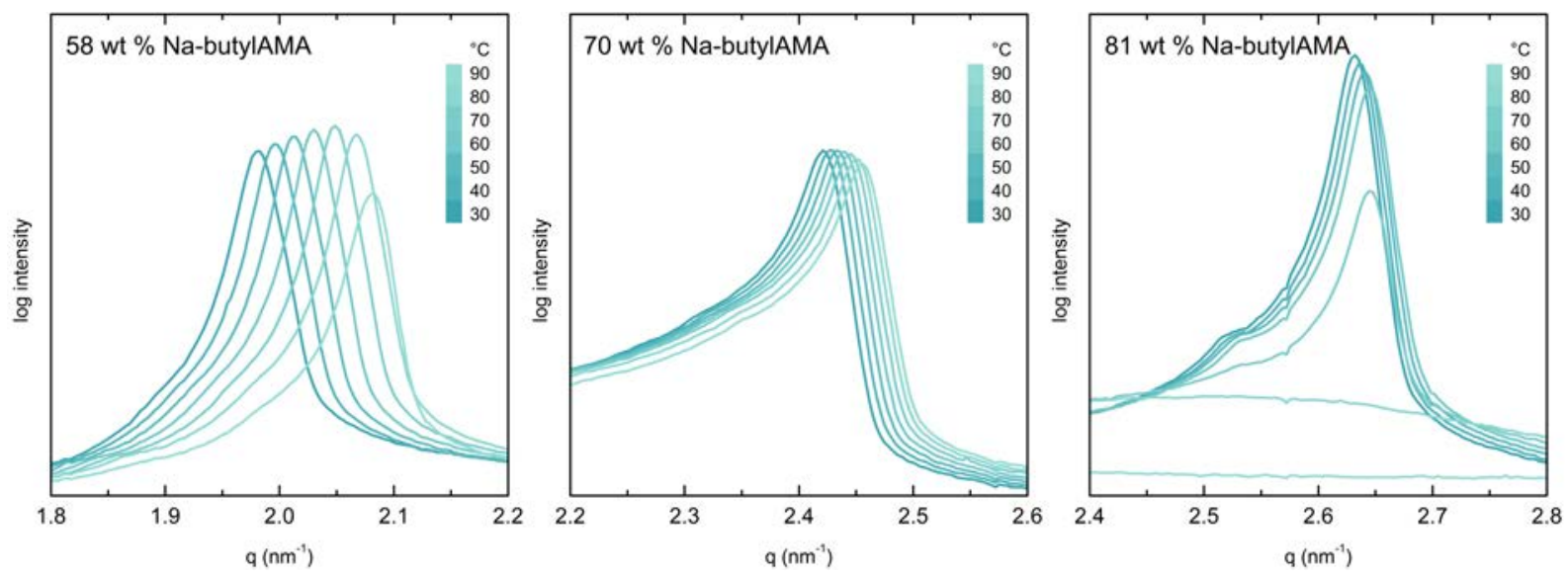

Figure 4.7: Evolution of the first-order Bragg peak with increasing temperature for Na-butyIAMA samples of 58,70 and $81 \mathrm{wt} \%$ surfactant. All samples show a shift to higher q spacing with increasing temperature, but the magnitude of the change decreases at higher concentrations. Note that changes in the peak intensity are only significant in the region of $\mathrm{L}_{\alpha}$ melting transition, and that this transition accounts for the loss of Bragg peaks at 80 and $90{ }^{\circ} \mathrm{C}$ for the sample of 81 wt \% Na-butylAMA.

that the small repeat distances in the Na-butylAMA system mean steric repulsion is the likely governing intermolecular force, the decrease in attraction of bilayers can be explained by a reduction in the magnitude of the steric hydration forces upon dehydration. Such changes in steric hydration have been reported elsewhere, ${ }^{131}$ but imply that any increase in the entropic component of this force is overcome by the change in dehydration. Na-butylAMA molecules do not have ability to protrude from the bilayer in the way that phospholipids do, and are not as bulky, so the effect of dehydration being able to overcome the relatively small steric contribution is feasible. Dehydration of the bilayer will be more favourable at low concentrations, which is consistent with the decreased effect of temperature on the repeat distance with increasing concentration.

\section{Sodium butyIAMA Optical Microscopy}

Concentration gradient POM textures for the Na-butylAMA system are shown in Figure 4.8. When compared with those obtained for Na-AOT (see Figure 3.15), ${ }^{\ddagger}$ there are obvious differences including the absence of a swollen lamellar phase. The Na-butylAMA images do not show a change in the optic sign of birefringence, uneven regions within the texture, or the formation of myelin structures at the $L_{1}-L_{\alpha}$ interface. The concentration gradient POM images are consistent with normal inverted surfactant systems, forming $H_{\| /}, V_{\| /}$and $L_{\alpha}$ phases. Within the $L_{\alpha}$ phase, mosaic and FCD-I structures can be observed, and flow-induced streaks are clearly visible at the interface with the micellar region.

Polarising optical microscopy images for the samples of Na-butylAMA are shown in Figure 4.9, and illustrate that the defect behaviour of the system is dominated by FCD-I defects. The

\footnotetext{
${ }^{\ddagger}$ Concentration gradient images of all the surfactant systems studied in this work are collated for comparison in Appendix B.
} 


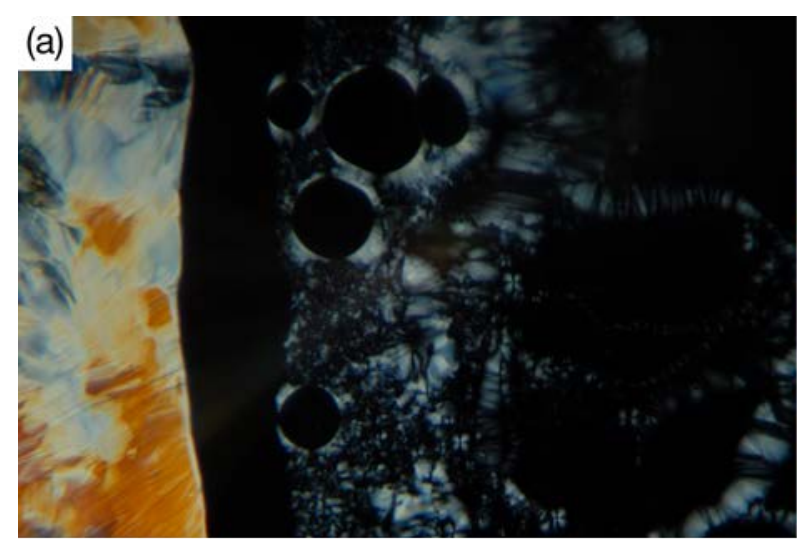

$\mathbf{H}_{\| l} \quad \mathbf{V}_{l l} \quad \mathrm{~L}_{a}$

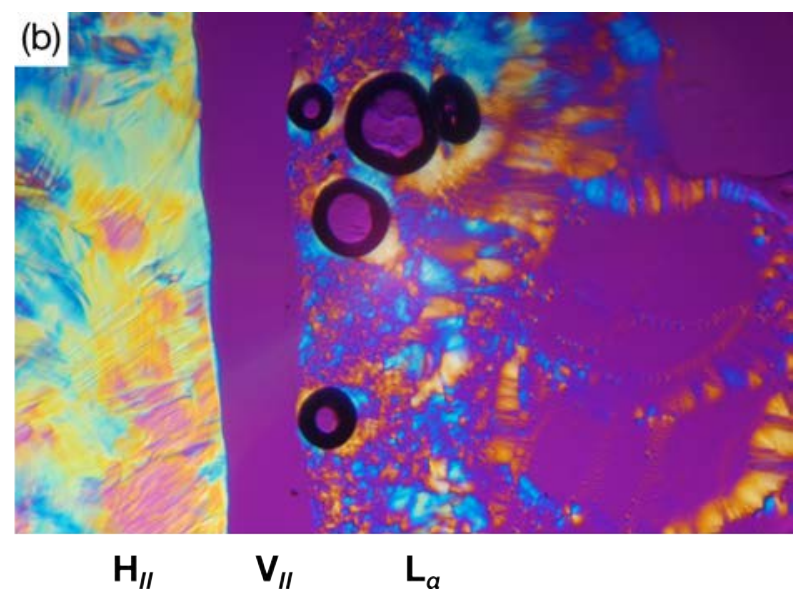

Figure 4.8: Concentration gradient of the Na-butylAMA system at $\times 40$ magnification and $\sim 25{ }^{\circ} \mathrm{C}$ viewed under (a) crossed polarised light and (b) with the addition of a quarter-wave plate.

assignment of these defects was confirmed through observation of the patterns upon rotation of the sample stage, with the negative Gaussian curvature confirmed by the rotation of the pattern in the opposite direction from the sample. The image for a sample of $47.5 \mathrm{wt} \% \mathrm{Na}$-butylAMA corresponds to the two-phase region at surfactant concentrations just below the lamellar phase. It is predominantly isotropic, but shows individual archetypal FCD-I defects, striated oily streaks and regions of distorted amalgamated defects indicating small lamellar domains within the isotropic domain. Upon entering the pure lamellar phase, the size of these defect patterns significantly decreases and the defects are distributed homogeneously throughout the textures.

At 52.5 wt \% Na-butylAMA, individual FCD-I defect points can be distinguished that on closer inspection often appear to be aligned in series with several neighbouring defects, indicating that they are components of oily streaks. There are also more examples of distorted FCD-I defects that form more obvious streaky features. The oily streaks contrast with the predominantly isotropic background that is likely the result of orientated domains of lamellar phase. As the surfactant concentration increases, the number of observable oily streaks also significantly increases, which reduces the size of the isotropic regions. The individual components of the streaks also become distorted, forming nodes at the junctions of oily streaks (57.5 wt \% Na-butylAMA) and then dominating the textures in mosaic-like patterns similar to those observed in the Na-AOT system at concentrations above $60 \mathrm{wt} \% \mathrm{Na}$-butylAMA. At $77.5 \mathrm{wt} \%$, in close proximity to the cubic phase, large individual defects are observed again, likely due to the defects playing a role in the $L_{\alpha}-V_{\|}$phase transition. Isotropic and fan-like textures are observed at higher concentrations, illustrating the formation of $V_{/ /}$and $H_{/ /}$phases.

The POM textures of the Na-butyIAMA lamellar phase are very similar to those observed in the Na-AOT system (see Figure 3.17) at similar concentrations. Given the similarities in the phase diagrams of the two systems at concentrations above 50 wt \% surfactant, with $\mathrm{L}_{\alpha}-\mathrm{V}_{\|}$and $\mathrm{V}_{\|}-\mathrm{H}_{\|}$ phase transitions occurring at similar concentrations, the formation of similar macroscopic defects is not surprising. This is because the two systems are stabilising the same type of phase and (based on the similar transitions) have similar curvature restraints within the $\mathrm{L}_{\alpha}$ region. However, the cryo-SEM images are able to provide more information about microstructural 

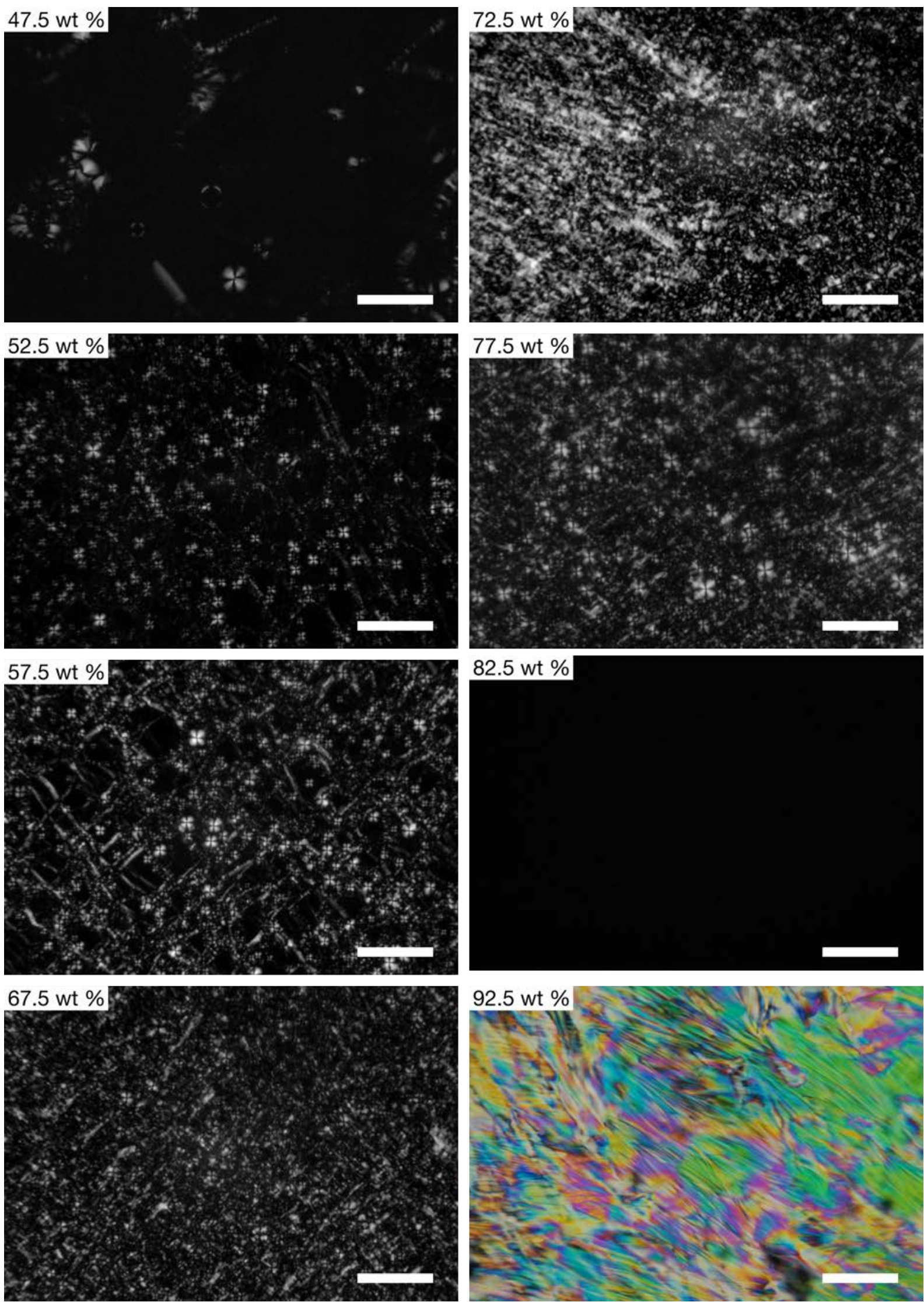

Figure 4.9: POM images of Na-butylAMA with increasing concentration at $\sim 25{ }^{\circ} \mathrm{C}$. FCD-I defects dominate the lamellar phase, producing textures with characteristic oily streaks and mosaic patterns upon increasing surfactant concentration. Isotropic and fan-like textures reveal the presence of $\mathrm{V}_{/ /}$and $\mathrm{H}_{/ /}$phases, respectively. Scale bars are $100 \mu \mathrm{m}$. 
differences between the systems and show how the size, shape and alignment of the FCD-I defects change within the Na-butylAMA system.

\section{Sodium butyIAMA Cryo-SEM}

The phase behaviour of the Na-butylAMA system shows that the reduced tail volume of the surfactant prevents the formation of a lamellar phase at concentrations below $50 \mathrm{wt} \%$ surfactant. In comparison to Na-AOT, the tails of Na-butylAMA do not exert the same level of steric bulk at the hydrophobic/hydrophilic interface and so the molecule, from a geometric point of view, is less inverted. From an elastic point of view, the molecule is less adaptable (i.e. is unable to form the variety of packing modes of Na-AOT). As such, it does not allow the variation in the bilayer elasticity that is required for deformations, both topological and undulatory, to lower the free energy of the disordered lamellar phase once the thickness of the water layer exceeds that of the bilayer. However, at concentrations above $50 \mathrm{wt} \%$ surfactant, the phase boundaries and POM images of the two systems are remarkably similar. This suggests that the additional tail volume of Na-AOT, responsible for the drastically different behaviour at low concentrations, is negated at high surfactant concentrations, resulting in the similar phase behaviours and POM textures. Cryo-SEM was used to determine if the microstructure of the Na-butylAMA system is comparable with that of the Na-AOT system, or if the difference in the flexibility of the bilayers formed in each system result in different defect behaviours.

Cryo-SEM images of sample of 45 wt \% Na-butylAMA, which is in the low concentration two-phase region that exists prior to the formation of a pure lamellar phase, are shown in Figure 4.10. In the lower magnification image - Figure 4.10 (a) - the majority of the image shows a lamellar structure with large channels or pockets of water, that exceed $500 \mathrm{~nm}$ in size. The lamellar structure is extremely swollen, and is clearly not present as a pure phase as the image also shows two small domains $(\sim 10 \mu \mathrm{m})$ of the disordered isotropic phase, marked in yellow. This phase consists of a disordered surfactant network comprised of small aggregated structures that are likely micellar or vesicular in nature. The regions of disordered phase also contain some larger aggregates that may be isolated lamellar droplets, in the form of MLV-type structures. Figure 4.10 (a) shows a region of the two-phase sample in which the lamellar phase is the dominant region. However, consistent with the POM images captured at the same concentration, the sample also showed regions consisting primarily of the isotropic phase, as shown in Figure 4.10 (b). The higher magnification images shown in Figure 4.10 (c) and (d) more clearly show the pockets and channels of water that exist as part of the lamellar structure, and it is apparent that these channels contain isolated regions of the disordered surfactant phase.

Cryo-SEM images of a sample of 55 wt \% Na-butylAMA are shown in Figure 4.11, and it is clear from both the low and high magnification images that the sample is a pure $L_{\alpha}$ phase. ${ }^{\ddagger}$

\footnotetext{
‡Small numbers of ice crystals observed in the low magnification image are an artefact of the freezing process and do not affect the composition of the sample or the majority structure. While it is extremely difficult to achieve no such artefacts upon freezing, the images are good representations of the actual structures.
} 

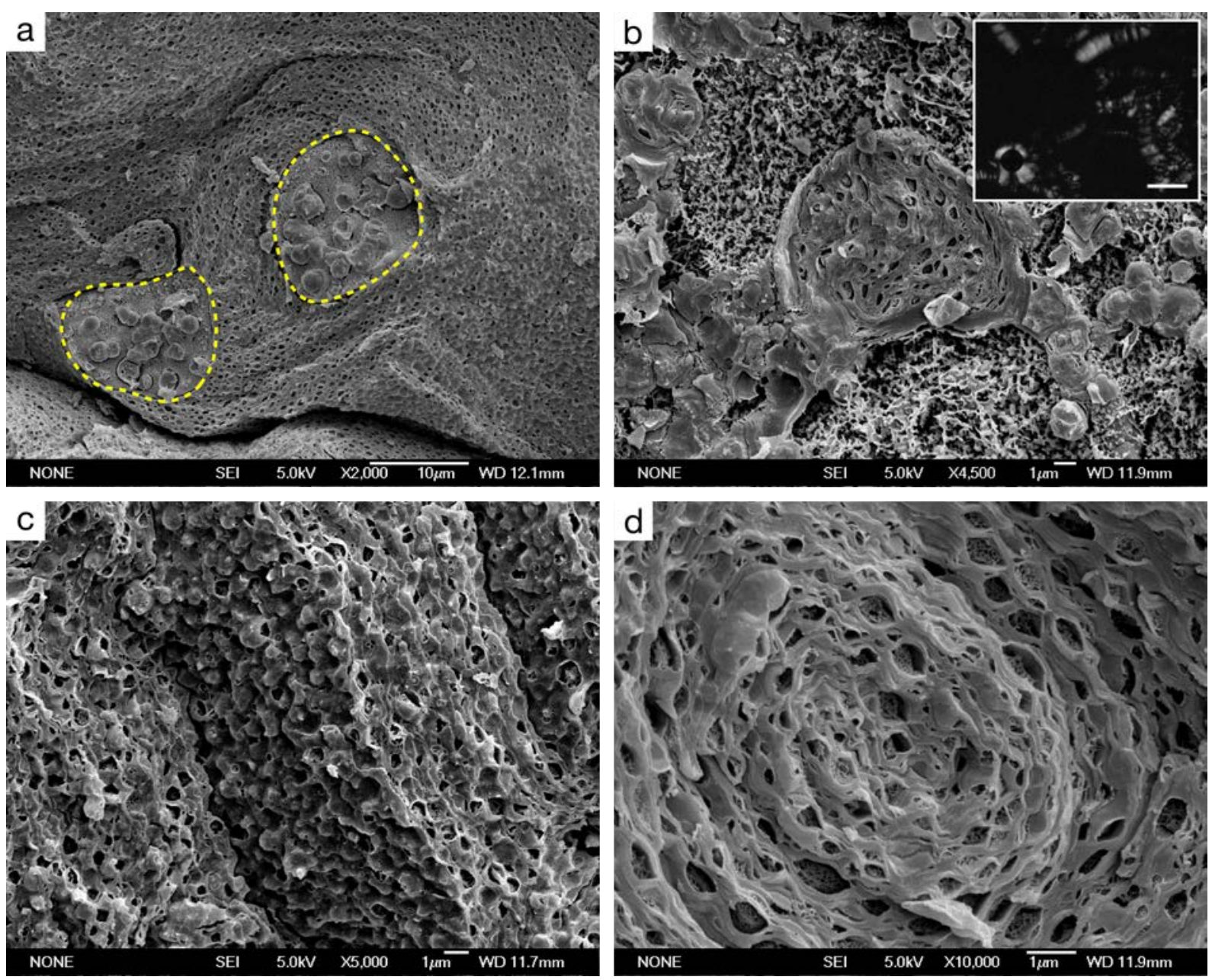

Figure 4.10: Cryo-SEM images of a sample of $45 \mathrm{wt} \%$ Na-butylAMA. Yellow dashed lines indicate small domains of a disordered isotropic phase within the swollen lamellar structure. The inset shows the corresponding POM image, with a scale bar of $50 \mu \mathrm{m}$.

The images are clearly in good agreement with the POM image shown for the same sample concentration, with the majority of the large defects in the sample being FCD-I defects, many of which are aligned into oily streaks. The image shown in Figure 4.11 (a) is at a very low magnification, but shows various domains that are generally between 30 and $50 \mu \mathrm{m}$ along their longest dimension. These ordered domains are broken up by the presence of both oily streaks and edge dislocations. Figure 4.11 (b) is an enlargement of the area marked in Figure 4.11 (a) and shows the edge of a relatively ordered domain that is broken by an obvious oily streak. At higher magnifications, several different types of defect behaviour are evident. Figure 4.11 (c) shows several tightly packed focal conic domains that could be interpreted as "leek-like" FCD-II cylinders, but could also be examples of the energetically ideal toroidal FCD-I defects. The hyperbola arising from the focal point between the two structures creates local environments of harsh curvature and results in regions of disorder. Figure 4.11 (e) shows paired line defects of $s= \pm 1 / 2$, and along with Figure 4.11 (d) and (f), shows the topology of the phase. The surface of the structure is clearly uneven and there is obvious connectivity between both the surfactant and water domains. This $55 \mathrm{wt} \% \mathrm{Na}$-butylAMA sample clearly shows a degree of bilayer undulation. 

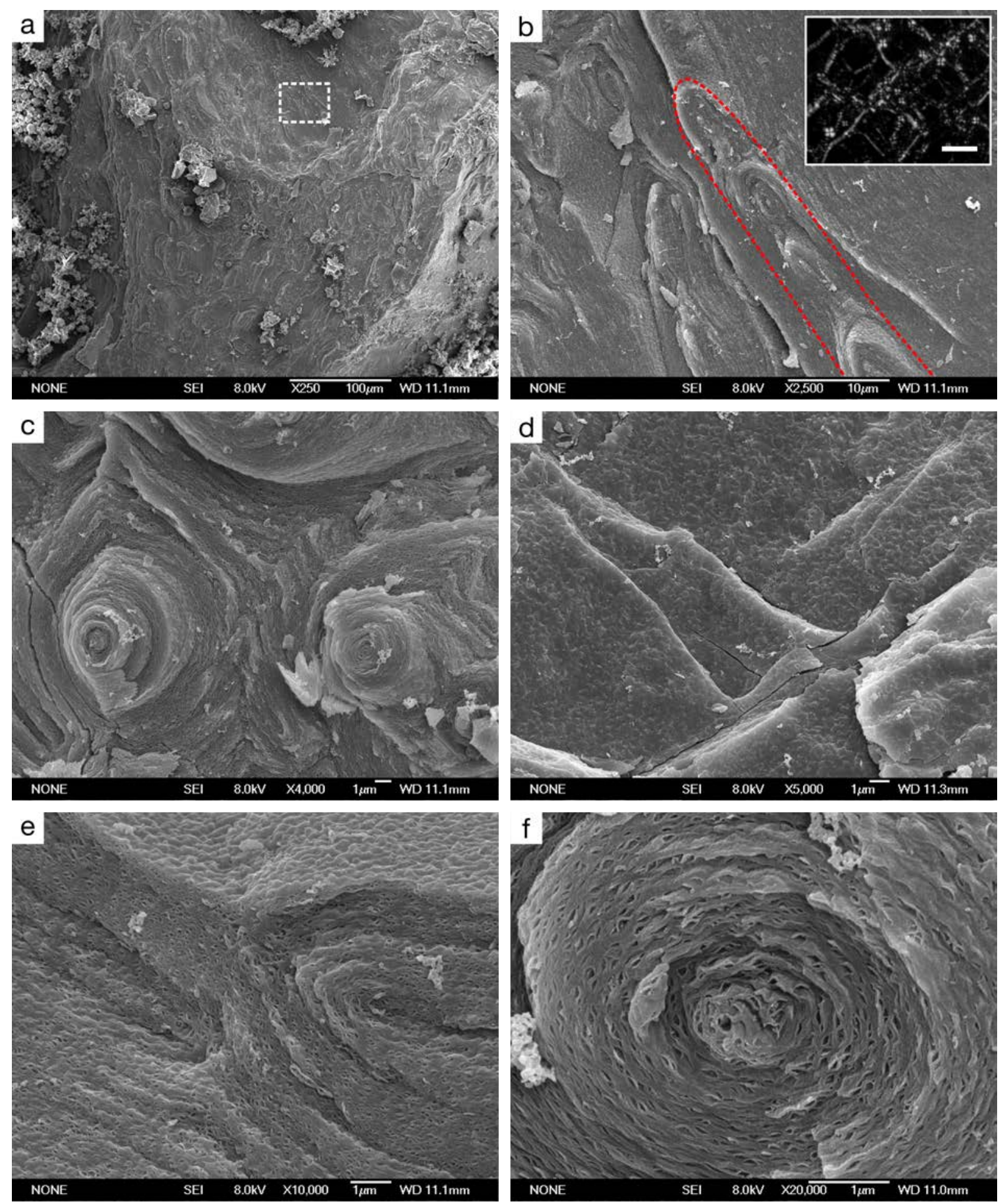

Figure 4.11: Cryo-SEM images of a sample of $55 \mathrm{wt} \%$ Na-butylAMA. Image (b) is an enlargement of the area indicated in (a). Red dashed line highlights an oily streak at the edge of an ordered domain. The inset shows the corresponding POM image, with a scale bar of $50 \mu \mathrm{m}$.

The phase diagram, SAXS and POM evidence indicates that the Na-butylAMA system does not stabilise a swollen, undulating lamellar phase like that observed in the Na-AOT system. However, it should be noted that at concentrations above the "crumpling transition" in the $\mathrm{Na}-\mathrm{AOT}$ system, the lamellar phase was still observed to show some evidence of fluctuations, and the SAXS patterns showed some diffuse scattering. Both the 1D and 2D SAXS profiles of the Na-butylAMA system also showed some broad scattering at concentrations below 
$\sim 62.5 \mathrm{wt} \%$ surfactant and thus some variation in the bilayer spacings should be expected. However, the Bragg peaks were very much the dominant feature of the SAXS spectra at all concentrations in the Na-butyIAMA lamellar region and so any fluctuations in the bilayer are not expected to be the dominant feature of the cryo-SEM images. This is consistent with the cryo-SEM images shown in Figure 4.11. Despite the evidence of undulations, the local bilayer structure is clearly ordered and the phase exhibits long-range ordering. When the sample is compared to the structure of the undulating Na-AOT lamellar phase (see Figure 4.12) it is clear that while the entropic forces contribute to the free energy of the Na-butylAMA phase, they are far from being the dominant force.
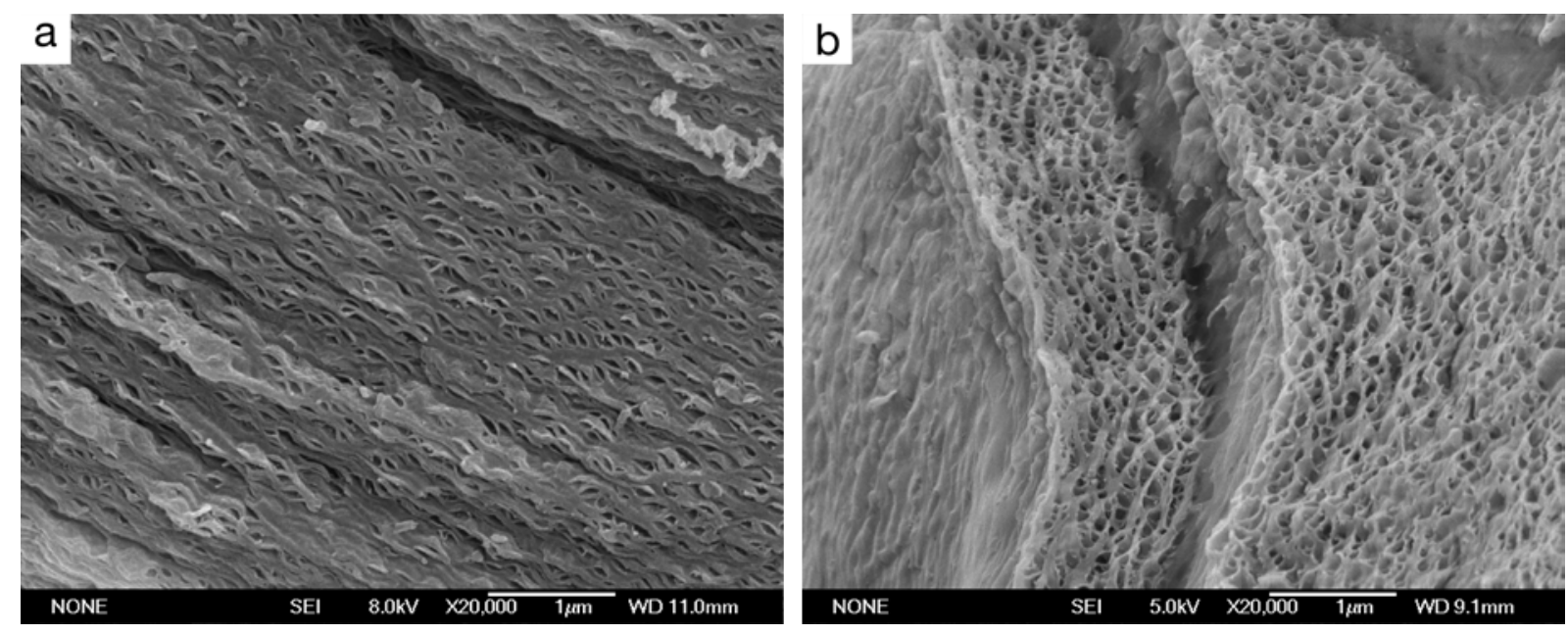

Figure 4.12: Comparison of the lamellar phase undulations at (a) $55 \mathrm{wt} \% \mathrm{Na}$-butylAMA and (b) 38 wt \% Na-AOT.

Cryo-SEM images for a sample of 60 wt \% Na-butylAMA are shown in Figure 4.13. This is only a concentration increase of $5 \mathrm{wt} \%$ from the images presented in Figure 4.11, and given that the SAXS spectra at $60 \mathrm{wt} \%$ still show evidence of diffuse scattering, it may have been expected that the images would not be appreciably different. However, there is a clear increase in order in the system upon moving from 55 to $60 \mathrm{wt} \%$ Na-butylAMA. The bilayers still show evidence of undulation at high magnifications, but this is decreased in comparison to the lower concentration images and the bilayers are clearly ordered, despite the connective defects. In general, however, the increase in order is evident through the alignment of the various domains and oily streaks. There are more streaks than at $55 \mathrm{wt} \% \mathrm{Na}$-butylAMA, and the majority are approximately $10-15 \mu \mathrm{m}$ in size, although some are as long as $30 \mu \mathrm{m}$. However, in comparison to those observed at $55 \mathrm{wt} \% \mathrm{Na}$-butylAMA, the streaks at $60 \mathrm{wt} \%$ are comprised of smaller individual components, and are generally of comparable size and aligned along the same director axis as their immediate neighbours. Between regions of aligned streaks there are still domains of idealised, well-ordered lamellar behaviour, which is consistent with the isotropic regions in the POM image that indicate areas of homeotropic alignment of the lamellar phase. Between some domains there is evidence of sharp domain boundaries. Such sudden transitions have a high energy cost, and while in some regions this is dissipated by the formation of localised regions of disorder/defects, in other regions this is not the case. This is an indication that the Na-butylAMA bilayer is flexible enough to accommodate some local regions of sharp curvature. 

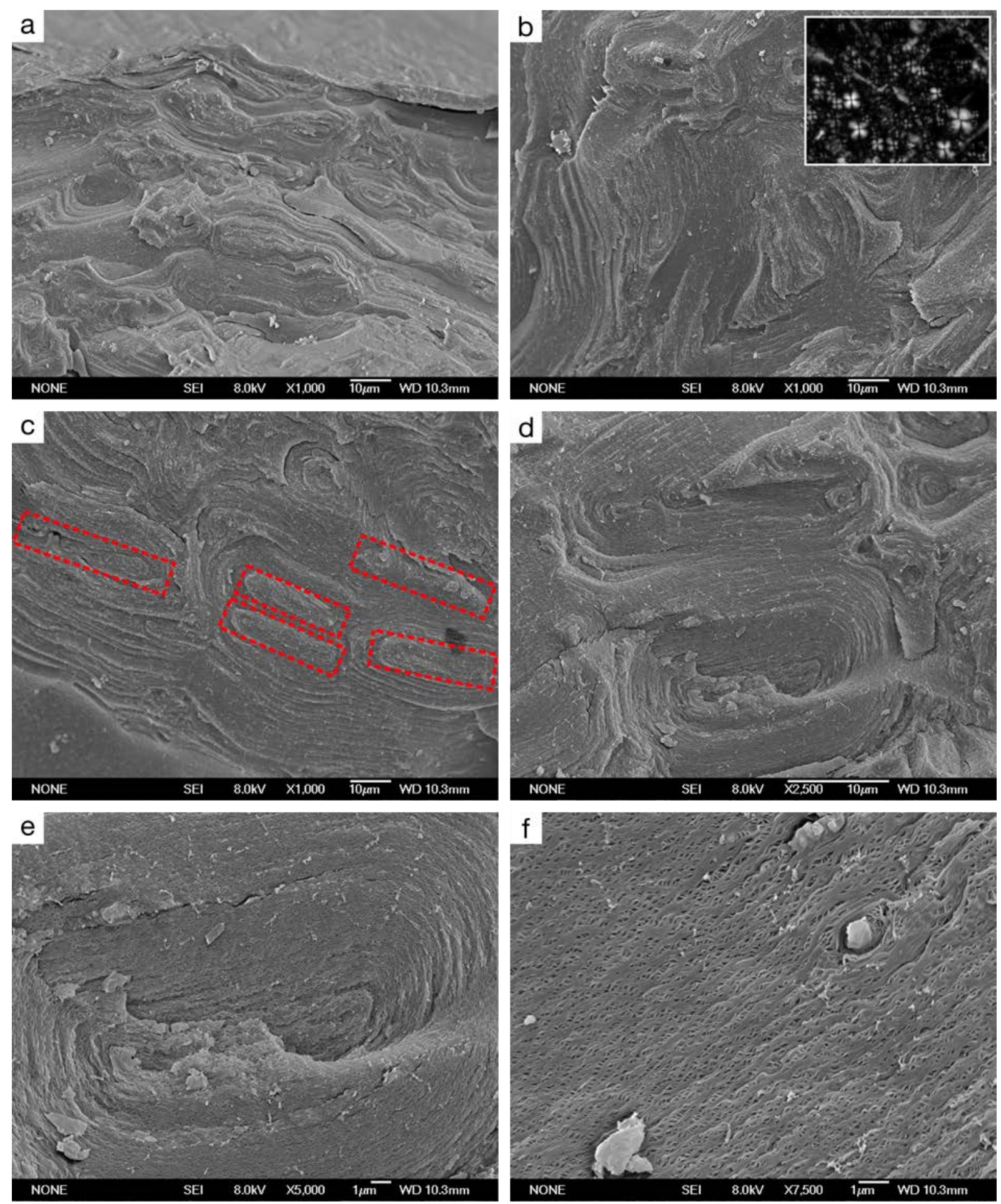

Figure 4.13: Cryo-SEM images of a sample of 60 wt \% Na-butyIAMA. Red dashed boxes show examples of aligned oily streaks. The inset shows the corresponding POM image, with a scale bar of $50 \mu \mathrm{m}$.

In general, the images shown for $60 \mathrm{wt} \% \mathrm{Na}$-butylAMA may be considered to be slightly more ordered than those obtained at comparable Na-AOT concentrations; however, the types of defects observed and the approximate domain sizes of 30-50 $\mu \mathrm{m}$ are consistent with observations of the Na-AOT system. The evidence for undulations in the bilayers for the two systems is very similar, with regions of concentrated/compressed bilayers separated by groups of connections between the bilayers. Although this is clearly a real feature, it should be noted 
that the effect is likely enhanced upon the sublimation of the sample, and so the undulations and water channels may appear larger than they truly are. It should also be considered that the images show a fluid system captured at one moment in time, and that such passages will continuously form and break up, a factor in facilitating flow in the system. While the SAXS profiles showed evidence of broad peaks/diffuse scattering at $60 \mathrm{wt} \% \mathrm{Na}$-butylAMA, it was also clear from 2D SAXS experiments that the scattering was monodirectional, and so even in terms of bilayer undulations the Na-butylAMA lamellar phase shows a degree of ordering, which is in stark contrast to the swollen structures formed at low concentrations in the Na-AOT system.

Moving from 60 wt \% Na-butyIAMA to 70 wt \% Na-butylAMA (shown in Figure 4.14), there remains a limited degree of porosity in the bilayers. In the main, the local bilayer structure is very ordered, which is consistent with the sharp Bragg reflections obtained in the SAXS experiments. However, the increasing progression towards defects of negative Gaussian curvature results in the structure appearing significantly more disordered on larger lengthscales than the structure shown at $60 \mathrm{wt} \%$ Na-butylAMA. The 70 wt \% sample still contains many oily streaks, which range in length from $\sim 5$ to $25 \mu \mathrm{m}$, but they are no longer at the centre of orientated domains of approximately equal size, as was observed in Figure 4.13 (c). Some of the oily streaks shown in Figure 4.14 can be grouped as aligning in the same direction, such as those shown in Figure 4.14 (c), which protrude from the surface and closely resemble the defect structures reported for the egg lecithin-phosphatidyl inositol/water system. ${ }^{179}$ However, many of the streaks are significantly distorted and form curved domains, giving rise to an increased number of saddle-splay surfaces and apparent random packing and disorder, as shown in Figure 4.14 (b). This disorder is due the increasing negative Gaussian curvature driving the system towards the formation of mosaic-like packing of highly curved domains, to dissipate the high energy cost of the increasingly inverted molecular shape of the tightly packed surfactant molecules and the increasing magnitude of the steric hydration repulsion between bilayers.

The images in Figure 4.14 clearly display a mixture of different behaviours. The sample shows some ordered regions bounded by oily streaks, and also highly curved domains of distorted defects. This is consistent with the POM images and also comparable with the observations of the Na-AOT system. This is because both systems are progressing towards mosaic-like arrays that are highly curved, in order to meet the energy costs incurred by retaining a bilayered structure in close proximity to the bicontinuous cubic phase. However, at 70 wt \% surfactant, the structures of these systems are intermediate to the highly curved arrays formed at higher concentrations (in proximity to the $\mathrm{V}_{/ /}$phase) and the highly ordered structures (more classical $\mathrm{L}_{\alpha}$ regions) at lower concentrations that contain ordered domains and oily streaks.

At 78 wt \% Na-butyIAMA, shown in Figure 4.15, the cryo-SEM images illustrate the highly curved lamellar domains formed by the system, in which oily streak defect chains pack closely together in excellent agreement with the mosaic-like POM image obtained for the same sample concentration. The individual FCD-I defects that make up the streaks are generally smaller than $1 \mu \mathrm{m}$, and the majority of the streaks are less than $10 \mu \mathrm{m}$ in length. The small size of these streaks is likely due to the close packing of domains, meaning that their growth is 

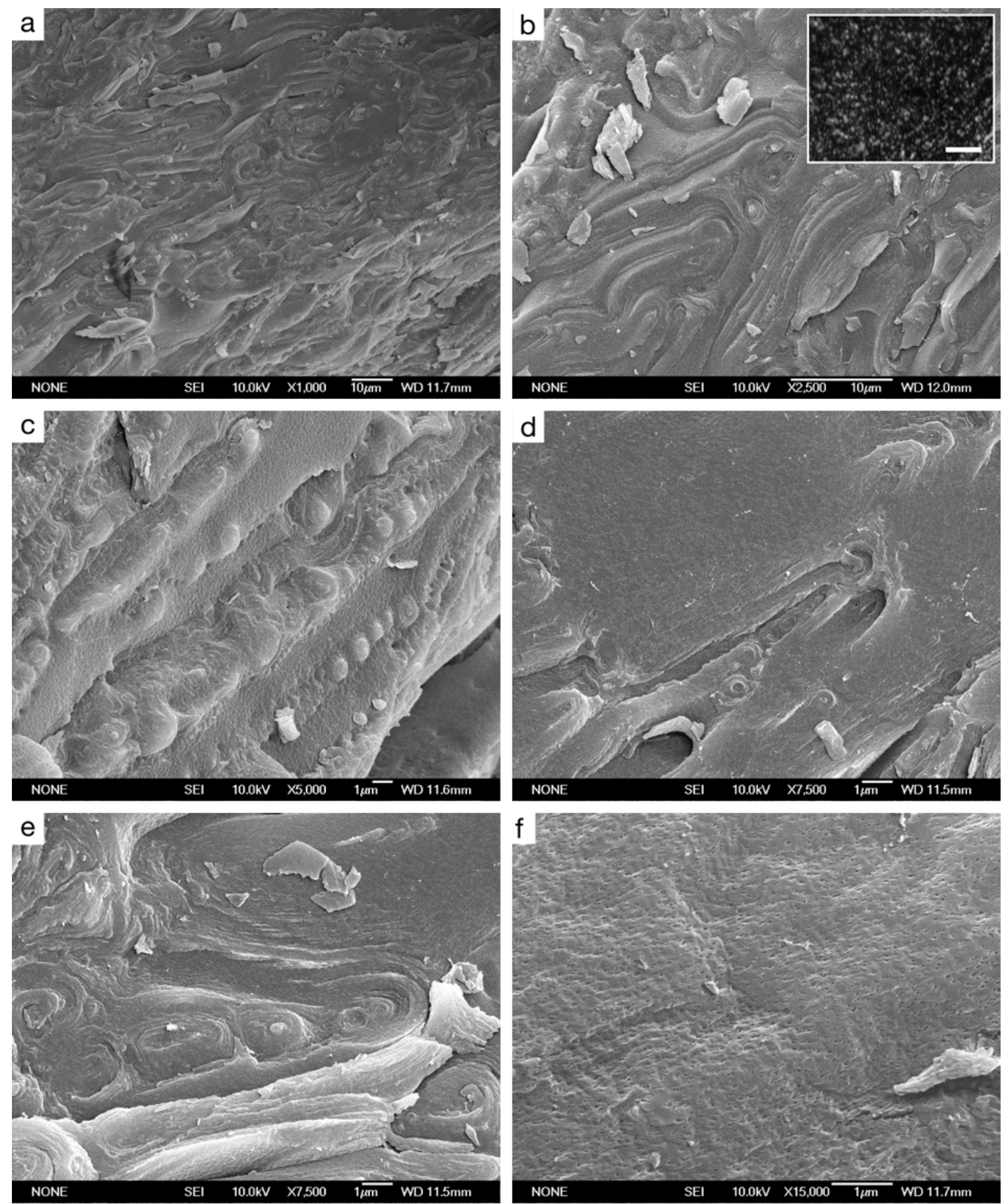

Figure 4.14: Cryo-SEM images of a sample of $70 \mathrm{wt} \% \mathrm{Na-butyIAMA.} \mathrm{The} \mathrm{inset} \mathrm{shows} \mathrm{the}$ corresponding POM image, with a scale bar of $50 \mu \mathrm{m}$.

limited by neighbouring domains. Figure 4.15 (c) is an enlargement of the area indicated in Figure 4.15 (a), and shows the ordered alignment of the streaks and the way in which the surfaces curve around them to form domains, while the low magnification image illustrates the uniformity of the sample. It is clear that the small bilayer repeat distance prevents any undulation of the bilayers, although the small bumps observed in Figure 4.15 (b) may indicate some form of topological defect structure. The images shown at $78 \mathrm{wt} \%$ are almost indistinguishable to those presented previously for 75 wt \% Na-AOT: see Figure $3.21(d-f)$. Both sets of images 

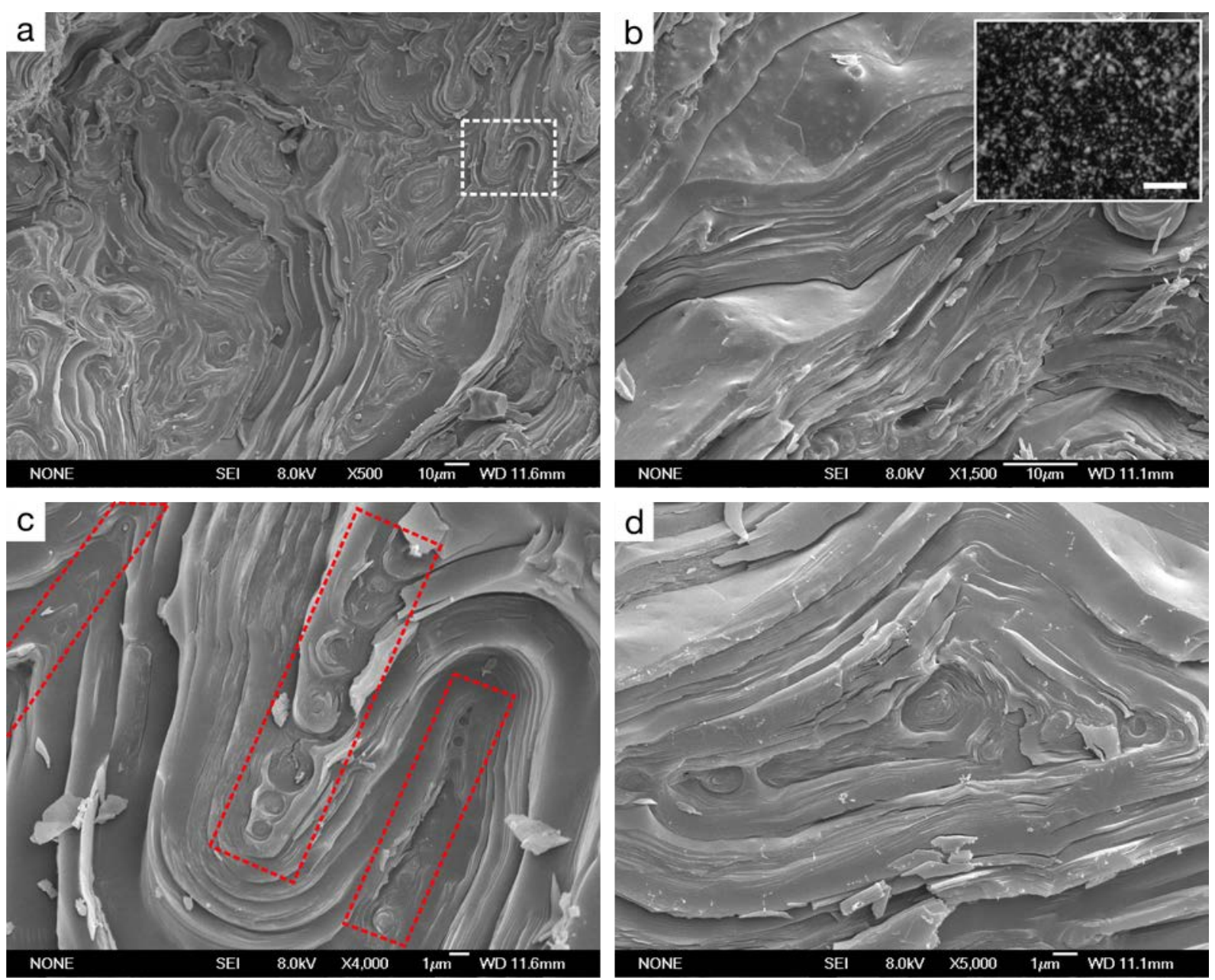

Figure 4.15: Cryo-SEM images of a sample of $78 \mathrm{wt} \%$ Na-butylAMA. Image (c) is an enlargement of the area marked in (a). Red dashed boxes highlight ordered and aligned oily streaks. The inset shows the corresponding POM image, with a scale bar of $50 \mu \mathrm{m}$.

show the structures of the lamellar phases at concentrations $\sim 3-4$ wt \% below the cubic phase boundary, and provide a clear indication that the two surfactants form comparable structures at high concentrations, indicating that the role of the increased tail branching is limited to (or at least more significant at) lower surfactant concentrations.

\section{Sodium butyIAMA: Discussion}

It is clear from the experimental results presented above that the Na-butylAMA system stabilises a micellar region rather than a $L_{\alpha}$ phase below $\sim 50 \mathrm{wt} \%$ surfactant. The system clearly has increased spontaneous curvature in comparison to the Na-AOT system. This can be explained purely using a geometric argument, in that the reduced tail volume means that the Na-butylAMA surfactant is less inverted and so the structure is more cone-like than that of Na-AOT. Hence, a packing type that produces micellar structures is energetically favoured. However, this argument is overly simplistic. The defect behaviour discussed above reveals that 
the Na-butyIAMA system almost exclusively forms FCD-I defects in preference to FCD-II defects in the lamellar phase. So, despite the geometric shape of the Na-butylAMA surfactant preferring a spherical packing arrangement (positive Gaussian curvature), the system forms a lamellar phase that has an energy cost associated with the curvature frustration of the bilayer that needs to be stabilised by the formation of defects with negative Gaussian curvature. This suggests that the point of dilution at which the lamellar phase of Na-butylAMA is no longer energetically favoured is not because the shape of the molecule is too cone-like to form bilayers, but that the bilayers become too fluid, and thus are not rigid enough to meet the required magnitudes of $\kappa$ and $\bar{\kappa}$ to stabilise a lamellar phase.

The reason for the change upon dilution is evident when the repeat distance at which the Na-butylAMA system does form a lamellar phase is considered. The pure $L_{\alpha}$ phase of the system is only formed with repeat distances at which the steric hydration repulsion forces are significantly strong. The system does not form a lamellar phase once the contribution of these forces to the free energy of the system becomes negligible. The system is not able to facilitate the "crumpling transition" observed in the Na-AOT system, where the order of the system alters abruptly as the governing force of the phase transition changes from steric hydration forces to Helfrich forces. This transition is facilitated in the Na-AOT system by a conformational rearrangement of the Na-AOT tails that is able to regulate the elasticity of the bilayers to allow the lamellar phase (in a disordered form) to be extended to lower concentrations. In the Na-butylAMA system, the molecule can clearly not regulate the elasticity to the same extent and so the requirement for this lamellar behaviour (that $\kappa$ is of similar magnitude to $k_{\mathrm{B}} T$ and $\bar{\kappa}$ is between the weakly negative and extremely negative values that stabilise $L_{3}$ or micelle formation over the $L_{\alpha}$ phase) is not met.

The effect of branching in the Na-butylAMA system is reduced in comparison to the Na-AOT system. Although both systems technically have two carbon atoms branched from the linear chain, their separate positions in the chain (and hence existence as methyl groups) in Na-butylAMA, rather than the ethyl groups of Na-AOT, alter their relative contribution, as does their relative distances from the surfactant headgroups. Using the empirical branching factor of Nave et al..$^{\ddagger}$ produces values of 1.5 for Na-butylAMA and 1.67 for Na-AOT (with a non-branched system having a value of 1 and higher numbers meaning a greater effect). ${ }^{48}$ The greater contribution in Na-AOT is a result of the added rotational freedom of ethyl groups in comparison to methyl groups, making them sterically bulkier. In addition, one of the $\mathrm{CH}_{3}$ groups of the Na-butylAMA molecule is terminal and hence is too far removed from the interfacial region to significantly affect it. The second $\mathrm{CH}_{3}$ group of Na-butylAMA is actually closer to the surfactant headgroup than the ethyl branches are in Na-AOT (and thus is able to contribute to the interfacial region of the headgroup). However, the reduced steric bulk and lack of rotational freedom means that this effect is minimised and it is likely that significantly splayed tail arrangements in Na-butylAMA may leave an energetically costly "void" between the splayed tails, that the ethyl moieties of Na-AOT would be able to adequately fill, but the methyl groups of Na-butyIAMA cannot. The ethyl branches of Na-AOT may also allow for sideways

\footnotetext{
${ }^{\ddagger}$ Nave’s branching factor is calculated by numbering the longest chain from the terminal end and using the equation $\Sigma$ (\# of carbons in branch $\times$ position on longest chain)/length of longest chain.
} 
interdigitating/tessellation of branches (that would increase the rigidity) that the methyl groups of Na-butyIAMA similarly would not.

The reduced branching in Na-butylAMA when compared with Na-AOT clearly increases the stability of the micellar phase, but removes all stability of the disordered lamellar phase formed by $\mathrm{Na}-\mathrm{AOT}$, and thus removes nearly all the behaviour that has previously been assigned as anomalous. It is clear that our conclusions in the previous chapter assigning these behavioural traits to the Na-AOT tail branching are justified, and that the role of the tail branching at the hydrophobic/hydrophilic interfacial region is fundamental to the self-assembly of these systems. However, it is also clear that the contribution of the branches is partially negated at high concentrations, as the Na-AOT and Na-butyIAMA systems show similar phase behaviours and similar microstructures in this region.

The behaviour of Na-AOT was rationalised in the previous chapter in terms of the tail branching introducing flexibility at the interfacial region of the surfactant, and providing the pathway towards different degrees of elasticity through rotational isomerism. The Na-AOT tails introduce disorder to the system by disrupting the efficiency of the packing of headgroups and altering the tail volume contribution and lateral pressure at the hydrophobic/hydrophilic interface. This disorder destabilises the formation of micellar aggregates and favours the localised instabilities of the disordered lamellar structure, allowing topological defects and undulations. These aid in the stabilisation of the system through entropic forces and are contributing factors in the values of $\kappa$ and $\bar{\kappa}$ being such that neither $\mathrm{L}_{3}$ phase or a vesicular phase are energetically preferred over the $L_{\alpha}$ phase. This disorder is normally a characteristic provided by the addition of a cosurfactant and is also the reason for the much-lauded emulsifying properties of the surfactant. The low concentration liquid crystalline behaviour is consistent with the microemulsifying behaviour investigated in detail by Nave et al., which allows us to draw several parallels with the other analogue systems reported in these publications. ${ }^{48,75,79,80}$ In particular, we can compare the Na-AOT and Na-butylAMA systems studied here with the straight-chained Na-hexyIAMA (the surfactant initially proposed for study in this work) and an isomer of Na-AOT containing unbranched octyl chains (Na-octylAOT).

The liquid crystalline phase behaviour was not the subject of the investigation by Nave et al., and so they did not produce phase diagrams for the series of surfactants they synthesised, and did not discuss any differences in lamellar structures that were formed. However, several concentration gradient POM images were produced and published in Nave's PhD thesis, including those of the unbranched hexyl and octyl systems. ${ }^{62}$ Obviously, no information can be inferred about the concentrations at which any transitions occur, but the images do provide information about the phase progression. From our own experimental work (based on all the systems presented in this thesis), we are able to conclude that the presence of the swollen lamellar phase stabilised by Helfrich forces can be identified in the concentration gradient images by a change in birefringence, formation of myelin structures, and transition to an uneven texture within the lamellar region. We can thus state that the images in Nave's thesis reveal that neither of the straight-chained systems (Na-hexyIAMA and Na-octyIAOT) form a disordered swollen lamellar phase. It is also a point of interest that the Na-octylAOT system does not form 
a $\mathrm{V}_{\text {// }}$ phase, but undergoes a $\mathrm{L}_{\alpha}-\mathrm{H}_{\text {// }}$ transition, which is likely a result of an increased bilayer thickness and thus increased rigidity and reduced spontaneous curvature.

Comparison of these four systems (Na-AOT, Na-octylAOT, Na-hexyIAMA and Na-butylAMA) allows us to show that the stabilisation of the disordered lamellar phase in Na-AOT is not solely a factor of either tail length or the volume provided by the number of carbon atoms in the tail region. The longest chains in Na-AOT are hexyl chains, while the other three systems have octyl, hexyl and butyl chains, and none of them are able to produce the disordered lamellar behaviour observed in Na-AOT. Similarly, if we consider the number of carbon atoms contributing to the volume of the tail region, the two AOT molecules have C8 chains, while the AMA molecules have C6 chains. The two C6 surfactants appear to produce similar behaviours because the branching of Na-butylAMA is not sufficient to introduce significant disorder at the interfacial region. In contrast, the two $\mathrm{C} 8$ systems are significantly different. The straightchained C8 analogue produces neither a disordered lamellar or cubic phase, while Na-AOT stabilises both. The branching in Na-AOT is clearly responsible, and has fundamental roles in introducing disorder into the system and increasing the flexibility and adaptability of the bilayer through the introduction of rotational isomerism.

The initial proposal of studying the behaviour of Na-hexylAMA as a comparison to Na-AOT would have also facilitated a discussion into the effect of chirality on the phase behaviours. The use of Na-butyIAMA, however, means that both systems have three chiral centres and thus are both composed of a range of diastereomers. However, the longer branches within the Na-AOT molecule mean it will form stereoisomers that are less equivalent than those formed in the Na-butylAMA system. A logical assumption would be that the more branched a system is (or the longer the branches it contains), the more likely the chirality is to play a significant role in providing disorder to the surfactant packing in the system. Structural differences enforced by different molecular configurations (stereoisomerism) persist at all concentrations and increase the system entropy, whereas conformational differences due to rotational isomerism are likely concentration dependent and different isomers will form only when energetically favourable. This means that mixtures of different rotational isomers can exist at different concentrations, and can induce localised packing environments through self-assembly that promote curvature in the bilayer. Thus, these rotamers can reduce the system entropy and energetic cost associated with maintaining a bilayered structure over large concentration ranges. It is likely that the large differences between the Na-AOT and Na-butyIAMA systems at low concentrations are a result of the difference in effectiveness of the rotational isomers formed in each system at promoting disorder at the interfacial hydrophobic/hydrophilic region. However, the smaller differences observed at high concentrations (i.e. the slight differences in the locations of the phase boundaries) are due to the different packing restraints of the stereoisomers present in each system, which depending on their molecular structures, conform to the desired packing arrangement of the whole to a greater or lesser extent. 


\section{Potassium butylAMA: Experimental Results}

As the extent of tail branching in the Na-butylAMA molecule is not sufficient to stabilise a swollen lamellar phase (such as that observed in the Na-AOT system), the question was asked as to whether this behaviour could be reintroduced using a further structural modification. This was achieved by manipulating the headgroup of the system, and hence its contribution at the hydrophobic/hydrophilic interface, by replacing the sodium counterion with a potassium cation, forming K-butyIAMA. There are no reports of this surfactant having been synthesised previously, but the the replacement of the sodium ion in Na-butylAMA with a potassium ion should result in a reduction in the effective size of the headgroup. Although the ionic radius of the $\mathrm{Na}^{+}$ ion is smaller than that of $\mathrm{K}^{+}(0.95$ and $1.33 \AA$, respectively $)$, the $\mathrm{K}^{+}$ion has a significantly reduced hydration shell, and so a fully hydrated $\mathrm{Na}^{+}$ion is larger than a fully hydrated $\mathrm{K}^{+}$ion (3.58 and $3.31 \AA$, respectively). ${ }^{180}$ Further to this, sodium and potassium lie either side of the chaotropic/kosmotropic divide, which describes the interactions with water as favourable or unfavourable. Favourable interactions with water result in the $\mathrm{Na}^{+}$ion being preferentially strongly hydrated. In comparison, the $\mathrm{K}^{+}$ion favours a partially dehydrated state, and readily loses its outer hydration shell. The difference between the steric contributions of the counterions is thus increased, a factor that is further enhanced by the two ions also differing in their affinities for the AMA headgroup.

According to Collins' law of matching water affinities, the sulfonate headgroup of the AMA ion will form a tighter ion pair with the similarly "soft" $\mathrm{K}^{+}$ion. ${ }^{49,181}$ The increased affinity between headgroup and counterion will result in a reduced capacity for the counterion to dissociate, while the effectively reduced size of the headgroup will result in a reduced headgroup contribution at the hydrophobic/hydrophilic interface. It follows that the role of the surfactant tail volume at this interface will then be increased, meaning that despite the reduced branching in comparison with $\mathrm{Na}$-AOT, the branches of K-butyIAMA should promote more flexibility when compared with the Na-butylAMA system. This should have the effect of promoting negative curvature at the interface and increasing the elasticity of the bilayer, meaning that the formation of a swollen lamellar phase and highly curved phases is more likely.

\section{Potassium butylAMA Phase Behaviour}

The phase diagram of K-butylAMA is presented in Figure 4.16, showing that $L_{\alpha}, V_{\|}$and $H_{\| l}$ phases are formed sequentially with increasing surfactant concentration. This is the same phase progression observed in the Na-AOT and Na-butylAMA systems described previously. With reference to these two systems, two features of the K-butylAMA phase diagram become immediately evident. Firstly, replacing the $\mathrm{Na}^{+}$ion with a $\mathrm{K}^{+}$ion results in the lamellar phase once again being stabilised at low surfactant concentrations, and so the room temperature phase behaviour of K-butyIAMA does not resemble that of its sodium analogue, Na-butylAMA, but rather it is more similar to that of the structurally different Na-AOT. However, it is also clear that the liquid crystalline phases of the K-butylAMA system are not as stable at high 


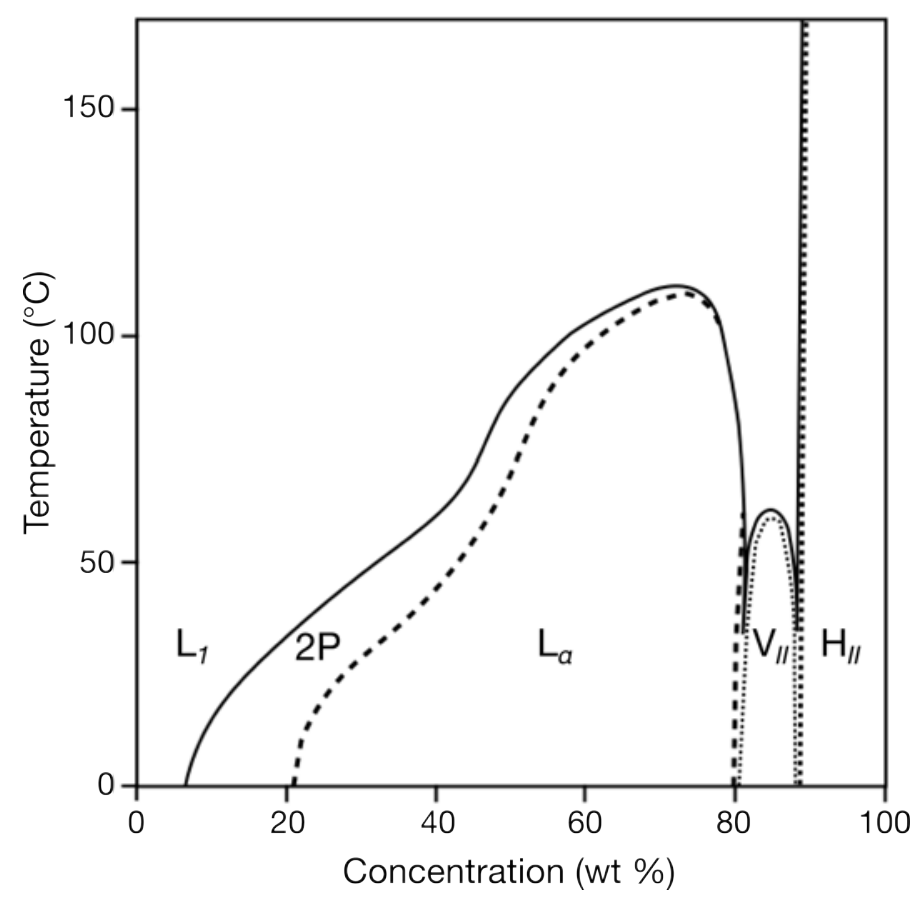

Figure 4.16: Phase diagram of K-butylAMA, showing a two-phase region (2P) and lamellar $\left(\mathrm{L}_{\alpha}\right)$, inverse bicontinuous cubic $\left(\mathrm{V}_{\| l}\right)$ and inverse hexagonal $\left(\mathrm{H}_{\| l}\right)$ phases.

temperatures as those of either of the previously described systems (with the exception of the $H_{/ /}$phase). The melting temperature of the $L_{\alpha}$ phase is significantly reduced in comparison to that of Na-AOT, particularly evident at low surfactant concentrations. The reason for this cannot be stated with confidence, but may be a result of $\mathrm{K}^{+}$having less favourable interactions with water than the $\mathrm{Na}^{+}$ion, or differences in the bilayer elasticity resulting in topological changes that facilitate the phase transition.

When viewed through crossed polarisers, bulk samples of K-butylAMA are similar in appearance to those of Na-AOT, with samples at low concentrations highly iridescent and samples at high concentrations appearing opaque. Notably, at room temperature and under standard lighting conditions, K-butyIAMA samples between $\sim 42$ and 43 wt \% surfactant appear blue. This colour could be induced to appear or disappear in samples with surfactant compositions within a few weight percent by gentle heating or cooling. From the work of Nallet et al., we can infer that the blue colour results from a change in the optic sign of birefringence upon dilution. ${ }^{111}$ It follows that the K-butylAMA system likely forms a swollen lamellar phase at low concentrations, similar to that of Na-AOT, with thermal fluctuations providing both disorder and a negative contribution to the birefringence. The change in birefringence was confirmed using POM.

A feature not shown in the phase diagram is the higher Krafft temperature of K-butylAMA when compared with that of Na-butyIAMA, evidenced by the slow formation of crystals in the mesophases of some K-butylAMA samples over time. The point of crystallisation was unable to be recorded accurately due to the formation/melting of the crystalline material being slower than the heating/cooling rate employed when investigating the phase behaviour. When stored at $25^{\circ} \mathrm{C}$, partial crystallisation was only observed in samples composed of over 
65 wt \% surfactant, although this was not apparent in all samples prepared within this range. Unfortunately, the existence of a small amount of crystallised surfactant within some samples means that the exact composition of the mesophasic portion of these samples is affected to a small, but unknown, extent. These crystals were observed directly in the POM images for this system and have an effect on the SAXS results, both of which are discussed below.

As the Krafft line indicating the temperature dependence of crystallisation is not shown, but is clearly higher than $0{ }^{\circ} \mathrm{C}$ for some surfactant concentrations, the phase diagram should be considered as preliminary. A fully elucidated phase diagram would indicate low temperature regions where, for some compositions, the $\mathrm{L}_{\alpha}, \mathrm{V}_{\text {/l }}$ and $\mathrm{H}_{/ /}$phases coexist with crystals. Of these three phases, crystallisation was most frequently observed in samples of the $V_{/ /}$phase. The synthesised pure surfactant was obtained as a waxy solid rather than a pure crystalline material, and after melting the surfactant to an isotropic fluid followed by cooling to $25^{\circ} \mathrm{C}$, a $\mathrm{H}_{/ I}$ phase was formed in which no crystals were observed. Although, as implied above, the lack of crystallisation could be due the rate of cooling rather than the true equilibration state.

\section{Potassium butyIAMA SAXS}

The SAXS behaviour of the K-butyIAMA system is presented in Figure 4.17, and it is clear from the spectra that the stabilisation of the lamellar phase at low concentrations results in non-classical lamellar behaviour similar to that observed for the Na-AOT system. Due to instrumentation problems, the SAXS data presented in Figure 4.17 were captured using a point collimated system and phosphor imaging plates rather than a line collimated system and CCD camera. These data were converted to $1 \mathrm{D}$ spectral representations by Azimuthal integration of the scattering intensity of the $2 \mathrm{D}$ images produced, and hence the resolution is lower than in the Na-AOT and Na-butylAMA spectra discussed previously. The spectra are also further complicated by crystallisation of the surfactant within samples at some surfactant concentrations, and by the presence of peaks due to long-lived shear-induced structures, which form upon loading of the extremely viscous $\mathrm{V}_{/ /}$and $\mathrm{H}_{/ /}$phases into the paste cell used in the analysis. The presence of a small amount of crystalline material coexisting within samples with the mesophases means that the exact surfactant composition of each component cannot be determined with certainty. The presence of crystals also results in several sharp peaks in the wide-angle scattering region above $8 \mathrm{~nm}^{-1}$ (not shown). The peaks due to shearinduced structures occur at q spacings below that of the main Bragg peak, and are evident at concentrations above $80 \mathrm{wt} \%$. Although these peaks could be reasoned to be due the presence of crystals within the samples, it is not likely as they were not observed to appear at the same q spacings when samples from the same batch were analysed. Similar peaks were also observed when the paste cell was used to examine similarly viscous materials produced in other surfactant systems (where no crystals were present).

Despite the issues highlighted above, the spectra are sufficient to characterise the phase behaviour of the system, and to draw several conclusions regarding the structure of the K-butyIAMA lamellar phase. Bulk observations of K-butylAMA samples between crossed 

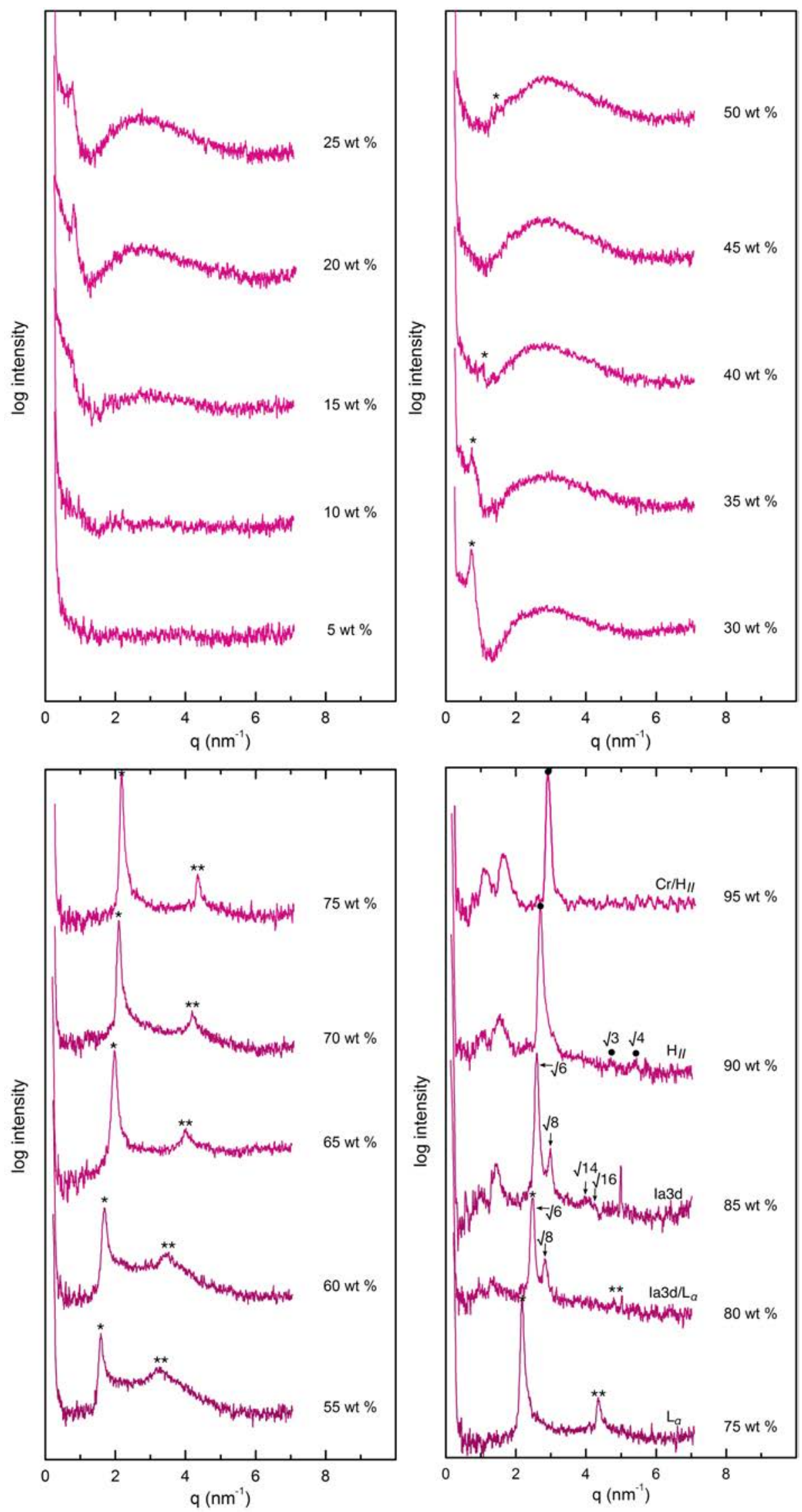

Figure 4.17: SAXS profiles of the K-butyIAMA system with increasing surfactant concentration. Bragg peaks due to the $\mathrm{L}_{\alpha}$ phase are evident up to $75 \mathrm{wt} \% \mathrm{~K}$-butylAMA (denoted by asterisks). A two-phase region is observed at $80 \mathrm{wt} \%$, and $\mathrm{V}_{/ /}$and $\mathrm{H}_{/ /}$phases are evident at 85 and $90 \mathrm{wt} \%$ (denoted by arrows and dots, respectively). 
polarisers at $25{ }^{\circ} \mathrm{C}$ showed clear phase separation of samples between $\sim 15$ and $30 \mathrm{wt} \%$ surfactant and an isotropic phase at lower concentrations. The profiles in Figure 4.17 are consistent with these observations, showing very little scattering at 5 and 10 wt $\%$ K-butylAMA, and only a small amount of diffuse scattering and a broad shoulder extending from the peak of the primary beam at $15 \mathrm{wt} \%$. More intense broad and diffuse scattering occurs between 1.5 and $5 \mathrm{~nm}^{-1}$ for concentrations of 20 and $25 \mathrm{wt} \% \mathrm{~K}$-butyIAMA, with a sharp peak also evident at $\sim 0.7 \mathrm{~nm}^{-1}$, which arises due to the stacked bilayers of lamellar structures within the two-phase region (confirmed by POM to be comprised of large MLVs).

Between 30 and $75 \mathrm{wt} \% \mathrm{~K}$-butylAMA, the system forms a pure lamellar phase; however, scattering that is characteristic of this phase (with Bragg peaks in the ratio of 1:2) is only observed at concentrations above $55 \mathrm{wt} \%$ surfactant. Between 30 and $50 \mathrm{wt} \% \mathrm{~K}$-butylAMA, diffuse scattering dominates the spectra, with a decrease in intensity (and the eventual disappearance) of the first-order Bragg peak occurring at slightly higher concentrations than is observed in the Na-AOT system. The diffuse scattering and loss of intensity of the Bragg peak imply that Helfrich forces have a role in stabilising the system, and thus the bilayers of the structure must undulate as they are highly elastic and have a low value of $\kappa\left(\kappa \approx k_{\mathrm{B}} T\right)$. At 55 and 60 wt \% K-butylAMA, diffuse scattering persists and is clearly still a significant feature of the spectra, despite the appearance of two Bragg peaks. This implies that the phase retains a significant amount of disorder at these concentrations, an observation that is supported by the fact that the second-order Bragg peak is both broad and low in intensity, indicating weak long-range ordering between bilayers.

At 80 wt $\%$ and 85 wt $\% \mathrm{~K}$-butylAMA, a $\mathrm{L}_{\alpha} / \mathrm{V}_{/ /}$mixture and then pure $\mathrm{V}_{/ /}$phase are observed. The noisy baseline and weak scattering mean a limited number of reflections can be assigned; however, those assigned are consistent with an la3d space group, also observed the Na-AOT and Na-butyIAMA systems (see Figure 4.18). At 90 wt \% $\mathrm{K}$-butylAMA, a $\mathrm{H}_{/ /}$phase is observed, although the shear-induced changes and crystallisation within the sample result in weak Bragg peaks and the assignment of the $\mathrm{V}_{/ /}$and $\mathrm{H}_{/ /}$phases is primarily based on POM observations.

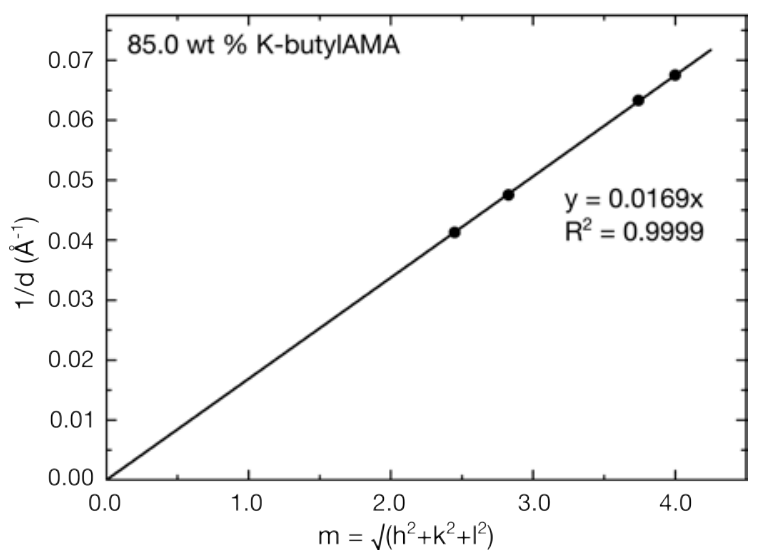

Figure 4.18: Plot showing the reciprocal d spacings vs. $\sqrt{ }\left(h^{2}+k^{2}+l^{2}\right)$ for the cubic phase of the K-butylAMA system, where hkl are the assigned Miller indices. Correct assignment of the lattice type and space group is confirmed by the linearity of the plot and the intercept of the fitted line at the origin. The lattice parameter is calculated as the reciprocal of the gradient of the fitted line and is $59.2 \AA$ for the sample concentration shown. 
Clearly the important feature of the spectra in Figure 4.17 is the obvious diffuse scattering that dominates the K-butylAMA lamellar phase and implies that the system is stabilised by Helfrich forces over a large concentration region. This indicates that the system forms highly defective and disordered lamellar structures based on undulating bilayers. The lack of longrange ordering in the system and weak scattering of the phase in comparison with equivalent spectra for Na-AOT or Na-butyIAMA (despite the $\mathrm{K}^{+}$ion being a larger and stronger scattering ion than $\mathrm{Na}^{+}$) imply that the degree of order in the K-butylAMA system is lower than in the previous systems. Increased disorder may result from differences in elasticity or spontaneous curvature that arise directly from the alterations in the molecular structure, although the chaotropic nature of the $\mathrm{K}^{+}$ion, which will destabilise the surrounding water structure, may also play a role by softening the bilayer at the water interface, resulting in increased undulation and increased entropy.

\section{Potassium butyIAMA Optical Microscopy}

Polarising optical microscopy images of K-butylAMA samples are shown in Figure 4.19 and illustrate a similar trend to those reported in Chapter 3 for the Na-AOT system. At 20 wt \% surfactant, samples of K-butylAMA fall within the two-phase region of the system, and bulk samples were observed to phase separate. The POM image of a sample of $20 \mathrm{wt} \% \mathrm{~K}$-butylAMA also shows the presence of two phases, with Maltese cross patterns on an isotropic background indicating the presence of many MLV structures within an isotropic fluid. This image is distinguishable from textures of a pure lamellar phase containing the same defect structures due to the flocculation of the MLV structures, the narrow distribution in their size, and the lack of any birefringence in the background due to smaller defects or domains of different ordering. In contrast, the image at $35 \mathrm{wt} \% \mathrm{~K}$-butylAMA shows the pure lamellar phase of the system near its lower concentration limit, and is dominated by FCD-II defects of varying size and degrees of distortion.

At 40 wt \% K-butylAMA, the POM texture appears dark due to the change from negative to positive birefringence. This is a result of the form and intrinsic birefringence components being of equal and opposite values, due to fluctuating bilayers stabilised by Helfrich interactions. At $45 \mathrm{wt} \% \mathrm{~K}$-butylAMA, within the positive birefringence region of the lamellar phase, many distorted FCD-II defects are retained. Upon rotation of the sample stage, some FCD-I defects were also identified, meaning the two defects types coexist at this concentration. These defects also coexist in Na-AOT samples of the equivalent concentration; however, the number of defects is considerably less in the Na-AOT system, the FCD-II defects are smaller, and the FCD-I defects form obvious oily streaks. At higher surfactant concentrations, the K-butylAMA textures are very similar to those observed in samples of both Na-AOT and Na-butylAMA. The image at 60 wt \% K-butylAMA shows short oily streaks, several individually discernible FCD-I defect points, and the beginning of the close packing of defects. At $75 \mathrm{wt} \%$ surfactant, the close packing of defects and highly curved domains results in a mosaic-like pattern. 

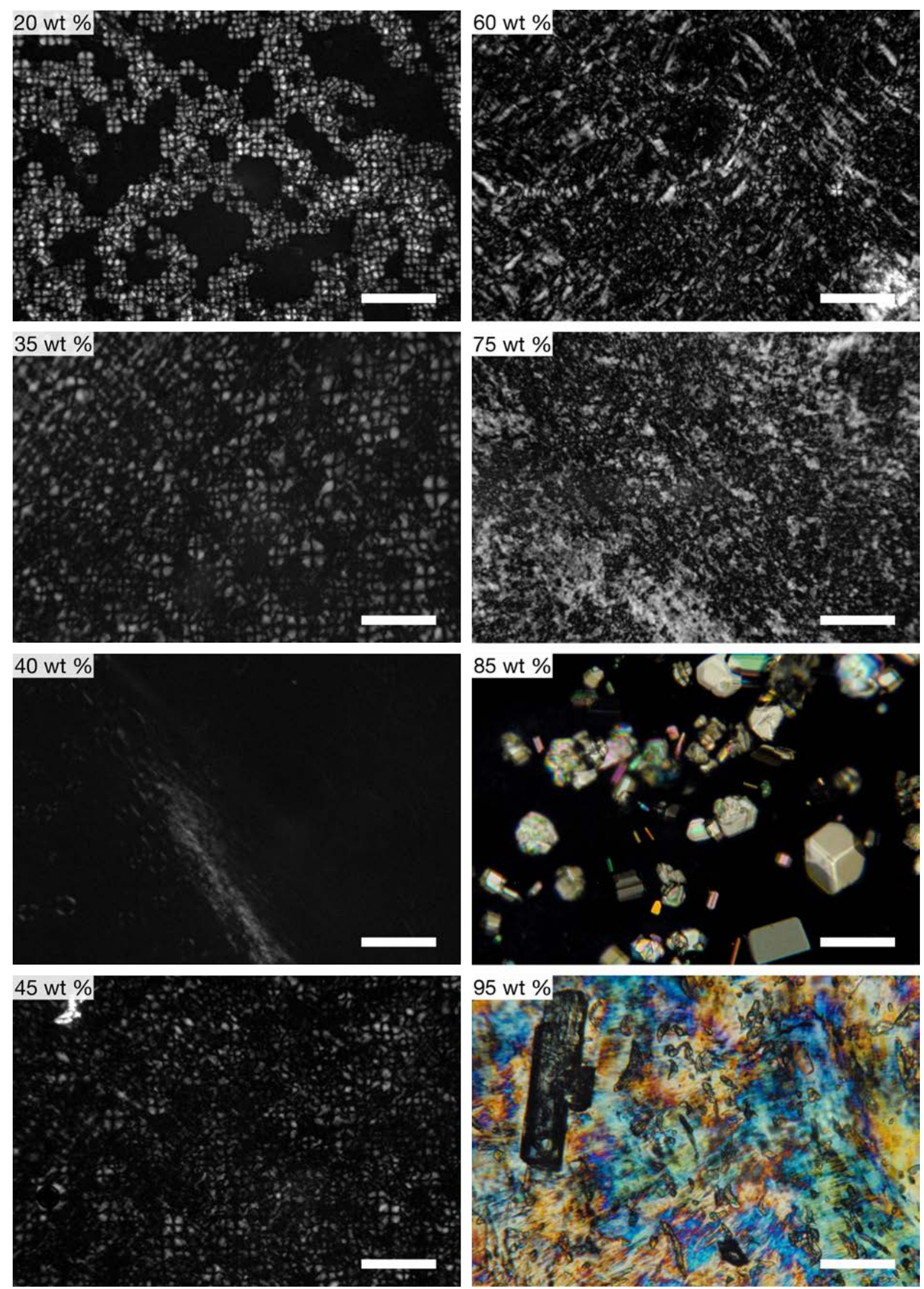

Figure 4.19: $\mathrm{POM}$ images of $\mathrm{K}$-butylAMA with increasing concentration at $\sim 25{ }^{\circ} \mathrm{C}$. A twophase region of flocculating MLVs gives way to a lamellar phase that undergoes a change from negative to positive birefringence, and a gradual change in defect behaviour from predominantly FCD-II to predominantly FCD-I defects, with increasing concentration. Crystalline material is observed to coexist with the mesophases within the isotropic and fan-like textures of the $V_{\text {II }}$ and $\mathrm{H}_{/ /}$samples. Scale bars are $100 \mu \mathrm{m}$. Additional images are available in Appendix $\mathrm{C}$. 
The POM textures at 85 and 95 wt \% K-butylAMA show isotropic and fan-like patterns characteristic of the $\mathrm{V}_{\| /}$and $\mathrm{H}_{\| /}$phases the system forms, but both textures also contain a significant amount of crystalline material. The images shown are representative of the samples viewed at those concentrations, but the surfactant and water compositions of the mesophases will be slightly different from the stated concentrations due to the crystallisation of some material. Once formed, the crystalline material was observed to be very stable, and was retained upon sample heating to temperatures in excess of those at which the mesophases were observed to melt. This non-equilibrium behaviour indicates an increase in the Krafft point relative to the $\mathrm{Na}$-AOT and $\mathrm{Na}$-butyIAMA systems. This can be rationalised by the comparatively shorter tails and/or smaller effective headgroup size (resulting from the tightly bound and poorly hydrated potassium ion).

The images shown in Figure 4.19 illustrate that the K-butylAMA system presents the same general trends as the Na-AOT system. The lamellar phase is highly defective and shows a gradual change towards negative Gaussian curvature, with FCD-II defects formed at low concentrations replaced by FCD-I defects in the form of oily streaks and mosaic patterns at high concentrations, where more classical lamellar behaviour is observed. However, while the images at high concentrations are very similar to those presented previously for the Na-AOT and Na-butylAMA systems, the images at lower concentrations show that the defects in the K-butylAMA system are generally larger than those observed in Na-AOT samples.

The larger defect structures and formation of a two-phase region, consisting of many stable MLV structures, at higher surfactant concentrations in the K-butylAMA system is an indication that the van der Waals contribution is dominant over the electrostatic and Helfrich forces for a larger concentration range than in the Na-AOT system. This suggests that while comparable, the elasticity of the K-butyIAMA system is not equivalent to that observed in the Na-AOT system. Both systems have a value of $\kappa$ in the approximate range of $k_{\mathrm{B}} T$ at some concentrations, but the monolayers composing the K-butylAMA bilayer likely have increased spontaneous curvature in comparison to those of $\mathrm{Na}$-AOT. This conclusion is supported by the reduced transition temperature upon heating in the low concentration lamellar phase region of the K-butylAMA system compared to that of Na-AOT. The bilayer structures of K-butylAMA are not as stable as those in the Na-AOT system. The K-butylAMA monolayers have a greater energy cost associated with retaining lamellar bilayers in the dilute region and a reduced capability to dissipate this cost, due to the decreased tail branching in proximity to the headgroup. As such, the $L_{\alpha}$ phase is energetically favoured over smaller temperature and concentration ranges than in the Na-AOT system. Replacing the $\mathrm{Na}^{+}$ion with a $\mathrm{K}^{+}$ion as the counterion for butyIAMA has effectively reproduced much of the low concentration Na-AOT phase behaviour; however, the differences in molecular structure mean that the behaviour of the K-butylAMA system is between that of the previous two systems. 


\section{Potassium butyIAMA Cryo-SEM}

Given the similarities between the K-butyIAMA and Na-AOT systems highlighted in the phase behaviour, SAXS and POM discussions above, it is unsurprising that the cryo-SEM images of the K-butylAMA lamellar phase are also comparable to those of the Na-AOT system. There are, however, also several differences between the microstructures of the two systems, which arise from the bilayers of each system having slightly different elasticities, and may also be a result of the chaotropic and kosmotropic counterions affecting the water structure of the system differently.

Cryo-SEM images obtained for samples of $30 \mathrm{wt} \% \mathrm{~K}$-butylAMA are presented in Figure 4.20, showing that the lamellar phase formed at this concentration is highly disordered and contains a large number of defects. Consistent with low concentration samples of the Na-AOT system, the K-butylAMA system appears to deviate from classical lamellar behaviour to varying degrees at different lengthscales, and at different magnifications the system can appear either more or less ordered. A sample concentration of $30 \mathrm{wt} \%$ surfactant was selected for the K-butylAMA system rather than a sample of 25 wt \% (which would have allowed for a direct comparison
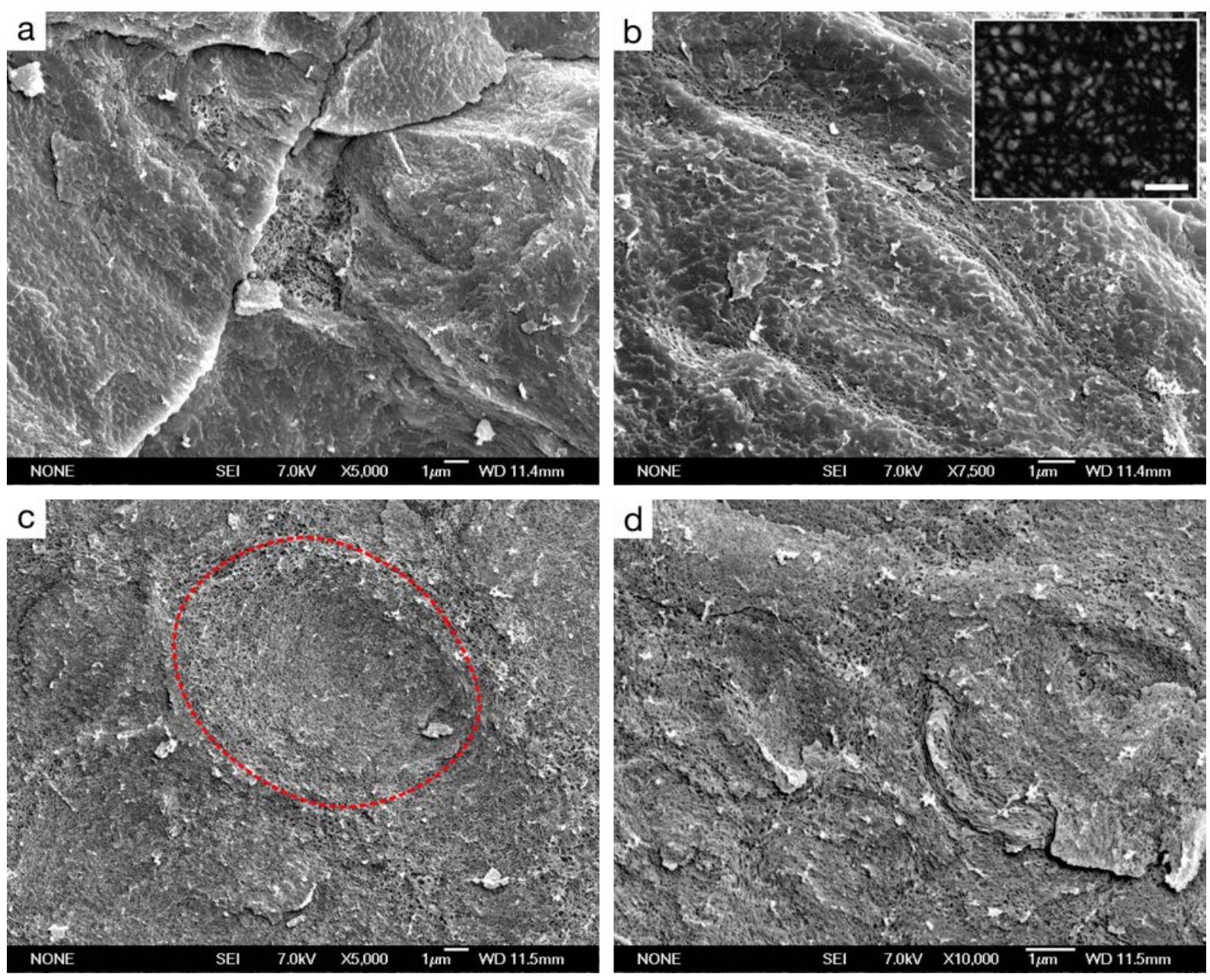

Figure 4.20: Cryo-SEM images of a sample of $30 \mathrm{wt} \% \mathrm{~K}$-butylAMA. Red dashed line indicates the approximate boundary of a disordered FCD-II defect. The inset shows the corresponding POM image, with a scale bar of $50 \mu \mathrm{m}$. 
with images of the Na-AOT system shown previously) as the K-butylAMA system produces a biphasic mixture at $25 \mathrm{wt} \%$ and our intent was to investigate the extent of connectivity within the pure $L_{\alpha}$ phase. However, it should be noted that at $25^{\circ} \mathrm{C}$, the $30 \mathrm{wt} \% \mathrm{~K}$-butylAMA sample is in close proximity to phase boundaries with respect to both reduced concentration and increased temperature.

Given that the K-butylAMA lamellar phase has reduced thermal stability in comparison with the Na-AOT system, likely due to the bilayer having more spontaneous curvature, it is not surprising that the lamellar phase is highly disordered. Given the close proximity of the sample to the $2 \mathrm{P}-\mathrm{L}_{\alpha}$ phase boundary it is also not surprising that different regions of the sample appear to show different amounts of order. All the images show the high degree of connectivity within the sample, within both the surfactant and water domains. The defects observed by cryo-SEM are consistent with those observed via POM, with highly distorted FCD-II defects evident. As is the case for the Na-AOT images at low surfactant compositions, obtaining an approximate value for the average domain size in the $30 \mathrm{wt} \% \mathrm{~K}$-butylAMA sample is difficult based on the cryo-SEM images. This is because the directional ordering of the lamellar phase is poorly defined, as it changes continuously without distinct walls or lines of concentrated defects separating regions of different order. Instead, Figure 4.20 shows that regions with different on average directions of order are separated from other regions by an area of greater disorder. This means that the separation of domains is gradual and pinpointing the phase boundary in such an area is somewhat arbitrary.

The images presented in Figure 4.21 for 50 wt \% K-butylAMA show a variety of different defect behaviours, and surfaces of both positive and negative Gaussian curvature are evident. This is consistent with the POM image (inset) obtained at the same surfactant concentration, which shows the phase contains a mixture of FCD-II and FCD-I defects. The low magnification image shown in Figure 4.21 (a) reveals different regions in which the system exhibits a range of behaviours. Areas with directional order averaged along a single director axis can be seen in the upper left of the image. Domains of $\sim 10-20 \mu \mathrm{m}$ in size that appear to have grown outwards from several defect singularities are observed in the centre of the image, and in addition, several large FCD-II defects can be observed throughout the image. These are also up to $20 \mu \mathrm{m}$ in size and would possibly resemble the domains in the centre of the image if fractured through the middle. In the lower right of the image streaky FCD-I defects are present. Figure 4.21 (b) is dominated by a partially visible FCD-II domain with obvious positive Gaussian curvature, and also clearly shows sharp boundaries separating different domains and possible screw defects. Figure 4.21 (c) and (d) are images captured at slightly higher magnifications - (d) is an enlargement of the centre of (c) - and are excellent indicators of the disorder within the system, also providing an excellent illustration of how some defects gradually merge with the bulk phase, while others form sharp boundaries. This region contains a high concentration of defects, which range in size from $\sim 0.3$ to $7 \mu \mathrm{m}$ and are obviously distorted and subjected to harsh curvature environments. The bilayers are clearly still highly connected and show clear evidence of undulations, consistent with the SAXS experiments carried out at this K-butylAMA concentration, which showed that diffuse scattering (arising from thermal fluctuations) dominates the spectra. 

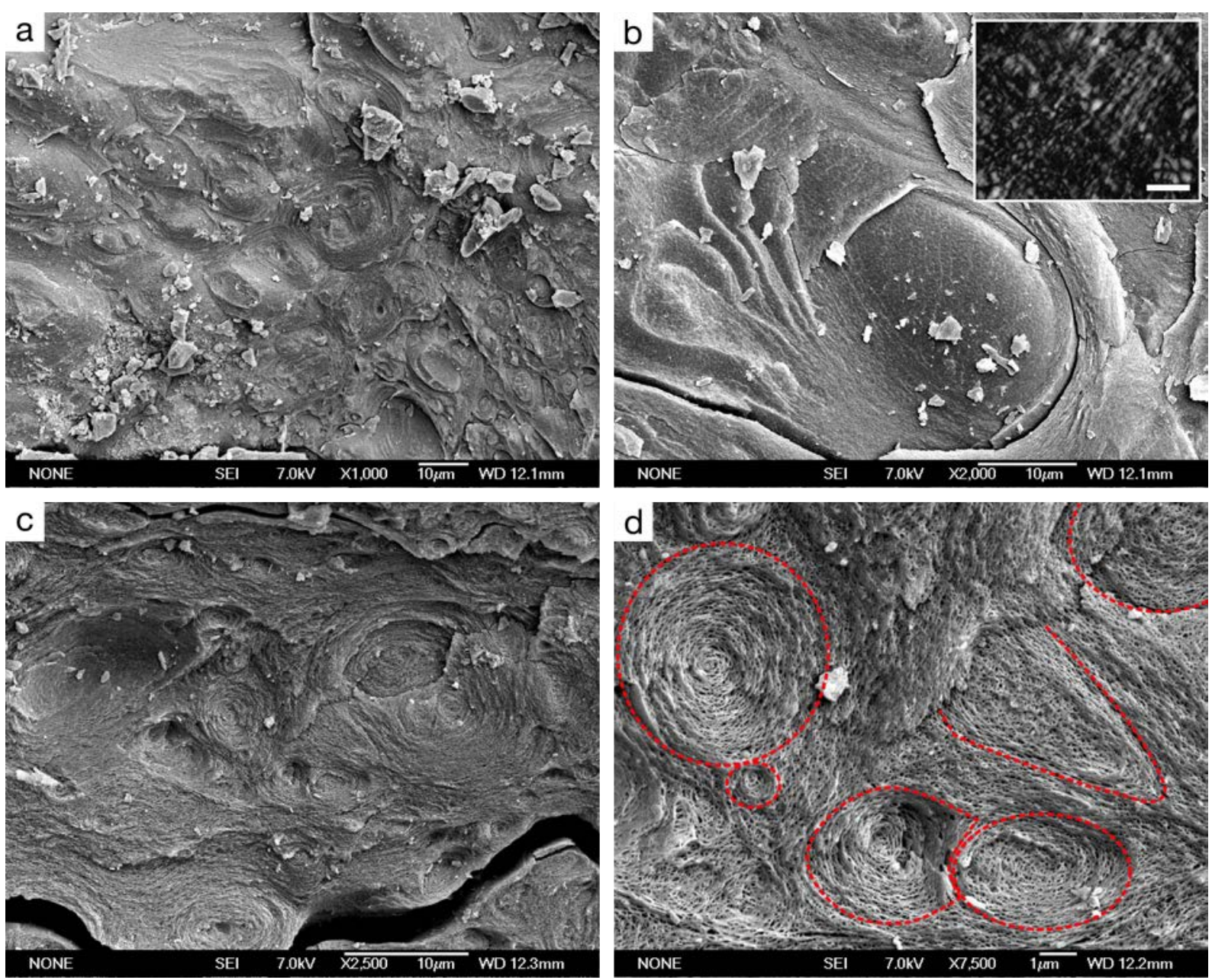

Figure 4.21: Cryo-SEM images of a sample of $50 \mathrm{wt} \% \mathrm{~K}$-butylAMA. Red dashed lines highlight examples of FCD defects of various size and distortion. The inset shows the corresponding POM image, with a scale bar of $50 \mu \mathrm{m}$.

At $70 \mathrm{wt} \% \mathrm{~K}$-butylAMA, there is a clear decrease in the local scale disorder of the bilayers (see Figure 4.22). However, when considering the images presented previously for the Na-AOT and Na-butylAMA systems, and the fact that at $70 \mathrm{wt} \% \mathrm{~K}$-butylAMA the phase is expected to be governed by strong steric hydration repulsion forces, the degree of connectivity in the phase is perhaps surprisingly still significant. Given that the Na-butylAMA system showed considerably less connectivity at an equivalent concentration, the disorder must result from the $\mathrm{K}^{+}$counterion. Given that the $\mathrm{K}^{+}$ion is both chaotropic and different in size to the $\mathrm{Na}^{+}$ion, it is logical that the $\mathrm{K}^{+}$ion will alter both the steric and hydration components of the short-range forces, and thus will display different degrees of bilayer elasticity. Apart from this local scale elasticity, the $70 \mathrm{wt} \% \mathrm{~K}$-butylAMA sample does not show any behaviour that is not expected from the POM investigation. The POM image of $70 \mathrm{wt} \% \mathrm{~K}$-butylAMA shows a mixture of features, with mosaic-like patterns and oily streaks both present, and the cryo-SEM images are consistent with this. Clear domain boundaries separating regions of different orientation are observed in all the images shown in the Figure 4.22. Some of these domains are relatively ordered and contain (or are separated by) oily streaks, while others are present as arrays of highly curved defects that are beginning to form the structures that give rise to the mosaic-like patterns observed by POM. 

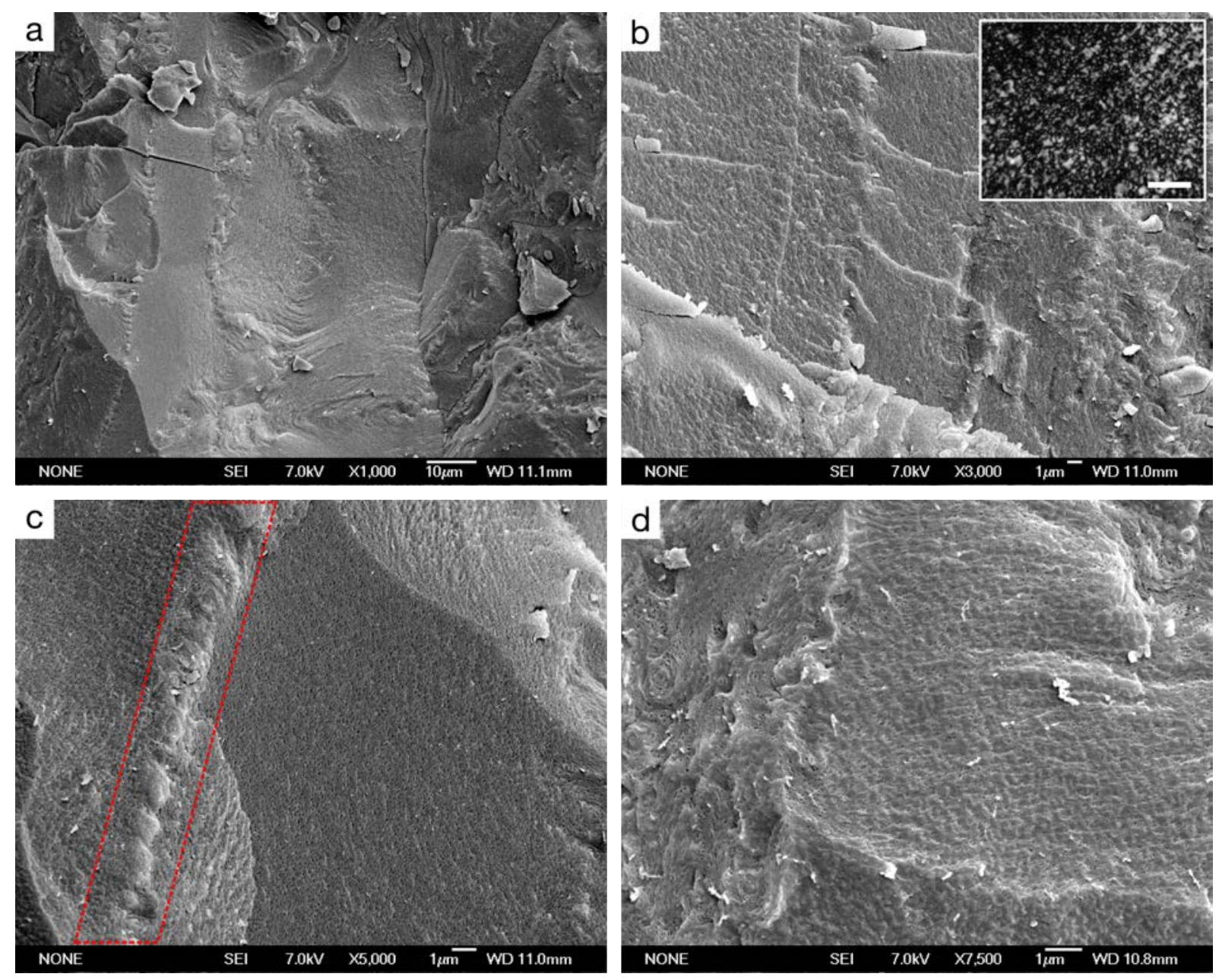

Figure 4.22: Cryo-SEM images of a sample of 70 wt \% K-butylAMA. Red box highlights an oily streak formed by many FCD-I defects. The inset shows the corresponding POM image, with a scale bar of $50 \mu \mathrm{m}$.

Compared with the images of the Na-AOT and Na-butylAMA systems shown previously, the images of the K-butylAMA system are clearly more similar to those of the Na-AOT system, following the same general trends at low and high surfactant concentrations (for an example see Figure 4.23). However, the behaviours observed in the Na-AOT system are shifted to slightly higher concentrations in the K-butylAMA system, presumably due to the apparent increase in disorder in the microstructure. A comparison of the phase diagrams of the three systems clearly indicates that the phase behaviour of the K-butylAMA system is more similar to the Na-AOT system than the Na-butylAMA system is. However, it is noticeable that while the cryo-SEM images show similar trends for all three systems, at high concentrations the K-butylAMA images show more disorder than the other systems, implying that the K-butylAMA bilayer retains a degree of flexibility upon packing that the Na-AOT and Na-butylAMA systems do not. This suggests that while the differences in the tail regions determine the relative microstructures of these systems at low concentrations, at higher surfactant concentrations the differences are minimised by the efficient packing of the tails, and differences in the headgroups (i.e. the role of the counterion) becomes the more important feature. As such, the Na-AOT system shows a greater resemblance to the Na-butyIAMA system at high concentrations than the K-butylAMA system. This is because the bound $\mathrm{K}^{+}$ion provides less steric bulk to the headgroup, resulting 
in weaker electrostatic repulsive forces between the headgroups and also increasing entropy through the disruption of the water structure.
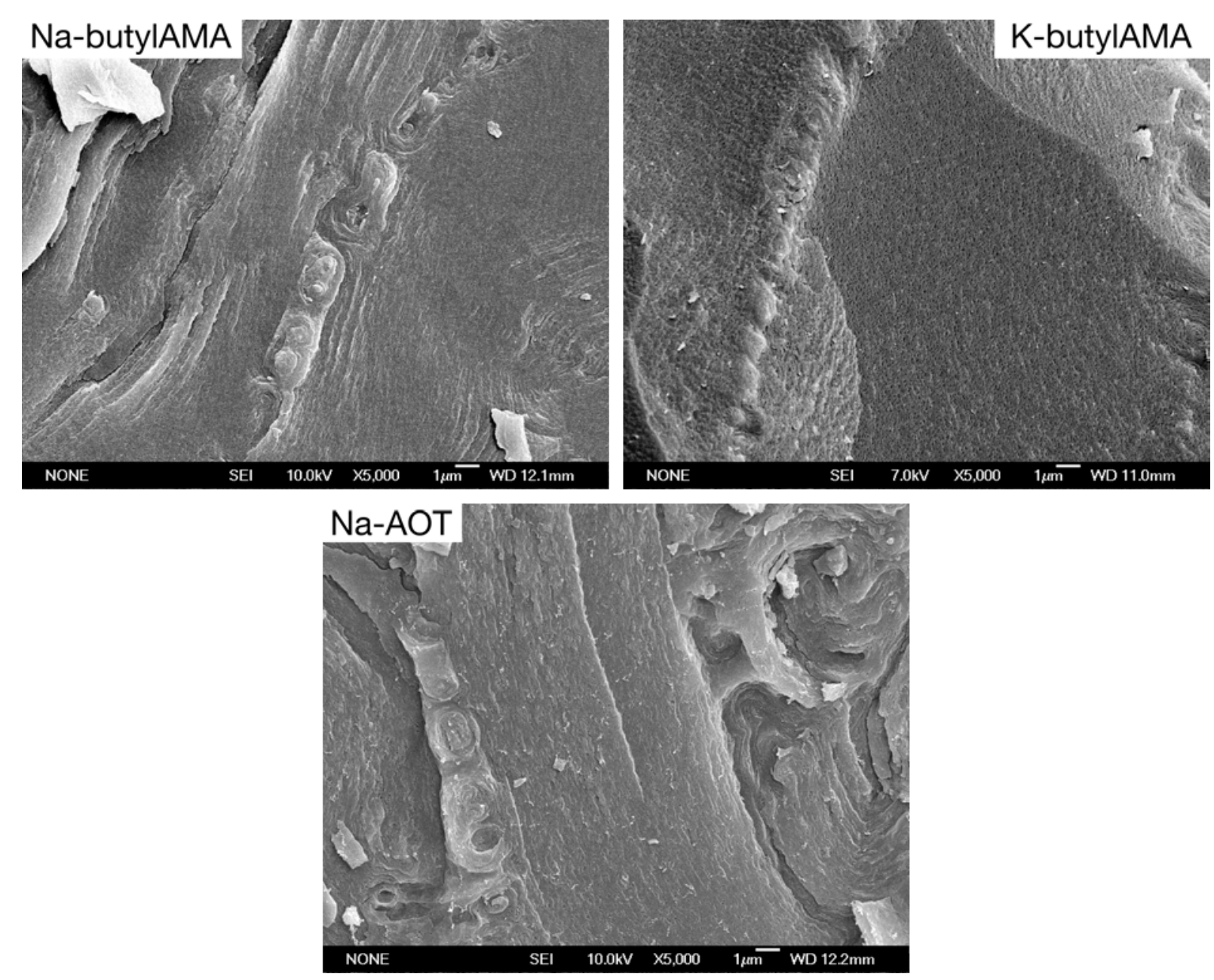

Figure 4.23: Comparative cryo-SEM images of $~ 70$ wt $\%$ surfactant samples of Na-butylAMA, K-butylAMA and Na-AOT at $\times 5000$ magnification.

\section{Potassium butyIAMA: Discussion}

In the comparison of Na-AOT and Na-butyIAMA presented previously in this chapter, it was concluded that the superior branching of Na-AOT in close proximity to the hydrophobic/hydrophilic interface is responsible for the different behaviour of the systems at low surfactant concentrations. The tail branching introduces flexibility and additional bulk at this interface, and the reduction of these properties in the Na-butylAMA system removes the ability of the molecule of stabilise a swollen and disordered lamellar phase. However, through the synthesis of K-butylAMA, we have reintroduced this flexibility by decreasing the effective steric bulk of the headgroup without changing the molecular composition of the butyIAMA anion itself.

The role of the potassium ion in producing a disordered lamellar phase in the K-butylAMA system is not limited to a reduced contribution of steric bulk at the headgroup. The $\mathrm{K}^{+}$ion 
also provides flexibility and disorder to the system in several other ways, as is evident in both the weak small-angle scattering of the system and the degree of connectivity in the structure in cryo-SEM images. The $\mathrm{K}^{+}$ion is chaotropic meaning that it generally exists in a partially dehydrated state, disturbs the surrounding water structure, and forms a closer ion pair with the sulfonate headgroup of the butyIAMA anion than the $\mathrm{Na}^{+}$ion does. The K-butylAMA lamellar bilayer is stabilised through a combination of factors that result from the chaotropic nature of the counterion, which each aid in introducing disorder to the micellar phase (and thus promoting the formation of the disordered lamellar structure), but also contribute to the decreased thermal stability of the phase and additional disorder at high concentrations.

The reduced branching in the butyIAMA anion compared to that of AOT (and thus the reduced tail contribution to the flexibility at the interface) is partially compensated for in K-butylAMA by the small and tightly bound $\mathrm{K}^{+}$ion, which reduces the contribution of the headgroup (and thus effectively increases the relative contribution of the tail region). However, the closer counterion binding of the $\mathrm{K}^{+}$ion also has the effect of increasing the shielding of the electrostatic repulsion forces in this system, which will increase the value of $\bar{\kappa}$ and promote topological variations in the bilayer. The disrupted water structure and reduced dehydration of the cation will likely result in an overall decrease in the hydration of the K-butylAMA headgroup, which has been shown previously to introduce additional conformational disorder into the tail region of similar systems. ${ }^{102}$ As well as dehydration, the tight counterion binding will also result in a modification of the tail region, which may affect the extent of interdigitating in the bilayer and allow for a change in bilayer thickness, thus altering the value of $\kappa$.

The Na-AOT lamellar phase is clearly more stable than that of the K-butylAMA system, with broader concentration and temperature ranges present. However, the $\mathrm{K}^{+}$ion provides enough disorder to the bilayer of K-butylAMA to allow the instabilities that promote the disordered lamellar phase to form over a concentration range of $\sim 30-50 \mathrm{wt} \%$ (i.e. the bilayer undulations and localised topological point defects). This work demonstrates the interplay between the degree of tail branching and steric contribution of the headgroup, and also highlights the important role of the counterion in the self-assembly of surfactant systems. A change in counterion in this system alters the hydrocarbon chain environment, the steric bulk of the headgroup, the hydration of the molecule, the magnitude of the electrostatic forces, and the entropy of the system through unfavourable interactions with water. Like the effect of branching in the hydrocarbon tail region, the role of counterions in surfactant self-assembly is often neglected. This role is further investigated in the following chapters, with the experimental results of replacing the $\mathrm{Na}^{+}$ion in $\mathrm{Na}-\mathrm{AOT}$ with other alkali metal and ammonium counterions discussed. 


\section{Chapter 5}

\section{Potassium and Lithium Aerosol OT}

\section{Potassium and Lithium AOT: Introduction}

The characterisation of the Na-AOT system revealed that molecular flexibility of the surfactant is a key factor in rationalising the phase and defect behaviours of the system. It also showed that the elasticity of the Na-AOT bilayer is susceptible to small changes in both entropic or electrostatic forces, due to the magnitudes of these forces being comparable at some surfactant concentrations. The relative contributions of these forces and the effect of their competition on the bilayer flexibility was further highlighted by the study of the Na-butyIAMA and K-butyIAMA systems. The different phase behaviours exhibited by the two butylAMA systems demonstrates the important role that the counterion plays in determining the thermodynamically preferred phase progression. The effect of the counterion is now discussed further with regards to its role in AOT systems. In this chapter the phase behaviours of the K-AOT and Li-AOT systems are presented and are shown to be significantly different to that of the Na-AOT system. This behaviour is then rationalised in terms of both the steric and electronic effects that the different cations have on the system, and also specific ion effects related to the different hydrations and water affinities, which cause differing degrees of counterion binding and freedom.

The effect on the phase and defect behaviours that will result from changing the counterion of a surfactant system are not easily predicted. This is because, although its role is often neglected, the counterion affects the other components of the system in a number of different ways, and how it does so changes with both concentration and temperature. Changes in the counterion can result in dramatic changes in the phase progression of surfactant systems, with one significant example being the difference in the phase progression of the chloride and bromide didodecyldimethylammonium systems. ${ }^{45,46}$ The first of these systems presents a lamellar phase that undergoes classical 1D swelling, while the second forms two different lamellar phases, that have a large region of coexistence. Such effects are difficult to explain, and are at present almost impossible to predict. However, we can state that the $\mathrm{K}^{+}$and $\mathrm{Li}^{+}$ ions used as counterions to AOT in the experimental work of this chapter have very different 
preferred hydration states. As discussed in the previous chapter, the chaotropic $\mathrm{K}^{+}$ion has a higher affinity for the sulfonate moiety of the AOT anion than the kosmotropic $\mathrm{Na}^{+}$ion, which can be explained in terms of reduced hydration compared with the $\mathrm{Na}^{+}$(the hydrated radii of $\mathrm{Na}^{+}$and $\mathrm{K}^{+}$are reported as 3.58 and $3.51 \AA$, respectively $\left.{ }^{180}\right)$. The hydrated ionic radius of the $\mathrm{Li}^{+}$ion is larger than that of either $\mathrm{Na}^{+}$or $\mathrm{K}^{+}$(at $3.82 \AA^{180}$ ) and so the ion will have favourable interactions with water, but a mismatched hydration shell to that of the sulfonate headgroup. Hence, the $\mathrm{Li}^{+}$ion will not form a strong ion pair, and thus will be the weakest bound of the three ions. The differences in hydration, binding and effective size will to varying degrees alter the tail packing, the steric bulk of the headgroup and all the intermolecular forces that govern the system.

Both K-AOT and Li-AOT have been produced previously; however, a literature search reveals only six articles that refer to Li-AOT and 23 that refer to K-AOT (by comparison the Na-AOT system has over 14,500 citations). ${ }^{\ddagger}$ It is worth noting that K-AOT does have some practical uses and is also cited in over 50 patents. These patents are primarily in the fields of pharmaceuticals, drug delivery and polymer formulations, although a number also report on the use of K-AOT in more traditional surfactant roles within industrial applications, such as the cosmetic, oil and ink industries. None of the literature articles or patents referring to either Li-AOT or K-AOT refer to any liquid crystalline behaviour, and so to our knowledge the experimental work reported within this thesis is the first example of this behaviour being studied for either surfactant. Of the literature articles that do reference the two surfactants, there are only three that provide any information that can be considered relevant to this study. Values for the CMCs of Li-AOT, $\mathrm{Na}$-AOT and K-AOT have been reported by two groups separately, and were first quoted as 3.0, 2.5 and $2.3 \times 10^{-3} \mathrm{M}$, respectively. ${ }^{182}$ However, they have more recently been reported as 2.66, 2.63 and $2.70 \times 10^{-3} \mathrm{M}$, and so with a different trend with respect to increasing value $\left(\mathrm{Na}^{+}<\mathrm{Li}^{+}<\mathrm{K}^{+}\right) .{ }^{183}$ The relative $\mathrm{CMC}$ values do not actually provide any significant information about the assembly behaviour of the surfactants at high concentration; however, of more relevance is that the second of these studies also reported calculated values for the hydration number and minimum area per molecule at the air/water interface for each ion. These follow the expected trend of the $\mathrm{Li}^{+}$ion being effectively largest due to a larger number of hydration waters, and $\mathrm{K}^{+}$being the smallest and least hydrated (hydration numbers are reported as 7.4, 6.5 and 5.1 for $\mathrm{Li}^{+}, \mathrm{Na}^{+}$and $\mathrm{K}^{+}$, respectively). It is also noteworthy that the vibrational spectra obtained for the pure surfactants by Moran et al. present peak positions consistent with the $\mathrm{Li}^{+}$ion having the smallest charge-to-radius ratio, showing that the $\mathrm{Li}^{+}$ion exists in very different hydration states at each concentration extreme of the system. ${ }^{101}$

‡Literature search conducted January 2017 . 


\section{Potassium AOT: Experimental Results}

\section{Potassium AOT Phase Behaviour}

The phase behaviour of the K-AOT system is significantly different to the Na-AOT and butyIAMA systems described in the previous chapters, a fact that was immediately apparent upon the preparation of bulk samples. K-AOT samples within a large concentration range, between $\sim 30$ and $\sim 74 \mathrm{wt} \%$, are isotropic at room temperature when viewed between crossed polarisers, and this region can be further separated into two regions based upon visual examination of their relative viscosities. Samples between 30 and $58 \mathrm{wt} \%$ surfactant have low viscosity (comparable to that of water), consistent with the properties of a sponge phase. In contrast, samples between 59 and 74 wt \% K-AOT are extremely viscous, indicative of a $V_{\text {/l }}$ phase. Samples prepared with concentrations at the boundary between the two regions generally appeared viscous, but upon significant agitation using a vortex mixer, were observed to separate into two phases with contrasting viscosities. Samples with K-AOT concentrations lower or higher than these regions were anisotropic when viewed between crossed polarisers, with those at low surfactant concentration producing coloured patterns and those at high surfactant concentration appearing opaque/white. These observations indicate the formation of $L_{\alpha}$ and $H_{\| /}$phases, respectively.

The assignment of the phases formed in the K-AOT system was confirmed using POM, SAXS and cryo-SEM, as discussed below. The system was also shown by POM and SAXS to exhibit several phase transitions with changes in temperature, which are shown in the elucidated phase diagram of the system presented in Figure 5.1. The formation of a $L_{3}$ phase between the $L_{\alpha}$ and $V_{/ /}$phases indicates that the counterion to $A O T$ has an important role in controlling the selfassembly of the molecules into specific phases. The fact that the K-AOT system stabilises the $L_{3}$ phase of continuous and on average negative Gaussian curvature, over the "flat" morphology of the lamellar phase (stabilised by undulations and many defects) formed in the Na-AOT system, reveals that the $\mathrm{K}^{+}$ion drives the formation of more negative interfacial curvature, which results in an increased positive contribution to the value $\bar{\kappa}$ and likely also a change in sign (from negative to positive). The $L_{3}$ phase is energetically favoured over a significant concentration range at room temperature (spanning a range of $27 \mathrm{wt} \% \mathrm{~K}-\mathrm{AOT}$ at $25{ }^{\circ} \mathrm{C}$ ); however, it is not the energetically stable high temperature phase. All samples within the $L_{3}$ phase region form a $L_{\alpha}$ phase with the addition of heat, showing that the K-AOT sponge phase is not stable at temperatures above $70^{\circ} \mathrm{C}$. Similarly, within the $\mathrm{K}$-AOT system many $\mathrm{V}_{\text {/I }}$ samples also transition to $a L_{\alpha}$ phase with increasing temperature (a transition observed in several other systems ${ }^{184-187}$ ). $A L_{3}-L_{\alpha}$ transition indicates that the system reverts to behaviour more akin to that observed in the Na-AOT system at high temperatures, and that upon increasing thermal energy the system (in some respects) undergoes a disorder-order transition. This is consistent with the SAXS results reported previously for the Na-AOT lamellar phase, which show the phase adopts smaller bilayer spacings at higher temperatures and that the general trend upon increasing temperature is the SAXS patterns mimicking those formed with increasing concentration. 


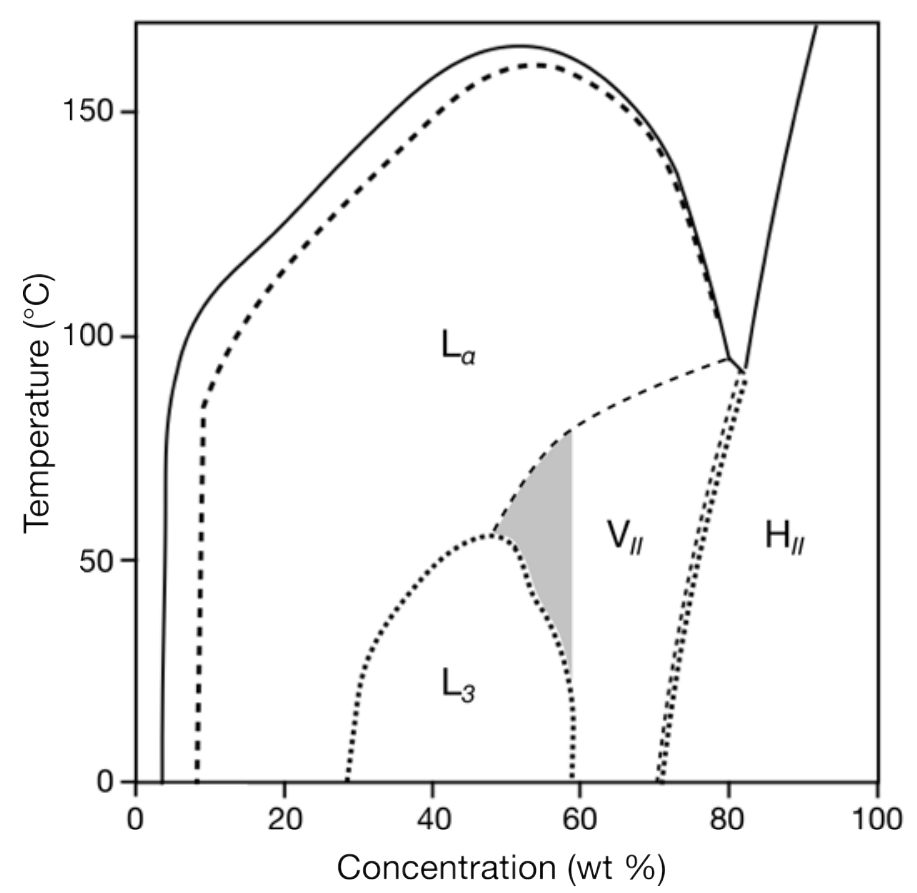

Figure 5.1: Phase diagram of the K-AOT system, showing sponge $\left(L_{3}\right)$, lamellar $\left(L_{\alpha}\right)$, inverse bicontinuous cubic $\left(\mathrm{V}_{I I}\right)$ and inverse hexagonal $\left(\mathrm{H}_{\| l}\right)$ phases. The region marked in grey is likely a continuation of the $V_{\text {II }}$ phase, but a single space group could not be definitively assigned in this region.

The increase in order with temperature is most obvious at concentrations between 52 and 58 wt \% surfactant, where the system undergoes an intermediate transition prior to the formation of a $L_{\alpha}$ phase (highlighted in grey in Figure 5.1). Samples within this intermediate region remain isotropic when viewed between crossed polarisers, but become noticeably more viscous. The structure within this region undoubtedly has 3D ordering (confirmed by SAXS measurements), but the formation of long-lived intermediate structures (which likely provide a low energy pathway between the $L_{3}, V_{l l}$ and $L_{\alpha}$ phases) prevented a single cubic space group being assigned to the region, likely due to a mixture of structures being formed. The disordered structure of the $L_{3}$ phase is often described as being that of a "melted" $V_{\text {II }}$ phase, a description that adequately depicts the connected nature of the $L_{3}$ phase, but may be misleading in this case given the observation of the $L_{3}$ phase forming $V_{\text {/I }}$ structures with increased temperature. This highlights the significant effect that temperature has on the system. The relative contributions of the intermolecular forces must change appreciably with temperature and, based on our study of $\mathrm{Na}$-AOT, result in a reorganisation of the conformational structure of the surfactant molecule. The phase transitions show that the relative values of $\kappa$ and $\bar{\kappa}$ are altered, and that (similarly to the Na-AOT system) the electrostatic forces of the system are in competition with the entropic contribution provided by the thermal energy and topological defects.

The formation of $a L_{3}$ phase in the K-AOT system is not the only change in phase behaviour resulting from the promotion of increasingly negative interfacial curvature brought about by the $\mathrm{K}^{+}$counterion. Although, as the $\mathrm{L}_{3}$ phase formation causes the disappearance of the lamellar phase at only $30 \mathrm{wt} \%$ surfactant, it is the most obvious. The relative stabilities of the $\mathrm{V}_{/ /}$and 
$\mathrm{H}_{/ /}$phases are also affected when compared with the Na-AOT and butylAMA systems reported in the previous chapters. The phase boundaries of both these phases are shifted to lower concentrations, forming at $\sim 59$ and $72 \mathrm{wt} \%$, respectively (compared with $\geqslant 78 \mathrm{wt} \%$ for $\mathrm{V}_{\text {/I }}$ and $\geqslant 82$ wt $\%$ for $\mathrm{H}_{/ /}$phase transitions in the Na-AOT and butylAMA systems).

\section{Potassium AOT SAXS}

SAXS profiles for the K-AOT system (1D and $2 \mathrm{D}$ at $25^{\circ} \mathrm{C}$ ) for concentrations between 5 and 55 wt \% surfactant are shown in Figure 5.2. The dominant feature of all the profiles is diffuse scattering; however, at low concentrations (below 30 wt \% K-AOT) a first-order Bragg peak is also present. The $2 \mathrm{D}$ profile of $25 \mathrm{wt} \% \mathrm{~K}$-AOT shows that this reflection has preferential ordering, while any scattering at similarly low q values occurring at higher concentrations does not. The disappearance of the first-order Bragg peak indicates the $L_{\alpha}-L_{3}$ transition (and the associated reduction in long-range ordering), but it should be noted that this occurs at

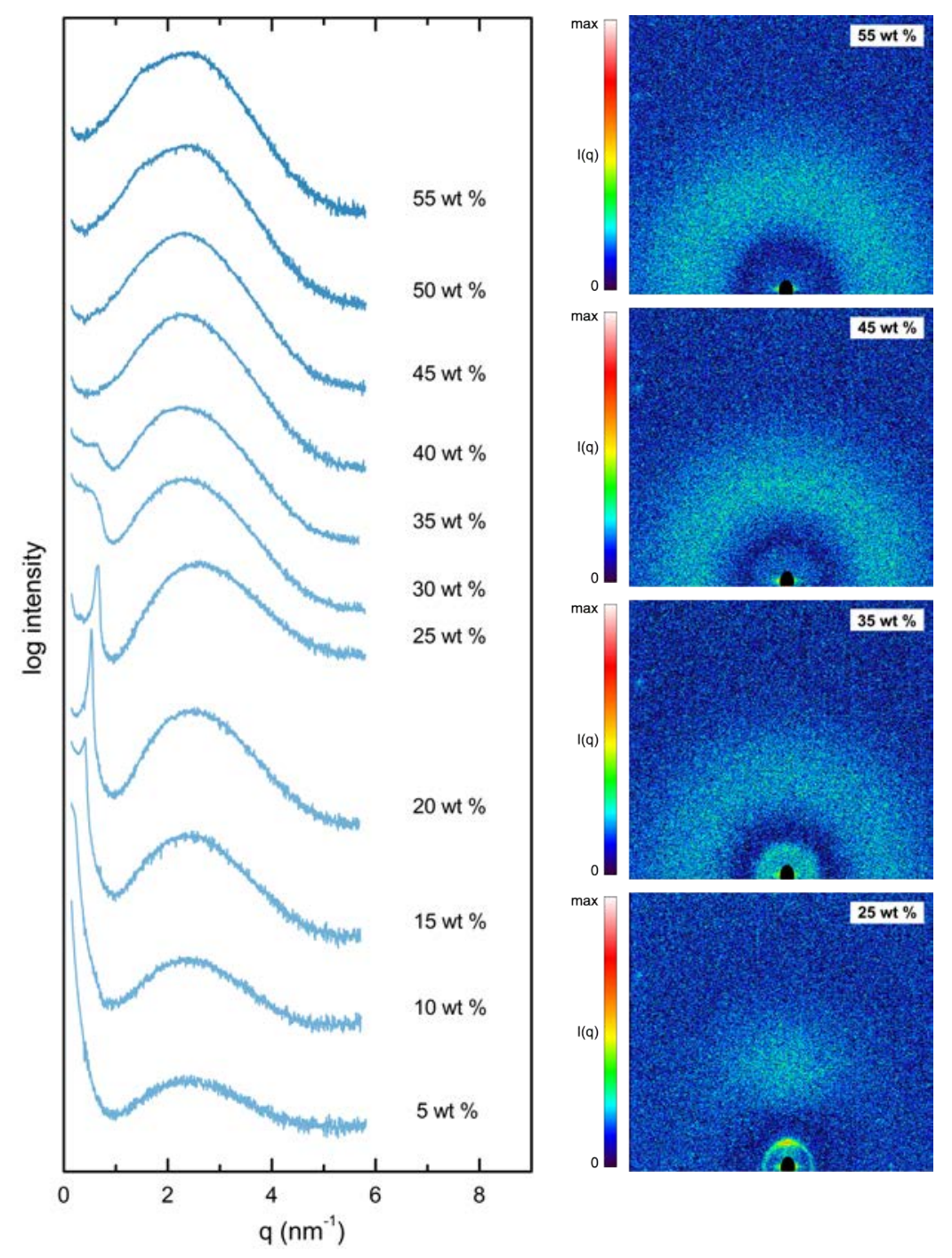

Figure 5.2: SAXS profiles of the K-AOT system, showing the $\mathrm{L}_{\alpha}-\mathrm{L}_{3}$ transition with increasing surfactant concentration. Both 1D (left) and 2D (right) profiles are shown, obtained using line and point collimation, respectively. 
approximately the same q spacing as the first-order Bragg peak of the Na-AOT system drops in intensity due to a minimum form factor contribution. In contrast to Na-AOT profiles, however, the K-AOT system does not produce a second-order Bragg peak and the loss of the first-order peak is accompanied by a shift in the diffuse scattering and very low angle scattering (indicating a change in form factor and increase in characteristic length/pore size). Moving from the $L_{\alpha}$ to the $L_{3}$ phase, there is a shift in the diffuse scattering towards lower q spacings, most obvious between concentrations of 25 and $30 \mathrm{wt} \% \mathrm{~K}$-AOT, where the centres/maximum intensities occur at $\sim 2.6$ and $\sim 2.2 \mathrm{~nm}^{-1}$, respectively. At higher concentrations within the $\mathrm{L}_{3}$ phase (at 50 and $55 \mathrm{wt} \% \mathrm{~K}-\mathrm{AOT}$ ), the diffuse scattering returns to higher $\mathrm{q}$ values and there is evidence of developing order through the appearance of a shoulder to the broad scattering at $\sim 1.5 \mathrm{~nm}^{-1}$, which is likely due to a change in topology approaching the $L_{3}-V_{/ /}$phase boundary.

SAXS spectra at higher surfactant concentrations are presented in Figure 5.3, showing that $\mathrm{V}_{\text {II }}$ and $\mathrm{H}_{\| /}$phases are formed. The characteristic $\mathrm{q}$ spacing ratios for an la3d space group $(\sqrt{ } 6: \sqrt{ } 8: \sqrt{ } 14 \ldots$, see Figure 5.4$)$ are observed at 60 and 65 wt \% K-AOT, although not all peaks are well-resolved. No other space groups are observed within the $\mathrm{V}_{\| /}$region, and the profile at 75 wt \% K-AOT shows the two-phase region to be a mixture of la3d and $\mathrm{H}_{/ /}$structures, and a pure $\mathrm{H}_{/ /}$phase with peaks in the ratio of $1: \sqrt{ } 3: \sqrt{ } 4$ is observed at higher concentrations.
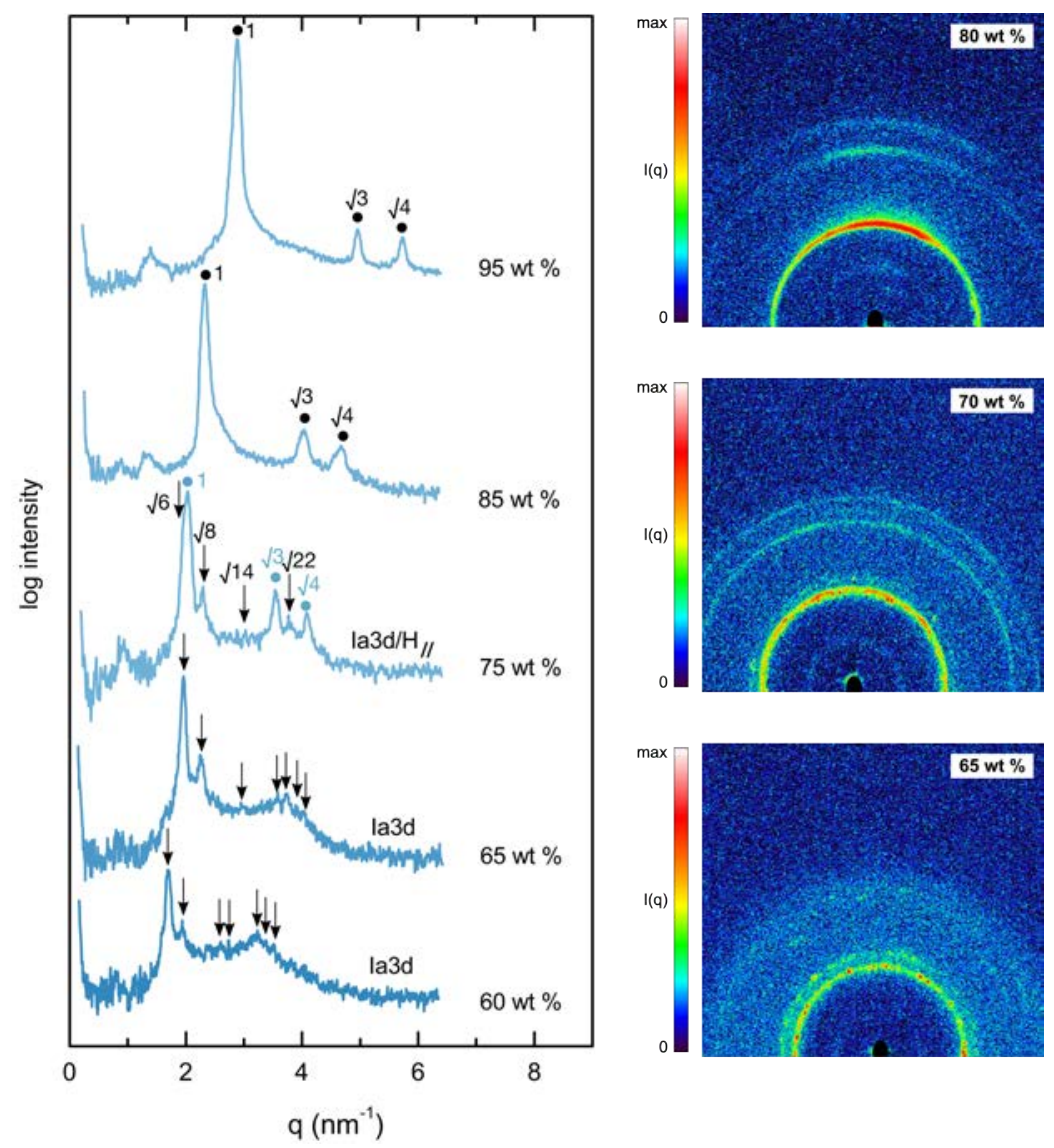

Figure 5.3: SAXS profiles of the K-AOT system, showing the formation of $\mathrm{V}_{/ /}$and $\mathrm{H}_{/ /}$phases. Both 1D (left) and 2D (right) profiles are shown, obtained using line and point collimation, respectively. The $V_{\| /}$reflections are denoted with arrows and the $\mathrm{H}_{/ /}$reflections are marked with dots. 

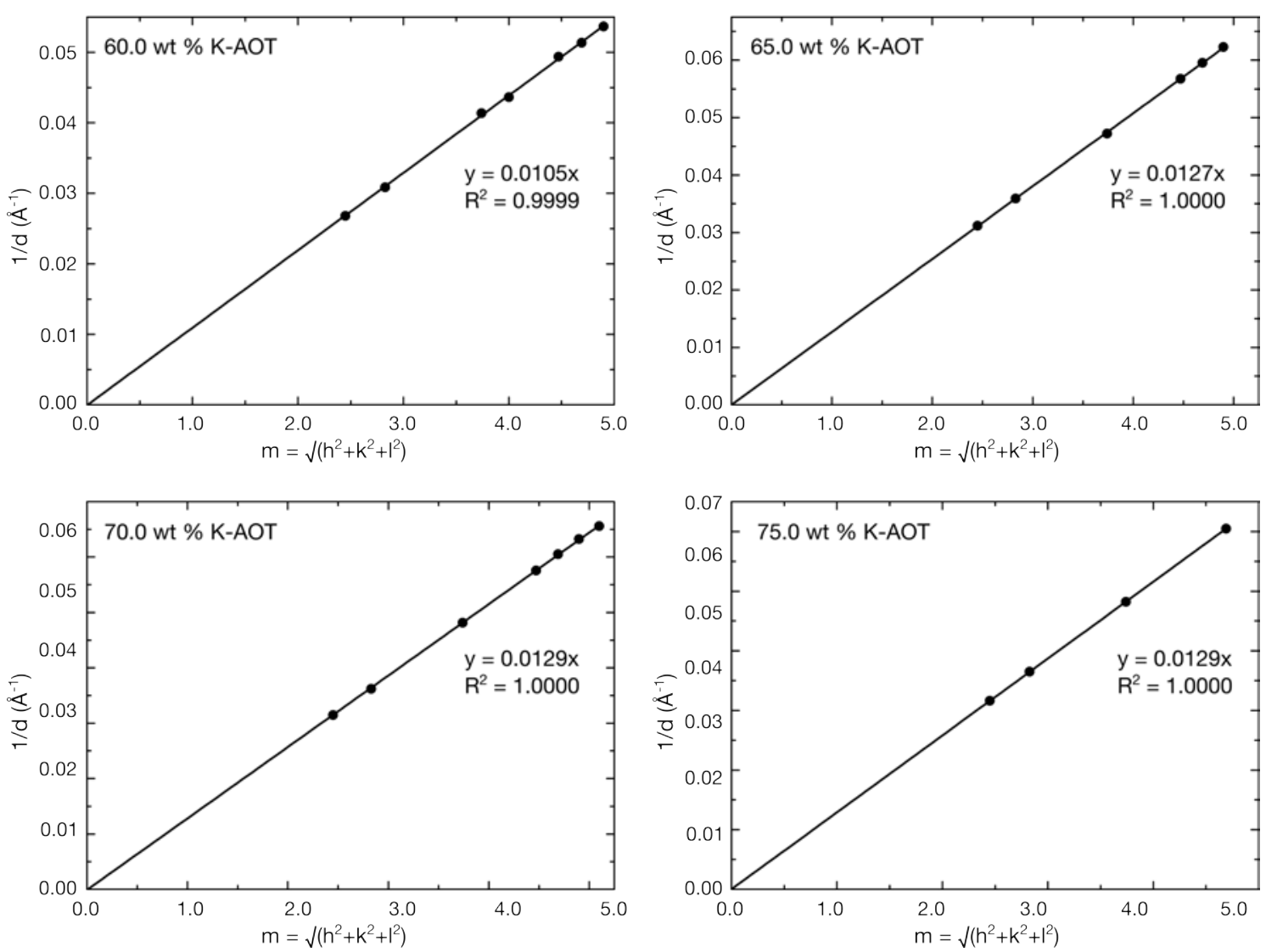

Figure 5.4: Plots showing the reciprocal $d$ spacings $v s . \sqrt{ }\left(h^{2}+k^{2}+l^{2}\right)$ for the cubic phases of the K-AOT system, where hkl are the assigned Miller indices. Correct assignment of the lattice type and space groups is confirmed by the linearity of each plot and the intercept of the fitted line at the origin. The lattice parameter for each graph can be calculated as the reciprocal of the gradient of each fit.

Figure 5.5 shows the evolution of scattering profiles with increasing temperature for samples of 30 and $35 \mathrm{wt} \% \mathrm{~K}$-AOT. The sets of spectra show similar trends, with a disorder-order transition evident from the appearance of Bragg peaks. At $30 \mathrm{wt} \% \mathrm{~K}-\mathrm{AOT}$, a sharp first-order peak (at temperatures of $35^{\circ} \mathrm{C}$ and above) and evidence of very weak second-order scattering can be seen. At 35 wt \% K-AOT, the second-order scattering is stronger and the first-order peak becomes significantly weaker with increasing temperature. Both sets of profiles show an intensity maximum in the first-order peak at $\sim 40 \mathrm{wt} \%$ surfactant, which implies that the factor behind the increased order involved in the $L_{3}-L_{\alpha}$ transition (likely a conformational change) is separate from the subsequent disorder upon further increases in temperature.

Figure 5.6 shows the effect of temperature at 50 and 55 wt \% K-AOT. At 50 wt \% surfactant, a trend similar to that observed at lower concentrations is evident, with first- and secondorder Bragg peaks appearing at the $\mathrm{L}_{3}-\mathrm{L}_{\alpha}$ transition point (occurring at $\sim 55^{\circ} \mathrm{C}$ ). At 55 wt \% surfactant, the profiles show a significant increase in order with temperature, evidenced by the formation of a series of up to seven sharp reflections between 0.8 and $1.4 \mathrm{~nm}^{-1}$. In contrast to samples at lower concentrations, which show a first-order transition from the $L_{3}$ to $L_{\alpha}$ phase with increasing temperature, samples between $\sim 52$ and $58 \mathrm{wt} \%$ form an extremely viscous isotropic phase prior to the formation of the anisotropic lamellar phase. This implies the formation of a structure with $3 \mathrm{D}$ ordering, such that the three refractive indices are equivalent. However, the 

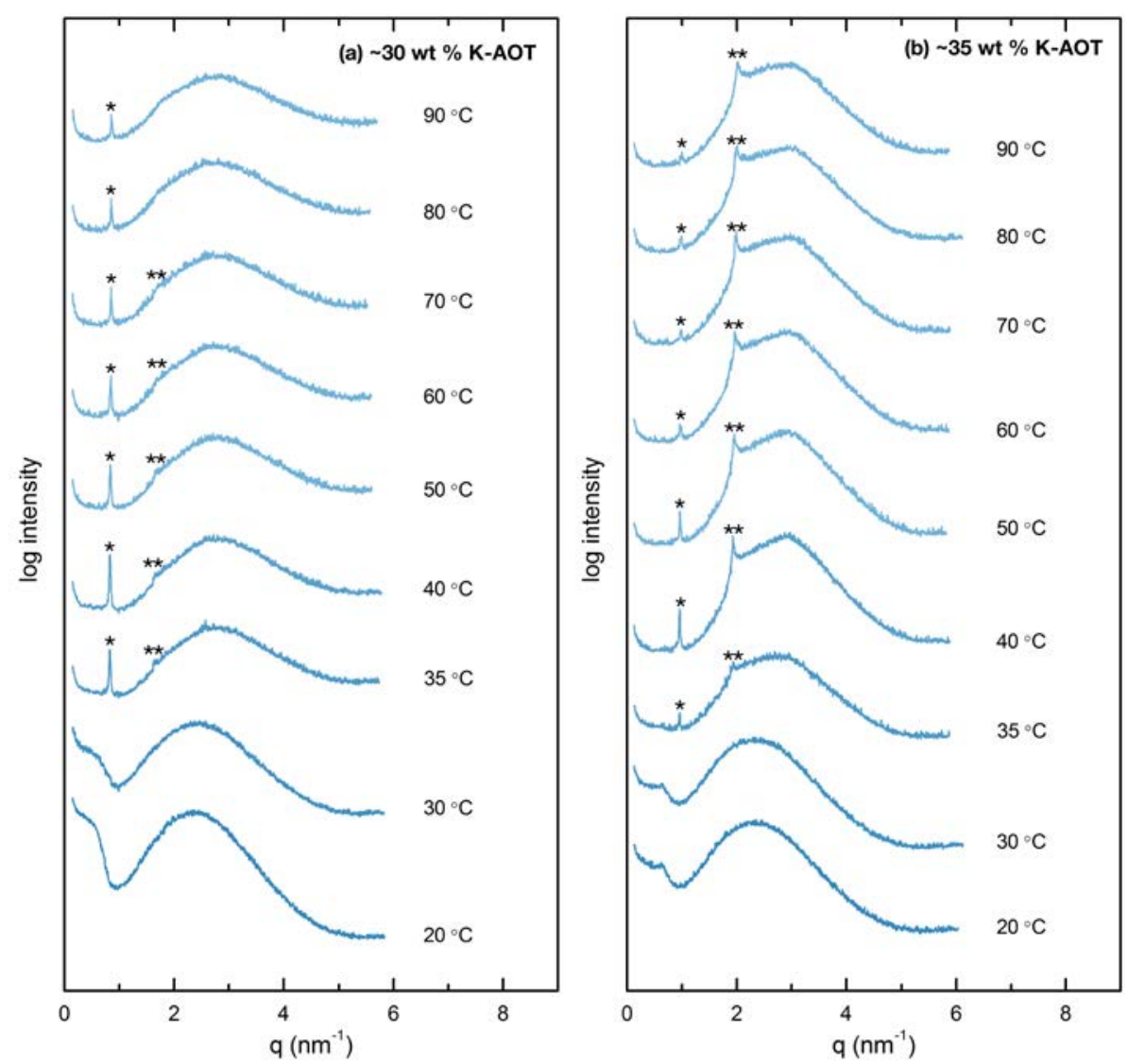

Figure 5.5: SAXS profiles of the K-AOT system with increasing temperature at (a) 30 wt \% $\mathrm{K}-\mathrm{AOT}$ and (b) $\sim 35 \mathrm{wt} \% \mathrm{~K}-\mathrm{AOT}$. Bragg peaks (denoted with asterisks) indicate the formation of the $\mathrm{L}_{\alpha}$ phase.

reflections shown in Figure 5.6 do not provide a satisfactory fit with a single cubic space group, and the broad scattering arising from the form factor of the bilayer (a factor of both the $L_{3}$ and $\mathrm{L}_{\alpha}$ phases at lower and higher temperatures, respectively) is retained in the SAXS patterns of this region. The relative $q$ spacings of up to five of the reflections are consistent with the la3d space group; however, this does not hold true at all temperatures and the assignment of peaks in this way results in the $\sqrt{ } 6$ reflection being weaker in intensity than the $\sqrt{ } 8$ reflection, an unlikely scenario in a pure $V_{/ /}$phase. The spectra show that the phase analysed is not a pure bicontinuous cubic phase, so it is likely highly distorted or a mixture of phases, and contains metastable intermediate structures.

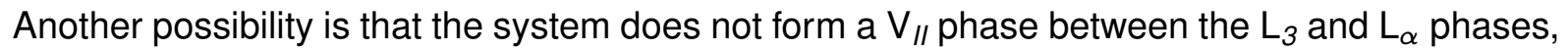
but instead forms a single intermediate phase, a term used to collectively refer to ribbon, mesh and non-cubic bicontinuous structures that can have rectangular (in the case of ribbon), random, tetragonal or rhombohedral space groups. ${ }^{188}$ The intensity pattern of the peaks formed at $55 \mathrm{wt} \% \mathrm{~K}-\mathrm{AOT}$ is consistent with the reported patterns of several mesh phases, which are all experimentally characterised with three strong reflections at low q spacings and a series of weaker reflections at higher q spacings. ${ }^{\ddagger 189-195}$ Of these reflections, the maximum intensity is normally observed in the second peak, which correlates to the lamellar stacking of the 2D ordered mesh aggregates. The patterns for 55 wt \% K-AOT shown in Figure 5.6 are consistent

\footnotetext{
‡See the work of Puntambekar for an extremely well-ordered example. ${ }^{189}$
} 

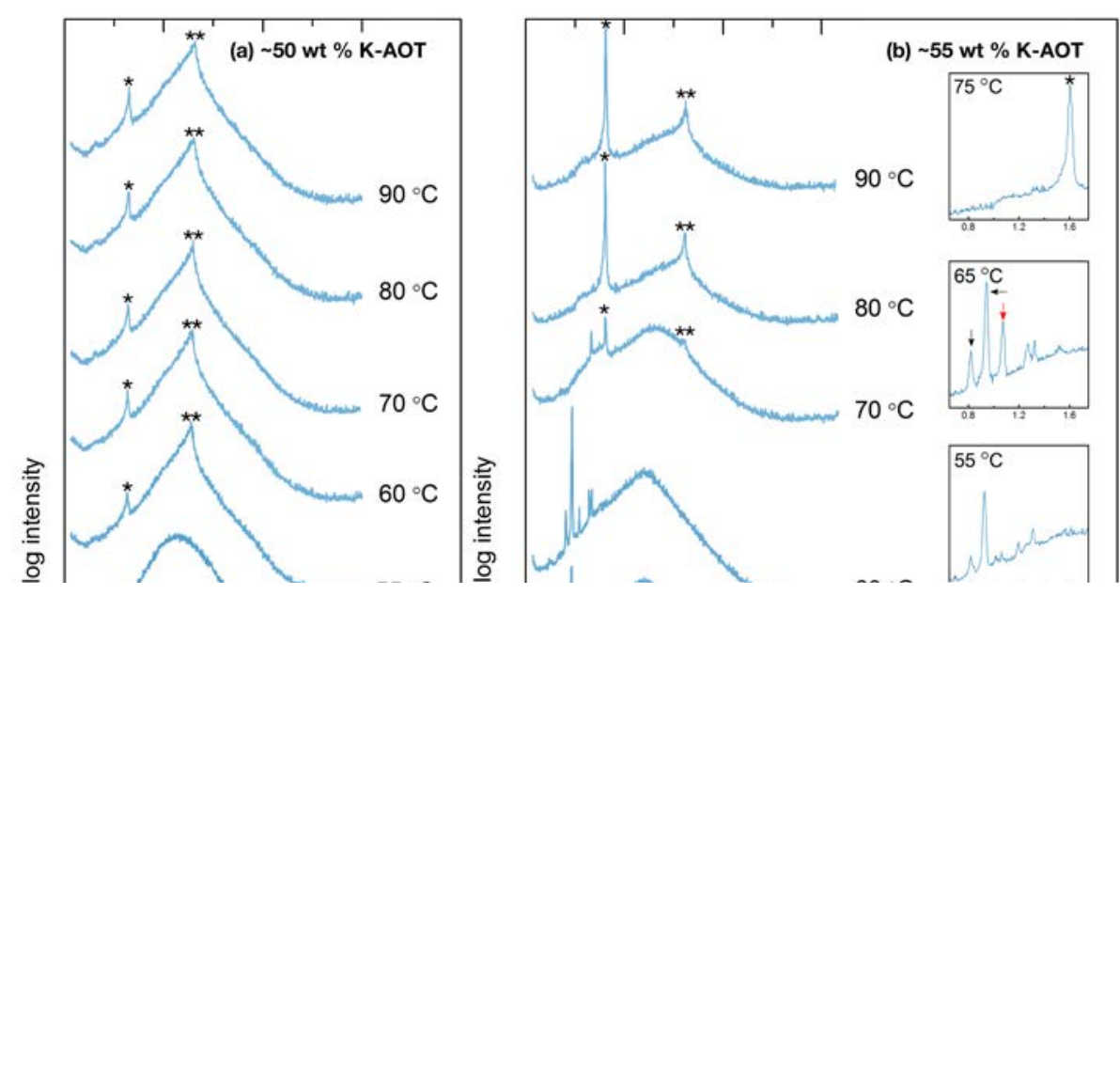

Figure 5.6: SAXS profiles of the K-AOT system with increasing temperature at (a) 50 wt \% $\mathrm{K}$-AOT and (b) 55 wt \% K-AOT. Bragg peaks due to the $\mathrm{L}_{\alpha}$ phase are marked with asterisks. Various peaks are observed with increasing temperature in (b) and are discussed in the text. The peaks marked by black arrows at $65^{\circ} \mathrm{C}$ can be indexed to the la3d space group or to several intermediate phases. However, the peak marked with a red arrow does not fit with any of these assignments.

with this, most obviously at $50-65^{\circ} \mathrm{C}$. However, the scattering pattern cannot be indexed to a tetragonal arrangement, and while attempts to index the pattern to a rhombohedral space group showed a reasonable fit for a number of peaks, the error in the positioning of the third peak in particular (in comparison to the expected position based on the calculated cell parameters) was too great, and hence rules out this possibility. Such phases arise due to the presence of nonuniform interfacial curvature, which (given the freedom of the AOT tails and the positioning of this region in the phase diagram) is a feasible scenario in the K-AOT system upon heating. This region borders the $L_{\alpha}$ phase and two bicontinuous phases $\left(V_{/ /}\right.$and $\left.L_{3}\right)$, and hence a non-cubic bicontinuous phase could logically aid the transitions between these phases. However, despite being reported several times, the existence of such a phase has not been unambiguously established, and its thermodynamic stability is doubted. An ordered mesh phase, which is essentially comprised of lamellar bilayers with a regular array of monodisperse pores could also facilitate these transitions.

The intermediate structure could also be one of several based upon polycontinuous domains that have been proposed recently. ${ }^{16,17}$ Figure 5.7 shows attempts to index the K-AOT SAXS spectra to various modelled space groups. While some assignments show a reasonable fit, they 

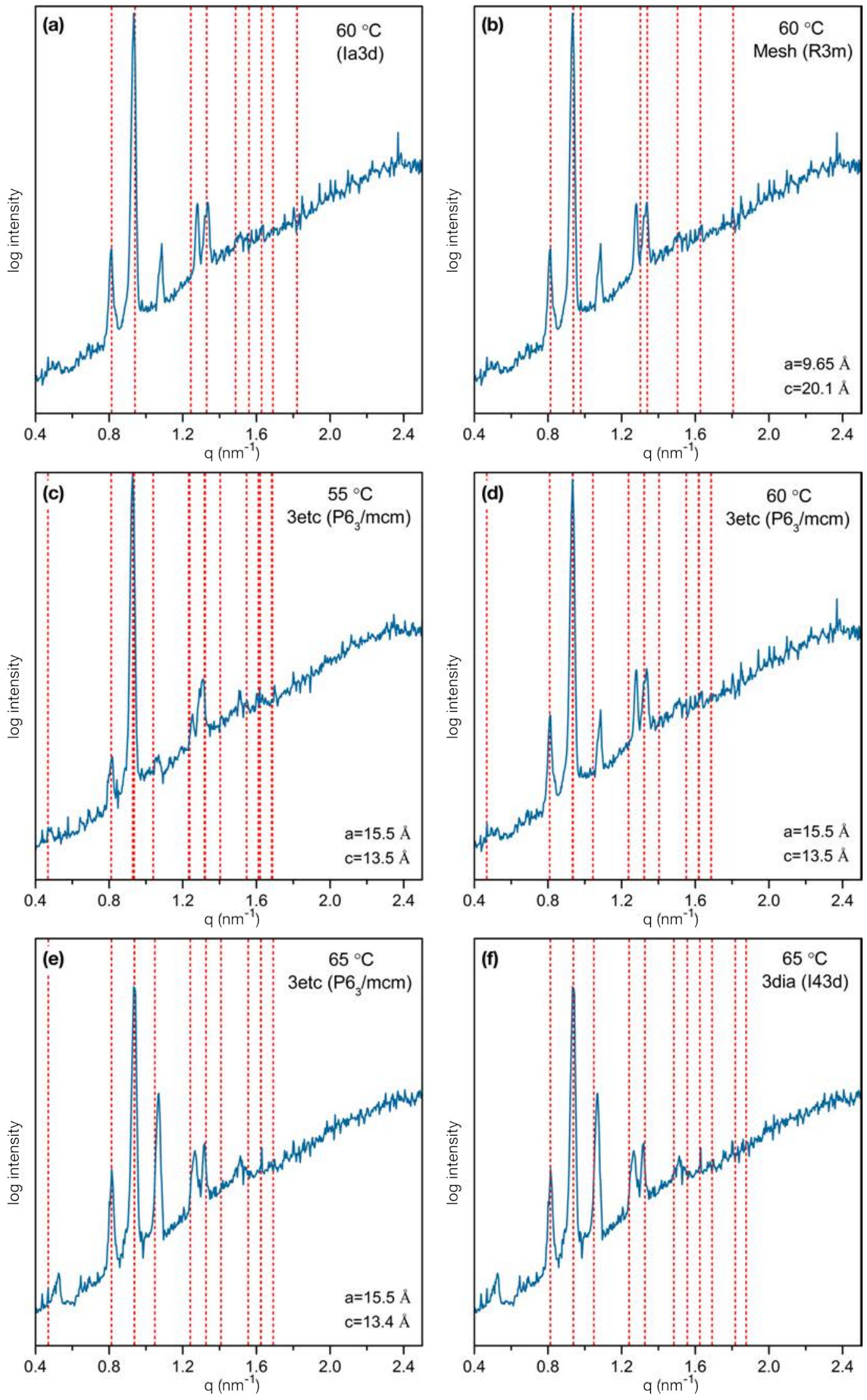

Figure 5.7: SAXS profiles for a sample of $55 \mathrm{wt} \% \mathrm{~K}$-AOT at various temperatures. Dotted red lines denote the expected peak positions for the labelled space groups, based upon all allowed Miller indices. The peak positions of the $\mathrm{R} 3 \mathrm{~m}$ and $\mathrm{P} 6_{3} / \mathrm{mcm}$ assignments are dependent upon the cell parameters a and $c$ (calculated values shown). Cell parameters were calculated using the equations $1 / d^{2}=4 / 3\left(h^{2}+h k+k^{2}\right) / a^{2}+l^{2} / c^{2}$ and $1 / d^{2}=4 / 3\left(h^{2}+h k+k^{2}\right)+\left(1 / R^{2}\right) I^{2}$ where $R=c / a$ for the $R 3 \mathrm{~m}$ and $P 6_{3} / \mathrm{mcm}$ phases, respectively. 
are inadequate to unambiguously assign the phase. The only tricontinuous/tetracontinuous ${ }^{\ddagger}$ phase to be observed experimentally is the 3etc structure with $\mathrm{P}_{3} / \mathrm{mcm}$ (hexagonal) symmetry, reported by Sorenson et al. ${ }^{17}$ We note that the evolution of the SAXS pattern with temperature observed for the 3etc structure is similar to that shown in Figure 5.6 for the K-AOT system and that our calculated unit cell ratio (c/a) of 0.87 is similar to those reported experimentally by Sorenson et al. and theoretically by Schröder-Turk et al. ${ }^{16}$

Intermediate structures are still poorly understood and notoriously difficult to assign (without promoting alignment of the phase and/or using a high-resolution synchrotron X-ray source for analysis). The additional prospect of thermally induced fluctuations within possible mesh structures would complicate this picture even further. As with the lamellar phase, there is likely a plethora of structural variations of each phase through various defects, meaning that our lack of understanding is in the underlying topology. Unfortunately, this is not a simple problem to investigate. While we have confidence in the cryo-SEM images produced in this work that detail the lamellar phase microstructure, these images were produced in samples that were frozen from their equilibrated state at room temperature. A methodology for reliably freezing samples for cryo-SEM analysis directly from high temperatures has not been established, and unless the samples are held at elevated temperatures for a significant time (days or weeks) prior to freezing, the true thermodynamic equilibration state may not be revealed. Such analysis was attempted in the experimental work undertaken for this thesis, with an example shown in Figure 5.8; however, we cannot be confident in the validity of the results obtained. Ideally, such intermediate structures should initially be investigated in systems that form such phases at room temperature, and any electron microscopy investigations supplemented with other techniques that probe the microstructure variations in the system, such as diffusion NMR techniques.
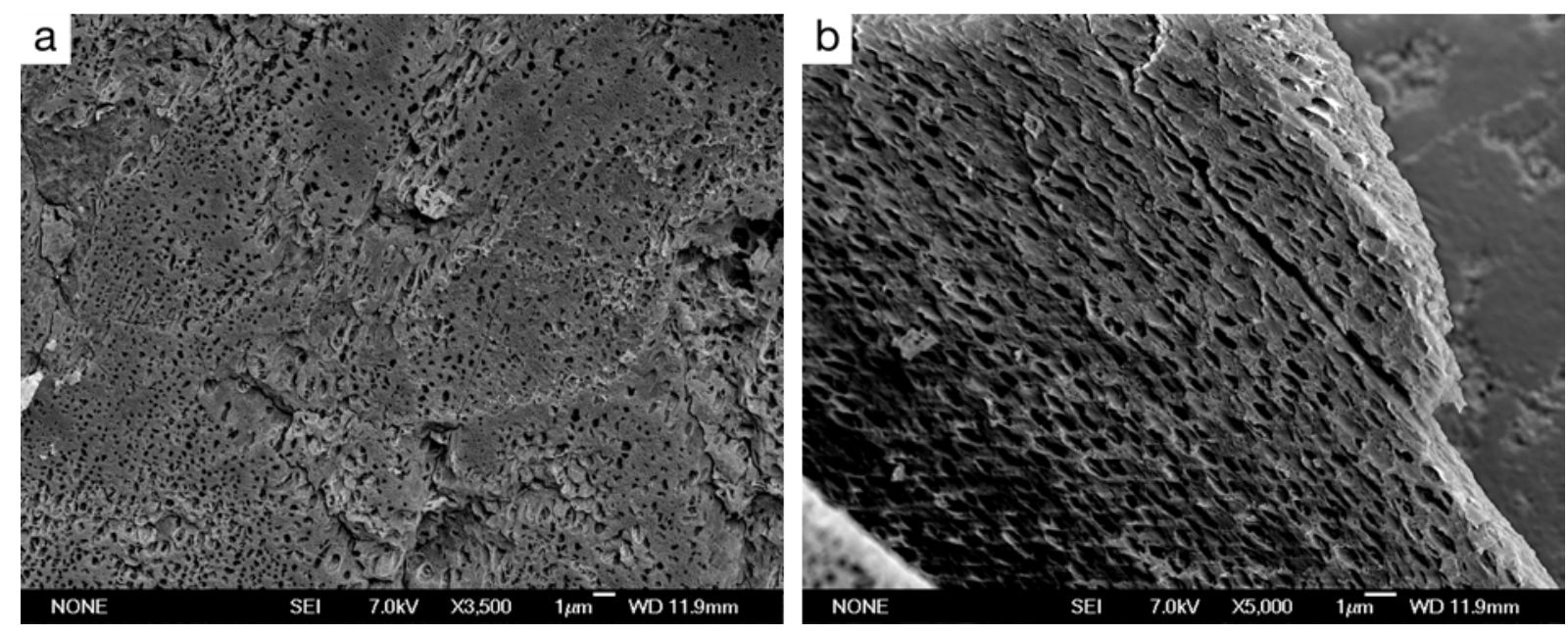

Figure 5.8: Cryo-SEM images of 40 wt \% K-AOT obtained by freezing a sample that had been held at $80^{\circ} \mathrm{C}$ for several days. We cannot be sure that our procedure was sufficiently reliable to be confident that the sample is truly representative of the equilibrated state at $80{ }^{\circ} \mathrm{C}$. However, the image does show a sample frozen from an elevated temperature, and directional ordering that would not be observed for the sponge phase is seen. Large holes within the structure may indicate a mesh-like topography.

\footnotetext{
${ }^{\ddagger}$ The naming convention for these structures is a current topic of debate. ${ }^{17,196,197}$
} 
From the SAXS data we are unable to unambiguously assign the elevated temperature region of the phase diagram between the $L_{3}$ and $L_{\alpha}$ phases, at 52-58 wt \% K-AOT. However, we do note that bulk samples within this temperature region were observed to be isotropic. This implies that the region is a $V_{\| l}$ phase, mixture of $V_{\| l}$ phases, or mixture of $V_{\| l}$ and $L_{3}$ phases. POM predominantly supports these observations; however, a few anisotropic regions were observed to form at temperatures slightly lower than the expected transition temperature. Whatever the transition between the $L_{3}$ and $L_{\alpha}$ phase, it is likely based on experimental data that various intermediate structures mediate the change. The dynamics of the $V_{/ /}$formation are slow, and so the 30 min equilibration time included in the SAXS procedure prior to data collection was likely insufficient for the true equilibration state to be reached. If the thermodynamically favoured state is a $V_{/ l}$ phase, then it is likely a la3d space group. However, the limited thermal stability of the phase likely means distortions in the idealised structure, and we note the presence of broad scattering at q spacings immediately below the first-order Bragg peak of $55 \mathrm{wt} \% \mathrm{~K}$-AOT in the high temperature lamellar phase shown in Figure 5.6. This scattering shows the retention of intermediate structures or significant disorder in the lamellar phase, akin to the disordered mesh structures reported elsewhere. ${ }^{146,189,191}$

\section{Potassium AOT Optical Microscopy}

Figure 5.9 shows a series of POM images of the K-AOT system captured using the concentration gradient method, and reveals the formation of isotropic phases alongside a $\mathrm{H}_{/ /}$phase at high surfactant concentration and $\mathrm{L}_{\alpha}$ phase at low concentration. Given that the neighbouring $V_{\text {II }}$ and $L_{3}$ phases of the K-AOT system are both isotropic, distinguishing between them in the images shown in Figure 5.9 (a) and (b) is difficult. However, while not obvious, the $\mathrm{V}_{\|}-\mathrm{L}_{3}$ and $\mathrm{L}_{3}-\mathrm{L}_{\alpha}$ phase boundaries can be differentiated using a quarter-wave plate and are marked with arrows in Figure 5.9 (b) (see Appendix $B$ for an enlarged image). The $L_{3}$ and $L_{\alpha}$ phases formed by the system are extremely fluid and the penetration of water into the sample occurred at a fast rate, causing interactions between the $L_{\alpha}$ phase and bulk water phase that resulted in rapid flow and breakdown of the structures formed. As such, only a few patterns characteristic of lamellar behaviour can be observed in Figure 5.9 (b). Once the rate of water penetration slowed, the lamellar phase of the system was observed to stabilise, forming the characteristic lamellar patterns and myelin structures shown in Figure 5.9 (c) and (d). ${ }^{\ddagger}$

POM textures of samples at fixed surfactant concentrations provide more information about the lamellar phase of the K-AOT system, and the progression of the phase with increasing concentration is shown in Figure 5.10. Large spherulites are formed at 10 wt \% K-AOT, indicative of MLV structures. At 15 wt \% surfactant, the spherulite patterns remain, but the POM texture is dominated by birefringent/white domains. This indicates the formation of on average ordered domains, likely consisting of small densely packed FCD-Ils. At 20 and 25 wt \% K-AOT, the textures shown in Figure 5.10 reveal the coexistence of FCD-I and FCD-II defects. At these concentrations, spherulite and oily streak patterns are discernible, with the streaks

\footnotetext{
${ }^{\ddagger}$ A single image showing the full progression of phases was not able to be obtained once the lamellar phase had stabilised, due to the expansion of the pattern beyond the microscope frame.
} 

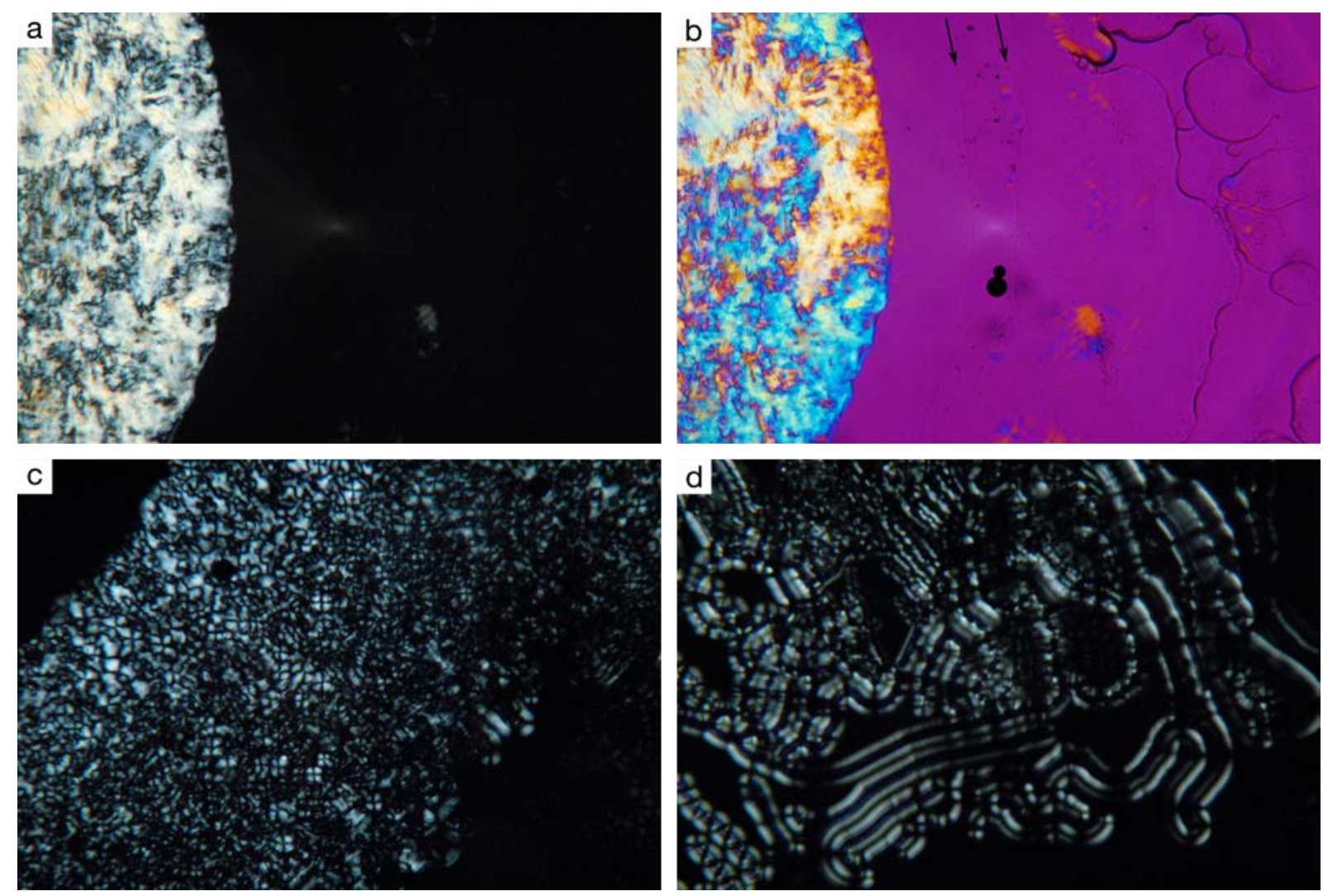

Figure 5.9: Concentration gradient of the K-AOT system at $\times 40$ magnification and $\sim 25{ }^{\circ} \mathrm{C}$ viewed under (a) crossed polarised light and (b) with the addition of a quarter-wave plate. Arrows denote phase boundaries. Image (c) shows characteristic lamellar patterns and (d) shows myelin structures.

appearing considerably smaller at the higher concentration. The coexistence of these patterns is at considerably lower surfactant concentration than in any of the systems reported in the previous chapters, showing that this system forms a bilayer with considerably greater negative curvature than the previous systems.

At 30 wt \% K-AOT, the texture shown in Figure 5.10 is remarkably different to the textures at lower concentrations (and those shown in the previous chapters). This is because the observed pattern formed rapidly under the microscope when the heating stage was stabilised at $25{ }^{\circ} \mathrm{C}$. The sample (which was initially isotropic upon preparation of the microscope slide) sits at the edge of the $L_{\alpha}-L_{3}$ phase boundary, with respect to both temperature and concentration. The large defect structures formed (which upon rotation of the sample stage were confirmed to be almost exclusively FCD-I defects) are due to the rapid growth of the lamellar phase in the confined area between the microscope slide and cover slip. The negative Gaussian curvature of the defect structures formed is consistent with the phase growing from the $L_{3}$ phase, which is also of predominantly negative Gaussian curvature. At 35 wt \% K-AOT, the phase is isotropic due to the disordered nature of the sponge phase. Upon heating, however, the sample transitions to a lamellar phase, becoming anisotropic and eventually producing characteristic textures composed of packed FCD-I defects. Figure 5.10 also shows a sample of $35 \mathrm{wt} \% \mathrm{~K}$-AOT at $120^{\circ} \mathrm{C}$, and it should be noted that the blue colour of this texture is consistent with the theory of Nallet et al. who propose that lamellar phases will appear blue in the region 

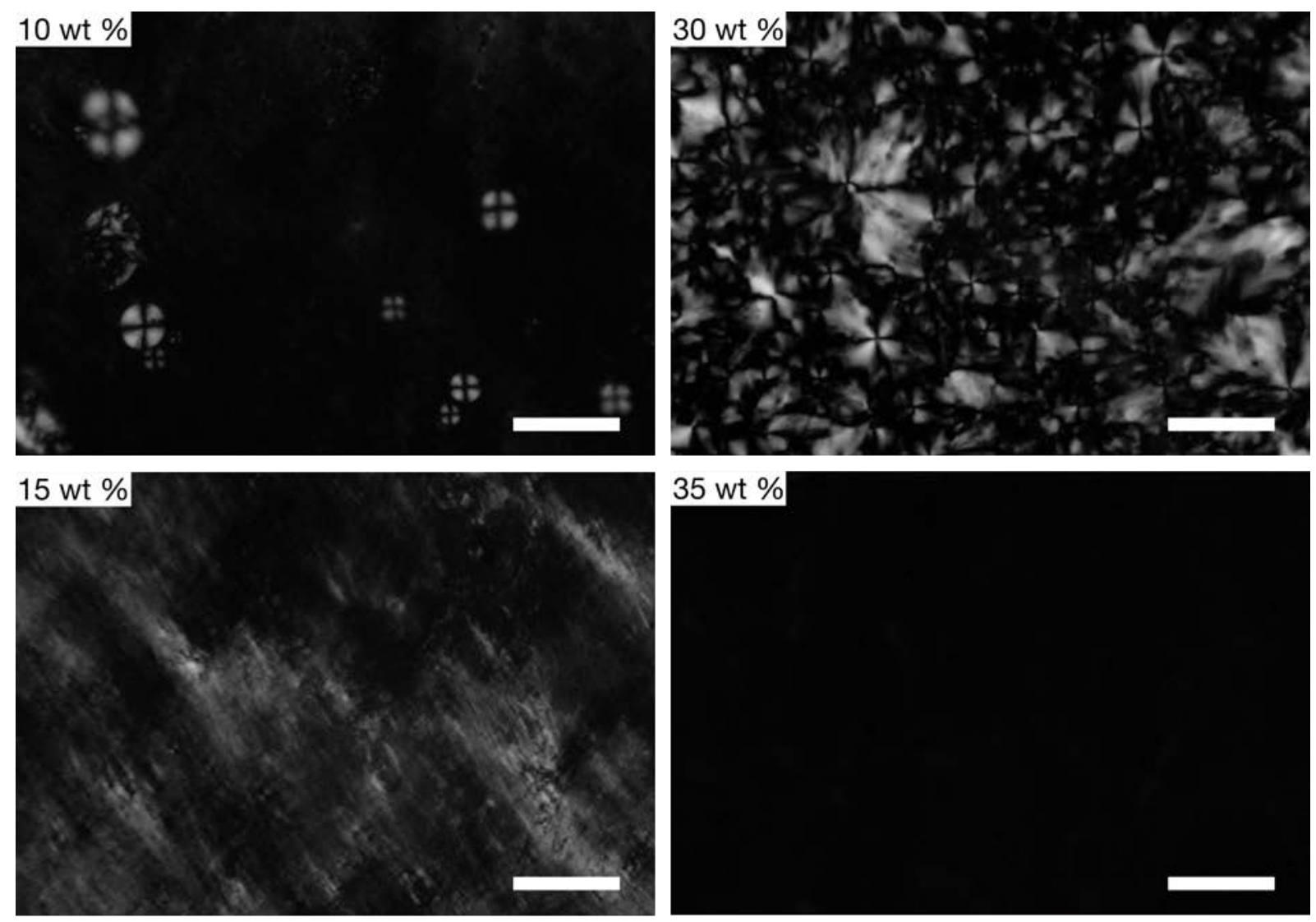

35 wt \%

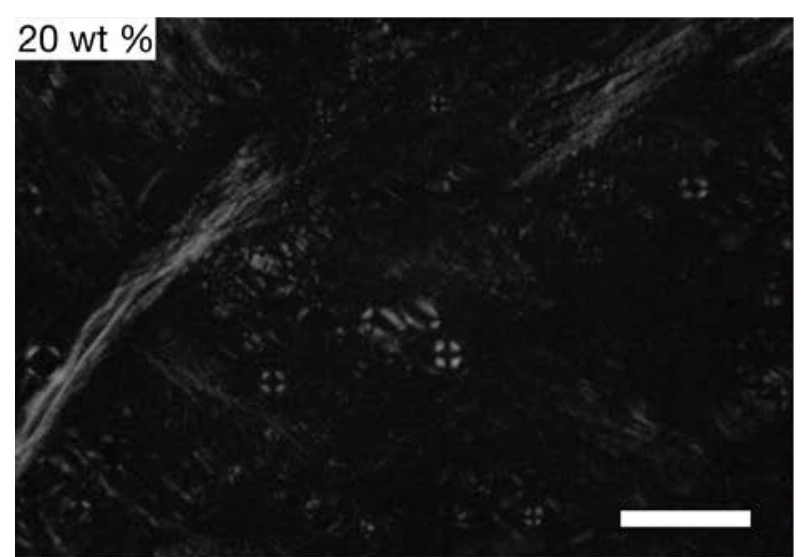

35 wt $\%$ at $120^{\circ} \mathrm{C}$
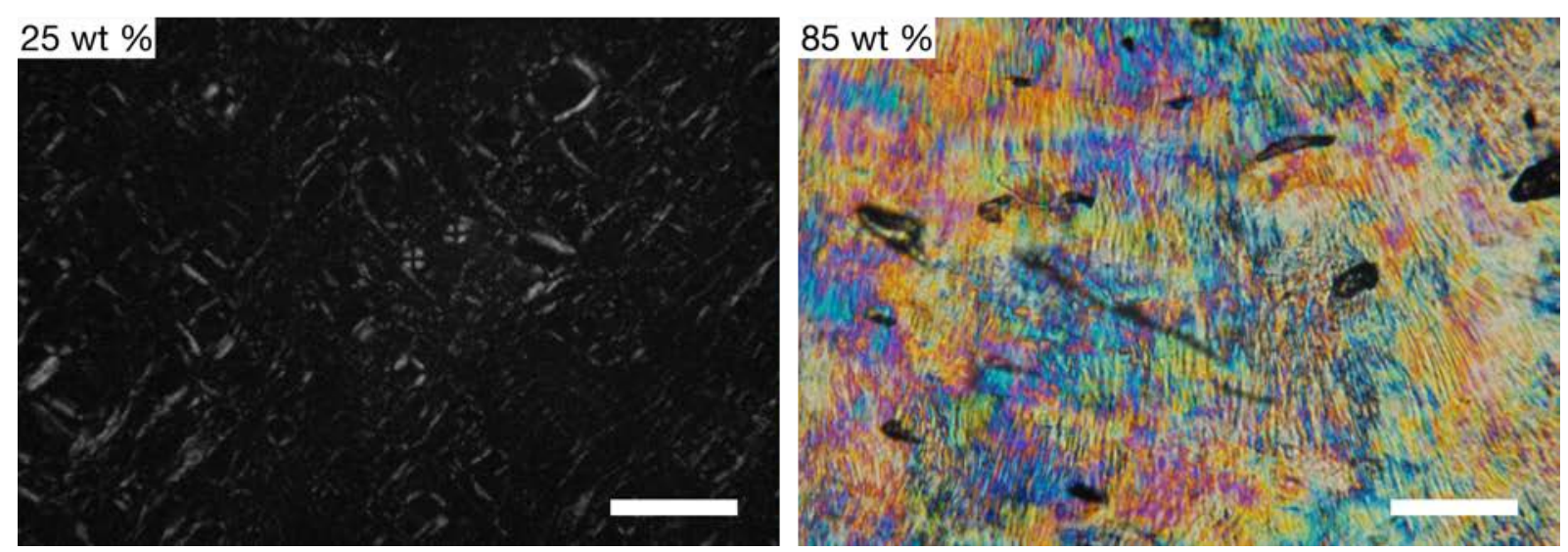

Figure 5.10: POM images of K-AOT with increasing concentration at $\sim 25^{\circ} \mathrm{C}$ (unless stated otherwise). Samples between 10 and $35 \mathrm{wt} \%$ surfactant show the progression from the $\mathrm{L}_{\alpha}$ to the $L_{3}$ phase. Also shown are a sample of $35 \mathrm{wt} \%$ surfactant at elevated temperature and a sample of 85 wt \% surfactant. Scale bars are $100 \mu \mathrm{m}$. 
at which they undergo a change in birefringence. ${ }^{111}$ This means that for K-AOT the value of $\kappa \approx k_{\mathrm{B}} T$ at this concentration (35 wt \% surfactant) and temperature $\left(120^{\circ} \mathrm{C}\right.$ ), and the bilayers of the K-AOT lamellar phase undergo significant fluctuations at very high temperatures.

Figure 5.11 illustrates the $L_{3}$ to $L_{\alpha}$ phase transition of a sample of $50 \mathrm{wt} \% \mathrm{~K}$-AOT upon heating. With increasing temperature, many separate nucleation sites were observed to appear within the isotropic phase. Most of the sites produced patterns characteristic of defects, which were confirmed upon rotation of the sample stage to be a mixture of $s=+1 / 2$ and $s=-1 / 2$ disclination strengths. These sites were not fixed in position, and collisions resulted in many annihilation reactions, which gave rise to three outcomes dependent upon the sign and strength of the interacting defects. In some cases, the defect structures broke down and lost their anisotropy. In other instances, the defects coalesced forming defects of different signs (predominantly FCD-I defects of $s=-1$ ), or multiple defects sites coalesced to produce small multiconnected domains. With increasing temperature, many of the domains broke down, forming FCD-I defects. These FCD-I defects then relaxed upon further increases in temperature into a polygonal array/mosaic structure composed of $s= \pm 1$ FCDs.
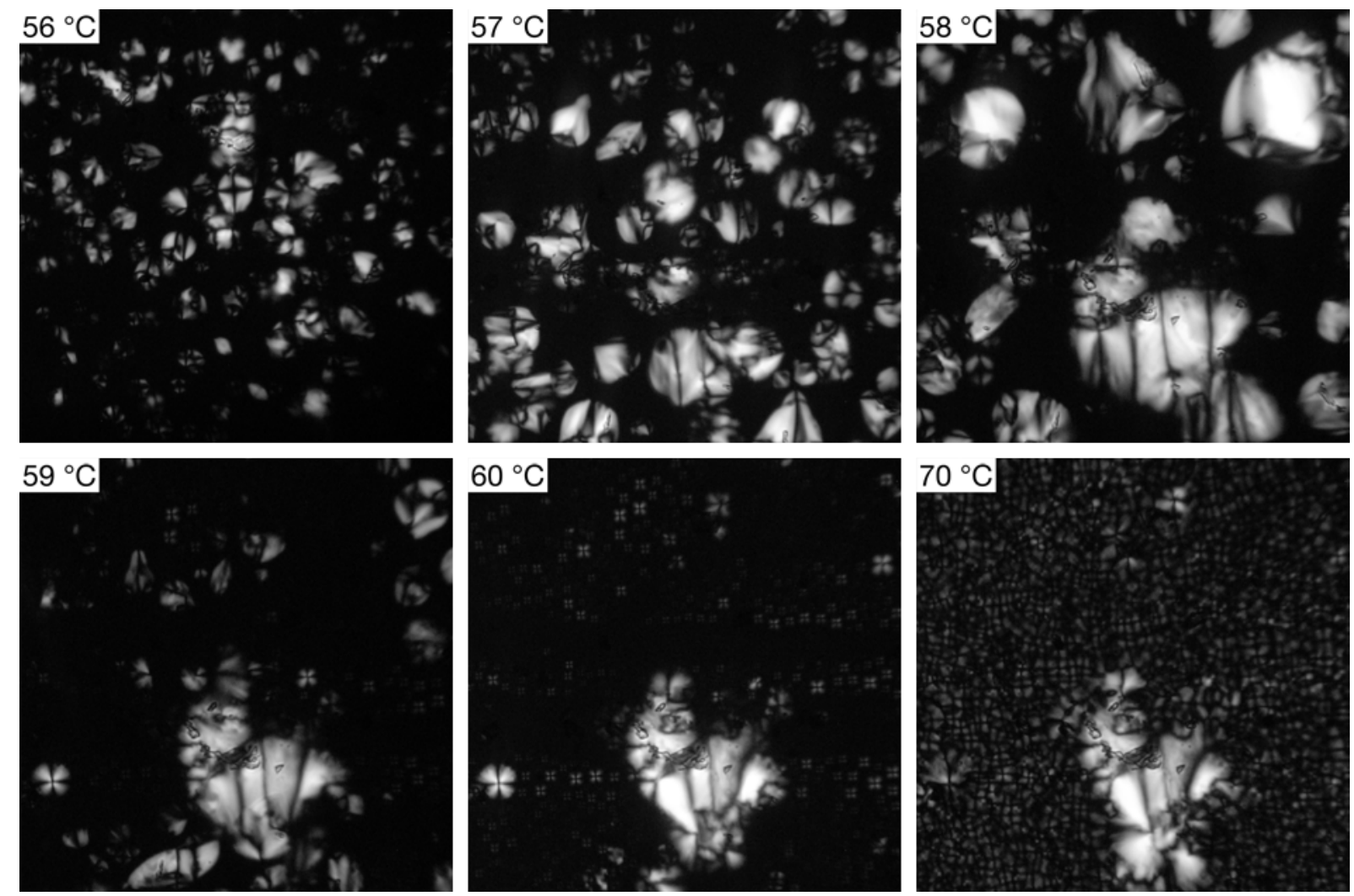

Figure 5.11: POM images of the $\mathrm{L}_{3}-\mathrm{L}_{\alpha}$ phase transition in a $35 \mathrm{wt} \% \mathrm{~K}$-AOT sample with increasing temperature.

The POM images of the K-AOT system show that this surfactant forms defects with negative Gaussian curvature at much lower concentrations than the Na-AOT, Na-butylAMA and K-butyIAMA systems reported in the previous chapters. The K-AOT bilayer clearly has a more positive value of $\bar{\kappa}$ and the formation of a sponge phase in this system shows that $\bar{\kappa} / \kappa$ is positive. It follows that the bilayer rigidity of the system is still low and of comparable magnitude 
to the thermal energy, $k_{\mathrm{B}} T$, but that the inherent curvature of the bilayer is altered through the reduced hydration and tight binding of the $\mathrm{K}^{+}$counterion to the sulfonate headgroup. The greater negative curvature in the K-AOT system also explains the small shift in the stabilisation of the lamellar phase to lower concentrations, as the micellar and MLV structures will be less energetically favoured.

\section{Potassium AOT Cryo-SEM}

Cryo-SEM images of the K-AOT system are presented in Figures 5.12 and 5.13, showing samples within the $L_{\alpha}$ and $L_{3}$ regions, respectively. The images of the K-AOT lamellar phase are noticeably different to those observed in the systems described previously at low concentrations. This is due to the presence of defects with negative Gaussian curvature and is consistent with the K-AOT POM images. Additionally, Figure $5.12(\mathrm{a}-\mathrm{c})$ show that despite there being no evidence of phase separation in bulk samples, regions of concentrated lamellar phase appear to coexist with highly swollen and disordered regions. This was initially attributed to poor freezing or sublimation artefacts; however, this result was reproducible in samples within a narrow K-AOT concentration range ( 15-20 wt \%), and was not observed in any of the other systems studied.

The progression between concentrated and swollen structures can be seen in Figure 5.12 (c) and it is evident that the swollen region also shows some lamellar ordering (note that the difference in spacings may appear more pronounced due to differences in depth of field). The SAXS scattering profiles did not show coexisting $L_{\alpha}$ phases; however, the more swollen/unbound phase may be at extremely low q values (and so not observable). The coexistence of lamellar phases with different swellings is a known phenomenon and has been reported to require weak electrostatic repulsion or enhanced dispersion forces between the layers, and is compatible with a pure electrostatic Poisson-Boltzmann theory. ${ }^{198}$ As such, the apparent lamellar-lamellar coexistence may be due to the binding of the $\mathrm{K}^{+}$ion, which would significantly shield electrostatic repulsion when bound, but not if dissociated. If real, this result may be an indication of large curvature frustration within the bilayer and is consistent with the POM images, which show the presence of different regions comprised of defects with opposing signs of Gaussian curvature. In Figure $5.12(d-f)$, which show the $L_{\alpha}$ phase at the higher concentration of $25 \mathrm{wt} \%$ $\mathrm{K}$-AOT, there is no evidence of two phases and rather than the distinct areas of swollen and highly ordered bilayers observed previously, the $L_{\alpha}$ phase is seen to be disordered and highly connected, which is likely due to the proximity of the phase to the $L_{\alpha}-L_{3}$ phase boundary.

Figure $5.13(\mathrm{a}-\mathrm{c})$ show samples of $40 \mathrm{wt} \% \mathrm{~K}-\mathrm{AOT}$, which is within the $\mathrm{L}_{3}$ phase region. The images are consistent with the assignment of a sponge phase, presenting an overlapping 3D-structure that is bicontinuous in nature. Both the surfactant and water domains are highly connected and continuous, and the low magnification images show that domain boundaries are not present. This is in contrast to the images presented previously in Figure 5.8, which show a sample of the same concentration frozen from an elevated temperature. Figure $5.13(a-c)$ are also significantly different from the image shown in Figure 5.13 (d), which is the Na-AOT 
lamellar phase at a similar surfactant concentration. The overall trend in the K-AOT system is increased negative interfacial curvature in comparison to the Na-AOT system. This can be rationalised by the $\mathrm{K}^{+}$ion resulting in increased electrostatic shielding and a reduction in the effective steric bulk. The K-AOT system shows a gradual and continuous increase in curvature with concentration, which is presumably due to the absence of any drastic changes in the counterion environment.
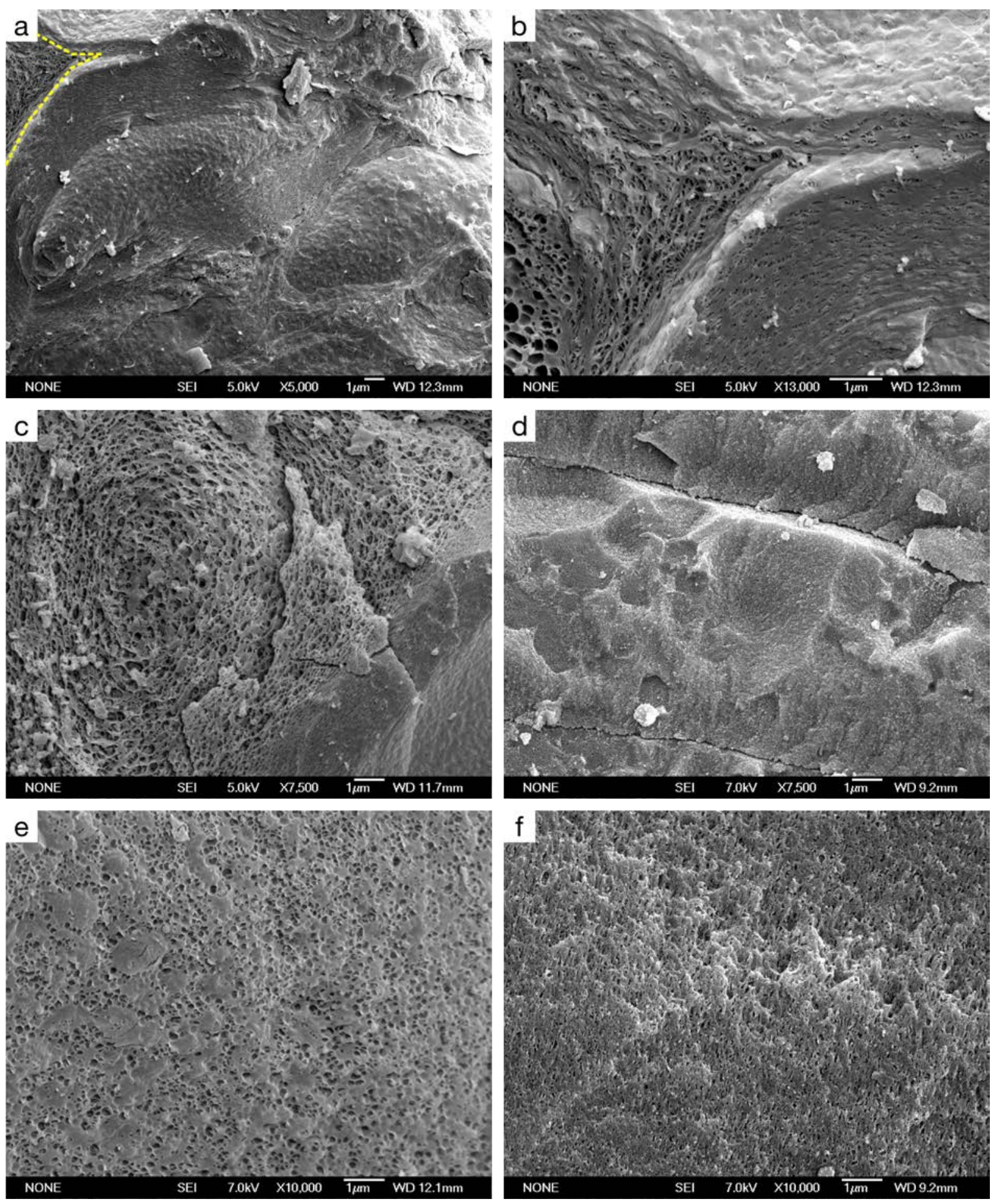

Figure 5.12: Cryo-SEM images of the K-AOT samples of (a-c) 17 and (d-f) 25 wt \% surfactant. Yellow dotted line in the top left corner of (a) shows approximate boundary between swollen and concentrated lamellar phases. 

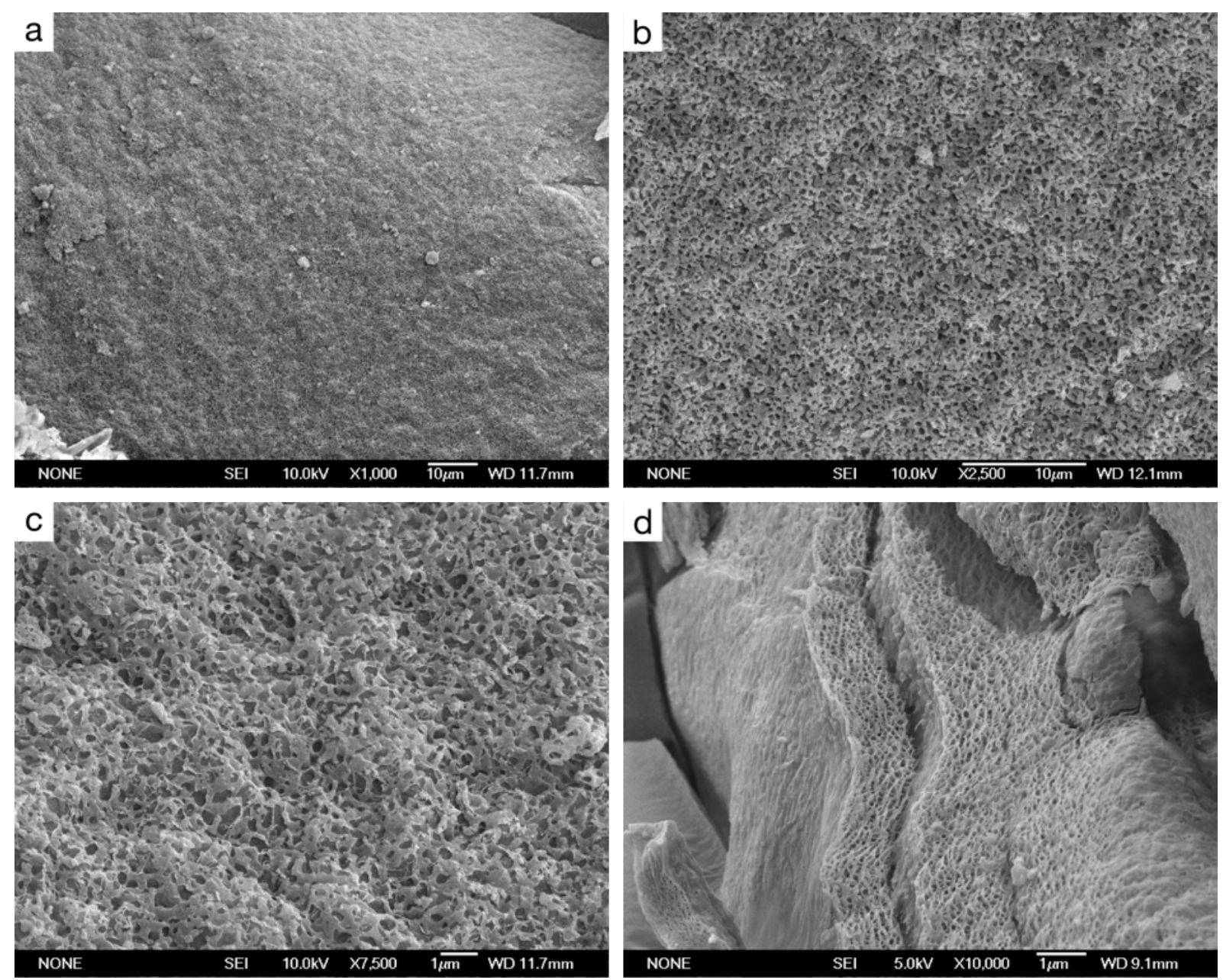

Figure 5.13: Cryo-SEM images of $(\mathrm{a}-\mathrm{c})$ the K-AOT sponge phase formed at $40 \mathrm{wt} \%$ surfactant and (d) an equivalent Na-AOT sample for comparison.

\section{Lithium AOT: Experimental Results}

\section{Lithium AOT Phase Behaviour}

The lithium ion is kosmotropic with a large hydration shell, and hence it is expected that $\mathrm{Li}^{+}$will not form a strong ion pair with the weakly hydrated sulfonate moiety within the headgroup of the AOT anion. As such, the phase behaviour of the Li-AOT system should more closely resemble that of Na-AOT than K-AOT ( $\mathrm{Na}^{+}$is also kosmotropic, whereas $\mathrm{K}^{+}$is chaotropic). Additionally, if the ions follow a logical trend rather than specific ion effects, the Na-AOT system is expected to present behaviour intermediate to the Li-AOT and K-AOT systems. However, the Li-AOT phase diagram shows that this is only partially true (see Figure 5.14).

At concentrations below 56 wt \% surfactant, the Li-AOT system is similar to that of Na-AOT, forming a dilute $L_{\alpha}$ phase rather than the $L_{3}$ phase formed in the K-AOT system (at $25{ }^{\circ} \mathrm{C}$ ). However, Type II phases are observed at concentrations above 56 wt \% Li-AOT, with two $\mathrm{V}_{\text {II }}$ phases (confirmed by SAXS) present at 56-77 wt \% and a $\mathrm{H}_{/ /}$phase at higher surfactant 


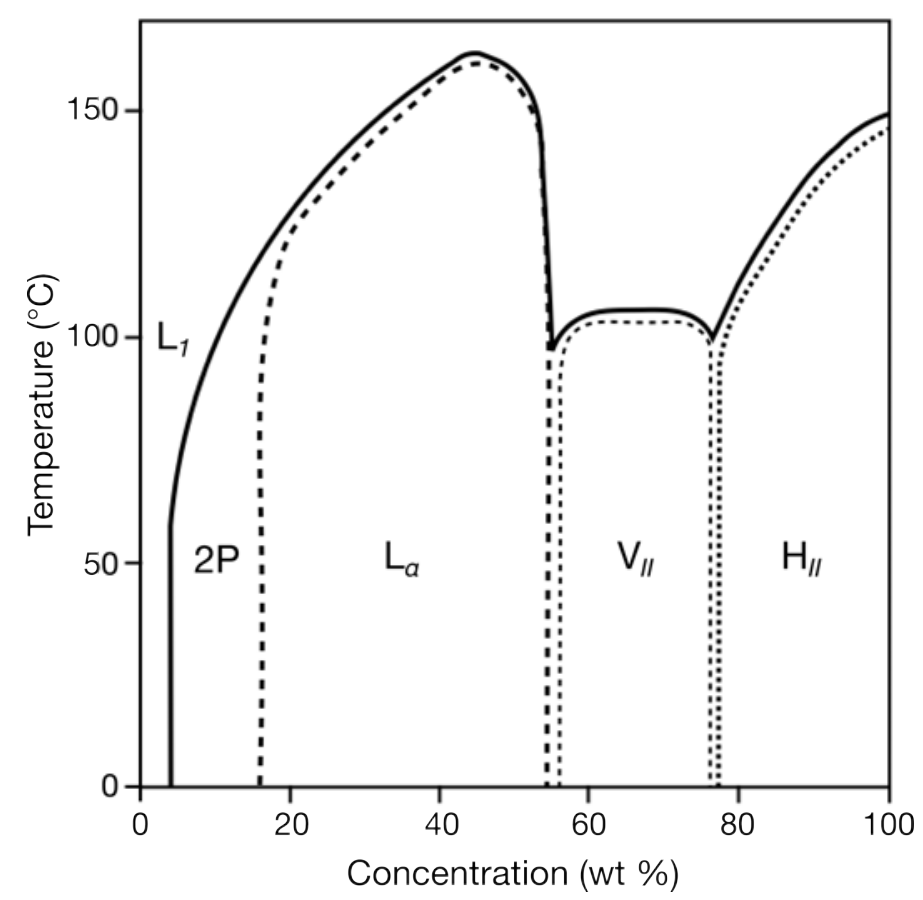

Figure 5.14: Phase diagram of the Li-AOT system, showing the positions of the two-phase (2P) region, and lamellar $\left(L_{\alpha}\right)$, inverse bicontinuous cubic $\left(V_{I I}\right)$ and inverse hexagonal $\left(H_{l l}\right)$ phases. Note that the $V_{/ l}$ region is shown by SAXS to contain two space groups at different concentrations.

concentrations. The $L_{\alpha}-V_{/ /}$transition occurs at a significantly lower concentration than in the $\mathrm{Na}$-AOT system, meaning that both $\mathrm{Li}^{+}$and $\mathrm{K}^{+}$ions result in increased curvature compared with the $\mathrm{Na}^{+}$ion. This implies that a simple trend is not followed and specific effects due to the different ions are contributing to the phase behaviour.

\section{Lithium AOT SAXS}

SAXS profiles for the Li-AOT lamellar phase are shown in Figure 5.15. An obvious trend towards more classical lamellar behaviour and increasing long-range order is evident with concentration; however, the low electron density of the $\mathrm{Li}^{+}$ion results in a weak scattering contribution. This limits the information available, especially at lower concentrations where the assignment of the $2 \mathrm{P}-\mathrm{L}_{\alpha}$ transition was inferred from bulk and POM observations rather than SAXS. It should be noted that at higher concentrations, where lamellar Bragg peaks are evident, diffuse scattering remains throughout the region. This can be seen in both the $1 \mathrm{D}$ and $2 \mathrm{D}$ data, with the $50 \mathrm{wt} \%$ Li-AOT 2D profile showing it to be monodirectional.

The SAXS profiles at higher Li-AOT concentrations, consistent with the formation of $\mathrm{V}_{\|}$and $\mathrm{H}_{\|}$ phases, show more variation. At the highest Li-AOT concentrations shown in Figure 5.16, the patterns show a sufficient number of sharp peaks at characteristic spacing ratios to assign a $\mathrm{H}_{\|}$ phase (85 wt \%), a $\mathrm{V}_{/ /} / \mathrm{H}_{/ /}$mixture (80 wt \%) and a $\mathrm{V}_{/ /}$phase with a Pn3m space group (70 and 72.5 wt \%) with confidence. At the lower concentrations shown (60 and 65 wt \% surfactant), only the first two peaks are sharp, while other peaks are either broad or weak. The two main 


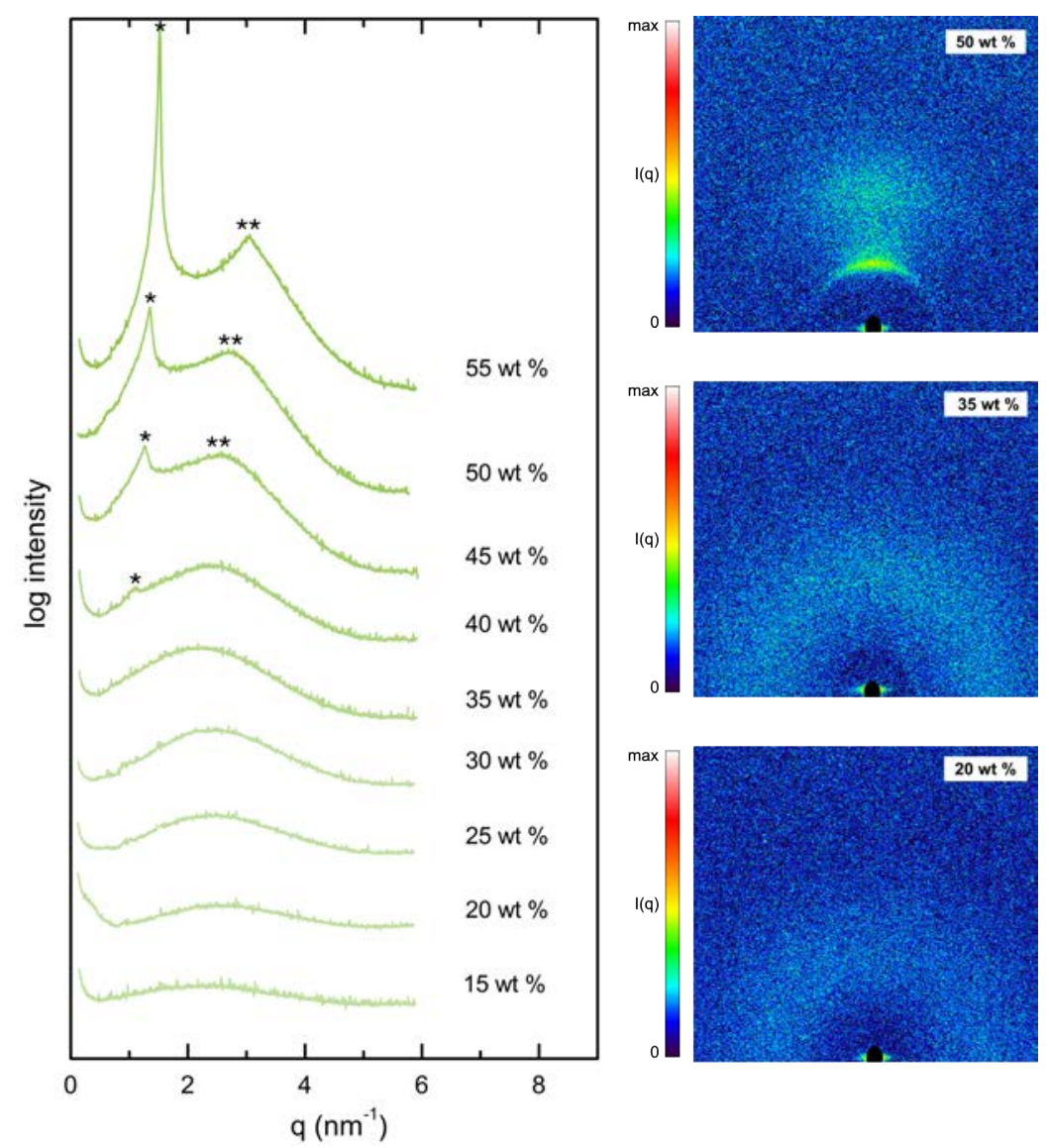

Figure 5.15: SAXS profiles of the Li-AOT lamellar phase with increasing surfactant concentration. Both 1D (left) and 2D (right) profiles are shown, obtained using line and point collimation, respectively. Identifiable Bragg peaks are denoted with single and double asterisks.

Bragg peaks are not in the same spacing ratio as those observed at $70 \mathrm{wt} \% \mathrm{Li}-\mathrm{AOT}$, and at all studied concentrations between 58 and $65 \mathrm{wt} \%$, the q values are consistently in the ratio of $\sqrt{ } 6: \sqrt{ } 8$ and the weak reflections are consistent with higher order peaks belonging to an la3d space group, except that the $\sqrt{ } 14$ reflection is noticeably absent (see Figure 5.17).

The progression from an la3d to a Pn3m space group would normally occur upon swelling, and so this transition would be expected to occur in the opposite direction from that observed in the Li-AOT system (the la3d space group would be expected at the higher surfactant concentration). To the best of our knowledge, the observed phase progression has not been reported in the literature. This presents two possibilities: either the indexing of the phase as an la3d space group is incorrect, or the Li-AOT system facilitates abnormal phase behaviour.

Given that the la3d phase appears out of the expected sequence, is missing the $\sqrt{ } 14$ reflection, and is primarily based upon only two strong signals, we cannot state with certainty that this assignment is correct. However, we do believe that it is the most logical assignment. Given that the sample is both isotropic and highly viscous, we can limit ourselves to considering 3D ordered cubic structures. The position of the cubic phases at concentrations between the $L_{\alpha}$ and $\mathrm{H}_{/ /}$phases implies that it is likely a bicontinuous structure rather than a cubic arrangement of discrete inverse micelles. Of the three known bicontinuous cubic space groups to exist in lyotropic systems, the data are only in good agreement with the la3d space group, as they fit 


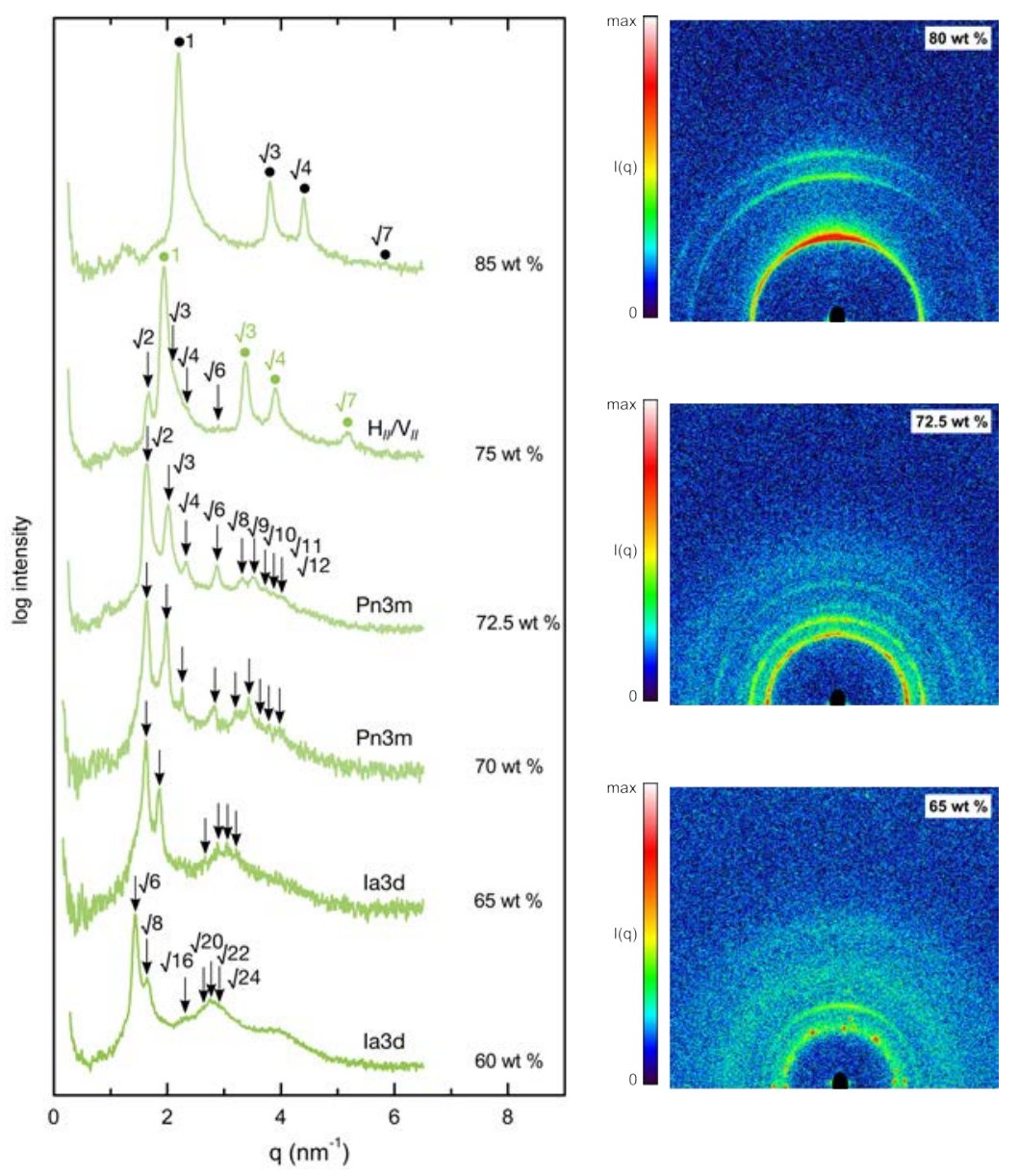

Figure 5.16: SAXS profiles of the Li-AOT system, showing the formation of $\mathrm{V}_{/ /}$and $\mathrm{H}_{/ /}$phases. Both 1D (left) and 2D (right) profiles are shown, obtained using line and point collimation, respectively. The $V_{/ /}$reflections are denoted with arrows and the $\mathrm{H}_{/ /}$reflections are marked with dots. Two different cubic space groups are apparent.

neither the Pn3m nor $\operatorname{Im} 3 m$ space groups (the ratios of $\sqrt{ } 2: \sqrt{ } 3$ and $\sqrt{ } 2: \sqrt{ } 4$ do not index to the two sharp peaks observed). It should be noted that the absence of the $\sqrt{ } 14$ reflection means that the spacings also fit the characteristic ratio of a face-centred array (the Fm3m space group has characteristic spacings of $\sqrt{ } 3: \sqrt{ } 4: \sqrt{ } 8: \sqrt{ } 11: \sqrt{ } 12$, which are equivalent to the $\sqrt{ } 6: \sqrt{ } 8: \sqrt{ } 16: \sqrt{ } 22: \sqrt{ } 24$ reflections of the la3d group). Cubic phases with this spacing have been reported; however, these phases are discrete inverse micellar phases with highly negative interfacial curvature and exist at concentrations above the $\mathrm{H}_{/ /}$phase, ${ }^{199-203}$ and thus are even more unlikely to exist in the region in question than the la3d phase. We cannot completely rule out the possibility that the phase is due to a space group or topological variant previously unreported in lyotropic systems; however, given that all of the sampled concentrations within this region are consistent with an la3d phase, we consider a gyroid structure to be the most likely candidate. We also note the work of Schwarz and Gompper, which states that although other bicontinuous cubic minimal surface types are known mathematically, they all require too large a deviation in Gaussian curvature to be observed experimentally. ${ }^{204}$ While the combined freedoms of both the tail region and the counterion in the Li-AOT system can be used to rationalise the formation of the $V_{\text {II }}$ phase over the $L_{\alpha}$ phase, we do not propose that this is sufficient to stabilise such surfaces. Other possible minimal surface structures are expected to be energetically stable 

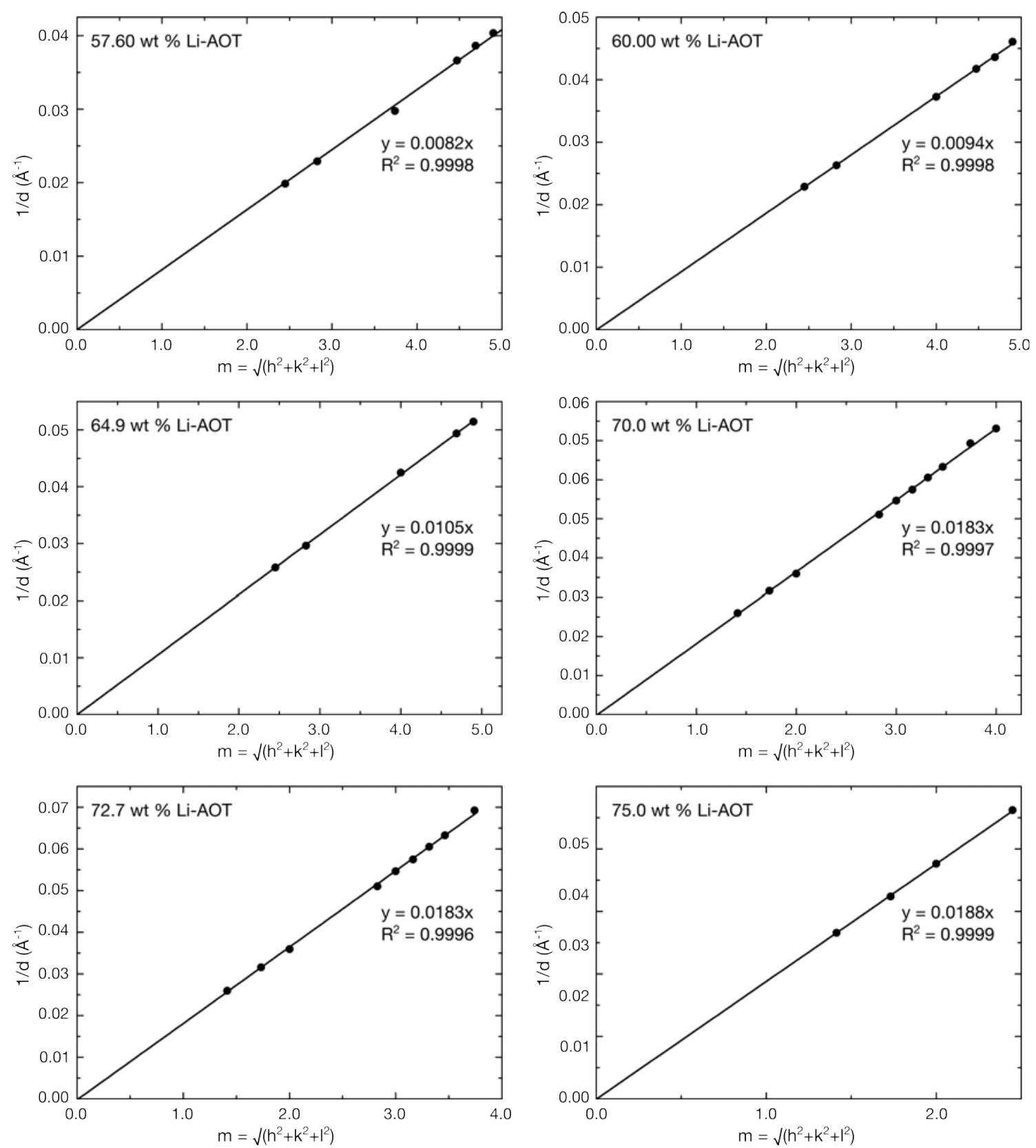

Figure 5.17: Plots showing the reciprocal d spacings $v s . \sqrt{ }\left(h^{2}+k^{2}+l^{2}\right)$ for the cubic phases of the Li-AOT system, where hkl are the assigned Miller indices. Correct assignment of the lattice type and space groups is confirmed by the linearity of each plot and the intercept of the fitted line at the origin. The lattice parameter for each graph is calculated as the reciprocal of the gradient of each fit.

in some lyotropic systems, ${ }^{16}$ and in particular, chiral structures are known but yet to be fully explored. ${ }^{205}$ However, our evidence strongly indicates an la3d structure.

The next question is whether we can rationalise the formation of the la3d $V_{\|}$phase and its appearance at lower surfactant concentrations than the Pn3m space group. The $L_{\alpha}-$ la3d transition is in itself unsurprising as this transition has been observed in three of the four systems already discussed (in the K-AOT system the la3d phase forms from a $L_{3}$ phase), meaning that it is perhaps the Pn3m phase in the Li-AOT system (the first example of a non-gyroid cubic structure in this work) that is unexpected. The calculated lattice parameters 
presented in Table 5.1 show that the la3d phase formed in the Li-AOT system is comparable to that of the K-AOT system and those reported for other systems, while the value for the Pn3m phase is also comparable with other systems. ${ }^{206,207}$ The calculated values of the water channel radii in the Li-AOT $\mathrm{V}_{\text {/I }}$ phases (as a function of tail length) show a steady decrease with increasing concentration, and thus a continual swelling upon dilution from the Pn3m to la3d space groups. Although this is not the expected progression, a swollen gyroid structure has been theorised previously. ${ }^{208}$ There are also several examples of surfactant systems in which phases appear in the "incorrect order" with respect to their theorised or expected positions, such as $V_{/ /}$phases appearing at higher concentrations than the $\mathrm{H}_{/ /}$phase. ${ }^{134}$ One of the most studied of these systems is that of monoolein, which presents $V_{\text {/I }}$ phases on the dilute side of an $\mathrm{L}_{\alpha}$ phase, and notably forms an la3d phase between the $\mathrm{L}_{\alpha}$ and $\mathrm{Pn} 3 \mathrm{~m}$ phases. The monoolein system has received considerable attention because of its use in drug delivery and was the subject of a recent review. ${ }^{44}$ The structure of the molecule itself is relatively simple, and so the abnormal phase behaviour must be a result of the kinked tail and different packing environments at different concentrations. Additionally, the $\mathrm{L}_{\alpha}-\mathrm{la} 3 \mathrm{~d}-\mathrm{Pn} 3 \mathrm{~m}-\mathrm{H}_{/ /}$progression has been reported with increasing chain length in gemini surfactants $\$ 209$ and with increasing addition of lauric acid and/or increased temperature in the dilauroyl phosphatidylcholine/water system. ${ }^{210}$ Although these sequences are not a function of increasing concentration, they imply that changes within the tail region of a surfactant can drive the formation of the space groups in this order.

Table 5.1: Structural parameters for the $\mathrm{V}_{/ /}$phases formed in the AOT and butylAMA systems.

\begin{tabular}{|c|c|c|c|c|}
\hline Surfactant & $\begin{array}{l}\text { Concentration } \\
\text { (wt \%) }\end{array}$ & Space group & $\begin{array}{l}\text { Lattice parameter } \\
\qquad(\AA)\end{array}$ & $\begin{array}{c}\text { Water channel radius } \\
(\AA \AA)\end{array}$ \\
\hline \multirow[t]{6}{*}{ Li-AOT } & 57.6 & la3d & 122.0 & $30.3-1$ \\
\hline & 60.0 & la3d & 106.4 & $26.3-1$ \\
\hline & 64.9 & la3d & 95.2 & $23.6-1$ \\
\hline & 70.0 & Pn3m & 54.6 & $21.1-1$ \\
\hline & 72.7 & Pn3m & 54.6 & $21.1-1$ \\
\hline & 75.0 & $\mathrm{Pn} 3 \mathrm{~m} / \mathrm{H}_{/ I}$ & 53.2 & $20.8-1$ \\
\hline \multirow[t]{4}{*}{ K-AOT } & 60.0 & la3d & 95.2 & $23.6-1$ \\
\hline & 65.0 & la3d & 78.7 & $19.5-1$ \\
\hline & 70.0 & la3d & 77.5 & $19.2-1$ \\
\hline & 75.0 & $\mathrm{la3d} / \mathrm{H}_{\|}$ & 77.5 & $19.2-1$ \\
\hline \multirow[t]{2}{*}{$\mathrm{Na}-\mathrm{AOT}$} & 78.1 & la3d & 69.1 & $17.1-1$ \\
\hline & 80.0 & la3d & 67.1 & $16.6-1$ \\
\hline \multirow[t]{3}{*}{ Na-butyIAMA } & 84.1 & la3d & 57.1 & $14.2-I$ \\
\hline & 84.6 & la3d & 56.8 & $14.1-I$ \\
\hline & 85.0 & la3d/ $/ \mathrm{H}_{/ I}$ & 56.8 & $14.1-1$ \\
\hline \multirow[t]{2}{*}{ K-butylAMA } & 82.5 & la3d & 60.4 & $15.0-1$ \\
\hline & 85.0 & la3d & 59.2 & $14.7-1$ \\
\hline
\end{tabular}

$\S$ We have not included values of I as changes to, or variation in, this parameter may facilitate the different structures observed in the Li-AOT system. Such calculations also neglect the surfactant tail branching.

\footnotetext{
${ }^{\ddagger}$ Gemini surfactants contain two hydrophilic headgroups each bearing a hydrophobic tail, linked together by a spacer moiety.
} 
The la3d, Pn3m and Im3m space groups are all based on minimal surfaces with zero mean curvature. As such, they are closely related in terms of both curvature and energy and it has been shown theoretically that zero curvature can be maintained through transformations between each of the phases facilitated only by bending transitions. ${ }^{23}$ The graph shown in Figure 5.18 (replicated from the work of $\mathrm{Hyde}^{14}$ ) shows the relative volume-to-area ratios required for each of the ideal minimal surfaces, for surfactants of various shape parameters. It is expected that as the concentration increases, dehydration of the headgroup results in an effective increase in the tail volume and thus the progression towards an la3d structure (with the most negative interfacial curvature). However, this assumes that there is not a significant change in geometric shape with increasing concentration. If upon increasing surfactant concentration there is a small increase in the shape parameter without a significant increase in the tail volume (a slight adjustment in the volume-to-area ratio) then an la3d phase may give way to the Pn3m phase. The $\mathrm{Li}^{+}$ion has a large hydration shell and unfavourable interactions with the sulfonate group; however, both of these factors will change significantly upon dehydration. We propose that a change in the effective size of the counterion and the degree of binding will alter both the headgroup area and tail configuration/interactions such that a small change in free energy required to stabilise the $\mathrm{Pn} 3 \mathrm{~m}$ phase over the la3d is feasible.

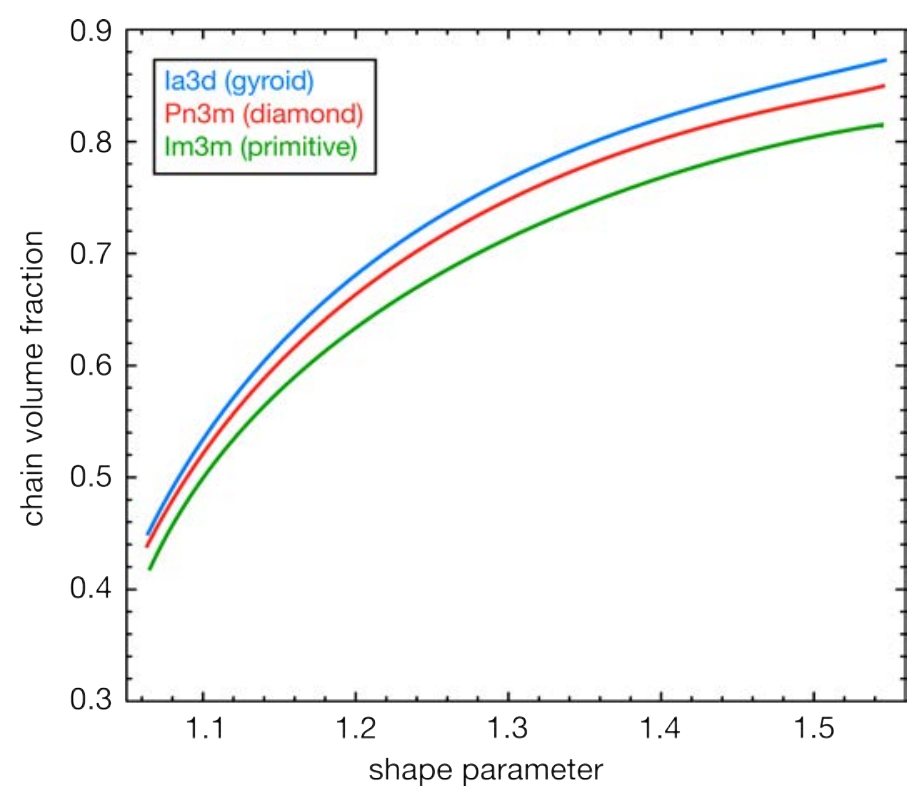

Figure 5.18: The theoretical stability of different $V_{\text {/I }}$ space groups as a function of chain volume and shape parameter, redrawn from the work of Hyde. ${ }^{14}$

Shearman et al. produced a mathematical model that predicts the progression of cubic phases sequentially in the order Im3m-Pn3m-la3d. ${ }^{208}$ To our knowledge this has so far been universally observed experimentally (with the exception that all three phases are rarely observed in a single system). Our results indicate that this may not be the case where one component of the system, such as the counterion, has a high degree of freedom and can dramatically alter the packing arrangement of the surfactant. The mathematical model assumes ideal minimal surfaces and so considers the mean curvature to remain unchanged. It therefore does not consider several major contributions to the free energy of the system that may significantly alter this parameter upon changes in dilution. We have already shown that the 
intermolecular forces change significantly with dilution in the AOT systems, and we note the broad SAXS peaks of the $L_{\alpha}$ phase at the $L_{\alpha}-V_{/ /}$boundary in the Li-AOT system, which imply that thermal undulations are a contributing factor (but are presumably not through the entire $V_{\text {II }}$ region). In the absence of any other logical explanation for our experimental data, we tentatively propose that the Li-AOT system may be the first system observed to disobey this model (or the first example of the "re-entrant" swollen gyroid phase that was also proposed). However, more experimental data are needed to definitively assign the space groups of the $V_{/ /}$phases. Such data should be obtained using samples equilibrated within sealed SAXS cells (avoiding the shear induced during the loading of paste cells used in this work), and given the relatively weak scattering of the lithium and AOT ions, may require the use of a synchrotron source.

\section{Lithium AOT Optical Microscopy}

Concentration gradient POM textures for the Li-AOT system are shown in Figure 5.19. The phases are consistent with the SAXS profiles, with a $\mathrm{H}_{/ /}$phase formed at high surfactant concentrations, and isotropic $\left(\mathrm{V}_{I I}\right)$ and lamellar bands sequentially formed at lower concentrations. Two further features are noteworthy. Firstly, a change in the optic sign of the birefringence is evident and the quarter-wave plate image reveals that the swollen lamellar phase is formed at dilute concentrations, thus the phase progression is similar to the Na-AOT system. However, the second point of note is the relative size of the isotropic $V_{/ l}$ band. This band is large due to the increased stability of the $\mathrm{V}_{\| /}$phase in comparison with the Na-AOT system, but there is no evidence that it is composed of two bands (as would be expected from the two different space groups observed via SAXS). This is presumably due to either the slow dynamics of the formation of the $V_{/ l}$ phase or because the change between space groups is continuous upon swelling.
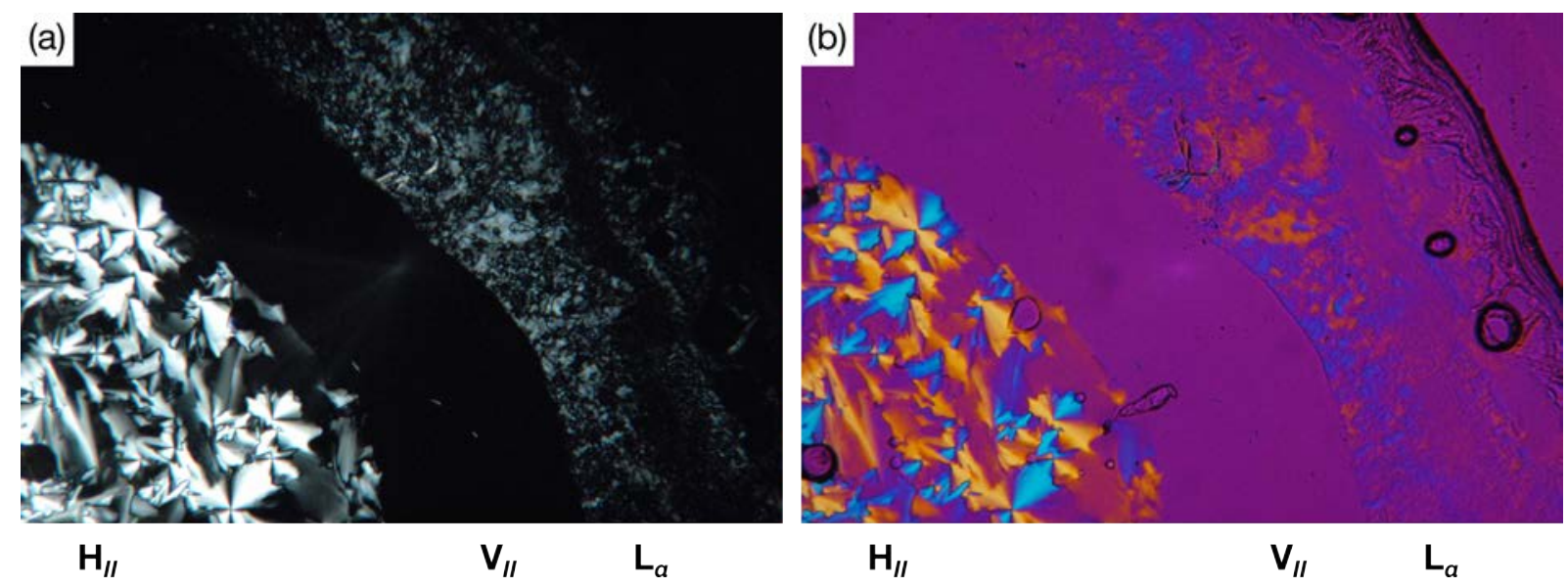

Figure 5.19: Concentration gradient of the Li-AOT system at $\times 40$ magnification and $\sim 25{ }^{\circ} \mathrm{C}$ viewed under (a) crossed polarised light and (b) with the addition of a quarter-wave plate.

POM images of the Li-AOT lamellar phase at room temperature with increasing concentration are shown in Figure 5.20. Defects of positive Gaussian curvature dominate the images at concentrations up to $35 \mathrm{wt} \% \mathrm{Li}$-AOT. As expected, this behaviour is more akin to the Na-AOT 


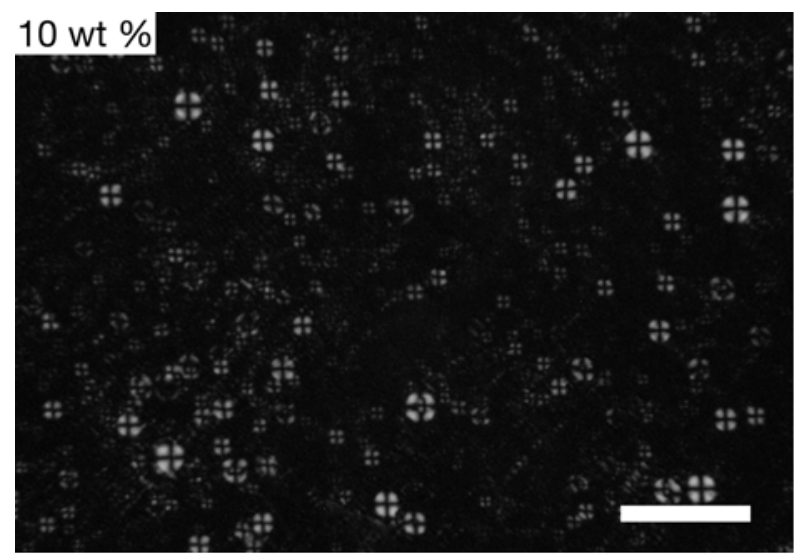

\section{0 wt \%}
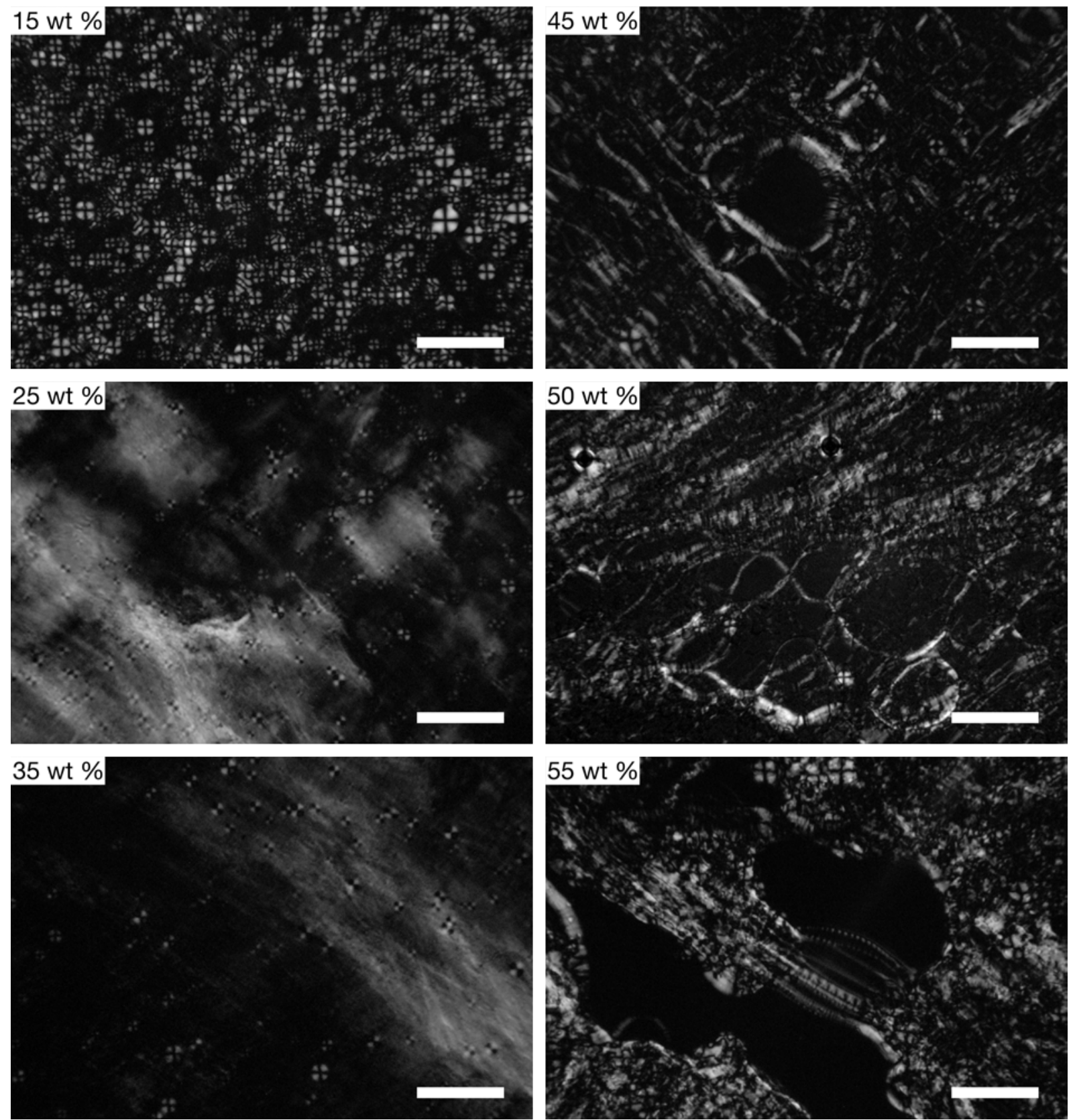

Figure 5.20: POM images of Li-AOT with increasing concentration at $\sim 25{ }^{\circ} \mathrm{C}$. Scale bars are $100 \mu \mathrm{m}$. 
system than the K-AOT system. At 25 and $35 \mathrm{wt} \%$ surfactant, large domains of anisotropy can be observed, indicating the close packing of small defects within averagely ordered domains. The large FCD-II defects present are generally observed to remain approximately spherical, but are reduced in number and size with increasing surfactant concentration. At 40 wt \% Li-AOT, the birefringence is weak due to a change in the optic sign, indicating a move from swollen to more classical lamellar behaviour. However, while the classical lamellar behaviour results in the formation of oily streaks (with some FCD-II defects retained), the system noticeably does not form mosaic patterns prior to the formation of a $V_{\text {/I }}$ phase.

\section{Lithium AOT Cryo-SEM}

Given the similarity of the POM images of the Li-AOT system with those of similar concentrations of Na-AOT, it should be expected that the cryo-SEM images will also show the two systems displaying similar behaviours. The Li-AOT and Na-AOT systems both form large FCD-II defects, and have regions of anisotropy arising from defects at lengthscales below the resolution of the optical microscope. The cryo-SEM images captured for a sample of $30 \mathrm{wt} \% \mathrm{Li}$-AOT, shown in Figure 5.21, are consistent with these observations and are also similar to those captured for Na-AOT at similar concentrations. Figure 5.21 (a) shows several FCD-II defects of various sizes, with the large $\sim 14 \mu \mathrm{m}$ defect surrounded by several smaller and more distorted domains. Figure 5.21 (b) shows the structure of the system at higher magnification and provides views of a $L_{\alpha}$ stack from different angles. At the bottom of the image the uneven surface can be seen, in the middle of the image the highly connected layers are evident, and the top of the image the angle of fracture or the gradual change in directional order show the phase to have a more globular appearance (similar to the Na-AOT images), which indicates many pockets of water caused by a disrupted bilayer structure.

Figure 5.21 (c) and (d) show further FCD-Il defects and it is observed that "space filling" defects are present at the interface between some larger domains. The images also show the presence of pores through the surfactant surface and undulations in the structure. The images presented in Figure 5.21 (e) and (f) further illustrate the points mentioned above. The stack of lamellar layers in Figure 5.21 (e) are highly defective and in many respects do not resemble a lamellar structure, but an aggregation of spherical particles. However, there is evidence of long-range ordering to produce a stacked structure, and upon close inspection, the structure does appear to be made of connected layers riddled with local scale defects that form isolated pockets of water. Thus, the connectivity of the phase is like that of Na-AOT samples of similar surfactant concentrations, which display severely restricted water diffusion. The region shown in Figure 5.21 (f) actually overlaps with the region shown in Figure 5.21 (c), and shows the curved layers of a FCD-II defect. It should be noted how as the layers of this defect curve towards the left side of the image (changing from an angle perpendicular to the imaging angle to a parallel angle) that a "top-down" rather than "side-on" view is obtained, again revealing the undulations and connectivity between the layers. Compared with the layers perpendicular to the page, the left side may appear more disordered; however, it is the same structural behaviour and thus 

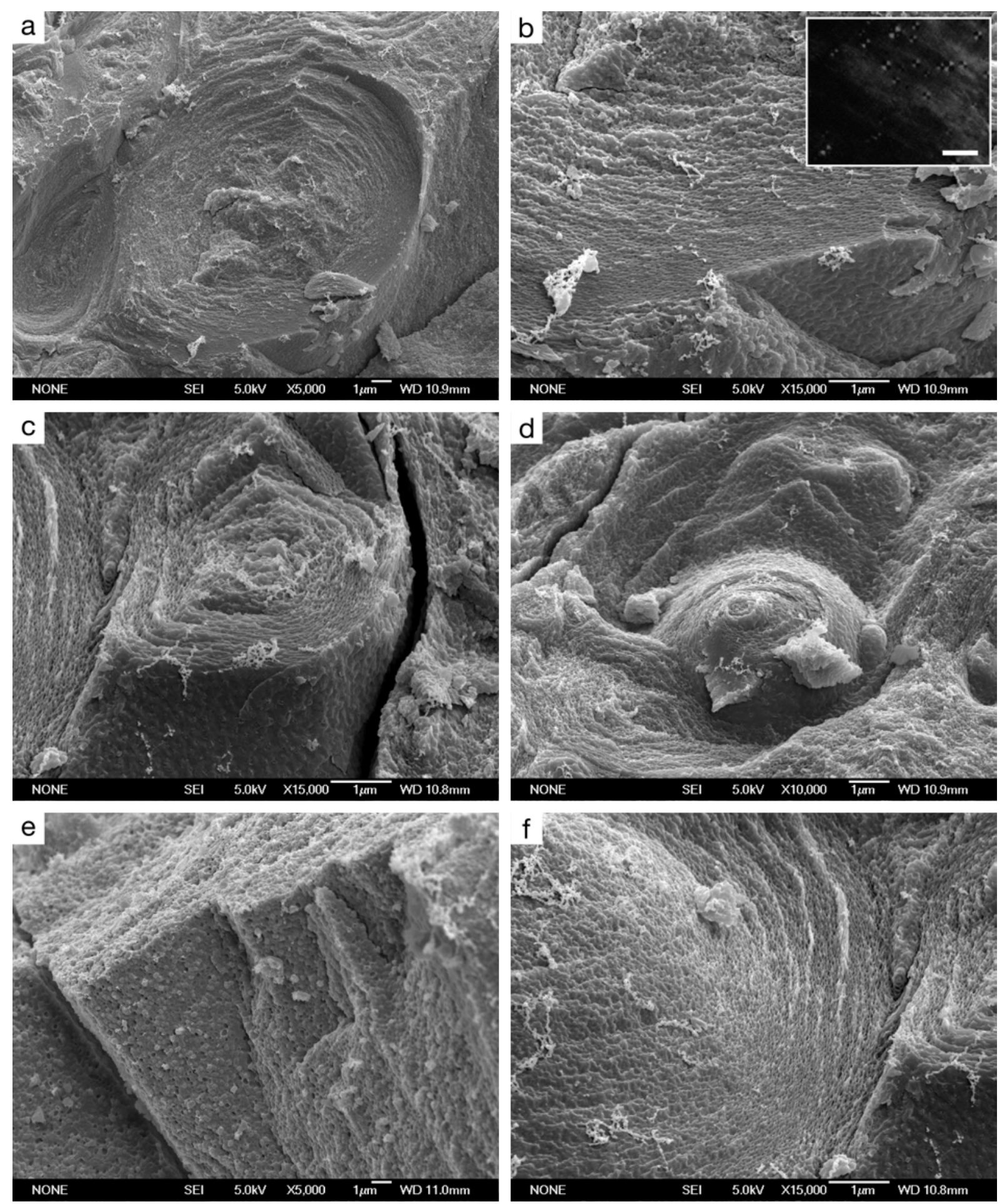

Figure 5.21: Cryo-SEM images of a sample of $30 \mathrm{wt} \% \mathrm{Li}$-AOT. The inset shows the corresponding POM image, with a scale bar of $50 \mu \mathrm{m}$.

illustrates the need for careful consideration when assigning the microstructure from cryo-SEM images.

Figure 5.22 shows cryo-SEM images obtained for a sample of $50 \mathrm{wt} \%$ Li-AOT. At this concentration, the system presents two sharp SAXS peaks, characteristic of a classical $L_{\alpha}$ phase, while the POM reveals that the main type of point defect is that of FCD-I defects aligned in oily streaks, but that some FCD-II defects also persist. The POM results showed that the 

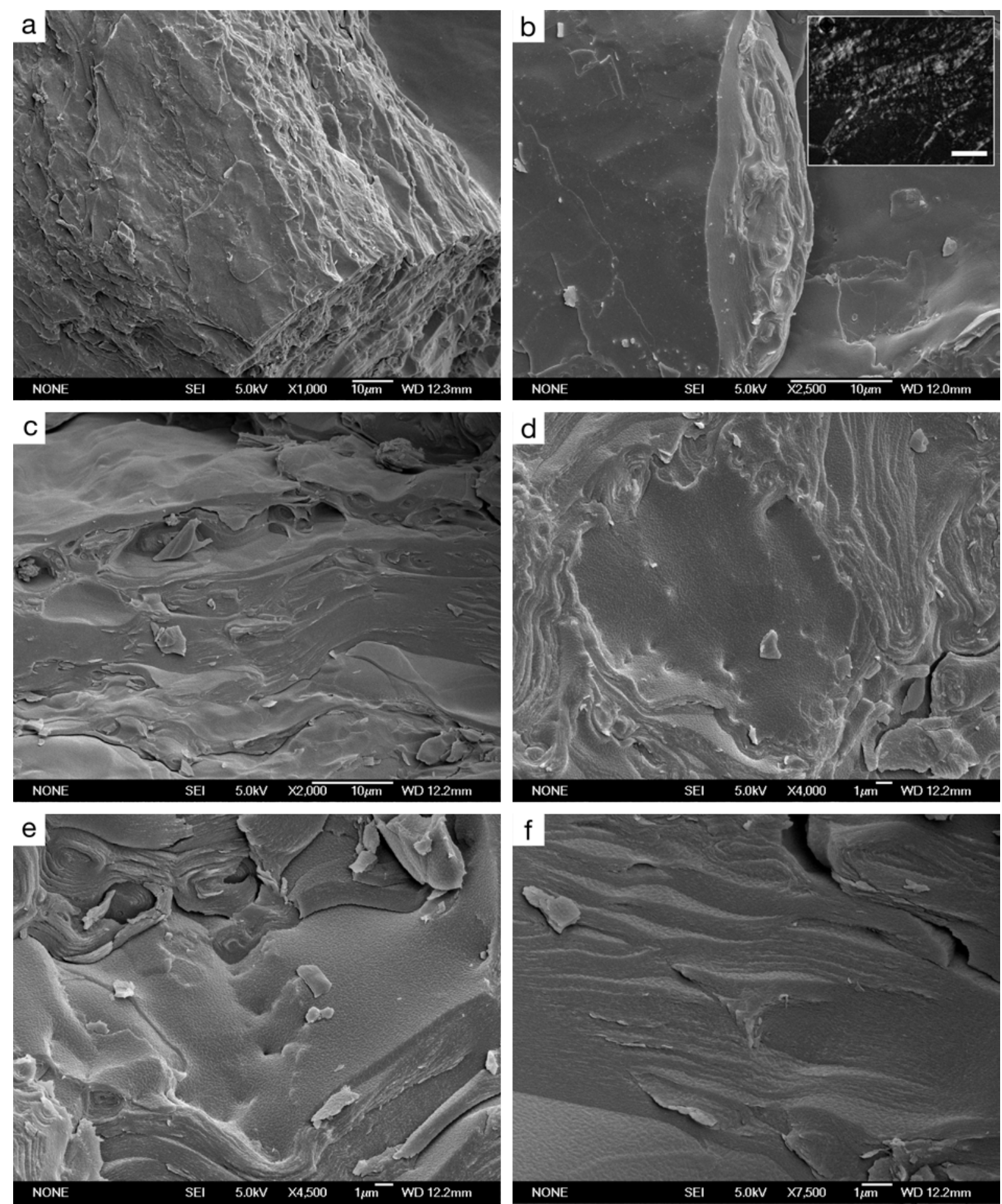

Figure 5.22: Cryo-SEM images of a sample of 50 wt \% Li-AOT. The inset shows the corresponding POM image, with a scale bar of $50 \mu \mathrm{m}$.

Li-AOT system does not form mosaic textures, but undergoes a drastic change at $~ 56 \mathrm{wt} \%$ to a viscous $V_{\|}$phase, formed via a small two-phase region of lower viscosity (due to the unfavourable mixing of two phases of different solubilities). The images in Figure 5.22 are consistent with the POM images, and the structure is observed to form domains of directional order, as well as small groups of oily streaks, particularly at the boundaries between domains. 
Figure 5.22 (a) is a low magnification image and shows that there are ordered stacks of lamellar bilayers in the Li-AOT system, but also that the domains are disrupted by various disordered regions and defective structures. The slightly higher magnification image, Figure 5.22 (b), shows what appears to be a relatively flat and ordered domain when viewed from above; however, a fracture plane reveals a cross-section and the domain is seen to be punctured by an array of disordered defects. Figure 5.22 (c) provides significantly more information about the system as it simultaneously shows many different fracture planes, and different types of defects. There are obvious regions of localised directional ordering; however, this is disrupted by clusters of defects and different domains, and the direction of the local ordering is frequently randomly altered due to series of edge defects.

The images in Figure $5.22(\mathrm{~d}-\mathrm{f})$ were captured at slightly higher magnification, and so more clearly resolve the surface topology, which is uneven due to some degree of bilayer undulation. However, in general they show the same behaviour, which is that the sample forms randomly orientated domains, bordered by grain boundaries composed of defects. Figure 5.22 (d) illustrates a small domain, which is surrounded by a range of defect structures and borders domains of different orientations, with regions ordered both approximately parallel and perpendicular to the central domain evident. While the phase does show ordered regions of relatively flat bilayers, there are also regions of curvature (predominantly negative); however, this is in the form of oily streaks or small clusters of distorted defects. They are not close-packed in mosaiclike arrays as observed prior to the $V_{\text {II }}$ phase for the systems detailed in Chapters 3 and 4 . Instead, the defect structures of the phase, such as the pits observed in Figure 5.22 (e) and the edge and screw dislocations in Figure 5.22 (f), are consistent with the defect structures reported in the previous systems at comparable concentrations.

In general terms, the structure appears slightly more ordered than those observed at equivalent surfactant concentrations in the Na-AOT and Na-butylAMA systems. However, bilayer connectivity and some degree of undulation are still evident, and the structure in no way resembles that of the Na-AOT, Na-butylAMA or K-butylAMA systems at similarly close proximity to their $\mathrm{L}_{\alpha}-\mathrm{V}_{\text {II }}$ phase boundaries. Some saddle-splay surfaces can be observed, particularly in Figure 5.22 (c) and (e), but overall there is not a high degree of curvature evident. Areas of directional order along a single director axis are evident and there is a relatively low number of point defects or curved domains present. This suggests that the Li-AOT system undergoes a radical change in curvature (and thus in the components of bilayer elasticity) at a certain $d$ spacing. For the interfacial curvature of the system to significantly change within a small concentration range indicates that the molecule likely undergoes a structural reorganisation. Given that the previous comparison of the Na-AOT and Na-butyIAMA systems indicates the effective flexibility induced by the AOT tail branches is limited to lower surfactant concentrations (where the Li-AOT and $\mathrm{Na}$-AOT systems are very similar), the most likely cause of a structural rearrangement at the higher concentration of $\sim 56 \mathrm{wt} \%$ is through a change in the counterion. This could be a change in effective size due to the partial loss of its hydration shell and/or due to an increase in binding affinity that results in decreased dissociation of the $\mathrm{Li}^{+}$ion (and thus also results in changes at the hydrophobic/hydrophilic interface arising from the altered geometric and intermolecular force contributions). 


\section{Potassium and Lithium AOT: Summary}

In this chapter, two new phase diagrams have been produced for AOT surfactant systems, which highlight the role of the counterion in phase stability. However, it should be noted that these AOT lamellar phases are highly defective and there are still several open questions regarding these systems. As such, these diagrams may at some point be altered to include the identification of different intermediate structures or to conform to new naming conventions. Surfactant systems lie at the boundary of physics, mathematics, chemistry and biology and so are of wide interest. As such, they are investigated with a range of different techniques and explained with different focuses. There exist many excellent theoretical studies of liquid crystals, and the number of proposed lyotropic phases and space groups far exceeds the number confirmed experimentally. One reason for this is that ideal structures do not truly exist. The primary focus of this work was the investigation of the lamellar structure, which is stabilised in AOT systems through the formation of many varied defect structures. With the absence of any first-order phase transitions between the different lamellar morphologies, all of these defective lamellar phases are designated with $L_{\alpha}$ notation, but discussed in terms of disorder and swelling. However, we note that other researchers occasionally choose to assign the disordered swollen lamellar phase with a specific notation.

Lamellar variants, such as swollen lamellar phases, lamellar gels and disordered mesh phases are all topologically different in their reported idealised forms. However, they all contain similar structural defects, and given that a $L_{\alpha}$ phase with no topological defects does not exist, how many pores must a $L_{\alpha}$ phase contain before its classification changes? At present, phases are often differentiated by X-ray data; however, the fundamental spacings in these phases produce the same main SAXS reflections. There is a need for experimental data that allow visualisation of the sample topography, and thus permit different classifications and different topological variants to be distinguished (rather than such classifications being dependent upon whether the X-ray data are of high enough quality to show significant deviation from ideal $L_{\alpha}$ scattering). Self-assembled structures are of fundamental importance and as new structural morphologies/variations are realised (there are likely many to be found based on complicated ordered interwoven domains or as a result of chirality ${ }^{16}$ ), studies such as this (which show the topology of samples) become increasingly important. We have deliberately included a large number of cryo-SEM images to truly show the sample topology at various lengthscales. These data are lacking within the literature, where reports often do not include EM images or supply only a few (likely carefully selected) images to confirm their assignments. It may be that the less ideal images provide additional information that can help unlock the topological unknowns. We have not been able to explain the phase behaviour of the Li-AOT and K-AOT systems in their entirety, and unfortunately it is the viscous and isotropic phases (that POM does not reveal the nature of) that we were unable to obtain reliable cryo-SEM images or temperature-based SAXS studies for. However, the work in this chapter significantly adds to that of the previous chapters, where we showed the AOT molecule to be highly flexible due to tail branching. This chapter shows that this molecular flexibility can be enhanced or restricted by altering the counterion, as 
the counterion can be selected to either increase or decrease variation in electrostatic shielding, hydration or steric bulk.

$\mathrm{Li}^{+}$and $\mathrm{K}^{+}$were selected for study as they lie either side of $\mathrm{Na}^{+}$in the Hoffmeister series. If this series was followed, then the phase behaviour of $\mathrm{Na}$-AOT would sit between that of the other two. This appears to be the case at concentrations below $~ 55$ wt \% surfactant. The Li-AOT system forms large MLVs and a swollen lamellar phase with positive Gaussian curvature, whereas the K-AOT system adopts more negative average interfacial curvature and favours the formation of $a L_{3}$ phase. However, above this concentration both K-AOT and Li-AOT form structures requiring negative curvature $\left(V_{/ /}\right.$phases $)$, while $a L_{\alpha}$ structure persists in the Na-AOT system. SAXS and POM data for the Li-AOT system imply a change occurs at a specific bilayer spacing that promotes a $L_{\alpha}-$ la3d transition.

The phase behaviour of the K-AOT system can be rationalised relatively simply. The small size of the hydrated ion and increased electrostatic shielding due to the high affinity for the sulfonate headgroup result in the $\mathrm{K}^{+}$ion forming phases with negative interfacial curvature. This combination of steric and electronic effects increases the negative spontaneous curvature of both monolayers that comprise the bilayer. The contribution of the monolayers to the magnitude of $\bar{\kappa}$ becomes more positive and the curvature frustration associated with being in a bilayer increases. Each monolayer has a greater need to curve away from the other and so in comparison with previous systems the K-AOT bilayer has a greater energy cost associated with the formation of bilayers. The highly defective lamellar structures observed in the Na-AOT and Li-AOT systems are no longer sufficient to meet the energy cost in the K-AOT system. The disordered $L_{3}$ morphology now has the lower energy cost and so becomes the energetically stable phase. As the surfactant concentration increases, the curvature becomes increasingly negative and the system becomes more ordered, thus $\mathrm{V}_{/ /}$and $\mathrm{H}_{/ /}$phases are formed.

The behaviours of $\mathrm{K}^{+}$and $\mathrm{Li}^{+}$ions in water are in stark contrast. The kosmotropic $\mathrm{Li}^{+}$ion has an affinity for water and its full hydration sphere (Stokes radius) is $\sim 6.4$ times larger than its ionic radius. The maximum hydration radius of $\mathrm{K}^{+}$is only $\sim 2.5$ times larger than its ionic radius (and the difference is generally further enhanced as the ion is chaotropic and readily loses its outer hydration sphere). The hydration of the $\mathrm{Li}^{+}$ion is poorly matched with the AOT sulfonate group and so the ion will bind weakly and is more likely to dissociate. When dissociated, $\mathrm{Li}^{+}$will not restrict the surfactant tail configuration and will provide poor shielding of electrostatic forces. When weakly bound (but hydrated) it will also provide large steric bulk to the surfactant headgroup. However, the $\mathrm{Li}^{+}$ion will not remain fully hydrated and weakly bound at all surfactant concentrations. As the ion becomes more dehydrated with increasing concentration, it drastically changes in effective size and will bind more efficiently to the AOT anion. At high concentration, the $\mathrm{Li}^{+}$ion likely provides the smallest steric bulk of the three counterions considered in this part of the thesis, in dramatic contrast to its behaviour at dilute concentrations. This large difference may explain the behaviour of the $\mathrm{Li}$-AOT system at concentrations between these extremes. Whereas the $\mathrm{K}^{+}$will remain tightly bound to the AOT headgroup at nearly all concentrations and will only change in size (due to dehydration) gradually and continuously, the $\mathrm{Li}^{+}$ion has a large degree of freedom and will 
display different behaviours dependent upon concentration. The variation in steric contributions (to the headgroup and to the tail configuration) and the van der Waals/electrostatic contributions result in the Li-AOT phase behaviour being harder to predict. This is due to the variation in the values of $\kappa$ and $\bar{\kappa}$, which alter the bilayer flexibility and drive the formation of nonlamellar phases at some concentrations. The sudden $\mathrm{L}_{\alpha}-\mathrm{V}_{\text {/I }}$ transition may be a result of enforced counterion binding and loss of freedom upon dehydration, or conversely a result of continued freedom allowing the ion to exist in different hydration states and bind nonhomogeneously along the bilayer (and thus stabilise a curved morphology). Our results show that the molecular flexibility of the AOT molecule is heavily dependent upon the counterion used, which is represented schematically in Figures 5.23 and 5.24. 


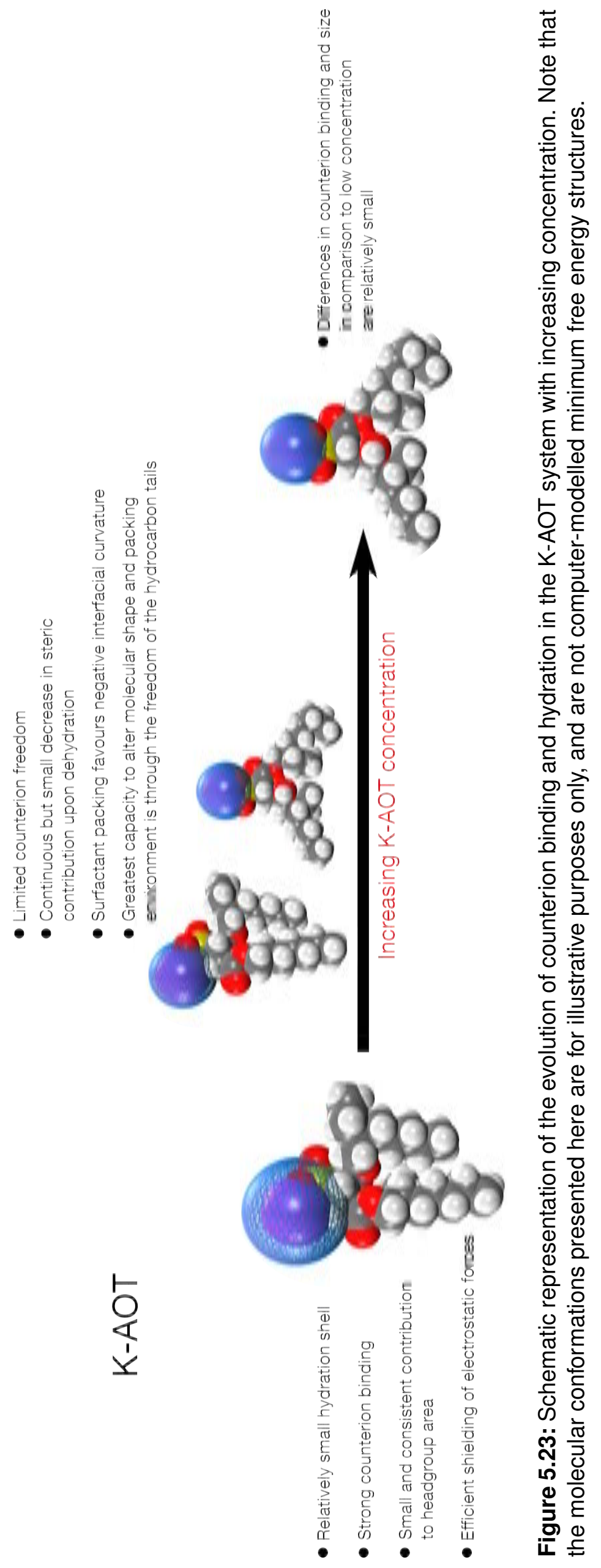




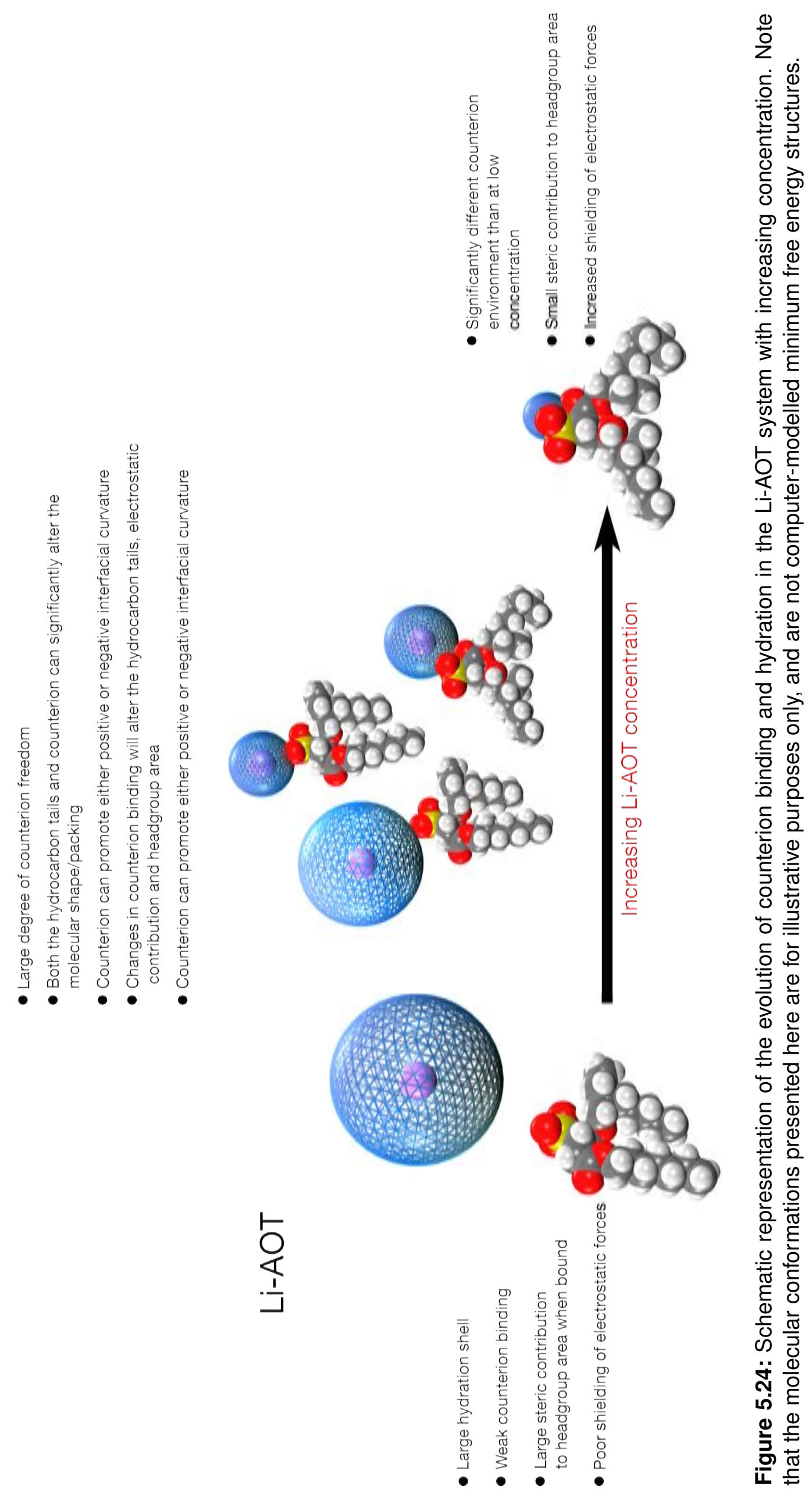




\section{Part II}

\section{Ammonium Counterions}




\section{Ammonium Counterions}

The motivation to extend the types of counterions investigated beyond the alkali metal cations to ammonium and quaternary ammonium ions stemmed from several places. Firstly, quaternary ammonium cations are already frequently used, and their role well understood, in surfactant science. They are the most common type of cationic surfactant, although they are rarely used as counterions. Moving from alkali metals to $\mathrm{NH}_{4}{ }^{+}$, and then larger molecules, also allowed us to continue the trend from the previous section of investigating the role of increasing steric bulk and electrostatic shielding of the counterion. Furthermore, ammonium ions present different degrees of hydration to the alkali metal ions, particularly due to the selection of molecules with the addition of hydroxyl groups and a carbonyl group in addition to the nitrogen-based cation.

The three ions selected - ammonium $\left(\mathrm{NH}_{4}^{+}\right)$, choline $\left(\mathrm{Ch}^{+}\right)$and acetylcholine $\left(\mathrm{AcCh}^{+}\right)$- all present the possibility of hydrogen bonding due to $\mathrm{N}-\mathrm{H}$ bonds, a hydroxyl group or a carbonyl group. The use of $\mathrm{NH}_{4}{ }^{+}$allows a direct comparison to the alkali metal systems as its reported hydrated ionic radius is the same as that of $\mathrm{K}^{+} .211$

Surfactants with quaternary ammonium cations have also been employed in two recent works that each suggest their potential use in promising fields of surfactant chemistry. Klein et al. recently used choline as a replacement counterion for sodium in the SDS system. ${ }^{60}$ This produced promising results in terms of solubility and toxicity, sparking the possibility of new "green surfactants" utilising quaternary ammonium ions that are known from biological uses to have low toxicity. However, this work was not extended to two-tailed systems such as AOT. Also, in a recent study Brown et al. reported the formation of several surfactant systems combining sulfonate anions (including AOT) with various tetraalkylammonium (TAA) cations, and asserted that such systems could be "super efficient" hydrocarbon amphiphiles. ${ }^{212}$ The systems were reported to have low surface tensions, equivalent if not superior to fluorocarbon analogues, and have the obvious benefit of being cheaper and safer alternatives to hazardous fluorinated surfactants. These systems were also reported to display lyotropic liquid crystal behaviour at room temperature upon the addition of water (in the form of optical microscopy concentration gradients), although this behaviour was not studied further in the form of phase diagrams.

Alongside any potential use in "green" chemistry applications, the three ions studied in this section were chosen due to their biological relevance. All three are known to have important roles in human biology, and both choline and acetylcholine are key components of important phospholipids. The additional functionalities in these two cations also introduces greater physiological degradability ${ }^{213}$ as compared with other quaternary ammonium ions, such as the TAA series investigated by Brown. ${ }^{212}$ 


\section{Chapter 6}

\section{Ammonium Aerosol OT}

\section{Ammonium AOT: Introduction}

The ammonium ion is of biological importance; however, a full discussion of its various roles is far beyond the scope of this work. A detailed review on the topic of metabolism of ammonium in the human body has been produced by Adeva et al. ${ }^{214}$ Although often referred to as a waste product, due to the removal of ammonium ions being an important step in the urea cycle, $\mathrm{NH}_{4}{ }^{+}$ ions are continuously being produced and consumed during cell metabolism. A source of free ammonium ions in the human body is vital for the synthesis of nitrogen-containing compounds such as amino acids. Ammonium is thought to be transported around the body by the same channels as $\mathrm{K}^{+}$ions, due to their similar size. The ability of the human body to differentiate between $\mathrm{Na}^{+}, \mathrm{K}^{+}$and $\mathrm{NH}_{4}{ }^{+}$is of fundamental importance to many cellular functions. ${ }^{215}$

Of the few publications containing $\mathrm{NH}_{4}$-AOT (a recent literature survey ${ }^{\ddagger}$ produced just 11 journal publications), only the work by Brown et al. ${ }^{212}$ alludes to any liquid crystal behaviour and only two others provide any information of direct relevance to the work reported here. One of these studies showed differing water dynamics in reverse micellar samples of AOT/isooctane/water systems with $\mathrm{NH}_{4}{ }^{+}$and $\mathrm{Na}^{+}$counterions. ${ }^{56}$ It was reported that water molecules in the former system were significantly more dynamic than those in the latter. Chakraborty et al. calculated CMC values showing a decreasing trend in the order $\mathrm{NH}_{4}{ }^{+}>\mathrm{Na}^{+}>\mathrm{Li}^{+}>\mathrm{K}^{+}$. The abnormal position of K-AOT was reasoned to be due to the formation of strong ion pairs. ${ }^{183}$

\footnotetext{
‡Literature search conducted October 2016.
} 


\section{Ammonium AOT: Experimental Results}

\section{Ammonium AOT Phase Behaviour}

A phase diagram for the $\mathrm{NH}_{4}$-AOT system is shown in Figure 6.1. At room temperature a twophase region presents below $20 \mathrm{wt} \%$ surfactant and a $\mathrm{L}_{\alpha}$ phase then extends from 20 to 72 wt \%. $A V_{/ l}$ phase then exists up to $83 \mathrm{wt} \%$, and a $\mathrm{H}_{/ /}$phase at all concentrations above this. The system presents one temperature-dependent transition between lyotropic phases. Samples at the more dilute end of the $V_{/ l}$ phase (72-78 wt \%) form a $L_{\alpha}$ phase at $\sim 90{ }^{\circ} \mathrm{C}$.

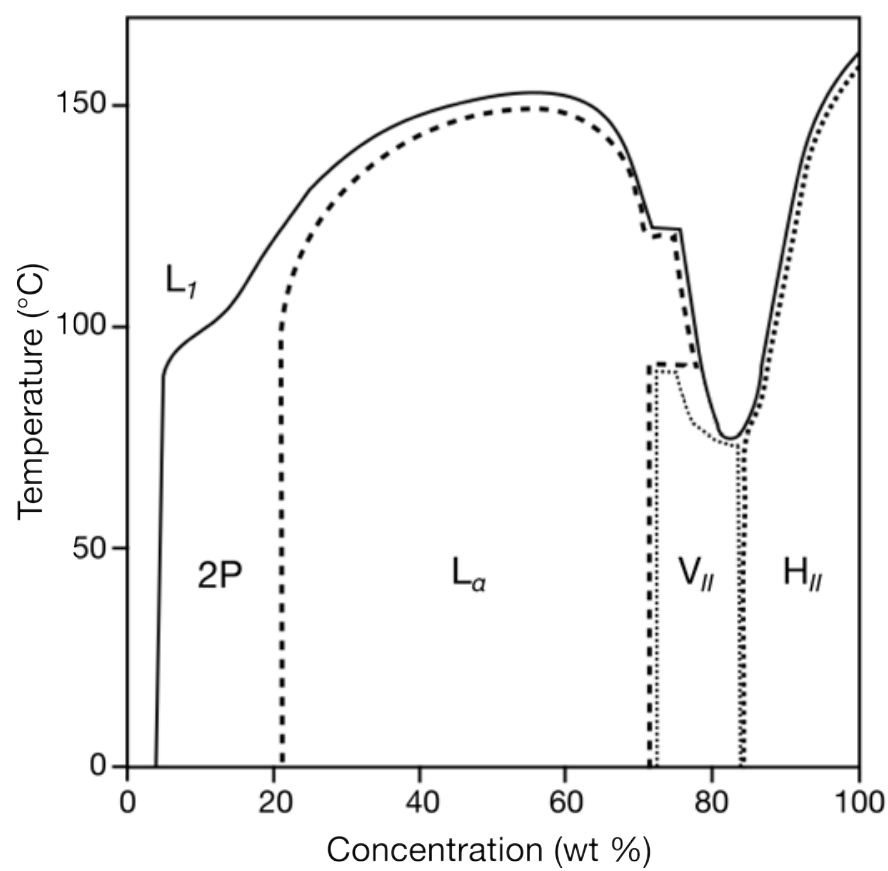

Figure 6.1: Phase diagram of $\mathrm{NH}_{4}$-AOT, showing a two-phase region and lamellar, inverse bicontinuous cubic and inverse hexagonal phases.

The reported behaviour of the $V_{\| l}$ phase at high temperature is clearly anomalous. The melting transition from the $V_{\|}$phase is discontinuous and samples at a concentration of $\sim 77.5 \mathrm{wt} \%$ were observed to form lamellar defects at lower temperatures than samples of lower $\mathrm{NH}_{4}$-AOT concentrations, while those at higher concentration formed an isotropic solution. This behaviour was reproducible upon an increase in temperature for bulk and POM samples. However, samples showed hysteresis upon cooling due to the kinetically slow formation of cubic phases. ${ }^{216}$ It is likely that the slow kinetics involved in the formation of cubic phases is the cause for the peculiar (and likely non-equilibrium) behaviour observed in the phase diagram. Cubic samples at lower surfactant concentrations, that form a lamellar phase upon heating, likely have a $V_{I I} V_{\text {II }}$ transition within the cubic region or form kinetically hindered intermediate structures, while those at higher concentrations do not. Samples at intermediate concentrations ( 77.5 wt \%) would likely be composed of a mixture of the two $V_{/ /}$phases, and so have different stability and kinetic behaviour upon heating. However, evidence for phase transitions within this 
region was not found via any method utilised here, although this was likely due to POM being insufficient to distinguish between two isotropic phases and equipment limitations preventing SAXS analysis of viscous phases at high temperatures.

Although the phase diagram indicates that the lamellar phase is stable up to temperatures as high as $150{ }^{\circ} \mathrm{C}$, bulk samples were observed to discolour at temperatures over $120^{\circ} \mathrm{C}$, developing a yellow or orange hue. This discolouration was not phase-dependent and was retained upon cooling. This is an indication that the surfactant could be prone to temperatureinduced degradation, possibly as a result of $\mathrm{NH}_{4}{ }^{+}$being slightly acidic. As such, the system is possibly not suitable for applications requiring high temperatures. Despite the observed discolouration of the sample, neither SAXS profiles nor POM images noticeably changed after sealed samples were kept at high temperatures for one week. ${ }^{1}{ }^{1} \mathrm{H}$ NMR spectrum obtained from a sample dried under vacuum after discolouration had occurred did not indicate any detectable changes to the molecule itself.

Literature values for the ionic radii of $\mathrm{K}^{+}$and $\mathrm{NH}_{4}{ }^{+}$are similar, and even identical in the case of the hydrated ion. ${ }^{180}$ As such, it would be expected that with no additional changes, these two systems would produce very similar phase diagrams. However, it is clear upon comparison of the phase diagrams (shown in Figure 6.2 along with that of Na-AOT) that this is not the case.
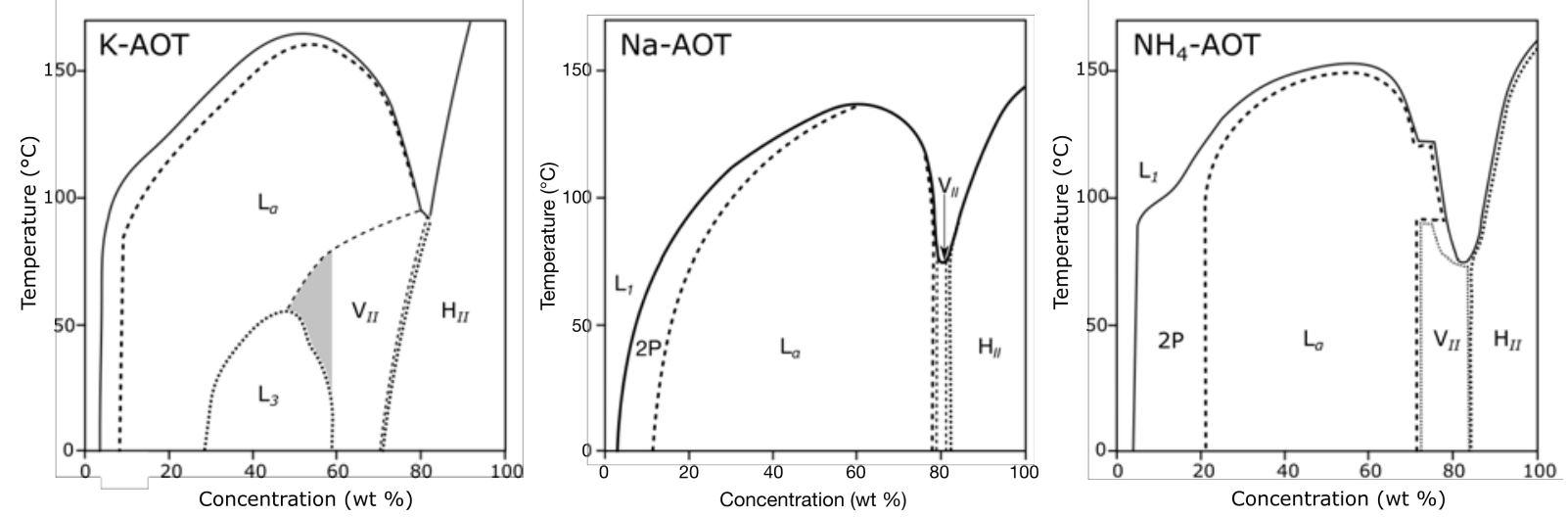

Figure 6.2: Phase diagrams of $\mathrm{K}-\mathrm{AOT}, \mathrm{Na}-\mathrm{AOT}$ and $\mathrm{NH}_{4}$-AOT.

Comparison of the Na-AOT, K-AOT and $\mathrm{NH}_{4}$-AOT phase diagrams indicates that the behaviour of the $\mathrm{NH}_{4}$-AOT system is closer to that of $\mathrm{Na}$-AOT than $\mathrm{K}$-AOT. The $\mathrm{NH}_{4}$-AOT system does not present a sponge phase, and the cubic and hexagonal phase boundaries are not extended to lower concentrations to the same extent as in the K-AOT system. At $25^{\circ} \mathrm{C}$, the only obvious differences between the $\mathrm{Na}$-AOT and $\mathrm{NH}_{4}$-AOT systems are small changes in the positioning of the $\mathrm{L}_{\alpha}$ phase boundaries. Additionally, samples in the two-phase region at low concentrations of $\mathrm{NH}_{4}$-AOT show clear phase separation and the phase extends to $20 \mathrm{wt} \%$, which is not the case for Na-AOT. However, it should also be noted that if left for several months, some of the prepared Na-AOT samples near the two-phase/lamellar boundary also exhibited phase separation. The $\mathrm{NH}_{4}$-AOT system also presents a cubic phase over a larger concentration range, extending from 72 to $84 \mathrm{wt} \%$ surfactant, in comparison to $78-82 \mathrm{wt} \%$ for the Na-AOT system, an indication that the bilayer of the $\mathrm{NH}_{4}$-AOT has more inherent curvature. 
At high temperatures, the $\mathrm{NH}_{4}$-AOT phase diagram is even more akin to that of $\mathrm{Na}-\mathrm{AOT}$, due to the presence of a $\mathrm{V}_{I I}-\mathrm{L}_{\alpha}$ transition for some concentrations of $\mathrm{NH}_{4}$-AOT. The presence of a similar transition in the K-AOT system, alongside the stabilisation of the $\mathrm{L}_{\alpha}$ phase over the $\mathrm{L}_{3}$ phase with increasing temperature, means that all three systems show similar behaviours above $85-90^{\circ} \mathrm{C}$ (notwithstanding the slight difference in melting temperature). The similarities of the phases at high temperature show that the forces stabilising the high temperature phases are less affected by the different counterions. The stabilisation of the $L_{\alpha}$ phases indicates that the bilayer curvature decreases upon heating. This is likely due to an increase in the ordering of the headgroups as a result of stronger electrostatic interactions upon dehydration of the headgroup and counterion, meaning that the counterion is less tightly bound to the sulfonate group of $\mathrm{AOT}^{217}$ and consequently has less influence in the self-assembly.

\section{Ammonium AOT SAXS}

The assignment of each phase was confirmed using SAXS, which produced results in good agreement with visual and microscopic observations. Figure 6.3 shows SAXS profiles obtained at concentration intervals of $5 \mathrm{wt} \% \mathrm{NH}_{4}$-AOT. The profiles are not significantly different in character from those observed in the alkali metal systems discussed previously.

The only sharp and assignable peak in the lower concentration region is the first-order Bragg reflection. Higher order Bragg peaks are masked by broad, diffuse scattering. With increasing concentration, the first-order peak is observed to start to diminish at $30 \mathrm{wt} \%$ and is absent at 40 wt \% surfactant. At concentrations of 45 wt \% and above, two sharp Bragg peaks are visible in a q spacing ratio of 1:2, which become sharper and increase in intensity with increasing concentration. A third Bragg peak is visible at some concentrations, but is primarily masked by diffuse scatter. With increasing concentration, the Bragg peaks are shifted to higher q spacings due to the reduction of the bilayer repeat distance. Similarly, peaks were observed to sharpen and were shifted to slightly higher q spacings with increases in temperature. This indicates a small increase in order upon heating, likely due to dehydration of the bilayer and counterion, and the subsequent changes to the headgroup conformation (increased headgroup repulsion along the bilayer makes it more rigid and less prone to curvature and undulations). However, increased motion of the hydrocarbon tails and the effective change in volume could also be contributing factors.

At $75 \mathrm{wt} \% \mathrm{NH}_{4}$-AOT, eight peaks are prominent in the spacing ratio of $\sqrt{ } 6: \sqrt{ } 8: \sqrt{ } 14: \sqrt{ } 16: \sqrt{ } 20$ : $\sqrt{ } 22: \sqrt{ } 24: \sqrt{ } 26$, representing la3d cubic symmetry. Despite the bicontinuous cubic phase spanning a concentration range of $\sim 12 \mathrm{wt} \%$, no other space groups were observed. The well resolved peaks in Figure 6.3 clearly indicate that this is the case for samples at $25{ }^{\circ} \mathrm{C}$; however, the discontinuity in the $\mathrm{V}_{\|}$melting transition upon increasing concentration (from POM observations) implies that different space groups, or intermediate phases, are formed at higher temperatures. Unfortunately, the high viscosity of the $V_{\text {/l }}$ samples necessitated the use of a paste cell for SAXS analysis, which did not provide sufficient sealing at high temperatures, and so changes in sample composition upon heating made the elucidation of different structures 

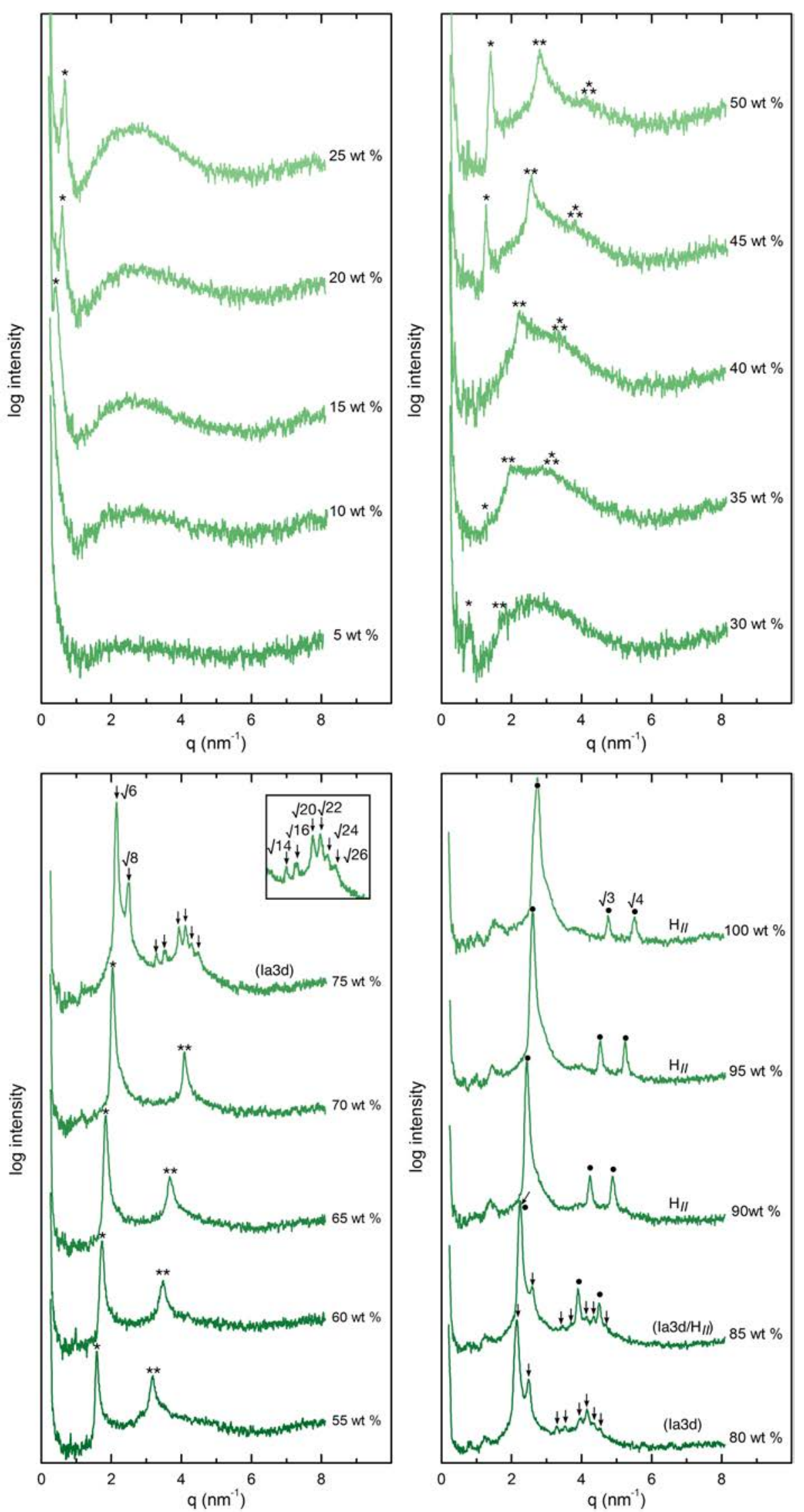

Figure 6.3: $\mathrm{SAXS}$ profiles of the $\mathrm{NH}_{4}$-AOT system with increasing surfactant concentration. Bragg peaks due to the $\mathrm{L}_{\alpha}$ phase are evident up to $70 \mathrm{wt} \% \mathrm{NH}_{4}-\mathrm{AOT}$ (asterisks). $\mathrm{A} \mathrm{V}_{\text {/I }}$ phase is observed at 75 and 80 wt \% (arrows), a mixture of $\mathrm{V}_{/ /}$and $\mathrm{H}_{/ /}$phases at $85 \mathrm{wt} \%$ and finally a pure $\mathrm{H}_{/ /}$phase exists up to $100 \mathrm{wt} \% \mathrm{NH}_{4}$-AOT (dots). Inset shows peak assignment of la3d space group at $75 \mathrm{wt} \%$. 
impossible. Attempts were made to load the less viscous, high temperature lamellar phase into a flow cell. However, these attempts were unsuccessful, in part due to the proximity of the transition region to the recommended temperature limit of the cell. If cubic phases of an la3d space group form another cubic phase upon heating, then the logical progression would imply that the phase formed would have a Pn3m space group and would form upon swelling of the la3d phase. ${ }^{14}$ Samples of higher concentrations may not form this phase due to an insufficient number of water molecules.

Moving from the cubic to hexagonal phase, the reflections of the cubic phase give way to three Bragg peaks in the ratio $1: \sqrt{ } 3: \sqrt{ } 4$. At $85 \mathrm{wt} \%$, in proximity to the $\mathrm{V}_{/ /}-\mathrm{H}_{/ /}$phase boundary, the system primarily displays hexagonal order, but also retains some low intensity peaks of the cubic phase (as evidenced by Figure 6.4). The gradual change in peak intensity shows the epitaxial relationship between the two phases and the growth of the hexagonal columns along the [111] plane of the cubic structure, as described elsewhere. ${ }^{177,218}$ Comparison of the profiles in the two phases indicates that the cubic reflections arising due to the [211], [420] and [431] planes gradually give way to the [100], [110] and [200] reflections of the hexagonal phase.
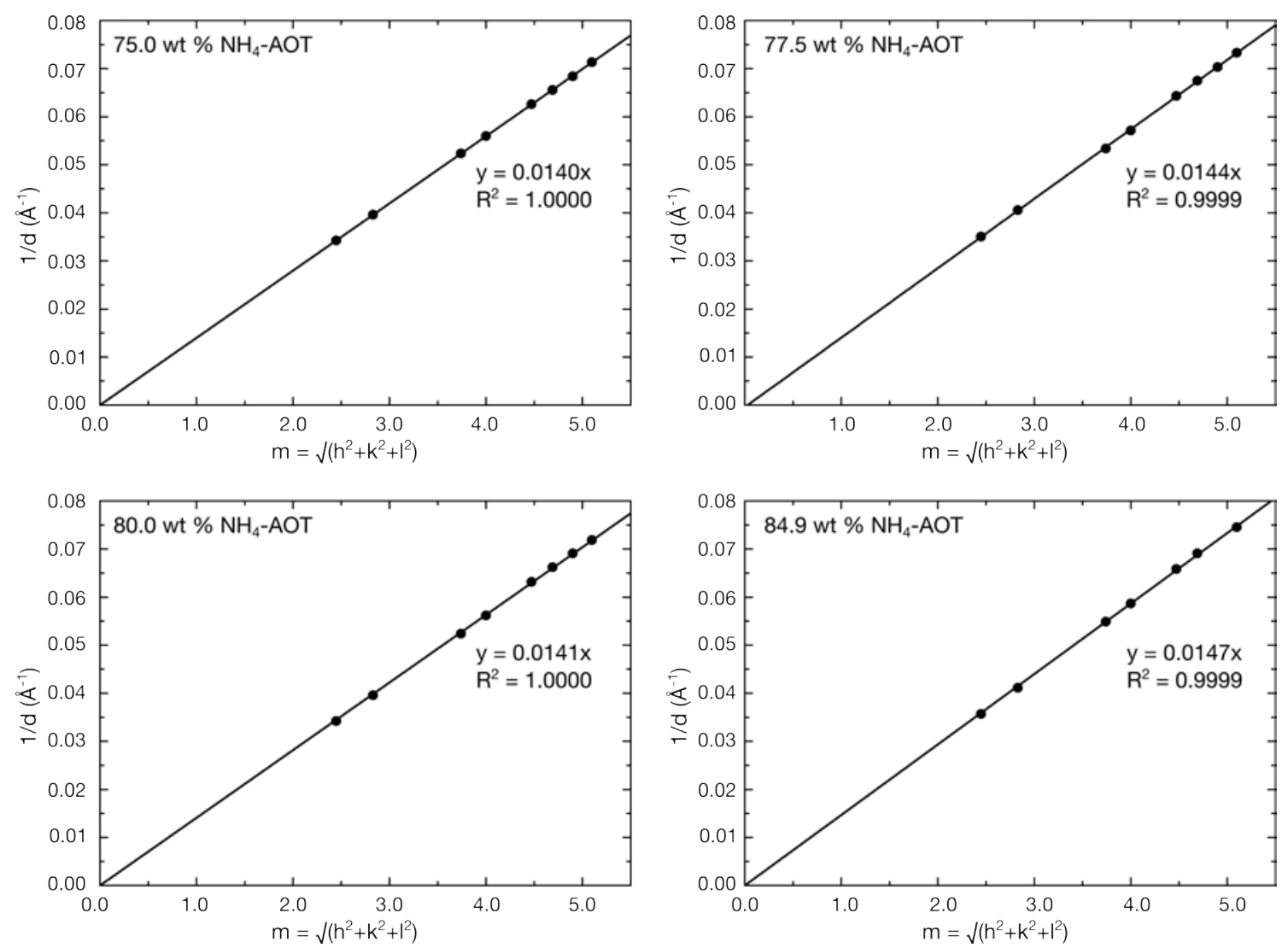

Figure 6.4: Plots showing the reciprocal d spacings vs. $\sqrt{ }\left(h^{2}+k^{2}+l^{2}\right)$ for the cubic phases of the $\mathrm{NH}_{4}$-AOT system, where hkl are the assigned Miller indices. Correct assignment of the lattice type and space groups is confirmed by the linearity of each plot and the intercept of the fitted line at the origin. The lattice parameter for each graph is calculated as the reciprocal of the gradient of each fit. 


\section{Ammonium AOT Optical Microscopy}

Polarising optical microscopy images of the $\mathrm{NH}_{4}$-AOT system are shown in Figure 6.5. The overall trend is recognisable as being the same as that observed for both the Na-AOT and K-butylAMA systems, with a gradual change from large focal conic onion defects to oily streaks of differing Gaussian curvature, and then mosaic-like textures due to close-packing of streaks.

At concentrations below $10 \mathrm{wt} \%$, the textures are predominantly isotropic with a few areas exhibiting anisotropy, but few singular defect points are discernible. ${ }^{\ddagger}$ With increasing concentration, the appearance of FCD-II defects of varying size is evident. These defects increase in number and become less isolated. Above $25 \mathrm{wt} \%$ they are significantly larger and reduced in number. At these concentrations the gradual appearance of oily streaks is also evident alongside the FCD-II defects. The oily streaks then start to prevail and the loss of birefringence seen in the previous systems is observed, although areas of each of the previous behaviours can be seen. Once positive birefringence is observed above $50 \mathrm{wt} \%$, only oily streaks are present. These streaks become shorter, and obvious nodes at the junctions of the streaks and the FCD-I defects that form them are seen. The streaks eventually give way to mosaic textures of tightly packed and distorted defects, as seen for the high concentration lamellar regions of all of the previous systems (excluding Li-AOT). At this point the curvature of the system has inverted and there are no longer any large isotropic domains due to ordered sheets of lamellae.

At concentrations between 72 and $83 \mathrm{wt} \%$, the textures appear black due to the isotropic nature of the pure bicontinuous phase. At higher concentrations, the textures are brightly coloured and exhibit the fan-like pattern of the hexagonal phase. Variations in colour are due to the thickness of the sample. The variation in domain sizes and the non-uniform alignment in the phase are due to the simultaneous growth of multiple nucleation points. Focal conic surfaces are formed to achieve minimal strain. Distinct lines between domains are due to the changing curvature around line disclinations of $s= \pm 1 / 2$. The degree of orientation in each texture varies greatly, but is defined by the formation of the phase on the slide rather than a changing property of the hexagonal phase. Due to the stability of the phase once formed, shear-induced structures are retained and so no information is obtained about how the defects in this system change with concentration.

As would be expected from the reported phase diagrams, comparison of POM images of the $\mathrm{NH}_{4}$-AOT system with those previously reported for the Na-AOT and K-AOT systems reveals the defect behaviour of the $\mathrm{NH}_{4}$-AOT lamellar phase to much more closely resemble that of the sodium system. Although these two systems follow the same general trends, there are several differences, primarily at lower surfactant concentrations. The FCD-II defects observed in $\mathrm{NH}_{4}$-AOT are predominantly larger in size and less distorted than those observed in Na-AOT. There appears to be significantly more FCD-II defects in the $\mathrm{NH}_{4}$-AOT system, although this is due to their larger size. The smaller Na-AOT defects are below the limit of the optical resolution of the microscope used and so are not as well resolved. The change in birefringence and

\footnotetext{
‡POM image of 5 wt \% $\mathrm{NH}_{4}$-AOT can be found in Appendix $\mathrm{C}$.
} 

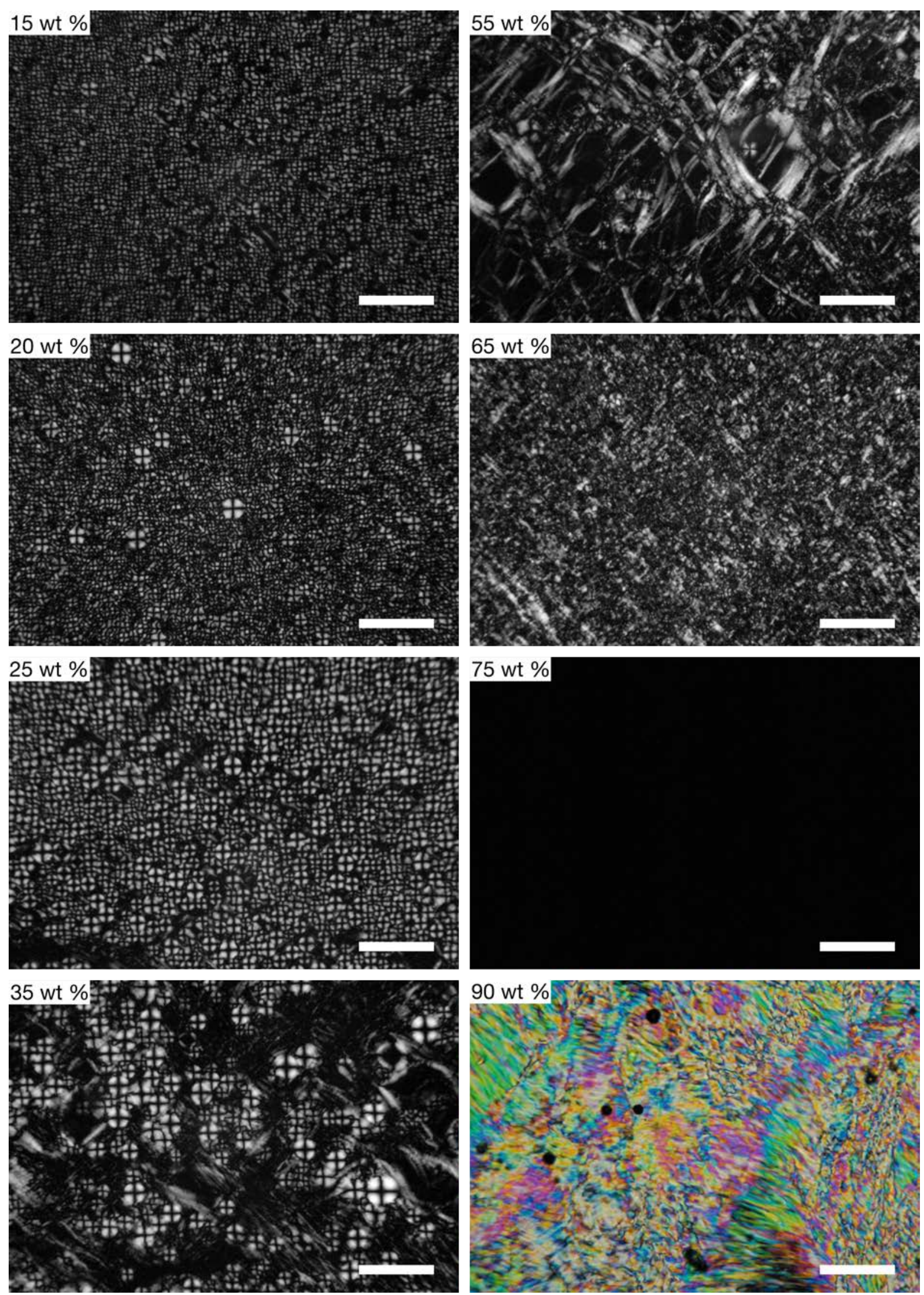

Figure 6.5: POM images of $\mathrm{NH}_{4}$-AOT with increasing concentration at $\sim 25^{\circ} \mathrm{C}$. FCD-II defects of varying sizes dominate the $L_{\alpha}$ phase at low concentrations, gradually giving way to streaky textures composed of FCD-I defects, then mosaic patterns due to densely packed defects with high degrees of curvature. The isotropic and coloured fan textures represent $V_{/ /}$and $H_{/ /}$phases, respectively. Scale bars are $100 \mu \mathrm{m}$. Additional images are available in Appendix C. 
emergence of oily streaks occur at higher surfactant concentrations in the $\mathrm{NH}_{4}$-AOT system. This can be attributed to small differences in the bilayer spacings of the two systems. The more tightly bound $\mathrm{NH}_{4}{ }^{+}$counterion will result in more efficient shielding of the charge on the sulfonate group, resulting in a reduction in electrostatic repulsion between headgroups. This will allow greater lateral movement of the headgroups in the $\mathrm{NH}_{4}$-AOT system, and so will result in reduced bilayer rigidity and increased out-of-plane fluctuations, and so a larger contribution of Helfrich forces. These differences can be visualised by comparing textures of $43 \mathrm{wt} \% \mathrm{NH}_{4}$-AOT and 50 wt \% Na-AOT, which are shown in Figure 6.6 and appear equivalent. These samples both show POM images characteristic of samples of low viscosity, with the textures dominated by features of flow. This decrease in viscosity indicates a defect-rich microstructure, where flow is promoted due to a sponge-like topology, and has been measured using rheology, which is discussed further in Appendix E.
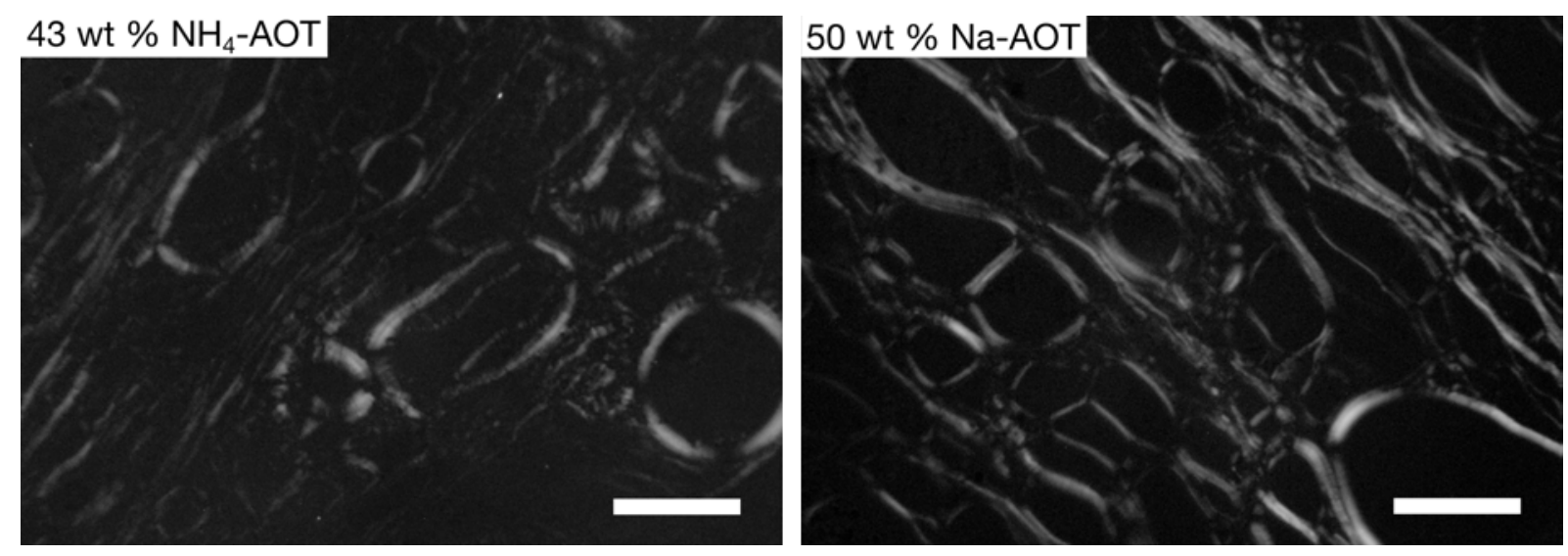

Figure 6.6: Comparison of the POM textures of $\mathrm{NH}_{4}$-AOT at 43 wt \% (left) and $\mathrm{Na}$-AOT at $50 \mathrm{wt} \%$ (right) at $\sim 25^{\circ} \mathrm{C}$. Scale bars are $100 \mu \mathrm{m}$.

The defects observed in the K-AOT lamellar phase are significantly different from those of both $\mathrm{Na}-\mathrm{AOT}$ and $\mathrm{NH}_{4}$-AOT. Defects with on average negative Gaussian curvature appear at much lower surfactant concentrations. FCD-I and FCD-II defects coexist at surfactant concentrations as low as $20 \mathrm{wt} \%$ and oily streaks dominate at $30 \mathrm{wt} \%$ facilitating the formation of a sponge phase. In the $\mathrm{NH}_{4}$-AOT system, oily streaks prevail from $50 \mathrm{wt} \%$.

\section{Ammonium AOT Cryo-SEM}

Cryo-SEM images, taken at a range of $\mathrm{NH}_{4}$-AOT concentrations, provide information about the local curvature of the system. This complements the POM investigation that revealed the nature of point defects and how they change with concentration. Figures 6.7-6.11, captured between 15 and 70 wt $\% \mathrm{NH}_{4}$-AOT, show a deviation from classic lamellar behaviour on sub-micrometre lengthscales with significant sponge-like characteristics observed at some concentrations.

Figure 6.7 shows cryo-SEM images at $15 \mathrm{wt} \% \mathrm{NH}_{4}$-AOT with a POM image of this concentration inset. This is within the two-phase region, and the POM image shows MLVs of 
approximately equal size. The cryo-SEM images are consistent with the POM, and reveal that these MLVs range between 0.2 and $5.0 \mu \mathrm{m}$. Between MLVs, a disordered surfactant phase is punctuated by a large water network comprised of channels of up to $0.5 \mu \mathrm{m}$.
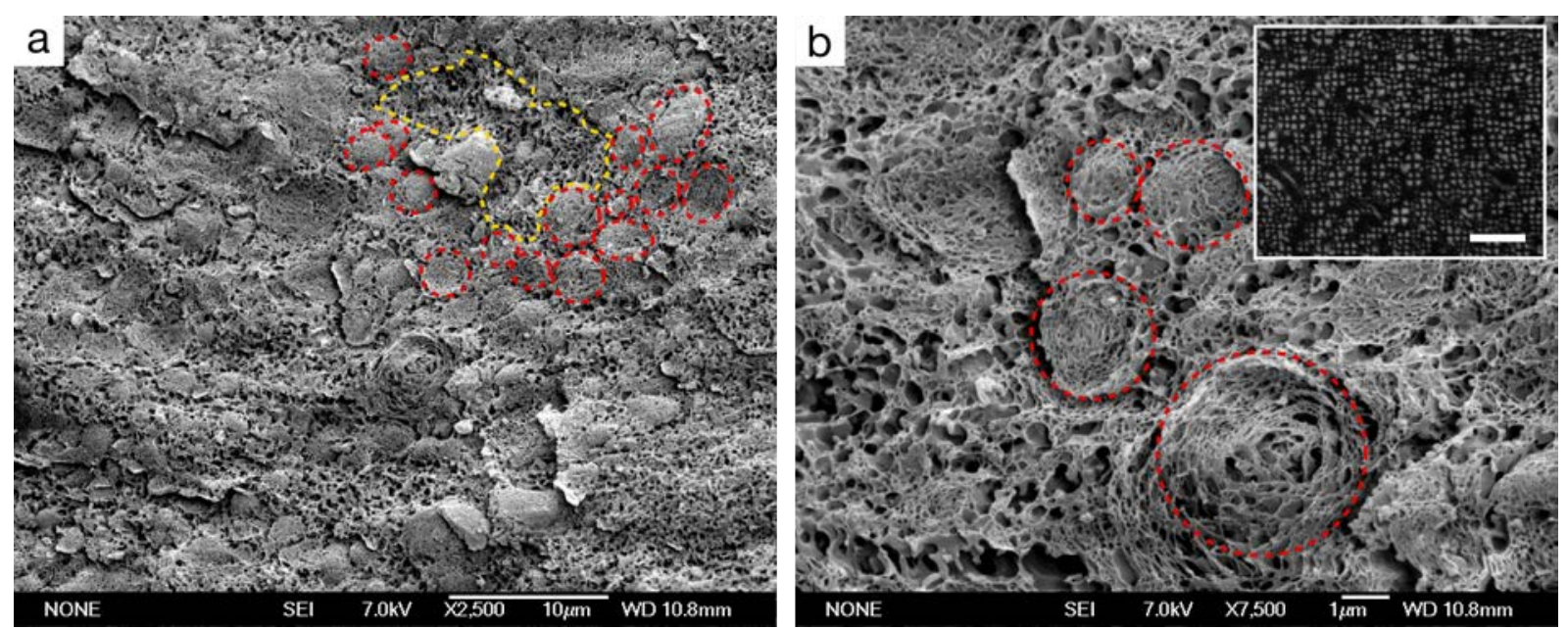

Figure 6.7: Cryo-SEM images of a sample of $15 \mathrm{wt} \% \mathrm{NH}_{4}$-AOT. The area highlighted in yellow shows a region consisting primarily of disordered surfactant phase, which is surrounded by MLVs (highlighted in red). The inset shows the corresponding POM image, with a scale bar of $50 \mu \mathrm{m}$.

At 25 wt \% $\mathrm{NH}_{4}$-AOT (see Figure 6.8), the two coexisting phases have been replaced by one continuous lamellar phase. Large water channels are no longer observed and low magnification images show lamellar regions with localised average orientational order, but clear phase boundaries are not observed. FCD-II defects are observed but are reduced in number compared with the vesicles of the two-phase region. Despite classification as a $L_{\alpha}$ phase, it is clear from the images that the phase is disordered, with high degrees of connectivity between both the bilayer and water networks. Although captured at the same magnification, Figure 6.8 (c) and (d) show differing amounts of disorder. In the former the average directional order is clear (as marked by the red arrow), whilst the latter is more disordered with a close resemblance to the sponge phase observed in the K-AOT system.

The local-scale connectivity is retained at $50 \mathrm{wt} \% \mathrm{NH}_{4}$-AOT, and is observable in the higher magnification images displayed in Figure 6.9. Although we have previously described the $\mathrm{Na}$-AOT lamellar phase as displaying $\mathrm{L}_{3}$-like characteristics, this behaviour is more pronounced in the $\mathrm{NH}_{4}$-AOT system, and is a sign of increased elasticity. Despite this difference at smaller lengthscales, lower magnification images show the $\mathrm{NH}_{4}$-AOT system contains point defects comparable to those observed at similar concentrations in the Na-AOT system. This is expected as the two systems have similar POM textures. Figure 6.9 reveals both oily streaks and individual focal conic defects up to $3 \mu \mathrm{m}$ in size. The oily streaks are not closely packed and appear to be randomly orientated.

Figure 6.10 shows cryo-SEM images of $60 \mathrm{wt} \% \mathrm{NH}_{4}$-AOT. Of all the concentrations studied, this appears the most ordered. It has the largest domains of ordered bilayers, which display clear 2D translational order. At this concentration large FCD-II defects are no longer present, 

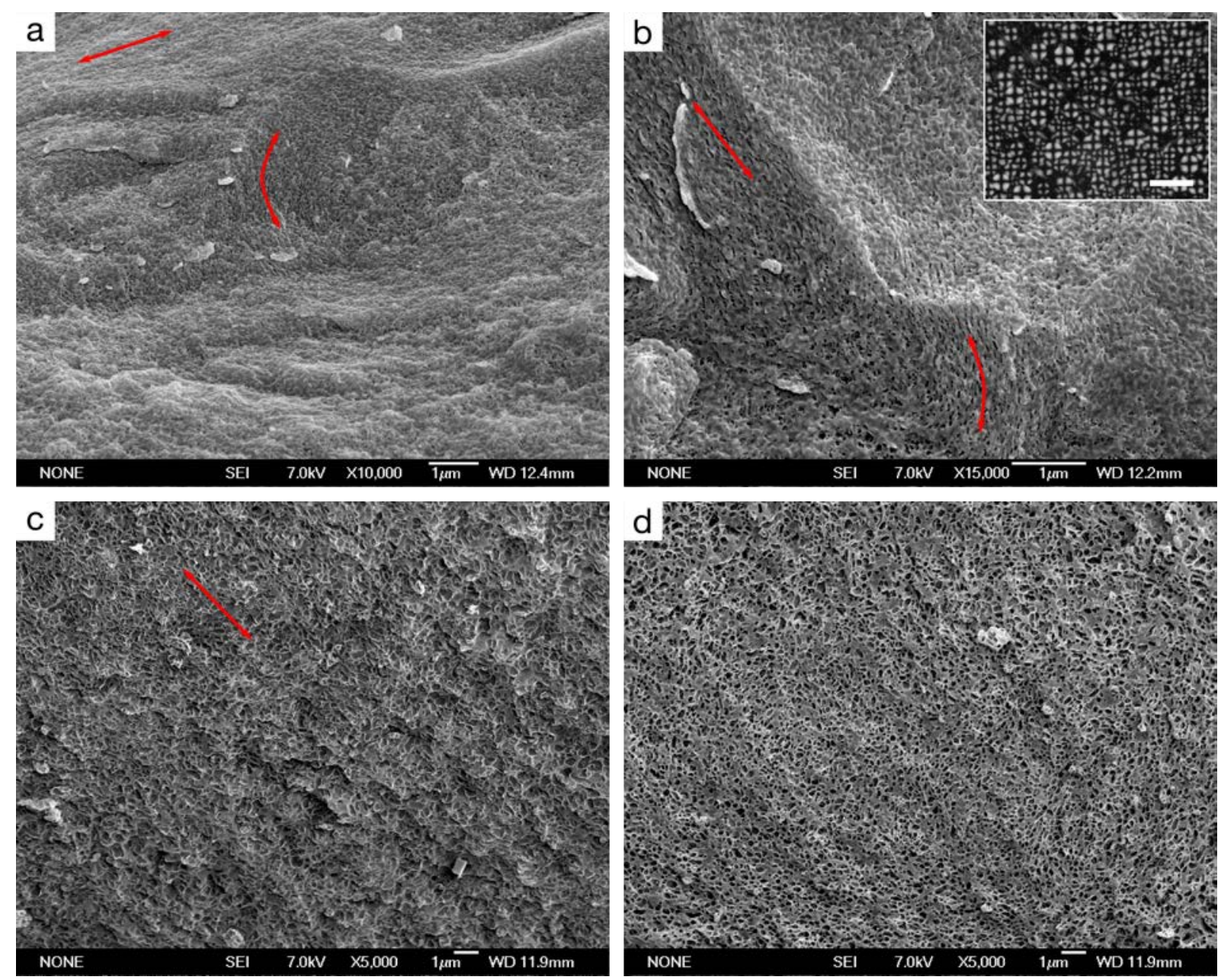

Figure 6.8: Cryo-SEM images of a sample of $25 \mathrm{wt} \% \mathrm{NH}_{4}$-AOT. Red arrows indicate localised average orientational order. The inset shows the corresponding POM image, with a scale bar of $50 \mu \mathrm{m}$.

but some oily streaks were observed. Despite samples at this concentration appearing the most ordered, bilayer undulations can still be observed from views both perpendicular and parallel to the bilayer. For the lamellar phase to transition from positive to negative Gaussian curvature with increasing concentration, it must transition through a state with on average zero Gaussian curvature. This accounts for the reduction in the number of defects observed; however, the undulations imply that the bilayer still has inherent elasticity governed by the electrostatic interactions, which differ between the systems studied. This implies a relatively low bending rigidity modulus $(\kappa)$ and a relative increase in the thermal energy contribution to the $\mathrm{NH}_{4}$-AOT system, which supports our earlier statement regarding Figure 6.6.

At 70 wt $\% \mathrm{NH}_{4}$-AOT, the images show dense arrays of close-packed point defects and oily streaks. Figure 6.11 shows a clear increase in curvature in comparison to samples of $60 \mathrm{wt} \%$. Despite the high concentration and resulting decrease in the bilayer repeat distance, bilayer undulation and significant amounts of connectivity between layers and domains is evident. This concentration is in close proximity to the $L_{\alpha}-V_{/ /}$phase boundary, which may explain the sharp grain boundaries separating domains, and also the high degree of curvature. 

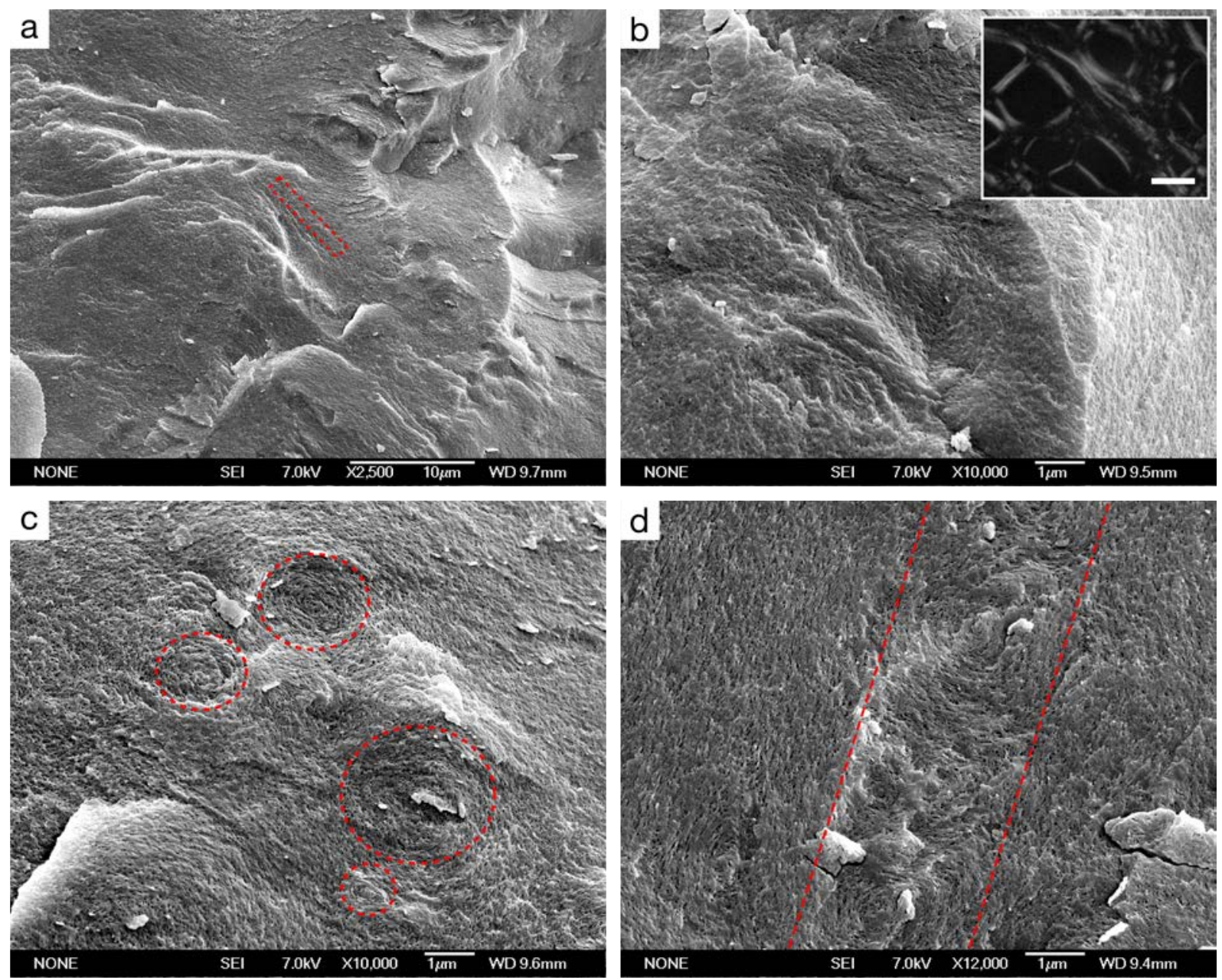

Figure 6.9: Cryo-SEM images of a sample of 50 wt \% $\mathrm{NH}_{4}$-AOT. Straight red lines indicate isolated oily streaks. Red circles indicate FCDs. The inset shows the corresponding POM image, with a scale bar of $50 \mu \mathrm{m}$.
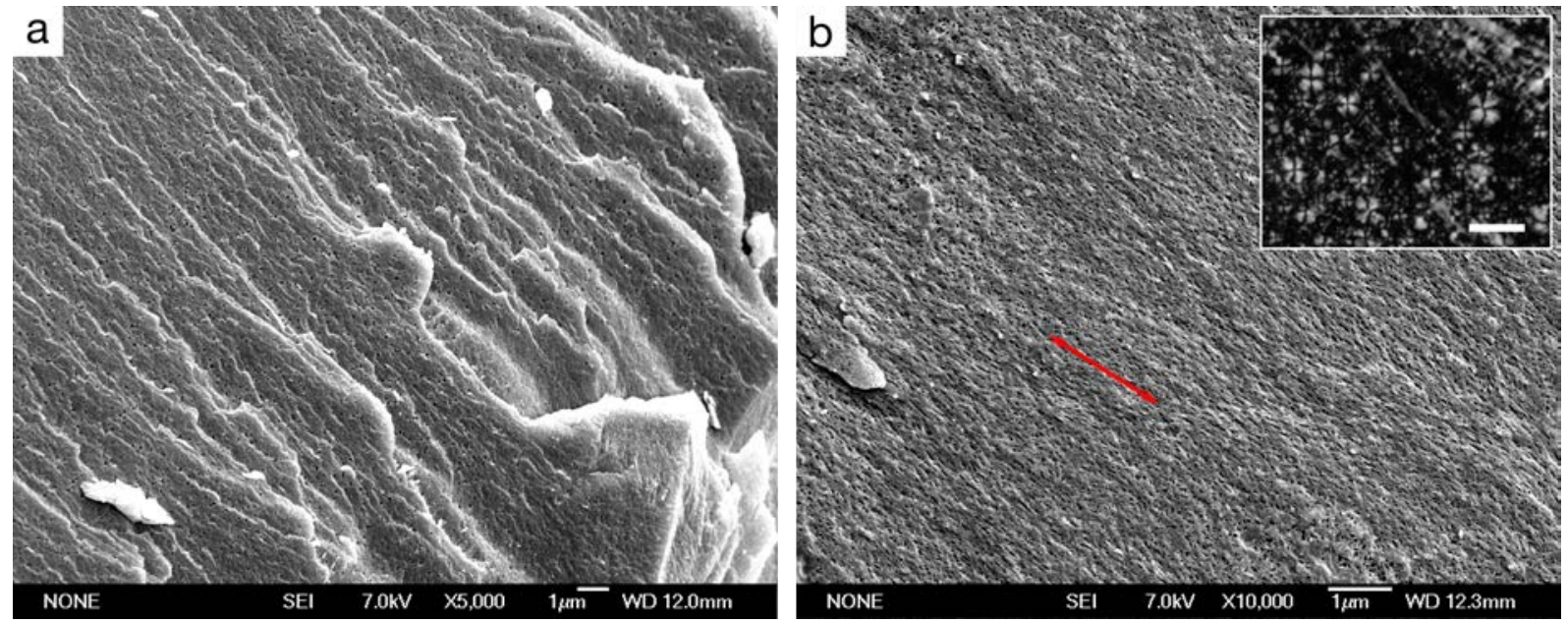

Figure 6.10: Cryo-SEM images of a sample of $60 \mathrm{wt} \% \mathrm{NH}_{4}$-AOT. Red arrow indicates directional order. The inset shows the corresponding POM image, with a scale bar of $50 \mu \mathrm{m}$. 

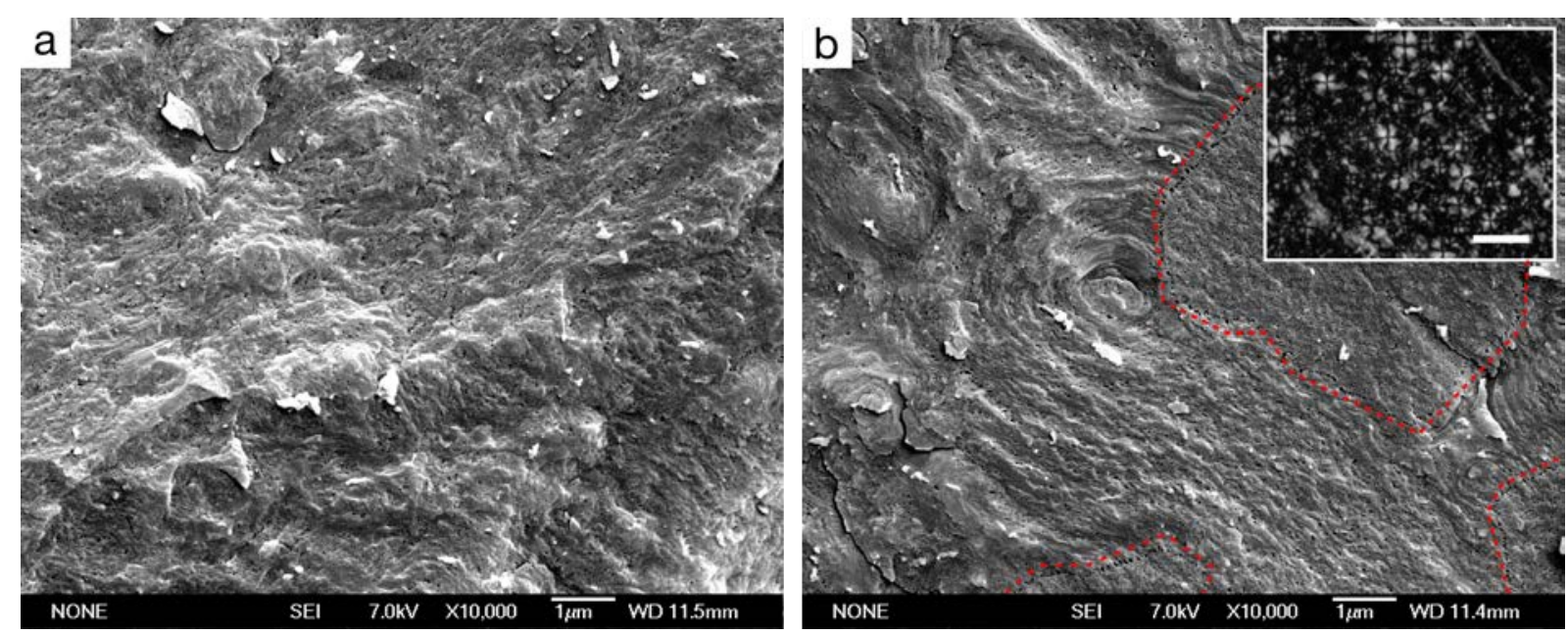

Figure 6.11: Cryo-SEM images of a sample of $70 \mathrm{wt} \% \mathrm{NH}_{4}$-AOT. Red lines indicate sharp domain boundaries. The inset shows the corresponding POM image, with a scale bar of $50 \mu \mathrm{m}$.

In comparison to the systems discussed previously, cryo-SEM images for all concentrations of the $\mathrm{NH}_{4}$-AOT lamellar phase show an overall increase in bilayer undulation and an increase in local connectivity between bilayers. The only exception is the K-AOT system, which forms a sponge phase. This indicates an overall increase in the elasticity of the $\mathrm{NH}_{4}$-AOT bilayer, both in terms of an alteration to the electrostatic contribution to the Gaussian curvature modulus $(\bar{\kappa})$ and a decrease in the bending rigidity modulus $(\kappa)$. This suggests that the steric repulsion and shielding effects of the $\mathrm{NH}_{4}{ }^{+}$ion lies between that of the $\mathrm{Na}^{+}$and $\mathrm{K}^{+}$ions.

\section{Ammonium AOT: Discussion}

It was anticipated that the phase behaviour of $\mathrm{NH}_{4}$-AOT would mirror that of the $\mathrm{K}$-AOT system. In terms of steric bulk, literature values give comparable ionic radii for the two ions, in both non-hydrated and hydrated forms (1.33 and $3.31 \AA$ for $\mathrm{K}^{+}$and 1.48 and $3.31 \AA$ for $\mathrm{NH}_{4}{ }^{+}$, respectively). ${ }^{180}$ Along with their size, several other specific ion properties are also well matched between the two ions (see Table 6.1 for a comparison with other alkali metals).

The ion mobility and Jones-Dole viscosity coefficient values for $\mathrm{NH}_{4}^{+}$and $\mathrm{K}^{+}$in aqueous solution are nearly identical. These are measures of the interactions between the ions and water, and the similar values indicate the ions are similar in size and have similar capability to stabilise or destabilise water structure. The negative Jones-Dole viscosity coefficients of $\mathrm{K}^{+}$ and $\mathrm{NH}_{4}{ }^{+}$indicate that they can both be considered to be chaotropes, whereas $\mathrm{Li}^{+}$and $\mathrm{Na}^{+}$ have positive values and are kosmotropes. The fact that $\mathrm{Na}^{+}$and $\mathrm{K}^{+}$lie on either side of the chaotropic/kosmotropic divide is considered important in biology, as this is thought to facilitate their differing roles in cellular functions. ${ }^{215}$ According to Collins' law of matching water affinities, chaotropic ions will form closer ion pairs with the soft sulfonate headgroup of AOT, and so $\mathrm{NH}_{4}{ }^{+}$ and $\mathrm{K}^{+}$will have significantly higher affinities for the headgroup than $\mathrm{Li}^{+}$or $\mathrm{Na}^{+}$, which are smaller ions with higher charge density and greater hydration. 
Table 6.1: Specific ion parameters for the ammonium ion and alkali metal ions.

\begin{tabular}{|c|c|c|c|c|c|c|c|c|}
\hline Ion & $\begin{array}{c}\text { Ionic } \\
\text { radius }(\AA)\end{array}$ & $\begin{array}{l}\text { Hydrated } \\
\text { radius }(\AA)\end{array}$ & \multicolumn{2}{|c|}{$\begin{array}{c}\Delta H_{\text {hydr }} \\
\left(\mathrm{kJ} \mathrm{mol}^{-1}\right)\end{array}$} & $\begin{array}{c}\Delta \sigma^{\mathrm{a}} \\
\left(\mathrm{mN} \mathrm{L} \mathrm{m} \mathrm{mol}^{-1}\right)\end{array}$ & $\begin{array}{c}\mu_{\mathrm{i}} \text { ion } \\
\left(\mathrm{cm}^{2} \mathrm{~V}^{-1} \mathrm{~s}^{-1} \times 10^{4}\right)\end{array}$ & $B$ & $\begin{array}{c}\alpha_{0} \\
\left(\AA^{3}\right)\end{array}$ \\
\hline $\mathrm{Li}^{+}$ & 0.60 & 3.82 & -519 & -531 & 1.63 & 4.01 & 0.147 & 0.028 \\
\hline $\mathrm{Na}^{+}$ & 0.95 & 3.58 & -409 & -416 & 1.64 & 5.19 & 0.086 & 0.131 \\
\hline $\mathrm{K}^{+}$ & 1.33 & 3.31 & -322 & -334 & 1.40 & 7.62 & -0.007 & 0.795 \\
\hline $\mathrm{Rb}^{+}$ & 1.48 & 3.29 & -293 & -308 & 1.56 & 7.92 & -0.029 & 1.348 \\
\hline $\mathrm{Cs}^{+}$ & 1.69 & 3.29 & -264 & -283 & 1.56 & 7.96 & -0.045 & 2.354 \\
\hline $\mathrm{NH}_{4}^{+}$ & 1.48 & 3.31 & -307 & -329 & 1.39 & 7.60 & -0.007 & 1.278 \\
\hline
\end{tabular}

Note: Shown are the ionic radius, ${ }^{180}$ hydrated radius, ${ }^{180}$ hydration enthalpy $\left(\Delta H_{\text {hydr }}\right),{ }^{211,219}$ surface tension increment $\left(\Delta \sigma^{\mathrm{a}}\right),{ }^{219}$ ion mobility $\left(\mu_{\mathrm{i}}\right),{ }^{220}$ Jones-Dole viscosity coefficient $(B),{ }^{49}$ and static polarisability $\left(\alpha_{0}\right) \cdot{ }^{221}$ lonic and hydrated radii have been calculated and measured using a variety of experimental methods, which have often yielded differing values, and so those quoted here may differ from other publications. The radii values listed are all taken from one source and were calculated using the same technique, allowing comparison between ions.

Despite the similarities, there are two notable differences between $\mathrm{K}^{+}$and $\mathrm{NH}_{4}{ }^{+}$ions. Firstly, $\mathrm{NH}_{4}{ }^{+}$has a slightly higher hydration enthalpy $\left(-307 \mathrm{~kJ} \mathrm{~mol}^{-1} \mathrm{vs} .-322 \mathrm{~kJ} \mathrm{~mol}^{-1}\right)$ and secondly, $\mathrm{NH}_{4}{ }^{+}$has been reported to have a significantly lower dissociation constant. ${ }^{220}$ Both of these properties indicate that of the two ions, it is the ammonium ion that should have the stronger binding affinity to the sulfonate headgroup of AOT. As such, it should form a closer ion pair with the sulfonate group, due to the soft-soft interactions that arise from their more similarly matched hydration enthalpies. In addition to this, when compared to the small hard sphere of $\mathrm{K}^{+}$, the tetrahedral arrangement of $\mathrm{NH}_{4}^{+}$gives the ion greater polarisability ${ }^{222}$ and its lower charge density means it is considered softer than any of the alkali metals in terms of Collins' water affinities. ${ }^{223}$

Given that many of the specific ion properties of $\mathrm{NH}_{4}^{+}$and $\mathrm{K}^{+}$are well matched, and those that do differ indicate that the $\mathrm{NH}_{4}{ }^{+}$ion should form a closer ion pair with the AOT headgroup than any of the alkali metal ions investigated, it was an unexpected result that the $\mathrm{NH}_{4}-\mathrm{AOT}$ system more closely resembles the Na-AOT system. The $\mathrm{NH}_{4}{ }^{+}$ion should form the closest ion pair with the AOT headgroup, and have the smaller effective headgroup size and greater shielding, meaning that it would be expected to stabilise more highly curved interfaces, such as the sponge phase. From our experimental evidence this is not the case, and so we must consider what factors would reduce the interfacial curvature of the bilayer.

Our first consideration is whether or not the assumption that $\mathrm{NH}_{4}^{+}$and $\mathrm{K}^{+}$are equivalent in size is valid. The quoted literature values for the hydrated ionic radii are based on calculations of the average hydration of ions in salt solutions. These values are subject to slight changes with different methods of measurement. The assumption that the values for an aqueous salt solution will hold true in a surfactant solution is also overly simplistic, as it ignores any external factors arising from the presence of the surfactant bilayer itself, and incorrectly assumes that the ions will be fully hydrated. Both $\mathrm{NH}_{4}^{+}$and $\mathrm{K}^{+}$are chaotropic and so have weakly bound outer hydration shells that are readily lost. 
Collins' paper on "sticky ions in biological systems" discusses the selective affinity of $\mathrm{K}^{+}$over $\mathrm{Na}^{+}$for binding to non-polar surfaces in biological systems. It is rationalised that increased binding affinity requires the partial dehydration of the ion, and that this process is facilitated by the ability of the $\mathrm{K}^{+}$ion to exist in this state. Similarly, molecular simulations have revealed that the $\mathrm{NH}_{4}{ }^{+}$ion is extremely labile due to its ability to offer several species with varying degrees of hydration. ${ }^{224}$ This is attributed to the $\mathrm{NH}_{4}{ }^{+}$ion having a tightly bound inner solvation shell, but a weakly bound external shell that changes depending on the environment.

If the $\mathrm{K}^{+}$ion were to exist in a more dehydrated form than the $\mathrm{NH}_{4}^{+}$ion, then it could result in increased counterion affinity for the AOT headgroup. This would result in reduced steric bulk and increased electrostatic shielding contributions, resulting in increased interfacial curvature. However, given that the hydration energies of $\mathrm{NH}_{4}{ }^{+}$and $\mathrm{K}^{+}$are well matched, and their JonesDole coefficients are the same, it is unlikely that either ion would lose significantly more of its hydration waters than the other to cause the observed change in phase behaviour. The two ions likely have similar hydration structures, as shown in molecular dynamic simulations. ${ }^{225}$ As such, we conclude that the ions can be assumed to be of equivalent size, meaning that the differences in phase behaviour must arise due to a different factor, which is most likely the ability of $\mathrm{NH}_{4}{ }^{+}$to hydrogen bond.

Unlike alkali metal counterions, $\mathrm{NH}_{4}{ }^{+}$can interact with the surfactant headgroup via directional hydrogen bonding. Depending on the orientation of the ion within the bilayer, each ion offers up to four such bonds, with the possibility of both intermolecular and intramolecular bonding. An increase in cation contribution to the hydrogen bonding network of the bilayer would result in increased bilayer rigidity, and thus reduced elasticity and interfacial curvature. This is due to stronger interactions, both between the counterion and headgroup, and laterally, with an increase in the attractive forces between adjacent headgroups. Electronic effects resulting in increased bilayer rigidity could compensate for the effects associated with an effective decrease in headgroup size that promotes the formation of the highly curved sponge phase in the K-AOT system, namely the reduced steric bulk and increased electrostatic shielding.

The hydrogen bonding capability of ions is not considered in Collins' law of matching water affinities, which is concerned with the strength of the ion pair formation rather than any lateral forces between adjacent molecules. Indeed, the $\mathrm{NH}_{4}{ }^{+}$ion has been observed to deviate from Collins' law previously. In a study by Kherb et al. on the ability of cations in metal chloride salts to bind to carboxylate groups on polypeptides, ${ }^{219}$ both $\mathrm{Li}^{+}$and $\mathrm{NH}_{4}{ }^{+}$were observed to bind significantly more tightly than expected.

The ability of the $\mathrm{NH}_{4}{ }^{+}$ion to hydrogen bond is important in biological systems. Offering four hydrogen bonds in a tetrahedral geometry is responsible for the ability of $\mathrm{NH}_{4}{ }^{+}$to stabilise the tertiary structures of ribosomal RNA molecules and results in a ten-fold increase in the affinity of RNA fragments for protein and antibiotic surfaces in comparison to $\mathrm{Na}^{+}{ }^{226}$

Comparison of the $\mathrm{NH}_{4}$-AOT and $\mathrm{K}$-AOT systems shows that hydrogen bonding can also have an important role in the self-assembly of surfactant systems. The hydrogen bonding of the 
$\mathrm{NH}_{4}{ }^{+}$counterion offers greater stability to the lamellar phase of the $\mathrm{NH}_{4}$-AOT system as it provides extra rigidity to the bilayer. This effect is enough to compensate for the reduced size of the hydrated ion and means the system is situated between the Na-AOT and K-AOT systems in terms of phase behaviour and local topology. The role of hydrogen bonding in surfactant counterions could be further investigated in the future by removing the ability of $\mathrm{NH}_{4}{ }^{+}$to hydrogen bond through the successive replacement of $\mathrm{H}$ atoms with either $\mathrm{CH}_{3}$ groups or deuterium atoms. An alternative would be a comparative study between ammonium and phosphonium ions, which do not offer the same degree of hydrogen bonding.

\section{Addition of Salt to the Ammonium AOT System: Experimental Results}

The cryo-SEM images of the Na-AOT and $\mathrm{NH}_{4}$-AOT systems indicate that their lamellar phases are not equivalent. The textures of the $\mathrm{NH}_{4}$-AOT system are observed to have more $\mathrm{L}_{3}$-like character than those of $\mathrm{Na}$-AOT, with domain boundaries less evident and the connectivity increased. We have attributed this to the bilayer having more inherent curvature (i.e. each monolayer has increased spontaneous curvature and thus increased packing frustration associated with the formation of the bilayer), a claim supported by the shift to lower surfactant concentration of the $\mathrm{L}_{\alpha}-\mathrm{V}_{\|}$phase transition. Although the phase diagram of the $\mathrm{NH}_{4}-\mathrm{AOT}$ system more closely resembles that of $\mathrm{Na}$-AOT, the curvature of the bilayer must be between that of $\mathrm{K}$-AOT and $\mathrm{Na}-\mathrm{AOT}$. To investigate this, electrolyte in the form of $\mathrm{NaCl}$ was added to the lamellar phase of $\mathrm{NH}_{4}$-AOT to observe whether phase transitions of higher curvature could be induced.

The $\mathrm{NH}_{4}{ }^{+}$ion has a greater affinity for the sulfonate group of the AOT molecule than the $\mathrm{Na}^{+}$ion does, so there should be limited or no exchange between them at the salt concentrations used in this study. This means that the composition of the bilayer should not change. The sodium ions will, however, provide additional shielding of the attractive forces between bilayers and the electrostatic forces between headgroups. This will result in a reduction in the projected $2 \mathrm{D}$ area of the headgroup at the hydrophobic/hydrophilic interface, and will promote more negative spontaneous curvature. As such, upon addition of salt we expect an increase in bilayer elasticity and eventually a loss in lamellar ordering, promoting a phase transition to a structural morphology with greater curvature.

The Na-AOT system is known to form a sponge phase upon addition of sufficient electrolyte to the system. The position of the $L_{\alpha}$ to $L_{3}$ phase transition upon $\mathrm{NaCl}$ addition has been studied previously, and the partial phase diagram for the $\mathrm{Na}-\mathrm{AOT} / \mathrm{NaCl} /$ water system is shown in Figure 6.12. A more complete diagram was compiled by Balinov et al., ${ }^{148}$ which also showed the formation of $V_{\| l}$ phases at high salt and surfactant concentrations. However, the $\mathrm{NaCl}$ concentration was quoted as a percentage of water and so makes for a less direct comparison to the results presented here. Highlighted on the phase diagram by Skouri et al. is the position of the $L_{\alpha}$ to $L_{3}$ transition at Na-AOT concentrations of 25 and 50 wt \% surfactant. In this 
study, samples of $\mathrm{NH}_{4}$-AOT at equivalent concentrations were prepared, containing increasing amounts of $\mathrm{NaCl}$. This allowed for direct comparison with the Na-AOT system.

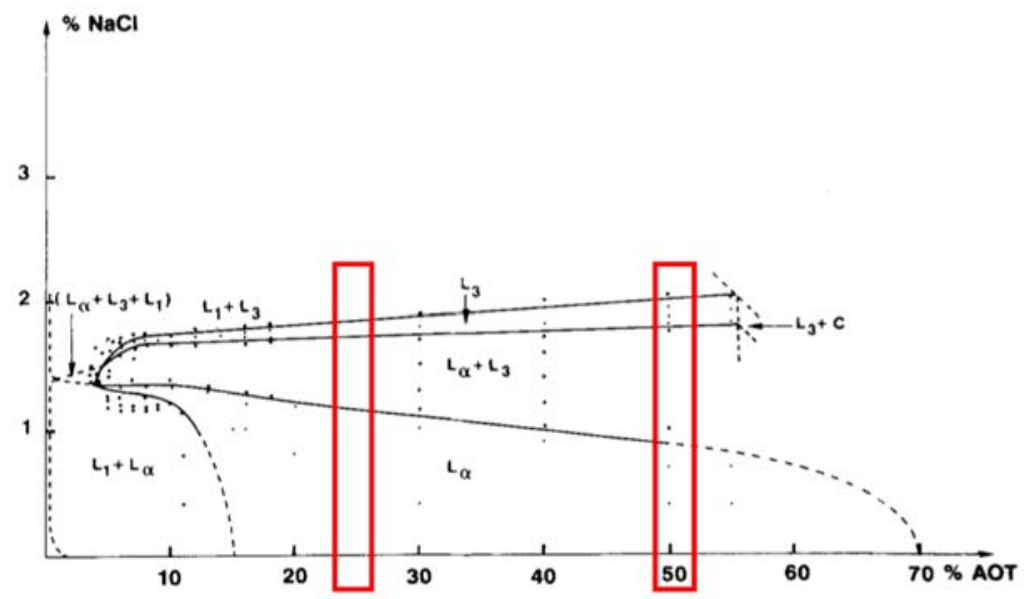

Figure 6.12: Phase diagram of the system AOT-NaCl-water at low salt content, duplicated from the work of Skouri et al. ${ }^{90}$ With permission of Springer, (C) Steinkopff Verlag 1991.

Upon the addition of sufficient $\mathrm{NaCl}$, samples composed of 25 and $50 \mathrm{wt} \% \mathrm{NH}_{4}$-AOT were observed to undergo phase transitions. In the case of $25 \mathrm{wt} \% \mathrm{NH}_{4}$-AOT, an isotropic phase of decreased viscosity was formed, consistent with an $L_{3}$ phase. At $50 \mathrm{wt} \%$ surfactant, a viscous isotropic phase was formed, indicative of bicontinuous cubic phase formation. Both of these phase changes transitioned through two-phase regions, where the $L_{\alpha}$ phase was able to be separated from the isotropic phases by centrifugation prior to analysis via SAXS. Although the cubic samples of $50 \mathrm{wt} \% \mathrm{NH}_{4}$-AOT were extremely viscous, upon exposure to air and under the shear involved in producing samples for analysis via SAXS, they were observed to become more fluid than would be expected for a pure cubic phase. This suggests that the samples were in close proximity to a phase boundary of a more fluid phase, which was confirmed using SAXS. Given that the two salt-induced phase transitions formed isotropic phases, POM cannot be used to assist in assigning the symmetry of the phases formed. The cryo-SEM images in Figure $6.13 \mathrm{do}$, however, support the assignment of the phase formed at $25 \mathrm{wt} \% \mathrm{NH}_{4}$-AOT upon the addition of $\mathrm{NaCl}$ as an isotropic $\mathrm{L}_{3}$ phase.

Figure 6.14 (a) shows the evolution of SAXS profiles for 25 wt \% $\mathrm{NH}_{4}$-AOT upon increasing the $\mathrm{NaCl}$ concentration, and confirms the visual observations of a $L_{\alpha}$ to $L_{3}$ transition. The firstorder Bragg peak of the lamellar phase is observed at $\mathrm{NaCl}$ concentrations below $1 \mathrm{wt} \%$. As the salt concentration increases, this peak is shifted to slightly lower q spacings, indicating a gradual swelling of the bilayer repeat distance prior to the phase transition. A shift from 0.71 to $0.64 \mathrm{~nm}^{-1}$ is observed when the $\mathrm{NaCl}$ concentration is increased from 0.20 to $0.85 \mathrm{wt} \%$, which equates to a rise in d spacing from 88.5 to $98.2 \AA$. At $1.00 \mathrm{wt} \% \mathrm{NaCl}$, two phases were observed and analysed separately. The lower layer, which was optically birefringent, retains the lamellar Bragg peak. The top layer, which was isotropic, shows only broad scattering and a sponge phase correlation peak at very low q values $\left(\sim 0.40 \mathrm{~nm}^{-1}\right)$. At higher salt concentrations only profiles due to the sponge phase are observed. SAXS profiles of both phases show similar broad scattering, indicating the two phases have similar form factors. This is because the two 

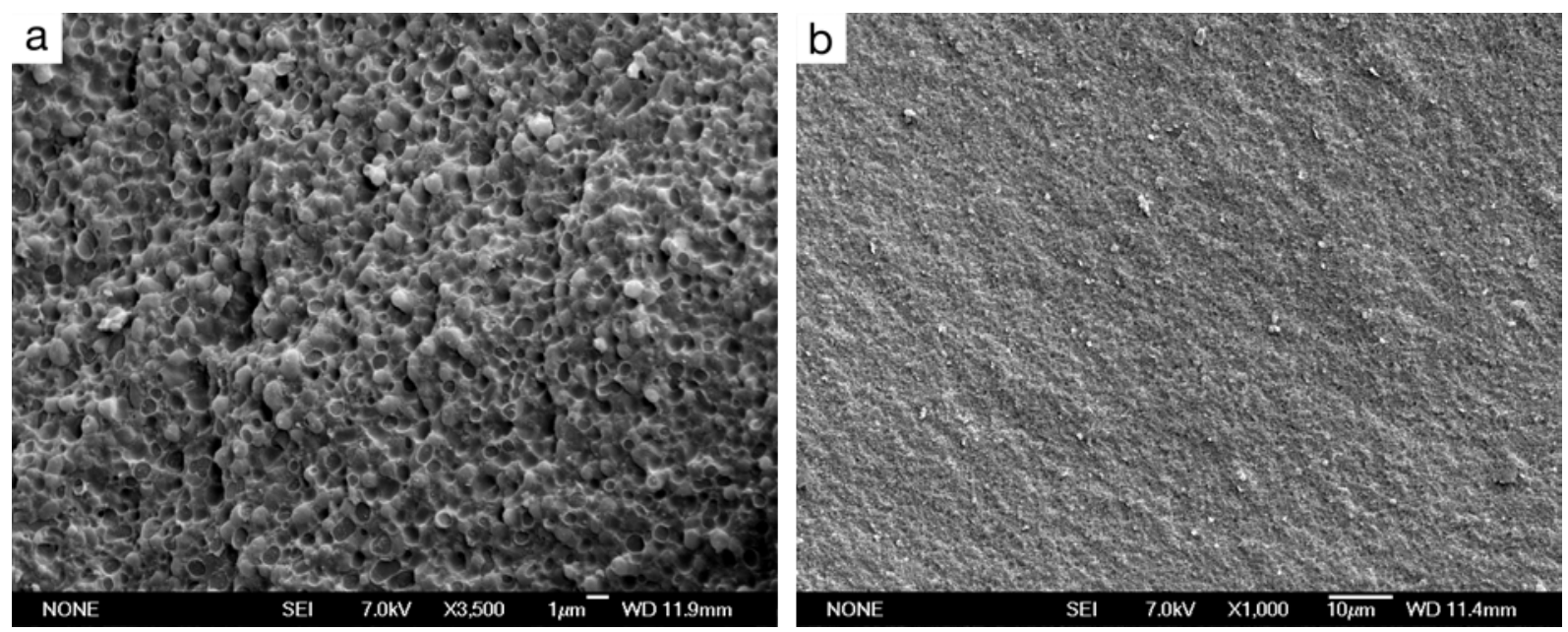

Figure 6.13: Cryo-SEM images of a sample of $25 \mathrm{wt} \% \mathrm{NH}_{4}$-AOT and $1.6 \mathrm{wt} \% \mathrm{NaCl}$ supporting the classification of the $L_{3}$ phase. A highly porous and bicontinuous structure is evident at a magnification of $\times 3,500$, and the lower magnification of $\times 1,000$ shows the absence of grain boundaries that would be expected in a lamellar phase.
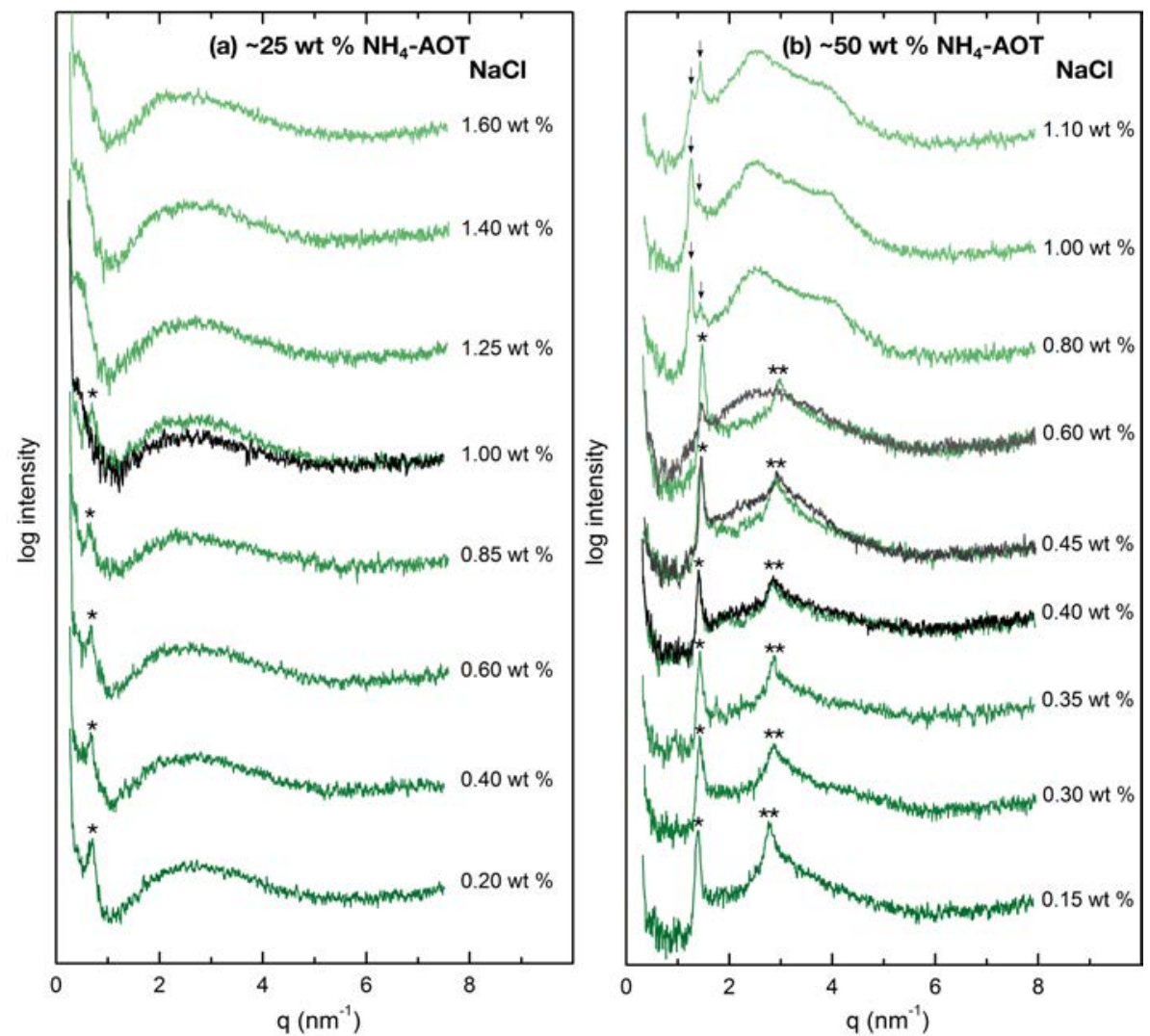

Figure 6.14: Stacked SAXS plots showing the effect on phase behaviour of the addition of $\mathrm{NaCl}$ to specified concentrations of $\mathrm{NH}_{4}$-AOT. Where samples presented two phases, they were separated by centrifugation and the isotropic layer is presented in black. Bragg peaks representing the lamellar phase are shown with single and double asterisks, indicating firstand second-order peaks respectively. Arrows indicate peaks that are tentatively assigned as representing the bicontinuous cubic phase and are consistent with the la3d space group. (a) At 25 wt \% $\mathrm{NH}_{4}$-AOT, increasing $\mathrm{NaCl}$ concentration results in a $\mathrm{L}_{\alpha}-\mathrm{L}_{3}$ transition. (b) At 50 wt \% $\mathrm{NH}_{4}$-AOT, increasing $\mathrm{NaCl}$ concentration results in the formation of a cubic phase and the loss of the lamellar Bragg peaks. 
phases are topologically very similar. The lamellar phase is highly defective on the nanometre lengthscale, with the elastic bilayer allowing high connectivity through the formation of many pores, as seen in the cryo-SEM images presented in Figure 6.8.

Figure 6.14 (b) shows the salt induced phase transition for 50 wt $\% \mathrm{NH}_{4}$-AOT. At low $\mathrm{NaCl}$ concentrations, two sharp Bragg peaks in the q spacing ratio of 1:2 indicate lamellar ordering. As the salt concentration increases, the intensity of the second-order peak decreases and there is a gradual shift of both peaks to higher values of $q$. The first-order Bragg peak shifts from 1.40 to $1.48 \mathrm{~nm}^{-1}$, a change in the average d spacing from 44.9 to $42.5 \AA$. This indicates that as the lamellar phase approaches the salt-induced cubic phase, the bilayer spacing decreases. This trend is opposite to that at $25 \mathrm{wt} \% \mathrm{NH}_{4}$-AOT, where the bilayer swelled when approaching the sponge phase.

At 50 wt $\% \mathrm{NH}_{4}$-AOT and $0.40,0.45$ or 0.60 wt $\% \mathrm{NaCl}$, two phases were observed. Despite efforts to analyse the two layers of each sample separately, the isotropic layer in each case retained some lamellar ordering in the corresponding SAXS profiles, indicating that efficient separation was not achieved. In addition to these Bragg peaks, the isotropic layers produced broad scattering at higher q space, similar to that of the sponge phase observed at lower concentrations. However, once a single viscous layer is formed at $0.80 \mathrm{wt} \% \mathrm{NaCl}$, the dominant feature is the presence of two peaks at 1.25 and $1.44 \mathrm{~nm}^{-1}$, which are in the ratio of $\sqrt{ } 6: \sqrt{ } 8$ and are consistent with the cubic space group la3d. However, any attempt to assign a space group here is tentative, as any reflections at higher q space are masked by the continued presence of diffuse scattering. This broad scattering suggests that the phase is not pure and is likely a mixture of both cubic and sponge phases. The relative intensities of the two peaks is also observed to change with $\mathrm{NaCl}$ concentration, another indication of a mixture of phases. The samples were observed to become more fluid upon loading of the cell used for SAXS analysis, so it is unlikely that the recorded profile is truly representative of the equilibrium state. Indeed, it is even feasible that one of the two sharp peaks could represent the presence of lamellar ordering due to the unavoidable shear induced upon loading the cell. These samples were reanalysed over the course of a week and showed the appearance of intense reflections at higher q spacings, characteristic of an la3d cubic space group. This may be due to the relaxation of the sample to the thermodynamically preferred structure; however, the cell used was not able to be adequately sealed, and so the sample composition may have changed over the time period.

Despite the apparent mixture of phases in the SAXS profile, the viscous and isotropic nature of the sample, combined with the presence of diffraction spots rather than diffraction rings in the $2 \mathrm{D}$ point collimated SAXS analysis, confirms the formation of a cubic phase. The mixture of cubic and sponge phases is due to the close proximity to the $L_{3}-V_{\text {II }}$ phase boundary. $A$ reduction in the surfactant concentration by $2 \mathrm{wt} \%$ (i.e. using a $48 \mathrm{wt} \% \mathrm{NH}_{4}$-AOT sample) was observed to remove the presence of the cubic phase.

SAXS profiles for a sample of 48 wt $\% \mathrm{NH}_{4}$-AOT and 1 wt $\% \mathrm{NaCl}$ are presented in Figure 6.15 (a), captured at a temperature range of $15-90{ }^{\circ} \mathrm{C}$. At 15,25 and $35^{\circ} \mathrm{C}$ only broad scattering is observed, due to the formation of a sponge phase. A small broad shoulder can 
be seen at a q spacing of $\sim 1.2 \mathrm{~nm}^{-1}$, either due to an average spacing of $\sim 52.4 \AA$ or the proximity of the $L_{3} / V_{/ /}$phase boundary and the emergence of some ordering within the sample, with the $L_{3}$ and $V_{/ /}$phases having related bicontinuous topologies. At $45{ }^{\circ} \mathrm{C}$, two sharp Bragg peaks in the ratio of 1:2 indicate the formation of a lamellar phase. These peaks are retained at higher temperatures, with a small shift to lower q spacings as the temperature is increased. The restabilisation of the lamellar phase over the sponge phase at high temperature again highlights the close resemblance of the two phases within the $\mathrm{NH}_{4}$-AOT system. At room temperature, the disruption of the electrostatic forces is sufficient to drive the transition to the sponge phase; however, when this shielding effect is reduced (due to an increase in temperature of $20^{\circ} \mathrm{C}$ ), the defective lamellar phase returns and is once again the energetically favoured phase.
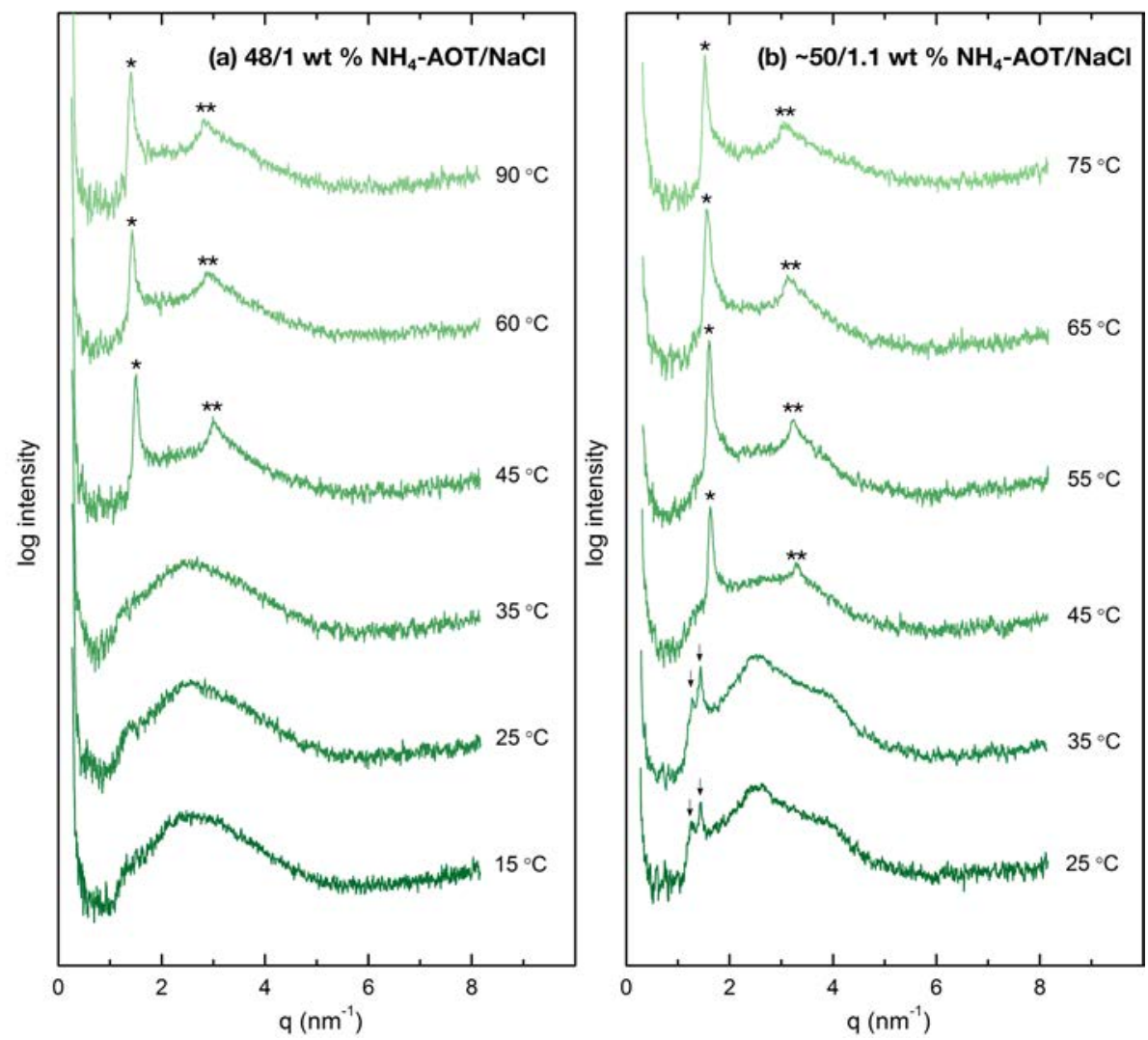

Figure 6.15: Stacked SAXS plots showing the effect on phase behaviour of increasing temperature for specified concentrations of $\mathrm{NH}_{4}$-AOT and $\mathrm{NaCl}$. Bragg peaks representing the lamellar phase are shown with single and double asterisks, indicating first- and second-order peaks, respectively. Arrows indicate peaks that are tentatively assigned as representing the bicontinuous cubic phase and are consistent with the la3d space group. (a) A temperature dependent $L_{3}-L_{\alpha}$ transition is observed for a sample of $48 \mathrm{wt} \% \mathrm{NH}_{4}$-AOT and $1.0 \mathrm{wt} \% \mathrm{NaCl}$. (b) A temperature dependent $\mathrm{V}_{\mathbb{I}}-\mathrm{L}_{\alpha}$ transition is observed for a sample of $50 \mathrm{wt} \% \mathrm{NH}_{4}-\mathrm{AOT}$ and $1.1 \mathrm{wt} \% \mathrm{NaCl}$.

Figure 6.15 (b) shows that for a sample of $50 \mathrm{wt} \% \mathrm{NH}_{4}$-AOT and $1.10 \mathrm{wt} \% \mathrm{NaCl}$, an increase in temperature also drives the transition from a cubic phase back to a $L_{\alpha}$ phase. Bragg peaks due to a lamellar phase appear at $45^{\circ} \mathrm{C}$ with a concomitant shift to lower q space with further increases in temperature. An increase from 45 to $85{ }^{\circ} \mathrm{C}$ results in a shift in q space from 1.62 to $1.46 \mathrm{~nm}^{-1}$, representing swelling of the bilayer repeat distance from 38.8 to $43.0 \AA$. 
The temperature-induced $\mathrm{L}_{3}-\mathrm{L}_{\alpha}$ and $\mathrm{V}_{I I}-\mathrm{L}_{\alpha}$ transitions were also confirmed using POM, with the birefringent lamellar phase appearing upon heating. The growth of the lamellar phase in a sample composed of $48 \mathrm{wt} \% \mathrm{NH}_{4}$-AOT and $1 \mathrm{wt} \% \mathrm{NaCl}$ is shown in Figure 6.16. Circular droplets with lamellar defects of $\mathrm{s}= \pm 1 / 2$ gradually appear within the isotropic background of the $\mathrm{L}_{3}$ phase. Upon continued heating, individual defects coalesce, forming the preferred defect type of $s= \pm 1$ and begin to form larger domains. Eventually, the lamellar phase dominates the texture and isotropic regions are no longer observed. A representative example of the $\mathrm{V}_{I I}-\mathrm{L}_{\alpha}$ transition ( $50 \mathrm{wt} \% \mathrm{NH}_{4}$-AOT and $0.8 \mathrm{wt} \% \mathrm{NaCl}$ ) can be found in Appendix $\mathrm{C}$.
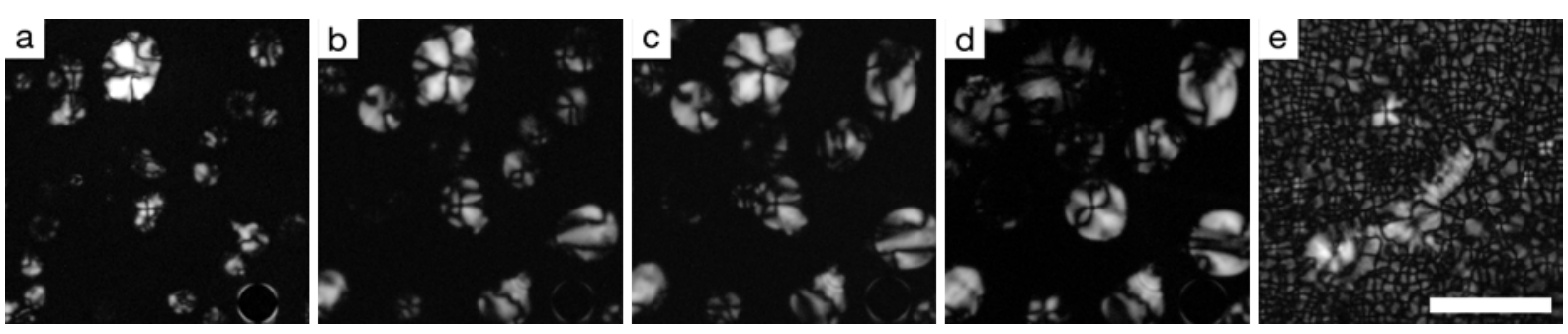

Figure 6.16: $\mathrm{POM}$ images showing the temperature-induced sponge to lamellar transition for a sample of $48 \mathrm{wt} \% \mathrm{NH}_{4}$-AOT and $1 \mathrm{wt} \% \mathrm{NaCl}$ at temperatures of (a) $32{ }^{\circ} \mathrm{C}$, (b) $35{ }^{\circ} \mathrm{C}$, (c) $37.5^{\circ} \mathrm{C}$, (d) $40^{\circ} \mathrm{C}$, (e) $90^{\circ} \mathrm{C}$. Scale bar is $100 \mu \mathrm{m}$.

\section{Addition of Salt to the Ammonium AOT System: Discussion}

We have shown that the $\mathrm{NH}_{4}$-AOT system undergoes salt-induced phase transitions upon the addition of sufficient amounts of $\mathrm{NaCl}$. The formation of $\mathrm{L}_{3}$ and $\mathrm{V}_{/ /}$phases show that the presence of the salt results in the stabilisation of bicontinuous topologies with higher degrees of curvature than the starting $\mathrm{L}_{\alpha}$ phase. When compared with the equivalent Na-AOT concentrations, there are two key differences. Firstly, the phase transitions occur in the $\mathrm{NH}_{4}$-AOT system at much lower $\mathrm{NaCl}$ concentrations. Secondly, the cubic phase is stabilised at significantly lower concentrations of surfactant.

In the sodium system, the lamellar phase formed at $25 \mathrm{wt} \% \mathrm{Na}$-AOT gives way to a two-phase mixture of sponge and lamellar phases at $1.18 \mathrm{wt} \% \mathrm{NaCl}$, and forms a pure sponge phase at 1.72 wt $\% \mathrm{NaCl}$. In contrast, the transitions in the $\mathrm{NH}_{4}$-AOT system are at the lower salt concentrations of $\sim 1.00$ and $\sim 1.25 \mathrm{wt} \%$, respectively. At the higher concentration of $50 \mathrm{wt} \%$ $\mathrm{Na}-\mathrm{AOT}$, the $\mathrm{L}_{\alpha}-2 \mathrm{P}$ and $2 \mathrm{P}-\mathrm{L}_{3}$ transitions occur at 0.86 and 1.79 wt \% respectively, whereas the $\mathrm{L}_{\alpha}-2 \mathrm{P}$ and $\mathrm{L}_{\alpha}-\mathrm{V}_{\text {/l }}$ transitions for the equivalent concentration of $\mathrm{NH}_{4}$-AOT were induced with half as much electrolyte added, observed at $\sim 0.40$ and $\sim 0.80 \mathrm{wt} \% \mathrm{NaCl}$ respectively.

The fact that the $\mathrm{NH}_{4}$-AOT transitions require significantly less salt is further evidence supporting our earlier assertion that the lamellar phase of the $\mathrm{NH}_{4}$-AOT system has more $\mathrm{L}_{3}$-like character than the Na-AOT system. The bilayer of the $\mathrm{NH}_{4}$-AOT lamellar phase has greater inherent curvature than that of the Na-AOT system, and so requires less modification of the 
electrostatic forces to be able to lose the translational order of the lamellar phase and adopt the sponge morphology. As such, the $\mathrm{NH}_{4}$-AOT system can be considered to display properties intermediate to those of the Na-AOT and K-AOT systems. The trend in terms of specific ion effects is therefore $\mathrm{Na}^{+}<\mathrm{NH}_{4}{ }^{+}<\mathrm{K}^{+}$, with respect to inducing bilayer elasticity, and in particular promoting negative Gaussian curvature, into the system. The position of $\mathrm{Na}^{+}$is rationalised through the greater size of its hydrated ion, while the smaller $\mathrm{NH}_{4}{ }^{+}$and $\mathrm{K}^{+}$ions are differentiated by the ability of $\mathrm{NH}_{4}{ }^{+}$to reduce the system entropy and provide rigidity to the bilayer through hydrogen bonding.

The formation of a cubic morphology at $50 \mathrm{wt} \%$ surfactant in the $\mathrm{NH}_{4}$-AOT system, rather than the sponge phase observed in the Na-AOT system, is further evidence of the increased negative Gaussian curvature in the $\mathrm{NH}_{4}$-AOT system. Although the two phases are topologically related, the $L_{3}$ phase is a translationally disordered variant of the $V_{/ /}$phase, and displays a greater variation in curvature and larger water channels. The $V_{/ /}$phase formed when switching to the $\mathrm{NH}_{4}{ }^{+}$ion is a periodic structure, forming a minimal surface where the two principle curvatures are equal (but opposite in sign) and is only stabilised for large negative values of the Gaussian curvature modulus. The formation of the $\mathrm{V}_{\text {/l }}$ phase in the $\mathrm{NH}_{4}$-AOT system demonstrates again that the shielding of electrostatic interactions through the addition of $\mathrm{NaCl}$ drives the behaviour of the system towards that displayed by the K-AOT system, which also forms a $V_{/ /}$phase at significantly lower concentrations than the Na-AOT system.

The SAXS reflections discussed above show that although $\mathrm{NaCl}$ promotes the formation of sponge and cubic phases at room temperature, the effect is negated upon heating, with the $L_{\alpha}$ phase being the lowest energy high temperature phase. It should be highlighted though that this does not mean that the effect of the salt is completely negated. The lamellar phase formed here is not equivalent to that formed at the same surfactant concentration and temperature without the addition of salt. This is most efficiently shown using the SAXS data, which show that the bilayer spacings and shifts are significantly changed upon addition of salt and changes in temperature. Selected values for $q$ spacings and the corresponding bilayer repeat distances are provided in Table 6.2 to illustrate this.

Table 6.2: Values of $q^{*}$ and $d$ for selected concentrations of $\mathrm{NH}_{4}-\mathrm{AOT}$ and $\mathrm{NaCl}$.

\begin{tabular}{ccccc}
\hline \multicolumn{2}{c}{ Concentration (wt \%) } & Temperature $\left({ }^{\circ} \mathrm{C}\right)$ & $\mathrm{q}^{*}\left(\mathrm{~nm}^{-1}\right)$ & $\mathrm{d}(\AA)$ \\
\cline { 1 - 2 } $\mathrm{NH}_{4}$-AOT & $\mathrm{NaCl}$ & & & \\
\hline 45.1 & - & 25 & 1.272 & 49.4 \\
50.0 & - & 25 & 1.396 & 45.0 \\
55.1 & - & 25 & 1.589 & 39.5 \\
50.0 & 0.45 & 25 & 1.462 & 43.0 \\
49.9 & 0.6 & 25 & 1.480 & 42.4 \\
49.9 & 1.1 & 45 & 1.629 & 38.5 \\
49.9 & 1.1 & 85 & 1.468 & 42.8 \\
48.0 & 1.0 & 45 & 1.505 & 41.7 \\
48.0 & 1.0 & 90 & 1.404 & 44.8 \\
\hline
\end{tabular}


The transition from either $a V_{/ /}$or $L_{3}$ phase to an $L_{\alpha}$ phase involves compression of the bilayer spacing. However, once the lamellar phase has formed, further increases in temperature result in the bilayer repeat distance increasing again. This trend is the opposite behaviour to that of the $\mathrm{NH}_{4}$-AOT system in the absence of salt, where increasing temperature results in smaller values of $\mathrm{d}$. For a sample of $50 \mathrm{wt} \% \mathrm{NH}_{4}$-AOT and $1.1 \mathrm{wt} \% \mathrm{NaCl}$, the $\mathrm{d}$ spacing is $38.5 \AA$ at the point the lamellar phase returns (at $45^{\circ} \mathrm{C}$ ). This value is comparable with that of $55 \mathrm{wt} \%$ $\mathrm{NH}_{4}$-AOT when no salt is added. An increase in temperature to $85^{\circ} \mathrm{C}$, results in an increase in the bilayer spacing to $42.8 \AA$.

The different trend in the way the bilayer repeat distances changes with temperature indicates that the presence of $\mathrm{NaCl}$ modifies the relative contributions to the free energy of the system, and so alters how the entropy of the system changes as a function of temperature. The addition of salt will affect the system in several ways, as it interacts with both the surfactant and water components of the system. The presence of $\mathrm{NaCl}$ will increase the system entropy by destabilising hydrogen bonding within the bulk water regions and will negatively affect the hydrophobic contribution of the hydrophobic regions. This second point is evidenced by the formation of a cubic phase at 50 wt \% surfactant, which requires either increased conformational freedom or volume within the tail region, which is only exhibited when salt is added. However, in terms of temperature, the main effect of salt is to increase the temperature at which significant dehydration occurs. At high temperatures the surfactant headgroups will be more hydrated in the $\mathrm{NH}_{4}-\mathrm{AOT} / \mathrm{NaCl}$ system, than for samples of corresponding surfactant concentrations when no salt is added. The effect of dehydration is to remove the screening of electrostatic forces by headgroups, and allow them to move closer together. With the range of hydration extended in the $\mathrm{NH}_{4}-\mathrm{AOT} / \mathrm{NaCl}$ system, the contribution of this screening is effectively increased, meaning the balance between competing forces is altered. In particular the screening of low frequency charge fluctuations results in the effective contribution of the van der Waals attraction forces being reduced. ${ }^{227}$ The change in balance between these competing forces explains how the $\mathrm{NH}_{4}$-AOT lamellar phase can constrict or swell with increasing temperature, depending on whether electrolyte has been added to the system. The extent of this swelling will depend on the size and charge of the electrolyte and on the initial $d$ spacing of the sample, as these factors will also affect the balance between interacting forces.

In general terms, the phase behaviour of the $\mathrm{NH}_{4}$-AOT system has been presented in this chapter for the first time. The behaviour has been shown to be similar to that of the Na-AOT system, but the bilayer of the lamellar phase has increased elasticity, resulting in the stabilisation of highly curved inverse structures over larger concentration ranges (including lower surfactant concentration). In particular, the la3d cubic phase persists for a large surfactant concentration range of $\sim 12 \mathrm{wt} \%$. The increased stabilisation of inverse phases, alongside the increase in local scale topological defects observed in the lamellar phase, show that the $\mathrm{NH}_{4}{ }^{+}$ion promotes behaviour intermediate to that of the $\mathrm{Na}^{+}$and $\mathrm{K}^{+}$ions (presented in Chapters 3 and 4). The increased rigidity of the $\mathrm{NH}_{4}$-AOT bilayer, in comparison to that of K-AOT (which forms a $\mathrm{L}_{3}$ phase), implies that the role of intermolecular hydrogen bonding in the $\mathrm{NH}_{4}$-AOT lamellar phase is to provide stability to the bilayer through increased ordering at the hydrophobic/hydrophilic interface. This increased rigidity allows the partial offset of the curvature frustration of the 
bilayer and so a decrease in spontaneous curvature resulting in retention of the lamellar phase. However, the increased elasticity in comparison to the Na-AOT system shows that the values of $\kappa$ and $\bar{\kappa}$ fall between those of K-AOT and Na-AOT bilayers.

The role of hydrogen bonding, clearly of fundamental importance in nature, is largely neglected in respect to counterions contributing to hydrogen bonding networks in systems such as these. This is clearly because most counterions do not have this capability. However, this does highlight one limitation in applying rules such as Collins' law of matching water affinities to a broad range of different systems and types of structures, when parameters that may significantly affect the local environment of the system are not considered. The observed differences between the $\mathrm{Na}$-AOT, $\mathrm{NH}_{4}$-AOT and K-AOT systems, a result of the different properties of the counterions, may be useful in the fine-tuning of surfactant systems. Switching between the ions allows the induction or restriction of curvature (altering the susceptibility of the system to added electrolyte) without changing the structure of surfactant molecule itself, or requiring the addition of extra components. In the $\mathrm{NH}_{4}$-AOT system, the $\mathrm{NH}_{4}{ }^{+}$ion is shown to be intermediate to the $\mathrm{Na}^{+}$and $\mathrm{K}^{+}$ions, which lie either side of the chaotropic/kosmotropic divide. 


\section{Chapter 7}

\section{Choline and Acetylcholine Aerosol OT}

In this chapter, the quaternary ammonium ions choline and acetylcholine are used as counterions to AOT. Both of these ions are biologically important and their relevance is briefly discussed before the phase behaviour is presented, and the defect behaviour characterised, for each system. According to Collins' law of matching water affinities, ${ }^{49,181,228}$ both choline and acetylcholine will form close contact ion pairs with the AOT headgroup. However, we show that their bulky size can more than compensate for their low levels of hydration, resulting in the stabilisation of the lamellar morphology in these systems to a greater extent than any of the systems discussed in previous chapters.

\section{Choline and Acetylcholine AOT: Introduction}

Choline (2-hydroxy- $N, N, N$-trimethylethanaminium) is a quaternary ammonium ion with the structure presented in Figure 7.1. It is a natural human metabolite, closely associated with the $B$ vitamins, and was classified as an essential nutrient for humans in 1998 with the establishment of the Dietary Reference Intakes by the Food and Nutrition Board of the Institute of Medicine in the USA. ${ }^{229-233}$ It has a diverse range of roles in human metabolism, and deficiency in choline is considered to be a contributing factor in several diseases, including atherosclerosis, ${ }^{234}$ liver dysfunction, ${ }^{230}$ and various neurological disorders. ${ }^{231,235}$ It has also been shown to have a critical role during foetal development, where it influences the structure and function of the spinal cord and brain. ${ }^{236,237}$ Low levels of choline during this period inhibit nerve and brain development, and lead to an increased risk of neural tube defects, such as spina bifida. ${ }^{238-240}$<smiles>C[N+](C)(C)CCO</smiles>

Figure 7.1: Molecular structure of the choline counterion. 
Choline is considered to have five main physiological roles:

- As a source of labile methyl groups in various synthetic pathways. ${ }^{213,231,241}$

- As a precursor in the production of acetylcholine, a principal neurotransmitter in the peripheral and central nervous systems. ${ }^{242,243}$

- As a component in several predominant phospholipids in cell membranes. ${ }^{231,244}$

- Controlling the phospholipid concentrations in the liver and in blood. ${ }^{231,245}$

- Intra- and inter-cell signalling. ${ }^{236,246}$

Two of the most predominant phospholipids within the human body are phosphatidylcholine and its sphingolipid analogue sphingomyelin, shown in Figure 7.2. Together they typically account for over $50 \%$ of membrane phospholipids. ${ }^{247-249}$ Both lipids contain choline attached to a phosphate within the headgroup, making them zwitterionic over a large $\mathrm{pH}$ range. In phosphatidylcholine, the phosphate group is linked to two fatty acid chains via a bridging glycerol component. In sphingomyelin, the phosphate is attached to a ceramide unit consisting of a longchain sphingosine base and an amide-bonded fatty acid chain. With similar headgroups and asymmetric configurations, there are clear structural similarities between the two, also indicated by some overlap in their functionality. However, the two lipids also serve separate roles within the human body and plasma membrane, due to structural differences in their interfacial and tail regions. ${ }^{249}$
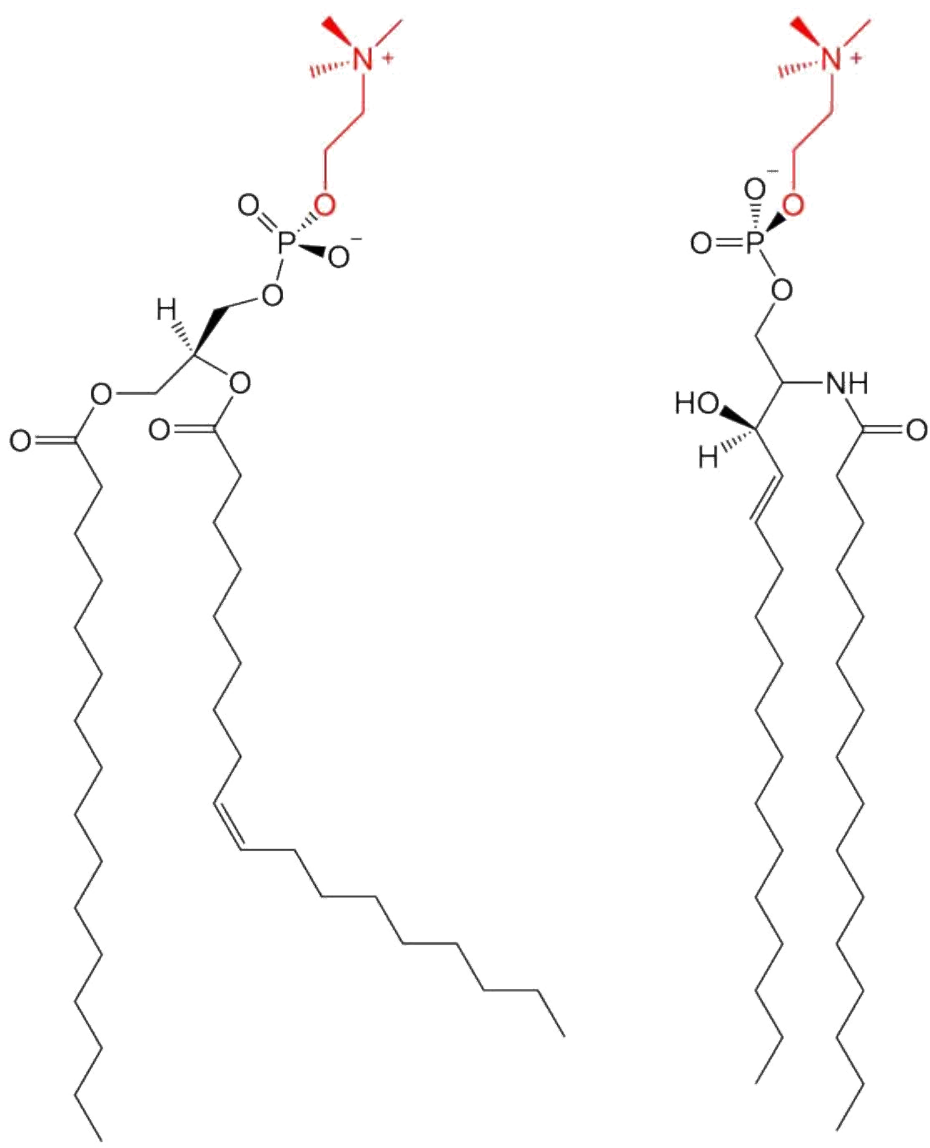

Figure 7.2: Molecular structures of phosphatidylcholine (left) and sphingomyelin (right). The choline component of each phospholipid is coloured red. The compositions of the hydrocarbon components will vary with dietary intake and between different tissues. 
The sn-1 chain of phosphatidylcholine is normally saturated, whilst the sn-2 chain is unsaturated, containing at least one double bond with a cis configuration. This kinked configuration lowers the sol-gel transition temperature and provides fluidity to a membrane. The comparable acyl chain in sphingomyelin is generally either saturated or trans-unsaturated, allowing for a tighter packing arrangement. The sol-gel transition temperature of sphingomyelin in membranes is regulated by a favourable interaction with cholesterol, which disrupts the local packing order and increases lateral diffusion. ${ }^{249}$

Although in this work we have used choline as a counterion to AOT rather than a covalently bonded component of the headgroup, investigating its role as a counterion is still of biological relevance. This is because choline within the human body is known to detach from the headgroup of phospholipids to drastically alter their properties and physical behaviour, and those of the structures they form. ${ }^{250}$ The release of choline is an important process in cell signalling, which is of significant importance in the understanding of cell growth and cell death, and also the targeting of cell growth in cancer therapies. ${ }^{251-254}$ Choline metabolites have also been shown to have a pivotal role in remyelination and have been proposed as potential substances for the treatment of multiple sclerosis, due to their regenerative effects. ${ }^{255}$

Aside from the role of choline in human metabolism, Klein et al. investigated the potential use of choline as a counterion in single chain carboxylate surfactants and in choline dodecyl sulfate (ChDS). ${ }^{60}$ ChDS was shown to present good solubility in water, with the Krafft temperature remaining below $0{ }^{\circ} \mathrm{C}$ for surfactant concentrations up to $65 \mathrm{wt} \%$. This increased solubility in comparison to its sodium analogue SDS, alongside the retention of equivalent counterion dissociation in the micellar phase - despite a trend observed within the alkali metal series that smaller ions induce lower Krafft temperatures and weaker ion-headgroup association - is postulated to be due to the choline ion providing greater hinderance to the efficient packing of surfactant molecules, presumably as a result of its steric bulk.

Quaternary ammonium ions are often reported to exhibit negative toxic impacts. ${ }^{256,257}$ However, ChDS was shown through cytotoxicity studies to present good biocompatibility, with studies on two human cell lines showing it to be no more toxic than the commonly used SDS and KDS. ${ }^{60}$ This low toxicity is likely due to the presence of the hydroxyl group. It also shows that some quaternary ammonium ions do have potential roles as counterions in green surfactants.

In this thesis, choline was employed as a counterion in the AOT system, and is shown to stabilise the lamellar phase of the system over a wider range of surfactant concentrations than any of the systems presented in the previous chapters. However, the $L_{\alpha}$ phase is also shown to lose stability at lower temperatures than the other systems studied. In addition, we show that the use of the choline ion produces a $L_{\alpha}$ phase capable of incorporating large amounts of electrolyte without undergoing drastic structural changes due to a phase transition.

As mentioned above, one of the physiological roles of choline is as the main precursor in the synthesis of the most common neurotransmitter within the human body, acetylcholine (2-acetoxy- $N, N, N$-trimethylethanaminium), shown in Figure 7.3. Acetylcholine functions in both 
the central and peripheral nervous systems, and all nerves leaving either the brain or spinal cord release acetylcholine as a neurotransmitter. ${ }^{258}$ In the peripheral nervous system it functions at neuromuscular junctions between motor nerves and skeletal muscles to activate muscle action. In the central nervous system, and in particular in the brain, it functions as both a neurotransmitter and a neuromodulator, meaning that it transmits directly over some synapse junctions, but also acts upon a variety of other neurons in the brain and regulates their activities. The concentration of acetylcholine within the human body has been shown to decrease with normal ageing, often resulting in short term memory loss. ${ }^{259}$ Where the drop in acetylcholine concentration is more severe, neurons become vulnerable to degradation. This is a contributing factor in several motor neuron diseases. ${ }^{260-263}$ The deterioration of one acetylcholine neural pathway has been associated with the onset of Alzheimer's disease. ${ }^{264,265}$ The disease is characteristically marked by a reduction in the concentration of acetylcholine of up to $90 \%$ and the subsequent degeneration of the cholinergic neurons that it acts at, such as those in the cerebral cortex. ${ }^{266-268}$ Current treatments for Alzheimer's disease target the breakdown of acetylcholine, acting to inhibit its enzyme-catalysed deactivation at synapses to maintain higher concentrations. ${ }^{269}$

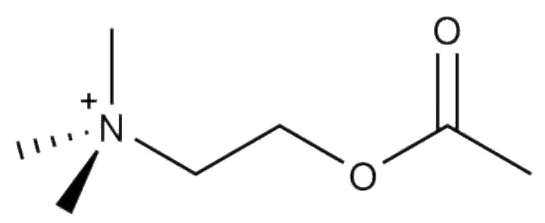

Figure 7.3: Molecular structure of the acetylcholine counterion.

Biosynthesis of acetylcholine occurs at the terminals of cholinergic neurons via the enzymecatalysed acetylation of choline. Choline acts as the immediate precursor of acetylcholine, and its abundance controls the rate of synthesis. ${ }^{242,263,270}$ Storage pools of choline exist in such neurons in the form of vesicles composed of choline-containing phospholipids, which are broken down via hydrolysis to produce choline. Levels of acetylcholine in the brain cannot be increased by external administration due to the polarity of the molecule and presence of the charged ammonium group preventing it from passing through the membranes of the bloodbrain barrier. ${ }^{271}$

Acetylcholine is clearly an important molecule within the human body; however, its inclusion within this study was due to its close structural resemblance to choline. The choice of acetylcholine allowed us to employ a second biogenic quaternary amine as a counterion to AOT, enabling a comparison with the choline system. The amine component of each molecule is identical; however, changing the functional group from an alcohol in choline to an ester in acetylcholine results in an increase in size and alters the physical properties, specifically the interaction of the molecule with water. Both molecules are polar and so offer dipole-dipole interactions alongside van der Waals dispersion forces. However, the ester group is less polar than the alcohol and so these attractions are reduced in acetylcholine, as is the capacity for hydrogen bonding. The hydroxyl group of choline allows it to act as both a hydrogen bond donor and acceptor, whereas the ester functionality of acetylcholine limits its role to hydrogen bond donation only. 
As both choline and acetylcholine are significantly bulkier cations than those used in previous chapters, we also introduce the possibility of forming ionic liquids. These can be generally defined as materials that consist entirely of ions and present a liquid state at a temperature below $100^{\circ} \mathrm{C}$ (below $25^{\circ} \mathrm{C}$ in the case of room temperature ionic liquids). This $100{ }^{\circ} \mathrm{C}$ melting point is entirely arbitrary and ionic liquids are defined in this way to represent their practical usefulness, rather than any inherent difference to salts that melt above this temperature. The low melting points of ionic liquids are due to poor coordination of the constituent salt ions. The formation of a stable crystal lattice is prevented by pairing bulky and unsymmetrical ions that do not pack efficiently or form strong ion pairs. Crystal lattices can be destabilised by increasing the distance between the charges and by decreasing the symmetry of the ions. As such, ionic liquids will generally consist of two bulky ions that are not able to pack closely, with at least one ion having a delocalised charge or significant charge shielding by large substituents. As such, many ionic salts contain one organic ion capable of charge distribution by resonance.

A list of common ions used in ionic salts can be found in a review by Hayes et al. and includes ammonium and sulfonate ions. ${ }^{272}$ As such, the hypothesis that Ch-AOT and AcCh-AOT could be ionic liquids is a reasonable one. The sulfonate group of AOT exhibits delocalisation of its negative charge and the positive charges of choline and acetylcholine are shielded through the alkyl groups attached to the nitrogen atoms. Ionic liquids have been a growing topic of interest over the last 15 years due to potential applications in a wide variety of fields. ${ }^{272}$ Generally considered good solvents and electrolytes, the added ease of fine-tuning the physicochemical properties by simple modifications to either the cation or anion component has led to them being termed "designer solvents" and "solvents of the future". 273,274 They are now widely used in both organic and inorganic chemistry to produce more environmentally friendly syntheses. ${ }^{275,276}$ So, although the objective of this work was not to produce ionic liquids, were the physical interactions between the chosen surfactant and counterions to produce such a result, it would be of academic interest and potential practical use. In fact, during 2014, Rao et al. also synthesised Ch-AOT ${ }^{277}$ (the first published synthesis) and stated that it is an ionic liquid. However, the results reported in this chapter suggest that this assignment is erroneous, which will be discussed in more detail below.

\section{Choline AOT: Experimental Results}

\section{Choline AOT Phase Behaviour}

The elucidated phase diagram for the Ch-AOT system is shown in Figure 7.4, and is markedly different from those of the alkali metal and ammonium systems. This is due to the absence of pure $\mathrm{V}_{/ /}$and $\mathrm{H}_{\| /}$phases. The phase diagram is clearly dominated by a lamellar phase, which extends from 20 to $98 \mathrm{wt} \%$ surfactant. Lamellar ordering exists as low as $5 \mathrm{wt} \%$ surfactant due to the presence of a two-phase region. This region is a mixture of the lamellar phase observed at higher concentrations and the isotropic liquid observed below $5 \mathrm{wt} \%$, which due to the bulky 
nature of both the head and tail components of the surfactant is likely a vesicular phase. The Ch-AOT phase diagram also differs from those presented previously in terms of the melting transition temperature of the lamellar phase. This is considerably lower in the Ch-AOT system, which is likely due to the disruption to the hydrogen bonding of the water network caused by the additional bulk of the choline cation upon dissociation.

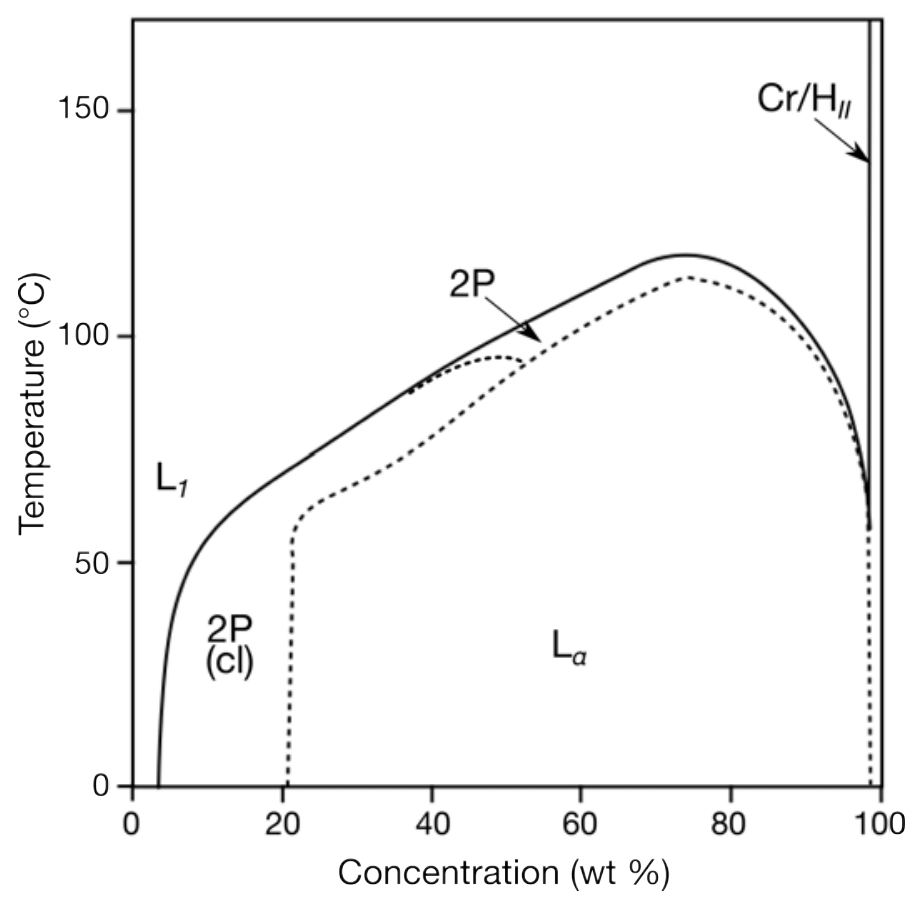

Figure 7.4: Phase diagram of the Ch-AOT system. The two-phase region is depicted by $2 \mathrm{P}$ and $2 \mathrm{P}(\mathrm{cl})$ to distinguish between the phase-separated and cloudy regions, respectively. Cr denotes the presence of crystals.

Bulk samples of the Ch-AOT system (see Figure 7.5), show that the two-phase region is more easily defined than in the Na-AOT system. This is due to the presence of a cloud point, which results in the samples within the two-phase region appearing cloudy at room temperature. The cloud point is evident upon heating lamellar samples of concentrations up to $\sim 47.5 \mathrm{wt} \%$. Above this concentration the optical birefringence of the bulk samples gives way to an isotropic texture, but a cloud point is no longer observed. The presence of a cloud point reveals strong counterion to headgroup binding, as observed in several other tetraalkylammonium surfactant systems. ${ }^{278-280}$

At room temperature, samples of concentrations higher than $20 \mathrm{wt} \%$ give rise to bright colours when viewed with crossed polarised light. They present the same trend in colour changes as observed in the alkali metal systems. This indicates ordering on the same lengthscales as the previous systems as the bright colours, which transition with increasing surfactant concentration through blue and black, before giving opaque textures more normally associated with a lamellar phase, are indicative of ordering on lengthscales comparable to that of the light shining through them. 


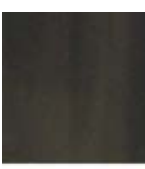

2.5

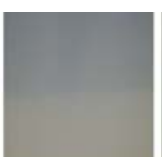

5

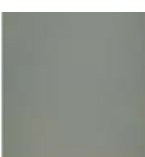

15

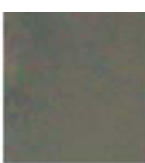

20

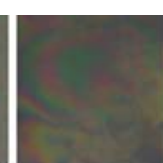

30

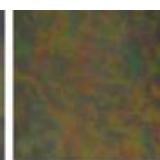

40

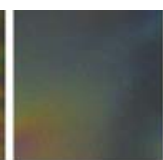

45

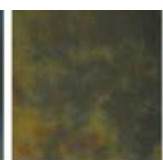

55

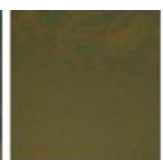

65

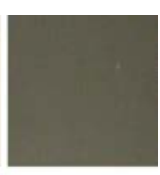

75

wt \% Ch-AOT samples

Figure 7.5: Bulk samples of Ch-AOT viewed under crossed polarised light. Samples at 5 and $15 \mathrm{wt} \%$ are cloudy in appearance. Samples at higher concentrations are within the lamellar phase and show the evolution from iridescent to opaque appearance with increasing surfactant concentration.

Approaching $100 \mathrm{wt} \%$ surfactant, the phase diagram shows a transition from a lamellar phase to a two-phase region. This is possibly an oversimplification, as moving from a lamellar phase to a mixture of hexagonal phase and crystals may involve several transition steps. However, it was not possible to isolate any such transitions in this work, due to the surfactant proving to be extremely hygroscopic. Any exposure of the pure surfactant to air resulted in the formation of a lamellar phase. Samples prepared under an inert atmosphere did not reveal any further phase behaviour, except that of the pure surfactant itself, which was revealed to be hexagonal by SAXS and POM. Concentration gradients viewed using POM, as shown in Figure 7.6, did not reveal an isotropic band to indicate the presence of a $V_{/ /}$phase between the $L_{\alpha}$ and $H_{/ /}$phases, as was observed in all of the alkali metal/AOT systems. As such, it is likely that there is a direct phase transition between the $\mathrm{L}_{\alpha}$ and $\mathrm{H}_{/ /}$phases, as has been observed elsewhere. ${ }^{281,282}$ It may be possible that a cubic phase could be induced by repeatedly cycling through the $L_{\alpha}$ to $H_{\text {// }}$ phase transition, as observed in other systems ${ }^{283,284}$ and that its absence is due to the slow equilibration time of the phase. However, this was not investigated further as studies in this part of the phase diagram are problematic and without practical application, due to the aforementioned hygroscopic nature of the surfactant at such high concentrations.

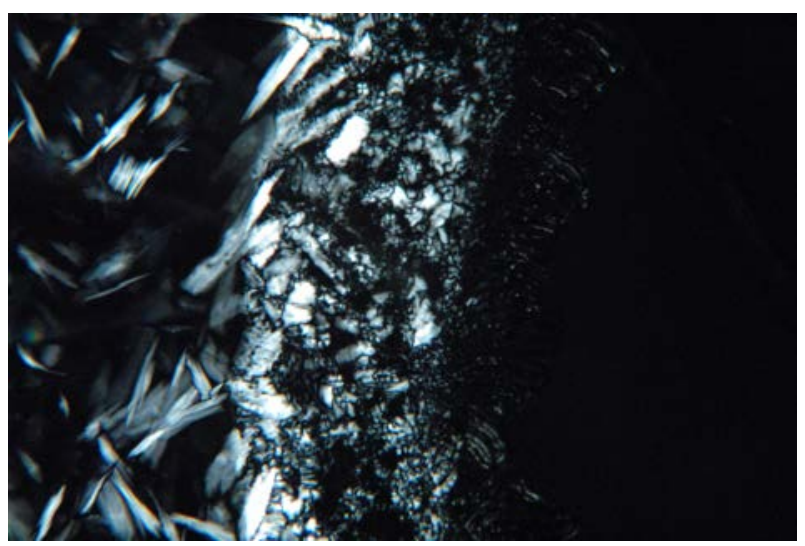

Figure 7.6: $\mathrm{POM}$ concentration gradient for Ch-AOT at $\sim 25{ }^{\circ} \mathrm{C}(\times 40$ magnification). The direction of water penetration is from right to left. There is no evidence of the formation of a $v_{/ l}$ phase. 


\section{Choline AOT SAXS}

Figure 7.7 shows the evolution of the SAXS profiles upon increasing surfactant concentration for the Ch-AOT system. Due to the large bilayer spacings within the two-phase region, firstorder lamellar peaks for samples below $15 \mathrm{wt} \%$ are in close proximity to the primary peak and so are visible only as a shoulder. Due to the dominance of the lamellar phase in the system, two Bragg peaks in the ratio of 1:2 can be observed for most samples, which upon increasing concentration become sharper and more intense due to increased ordering. An exception to this occurs between 35 and $45 \mathrm{wt} \%$, where intensity of the first-order peak is diminished due to the zero form factor (as observed for the previous systems). At low concentrations, the secondorder Bragg peak is masked by diffuse scattering. The relative contribution of this scattering is reduced at higher concentrations due to the reduction in Helfrich repulsion forces at lower $d$ spacings. This reduces the diffuse scattering and results in increased bilayer ordering and so sharper second-order Bragg peaks.

At $100 \mathrm{wt} \%$, the Ch-AOT system forms a mixture of a hexagonal phase and crystals. The hexagonal ordering is confirmed by Bragg peaks in the ratio $1: \sqrt{ } 3: \sqrt{ } 4$. The crystallinity is confirmed by the WAXS region. The insets in Figure 7.7 show the scattering at wide angles for concentrations of 90 and $100 \mathrm{wt} \%$ Ch-AOT. At $90 \mathrm{wt} \%$, fluid alkyl chains result in a characteristic broad scattering centred at $13.6 \mathrm{~nm}^{-1}$, indicating liquid crystalline behaviour. At $100 \mathrm{wt} \%$, both broad scattering due to the liquid crystalline hexagonal phase and sharp peaks due to the crystalline phase are evident, confirming the assignment as biphasic. The sharp peak at $8.3 \mathrm{~nm}^{-1}$ correlates to $\sim 7.5 \AA$, and can be assigned as arising from headgroup correlations between dehydrated headgroups. ${ }^{285,286}$ Peaks at $13.4,14.1$ and $14.4 \mathrm{~nm}^{-1}$ are likely due to solidified alkyl chains within the crystalline solid.

In the lamellar phase, the relative width and position of the broad peak arising due to the fluid surfactant tails gives an indication of the average spacing between the alkyl chains. With increasing concentration, this broad peak shifted to lower q values (14.9 to $13.6 \mathrm{~nm}^{-1}$ between 50 and $90 \mathrm{wt} \% \mathrm{Ch}-\mathrm{AOT}$ ), indicating an increasingly splayed conformation of the tails. The peak also significantly narrows at high concentrations, reflecting more regular interactions between chains. An increasingly splayed conformation of the tail region within the lamellar phase implies the formation of a highly curved, defect rich microstructure.

Figure 7.8 compares the bilayer repeat distances of the Ch-AOT system to those observed in Na-AOT. Both systems show normal one dimensional swelling behaviour, although Ch-AOT shows lamellar behaviour over a larger concentration range. The sterically larger and weakly hydrated $\mathrm{Ch}^{+}$ion should result in an increased contribution of attractive (over repulsive) intermolecular forces. However, the Ch-AOT system displays larger d spacings than Na-AOT at comparable concentrations. This is probably the result of a combination of factors. Firstly, despite $\mathrm{Ch}^{+}$forming a closer ion pair with the sulfonate group, the steric bulk of the $\mathrm{Ch}^{+}$ion will result in a thicker bilayer, and thus a larger contribution to the $\mathrm{d}$ spacing. In addition to this, the Ch-AOT system will have more bulk water between the bilayers. The chaotropic $\mathrm{Ch}^{+}$ion will promote a dehydrated bilayer, whereas the kosmotropic $\mathrm{Na}^{+}$ion will be strongly hydrated. 

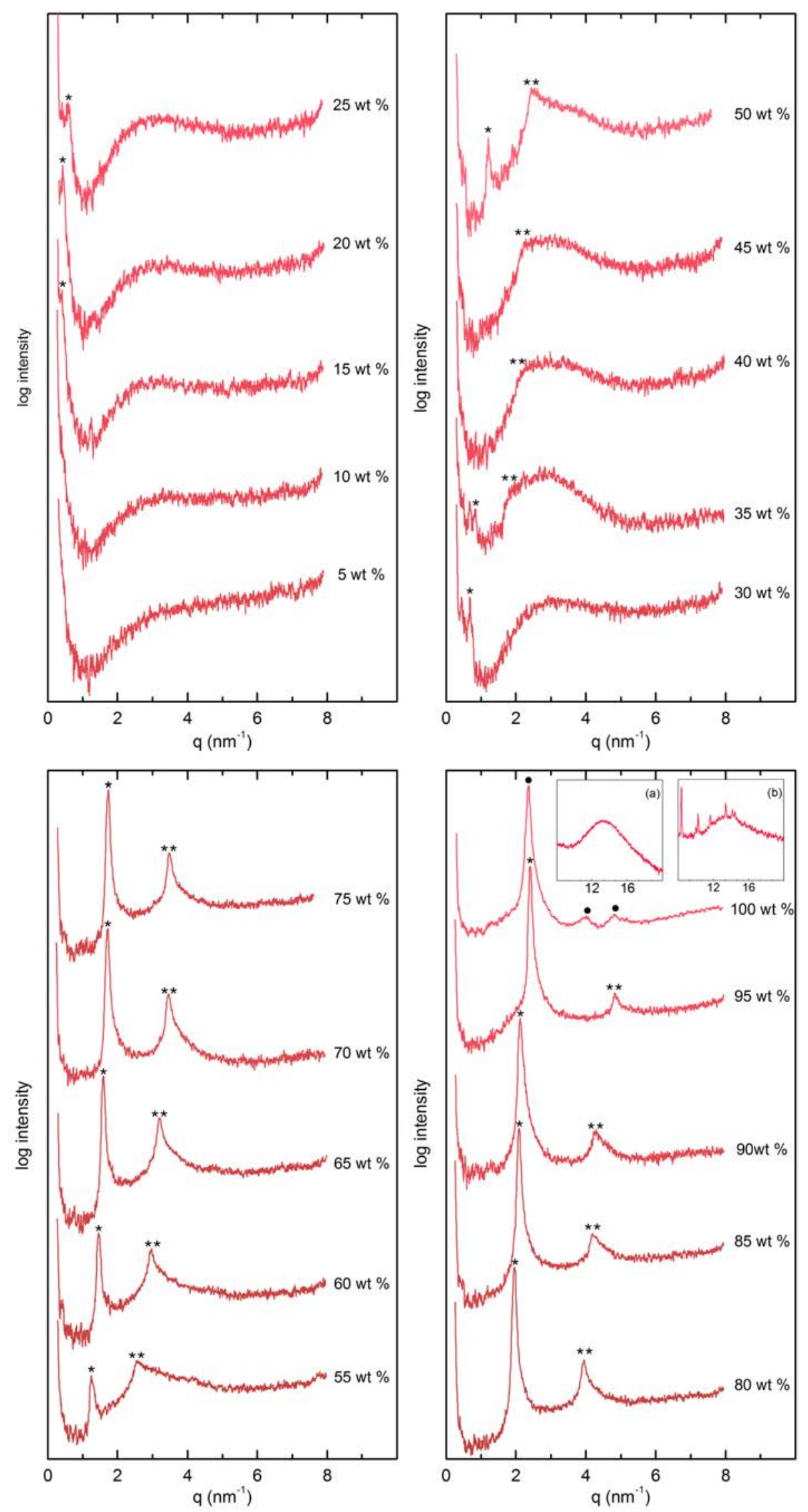

Figure 7.7: SAXS profiles of the Ch-AOT system with increasing surfactant concentration. Peaks marked by asterisks are Bragg peaks due to the lamellar phase. Peaks marked with dots, seen at $100 \mathrm{wt} \% \mathrm{Ch}$-AOT, are due to hexagonal ordering and are in the ratio $1: \sqrt{ } 3: \sqrt{ } 4$. The insets show the WAXS region for (a) 90 and (b) 100 wt \% Ch-AOT. 


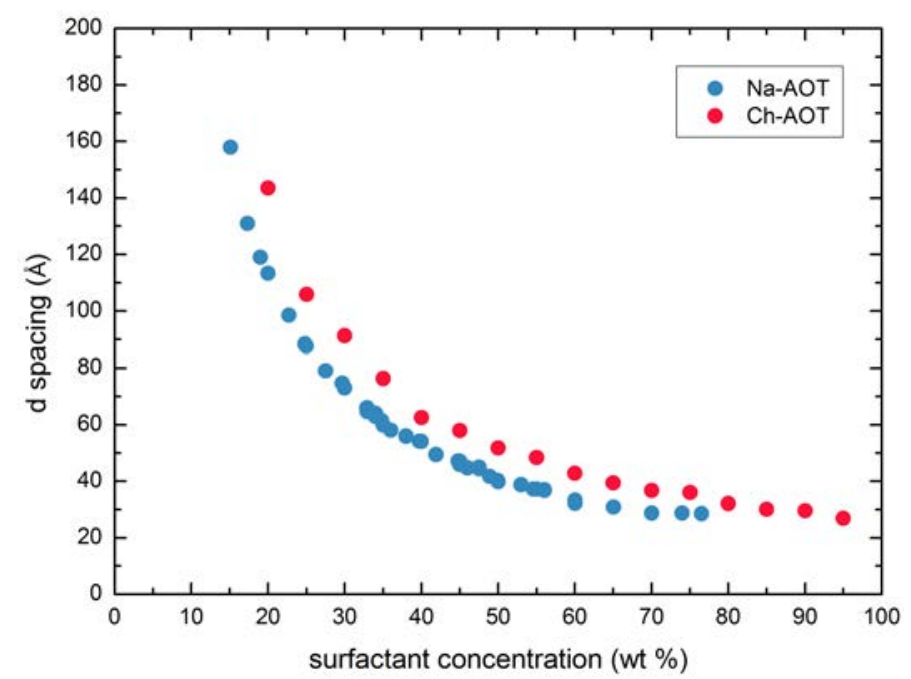

Figure 7.8: Changes in d spacing with increasing concentration for Ch-AOT and Na-AOT systems. Where the form factor interference resulted in the disappearance of the first-order Bragg peak, the $d$ spacing was calculated using the position of the second-order Bragg peak.

Also, the relative number of water molecules per AOT molecule is larger in the Ch-AOT system for any given weight percent (e.g. 29.2 vs. $24.7 \mathrm{H}_{2} \mathrm{O}$ molecules for Ch-AOT and Na-AOT, respectively, at $50 \mathrm{wt} \%$ surfactant concentration).

At high concentrations, the two systems trend towards a similar limit in bilayer spacing. Within this region, the close proximity of the bilayers means that the $d$ spacing is governed by steric hydration forces, which are far greater than the van der Waals attractive forces. With increasing concentration, this strong repulsion between bilayers promotes structural transformations towards inverse morphologies, such as the $\mathrm{V}_{\text {/l }}$ phase adopted by the Na-AOT system. However, the lamellar phase in the Ch-AOT system persists up to $98 \mathrm{wt} \%$ surfactant. The steric bulk of the $\mathrm{Ch}^{+}$ion results in an increased headgroup area in the Ch-AOT system, which promotes columnar packing, increased bending rigidity and increased van der Waals attractions. Also, the chaotropic nature of $\mathrm{Ch}^{+}$results in weak hydration and so a reduction in the repulsive forces. As such, the Ch-AOT system retains a $L_{\alpha}$ phase at concentrations approaching $100 \mathrm{wt} \%$. The formation of a hexagonal/crystalline phase only occurs upon changes in the intermolecular forces and packing arising from the unfavourable dehydration of the hydroxyl group, which is rapidly reversed upon exposure to moisture.

\section{Choline AOT Optical Microscopy}

Figure 7.9 shows POM textures of the Ch-AOT system with increasing concentration captured at $25^{\circ} \mathrm{C}$. At the boundary between the isotropic and two-phase regions, the textures are predominantly isotropic with the presence of some isolated FCD-II defects. As the concentration increases through 10 and 15 wt \%, the number of FCD-II defects dramatically increases, although they remain spherical and appear uniform in size. At this stage defect centres remain isolated and the background remains isotropic. At $20 \mathrm{wt} \%$, where bulk samples were observed to transition from a cloudy two-phase mixture to a single pure $L_{\alpha}$ phase, a change can 

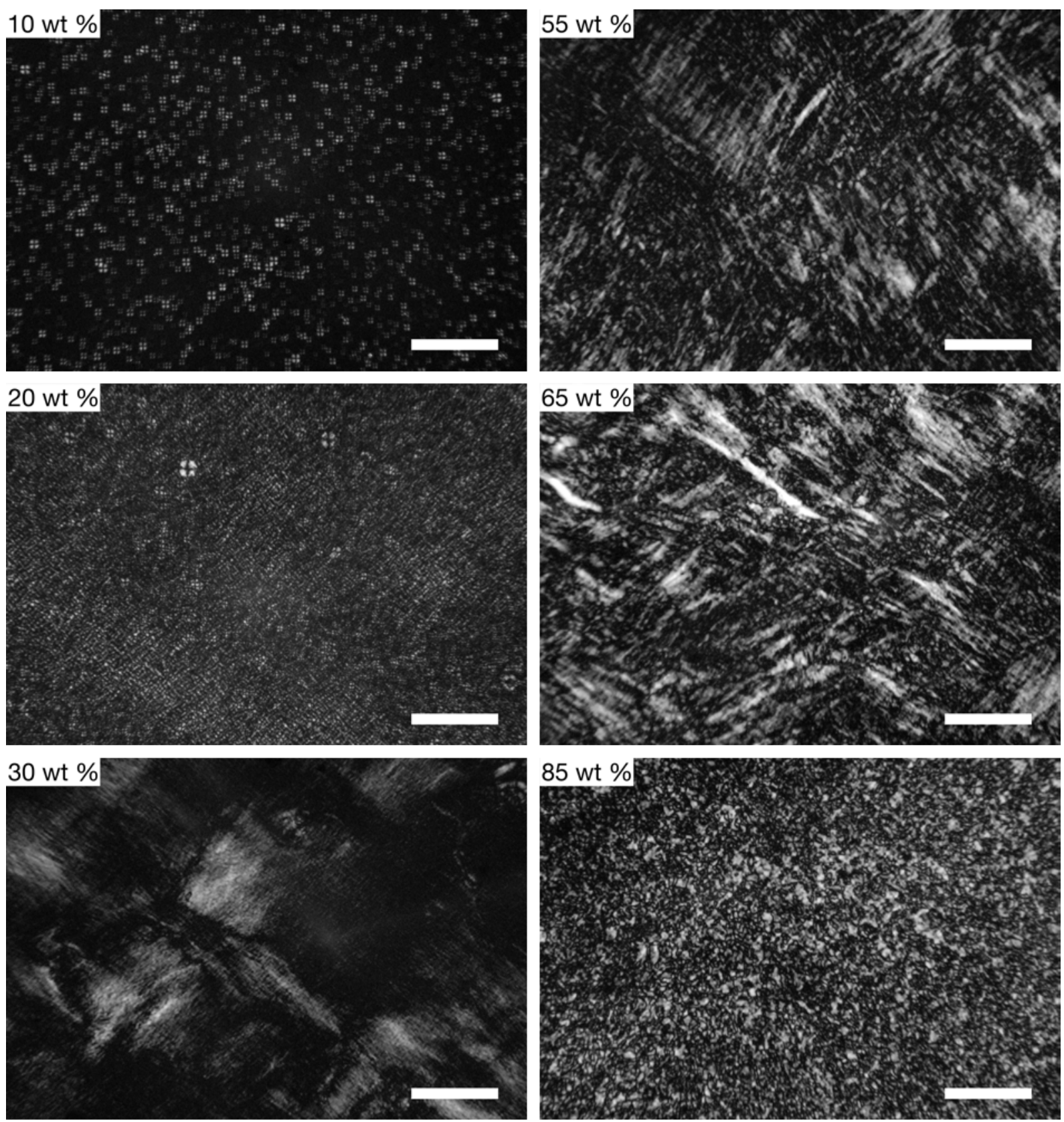

45 wt \%
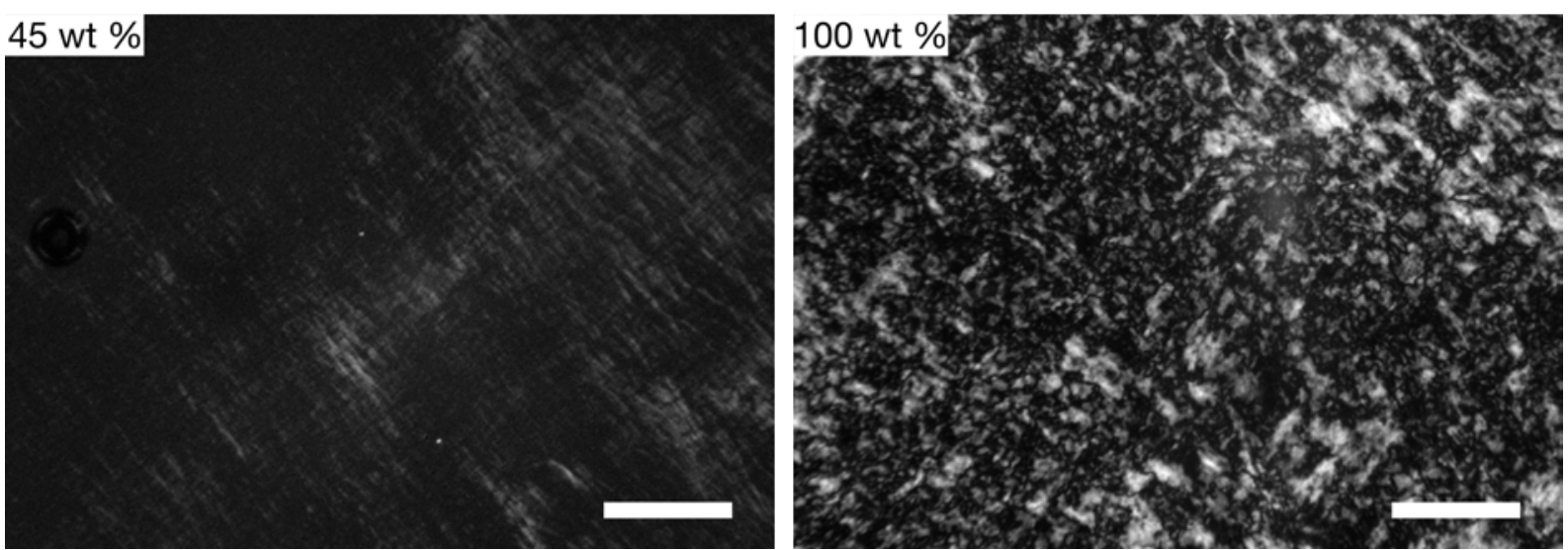

Figure 7.9: $\mathrm{POM}$ images of Ch-AOT with increasing concentration at $\sim 25^{\circ} \mathrm{C}$. A gradual move from positive to negative Gaussian curvature results in change from isolated FCD-II defects at low concentrations to streaky textures and mosaic patterns at high concentrations. A change from negative to positive birefringence occurs at $\sim 45 \mathrm{wt} \%$. Scale bars are $100 \mu \mathrm{m}$. Additional images are available in Appendix C. 
be observed in the FCDs. They are smaller in size and become visibly distorted in shape. The presence of orientated domains is also observed, appearing white against an isotropic background. Upon rotation of the sample stage $90^{\circ}$ these domains fade, appearing to become isotropic, and the previously isotropic regions appear as orientated domains.

Between 20 and 40 wt \% Ch-AOT a gradual change was observed. Individual FCD-II defects became drastically distorted and decreased in number, until finally they were no longer observed. Instead, the textures are dominated by the ordered domains that were first observed at $20 \mathrm{wt} \%$. These domains gradually decrease in size within this concentration region. The change from negative to positive birefringence, reported in the previously studied alkali metal systems, was also observed at $\sim 40 \mathrm{wt} \%$ surfactant, resulting in near isotropic textures. It becomes difficult to accurately define the defect behaviour at this concentration, although there also appears to be a decrease in the number of defects present.

Above 45 wt \% the switch to positive birefringence results in brighter textures, which are more easily assigned. The orientated domains have developed into the more classical streaky textures which were observed at similar concentrations in the previously discussed systems. This indicates a general switch from positive to negative Gaussian curvature and the formation of oily streaks (FCD-I defects) that obey Friedel's laws of association. With increasing concentration, the ordering of these streaky domains is increased and they become more uniformly aligned. The background of these textures is also observed to gradually change, switching from isotropic to mosaic patterns consistent with point defects of different signs packing together.

At Ch-AOT concentrations of $80 \mathrm{wt} \%$ and above, the mosaic pattern is the dominating feature of the textures. The distortion of the individual defect points within this pattern shows that the size and shape of each defect is defined by the interactions with those that surround it. As such, they are forced to adopt morphologies that differ from their ideal, energetically favoured states. Such a pattern suggests a large amount of curvature in the system with the structure of the phase on a micrometre lengthscale much deviated from the "ideal" of indefinite flat domains, whilst bilayers still exhibit lamellar ordering on a local lengthscale.

To characterise the behaviour at $100 \mathrm{wt} \% \mathrm{Ch}-\mathrm{AOT}$, the pure surfactant was slowly cooled to room temperature from an isotropic liquid to obtain a thin texture which allowed an assignment of the phase behaviour. This texture is shown in Figure 7.10 and the presence of needle-like crystals is observed overlaying a characteristic splayed fan texture arising due to the columnar focal conic domains of the $\mathrm{H}_{/ /}$phase.

In general, the nature of the defects observed in the Ch-AOT system is very similar to that already observed in the alkali metal and $\mathrm{NH}_{4}$-AOT systems. In particular, the textures are very similar to those of the Na-AOT system. The Na-AOT system stabilises the $L_{\alpha}$ phase over a larger concentration region than the $\mathrm{Li}$-AOT, K-AOT and $\mathrm{NH}_{4}$-AOT systems and so exhibits a greater range of defect behaviours, ranging from highly positive to highly negative Gaussian curvature. The concentration range of the $L_{\alpha}$ phase in the Ch-AOT system is increased further in comparison to the Na-AOT system, however no additional defect types are observed, but 


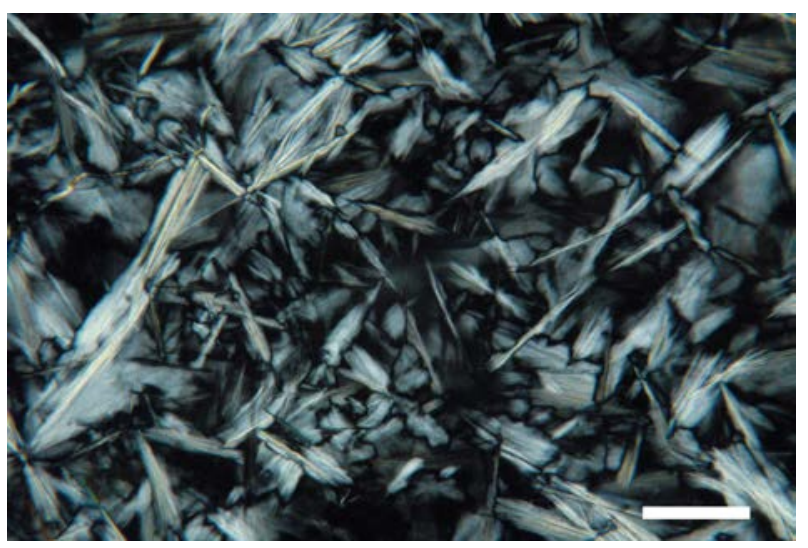

Figure 7.10: POM texture of pure Ch-AOT at $25^{\circ} \mathrm{C}$, obtained by slow cooling from an isotropic melt. Scale bar is $100 \mu \mathrm{m}$.

the distribution of the behaviours is extended over a larger concentration region. The main differences in the appearance of textures between the Ch-AOT and the Na-AOT systems are observed in the two-phase region. This is because the maximum size of the large MLVs formed is not dictated by the intrinsic curvature stress of the bilayer, but by the boundary conditions governed by the hydrophobic effect, which are controlled by the varying contributions of the different counterions. ${ }^{287}$ The difference in the relative contributions of the sodium and choline ions will be large, as $\mathrm{Na}^{+}$is a small, hard kosmotrope while $\mathrm{Ch}^{+}$is a large chaotrope and is hydrophobically hydrated. ${ }^{288}$

\section{Choline AOT Cryo-SEM}

The interactions of defects and the progression through different defect types within the Ch-AOT system was investigated further using cryo-SEM. Figure 7.11 shows images of a $15 \mathrm{wt} \%$ sample, which is within the two-phase region of the system, at various magnifications. Low magnification images shown in Figure 7.11 (a) and (b) confirm the presence of the MLVs (or FCD-II defects) reported in the POM textures, and indicate a distribution in size of 1-20 $\mu \mathrm{m}$. The distinction between the two phases is clear even at these low magnifications, with the defined MLVs in contrast to the highly connected and disordered surfactant and water domains. Figure 7.11 (c) and (d), at higher magnifications, show the ordered periodicity due to stacked bilayers within the MLV structure. Also evident at these magnifications is the high number of connections between the bilayers, which disrupt the water diffusion in three dimensions by creating barriers that restrict significant water movement over the measured timescale, as reported for Na-AOT via diffusion NMR. ${ }^{47}$ Both images also show the extent of the water connectivity within the continuous surfactant domain, with channels as large as $1 \mu \mathrm{m}$ in size discernible. Figure 7.11 (e) and (f) present images at even higher magnifications, and reveal that the continuous surfactant phase is also consistent with a bilayered structure. They also show that the connecting nodes within the continuous structure are often globular and resemble small MLVs in the range of 100-500 nm. These are held within the continuous surfactant network, but would be too small to be observed via POM. The connective nature of this two-phase region, with large isolated FCD-II defects distributed throughout, is similar to the structures observed 

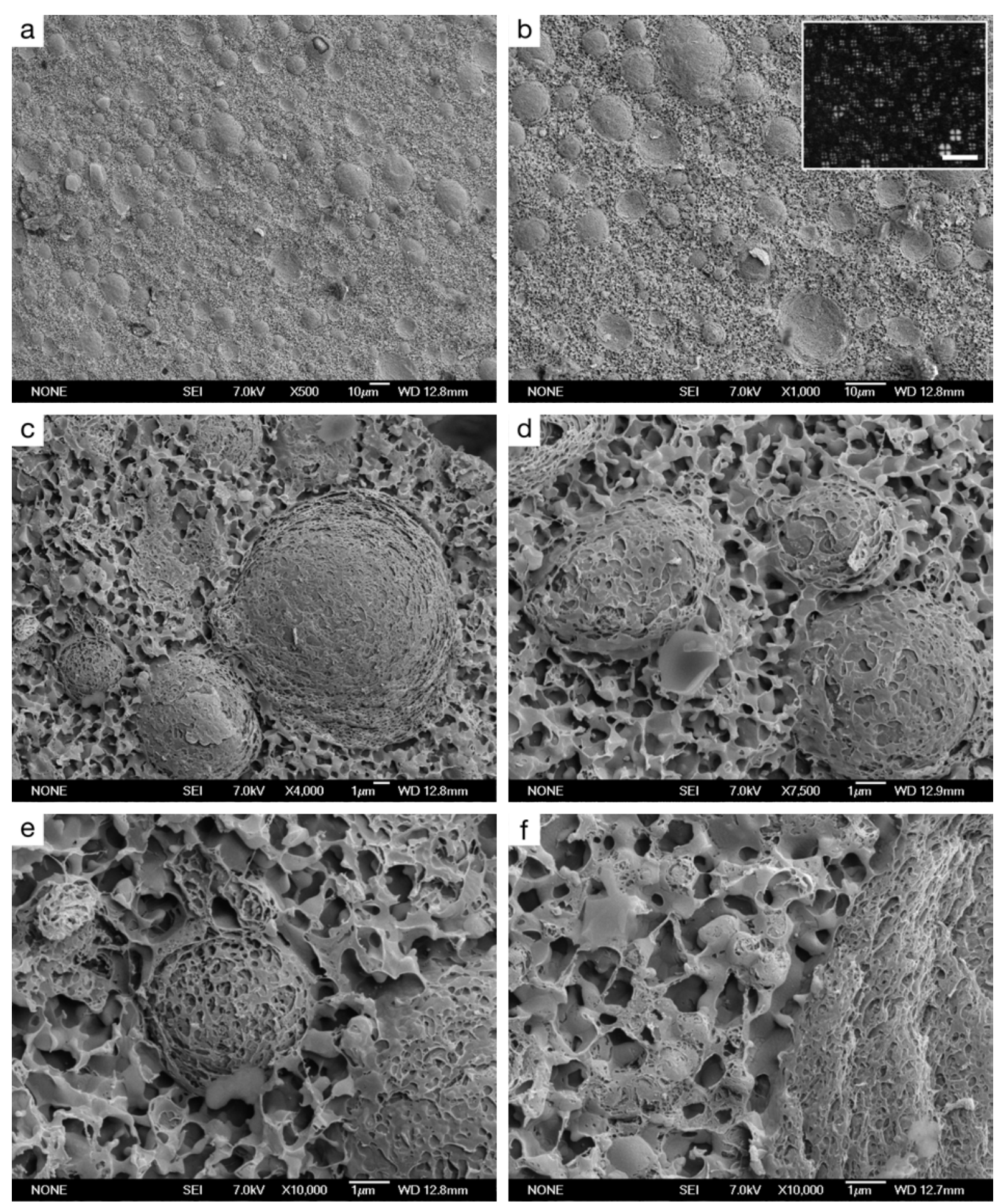

Figure 7.11: Cryo-SEM images of a sample of $15 \mathrm{wt} \%$ Ch-AOT. The inset shows the corresponding POM image, with a scale bar of $50 \mu \mathrm{m}$.

in the alkali metal systems; however, the distribution in size and number of FCD-II defects is increased.

Figure 7.12 shows cryo-SEM images of a sample of $25 \mathrm{wt} \%$ Ch-AOT. This concentration is within the pure $L_{\alpha}$ region of the phase diagram presented above, and as expected the images are significantly different from those presented in Figure 7.11. Both figures show the presence of focal conic defects, however at 25 wt \% surfactant the number of defects is increased and 
they are no longer scattered throughout a disordered surfactant phase. Some large channels of water up to $0.5 \mu \mathrm{m}$ in size remain. However, most of these channels have directional order, showing an increase in order compared to the two-phase region. The focal conic defects also show an increase in order, with the defects no longer being spherical. Instead they are elongated along one axis, with neighbouring defects elongated along the same direction. This elongation of defects is consistent with the observations made for the same concentration of Ch-AOT during the POM investigation, where the spherulite defects were observed to be distorted and domains of differing birefringence were observed. The cryo-SEM images presented here reveal that these domains are formed of groups of defects with on average directional ordering. Despite being orientated in the same direction, many of the defects are separated by the large water channels and so are not closely packed together.
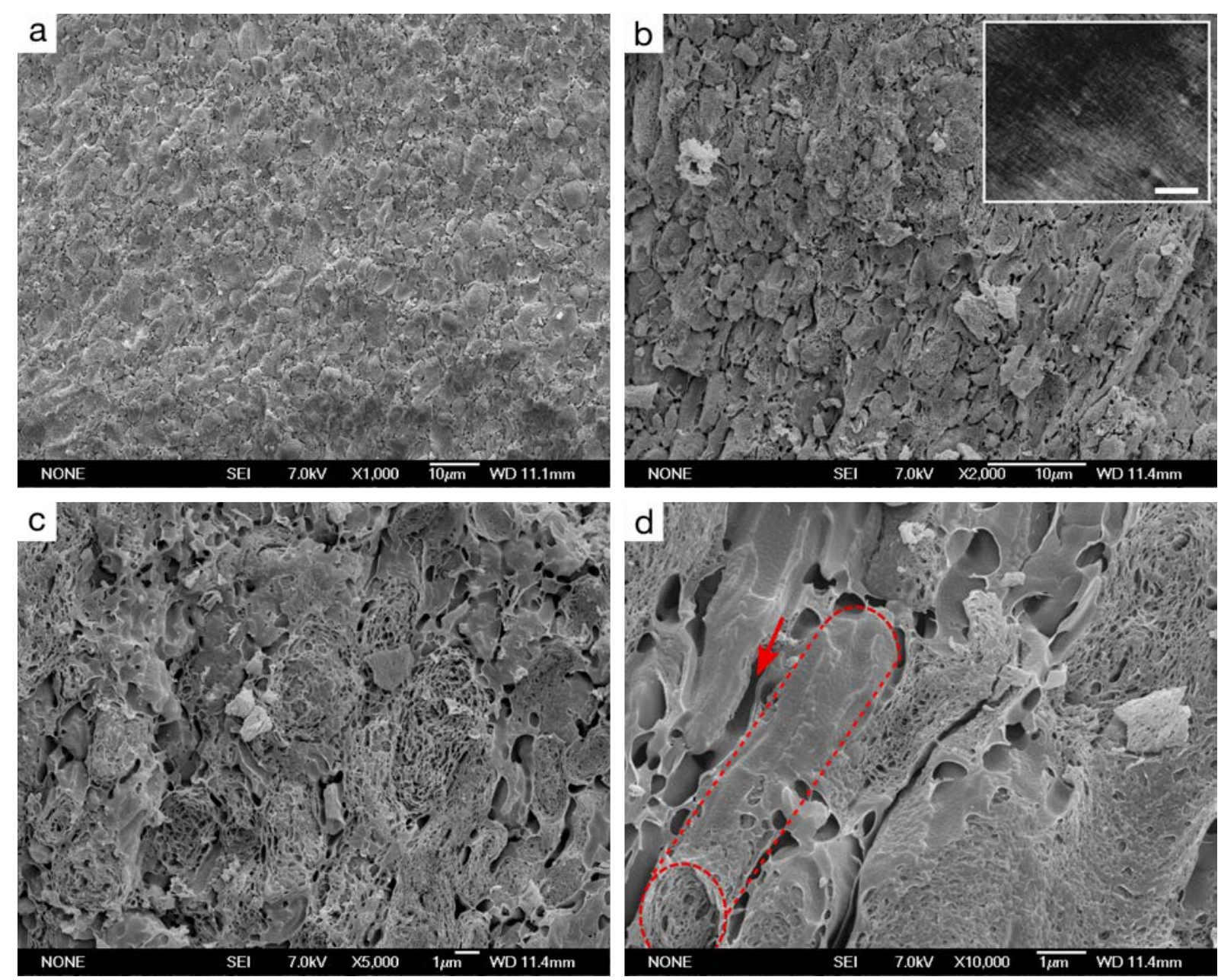

Figure 7.12: Cryo-SEM images of a sample of 25 wt \% Ch-AOT. The area highlighted in red shows one example of an elongated FCD-II defect. The red arrow indicates a large water channel. The inset shows the corresponding POM image, with a scale bar of $50 \mu \mathrm{m}$.

The size of the FCD-II defects range from 0.5 to $4 \mu \mathrm{m}$; however, the majority are between 1 and $2 \mu \mathrm{m}$. Along the length of the longer axis, defects are observed to be as large as $12 \mu \mathrm{m}$, although again the majority are smaller and range between approximately 4 and $6 \mu \mathrm{m}$. On local lengthscales, a high degree of connectivity between layers is evident, alongside pores of water. The constant formation and breakdown of bilayer connections (a characteristic of the $L_{3}$ phase), alongside the large channels of bulk water which separate many of the defects, accounts for the 
low viscosity of the bulk samples, which was evident upon handling (and is discussed further in Appendix E).

It should be noted that formation of elongated defects at this concentration was a reproducible result and they were observed to be thermodynamically stable over several months. They were not observed to relax into the classical spherical form over time, and as such, should not be confused with the short-lived kinetically stable elliptical defects that are often observed to briefly form during slide preparation for POM studies. The good correlation between the POM and cryo-SEM techniques, which involve different methods of sample preparation, also indicate that despite some similarities with the "leeks" formed by some lamellar phases under shear, the results here are not a product of shearing of the sample. Cryo-SEM images of the Ch-AOT system under controlled shear are presented in Appendix $E$ and differ significantly from those presented in Figure 7.12.

At 50 wt \% Ch-AOT (see Figure 7.13), images show that the defects have started to pack together and their shapes deviate from the spherical ideal, due to the constraints imposed by neighbouring defects. The number of defects is very high and their presence dominates the images, although there is also some evidence of orientated domains. This is particularly true in the lowest magnification images, which show a mixture of grouped defects aligned upon the same director axis, and flat sheet-like areas where the fracture along a grain boundary has removed domains aligned in other directions. However, the unilateral direction of the fracture limits our ability to comment upon their size. In conjunction with the POM discussed previously, we can conclude that the sample is comprised of domains consisting of a large number of defects aligned roughly along one directional axis. The various domains are orientated in different directions and their energetically unfavourable adjoining boundaries are prone to fracture upon cryo-SEM preparation.

At the higher magnifications shown in Figure $7.13(\mathrm{~d})-(\mathrm{f})$, point defect centres of both positive and negative sign are observed. The strain of packing upon the elasticity of individual defects is seen, as they are forced to adopt regions of sharp curvature to accommodate neighbouring defects. It is also clear in these images that the structure retains a high degree of connectivity between layers. In contrast to the alkali metal systems, the alignment of defects into oily streaks is not evident at this concentration. Indeed, the phase is more comparable to that of a shearinduced onion phase. However, the results reported here were reproducible and care was taken not to introduce shear into the samples. The samples were also prepared via the same methods as the systems discussed previously. This indicates either the Ch-AOT system is more prone to the formation of stable defects during the sample equilibration process or their formation is not shear dependent.

The alignment of defects to form oily streaks is clearly evident in images of $75 \mathrm{wt} \% \mathrm{Ch}$-AOT shown in Figure 7.14. The previous figures reveal a gradual progression, as isolated point defects within a medium of continuous surfactant and water domains grow in both size and number, before beginning to deform, align and pack together. Figure 7.14 however, presents a significantly different texture with increased long-range ordering. The reduction in 

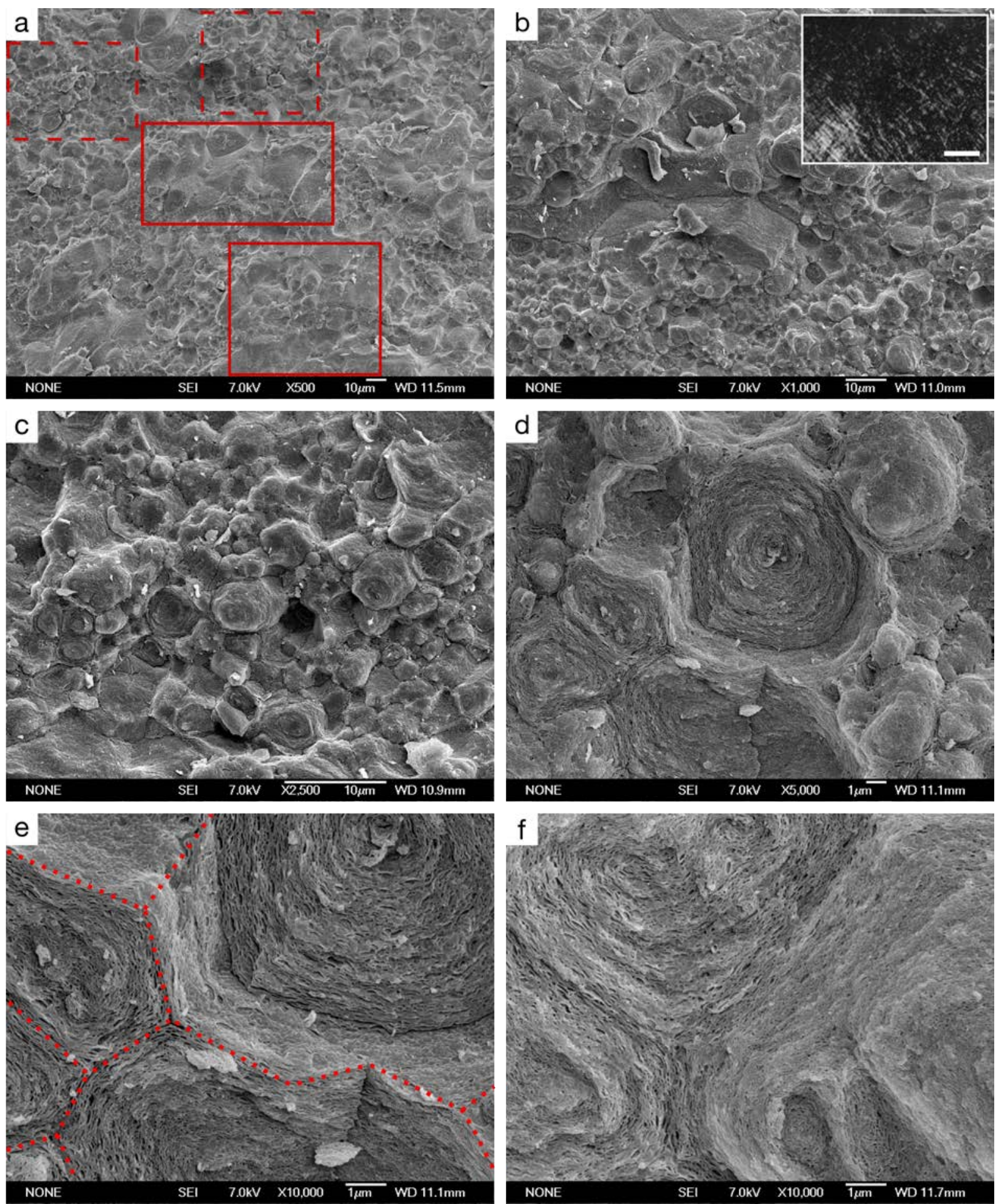

Figure 7.13: Cryo-SEM images of a sample of $50 \mathrm{wt} \%$ Ch-AOT. Red boxes indicate regions of grouped defects (dashed lines) and orientated domains (solid lines). The dotted line in (e) highlights the close packing of defects. The inset shows the corresponding POM image, with a scale bar of $50 \mu \mathrm{m}$.

bilayer spacing results in a significant decrease in the bilayer elasticity, shown by the loss of connectivity and water channels between layers (evident at lower concentrations), due to the phase being governed by electrostatic rather than entropic forces. The inherent curvature of the bilayer has also clearly changed, with a switch to defects of negative Gaussian curvature and ordered domains of up to $40 \mu \mathrm{m}$ in size. There is also a reduction in the size of individual defects, and oily streaks have formed and are aligned with neighbouring streaks. When 

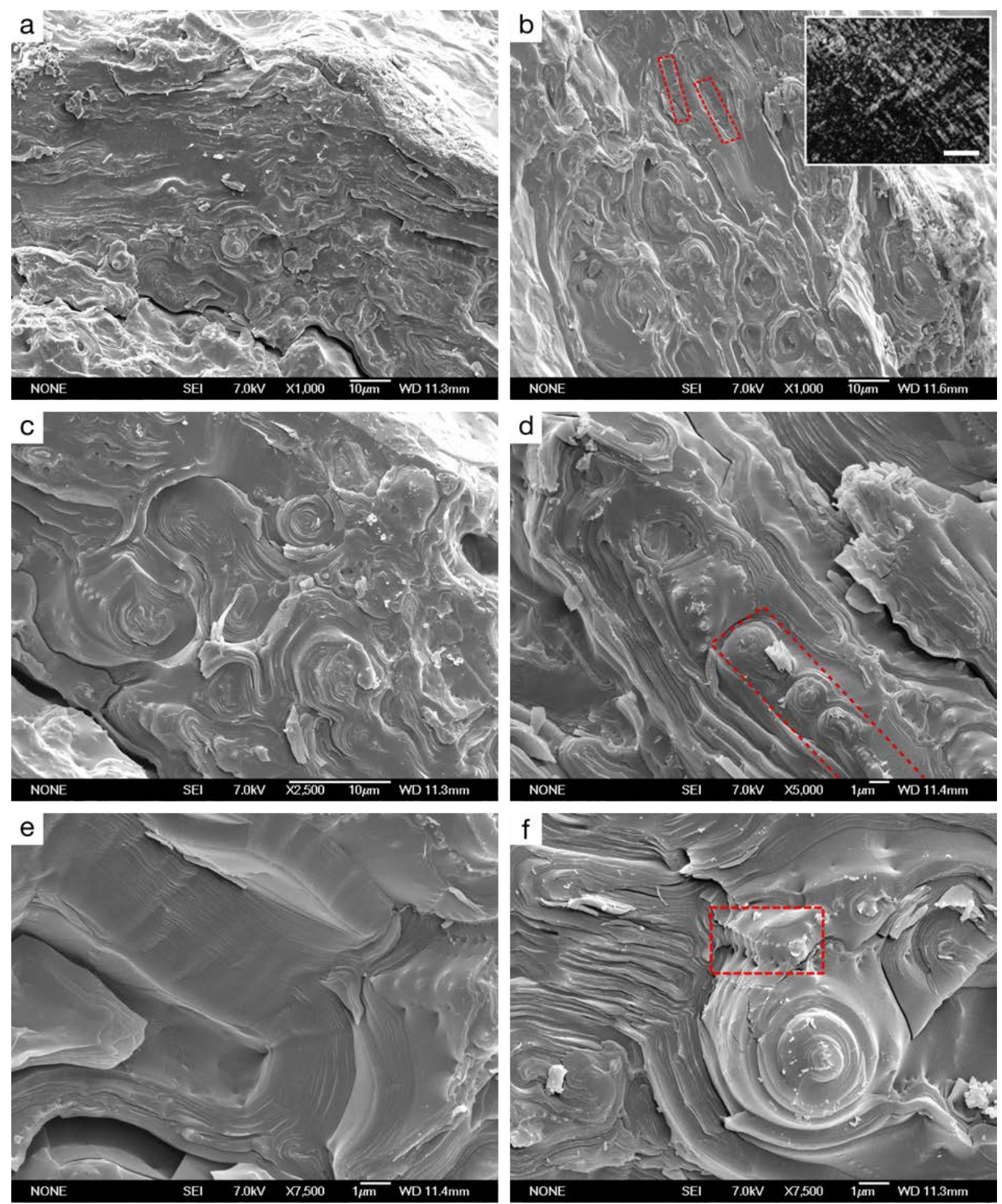

Figure 7.14: Cryo-SEM images of a sample of $75 \mathrm{wt} \%$ Ch-AOT. Red boxes show examples of oily streaks. The inset shows the corresponding POM image, with a scale bar of $50 \mu \mathrm{m}$.

comparing different oily streaks, the size of their defect components varies drastically (from 0.3 to $3 \mu \mathrm{m}$ ), although with both the large depth of field shown in low magnification images and the fact that different streaks have fractured along different planes, this may be misleading. Whilst the oily streaks are aligned in similar directions to the neighbouring streaks, there are still ordered domains revealed along different directional axes. Large point defects of both positive and negative Gaussian curvature also remain; however, the majority are of negative Gaussian curvature. 
At 95 wt \% Ch-AOT, shown in Figure 7.15, the mosaic pattern observed in POM images is confirmed to be due to the close packing of highly curved FCD-I defects. The oily streaks are significantly shorter than those observed at $75 \mathrm{wt} \%$ ( 2-3 $\mu \mathrm{m}$ instead of up to $20 \mu \mathrm{m})$ and are highly curved and packed together with no obvious average direction. This confirms that as the surfactant concentration has increased, the Gaussian curvature of the system has become increasingly negative. Low magnification images do show some larger domains where the system is presenting more classical lamellar behaviour, although these too contain some FCD-I defects.
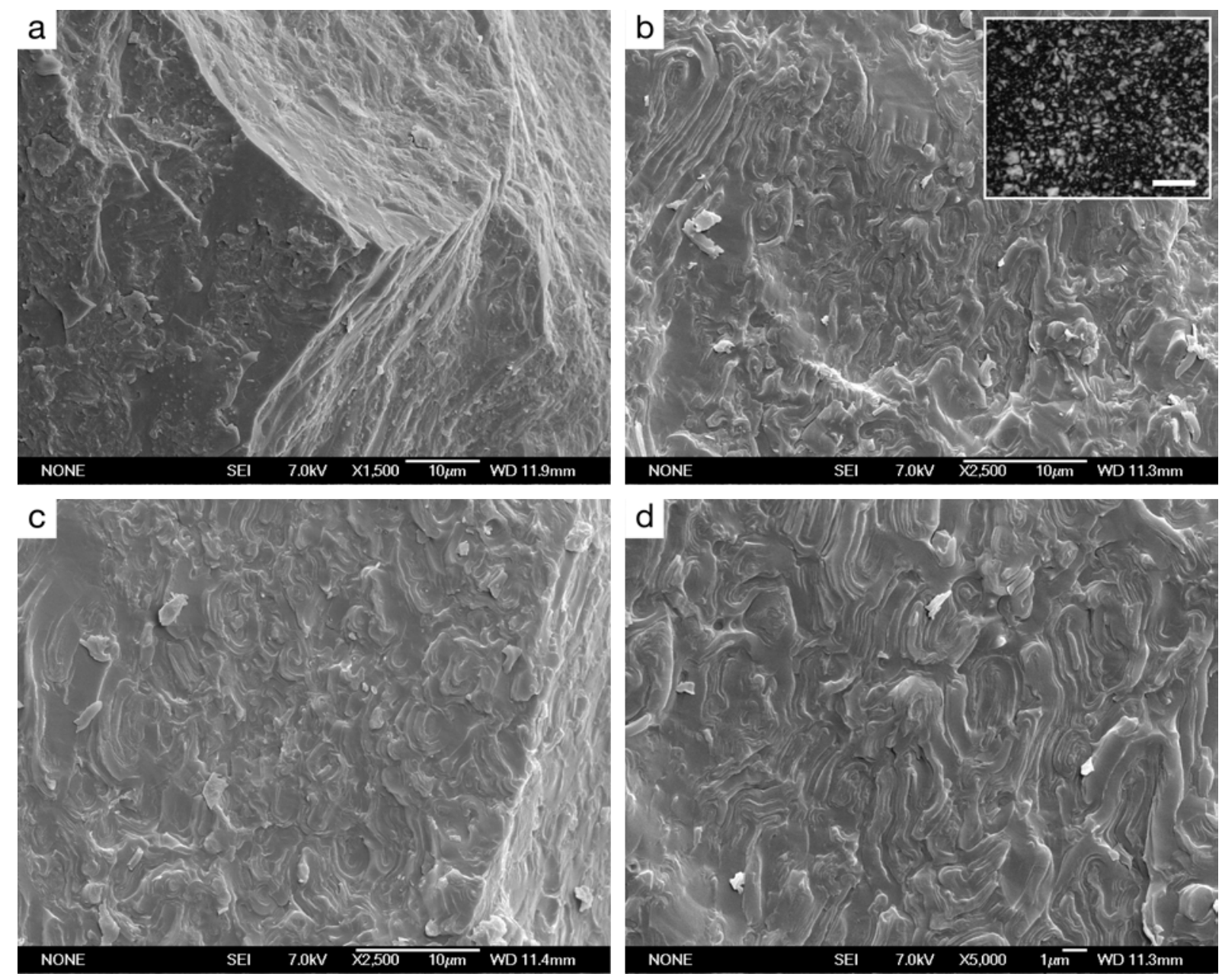

Figure 7.15: Cryo-SEM images of a sample of $95 \mathrm{wt} \%$ Ch-AOT. The inset shows the corresponding POM image, with a scale bar of $50 \mu \mathrm{m}$.

\section{Choline AOT: Summary}

In Chapters 5 and 6, it was shown that the alkali metal and ammonium systems of AOT transition from a multilamellar vesicular phase, through a lamellar phase and into inverted structures, with increasing concentration. This is due to AOT molecules becoming more closely packed and adopting an increasingly inverted shape. It is also facilitated by a decrease in headgroup area, and changes to the elastic constants of the bilayer, which arise due to the 
variation in hydration and electrostatic forces that govern the free energy. The stability of the lamellar phases in these systems was mediated through the formation of defect structures with increasingly negative Gaussian curvature. However, this progression was subject to change upon manipulation of the intermolecular forces, through changes in counterion, temperature or by addition of salt.

The use of the bulky and weakly hydrated $\mathrm{Ch}^{+}$counterion in the Ch-AOT system has increased the stability of the $L_{\alpha}$ phase, with the system retaining a lamellar morphology over most of the phase diagram. The larger effective headgroup size in Ch-AOT restricts the ability of the molecule to adopt a non-cylindrical shape. This results in increased bilayer rigidity and almost removes the formation of inverted structures entirely. Despite this increased bilayer rigidity, the system follows the same general trend in defect behaviour as the previously studied systems. The POM and cryo-SEM images are in good agreement and show the formation of highly curved defects at both high and low concentrations. This progression from FCD-II to FCD-I defects upon increasing surfactant concentration is accompanied by a reduction in connectivity, which is evident at low concentrations. This connectivity is also a feature of the SAXS profiles of the system, which show broad scattering and weak second-order lamellar reflections due to long-range undulation forces between the bilayers.

Despite the induced rigidity in the Ch-AOT bilayer, the formation of defect-rich morphologies ranging from MLVs to mosaic-like structures shows that the bilayer still has a curvature frustration cost associated with the spontaneous curvature of each monolayer being restricted. It also shows that the packing within the bilayer is flexible, and that the surfactant molecules are able to accommodate the demands of drastically different interfacial curvatures, despite being restricted to a lamellar phase. This is likely enabled by the range of packing environments available to the surfactant tails.

Upon increasing temperature, the lamellar phase of the Ch-AOT system is observed to transition to an isotropic liquid at a lower temperature than the previously studied systems. This is presumably a result of the dissociation of the bulky $\mathrm{Ch}^{+}$ion disrupting the water network. However, no lyotropic phase transitions were observed on increasing temperature, and the defects were not observed by POM to appreciably change until the melting transition occurs. This is further indication of the rigidity of the Ch-AOT bilayer and suggests that due to the large steric effects, the system is stable upon changes to the electrostatic forces. This was further investigated through the addition of salt to the system.

\section{Addition of Salt to the Choline AOT System: Experimental Results}

Na-AOT has often been cited as a good system for investigating the behaviour of lamellar phases, due to it presenting the phase over such a large concentration range. Given that the Ch-AOT system described above shows an extended stability of this phase, beyond that exhibited by Na-AOT, a further investigation was carried out to determine if this increased phase 
stability is retained upon the addition of electrolyte. Ch-AOT concentrations of approximately $20,40,60$ and $80 \mathrm{wt} \%$ were prepared with the addition of various concentrations of $\mathrm{NaCl}$, as presented in Table 7.1. As the Na-AOT ${ }^{90}$ and $\mathrm{NH}_{4}$-AOT (see Chapter 6) systems form sponge phases at $\sim 1.4$ and $1.2 \mathrm{wt} \% \mathrm{NaCl}$, respectively, the Ch-AOT samples prepared initially contained between 1 and $2 \mathrm{wt} \% \mathrm{NaCl}$. All of these samples presented optical birefringence under crossed polarised light indicative of a lamellar phase, and so samples of increasing $\mathrm{NaCl}$ concentration were prepared until a change in the phase behaviour was induced.

Figure 7.16 shows the bulk samples prepared for $~ 40 \mathrm{wt} \%$ Ch-AOT between crossed polarisers. Moving from 4 to $5 \mathrm{wt} \% \mathrm{NaCl}$, the bulk sample is observed to separate into two layers. The bottom layer continues to show optical birefringence consistent with a lamellar phase, whilst the top layer is optically isotropic. The addition of $6 \mathrm{wt} \% \mathrm{NaCl}$ resulted in a single isotropic phase of low viscosity, and by $7 \mathrm{wt} \% \mathrm{NaCl}$, two isotropic phases were observed. Similar trends were observed for 20 and 60 wt \% Ch-AOT. At 80 wt \% Ch-AOT an isotropic phase induced by the addition of 4 wt $\% \mathrm{NaCl}$ was of higher viscosity rather than lower viscosity than the preceding lamellar phase. Further addition of $\mathrm{NaCl}$ at this surfactant concentration surpassed the solubility limit of $\mathrm{NaCl}$ in water ( $35.9 \mathrm{~g}$ per $100 \mathrm{~g}$ water at $20^{\circ} \mathrm{C}$ ) and resulted in the retention of the viscous phase plus undissolved $\mathrm{NaCl}$.

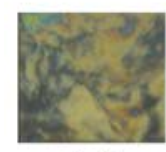

1.0

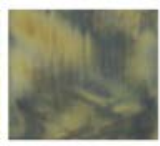

1.4

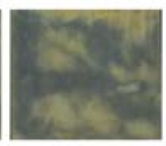

1.5

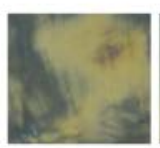

1.8

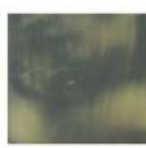

2.1

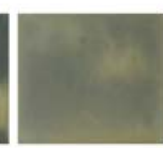

3.0

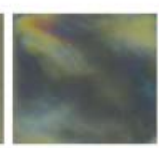

4.2

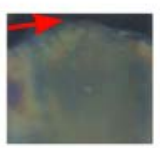

5.0

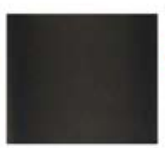

6.0

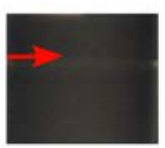

7.0

wt $\% \mathrm{NaCl}$ in 40 wt \% Ch-AOT samples

Figure 7.16: Bulk samples of $\sim 40$ wt $\%$ Ch-AOT with increasing $\mathrm{NaCl}$ concentrations viewed under crossed polarised light. Samples of 5.0 and $7.0 \mathrm{wt} \% \mathrm{NaCl}$ present two phases (interfaces indicated by red arrows) and $6.0 \mathrm{wt} \%$ presents a single optically isotropic phase.

The effect of the increasing salinity for each concentration was monitored using SAXS and is presented as stacked plots in Figure 7.17. In cases where samples presented phase separation, the layers were further separated by centrifugation and analysed individually. The most obvious changes in the SAXS profiles are observed at high Ch-AOT concentrations. Figure 7.17 (d) shows that 80 wt \% Ch-AOT exhibits a $L_{\alpha}-V_{/ /}$transition, with sharp Bragg peaks in the ratio of $1: 2$ giving way to a series of peaks in the ratio $\sqrt{ } 6: \sqrt{ } 8: \sqrt{ } 12: \sqrt{ } 14: \sqrt{ } 16: \sqrt{ } 20: \sqrt{ } 22: \sqrt{ } 24$, indicative of a bicontinuous cubic phase with an la3d space group of a gyroid arrangement. Upon further salt addition, a change in peak ratios reveals a $\mathrm{V}_{I /}-\mathrm{V}_{\text {II }}$ transition, with peaks giving rise to the ratio $\sqrt{ } 2: \sqrt{ } 3: \sqrt{ } 4: \sqrt{ } 6: \sqrt{ } 9: \sqrt{ } 10$ of a $P n 3 m$ diamond space group. The assignment of peaks to the correct Miller indices and hence the correct space group is confirmed by the linear plots in Figure 7.18, which allow the calculation of lattice parameters for each sample shown in Table 7.1. The two space groups are based on the infinite periodic minimal surface and transitions between the two can be brought about by small changes in composition, shape parameter or temperature. ${ }^{14}$ 
Table 7.1: Structural parameters for various compositions of $\mathrm{Ch}-\mathrm{AOT}$ and $\mathrm{NaCl}$.

\begin{tabular}{|c|c|c|c|c|c|}
\hline $\begin{array}{l}\text { Concentration of } \\
\text { Ch-AOT (wt \%) }\end{array}$ & $\begin{array}{l}\text { Concentration of } \\
\mathrm{NaCl} \text { (wt \%) }\end{array}$ & $\begin{array}{c}\text { Phase } \\
\text { Classification }\end{array}$ & $\begin{array}{c}\mathrm{q}^{*} \\
\left(\mathrm{~nm}^{-1}\right)\end{array}$ & hkl & $\begin{array}{c}\text { Characteristic } \\
\text { Length }(\AA)\end{array}$ \\
\hline 20.04 & 0.00 & $\mathrm{~L}_{\alpha}$ & 0.442 & 001 & $d_{\alpha} 142$ \\
\hline 19.79 & 1.04 & $\mathrm{~L}_{\alpha}$ & 0.489 & 001 & $d_{\alpha} 128$ \\
\hline 19.90 & 1.35 & $\mathrm{~L}_{\alpha}$ & - & - & - \\
\hline 19.91 & 1.49 & $\mathrm{~L}_{\alpha}$ & - & - & - \\
\hline 19.95 & 1.83 & $\mathrm{~L}_{\alpha}$ & - & - & - \\
\hline 19.90 & 2.04 & $\mathrm{~L}_{\alpha}$ & 0.461 & 001 & $d_{\alpha} 138$ \\
\hline 20.00 & 3.03 & $\mathrm{~L}_{\alpha}$ & 0.379 & 001 & $d_{\alpha} 166$ \\
\hline 19.99 & 3.96 & $\mathrm{~L}_{3} / \mathrm{L}_{1}$ & $0.358 /-$ & - & $\mathrm{d}_{3} 175 /-$ \\
\hline 19.09 & 5.01 & $\mathrm{~L}_{3} / \mathrm{L}_{1}$ & $0.354 /-$ & - & $\mathrm{d}_{3} 177 /-$ \\
\hline 19.20 & 6.02 & $\mathrm{~L}_{3} / \mathrm{L}_{1}$ & - & - & - \\
\hline 40.04 & 0.00 & $\mathrm{~L}_{\alpha}$ & 0.988 & 001 & $d_{\alpha} 63.6$ \\
\hline 39.91 & 0.98 & $\mathrm{~L}_{\alpha}$ & 0.902 & 001 & $d_{\alpha} 61.8$ \\
\hline 39.49 & 1.38 & $\mathrm{~L}_{\alpha}$ & - & - & - \\
\hline 39.89 & 1.45 & $\mathrm{~L}_{\alpha}$ & - & - & - \\
\hline 39.89 & 1.77 & $\mathrm{~L}_{\alpha}$ & - & - & - \\
\hline 39.91 & 2.12 & $\mathrm{~L}_{\alpha}$ & - & - & - \\
\hline 39.91 & 3.02 & $\mathrm{~L}_{\alpha}$ & - & - & - \\
\hline 39.90 & 4.15 & $\mathrm{~L}_{\alpha}$ & - & - & - \\
\hline 39.81 & 4.96 & $\mathrm{~L}_{\alpha} / \mathrm{L}_{3}$ & - & - & - \\
\hline 38.05 & 5.99 & $\mathrm{~L}_{3}$ & - & - & - \\
\hline 40.02 & 7.02 & $\mathrm{~L}_{3} / \mathrm{L}_{1}$ & - & - & - \\
\hline 36.51 & 8.83 & $\mathrm{~L}_{3} / \mathrm{L}_{1}$ & - & - & - \\
\hline 60.00 & 0.00 & $\mathrm{~L}_{\alpha}$ & 1.470 & 001 & $d_{\alpha} 42.7$ \\
\hline 59.97 & 1.07 & $\mathrm{~L}_{\alpha}$ & 1.420 & 001 & $\mathrm{~d}_{\alpha} 44.2$ \\
\hline 60.07 & 1.25 & $\mathrm{~L}_{\alpha}$ & - & - & - \\
\hline 60.06 & 1.51 & $\mathrm{~L}_{\alpha}$ & - & - & - \\
\hline 59.94 & 1.78 & $\mathrm{~L}_{\alpha}$ & - & - & - \\
\hline 60.14 & 2.02 & $\mathrm{~L}_{\alpha}$ & 1.440 & 001 & $d_{\alpha} 43.6$ \\
\hline 59.64 & 3.05 & $\mathrm{~L}_{\alpha}$ & 1.427 & 001 & $d_{\alpha} 44.0$ \\
\hline 58.50 & 4.07 & $\mathrm{~L}_{\alpha}$ & 1.382 & 001 & $d_{\alpha} 45.5$ \\
\hline 57.80 & 4.97 & $\mathrm{~L}_{\alpha} / \mathrm{L}_{3}$ & $1.397 / 1.345$ & $001 /-$ & $\mathrm{d}_{\alpha} 45.0 / \mathrm{d}_{\alpha}^{\prime} 46.7$ \\
\hline 57.33 & 6.07 & $\mathrm{~L}_{3}$ & 1.193 & - & $d_{3} 52.7$ \\
\hline 60.00 & 7.08 & $\mathrm{~L}_{3} / \mathrm{L}_{1}$ & $1.433 /-$ & - & $d_{3} 43.8 /-$ \\
\hline 80.00 & 0.00 & $\mathrm{~L}_{\alpha}$ & 1.957 & 001 & $d_{\alpha} 32.1$ \\
\hline 79.91 & 1.05 & $\mathrm{~L}_{\alpha}$ & 1.949 & 001 & $d_{\alpha} 32.3$ \\
\hline 80.10 & 1.22 & $\mathrm{~L}_{\alpha}$ & - & - & - \\
\hline 80.01 & 1.49 & $\mathrm{~L}_{\alpha}$ & - & - & - \\
\hline 80.02 & 1.80 & $\mathrm{~L}_{\alpha}$ & - & - & - \\
\hline 80.03 & 2.00 & $\mathrm{~L}_{\alpha}$ & 1.956 & 001 & $d_{\alpha} 32.1$ \\
\hline 80.05 & 3.01 & $\mathrm{~L}_{\alpha}$ & 1.834 & 001 & $d_{\alpha} 34.3$ \\
\hline 80.04 & 3.97 & $\mathrm{~V}_{I I}(\mathrm{la} \mathrm{d} \mathrm{d})$ & 1.728 & 211 & a 89.3 \\
\hline 80.03 & 4.59 & $\mathrm{~V}_{I I}(\mathrm{la3d})$ & 1.718 & 211 & a 89.3 \\
\hline 77.16 & 5.30 & $\mathrm{~V}_{\| /}(\mathrm{Pn} 3 \mathrm{~m})$ & 1.489 & 110 & a 54.9 \\
\hline 75.75 & 6.59 & $\mathrm{~V}_{I I}(\mathrm{Pn} 3 \mathrm{~m})$ & 1.617 & 110 & a 56.8 \\
\hline
\end{tabular}

Note: $d_{\alpha}, d_{3}$ and a refer to the $d$ spacing of the lamellar phase, the average bilayer correlation of the sponge phase, and the cubic lattice parameter, respectively. $d_{\alpha}^{\prime}$ refers to the $d$ spacing of a lamellar impurity present in the sponge phase due to mutual solubilities. Where no values for $\mathrm{q}^{*}, \mathrm{hkl}$ and characteristic length are given, samples were characterised solely using POM or the $\mathrm{q}^{*}$ peak was not discernible from SAXS data. 
It should be noted that while samples presenting the Pn3m space group are at higher $\mathrm{NaCl}$ concentrations, they are also at slightly lower surfactant concentrations than the la3d samples (77.2 and $75.8 \mathrm{wt} \%$ rather than $80.0 \mathrm{wt} \%)$. This means the change in phase behaviour could be a result of either salt concentration or the dilution of the surfactant, or both. As such, the driving force cannot be determined with confidence. A transition from la3d to $\mathrm{Pn} 3 \mathrm{~m}$ upon dilution is consistent with the expected phase progression in surfactant systems, having been observed experimentally and theorised previously. ${ }^{14,204,289,290}$ However, an la3d to Pn3m transition can also be rationalised upon the addition of salt, and has been observed previously with other surfactant systems. ${ }^{291}$ The phase progression observed in the $\mathrm{Ch}-\mathrm{AOT} / \mathrm{NaCl}$ system is likely to be a combination of the two factors, with the increased $\mathrm{NaCl}$ concentration resulting in screening of van der Waals and electrostatic forces between layers, and a reduction in the repulsion between adjacent headgroups. This results in a smaller headgroup area, which promotes increasingly negative Gaussian curvature and increases the spontaneous curvature of the bilayer. The progression from $L_{\alpha}$ to la3d and $P n 3 m$ is commonly observed upon increased temperature due to a continued swelling of the phase and an epitaxial relationship has been shown between the (001), (220) and (111) planes, respectively. ${ }^{292}$ The close relationship between cubic phases means that such transitions would result from only subtle changes to the composition of the sample. Although a full phase diagram of this system has not been produced, it is likely that such a phase diagram would reveal the formation of more phases, such as an Im3m cubic phase, and a hexagonal phase at higher surfactant concentrations.

Figure 7.17 (c) shows the recorded profiles for $60 \mathrm{wt} \%$ Ch-AOT samples. Two sharp Bragg peaks are clearly defined for the system, before the addition of $\mathrm{NaCl}$, at 1.47 and $2.94 \mathrm{~nm}^{-1}$. These reflections remain for $\mathrm{NaCl}$ concentrations up to at least $4.1 \mathrm{wt} \%$ and are also present in the bottom layer of the $5.0 \mathrm{wt} \% \mathrm{NaCl}$ sample. These peaks confirm the retention of lamellar ordering, and a gradual shift to lower $q$ indicates a small increase in bilayer spacing with increasing $\mathrm{NaCl}$ concentration, consistent with an increase in shielding of electrostatic interactions. The loss of intensity and broadening of the second-order peak indicate a decrease in structural ordering, and hence greater bilayer fluctuation, consistent with the increase in bilayer spacing. The reduced order also suggests a change in defect behaviour on a local lengthscale, likely an increase in size and/or number of connective passages or holes within the bilayer. Such a conclusion is consistent with the phase transition observed upon the further addition of $\mathrm{NaCl}$ (i.e. the top layer of the $5.0 \mathrm{wt} \%$ and the entirety of the $6.1 \mathrm{wt} \% \mathrm{NaCl}$ samples). These isotropic samples have similar SAXS spectra, indicating that they are the same phase. Both present broad scattering between 0.66 and $4.45 \mathrm{~nm}^{-1}$, consistent with bilayer correlations of $a L_{3}$ phase.

The spectra of the 5.0 and $6.1 \mathrm{wt} \% \mathrm{NaCl}$ samples also both display a peak in the same region as the first-order lamellar peak observed at lower salt concentrations. In the case of $5.0 \mathrm{wt} \%$ $\mathrm{NaCl}$, this peak is sharp and can be explained by the presence of some lamellar phase due to the mutual solubility of lamellar and sponge phases, meaning that the separation of the two layers is likely inefficient. At $6.1 \mathrm{wt} \% \mathrm{NaCl}$, where the bulk sample was observed to be a single isotropic phase, this peak is broader, although its presence is still likely due to the retention of 

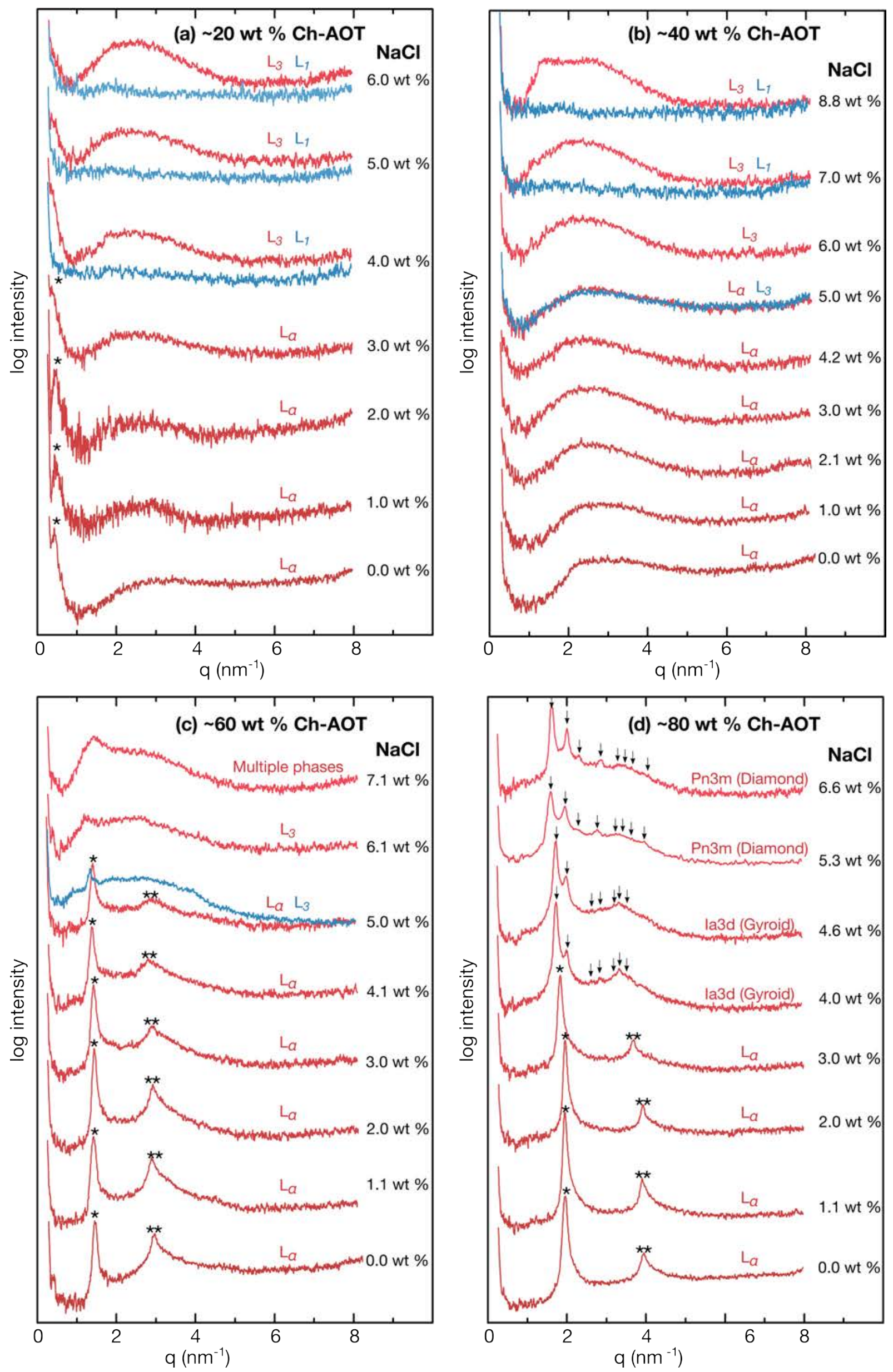

Figure 7.17: (Continued on the following page.) 
Figure 7.17: Stacked SAXS plots showing the effect of increasing $\mathrm{NaCl}$ concentration at surfactant concentrations of (a) 20, (b) 40, (c) 60 and (d) 80 wt \% Ch-AOT (exact compositions of each sample can be found in Table 7.1). Where samples presented two phases, they were separated by centrifugation - the lower layer is presented in blue and the upper layer is shown in red. Lamellar peaks are denoted by asterisks, with single and double asterisks indicating first- and second-order peaks respectively. Peaks arising due to cubic reflections are denoted by arrows and follow the ratios defined by the Miller indices of the assigned space groups as labelled.

(a) At 20 wt \% Ch-AOT, the loss in intensity of the first-order lamellar peak due to a $L_{\alpha}-L_{3}$ transition is seen.

(b) At 40 wt \% Ch-AOT, the first-order lamellar peak is not always discernible and so the scattering of $L_{\alpha}$ and $L_{3}$ phases in this region are very similar, although the coexistence of both phases at $5.0 \mathrm{wt} \% \mathrm{NaCl}$ (see Figure 7.16) confirms the transition, and the $\mathrm{L}_{3}$ phase is the only phase existing at $6.0 \mathrm{wt} \% \mathrm{NaCl}$.

(c) At $60 \mathrm{wt} \%$ Ch-AOT, the clear loss of the second-order $L_{\alpha}$ peak and a rise in diffuse broad scattering is evident upon transition from a $L_{\alpha}$ phase to a $L_{3}$ phase. The first-order $L_{\alpha}$ peak is also diminished but does not disappear entirely. At 5.0 and $6.1 \mathrm{wt} \% \mathrm{NaCl}$ this is likely due to the retention of some regions of lamellar ordering due to difficulties in separating samples of mutual solubility, see text for a fuller description of the scattering pattern. At a concentration of $7.1 \mathrm{wt} \%$ this is due to a salting out process occurring and the formation of multiple phases within the sample.

(d) At 80 wt \% Ch-AOT, the transition from $L_{\alpha}$ to successive $V_{/ /}$phases of la3d and Pn3m space groups is clear. The values and assignments of the marked reflections are listed in Table 7.1.
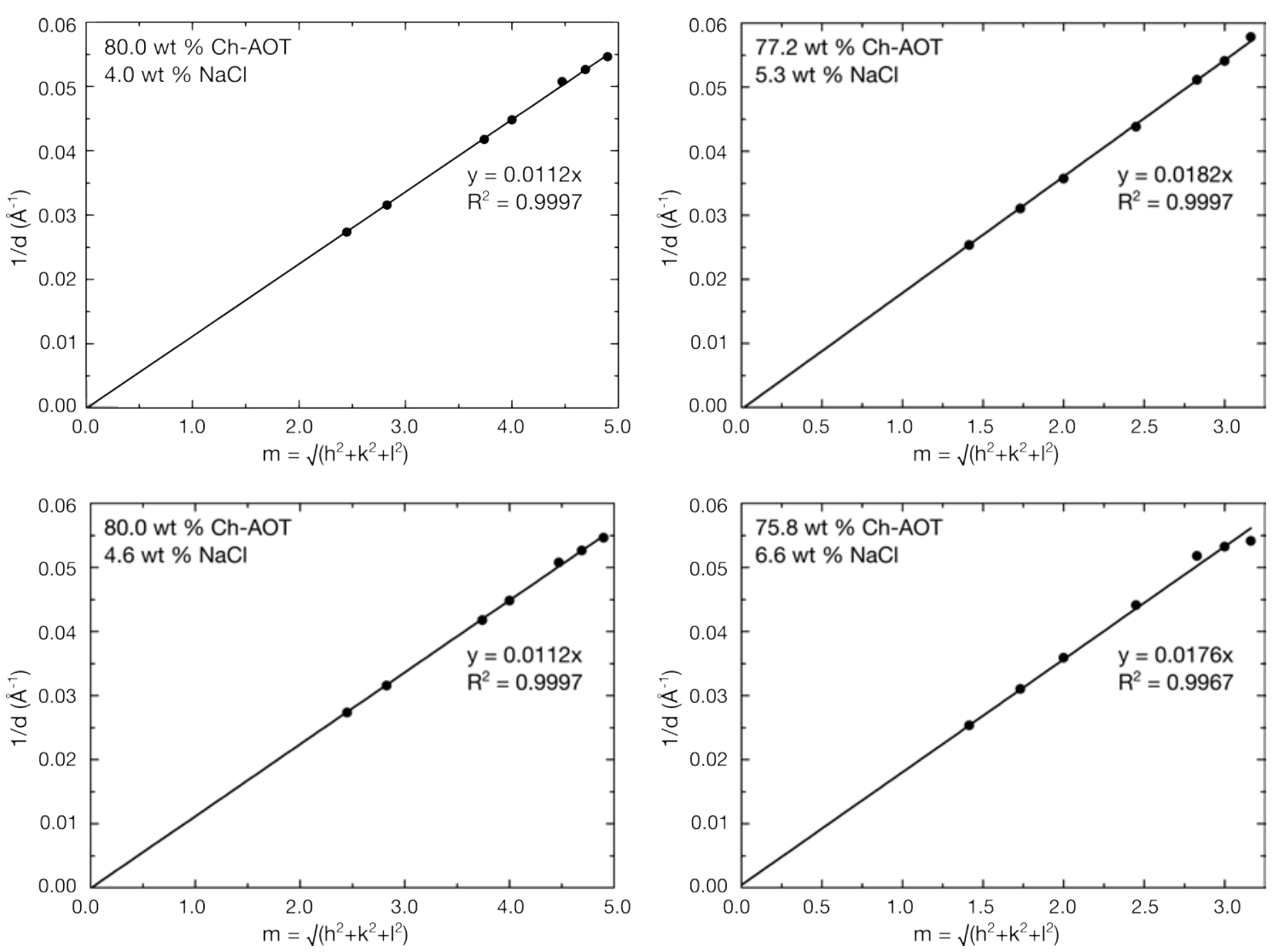

Figure 7.18: Plots showing the observed reciprocal d spacings $v s . \sqrt{ }\left(h^{2}+k^{2}+l^{2}\right)$ for cubic phases of the Ch-AOT system, where hkl are the assigned Miller indices. Correct assignment of the lattice type and space groups is confirmed by the linearity of each plot and the intercept of the fitted line at the origin. The lattice parameter for each graph is calculated as the reciprocal of the gradient of each fit. 
small quantities of lamellar phase. This sample was analysed again after seven days rest, and presented no distinguishable differences in scattering. This suggests the peak is not caused by a metastable $L_{\alpha}$ phase formed due to shear upon loading, but does not entirely rule it out as these phases can persist for long periods of time. ${ }^{293}$ The peak may be due to sponge cellcell correlations; however, if observed, such a peak would be expected to appear at a slightly lower $q$ value $\left(d_{3}\right.$ is generally observed to be $1.2-1.5$ times the size of $\left.d_{\alpha}\right){ }^{26,27}$ Although, Beck and Hoffmann have presented several examples of ionic systems forming non-classical sponge phases where the broad correlation peak is observed at the same value of $q$ as the $L_{\alpha}$ peak. ${ }^{29,30,294}$ A similar system was also reported by Pal et al., although they opted to describe the phase as a coacervate rather than a sponge phase. ${ }^{295}$ These non-classical sponge phases have so far only been reported for ionic surfactant systems containing cosurfactants. We should consider that a sample of $60 \mathrm{wt} \% \mathrm{Ch}$-AOT and $6.1 \mathrm{wt} \% \mathrm{NaCl}$ equates to $0.91 \mathrm{Na}^{+}$ions per AOT molecule. This is a significant amount of $\mathrm{Na}^{+}$ions, which could allow the organic $\mathrm{Ch}^{+}$ion to adopt the role of a cosurfactant. At $7.1 \mathrm{wt} \% \mathrm{NaCl}$ the bulk sample was no longer isotropic and was observed to have undergone a "salting out" process, and as such is considered to contain multiple phases. Both the broad scattering and the peak at approximately $1.45 \mathrm{~nm}^{-1}$ are, however, retained.

For concentrations of $\sim 40 \mathrm{wt} \% \mathrm{Ch}$-AOT, the switch from anisotropy to isotropy that is clearly evident under crossed polarisers is less obvious when looking at the SAXS profiles shown in Figure 7.17 (b). At 40 wt \% Ch-AOT, the intensity of the first-order Bragg peak is masked due to the peak coinciding with the form factor minimum, and so all that is observed in both the $L_{\alpha}$ and $L_{3}$ phases is broad diffuse scattering. However, there is a general trend with the broad scattering shifting to lower values of q, with $q_{\max }$ decreasing from 2.98 to $2.26 \mathrm{~nm}^{-1}$ when comparing samples with salt concentrations of 1.0 and $7.0 \mathrm{wt} \% \mathrm{NaCl}$. This trend equates to a swelling of the phase, and an increase in the average pore size within the lamellar or sponge bilayer due to extra curvature induced by the shielding of electrostatic forces. At $5.0 \mathrm{wt} \% \mathrm{NaCl}$ the two phases are present in the same sample. When analysed separately, the two layers give superimposable SAXS spectra, a clear indication of the similar structure of the two phases. This further highlights the degree of geometric distortion in the lamellar phase compared to its ideal structure and thus confirms its sponge-like character on sub-micrometre lengthscales as observed with cryo-SEM and discussed previously.

The SAXS profiles for $20 \mathrm{wt} \%$ Ch-AOT- see Figure 7.17 (a) - are similar to those observed for 40 wt \% Ch-AOT, with the broad scattering of both the $L_{\alpha}$ and the $L_{3}$ phases being very similar. However, the first-order lamellar peak is more evident at these surfactant concentrations, and therefore so is the transition between the two phases in the SAXS spectra. The $q_{\max }$ of the broad scattering shows a gradual shift to lower q numbers, indicating a general swelling of the lamellar phase and increase in the average pore size within the bilayer when moving towards the $L_{\alpha}-L_{3}$ transition. The pores, that become the characteristic distance of the sponge phase, continue to swell upon increasing salt concentration.

Further evidence for the formation of $a L_{3}$ phase is provided by cryo-SEM, with representative images shown in Figure 7.19. Figure 7.19 (a) and (b) show the sponge phase formed in a 

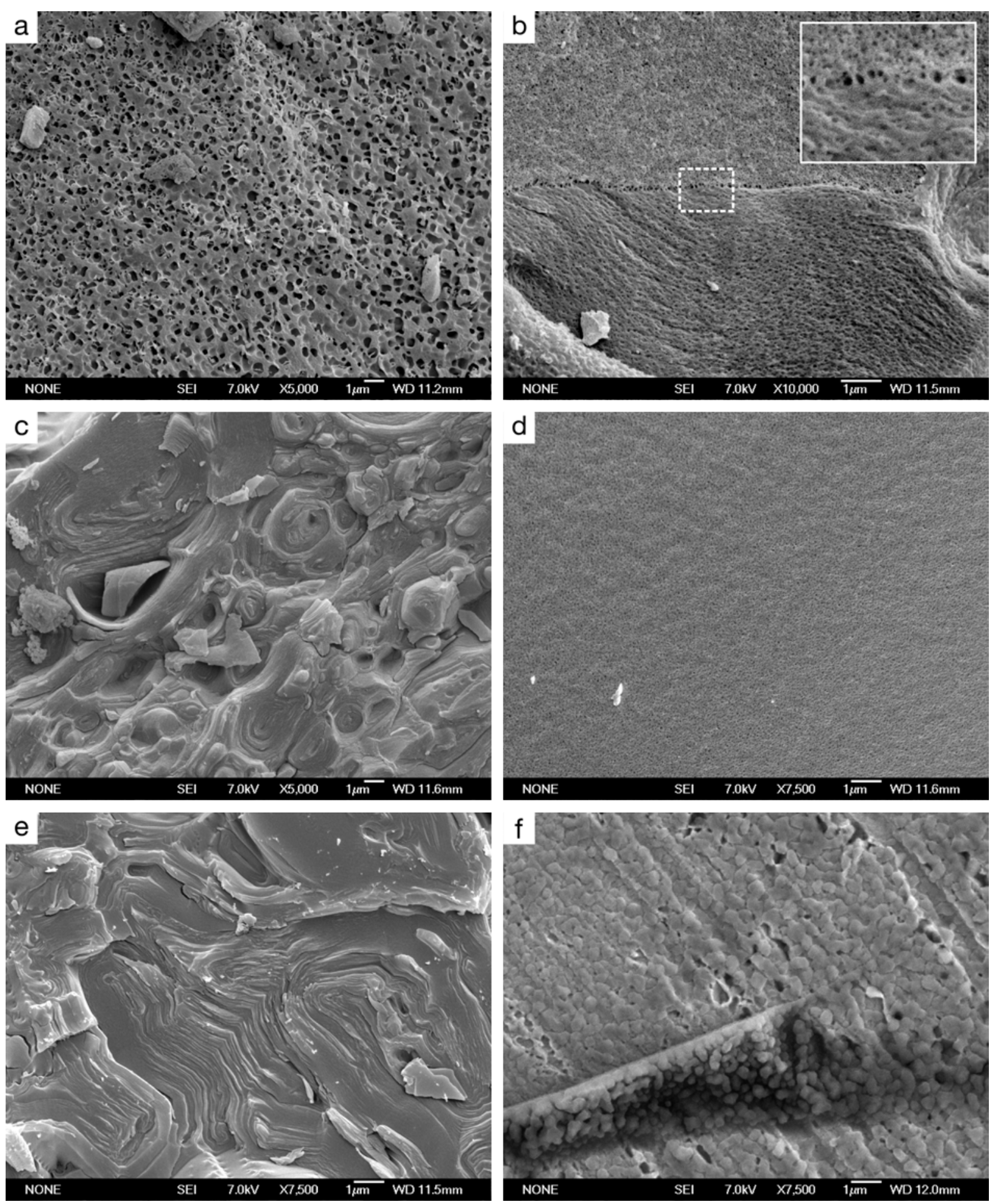

Figure 7.19: Cryo-SEM images showing the variety of phases formed in samples of varying Ch-AOT/NaCl/water composition. SEM allows the distinction between phases, with the $L_{3}$ phase showing a bicontinuous structure and the absence of the domains and point defects observed in the $\mathrm{L}_{\alpha}$ phase. Ch-AOT/NaCl concentrations of (a) and (b) 38.1/6.0 wt \% ( $\mathrm{L}_{3}$ phase), (c) $60.0 / 1.1 \mathrm{wt} \%$ ( $\mathrm{L}_{\alpha}$ phase), (d) 57.3/6.1 wt \% ( $\mathrm{L}_{3}$ phase), (e) 75.0/0.0 wt \% ( $\mathrm{L}_{\alpha}$ phase) and (f) $75.8 / 6.6 \mathrm{wt} \%\left(\mathrm{~V}_{/ /}\right.$phase). The inset in (b) shows an enlargement of the section indicated by dashed line.

sample of $38.1 \mathrm{wt} \% \mathrm{NaCl}$ with the addition of $6.0 \mathrm{wt} \% \mathrm{NaCl}$. Figure 7.19 (a) presents the expected highly connected structure of the sponge phase. The structure is bicontinuous and it is possible to trace a connected path throughout the image over several layers and over many 
micrometres. The variation in pore size giving rise to the broad SAXS scattering is evident, as is the high degree of curvature characteristic of the sponge phase. Figure 7.19 (b) shows a small region (not representative of the whole sample) that contains both $L_{3}$ and $L_{\alpha}$ ordering. The small $\mathrm{L}_{\alpha}$ domain $(\sim 10 \mu \mathrm{m})$ is likely a metastable region induced by shear during sample preparation and preserved during freezing. The lamellar ordering exhibited in this image is weak, with layers clearly fluctuating and riddled with bridging defects. This highly connective structure and the presence of large water channels indicate the phase has a bending rigidity close to that needed for the neighbouring sponge phase. The boundary between the two domains is sharp, with the domains connected via a series of bridging defects. Sharp boundaries between coexisting $L_{3}$ and $L_{\alpha}$ phases have previously been reported ${ }^{296}$ and the connection of phases through bridging defects is also consistent with previous observations. ${ }^{297,298}$

Figure 7.19 (c) and (d) show $\sim 60 \mathrm{wt} \%$ Ch-AOT with the addition of 1.1 and $6.1 \mathrm{wt} \% \mathrm{NaCl}$, respectively. At $1.1 \mathrm{wt} \% \mathrm{NaCl}$, the system presents a packed array of defined point defects ranging in size from $100 \mathrm{~nm}$ to $10 \mu \mathrm{m}$, and suggests little deviation from the images shown in the previous section for the Ch-AOT system without the addition of salt. However, in stark contrast, the image at $6.1 \mathrm{wt} \% \mathrm{NaCl}$ shows no lamellar defects or domains, instead presenting the continuous disordered structure of the $L_{3}$ phase. Although the images show different magnifications, as expected there is a clear reduction in the size of the channels of the sponge phase moving from 40 to $60 \mathrm{wt} \% \mathrm{Ch}$-AOT.

Figure 7.19 (e) and (f) show samples of $\sim 75 \mathrm{wt} \%$ Ch-AOT - the former in the absence of salt and the latter with the addition of $6.6 \mathrm{wt} \% \mathrm{NaCl}$. In the absence of salt, ordered layering of bilayers is evident due to a $L_{\alpha}$ phase. The fracture is predominantly perpendicular to the bilayer and reveals the packing of a variety of domains of approximately equal size. For the sample containing $\mathrm{NaCl}$ there is no evidence of lamellar ordering. Instead, the appearance of a continuous but globular structure with obvious water channels, is consistent with the assignment of the cubic phase from SAXS. The image shows similarities to SEM images presented by Rizwan et al. of the Pn3m cubic phase and cubosomes formed by phythanol/water systems, which were described as tortuous and nodular. ${ }^{299}$ However, the observation of cubic ordering at the magnifications used and resolution achieved is not necessarily expected. As such, we consider the possibility that the high surfactant content and 3D ordering in the cubic phase could result in crystallisation upon freezing, resulting in the ordered structure observed.

The effect of increased salinity on the temperature and defect behaviour of the surfactant was evaluated using polarising optical microscopy. It was observed that upon increasing the concentration of $\mathrm{NaCl}$, the transition temperature of the lamellar phase was increased. For $\sim 60$ wt \% Ch-AOT, the lamellar phase was observed to melt at 110,117 and $131{ }^{\circ} \mathrm{C}$ at $\mathrm{NaCl}$ concentrations of 0,2 and $4 \mathrm{wt} \%$, respectively. ${ }^{\ddagger} \mathrm{A}$ similar increase of phase transition temperature was reported by Sutton et al. for phospholipid mixtures of phosphatidylethanolamine and phosphatidylglycerol, ${ }^{300}$ a possible indication that the behaviour of the Ch-AOT system could be biologically relevant or analogous to biological systems. Upon heating of $L_{3}$ phase samples, no evidence of a temperature driven $L_{3}$ to $L_{\alpha}$ transition was observed. These observations are

\footnotetext{
${ }^{\ddagger}$ Example POM images can be found in Appendix C.
} 
in contrast to the sponge phase observed in the $\mathrm{Na}-\mathrm{AOT} / \mathrm{NaCl} /$ water system $^{90}$ and those of the $\mathrm{K}$-AOT and $\mathrm{NH}_{4}$-AOT systems discussed previously, in Chapters 5 and 6 , which presented high temperature $\mathrm{L}_{\alpha}$ phases.

Figure 7.20 shows the change in defect behaviour observed using polarising optical microscopy upon increasing $\mathrm{NaCl}$ concentration at 60 wt \% Ch-AOT. Up to a concentration of 2.0 wt \% $\mathrm{NaCl}$, the lamellar phase is unchanged from samples without salt. Small, orientated, streaky domains are observed alongside mosaic regions, which upon rotation of the sample stage are revealed to contain streaky domains oriented along a different director axis. As shown in cryo-SEM Figure 7.19 (c), these domains are regions of packed, distorted and interacting point defects. At a $\mathrm{NaCl}$ concentration of $3.0 \mathrm{wt} \%$, these small domains have clearly changed, with the oily streaks appearing more separated and longer in length. The appearance of central nodes from which oily streaks propagate implies that the sample has undergone flow upon placement of the cover slip, an indication of the decreasing viscosity and gradual loss of long-range order upon increasing $\mathrm{NaCl}$ concentration. Upon increasing the $\mathrm{NaCl}$ concentration further, this trend continues, with individual defect centres within oily streaks discernible at $4.0 \mathrm{wt} \% \mathrm{NaCl}$. The defects are clearly no longer tightly packed, an indication of both increasing disorder in the phase and a thinner texture due to a decrease in viscosity upon approaching the $\mathrm{L}_{\alpha}-\mathrm{L}_{3}$ phase boundary. The decrease in viscosity upon increasing $\mathrm{NaCl}$ concentration was quantified used rheology, and is shown in Figure 7.21. At $5.0 \mathrm{wt} \% \mathrm{NaCl}$, large isotropic regions are presented between regions of lamellar defects due to the presence of the $L_{3}$ phase in this
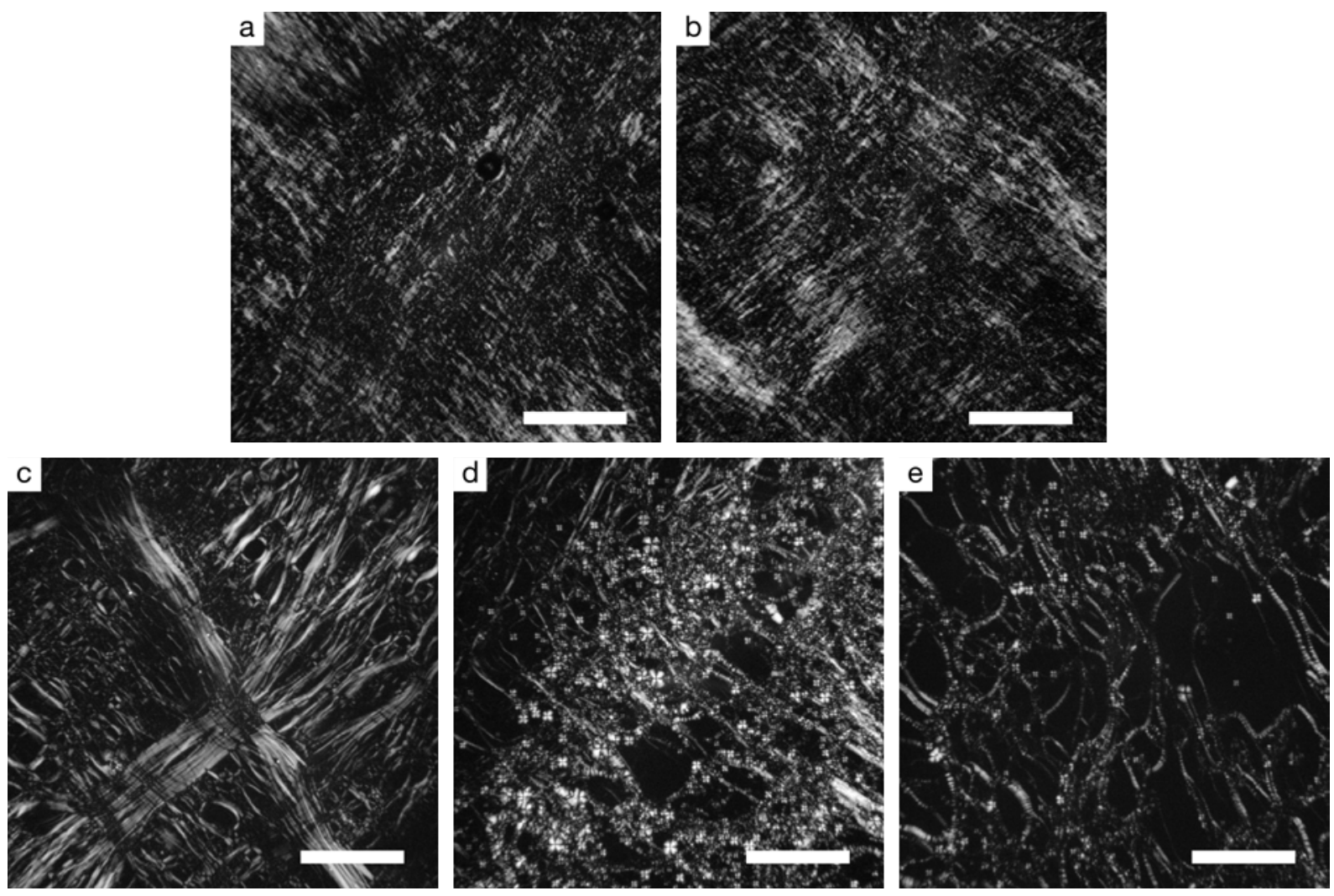

Figure 7.20: POM images showing the change of defect behaviour in the lamellar phase at $\sim 60$ wt \% Ch-AOT upon increasing salt concentration (at $\sim 25{ }^{\circ} \mathrm{C}$ ). The $\mathrm{NaCl}$ concentrations shown are (a) 1.1, (b) 2.0, (c) 3.0, (d) 4.1 and (e) $5.0 \mathrm{wt} \%$. Scale bars are $100 \mu \mathrm{m}$. 
biphasic sample. The change in optical textures show that the loss of long-range order in the $L_{\alpha}$ phase is continuous upon increasing the salt concentration. This is due to the gradual change in the spontaneous curvature of the bilayer induced with increasing salt concentration.

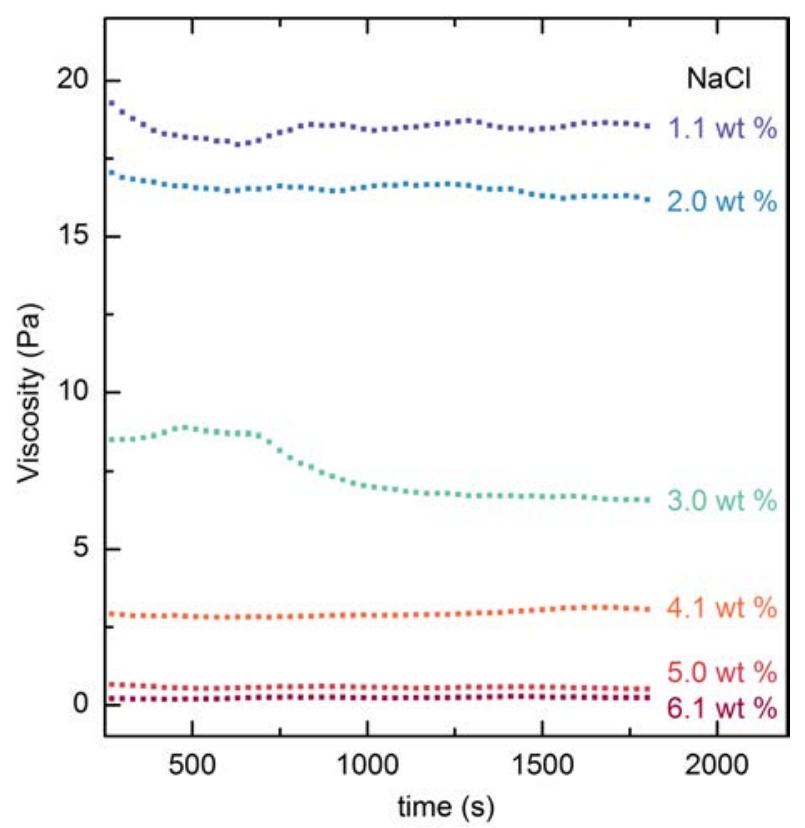

Figure 7.21: A plot of viscosity vs. time for samples of $\sim 60 \mathrm{wt} \%$ Ch-AOT with the stated $\mathrm{NaCl}$ concentrations. The viscosity was measured by rheology at a constant shear rate of $1 \mathrm{~s}^{-1}$ and decreases with increasing salt concentration.

\section{Addition of Salt to the Choline AOT System: Summary}

The ability of the Ch-AOT system to retain lamellar behaviour upon the addition of up to 5 wt $\% \mathrm{NaCl}$, which equates to a salt concentration in excess of $1 \mathrm{~mol} \mathrm{~L}^{-1}$, indicates the phase is very stable and has the ability to accommodate the presence of salt straightforwardly - perhaps due to the richness of defects and hugely varying curvature. The addition of salt to a bilayered system increases the electrostatic shielding between the surfactant headgroups and thus results in increased negative Gaussian curvature and enhanced bilayer elasticity. For an ordered bilayer system to still be energetically favoured at high salt concentrations, over almost the whole concentration range of surfactant (even at low surfactant concentrations), implies that the balance between headgroup and tail volume in the Ch-AOT system is close to ideal. We have demonstrated that it takes a significant alteration in the energy of the system to induce a change in the phase behaviour. This further highlights the efficient and flexible surfactant packing in the Ch-AOT system discussed above. Even when a salt-induced phase transition occurs in the system, at most concentrations the bilayered structure of the sponge phase is formed, itself a highly disordered variant of the lamellar phase.

The superior stability of the Ch-AOT system is in stark contrast to the two systems presented in previous chapters that paired the sulfonate AOT headgroup with other chaotropic ions. The 
$\mathrm{K}$-AOT and $\mathrm{NH}_{4}$-AOT systems produce a multitude of different phases. Like the $\mathrm{Ch}^{+}$ion, both $\mathrm{K}^{+}$and $\mathrm{NH}_{4}{ }^{+}$are weakly hydrated and form closer ion pairs with the AOT headgroup than the $\mathrm{Na}^{+}$ion does. However, these systems can be manipulated by tuning electrostatic forces, and transitions between $L_{3}, L_{\alpha}$ and $V_{/ l}$ phases can be induced with adjustments to the temperature or salt concentration. This is because the elastic constants ( $\kappa$ and $\bar{\kappa}$ ) are susceptible to changes in the intermolecular forces and so when the electrostatic repulsion between headgroups is increased, the increasingly positive $\bar{\kappa}$ causes the formation of the highly curved sponge phase. In comparison, the tightly bound $\mathrm{Ch}^{+}$ion, which also results in reduced headgroup repulsion and increased van der Waals attraction, provides significant bulk and steric repulsion to the headgroup to overcome the electrostatic forces. This has the effect of increasing the elastic constant $\kappa$ (the bending rigidity) and so changes in the surfactant concentration and temperature are not sufficient to induce phase transitions, and only the addition of large amounts of salt can drive the system away from the energetically preferred lamellar phase.

\section{Acetylcholine AOT: Experimental Results}

Given the impressive stability of the lamellar phase in the Ch-AOT system (which was the dominant phase over a large concentration range and persisted upon the addition of large amounts of salt), it should be expected that the AcCh-AOT system may exhibit similar behaviour. The inherent bilayer curvature is clearly restricted in the Ch-AOT lamellar phase due to the strong binding of the bulky choline counterion, and replacing it with another bulky counterion will likely produce the same effect. However, despite the enforced bilayer rigidity in the Ch-AOT system resulting in the adoption of the on average "flat" morphology of the $L_{\alpha}$ phase, the changes in defect behaviour revealed that the Gaussian curvature still changed from positive to negative with increasing concentration. This resulted in the formation of highly curved structures due to increasing inversion of the molecule. Moving from choline to acetylcholine introduces an even larger counterion to the molecule, and thereby introduces the potential for the additional bulk to alter the surfactant packing sufficiently to allow a reversion back to Type I morphologies, or for the coordination between the counterion and surfactant to be further disrupted, promoting ionic liquid properties.

\section{Acetylcholine AOT Phase Behaviour}

The phase behaviour of the AcCh-AOT system is presented alongside that of Ch-AOT in Figure 7.22. The two systems are clearly similar, with the lamellar phase dominating the phase diagrams of both systems. Neither a reversion to Type I morphologies or the low melting point of an ionic liquid is observed in either system. 
Comparison of the two diagrams shown in Figure 7.22 yields several areas of distinction:

- The two-phase region of the AcCh-AOT system is extended to higher concentrations (25 wt \% vs. 20 wt \% surfactant at $25^{\circ} \mathrm{C}$ ).

- The two-phase region of the AcCh-AOT diagram is also marked as two regions, distinguishing between different observed behaviours with regard to the cloud point of the surfactant above and below 15 wt \%.

- A crystalline phase is formed in the AcCh-AOT system at a concentration of $\sim 92 \mathrm{wt} \%$ surfactant, which transitions to a lamellar phase upon heating.

- The stability of the lamellar phase is reduced at high temperatures in the AcCh-AOT system.
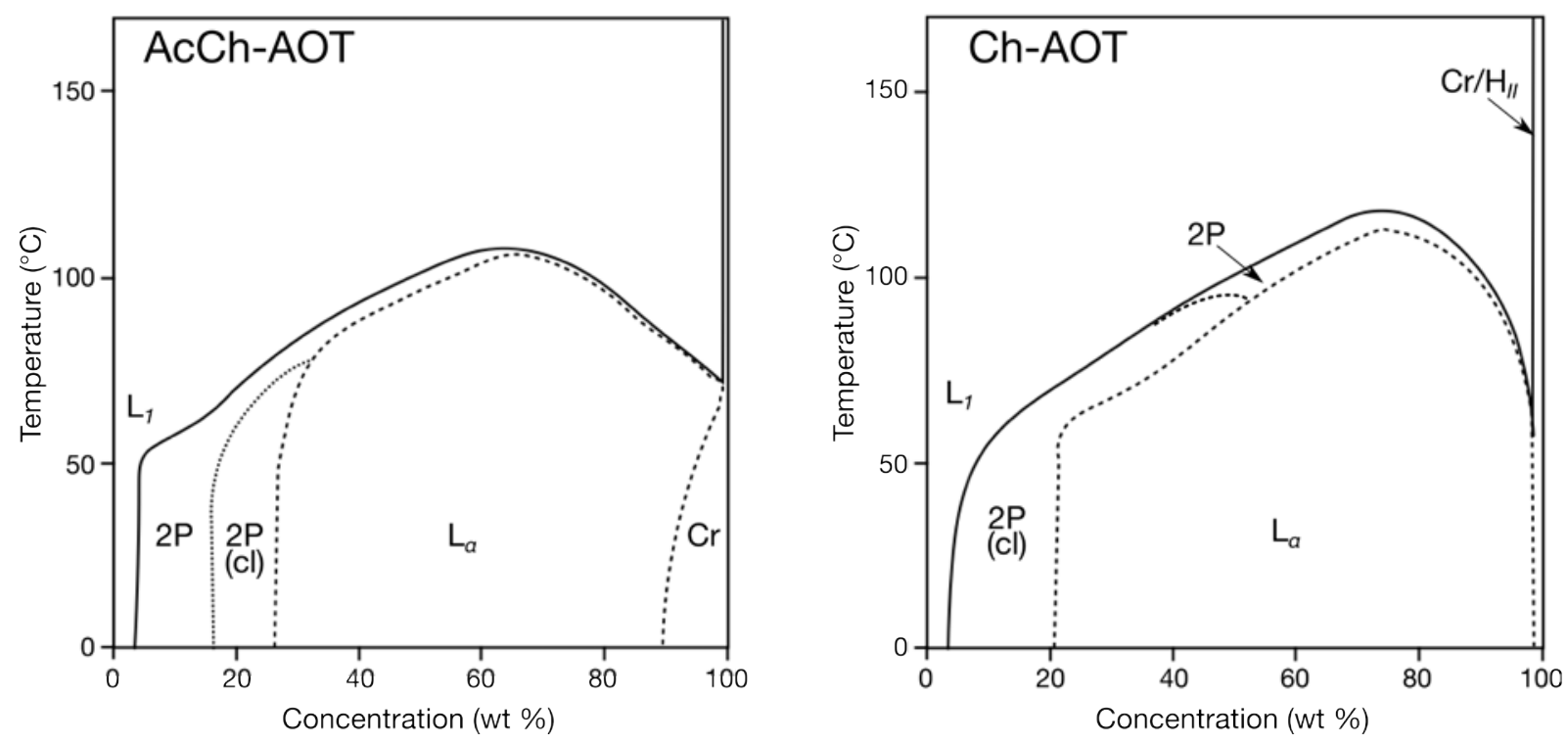

Figure 7.22: Phase diagrams of AcCh-AOT (left) and Ch-AOT (right). The two-phase region is depicted by $2 \mathrm{P}$ and $2 \mathrm{P}(\mathrm{cl})$ to distinguish between the phase-separated and cloudy regions, respectively.

Similar to the Ch-AOT system, the AcCh-AOT system displayed a cloud point in the two-phase region prior to the formation of a pure lamellar phase. In the AcCh-AOT system this can be further split into two regions. Between 15 and 25 wt \% surfactant, samples appeared cloudy. At lower concentrations clear separation was achieved by centrifugation, yielding a surfactantrich cloudy layer and a water-rich isotropic layer. The structural differences between the $2 \mathrm{P}$ and $2 \mathrm{P}(\mathrm{cl})$ areas on the phase diagram is likely small. The observed change from two layers to one cloudy layer may be due to either increasing solubility with increasing concentration or simply due to increased proportion of the cloudy phase to water phase. Although the cloudy phase could not be separated by centrifugation, it was confirmed to be biphasic by POM, readily forming lamellar and isotropic domains.

As with the Ch-AOT system, the existence of a cloud point in the AcCh-AOT system reveals information about the interactions between the counterion and the headgroup. The existence of cloud points in anionic surfactants is not standard behaviour and has primarily been reported for systems containing a tetraalkylammonium counterion. ${ }^{278-280}$ These bulky quaternary 
ammonium cations (including both acetylcholine and choline) are soft cations. The positive charge on the nitrogen atom is shielded by the surrounding alkyl groups, resulting in weakly hydrated ions and hydrophobic interactions with water. As such, they form strong ion pairs with soft headgroups, such as the sulfonate moiety in AOT. This results in the formation of structures with hydrophobic interfaces (in this case the formation of MLVs), which aggregate together resulting in poor solubility in the water phase, producing turbid cloudy samples. The presence of a cloud point in this system confirms that the acetylcholine counterion forms a strong ion pair with the AOT headgroup and has poor interactions with bulk water.

At the opposite end of the phase diagram, the pure crystalline phases of Ch-AOT and AcCh-AOT are stable to high temperatures. However, the stability of the crystal lattice is severely disrupted upon the addition of water. AcCh-AOT can incorporate up to 8 wt \% water (at $25^{\circ} \mathrm{C}$ ) into its crystalline phase; however, this occurs at a cost in terms of entropy with the water clearly destabilising the crystalline order upon heating. This results in the reappearance of the $L_{\alpha}$ phase. This transition from a crystalline phase to a $L_{\alpha}$ phase upon heating, in conjunction with the SAXS profiles of the crystalline phase presenting Bragg spacings indicative of layered ordering, suggests that the crystalline solid could be a crystalline lamellar $\left(L_{c}\right)$ phase. This would mean that the phase transition between the lamellar and crystalline phases is not a major structural reorganisation, but is due to solidification of the alkyl tails, which then return to their molten form upon heating.

At concentrations up to $60 \mathrm{wt} \%$ surfactant, the transition temperatures between the $\mathrm{L}_{\alpha}$ phase and the isotropic fluid match very well between the AcCh-AOT and Ch-AOT systems. Above this concentration, the $L_{\alpha}$ phase of the Ch-AOT system is retained at temperatures up to $15^{\circ} \mathrm{C}$ higher than that of the AcCh-AOT system. Although both ions are weakly hydrated, the increased stability of the Ch-AOT system is likely a result of hydrogen bonding with the water network. This was observed in the $\mathrm{NH}_{4}$-AOT system in Chapter 6, where the melting transition of the lamellar phase was higher than that of any of the other systems presented in this work. The choline counterion has greater hydrogen bonding capability (through the hydroxyl group) than the ester functionality of the acetylcholine ion. A reduced contribution through hydrogen bonding, combined with the possible additional steric bulk if the counterion dissociates, results in greater disruption to the water network in the AcCh-AOT system.

One observation that is not evident from the phase diagrams, and is a possible indication of their relative hydrogen bonding abilities, is the hygroscopic nature of each surfactant. Although both form crystalline phases when pure and sufficiently dried, in the case of Ch-AOT, achieving this required the use of inert atmosphere. Upon exposure of Ch-AOT to moisture in the air, it was rapidly and visibly altered, forming the concentrated lamellar phase. In contrast, a pure AcCh-AOT sample showed negligible change by weight after exposure to the air for one hour. This is an indication that the $\mathrm{Ch}^{+}$hydroxyl group is readily available for hydrogen bonding, while the ester of $\mathrm{AcCh}^{+}$is not. 


\section{Acetylcholine AOT SAXS}

SAXS profiles for samples of increasing AcCh-AOT concentration are shown in Figure 7.23, confirming the assignment of the lamellar phase that exists from 25 to $92 \mathrm{wt} \%$. Samples below 15 wt \% are of weak intensity and show only broad scattering. Samples at 30 and 35 wt \% coincide with the form factor minimum and do not display a first-order peak, as has also been observed in previously described systems. However, the binding of the acetylcholine counterion does significantly alter the form factor of the bilayer. There is a significant reduction in diffuse scattering, resulting in the appearance of sharp and well-defined third order Bragg peaks. The reduction in diffuse scattering implies a decrease in the long-range Helfrich repulsion forces that arise due to out-of-plane fluctuations. This results in decreased bilayer elasticity. ${ }^{301}$

The SAXS spectra at 95 and $100 \mathrm{wt} \%$ show the formation of a crystalline phase at high surfactant concentrations of AcCh-AOT. At $100 \mathrm{wt} \%$, a sharp and intense reflection at a $\mathrm{q}$ spacing of $2.89 \mathrm{~nm}^{-1}$ is accompanied by a sharp peak at $5.78 \mathrm{~nm}^{-1}$, a broad hump consisting of a number of peaks centred at $\sim 4 \mathrm{~nm}^{-1}$ and a significant tailing of the primary peak itself. The pattern does not fit with the scattering of any typical lyotropic phase, although the peak at $5.78 \mathrm{~nm}^{-1}$ is consistent with being either a second-order $L_{\alpha}$ peak or the $\sqrt{ } 4$ reflection due to hexagonal ordering. This implies the existence of a periodic repeating unit in one direction, with a d spacing of $21.8 \AA$ that correlates to the $\mathrm{q}^{*}$ peak.

The shift of $q^{*}$ to a higher value in comparison with that observed in the lamellar phase results in a reduction in the characteristic length ( 21.8 vs. $24.5 \AA$ at 100 and $90 \mathrm{wt} \%$, respectively). This allows the inference of several conclusions. Firstly, it rules out the formation of any rippled or gel lamellar phases, as these phases result in an increase in bilayer thickness in comparison with the fluid state. $\ddagger$ However, it does not rule out a crystalline lamellar or hexagonal arrangement. The reduction in d also results in a spacing comparable to the thickness of the bilayer observed in the lamellar phase, and so if the crystallised morphology is based on a bilayered structure, then the layers are close to aggregation. Such a low value indicates that the hydrated steric repulsion is significantly reduced upon formation of the crystalline phase, which implies that the bulky $\mathrm{AcCh}^{+}$ion is embedded within the surfactant headgroups, that the alkyl chains are efficiently packed and ordered, and that the headgroup and counterion are dehydrated. The headgroup dehydration is confirmed by the sharp peaks at 7.14 and $7.70 \mathrm{~nm}^{-1}$, which arise due to headgroup correlations. ${ }^{285,286}$

The absence of a $\sqrt{ } 3$ reflection rules out a hexagonal arrangement, but the possibility of a crystalline structure based on lamellar ordering is supported by the temperature-induced crystalline to $L_{\alpha}$ transition. However, the presence of other peaks means that the structure is likely to be more complicated and the defect behaviour at high concentrations (shown in the cryo-SEM section below) implies an inherent desire of the bilayer to curve (i.e. for the alkyl chains to splay), which will be further enhanced upon dehydration of the headgroup. This indicates a deviation from ideal flat layers, but it also shows that the preferred packing

\footnotetext{
${ }^{\ddagger}$ An increase in interdigitating of surfactant molecules allowing a condensed rippled phase is unlikely as the AOT tail groups are already in an interdigitated configuration.
} 

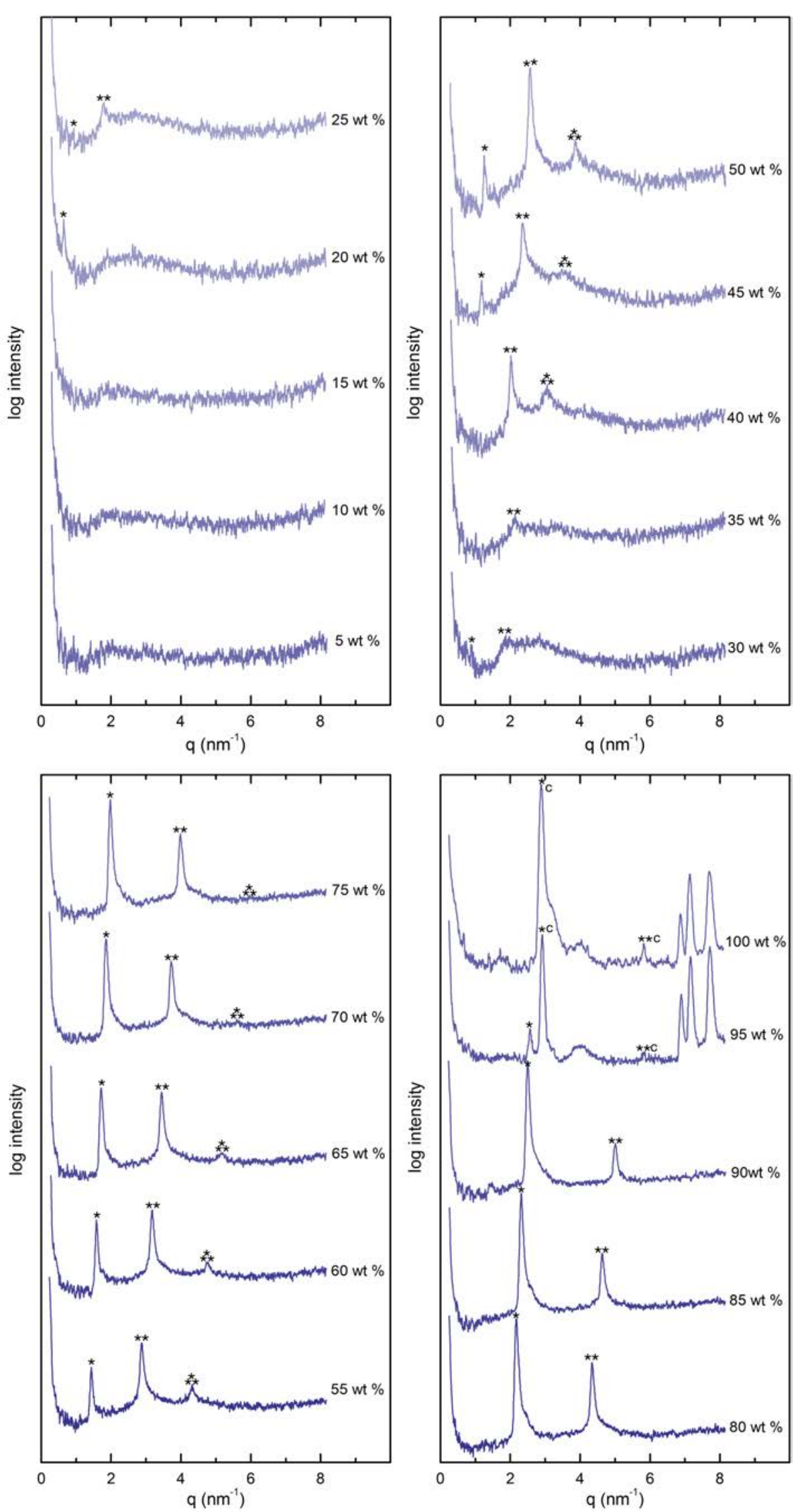

Figure 7.23: SAXS profiles of the AcCh-AOT system with increasing surfactant concentration. Peaks marked by asterisks are Bragg peaks due to the $L_{\alpha}$ phase. Samples at 95 and $100 \mathrm{wt} \%$ AcCh-AOT are crystalline and the space group has not been assigned. The labelling of peaks as $L_{c}$ (crystalline lamellar) is to indicate one possible space group. 
arrangement of the molecules is that of a bilayer. Some support for a lamellar arrangement is shown in the 2D SAXS profiles shown in Figure 7.24. At $90 \mathrm{wt} \%$ AcCh-AOT, the scattering of the lamellar phase has orientational order due an ordered lamellar phase. At $95 \mathrm{wt} \%$, some traces of this lamellar phase remain, along with the $\mathrm{q}^{*}$ reflection of the crystalline phase. Both of these phases show ordered scattering in one direction that is consistent with a lamellar phase, as do the weak second-order reflections. At $100 \mathrm{wt} \%$ AcCh-AOT, all reflections present as isotropic Debye-Scherrer rings, indicative of a polycrystalline sample with crystallites of varying size and orientation. However, the positions of the peaks at $100 \mathrm{wt} \%$ are not shifted from those at $95 \mathrm{wt} \%$. As such, the reflections observed are due to the same phase that presented the clear orientation at $95 \mathrm{wt} \%$.
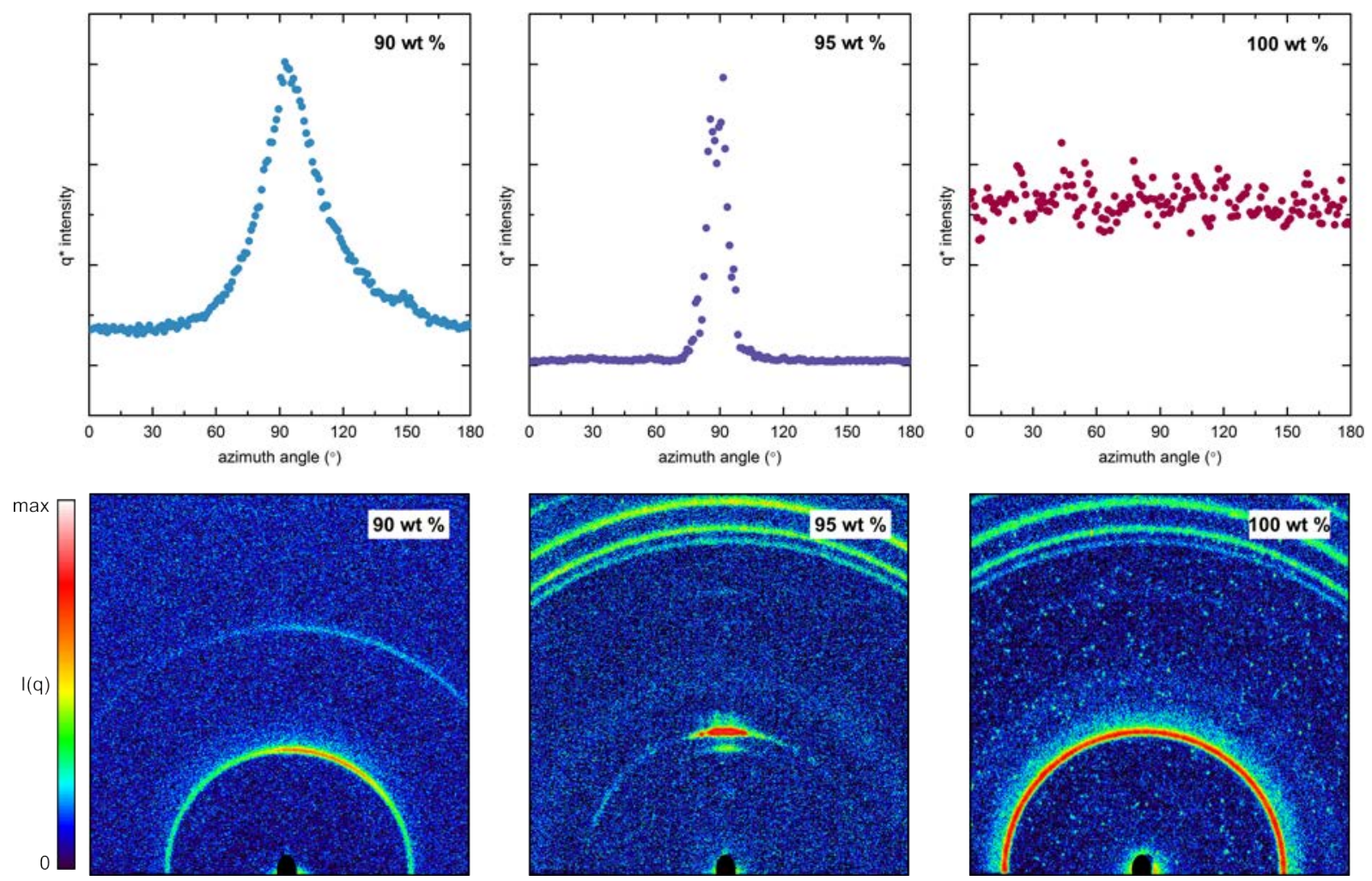

Figure 7.24: Plots of $q^{*}$ intensity vs. azimuth angle for concentrations of 90,95 and 100 wt \% AcCh-AOT, and the corresponding 2D SAXS profiles.

The correlation between the scattering at 95 and $100 \mathrm{wt} \%$ AcCh-AOT is also evident in the WAXS region, where both present an array of sharp and intense peaks at equivalent $q$ values. Figure 7.25 shows this region for 90 and 100 wt \% AcCh-AOT, alongside those of Ch-AOT, representing the lamellar and crystalline phases of each system. The crystalline phase of AcCh-AOT does not result in broad scattering due to fluid alkyl chains, but instead results in a far more complex series of sharp peaks in comparison with the biphasic and extremely hygroscopic Ch-AOT. These peaks indicate the complete dehydration and ordered packing of the AcCh-AOT headgroup, tails and counterion, which allows the steric hydration forces to be overcome. The complex array of diffraction peaks upon dehydration is rational, as AOT has three chiral centres and so exists as four enantiomeric pairs, while acetylcholine has four rotational bonds, giving nine distinct conformations. This results in a large range of possible interactions upon crystallisation. Comparison of the WAXS region with the powder 

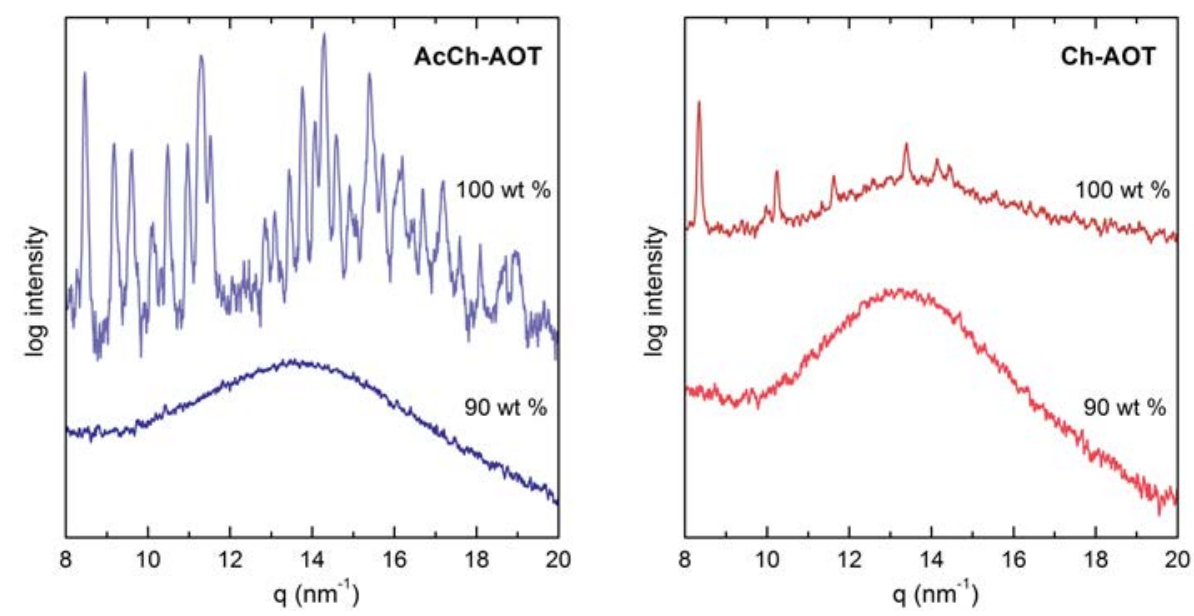

Figure 7.25: WAXS profiles for AcCh-AOT and Ch-AOT at concentrations of 90 and $100 \mathrm{wt} \%$.

X-ray diffraction pattern of acetylcholine chloride did not allow the assignment of specific peaks to the acetylcholine counterion. ${ }^{302}$

The centring of the broad scattering of the fluid alkyl chains in the lamellar phase is lower than the expected $14.9 \mathrm{~nm}^{-1} .{ }^{14}$ This is representative of the inter-surfactant correlations between alkyl chains and implies the tails in both systems are splayed due to an inverted surfactant shape. This is confirmed through the formation of defects presented in the microscopy sections. The lower value for Ch-AOT (13.2 vs. $13.8 \mathrm{~nm}^{-1}$ ) suggests that the chains are further apart in this system, consistent with the formation of a hexagonal phase above $98 \mathrm{wt} \%$ in Ch-AOT, but AcCh-AOT remaining layered.

The swelling behaviour of AcCh-AOT upon dilution is represented in Figure 7.26, which shows the change in $d$ spacing of the lamellar phase with concentration. The $d$ values of Na-AOT and Ch-AOT are also shown for comparison. The graph can be split into three regions, with AcCh-AOT deviating from ideal 1D swelling at the high and low concentration ranges.

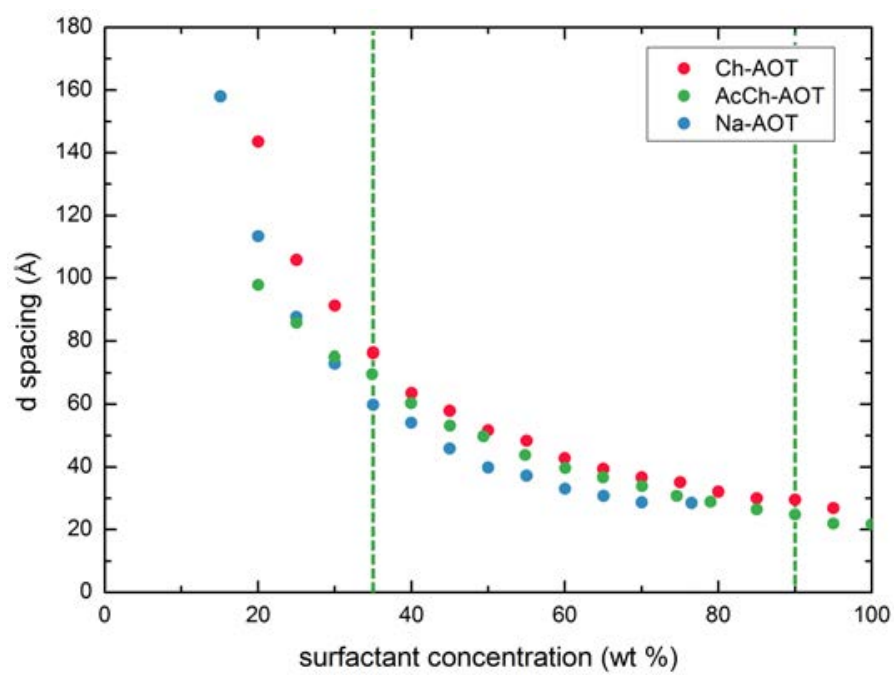

Figure 7.26: Changes in d spacing with increasing concentration for the AcCh-AOT, Ch-AOT and $\mathrm{Na}-\mathrm{AOT}$ systems. The dotted green lines indicate the limits of 1D swelling in the AcCh-AOT system. 
At the high concentrations, a deviation from 1D swelling occurs when approaching and surpassing the lamellar to crystalline phase boundary. Although the crystalline structure has not been confirmed to consist of stacked bilayers, the values of $d$ for this phase are included to illustrate the efficiency of the AcCh-AOT system in overcoming interbilayer repulsion forces at high concentrations. Given that the contribution of the bilayer thickness to the $d$ spacing is $\sim 20 \AA$, then the measured d spacing of $21.8 \AA$ in the pure surfactant, and $24.8 \AA$ in the $L_{\alpha}$ phase, indicate that the bilayers are separated by less than $5 \AA . \ddagger$ Israelachvili states that "under natural conditions, fluid bilayers are unlikely to approach closer than $20 \AA^{\prime \prime}{ }^{132}$ For AcCh-AOT bilayers in the $\mathrm{L}_{\alpha}$ phase to stack so closely, they must overcome both the electrostatic repulsion and steric repulsion forces that prevent the aggregation of bilayers and drive the formation of curved mesophases in the systems discussed previously. The headgroup and counterion must be strongly dehydrated, and the $\mathrm{AcCh}^{+}$ion must efficiently shield the charge of the $\mathrm{SO}_{3}{ }^{-}$moiety and pack within the bilayer.

At lower concentrations (between the lines indicated in Figure 7.26), the AcCh-AOT system shows 1D swelling and follows the values for the Ch-AOT system closely, although the $\mathrm{d}$ spacing is consistently $\sim 4 \AA$ smaller. This difference can be explained in two ways, both of which are a result of the $\mathrm{AcCh}^{+}$counterion forming a closer ion pair with the sulfonate headgroup. Firstly, from a purely steric point of view, the tighter binding of the $\mathrm{AcCh}^{+}$counterion may result in a smaller bilayer thickness than the $\mathrm{Ch}^{+}$ion, and hence a reduced bilayer contribution to the $\mathrm{d}$ spacing. Of the two ions, $\mathrm{AcCh}^{+}$is the larger and so must pack more efficiently than the $\mathrm{Ch}^{+}$ ion for this to be the case. Both counterions are predominantly hydrophobic and so a packing arrangement penetrating the bilayer is feasible. However, in the case of the $\mathrm{Ch}^{+}$ion, this may be compromised by the terminal hydroxyl group, which has good affinity with water, making the $\mathrm{Ch}^{+}$ ion amphiphilic. As such, it is likely that the hydrophobic effect would promote a configuration in which the $\mathrm{OH}$ group is orientated towards the water, possibly protruding from the headgroup, where it will increase the thickness of the bilayer.

The second explanation considers the effect of counterion binding on the forces that govern the repeat distance. Bilayer swelling is caused by long-range repulsive forces that outweigh the long-range attractive forces. If the relative contribution of long-range attractive forces is greater in the AcCh-AOT system than in the Ch-AOT system, then the value of $d$ will be lower. This is likely, as the $\mathrm{AcCh}^{+}$ion will alter the intermolecular forces in the following ways:

- Electrostatic repulsion: The reduced hydration of the $\mathrm{AcCh}^{+}$ion will result in a reduction of the electrostatic repulsive forces both between adjacent headgroups and between bilayers. A reduction between bilayers would result in a decrease in d spacing. A reduction between headgroups would normally lower the bending rigidity of the bilayer and increase the entropic repulsion due to undulation. However, the incorporation of such a large counterion into the headgroup would more than compensate for this through steric hinderance, resulting in a more rigid bilayer.

\footnotetext{
¥The approximate value of the bilayer thickness is based on that quoted for Na-AOT. ${ }^{63}$ Given the comparative bulk of the $\mathrm{AcCh}^{+}$ion and the higher d spacings at equivalent concentrations, the bilayer thickness of AcCh-AOT will likely exceed the $20 \AA$ quoted.
} 
- van der Waals attraction: The increased atomic mass and polarisability of the $\mathrm{AcCh}^{+}$ion will result in an increase in the van der Waals attraction forces between bilayers.

- Steric hydration repulsion: The steric hydration forces will only be significant at interbilayer distances within $30 \AA$; however, the reduced hydration will result in $\mathrm{AcCh}^{+}$being more effective at negating these forces. ${ }^{132}$

- Helfrich repulsion: The increased steric repulsion of the tightly bound and bulky $\mathrm{AcCh}^{+}$ion will increase the bending rigidity of the bilayer, and hence the energy cost of undulation, resulting in a reduction in Helfrich repulsion.

Despite the above interactions, which promote greater attraction between bilayers (and also apply to the $\mathrm{Ch}^{+}$ion when compared to the small, hard ions of the alkali metal systems), the AcCh-AOT and Ch-AOT systems display larger d spacings than the Na-AOT system over the same concentration range. This can be attributed to the contribution of the ions to the bilayer thickness. Despite the evidence for efficient packing and strong ion pair formation, they will still result in increasing the size of the headgroups (in both monolayers) as they still possess significant bulk in comparison to the sodium ion (the hydrated radius of $\mathrm{Na}^{+}$is $3.58 \AA$ when fully hydrated, $\mathrm{AcCh}^{+}$is an organic molecule at least twice this size and comparable in size to one of the AOT alkyl chains).

Within the cloudy two-phase region, and at the lower concentration end of the AcCh-AOT lamellar region, deviation from 1D swelling is observed. The AcCh-AOT system shows comparatively limited swelling and at concentrations of $20 \mathrm{wt} \%$, both the Ch-AOT and Na-AOT systems are considerably more swollen, with the difference between AcCh-AOT and Ch-AOT systems being $\sim 45 \AA$ (143.6 and $97.9 \AA$ respectively). This can also be explained by the increase in the relative contribution of the attractive rather than repulsive intermolecular forces. The larger differences at low concentrations (high d spacings) is due to the reduced importance of steric and electrostatic effects in comparison with Helfrich and van der Waals forces. A possible cause is the bilayer approaching its swelling limit due to Helfrich undulations being less favourable, and so excess water is not stored within bilayers but expelled into the water-rich phase (likely a disordered micellar or vesicular solution). This would result in the surfactantrich phase having a higher effective concentration with reduced d spacing. Similar retarded swelling behaviour has been reported in other binary surfactant and water systems. ${ }^{303-305}$ In the case of the anionic surfactant caesium dodecylbenzenesulfonate, this was also caused by the tight counterion binding of a large and poorly hydrated counterion, and resulted in "mildly flocculated" lamellar droplets similar to those reported in the POM section below. ${ }^{305}$ In the didodecyldimethylammonium bromide system it was attributed to an osmotic effect, where the equilibrium between the pressures of the two phases restricted the swelling of the lamellar phase, due to the compression of the micellar phase being unfavourable. ${ }^{304}$

\section{Acetylcholine AOT Optical Microscopy}

POM images for AcCh-AOT are shown in Figure 7.27. Given the similarities between the AcCh-AOT and Ch-AOT phase diagrams, and the close agreement of the lamellar repeat 
distances at concentrations above $40 \mathrm{wt} \%$ surfactant, it is not surprising that the microstructure of the systems also share a close resemblance. Between 37 and $41 \mathrm{wt} \%$, AcCh-AOT samples pass through a period of zero birefringence, as observed in most of the systems studied in this body of work. This is due to a decrease in the negative contribution to the bilayer birefringence, owing to a transition from fluctuating bilayers to more ordered stacks. This occurs upon reaching the bilayer repeat distance where electrostatic forces begin to stabilise the system over the entropic Helfrich repulsion.

At concentrations above 40 wt \% AcCh-AOT, the optical textures show a continuous change in defect type upon an increase in concentration, with defects averaging negative Gaussian curvature becoming increasingly dominant. Between 45 and $60 \mathrm{wt} \%$, these defects were observed to increase in number, decrease in size and begin to pack together. Many also formed small groups of oily streaks. Above $60 \mathrm{wt} \%$, this increased interaction between defects continued, with streaky textures gradually giving way to mosaic patterns formed by a rich array of tightly packed and distorted defects, indicating a highly curved structure of small and randomly orientated domains.

At AcCh-AOT concentrations above 92 wt \%, samples were highly iridescent under polarised light and were comprised of small hydrated crystals. At $100 \mathrm{wt} \%$, the pure surfactant presented larger plate-like crystals. However, the crystal morphology was dependent upon the crystallisation method. When allowed to slowly cool from solution, bâtonnet-shaped crystals were formed.

At low concentrations (below $\sim 35 \mathrm{wt} \%$ ), the presented POM textures of the AcCh-AOT system differ significantly from those presented previously for the Ch-AOT system (see Figure 7.9) and the $\mathrm{NH}_{4}$-AOT and alkali metal systems. In this concentration range, the $\mathrm{d}$ spacings obtained by SAXS are dramatically different for the AcCh-AOT and Ch-AOT systems. This is due to the different hydrophobic effects of the counterions resulting in different cloud point behaviours. The $\mathrm{AcCh}^{+}$ion promotes more efficient phase separation, seen through the formation of separate layers in samples and with the extension of the two-phase region to higher concentrations. This is likely due to the terminal $\mathrm{OH}$ group of the $\mathrm{Ch}^{+}$ion, which will increase the solubility of the headgroup and stabilise the formation of lamellar aggregates in the Ch-AOT system.

At concentrations below 15 wt \% surfactant, AcCh-AOT samples separate into two clearly different layers. The POM textures within this region show some lamellar defects within a predominantly isotropic texture. The use of a full wave retardation plate revealed that the defects are not incorporated within the same phase as the isotropic background, but exist as large droplets with a clear interface. This shows the clear separation between the phases and indicates the possible formation of multivesicular bodies. As the concentration was increased, these lamellar droplets began to aggregate, forming microdomains comprised of MLVs. Between 15 and 25 wt \%, where samples appear as one cloudy layer, the samples continue to show separate domains under the microscope. However, the domains are larger and appear as packed arrays of defects of both negative and positive Gaussian curvature. Over time, connections form between the domains through the formation of oily streaks. 

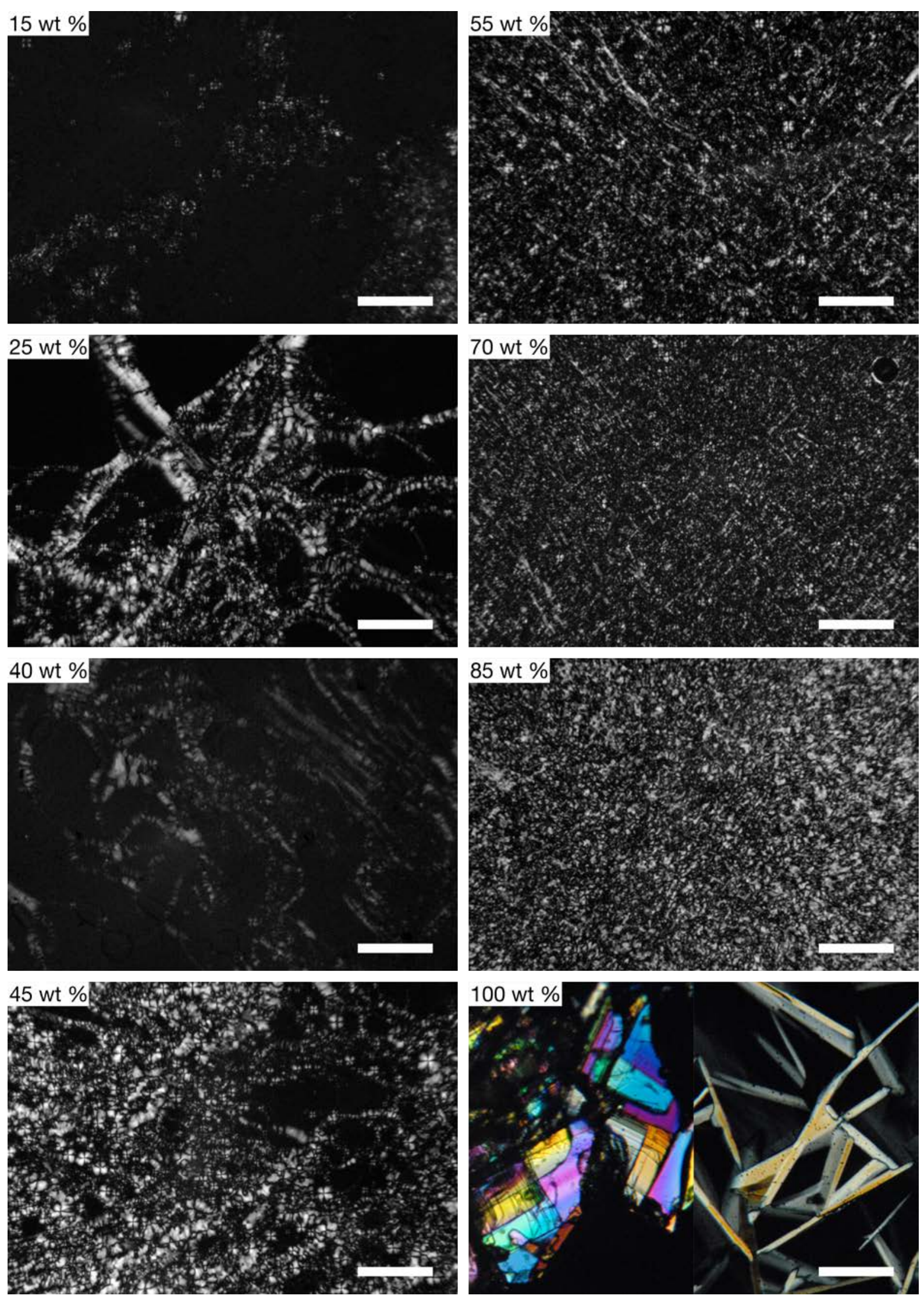

Figure 7.27: POM images of AcCh-AOT with increasing concentration at $\sim 25^{\circ} \mathrm{C}$. Deviation from the behaviour observed in previously discussed systems is seen at lower concentrations, due to the cloud point of the surfactant. With increasing concentration, oily streaks, mosaic patterns and crystals are observed. At $100 \mathrm{wt} \%$, the left and right images show the structure at $25^{\circ} \mathrm{C}$ before and after melting, respectively. Scale bars are $100 \mu \mathrm{m}$. Additional images can be found in Appendix $\mathrm{C}$. 
Figure 7.28 shows a sample of $20 \mathrm{wt} \%$ AcCh-AOT. The image shows the dynamic interface between the surfactant-rich and water-rich phases. The two regions were observed to evolve over time. MLV defects of varying size were continuously formed at the edge of the surfactantrich phase, before moving into the water-rich phase, and then breaking down and becoming isotropic. The average lifetime of the MLVs was approximately $200 \mathrm{~s}$.

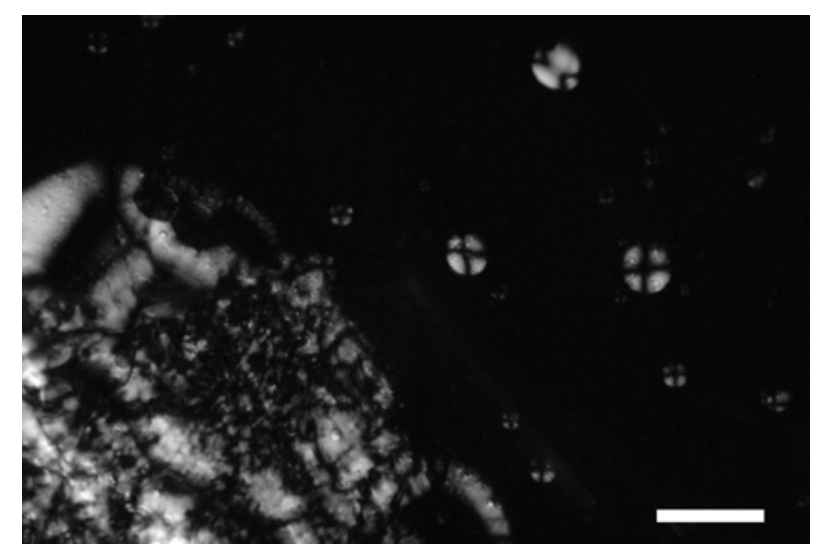

Figure 7.28: POM image of a 20 wt \% AcCh-AOT sample at $\sim 25{ }^{\circ} \mathrm{C}$, showing the interface between the two phases. Multilamellar vesicles are formed at the edge of the surfactant phase and released into the bulk water phase, where they subsequently breakdown within 200 s. Scale bar is $100 \mu \mathrm{m}$.

At 30 to 35 wt \% AcCh-AOT, the bulk samples were observed to be a single lamellar phase. However, they were also evident upon handling to be of low viscosity. As such, flow behaviour was evident under the microscope upon the placement of the cover slip, forming streaky textures and at $30 \mathrm{wt} \%$, some phase separation. This formation of defects with negative Gaussian curvature at low concentrations, and subsequent separation, are an indication of the low viscosity of the phase. This is a result of the hydrophobic nature of the $\mathrm{AcCh}^{+}$counterion. However, it does not represent the true equilibrium system. Such separation under flow may explain the low d spacing values obtained via SAXS, as samples were loaded using a flow cell.

\section{Acetylcholine AOT Cryo-SEM}

The cryo-SEM images of AcCh-AOT show a now familiar trend. High degrees of positive curvature and interbilayer connectivity at lower concentrations gradually give way to tightly packed layers, forming concentrated defect arrays of predominantly negative Gaussian curvature. Although this system has increased bilayer rigidity and steric bulk, which prevent formation of the inverse phases observed in the alkali metal and $\mathrm{NH}_{4}$-AOT systems, the cryo-SEM images show that similarly to the Ch-AOT system, the bilayer is far from the flat ideal. Each monolayer within the bilayer still has an inherent need to curve away from the other, and the energy cost associated with adopting the bilayer structure is met through the formation of highly curved defects that stabilise the phase. 
The cryo-SEM images of 15 wt \% AcCh-AOT, shown in Figure 7.29, are in good agreement with the POM observations. Both techniques clearly show separation into two phases, with the major phase being the bulk water phase, which was removed by sublimation revealing large voids and channels of up to $5 \mu \mathrm{m}$ in size. The surfactant phase is clearly comprised of MLVs ranging from 0.5 to $4 \mu \mathrm{m}$. In some regions the larger of these MLVs are observed to have aggregated, forming microdomains of $L_{\alpha}$ phase. Many of the smaller MLVs also show evidence of aggregation, appearing to create a randomly interconnected network. Given that this is a fluid system, such connections are likely continually forming and breaking, but will lead to increasing aggregation, promoting the formation of larger MLVs and eventually lamellar structures upon increasing concentration.
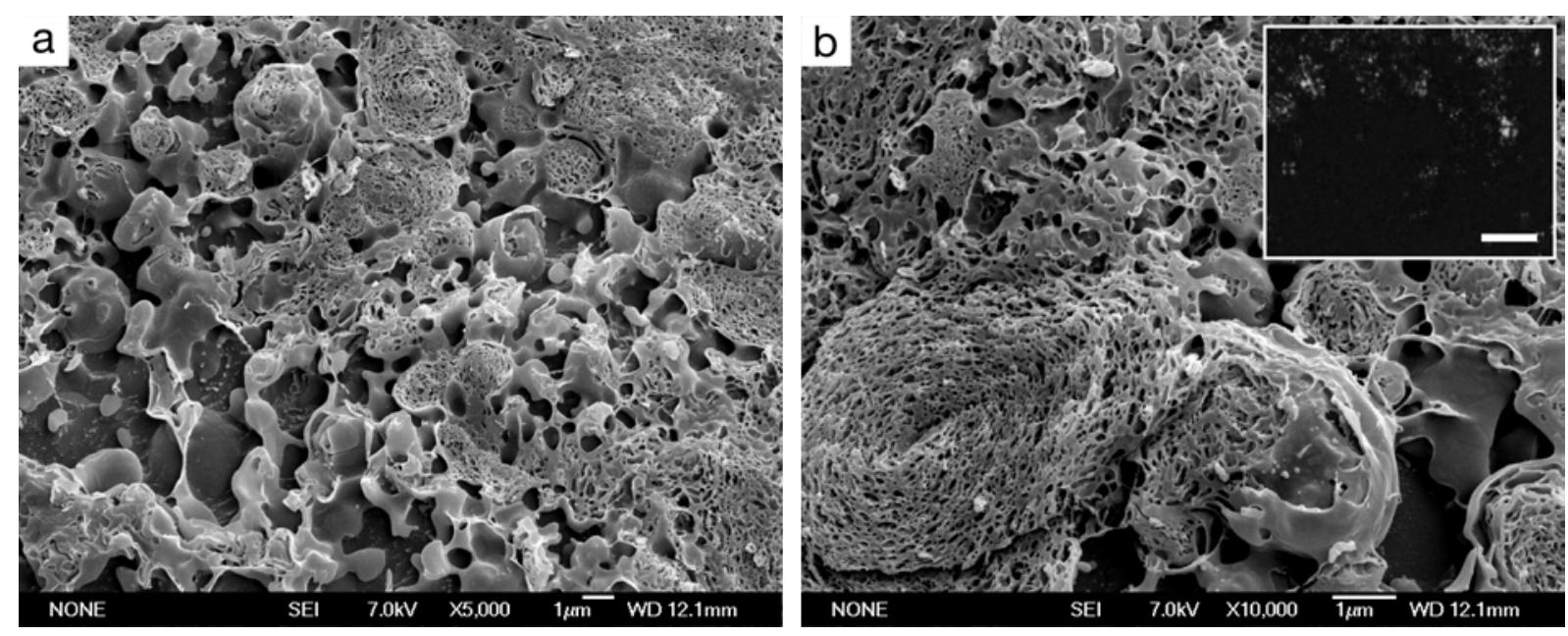

Figure 7.29: Cryo-SEM images of a sample of $15 \mathrm{wt} \%$ AcCh-AOT. The inset shows the corresponding POM image, with a scale bar of $50 \mu \mathrm{m}$.

These images are similar to those of Ch-AOT at the same concentration (see Figure 7.11), in terms of the surfactant phases being comprised of MLVs. However, in the AcCh-AOT system, their maximum size is smaller $(4 \mathrm{vs} .10 \mu \mathrm{m})$ and the surfactant network is less continuous. At this concentration the cloudy Ch-AOT samples did not separate into layers, whereas the AcCh-AOT samples did. If the surfactant phase of AcCh-AOT is less connected, then this could explain the greater propensity for the system to separate. Conversely, the separation may be the cause of the observed lack of connectivity. The separation of samples into layers means that we cannot have confidence that the composition of the observed region truly represents $15 \mathrm{wt} \%$ surfactant. Within the two-phase regions, calculated values for the $\mathrm{d}$ spacing were significantly lower for the AcCh-AOT system than for Ch-AOT, implying that the bilayers are closer together and that more water is expelled into the bulk water phase. This could explain the larger water channels observed in the AcCh-AOT system, although again, the reduced $d$ spacing could be a result of the separation rather than the cause.

At the higher concentration of $25 \mathrm{wt} \%$ AcCh-AOT, shown in Figure 7.30, only one phase is observed. Large channels due to the bulk water phase are not present. Instead, a continuous and highly defective lamellar phase is observed. On a local scale there is a large amount of connectivity, which combined with the lack of defined domain boundaries, is similar to the swollen $L_{\alpha}$ phases with sponge-like character observed in the alkali metal and ammonium 
systems at similar concentrations. The lack of defined domain boundaries indicates a fast and continuous rearrangement of the lamellar directional ordering and implies a low viscosity phase. FCD-II defects up to $5 \mu \mathrm{m}$ in size exist throughout the structure.
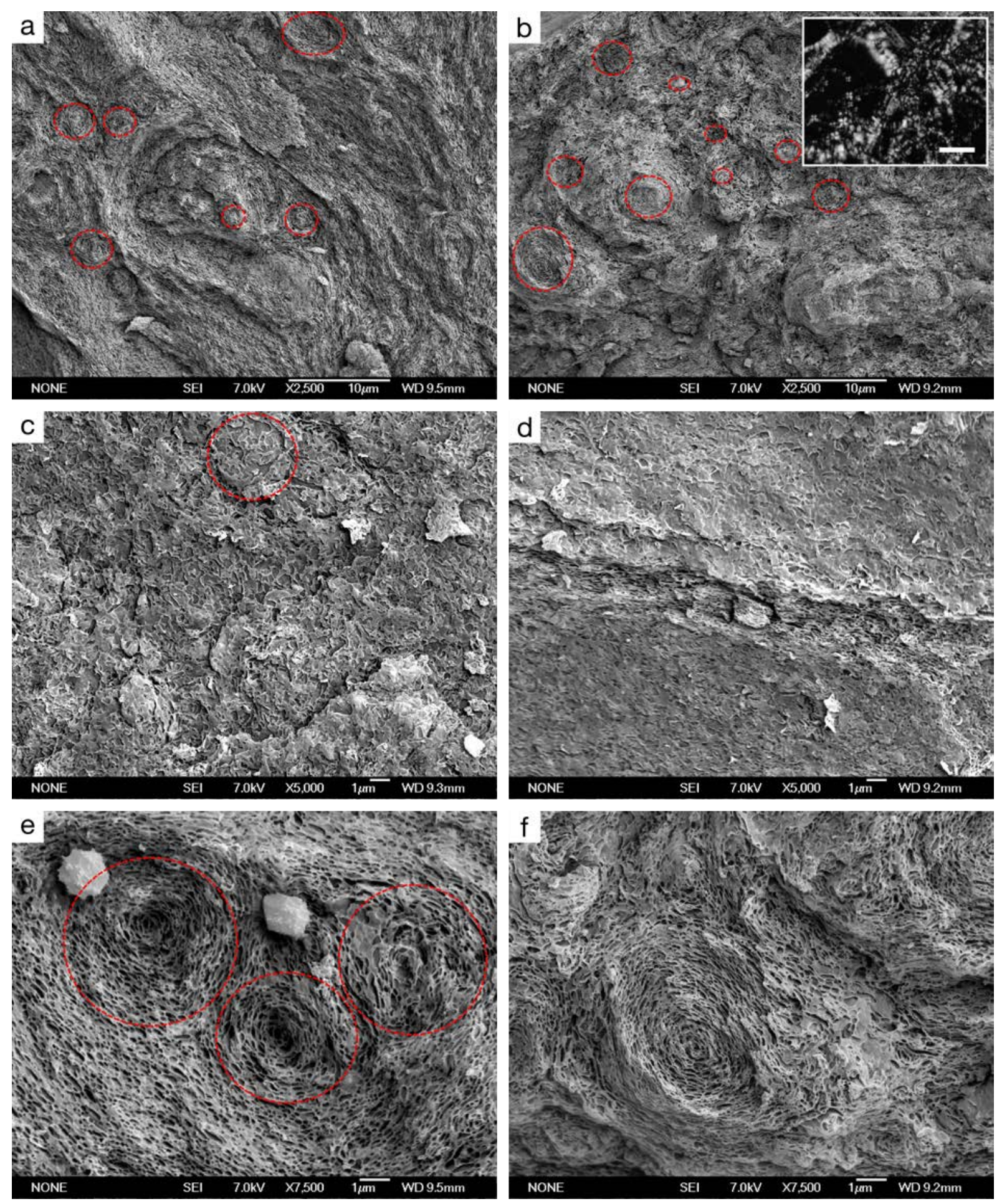

Figure 7.30: Cryo-SEM images of a sample of 25 wt \% AcCh-AOT. Red circles identify a selection of FCD-II defects of varying size. The inset shows the corresponding POM image, with a scale bar of $50 \mu \mathrm{m}$.

The FCD-II defects present at $25 \mathrm{wt} \%$ AcCh-AOT are different in appearance to those of the Ch-AOT system, which were often elongated along one axis and existed alongside large water 
channels. Differences between the two systems at this concentration are expected and were also observed by POM. The systems have very different $d$ spacings ( $86 \AA$ in AcCh-AOT and $106 \AA$ in Ch-AOT), and for AcCh-AOT this concentration is in close proximity to the $2 \mathrm{P}-\mathrm{L}_{\alpha}$ phase boundary.

Figure 7.31 shows cryo-SEM images of $50 \mathrm{wt} \%$ AcCh-AOT, and the continuity with the $25 \mathrm{wt} \%$ sample is obvious. The phase still displays a high amount of connectivity and some FCD-II defects. However, the phase is also more ordered, displaying regions with a clear average direction and the formation of FCD-I defects. The FCD-I defects are observed to form oily streaks of up to $8 \mu \mathrm{m}$ in length. Examples of each of these behaviours are marked on the figure.

The differences between AcCh-AOT and Ch-AOT at 50 wt \% are not as striking as at lower concentrations, which correlates with them having similar POM textures and $d$ values within $2 \AA$. However, it is evident at high magnifications that the Ch-AOT system contains areas with larger and more closely packed defects than those observed for AcCh-AOT. The distortion of neighbouring defects resulted in hexagonal-like packing, requiring small regions of extreme bilayer curvature. If the binding of $\mathrm{AcCh}^{+}$to the AOT headgroup results in even greater bilayer rigidity than in the Ch-AOT system, then such extreme curvature would be even less energetically favourable.

The images at $75 \mathrm{wt} \%$ AcCh-AOT (see Figure 7.32) show various behaviours depending upon the angle along which the region has fractured. In general, the behaviour is clearly similar to that observed in the other systems, and matches well with the mosaic patterns shown by POM. There is a clear decrease in the bilayer connectivity due to the loss of undulations upon a reduction in d spacing. Many FCD-I defects of negative Gaussian curvature are present. These defects are packed into oily streaks, which appear in orientated groups as large as $20 \mu \mathrm{m}$, or as shorter streaks of $\sim-4 \mu \mathrm{m}$ in length, which are packed into the highly curved mosaic structure. The individual defect components are mostly below $0.5 \mu \mathrm{m}$ in size.

In some regions, we observe what appear as ordered and relatively flat domains. These are up to $20 \mu \mathrm{m}$ in size and are segregated by highly defective grain boundaries composed of small defects, which are presumably also oily streaks. At higher magnifications and when viewed from above - see Figure 7.32 (f) - we see that these ordered "flat" domains are punctured with indentations or "pits". The grouping and size of these indentations implies that they are not connective channels through the bilayer, but are the result of the defect behaviour in the layers below. If we consider the nature of the formation of oily streaks, we can conclude that these indentations likely result from the edge dislocations that connect the individual FCD-I defects.

The cryo-SEM images for the Ch-AOT and AcCh-AOT systems at $75 \mathrm{wt} \%$ are very similar, and both show good agreement with their POM textures. In general, the defect behaviour of the two systems is observed by cryo-SEM to become more similar with increasing concentration. This is logical, as the calculated $d$ spacings of the two systems differ significantly at low concentrations, but trend towards the same value at higher concentrations. As the surfactant 

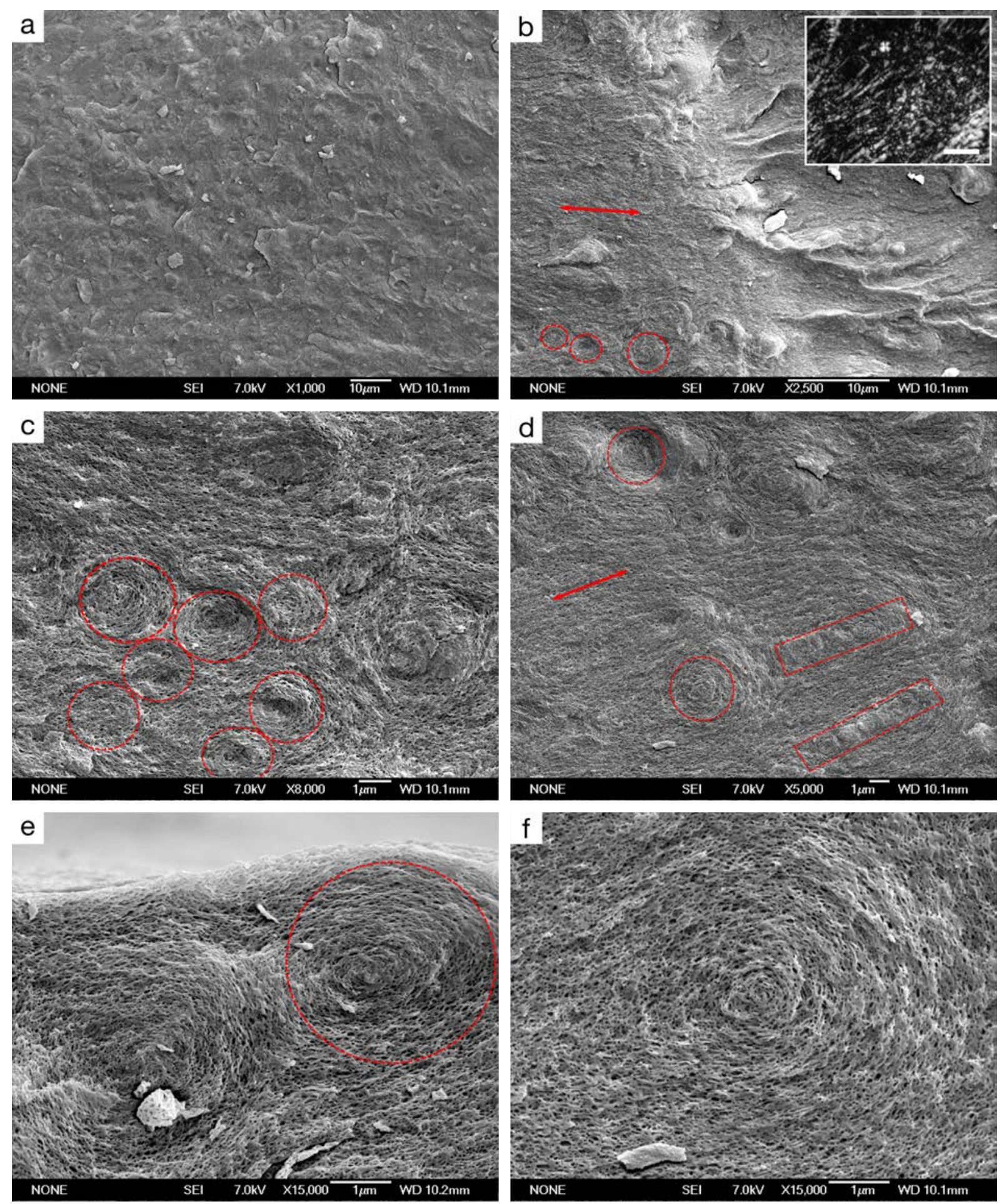

Figure 7.31: Cryo-SEM images of a sample of $50 \mathrm{wt} \%$ AcCh-AOT. Examples of different defect types are marked in red, with circles indicating individual FCD defects and boxes indicating oily streaks composed of FCD-I defects. Arrows indicate the average directional order of local bilayers. The circles in (c) indicate the packing of the FCD defects. The inset shows the corresponding POM image, with a scale bar of $50 \mu \mathrm{m}$.

concentration increases, any differing contributions of the counterions to the balance between long-range repulsion and van der Waals attraction forces become negligible in comparison with the electrostatic repulsion and steric hydration forces that start to dominate. 

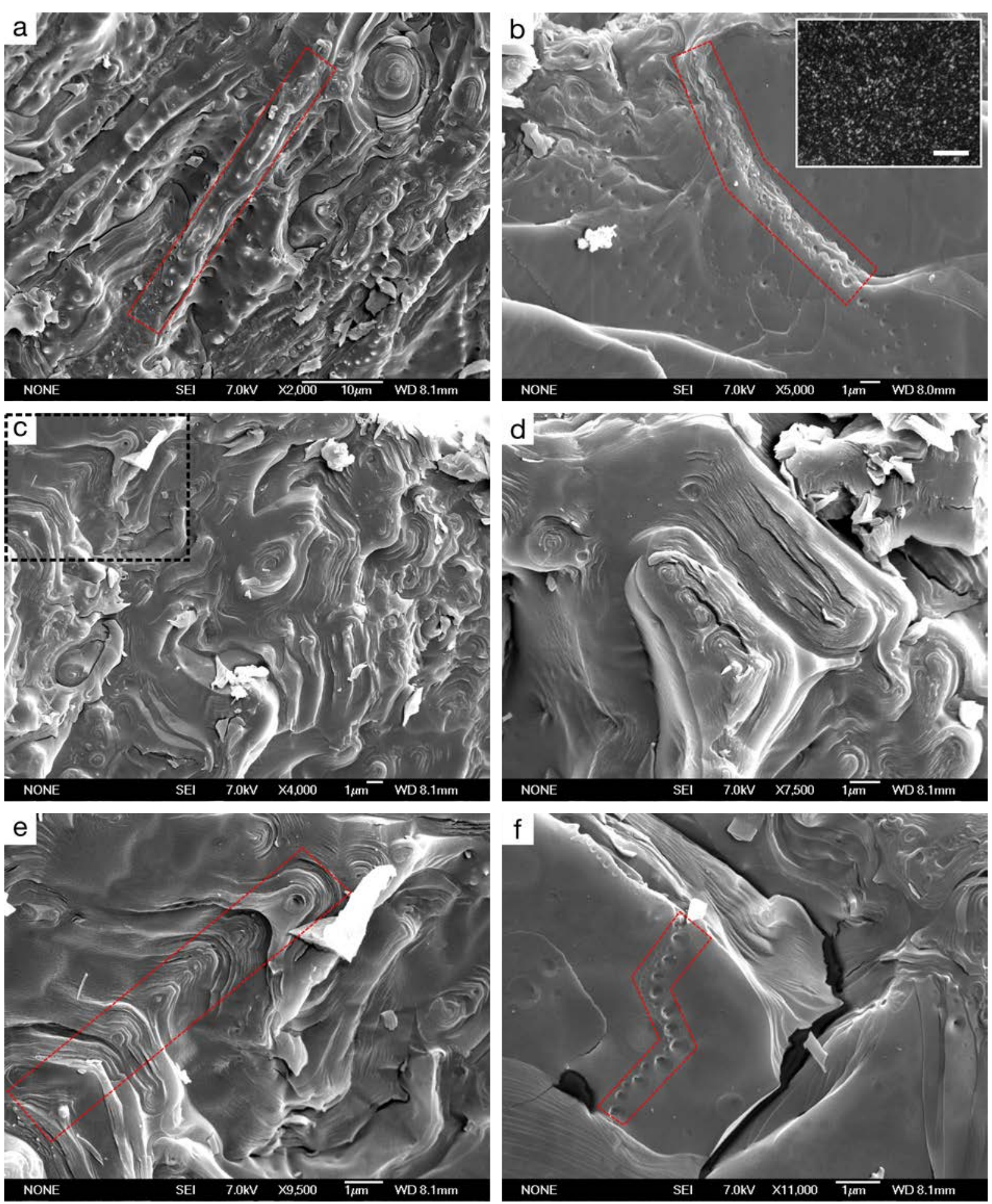

Figure 7.32: Cryo-SEM images of a sample of $75 \mathrm{wt} \%$ AcCh-AOT. Red boxes highlight oily streaks, grain boundaries and pitted defects as described in the text. The image in (e) is an enlargement of the area marked in (c). The inset shows the corresponding POM image, with a scale bar of $50 \mu \mathrm{m}$.

\section{Acetylcholine AOT: Summary}

Over the previous sections the characterisation of the mesophase behaviour was reported for the novel surfactant AcCh-AOT. The pairing of a bulky and hydrophobic counterion with AOT resulted in a system with an extremely stable lamellar phase, which was the only lyotropic 
phase formed. At high surfactant concentrations the system formed a crystalline structure that showed evidence of lamellar periodicity. Upon dilution with $\sim 75 \mathrm{wt} \%$ water, the lamellar phase gave way to a water-rich phase and a surfactant-rich phase comprised of MLVs. The system showed similarities to the systems presented previously, primarily in the type and progression of the defect structures formed in the lamellar region. The presented phase behaviour is very similar to that of Ch-AOT, with neither system favouring the formation of inverted structures, although the two systems do show structural differences at lower concentrations. Despite the absence of Type II structures in the phase diagram of AcCh-AOT, the use of the bulky $\mathrm{AcCh}^{+}$ion did not introduce Type I morphologies or the formation of an ionic liquid. Although, the extension of the two-phase region to higher concentrations indicates a trend towards the former.

Despite the assignment of a pure $\mathrm{L}_{\alpha}$ phase from 25 to $92 \mathrm{wt} \%$ AcCh-AOT, some samples in the region of 25-35 wt \% were seen to separate during POM observations, and their SAXS reflections indicated a deviation from $1 \mathrm{D}$ swelling. This brings into question the thermodynamic stability of the phase within this region, and whether the assigned phase boundary is in the correct position. However, cryo-SEM imaging at $25 \mathrm{wt} \%$ showed no evidence of a biphasic sample and revealed the phase to contain features of a lamellar phase, rather than just coalescence of MLVs. Observations of sealed bulk samples showed no evidence of separation when stored for several months, repeatedly centrifuged, or when subjected to heating and cooling cycles within the temperature boundaries of the phase. These observations, and that of a cloud point at higher temperatures, indicate that assignment of the equilibrium structure as a $L_{\alpha}$ phase is correct.

\section{Choline and Acetylcholine AOT: Discussion}

In this chapter, two new phase diagrams have been presented, which show the formation of lamellar phases that are stable over large concentration ranges, on increasing temperature and (as shown for Ch-AOT) upon the addition of salt. This behaviour can be explained very simply in terms of the packing parameter. A close to ideal balance has been achieved at the hydrophobic/hydrophilic interface, between the surfactant headgroups and tail regions, by providing the AOT molecule with a bulky counterion that results in a larger effective headgroup (and thus larger interfacial surface area). As a result, the molecules favourably pack into a bilayer structure. This is undoubtedly the overriding factor of the phase behaviour of these two systems. However, this explanation also misses several key points. The bilayer is not just the preferred packing motif, but is also extremely stable within these two systems. Bilayered structures are formed at just a few weight percent surfactant and persist as a lamellar phase over nearly the whole phase diagram, and have been shown to be resistant to major changes to the intermolecular forces that govern self-assembly.

The choice of large, hydrophobic and weakly hydrated counterions results in the formation of close ion pairs between the quaternary ammonium ions and the sulfonate moiety of AOT. In a normal bilayered system, the average size of the headgroup area (and thus interfacial 
curvature) decreases significantly with increasing concentration. This is mediated through the dissociation or dehydration of the counterion, and through the changes in bilayer repeat distance, all of which result in changes to the intermolecular forces that control self-assembly. However, changes to headgroup area are limited in the cases of the Ch-AOT and AcCh-AOT systems. The cloud points observed at low concentrations in these systems reveal that the counterions are strongly bound to the sulfonate headgroup and have unfavourable interactions with water. As such, it is favourable for the $\mathrm{Ch}^{+}$and $\mathrm{AcCh}^{+}$ions to dehydrate and to remain bound to the headgroup at nearly all surfactant concentrations. The tight counterion binding and embedding of such bulky counterions into the bilayer increases the headgroup area, but also adds significant stability through lateral steric repulsion between headgroups and increased van der Waals attraction between bilayers, which both result in a reduction in the elasticity of the bilayer.

If the lamellar bilayers of the AcCh-AOT and Ch-AOT systems are each considered as a compression of two monolayers, then the efficient geometric packing of the surfactant molecules results in each of the monolayers having a spontaneous curvature close to zero. This relieves the bilayers of the energy cost that arises from the curvature frustrations of two monolayers with an inherent need to curve away from each other. As such, the formation of inverted structures is energetically less favoured. However, the intriguing behaviour of the Ch-AOT and AcCh-AOT systems is not just the preferred formation of the $L_{\alpha}$ phase, but that this phase remains the lowest free energy form upon drastic changes in the intermolecular energy contributions. Changes in concentration, temperature and the addition of electrolyte all change the relative contributions of the free energy, and the systems are at various points governed by Helfrich, van der Waals, electrostatic and steric hydration forces, all of which have the capacity to alter the headgroups of surfactants and thus change the spontaneous curvature. To rationalise the stability of the $L_{\alpha}$ phases of these systems, the role of the counterion must be considered, both in terms of how it negates many of the changes in energy and in the way it affects the curvature of the bilayer. Both of these factors depend not only on the addition of steric bulk to the packing parameter, but also on the specific ion effects of the cation.

The value of the bending rigidity, $\kappa$, is associated with fluctuations and can be considered as the energy cost to bend the bilayer components (each monolayer) away from their desired spontaneous curvature. The tight binding of a bulky quaternary ammonium ion within the surfactant headgroup will have several effects on the value of $\kappa$. The ion will provide more efficient shielding of the electrostatic forces both between and along the bilayers. The former results in a net gain of interbilayer attractive forces, negating the electrostatic contribution to the overall repulsion. The second would have the effect of decreasing the value of $\kappa$ due to the increased freedom of the headgroups to move. However, the tight binding of such a large, bulky cation, that has the possibility of penetrating the interfacial region, results in a large steric repulsion that outweighs any result of reduced electrostatic repulsion (the headgroups cannot approach each other because the ammonium ion increases the headgroup area and the space is filled due to close packing). The result is an increase in the lateral pressure across the monolayer and thus a decrease in the freedom of movement of each molecule. As such the value of $\kappa$ is large due to a rigid bilayer, which resists curvature. The effect of this is seen in 
both Ch-AOT and AcCh-AOT at low concentrations, where the systems produced concentrated MLV phases, i.e. a far greater number of MLVs is observed than in the alkali metal systems. The formation of MLVs over unilamellar vesicles occurs only if the net bilayer interaction is attractive, which requires the system to overcome the undulation forces of Helfrich repulsion, meaning that the bilayer needs minimal spontaneous curvature and a large value of $\kappa$. $^{306}$

The value of $\kappa$ for a bilayer is simply twice the cost of bending an individual monolayer, whereas the second contribution to the bilayer elasticity, the topology-controlling Gaussian curvature modulus $\bar{k}$, is in part related to the spontaneous curvature $\left(c_{0}\right)$, the monolayer thickness $\left(I_{m}\right)$ and the already discussed bending rigidity, such that:

$$
\bar{\kappa}_{\text {bilayer }}=2 \bar{\kappa}_{\text {monolayer }}-2 \mathrm{I}_{\mathrm{m}} \mathrm{c}_{0 \text { monolayer }} \kappa_{\text {monolayer }}
$$

The bound $\mathrm{Ch}^{+}$or $\mathrm{AcCh}^{+}$counterions result in an increase in $\kappa$, and promote a near zero spontaneous curvature, resulting in a reduction in the elasticity contribution of $\bar{\kappa}$. If the spontaneous curvature of each monolayer is prevented from becoming sufficiently negative to promote a negative interfacial curvature, then $\bar{\kappa}$ does not need to relieve the energy demands through the formation of saddle-splay surfaces. The spontaneous curvature of a bilayer is normally sensitive to the composition of the phase and changes to the intermolecular energy contributions of the system result in changes to the Gaussian curvature and thus the topology of the system. The alkali metal systems discussed previously are prone to changes in elastic constants, especially changes in $\bar{\kappa}$ due to increased temperature or electrolyte concentration. These changes result in an increase in the value of $\bar{\kappa}$ and result in topological changes that promote the formation of $\mathrm{L}_{3}$ or $\mathrm{V}_{\text {// }}$ phases. $\bar{\kappa}$ is a key component in governing the formation of bicontinuous phases, in which the bilayer retains zero mean curvature but the Gaussian curvature changes everywhere along the minimal surface. However, if the rigidity imposed in the bilayer and the reduction in the overall repulsive forces in the system caused by the tightly bound quaternary ammonium ions prevent the spontaneous curvature from becoming significantly negative, then formation of inverse phases does not become energetically favoured.

Upon using either $\mathrm{Ch}^{+}$or $\mathrm{AcCh}^{+}$as the counterion in the surfactant, the net gain of the bilayer is a reduction in repulsive forces, an increase in attractive forces, decreased elasticity and minimal mean curvature. Thus, the bulky quaternary ammonium ions promote planar bilayers with large energy costs associated with either bending or stretching deformations. As such, the lamellar phases produced are stable and can withstand significant changes in intermolecular energy contributions. These range from Helfrich undulations at low concentrations (overcome due to the large value of $\kappa$ and result in the formation of MLVs) to the steric hydration repulsion forces at high concentrations (overcome by the superior dehydration of the bilayer). It is more energetically favourable for the bilayers to approach within 5-10 $\AA$, than it is for the monolayers to each adopt the negative interfacial curvature of the hexagonal phase, which is far removed from their values of spontaneous curvature. However, the microscopy observations for both systems revealed rich defect behaviours of curved point defects and topological connectivity. This is because the preferred free energy structure is a result of many 
different competing forces. The lamellar phases in AcCh-AOT and Ch-AOT have large energy costs associated with deforming the bilayers to the point of a phase transition. These costs are far greater than the curvature frustration of the monolayers within each bilayer, and so the bilayer structure is retained and the energy demands of the curvature frustration are met. Although the spontaneous curvature of the bilayers is minimal in comparison to the previously studied systems and less sensitive to the changes in intermolecular forces, it does not remain unchanged with concentration. This means that the elasticity of the two systems is situated such that the Gaussian curvature can satisfy the energy demands by formation of defects of positive and negative values, but could not be manipulated to drive a phase transition except upon the addition of large quantities of salt.

The topological changes in Gaussian curvature are likely aided by the hitherto neglected alkyl chains. The second term of Equation 7.1, also showed the contribution of the tail length to the value of $\bar{k}$. In comparison to phospholipids, the AOT bilayer is thin, which will increase the elasticity. The WAXS scattering of the AcCh-AOT and Ch-AOT showed the broad peak due to the fluid alkyl chains, was shifted to lower q values and significantly narrowed on increasing concentration. The alkyl chains are often assumed to have an insignificant role in comparison to the changes in intermolecular forces that govern the headgroup area, however they are likely a key component in the stability of the Ch-AOT and AcCh-AOT lamellar phases. The AOT chains are short, but also bulky due to branching and able to adopt different configurations. The flexibility of the alkyl chains to adopt different configurations allows the bilayer to relieve the range of curvature and packing frustrations imposed by the lamellar phase existing over such a large range of concentrations. This was shown in Chapter 4, where the Na-AOT system was able to stabilise a dilute lamellar phase, but the Na-butylAMA system (with reduced tail branching) was not.

The phase behaviour of the AcCh-AOT and Ch-AOT are described together above due to their similar behaviours. However, the results presented in this chapter also showed that the systems differ at low concentrations, both in terms of two-phase regions and the defect behaviour of each system. In comparison with the $\mathrm{Ch}^{+}$ion, the $\mathrm{AcCh}^{+}$ion is larger, has greater polarisability, is more hydrophobic, is more readily dehydrated and so forms a closer ion pair with the sulfonate ion. The $\mathrm{Ch}^{+}$ion is more polar and so amphiphilic in nature and likely has the terminal $\mathrm{OH}$ group protruding from the bilayer headgroup. The difference between the systems can be attributed to the hydrogen bonding capabilities of the $\mathrm{Ch}^{+}$ion providing more favourable interactions with the water. The more efficient separation of phases in the AcCh-AOT system can be rationalised in terms of it being the more hydrophobic, and the larger and deformed MLVs observed in the Ch-AOT system (compared with the AcCh-AOT system) are due to differences in their spontaneous curvatures.

Although the $L_{\alpha}$ phases of the two systems have low melting temperatures at high concentrations, the pure surfactants of both AcCh-AOT and Ch-AOT were observed to crystallise upon sufficient drying, and had melting points in excess of $200^{\circ} \mathrm{C}$. As such, neither system can be classified as an ionic liquid. However, Kumar and coworkers have recently reported the first published synthesis of Ch-AOT and used it to encapsulate the enzyme cytochrome $c$ within 
vesicles, biomimicking protein-membrane binding. ${ }^{277,307,308}$ In these publications, they classify $\mathrm{Ch}$-AOT as an ionic liquid with a gel-like appearance and a melting point of $-47.4^{\circ} \mathrm{C}$. The observations reported here are contrary to this, with the formation of crystals and a waxy solid. This discrepancy is likely a result of the different methodologies used to dry the surfactant. Due to the extremely hygroscopic nature of Ch-AOT, we employed air-sensitive techniques and the surfactant was dried for 72 hours and transferred to a glove box for storage and sample preparation. The drying time of several hours quoted by Rao et al. ${ }^{277}$ is likely insufficient to fully dry the surfactant and so the observed "gel" was probably within the lamellar phase. The published ${ }^{1} \mathrm{H}$ NMR that was used to characterise the surfactant contained an unassigned peak a $3.33 \mathrm{ppm}$ which integrated for around six protons and is characteristic of water in DMSO- $d_{6}$. This integration is equivalent to a water concentration of $\sim 9 \mathrm{wt} \%$, although water could have been present in the solvent, which is also notoriously hygroscopic.

Given that Ch-AOT is extremely hygroscopic and rapidly forms a lamellar phase upon exposure to air, the classification of it as a crystalline salt and the distinction between this and the reported ionic liquid properties are likely inconsequential. Most anionic surfactant ionic liquids are used for their self-assembly properties, and so the likely use of Ch-AOT would be in the formation of vesicles for biological applications, where water is generally employed as a solvent. Aside from casting doubt over the concentrations quoted in the aforementioned articles, the physical state of the pure surfactant is probably of little practical interest. The formation of stable crystals in the AcCh-AOT and Ch-AOT systems indicates that they form strong ion pairs with the AOT sulfonate group. lonic liquid formation would likely prevail if the nitrogen atom of these cations was further shielded by switching the methyl groups for longer, bulkier alkyl chains, similar to the two TAA-AOT ionic liquids produced by Brown et al. ${ }^{212}$

In general terms, this chapter can be summarised as presenting two new phase diagrams that show that the contributions of the various electrostatic forces that govern self-assembly can be overcome by combining the steric effects of a bulky cation with the formation of a close ion pair with the surfactant headgroup by following Collins' law of matching water affinities. ${ }^{49,181}$ However, as stated in Chapter 6, the ability of the ion to hydrogen bond is not accounted for within these laws, and if the selection of counterion is an organic ion rather than a point charge, the asymmetry of the ion and location of the functional groups will affect the efficiency of the packing and the ion pair formed. 


\section{Chapter 8}

\section{Concluding Remarks}

The current state of surfactant science is far from ideal. Surfactants are used globally in quantities of billions of tonnes annually, and with respect to chemical and biological sciences they are used in some form in almost all branches of research. However, the subtleties of surfactant behaviour are not generally well understood by the researchers that use them, potentially adversely affecting their performance. Models that exist to explain or predict surfactant behaviour are often either overly simplistic and unhelpfully make general assumptions that neglect the key roles of various system components (e.g. chirality, tail branching or counterions) or require a knowledge of theoretical physics beyond that of the average user of surfactants.

Manufacturers generally quote a value for the CMC of a surfactant, but this actually provides very little information about how any given surfactant will behave or pack at an interface, other than when it is present in very low (and generally irrelevant) concentrations. The other commonly cited concept - the surfactant packing parameter - at least provides a starting point for the type of curvature a surfactant may induce, but without knowledge of how this shape will change with the environmental conditions, this too provides very little useful information. For surfactants to be used efficiently in the wide range of fields in which they are currently employed, the users need to be provided with the appropriate information that will allow them to make informed decisions about the type of surfactant and methods they use. Steven Abbott's recently launched website (www.stevenabbott.co.uk) provides several digital applications that allow the user to model the predicted emulsifying behaviour for different compositions of various surfactant mixtures, and is an excellent resource that begins to address the above issues. However, these models rely heavily on work that may have unknowingly used Na-butylAMA that was sold as Na-hexylAMA (as described in Chapter 4).

To expand our knowledge of how surfactants behave and how their assembled structures are modified by the various forces that control them, more experimental data are required in the form of phase diagrams. The phase progression then needs to be understood and explained in terms of how the structure of the surfactant facilitates the microstructural changes that result in different behaviours, and how the surfactant will adapt to different environmental conditions and 
changes in the governing intermolecular forces. Ideally, phase diagrams are needed for several series of surfactants in which a single structural property is systematically changed. This will allow some general "rules of thumb" and guidelines to be formed, that will enable users of surfactants in other scientific disciplines to make informed choices regarding the molecular structure and chemical functionality of the surfactant they use.

A significant example of this issue is the role surfactants play in the self-assembly that controls the shape and size of nanoparticles/nanomaterials. Often, the choice of this surfactant is controlled by what is cheap and readily available, resulting in the use of common industrial surfactants. Providing a greater level of understanding to this selection process, and the knowledge and ability to manipulate the properties of the selected surfactant in a predictable way, may result in increased control and efficiency. However, the acquisition of phase diagrams is not glamorous research and rarely gains funding, while phase diagrams formulated within industrial companies are not made freely available. We have produced a family of AOTbased surfactants that differ in either the tail branching or the counterion. The different defect structures and specific microstructural changes of each system are detailed and discussed in each chapter. In this section, we focus on summarising the general trends and conclusions, and attempt to provide a list of key considerations that may aid other researchers to make more informed decisions.

Branched alkyl chains and chirality (within either the tail region or headgroup) introduce both stereoisomerism and rotational isomerism to surfactants. By comparing the Na-AOT, Na-butylAMA and K-butylAMA systems we have shown that this isomerism promotes disorder by enabling the surfactant to significantly alter its tail volume in response to changes in the environmental conditions. It is well known that changing the length of alkyl chains alters the flexibility of a bilayer due to changes in bilayer thickness; however, it may be of more practical use to be able to tune this flexibility by introducing, and adjusting the length and position, of tail branches. The work in this thesis shows that these factors can produce systems that are not truly binary and so can act as their own cosurfactants. It would be interesting to produce an enantiomerically pure chiral surfactant to see if the stereoisomerism has any quantifiable effect. However, this would be challenging and the practical purpose is questionable. Our results imply that stereoisomerism is likely responsible for small changes to the positioning of phase boundaries at high concentrations, while it is the formation of different rotational isomers that control the low concentration and thermal behaviours, which vary greatly between systems with different levels of branching.

The use of branched surfactants with an inherent flexibility could negate the need for certain additives that are normally required to promote this property, and thus significantly simplify formulations. If this work can be extended to the point where we are able to achieve predictable changes in interfacial curvature through adjusting the degree of tail branching, then we introduce a way of tuning the efficiency of surfactants and can improve their suitability for specific applications through structural modifications. The possible industrial importance of branched surfactants is illustrated by the widespread use of Na-AOT, which has long been considered to have superior properties to other systems, although it was not understood that the 
branched chains were responsible for this behaviour. The role and importance of branching in surfactants is now being recognised and is a recent active field of research. Of particular note is the work of the Eastoe group, who have reported encouraging results in the production of highly branched "hedgehog surfactants", in which the branching has been shown to efficiently reduce the surface tension and hence may provide environmentally friendly alternatives to fluorinated surfactants. ${ }^{18,147,309}$

Developing a better understanding of how the degree of branching can be used to tune the properties of surfactants may be a significant step towards the desired goal of producing surfactants via rational design. However, surfactants with systematic variations in branching are not generally commercially available, and their synthesis is neither simple nor cheap. Thus, adjusting surfactant systems by changing the extent of tail branching is not a convenient way for the "average user" to manipulate or control self-assembly or desired properties. In contrast, ion-exchange is a relatively simple and cheap process, and with the number of ions available to choose from, it provides an array of new behaviours for any given surfactant. lon-exchange offers an alternative way of manipulating the interfacial curvature and packing properties of the surfactant, without changing the molecular structure of the main component. However, while the effect of changing the length, number, or position of tail branches will likely yield results that will soon be predictable, the role of the counterion is significantly more complex.

The surfactant counterion is capable of altering every other contribution to the thermodynamically favoured structure. Many of these contributions cannot be measured or modelled directly, and the effect that changing the counterion has upon them cannot be quantified or evaluated independently of the changes it promotes in the other contributing forces. This is further complicated by the fact that different ions behave in different ways, and even for a single ion the effects are both concentration- and temperature-dependent. This point is illustrated by the $\mathrm{Li}^{+}$ion used in this work, which can be considered as either the largest or smallest ion used depending on the state of hydration.

The complexity of the roles of ions is evidenced by the number of experimentally observed phenomena that are accounted for by the term "specific ion effects". The point is perhaps best made by Kunz and Tiddy, who said in relation to ions in solution: "The specific behaviour of an ion cannot be described by only one parameter. In fact, the specificity of a certain type of ion is always dependent on the environment, be it the counterion, the interface or the interacting part of a macromolecule... it is utopian to describe the ions only with a single set of parameters. This is also the reason why correlations of specific ion effects with ion radii, polarisabilities, viscosity $B$ coefficients, lyotropic number etc. never give satisfactory results for a broad range of experimental findings". ${ }^{310}$ We have at times in this thesis been critical of the oversimplifications that are frequently used to explain surfactant self-assembly. So, given that we cannot qualitatively predict the ion effects of any given counterion on a wide range of different surfactants, how do we summarise the results of this work in a way that will prove useful to general scientists, without being unhelpfully simplistic ourselves? 
Self-assembly is by definition the collective result of every interaction between every component of a system, with the resulting formation of an equilibrium structure of minimum free energy. Too often, the role of entire components or forces within a system that actually play key roles in their assembly are neglected, or the results are described in a way that does not truly represent the structure formed, both of which are unhelpful and misleading. Our results clearly demonstrate that the counterion and tail branching are key factors in determining the selfassembly of surfactants, and that changes to them can have dramatic effects. Similarly, we have demonstrated that defect structures are fundamental to both the structural and physical properties of the system. The rationalisation of experimental results with simple concepts, or based on a single principle, that ignore the fundamentals of self-assembly is unhelpful. However, a knowledge of general trends and typical behaviours is fundamental to making informed decisions, and simple guidelines indicating likely behaviours can be a powerful tool for the general surfactant user. We do not attempt to quantify the trends we have observed in our experimental work by assigning arbitrary values to any factors, and we stress that we are not proposing formal rules with respect to any of the effects we have reported. The results of this experimental work are a direct factor of their specific environment, and would rapidly fail to hold true if applied to other surfactant systems without considering how the conditions of that system will impact upon them.

Here we provide, based on our experimental observations, a list of key considerations that we believe will assist a general surfactant user in tuning a surfactant to promote the desired properties through changing the counterion. These guidelines will allow the user to consider the role of various intermolecular forces within a system and how different counterions will likely affect them. The goal is to facilitate other scientists in making informed decisions regarding how changing the chemical structure of a given surfactant may alter the behaviour of the system in a predictable and desirable way, through the promotion or restriction of interfacial curvature.

- Matching of ions: Collins' general rules for matching water affinities (strong inner sphere ion pairs form between ions of opposite charge when they are of similar size and hydration) is an excellent and easily understood concept, which holds true for the systems investigated in this thesis. Choosing mismatched ions promotes counterion dissociation, adding an extra degree of freedom to the system, while pairing like ions results in tight binding and removes the ability of the counterion to selectively bind with concentration or bind inhomogeneously. Thus, when ions are well matched (such as in the Ch-AOT and AcCh-AOT systems) the electrostatic and van der Waals forces in the system, both along and across bilayers, are unlikely to change unpredictably. In contrast, when the ions are not well matched (such as in the Li-AOT system), the counterion has a greater role in governing the defect and phase behaviour of the system.

- Hydration: The hydration of the ion is clearly related to the first point; however, the choice of an ion with a large hydration shell again introduces a degree of freedom into the system that is controlled by the counterion. When fully hydrated, the $\mathrm{Li}^{+}$ion has a larger effective size than any of the other ions used in this work (including the bulky organic ions). However, when fully dehydrated, the $\mathrm{Li}^{+}$ion is the smallest ion. So, in contrast to the ions with small hydration shells (that do not change considerably upon dehydration), 
the $\mathrm{Li}^{+}$ion can provide contrasting behaviours at each concentration extreme, and induce either positive or negative curvature at the interface.

- Steric bulk: The effective steric bulk of an ion can be partially determined by the previous two points, which govern whether this factor can change significantly with concentration or temperature. However, the fundamental size of the charged species is still a key consideration in determining the steric bulk/repulsion added to the headgroup. If we consider that both AcCh-AOT and K-AOT preferentially dehydrate and bind strongly to the sulfonate headgroup of AOT, then the drastic differences in phase behaviour are due to size. These differences will result in differing electrostatic and van der Waals contributions, but the overriding factor is steric repulsion. The bulky size of $\mathrm{AcCh}^{+}$prevents the close packing of AOT headgroups, negating the decreased electrostatic contribution to the bilayer rigidity, and restricting the bilayer to form a stable lamellar phase. Conversely, the smaller $\mathrm{K}^{+}$ion promotes bilayer flexibility and negative interfacial curvature, as it does not restrict the close packing of headgroups, so the shielding of electrostatic forces is more important and drives the formation of a sponge phase.

- Shape: Unsymmetric ions (i.e. not simple point charges) will bind with different efficiencies to the surfactant headgroup, depending on their molecular structures and chemical functionalities. They will have different polarisabilities and so will result in different van der Waals contributions and degrees of electrostatic shielding.

- Hydrogen bonding: The ability of a counterion to hydrogen bond will undoubtedly alter the surfactant/water interactions within a lyotropic system. This is an effect that is not common in counterions and is not considered in any models of surfactant behaviour. However, our investigations indicate that the $\mathrm{K}^{+}$and $\mathrm{NH}_{4}{ }^{+}$ions, which were expected to produce similar behaviours are significantly different due to a contribution of hydrogen bonding to the bilayer rigidity in $\mathrm{NH}_{4}$-AOT resulting in $\mathrm{L}_{\alpha}$ formation rather than a $\mathrm{L}_{3}$ phase.

- Hydrophobicity: The hydrophobic effect is the underlying principle behind the selfassembly of amphiphilic molecules into lyotropic mesophases. Hence, the use of counterions that are slightly amphiphilic or differ in relative polarities will alter the system at the headgroup/water interface. This may be through the hydrophobic regions of the counterion having a greater desire to penetrate the tail region (rather than protrude from the surface), or it may result in the interfacial surface having a degree of hydrophobicity and the structures formed will resist aggregation. It is likely that the amphiphilic natures of Ch-AOT and AcCh-AOT explain their swelling behaviours and the size and distortion of the MLV structures formed.

The ultimate goal of surfactant science is the rational design of molecules for specific purposes, with control over the final properties. The complex nature of ions in solution mean that predicting the exact outcome of using a specific counterion is currently not feasible. However, the results obtained in this work do allow for a higher degree of predictability. Changing the counterion of a surfactant system will result in many alterations (including in the hydrocarbon tail region), but by considering how each of the above factors is likely to affect the environment of a known surfactant system for a given ion, the question of whether the change promotes increased counterion freedom or limits its effect can be rationalised. As such, a general surfactant user should be able to promote changes in the hydrophobic/hydrophilic interfacial region that drive 
the curvature of the system in the desired direction. For point charges, such as the alkali metal ions investigated in Part I, the last three considerations listed above are not applicable. However, the use of unsymmetric ions, such as the biologically relevant ammonium ions investigated in Part II, can alter the systems in different ways, and so careful selection of a counterion could potentially introduce more desirable properties. For example, could the super-efficient "hedgehog" surfactants reported by the Eastoe group be further improved by the selection of a counterion that also promotes a reduction in surface tension? Suitable candidates for this purpose include the $\mathrm{Ch}^{+}$and $\mathrm{AcCh}^{+}$ions used in this work.

This thesis reports on a study into how systematic variations in the molecular structure of a family of surfactants (through changing the counterion and tail branching) alters the interactions of the system on local lengthscales, and how this drives the global self-assembly of the systems, resulting in different phase behaviours. For the commonly used Na-AOT surfactant, we have shown that the tail branching and defect structures can be used to rationalise previously anomalous behaviours. We have also shown that for the two-tailed, branched AOT anion, a combined increase in both counterion binding and steric bulk can effectively inhibit the forces that promote non-zero spontaneous curvature. This produces extremely stable lamellar systems that are capable of accommodating changes in concentration, temperature and the addition of large amounts of electrolyte through alterations in defect structures rather than phase transitions. For smaller counterions, electrostatic interactions have a significant role, and the choice of counterions with different binding affinities can induce different elasticity behaviours, promoting either positive or negative Gaussian curvature at the hydrophobic/hydrophilic interface. The combination of increased hydration and reduced counterion binding results in a large degree of counterion freedom and thus a rise in unpredictable specific ion effects. To improve our ability to predict how such changes will affect other systems, further similar studies need to be carried out. In particular, it would be helpful to study a further family of two-tailed anionic surfactants, and incorporate a "hard" headgroup with a large hydration shell, so that the degree of freedom as a result of hydration does not correlate with the freedom given by counterion dissociation.

Going forwards, the use of surfactants in new and exciting applications in wide-ranging fields of research will continue to grow. It is important that our collective understanding of how they work grows at a similar rate, so that we can design superior surfactant molecules that more efficiently fulfil their designated role. For this to happen, the fundamental science behind their properties must continue to be probed, both theoretically and experimentally. It is our hope that the new concepts and understanding that will result are then shared freely, and communicated in a way that facilitates users from all scientific disciplines to apply this knowledge in beneficial ways and that deeper understanding leads to exciting new outcomes. 


\section{Bibliography}

1. I. W. Hamley, Liquid crystal phase formation by biopolymers, Soft Matter, 2010, 6, 18631871.

2. E. E. Magat, Fibres from extended chain aromatic polyamides, Philos. Trans. R. Soc., A, 1980, 294, 463-472.

3. J. Grdadolnik, F. Merzel and F. Avbelj, Origin of hydrophobicity and enhanced water hydrogen bond strength near purely hydrophobic solutes, Proc. Natl. Acad. Sci. USA, 2017, 114, 322-327.

4. G. S. Attard, J. C. Glyde and C. G. Göltner, Liquid-crystalline phases as templates for the synthesis of mesoporous silica, Nature, 1995, 378, 366-368.

5. D. Constantin and P. Davidson, Lamellar $L \alpha$ mesophases doped with inorganic nanoparticles, ChemPhysChem, 2014, 15, 1270-1282.

6. T. Ahmed, A. O. Kamel and S. D. Wettig, Interactions between DNA and Gemini surfactant: Impact on gene therapy: Part I, Nanomedicine (London, UK), 2016, 11, 289-306.

7. T. Ahmed, A. O. Kamel and S. D. Wettig, Interactions between DNA and gemini surfactant: Impact on gene therapy: Part II, Nanomedicine (London, UK), 2016, 11, 403-420.

8. D.-H. Kim, A. Jahn, S.-J. Cho, J. S. Kim, M.-H. Ki and D.-D. Kim, Lyotropic liquid crystal systems in drug delivery: A review, J. Pharm. Invest., 2014, 45, 1-11.

9. J. N. Israelachvili, D. J. Mitchell and B. W. Ninham, Theory of self-assembly of hydrocarbon amphiphiles into micelles and bilayers, J. Chem. Soc., Faraday Trans. 2, 1976, 72, 1525-1568.

10. J. N. Israelachvili, D. J. Mitchell and B. W. Ninham, Theory of self-assembly of lipid bilayers and vesicles., Biochim. Biophys. Acta, 1977, 470, 185-201.

11. C. Tanford, Micelle shape and size, J. Phys. Chem., 1972, 76, 3020-3024.

12. W. Helfrich, Elastic properties of lipid bilayers: Theory and possible experiments, $Z$. Naturforsch., C, 1973, 28, 693-703.

13. G. Porte, J. Appell, P. Bassereau and J. Marignan, L $\alpha$ to $L 3$ : A topology driven transition in phases of infinite fluid membranes, J. Phys. France, 1989, 50, 1335-1347. 
14. S. T. Hyde, in Handbook of Applied Surface and Colloid Chemistry, ed. K. Holmberg, John Wiley \& Sons Ltd, UK, 1st edn, 2001, vol. 1-2, ch. 16, pp. 299-332.

15. S. T. Hyde, On swelling and structure of composite materials. Some theory and applications of lyotropic mesophases, Colloids Surf., A, 1995, 103, 227-247.

16. G. E. Schröder-Turk, L. de Campo, M. E. Evans, M. Saba, S. C. Kapfer, T. Varslot, K. Grosse-Brauckmann, S. Ramsden and S. T. Hyde, Polycontinuous geometries for inverse lipid phases with more than two aqueous network domains, Faraday Discuss., 2013, 161, 215-247.

17. G. P. Sorenson, A. K. Schmitt and M. K. Mahanthappa, Discovery of a tetracontinuous, aqueous lyotropic network phase with unusual 3D-hexagonal symmetry, Soft Matter, 2014, 10, 8229-8235.

18. M. Sagisaka, T. Narumi, M. Niwase, S. Narita, A. Ohata, C. James, A. Yoshizawa, E. Taffin de Givenchy, F. Guittard, S. Alexander and J. Eastoe, Hyperbranched hydrocarbon surfactants give fluorocarbon-like low surface energies, Langmuir, 2014, 30, 6057-6063.

19. J.-P. Douliez, Self-assembly of hollow cones in a bola-amphiphile/hexadiamine salt solution, J. Am. Chem. Soc., 2005, 127, 15694-15695.

20. A. Bhadani, T. Okano, T. Ogura, T. Misono, K. Sakai, M. Abe and H. Sakai, Structural features and surfactant properties of core-shell type micellar aggregates formed by gemini piperidinium surfactants, Colloids Surf., A, 2016, 494, 147-155.

21. X. Li, F. Xing, Q. Xu, X. Sun, Y. Wang, L. Wang and P. Wang, Synthesis and surface-active properties of a homologous series of star-like triple-chain anionic surfactants derived from 1,1,1-tris(hydroxymethyl)ethane, J. Surfactants Deterg., 2016, 19, 129-135.

22. D. Lombardo, M. A. Kiselev, S. Magazù and P. Calandra, Amphiphiles self-assembly: Basic concepts and future perspectives of supramolecular approaches, Adv. Condens. Matter Phys., 2015, 2015, 151683.

23. S. Andersson, S. T. Hyde, K. Larsson and S. Lidin, Minimal surfaces and structures: from inorganic and metal crystals to cell membranes and biopolymers, Chem. Rev., 1988, 88, 221-242.

24. T. Castle, M. E. Evans, S. T. Hyde, S. Ramsden and V. Robins, Trading spaces: Building three-dimensional nets from two-dimensional tilings, Interface Focus, 2012, 2, 555-566.

25. A. Angelova, B. Angelov, R. Mutafchieva, V. M. Garamus, S. Lesieur, S. S. Funari, R. Willumeit and P. Couvreur, Swelling of a Sponge Lipid Phase via Incorporation of a Nonionic Amphiphile: SANS and SAXS Studies, Prog. Colloid Polym. Sci., 2011, 138, $1-6$.

26. W. A. Hamilton, L. Porcar, P. D. Butler and G. G. Warr, Local membrane ordering of sponge phases at a solid-solution interface, J. Chem. Phys., 2002, 116, 8533. 
27. A. Maldonado, W. Urbach, R. Ober and D. Langevin, Swelling behavior and local topology of an $\mathrm{L}_{3}$ (sponge) phase, Phys. Rev. E: Stat. Phys., Plasmas, Fluids, Relat. Interdiscip. Top., 1996, 54, 1774-1778.

28. B. Angelov, A. Angelova, R. Mutafchieva, S. Lesieur, U. Vainio, V. M. Garamus, G. V. Jensen and J. S. Pedersen, SAXS investigation of a cubic to a sponge $\left(L_{3}\right)$ phase transition in self-assembled lipid nanocarriers, Phys. Chem. Chem. Phys., 2011, 13, 3073-3081.

29. R. Beck and H. Hoffmann, Phase behaviour and physical properties of mixtures of calcium salts of $\alpha$-sulfonated fatty acid methyl ester and a cosurfactant, Phys. Chem. Chem. Phys., 2001, 3, 5438-5443.

30. R. Beck, Y. Abe, T. Terabayashi and $H$. Hoffmann, A novel $L_{3}$-phase from a Ca-salt of an anionic surfactant and a cosurfactant, J. Phys. Chem. B, 2002, 106, 3335-3338.

31. D. Roux, M. E. Cates, U. Olsson, R. C. Ball, F. Nallet and A. M. Bellocq, Light scattering from a surfactant "sponge" phase: Evidence for a hidden symmetry, Europhys. Lett., 1990, 11, 229-234.

32. W. Helfrich, Steric interaction of fluid membranes in multilayer systems, Z. Naturforsch., A, 1978, 33, 305-315.

33. R. Strey, R. Schomäcker, D. Roux, F. Nallet and U. Olsson, Dilute lamellar and $L_{3}$ phases in the binary water- $\mathrm{C}_{12} \mathrm{E}_{5}$ system, J. Chem. Soc., Faraday Trans., 1990, 86, 2253-2261.

34. S. Chandrasekhar, Liquid Crystals: Second Edition, Cambridge University Press, UK, 1992.

35. M. Kléman and O. D. Lavrentovich, Grain boundaries and the law of corresponding cones in smectics, Eur. Phys. J. E, 2000, 2, 47-57.

36. C. Blanc and M. Kléman, Curvature walls and focal conic domains in a lyotropic lamellar phase, Eur. Phys. J. B, 1999, 10, 53-60.

37. P. Boltenhagen, O. D. Lavrentovich and M. Kléman, Focal conic domains with positive Gaussian curvature and saddle-splay rigidity of smectic $L_{\alpha}$ phases, Phys. Rev. A, 1992, 46, R1743-R1746.

38. M. Kléman, C. Meyer and Y. A. Nastishin, Imperfections in focal conic domains: the role of dislocations, Philos. Mag., 2006, 86, 4439-4458.

39. M. Kléman and O. D. Lavrentovich, Liquids with conics, Liq. Cryst., 2009, 36, 1085-1099.

40. R. D. Kamien and T. C. Lubensky, Minimal surfaces, screw dislocations, and twist grain boundaries, Phys. Rev. Lett., 1999, 82, 2892-2895.

41. D. Constantin and P. Oswald, Diffusion coefficients in a lamellar lyotropic phase: Evidence for defects connecting the surfactant structure, Phys. Rev. Lett., 2000, 85, 4297-4300.

42. M. Kléman, Defects in liquid crystals, Rep. Prog. Phys., 1989, 52, 555-654. 
43. M. Kléman and O. D. Laverntovich, Soft Matter Physics: An Introduction, Springer-Verlag New York, Inc., USA, 2003.

44. C. V. Kulkarni, W. Wachter, G. Iglesias-Salto, S. Engelskirchen and S. Ahualli, Monoolein: A magic lipid?, Phys. Chem. Chem. Phys., 2011, 13, 3004-3021.

45. Th. Zemb, D. Gazeau, M. Dubois and T. Gulik-Krzywicki, Critical behaviour of lyotropic liquid crystals, Europhys. Lett., 1993, 21, 759-766.

46. C. Kang and A. Khan, Self-assembly in systems of didodecyldimethylammonium surfactants: Binary and ternary phase equilibria and phase structures with sulphate, hydroxide, acetate, and chloride counterions, J. Colloid Interface Sci., 1993, 156, 218228.

47. P. T. Callaghan and O. Söderman, Examination of the lamellar phase of aerosol OT/water using pulsed field gradient nuclear magnetic resonance, J. Phys. Chem., 1983, 87, 17371744.

48. S. Nave, J. Eastoe and J. Penfold, What is so special about aerosol-OT? 1. Aqueous systems, Langmuir, 2000, 16, 8733-8740.

49. K. D. Collins, lons from the Hofmeister series and osmolytes: Effects on proteins in solution and in the crystallization process, Methods, 2004, 34, 300-311.

50. W. Kunz and R. Neueder, in Specific lon Effects, ed. W. Kunz, World Scientific Publishing Co. Pte. Ltd., Singapore, 1st edn, 2010, ch. 1, pp. 3-54.

51. Y.-D. Dong, A. W. Dong, I. Larson, M. Rappolt, H. Amenitsch, T. Hanley and B. J. Boyd, Impurities in commercial phytantriol significantly alter its lyotropic liquid-crystalline phase behavior, Langmuir, 2008, 24, 6998-7003.

52. P. Kékicheff, C. Grabielle-Madelmont and M. Ollivon, Phase diagram of sodium dodecyl sulfate-water system: 1. A calorimetric study, J. Colloid Interface Sci., 1989, 131, 112132.

53. J. K. Cline, US Pat., US 1957443 A, 1934.

54. H. E. Gottlieb, V. Kotlyar and A. Nudelman, NMR chemical shifts of common laboratory solvents as trace impurities, J. Org. Chem., 1997, 62, 7512-7515.

55. J. Eastoe, T. F. Towey, B. H. Robinson, J. Williams and R. K. Heenan, Structures of metal bis(2-ethylhexylsulfosuccinate) aggregates in cyclohexane, J. Phys. Chem., 1993, 97, 1459-1463.

56. R. E. Riter, E. P. Undiks and N. E. Levinger, Impact of counterion on water motion in aerosol OT reverse micelles, J. Am. Chem. Soc., 1998, 120, 6062-6067.

57. M. L. Stahla, B. Baruah, D. M. James, M. D. Johnson, N. E. Levinger and D. C. Crans, ${ }^{1} \mathrm{H}$ NMR studies of aerosol-OT reverse micelles with alkali and magnesium counterions: Preparation and analysis of MAOTs, Langmuir, 2008, 24, 6027-6035. 
58. H. R. Rabie and J. H. Vera, Counterion binding to ionic reverse micellar aggregates and its effect on water uptake, J. Phys. Chem. B, 1997, 101, 10295-10302.

59. J. Eastoe, D. C. Steytler, B. H. Robinson, R. K. Heenan, A. N. North and J. C. Dore, Structure of cobalt aerosol-OT reversed micelles studied by small-angle scattering methods, J. Chem. Soc., Faraday Trans., 1994, 90, 2479-2504.

60. R. Klein, M. Kellermeier, D. Touraud, E. Müller and W. Kunz, Choline alkylsulfates - New promising green surfactants, J. Colloid Interface Sci., 2013, 392, 274-280.

61. Sigma-Aldrich Inc., Dihexyl Sulfosuccinate Sodium Salt Solution, www.sigmaaldrich.com/catalog/product/aldrich/86146?lang=en\&region=GB, accessed December 2016.

62. S. Nave, PhD thesis, University of Bristol, 2001.

63. K. Fontell, The structure of the lamellar liquid crystalline phase in aerosol OT-water system, J. Colloid Interface Sci., 1973, 44, 318-329.

64. J. Rogers and P. A. Winsor, Optically positive, isotropic and negative lamellar liquid crystalline solutions, Nature, 1967, 216, 477-479.

65. Olympus America Inc. ${ }^{\odot}$, Microscopy Resource Center: Optical Birefringence, www.olympusmicro.com/primer/lightandcolor/birefringence.html, accessed January 2017.

66. L. Belkoura, C. Stubenrauch and R. Strey, Freeze fracture direct imaging: A new freeze fracture method for specimen preparation in cryo-transmission electron microscopy, Langmuir, 2004, 20, 4391-4399.

67. D. Danino and Y. Talmon, in Molecular Gels: Materials with Self-Assembled Fibrillar Networks, ed. R. G. Weiss and P. Terech, Springer, The Netherlands, 1st edn, 2006, ch. 9, pp. 253-274.

68. G. Tan, P. Xu, V. T. John, J. He, G. L. McPherson, V. Agarwal and A. Bose, Cryofield emission scanning electron microscopy imaging of a rigid surfactant mesophase, Langmuir, 2008, 24, 10621-10624.

69. L. Issman and Y. Talmon, Cryo-SEM specimen preparation under controlled temperature and concentration conditions, J. Microsc., 2012, 246, 60-69.

70. T. K. De and A. Maitra, Solution behaviour of aerosol OT in non-polar solvents, Adv. Colloid Interface Sci., 1995, 59, 95-193.

71. Grand View Research, Surfactants Market Analysis By Product (Cationic, Nonionic, Anionic, Amphoteric), By Application (Home Care, Personal Care, Industrial \& Institutional Cleaners, Food Processing, Oilfield Chemicals, Agricultural Chemicals, Textiles, Emulsion Polymerization, Paints \& Coatings, Construction) And Segment Forecasts To 2022: Report Summary, www.grandviewresearch.com/industry-analysis/surfactantsmarket, accessed November 2016. 
72. World Health Organization, WHO Model List of Essential Medicines: 19th List (April 2015), WHO Press, Switzerland, 2015.

73. P. Flynn, Multidimensional multinuclear solution NMR studies of encapsulated macromolecules, Prog. Nucl. Magn. Reson. Spectrosc., 2004, 45, 31-51.

74. Z. X. Li, J. R. Lu, R. K. Thomas and J. Penfold, Neutron reflectivity studies of the adsorption of aerosol-OT at the air-water interface: The structure of the sodium salt, $J$. Phys. Chem. B, 1997, 101, 1615-1620.

75. S. Nave, J. Eastoe, R. K. Heenan, D. Steytler and I. Grillo, What is so special about aerosol-OT? 2. Microemulsion systems, Langmuir, 2000, 16, 8741-8748.

76. R. K. Mitra and B. K. Paul, Effect of $\mathrm{NaCl}$ and temperature on the water solubilization behavior of AOT/nonionics mixed reverse micellar systems stabilized in IPM oil, Colloids Surf., A, 2005, 255, 165-180.

77. M. S. Hellsing, A. R. Rennie and A. V. Hughes, Adsorption of aerosol-OT to sapphire: Lamellar structures studied with neutrons, Langmuir, 2011, 27, 4669-4678.

78. R. W. Mattoon and M. B. Mathews, Micelles in non-aqueous media, J. Chem. Phys., 1949, 17, 496-497.

79. S. Nave, J. Eastoe, R. K. Heenan, D. Steytler and I. Grillo, What is so special about aerosol-OT? Part III - Glutaconate versus sulfosuccinate headgroups and oil-water interfacial tensions, Langmuir, 2002, 18, 1505-1510.

80. S. Nave, A. Paul, J. Eastoe, A. R. Pitt and R. K. Heenan, What is so special about aerosolOT? Part IV. Phenyl-tipped surfactants, Langmuir, 2005, 21, 10021-10027.

81. W.-C. Chien, Synthesis of $\mathrm{Y}_{2} \mathrm{O}_{3}$ :Eu phosphors by bicontinuous cubic phase process, J. Cryst. Growth, 2006, 290, 554-559.

82. L. Huang, H. Wang, Z. Wang, A. Mitra, D. Zhao and Y. Yan, Cuprite nanowires by electrodeposition from lyotropic reverse hexagonal liquid crystalline phase, Chem. Mater., 2002, 14, 876-880.

83. A.-H. Ranneh, Y. Iwao, S. Noguchi, T. Oka and S. Itai, The use of surfactants to enhance the solubility and stability of the water-insoluble anticancer drug SN38 into liquid crystalline phase nanoparticles, Int. J. Pharm., 2016, 515, 501-505.

84. L. Tavano, L. Gentile, C. O. Rossi and R. Muzzalupo, Novel gel-niosomes formulations as multicomponent systems for transdermal drug delivery, Colloids Surf., B, 2013, 110, 281-288.

85. R. Nagarajan, Molecular packing parameter and surfactant self-assembly: The neglected role of the surfactant tail, Langmuir, 2002, 18, 31-38.

86. J. Rogers and P. A. Winsor, Change in the optic sign of the lamellar phase (G) in the aerosol OT/water system with composition or temperature, J. Colloid Interface Sci., 1969, 30, 247-257. 
87. P. G. Petrov, S. V. Ahir and E. M. Terentjev, Rheology at the phase transition boundary: 1. Lamellar $L_{\alpha}$ phase of AOT surfactant solution, Langmuir, 2002, 18, 9133-9139.

88. P. Ekwall, L. Mandell and K. Fontell, Some observations on binary and ternary aerosol OT systems, J. Colloid Interface Sci., 1970, 33, 215-235.

89. G. Ungar, V. Tomašić, F. Xie and X.-b. Zeng, Structure of liquid crystalline aerosol-OT and Its alkylammonium salts, Langmuir, 2009, 25, 11067-11072.

90. M. Skouri, J. Marignan and R. May, X-ray and neutron-scattering study of the lamellar and $\mathrm{L}_{3}$ phases of the system aerosol-OT-water: effect of $\mathrm{NaCl}$ and decane, Colloid Polym. Sci., 1991, 269, 929-937.

91. E. Y. Sheu, S. H. Chen and J. S. Huang, Structure and growth of bis (2-ethylhexyl) sulfosuccinate micelles in aqueous solutions, J. Phys. Chem., 1987, 91, 3306-3310.

92. H. N. Patrick and G. G. Warr, in Specialist Surfactants, ed. I. D. Robb, Chapman \& Hall, UK, 1st edn, 1997, ch. 2, pp. 7-36.

93. A. I. Fajalia, E. Antoniou, P. Alexandridis and M. Tsianou, Self-assembly of sodium bis(2-ethylhexyl) sulfosuccinate in aqueous solutions: Modulation of micelle structure and interactions by cyclodextrins investigated by small-angle neutron scattering, J. Mol. Liq., 2015, 210, 125-135.

94. P. L. Hubbard, K. M. McGrath and P. T. Callaghan, Evolution of a lamellar domain structure for an equilibrating lyotropic liquid crystal, J. Phys. Chem. B, 2006, 110, 20781-20788.

95. P. L. Hubbard, K. M. McGrath and P. T. Callaghan, Orientational anisotropy in the polydomain lamellar phase of a lyotropic liquid crystal, Langmuir, 2006, 22, 3999-4003.

96. W. Philippoff and J. W. McBain, Expansion of the lamellar crystal lattice of aerosol OT upon the addition of water, Nature, 1949, 164, 885.

97. D. Park, J. Rogers, R. W. Toft and P. A. Winsor, The structure of micellar solutions of ionic amphiphiles: The lamellar phase. X-ray diffraction measurements with the aerosol OT/water system, J. Colloid Interface Sci., 1970, 32, 81-89.

98. I. Lundström and K. Fontell, Lateral electrical conductivity in aerosol-OT-water systems, J. Colloid Interface Sci., 1977, 59, 360-370.

99. R. Faiman, I. Lundström and K. Fontell, A Raman spectroscopic study of the Aerosol OT-water system, Chem. Phys. Lipids, 1977, 18, 73-83.

100. A. Khan, K. Fontell and B. Lindman, Liquid crystallinity in systems of magnesium and calcium surfactants: Phase diagrams and phase structures in binary aqueous systems of magnesium and calcium di-2-ethylhexylsulfosuccinate, J. Colloid Interface Sci., 1984, 101, 193-200.

101. P. D. Moran, G. A. Bowmaker, R. P. Cooney, J. R. Bartlett and J. L. Woolfrey, Vibrational spectra of metal salts of bis(2-ethylhexyl)sulfosuccinate (AOT), J. Mater. Chem., 1995, 5, 295-302. 
102. Y. Nagasoe, N. Ichiyanagi, H. Okabayashi, S. Nave, J. Eastoe and C. J. O'Connor, Raman scattering spectra of Aerosol-OT homologous sodium dialkylsulfosuccinates and the environment of their hydrophobic chains, Colloid Polym. Sci., 1999, 277, 947-956.

103. M. A. Arunagirinathan, M. Roy, A. K. Dua, C. Manohar and J. R. Bellare, Micro-Raman investigations of myelins in aerosol-OT/water system, Langmuir, 2004, 20, 4816-4822.

104. Y. Nagasoe, N. Ichiyanagi, H. Okabayashi, S. Nave, J. Eastoe and C. J. O'Connor, Infrared absorption spectra of Aerosol-OT homologous sodium dialkylsulfosuccinates and the effect of crystal polymorphism on the environment of the succinate segment, Colloid Polym. Sci., 1999, 277, 1051-1057.

105. Y. Nagasoe, H. Okabayashi, M. Abe, J. Eastoe and C. J. O'Connor, Vibrational spectra of Aerosol-OT homologous sodium dialkylsulfosuccinates - normal coordinate analyses of sodium diethylsulfosuccinate and sodium dimethylsulfosuccinate and their application to longer homologues, Vib. Spectrosc., 2000, 23, 151-168.

106. C. Tanford, The Hydrophobic Effect: Formation of Micelles and Biological Membranes, John Wiley \& Sons Inc., USA, 1973.

107. C. Boissière, J. B. Brubach, A. Mermet, G. de Marzi, C. Bourgaux, E. Prouzet and P. Roy, Water confined in lamellar structures of AOT surfactants: An infrared investigation, J. Phys. Chem. B, 2002, 106, 1032-1035.

108. E. Prouzet, J.-B. Brubach and P. Roy, Differential scanning calorimetry study of the structure of water confined within AOT lamellar mesophases, J. Phys. Chem. B, 2010, 114, 8081-8088.

109. K. Czarniecki, A. Jaich, J. M. Janik, M. Rachwalska, J. A. Janik, J. Krawczyk, K. Otnes, F. Volino and R. Ramasseul, Calorimetric, nuclear magnetic resonance, and quasi-elastic neutron scattering studies of the aerosol OT- $\mathrm{D}_{2} \mathrm{O}$ system, J. Colloid Interface Sci., 1983, 92, 358-366.

110. N. Casillas, J. E. Puig, R. Olayo, T. J. Hart and E. I. Franses, State of water and surfactant in lyotropic liquid crystals, Langmuir, 1989, 5, 384-389.

111. F. Nallet and P. Barois, Anomalous birefringence of swollen lamellar phases: Blue smectics, J. Phys. II, 1994, 4, 1049-1060.

112. F. Nallet, R. Laversanne and D. Roux, Modelling X-ray or neutron scattering spectra of lyotropic lamellar phases: Interplay between form and structure factors, J. Phys. II, 1993, 3, 487-502.

113. A. Caillé, Physique cristalline - Remarques sur la diffusion des rayons $X$ dans les smectiques A, C. R. Acad. Sci., Paris, Sér. B, 1972, 274, 891-893.

114. W.-c. Zhuang, X. Chen, C.-j. Yang, L.-y. Wang and Y.-c. Chai, Study of ordering for AOT/water lamellar lyotropic liquid crystal: Small-angle X-ray scattering experiments, Acta Phys.-Chim. Sin., 2005, 21, 1055-1058. 
115. E. I. Franses and T. J. Hart, Phase behavior and molecular motion of aerosol OT in liquidcrystalline phases with water, J. Colloid Interface Sci., 1983, 94, 1-13.

116. G. Chidichimo, C. La Mesa, G. A. Ranieri and M. Terenzi, NMR investigation of the lamellar mesophase occurring in the system aerosol OT-water, Mol. Cryst. Liq. Cryst., 1987, 150, 221-236.

117. L. Coppola, R. Muzzalupo, G. A. Ranieri and M. Terenzi, Characterization of the lamellar phase aerosol OT/water system by NMR diffusion measurements, Langmuir, 1995, 11, 1116-1121.

118. C. Garza, L. T. Thieghi and R. Castillo, Atomic force microscopy images of lyotropic lamellar phases, J. Chem. Phys., 2007, 126, 051106.

119. C. Yang, X. Chen, H. Qu, W. Zhuang, Y. Chai and J. Hao, Dissipative particle dynamics simulation of phase behavior of aerosol OT/water system, J. Phys. Chem. B, 2006, 110, 21735-21740.

120. K. Sengupta, V. A. Raghunathan and J. Katsaras, Structure of the ripple phase of phospholipid multibilayers, Phys. Rev. E, 2003, 68, 031710.

121. B. A. Cunningham, A.-D. Brown, D. H. Wolfe, W. P. Williams and A. Brain, Ripple phase formation in phosphatidylcholine: Effect of acyl chain relative length, position, and unsaturation, Phys. Rev. E, 1998, 58, 3662-3672.

122. T. Mori, T. Tada and T. Matsumoto, Structure and viscoelastic properties of lamellar phase formed in aerosol OT/water binary systems, Nihon Reoroji Gakkaishi, 1997, 25, 223-225.

123. P. T. Callaghan, Translational Dynamics and Magnetic Resonance: Principles of Pulsed Gradient Spin Echo NMR, Oxford University Press, UK, 2011.

124. M. M. Kozlov, S. Leikin and R. P. Rand, Bending, hydration and interstitial energies quantitatively account for the hexagonal-lamellar-hexagonal reentrant phase transition in dioleoylphosphatidylethanolamine, Biophys. J., 1994, 67, 1603-1611.

125. V. I. Gordeliy, V. Cherezov and J. Teixeira, Strength of thermal undulations of phospholipid membranes, Phys. Rev. E, 2005, 72, 061913.

126. V. Kocherbitov, Driving forces of phase transitions in surfactant and lipid systems, J. Phys. Chem. B, 2005, 109, 6430-6435.

127. A. Pal, P. Bharath, S. G. Dastidar and V. A. Raghunathan, Thermal unbinding and ordering of amphiphile bilayers in the presence of salt, J. Colloid Interface Sci., 2013, 402, 151156.

128. S. C. Costigan, P. J. Booth and R. H. Templer, Estimations of lipid bilayer geometry in fluid lamellar phases, Biochim. Biophys. Acta, Biomembr., 2000, 1468, 41-54.

129. P. J. Quinn and C. Wolf, Hydrocarbon chains dominate coupling and phase coexistence in bilayers of natural phosphatidylcholines and sphingomyelins, Biochim. Biophys. Acta, Biomembr., 2009, 1788, 1126-1137. 
130. J. Pan, S. Tristram-Nagle, N. Kučerka and J. F. Nagle, Temperature dependence of structure, bending rigidity, and bilayer interactions of dioleoylphosphatidylcholine bilayers, Biophys. J., 2008, 94, 117-124.

131. A. Pertsin and M. Grunze, Temperature dependence of the short-range repulsion between hydrated phospholipid membranes: A computer simulation study, Biointerphases, 2007, 2, 105-108.

132. J. N. Israelachvili, Intermolecular and Surface Forces, Third Edition, Academic Press, USA, 2011.

133. T. Sottmann and R. Strey, in Fundamentals of Interface and Colloid Science: Volume 5 Soft Colloids, ed. J. Lyklema, Elsevier Ltd., UK, 1st edn, 2005, ch. 5, pp. 5.1-5.96.

134. J. M. Seddon and R. H. Templer, in Handbook of Biological Physics: Structure and Dynamics of Membranes, ed. R. Lipowsky and E. Sackmann, Elsevier Science B.V., The Netherlands, 1st edn, 1995, ch. 3, pp. 97-160.

135. C. Stubenrauch, S. Burauer, R. Strey and C. Schmidt, A new approach to lamellar phases $\left(L_{\alpha}\right)$ in water - Non-ionic surfactant systems, Liq. Cryst., 2004, 31, 39-53.

136. A. Maitra and T. K. Jain, Study of the vibrational characteristics of aerosol OT by laser raman spectroscopy, Colloids Surf., 1987, 28, 19-27.

137. A. Maitra, D. Varshney and M. Varshney, Revisiting the rotational isomerism of aerosol OT from the selective heteronuclear nuclear overhauser effect, Colloids Surf., 1987, 24, 119-126.

138. A. M. Amorim da Costa, L. A. E. Batista de Carvalho, J. J. C. Teixeira-Dias, E. F. G. Barbosa and I. M. S. Lampreia, The temperature dependence of the Raman spectrum and gauche interactions of tri-N-butylamine: A conformational study, Can. J. Chem., 1987, 65, 384-389.

139. M. Kléman, The lamellar and sponge phases of dilute surfactant systems: Structures and defects at equilibrium and under shear, Pramana, 1999, 53, 107-119.

140. M. Allain, Possible defect-mediated phase transition in a lyotropic liquid crystal. Electron microscopy observations, Europhys. Lett., 1986, 2, 597-602.

141. L. Golubović, Passages and droplets in lamellar fluid membrane phases, Phys. Rev. E, 1994, 50, R2419-R2422.

142. S. S. Funari, M. C. Holmes and G. J. T. Tiddy, Microscopy, x-ray diffraction, and NMR studies of lyotropic liquid crystal phases in the C22EO6/water system: A new intermediate phase, J. Phys. Chem., 1992, 96, 11029-11038.

143. S. S. Funari, M. C. Holmes and G. J. T. Tiddy, Intermediate lyotropic liquid crystal phases in the C16EO6/water system, J. Phys. Chem., 1994, 98, 3015-3023.

144. P. Pincus, J. F. Joanny and D. Andelman, Electrostatic interactions, curvature elasticity, and steric repulsion in multimembrane systems, Europhys. Lett., 1990, 11, 763-768. 
145. D. Roux and C. R. Safinya, A synchrotron X-ray study of competing undulation and electrostatic interlayer interactions in fluid multimembrane lyotropic phases, J. Phys. France, 1988, 49, 307-318.

146. J. Gustafsson, G. Orädd, G. Lindblom, U. Olsson and M. Almgren, A defective swelling lamellar phase, Langmuir, 1997, 13, 852-860.

147. A. Czajka, G. Hazell and J. Eastoe, Surfactants at the design limit, Langmuir, 2015, 31, 8205-8217.

148. B. Balinov, U. Olsson and O. Soederman, Structural similarities between the $L_{3}$ and bicontinuous cubic phases in the AOT-brine system, J. Phys. Chem., 1991, 95, 59315936.

149. J. Bergenholtz and N. J. Wagner, Formation of AOT/brine multilamellar vesicles, Langmuir, 1996, 12, 3122-3126.

150. CYTEC Industries Inc., Specialty Additives AEROSOL ${ }^{\circledR}$ Surfactants, www.cytec.com/sites/default/files/files/AEROSOL\%20Surfactants\%20Brochure.pdf, accessed December 2016.

151. Colonial Chemical, Inc., Cola ${ }^{\circledR}$ Wet MA-80 Technical Data Sheet, www.colonialchem.com/fullpanel/uploads/files/colawet-ma-80.pdf, accessed December 2016.

152. R. J. Vetter, Micelle structure in aqueous solutions of colloidal electrolytes, J. Phys. Colloid Chem., 1947, 51, 262-277.

153. R. F. Stamm, T. Mariner and J. K. Dixon, Turbidimetric determination of the molecular weight of micelles of dihexyl sodium sulfosuccinate in water, J. Chem. Phys., 1948, 16, 423-424.

154. P. A. Winsor, Binary and multicomponent solutions of amphiphilic compounds. Solubilization and the formation, structure, and theoretical significance of liquid crystalline solutions, Chem. Rev., 1968, 68, 1-40.

155. V. Luzzati and F. Husson, The structure of the liquid-crystalline phases of lipid-water systems, J. Cell Biol., 1962, 12, 207-219.

156. F. Husson, H. Mustacchi and V. Luzzati, La structure des colloïdes d'association. II. Description des phases liquide-cristallines de plusieurs systèmes amphiphile-eau: Amphiphiles anioniques, cationiques, non-ioniques, Acta Crystallogr., 1960, 13, 668-677.

157. J. R. Baran Jr., G. A. Pope, W. H. Wade and V. Weerasooriya, Phase behavior of water/perchloroethylene/anionic surfactant systems, Langmuir, 1994, 10, 1146-1150.

158. E. J. Acosta, J. S. Yuan and A. S. Bhakta, The characteristic curvature of ionic surfactants, J. Surfactants Deterg., 2008, 11, 145-158.

159. E. Acosta, E. Szekeres, D. A. Sabatini and J. H. Harwell, Net-average curvature model for solubilization and supersolubilization in surfactant microemulsions, Langmuir, 2003, 19, 186-195. 
160. E. Acosta, H. Uchiyama, D. A. Sabatini and J. H. Harwell, The role of hydrophilic linkers, J. Surfactants Deterg., 2002, 5, 151-157.

161. T. Doan, E. Acosta, J. F. Scamehorn and D. A. Sabatini, Formulating middle-phase microemulsions using mixed anionic and cationic surfactant systems, J. Surfactants Deterg., 2003, 6, 215-224.

162. A. Upadhyaya, E. J. Acosta, J. F. Scamehorn and D. A. Sabatini, Microemulsion phase behavior of anionic-cationic surfactant mixtures: Effect of tail branching, J. Surfactants Deterg., 2006, 9, 169-179.

163. S. K. Kiran and E. J. Acosta, HLD-NAC and the formation and stability of emulsions near the phase inversion point, Ind. Eng. Chem. Res., 2015, 54, 6467-6479.

164. T. T. Nguyen and D. A. Sabatini, Formulating alcohol-free microemulsions using rhamnolipid biosurfactant and rhamnolipid mixtures, J. Surfactants Deterg., 2008, 12, $109-115$.

165. CYTEC Industries Inc., AEROSOL ${ }^{\circledR} M A 80 \%$ Surfactant Product Safety Summary, www.cytec.com/sites/default/files/files/AEROSOL_MA_80percent_PSS.pdf, accessed December 2016.

166. CYTEC Industries Inc., AEROSOL ${ }^{\circledR} M A$ 80-I Surfactant Safety Data Sheet, www.sfm.state.or.us/cr2k_subdb/MSDS/AEROSOL_MA_80.PDF, accessed December 2016.

167. S. Ghasemi, M. T. Darestani and Z. Abdollahi, Electrical impedance spectroscopy for determining critical micelle concentration of ionic emulsifiers, Colloids Surf., $A, 2014, \mathbf{4 4 1}$, 195-203.

168. J. Greener, B. A. Contestable and M. D. Bale, Interaction of anionic surfactants with gelatin: Viscosity effects, Macromolecules, 1987, 20, 2490-2498.

169. A. P. Brady and D. J. Salley, The self-diffusion coefficients and transference numbers of the small ions in colloidal electrolytes, J. Am. Chem. Soc., 1948, 70, 914-919.

170. A. Franzetti, P. Di Gennaro, A. Bevilacqua, M. Papacchini and G. Bestetti, Environmental features of two commercial surfactants widely used in soil remediation, Chemosphere, 2006, 62, 1474-1480.

171. C. A. Ramsburg, K. D. Pennell, T. C. G. Kibbey and K. F. Hayes, Refinement of the densitymodified displacement method for efficient treatment of tetrachloroethene source zones, J. Contam. Hydrol., 2004, 74, 105-131.

172. K. Kostarelos, S. R. Lenschow, M. A. Stylianou, P. C. de Blanc, M. M. Mygind and A. G. Christensen, Jet A fuel recovery using micellar flooding: Design and implementation, Sci. Total Environ., 2016, 563-564, 890-898.

173. A. A. Kmetz, M. D. Becker, B. A. Lyon, E. Foster, Z. Xue, K. P. Johnston, L. M. Abriola and K. D. Pennell, Improved mobility of magnetite nanoparticles at high salinity with polymers and surfactants, Energy Fuels, 2016, 30, 1915-1926. 
174. L. Farías-Cepeda, J. Herrera-Ordonez and E. Saldívar-Guerra, On the kinetics and particle size polydispersity of the styrene emulsion polymerization using aerosol MA80 and sodium dodecyl sulfate as surfactants, Colloid Polym. Sci., 2010, 288, 1401-1409.

175. S. Sajjadi, Particle formation in interval III of the emulsion polymerization of styrene with aerosol-MA as an emulsifier, J. Polym. Sci., Part A: Polym. Chem., 2002, 40, 1652-1663.

176. C. D. Wilfred, G. D. Nair and A. K. Ziyada, Synthesis and characterisations of Aliquat $336^{\circledR}$ and cetylpyridinium ionic liquids incorporated with sulfonate-based anions, AIP Conf. Proc., 2012, 1482, 214-218.

177. M. Clerc, A. M. Levelut and J. F. Sadoc, Transitions between mesophases involving cubic phases in the surfactant-water systems. Epitaxial relations and their consequences in a geometrical framework, J. Phys. II, 1991, 1, 1263-1276.

178. C. E. Carraher Jr., Seymour/Carraher's Polymer Chemistry: Sixth Edition, Marcel Dekker Inc., USA, 2005.

179. M. Kléman, C. E. Williams, M. J. Costello and T. Gulik-Krzywicki, Defect structures in lyotropic smectic phases revealed by freeze-fracture electron microscopy, Philos. Mag., 1977, 35, 33-56.

180. E. R. Nightingale Jr., Phenomenological theory of ion solvation. Effective radii of hydrated ions, J. Phys. Chem., 1959, 63, 1381-1387.

181. K. D. Collins, G. W. Neilson and J. E. Enderby, lons in water: Characterizing the forces that control chemical processes and biological structure, Biophys. Chem., 2007, 128, 95-104.

182. Y. Sato, M. Ueno and H. Kishimoto, Micelle formations of bis(2-ethylhexyl) sodium 2sulfonatosuccinate (Aerosol OT) and its lithium or potassium substituted salt in water, Yakugaku Zasshi, 1975, 95, 1286-1291.

183. A. Chakraborty, S. Chakraborty and S. K. Saha, Temperature dependant micellization of AOT in aqueous medium: Effect of the nature of counterions, J. Dispersion Sci. Technol., 2007, 28, 984-989.

184. H. Takahashi, A. Matsuo and I. Hatta, Effects of salt on the lamellar and bicontinuous cubic phases of fully hydrated monoacylglycerol (monoelaidin), Phys. Chem. Chem. Phys., 2002, 4, 2365-2370.

185. A. M. Squires, R. H. Templer, J. M. Seddon, J. Woenckhaus, R. Winter, S. Finet and $\mathrm{N}$. Theyencheri, Kinetics and mechanism of the lamellar to gyroid inverse bicontinuous cubic phase transition, Langmuir, 2002, 18, 7384-7392.

186. P. M. Duesing, J. M. Seddon, R. H. Templer and D. A. Mannock, Pressure effects on lamellar and inverse curved phases of fully hydrated dialkyl phosphatidylethanolamines and $\beta$-D-xylopyranosyl-sn-glycerols, Langmuir, 1997, 13, 2655-2664.

187. C. V. Kulkarni, Lipid crystallization: From self-assembly to hierarchical and biological ordering, Nanoscale, 2012, 4, 5779-5791. 
188. M. C. Holmes and M. S. Leaver, in Bicontinuous Liquid Crystals, ed. M. L. Lynch and P. T. Spicer, CRC Press, USA, 1st edn, 2005, vol. 127, ch. 2, pp. 15-40.

189. S. Puntambekar, M. C. Holmes and M. S. Leaver, Novel intermediate phases in an ionic fluorocarbon surfactant/water system, Liq. Cryst., 2000, 27, 743-747.

190. R. Zhou, M. Leaver, R. McCabe and M. Holmes, Mesh phases in tetramethylammonium perfluorodecanoate-water ternary systems, Soft Matter, 2012, 8, 11255-11261.

191. V. A. Raghunathan, Mesh phases of surfactant-water systems, J. Indian Inst. Sci., 2012, 88, 197-210.

192. S. K. Ghosh, R. Ganapathy, R. Krishnaswamy, J. Bellare, V. A. Raghunathan and A. K. Sood, Structure of mesh phases in a cationic surfactant system with strongly bound counterions, Langmuir, 2007, 23, 3606-3614.

193. C. E. Fairhurst, M. C. Holmes and M. S. Leaver, Structure and morphology of the intermediate phase region in the nonionic surfactant C16EO6/water system, Langmuir, 1997, 13, 4964-4975.

194. Y. Wang, M. C. Holmes, M. S. Leaver and A. Fogden, Mesh phases in a ternary nonionic surfactant, oil, and water system, Langmuir, 2006, 22, 10951-10957.

195. J. Burgoyne, M. C. Holmes and G. J. T. Tiddy, An extensive mesh phase liquid crystal in aqueous mixtures of a long chain nonionic surfactant, J. Phys. Chem., 1995, 99, 60546063.

196. M. G. Fischer, S. T. Hyde and G. E. Schröder-Turk, Comment on "Discovery of a tetracontinuous, aqueous lyotropic network phase with unusual 3D-hexagonal symmetry" by M. Mahanthappa, G. Sorenson and A. Schmitt., Soft Matter, 2015, 11, 1226-1227.

197. G. P. Sorenson and M. K. Mahanthappa, Reply to the 'Comment on "Discovery of a tetracontinuous, aqueous lyotropic network phase with unusual 3D-hexagonal symmetry" ' by G. Schröder-Turk, M. Fischer and S. Hyde, Soft Matter, 2015, 11, 1228-1230.

198. G. Brotons, M. Dubois, L. Belloni, I. Grillo, T. Narayanan and Th. Zemb, The role of counterions on the elasticity of highly charged lamellar phases: A small-angle x-ray and neutron-scattering determination, J. Chem. Phys., 2005, 123, 024704.

199. J. M. Seddon, N. Zeb, R. H. Templer, R. N. McElhaney and D. A. Mannock, An Fd3m lyotropic cubic phase in a binary glycolipid/water system, Langmuir, 1996, 12, 5250-5253.

200. M. Kumar, N. G. Patil, C. K. Choudhury, S. Roy, A. V. Ambade and G. Kumaraswamy, Compact polar moieties induce lipid-water systems to form discontinuous reverse micellar phase, Soft Matter, 2015, 11, 5417-5424.

201. M. Kumar and G. Kumaraswamy, Phase behaviour of the ternary system: Monooleinwater-branched polyethylenimine, Soft Matter, 2015, 11, 5705-5711.

202. I. Martiel, L. Sagalowicz and R. Mezzenga, A reverse micellar mesophase of facecentered cubic $F m \overline{3} m$ symmetry in phosphatidylcholine/water/organic solvent ternary systems, Langmuir, 2013, 29, 15805-15812. 
203. I. Martiel, L. Sagalowicz and R. Mezzenga, Phospholipid-based nonlamellar mesophases for delivery systems: Bridging the gap between empirical and rational design, Adv. Colloid Interface Sci., 2014, 209, 127-143.

204. U. Schwarz and G. Gompper, Stability of inverse bicontinuous cubic phases in lipid-water mixtures, Phys. Rev. Lett., 2000, 85, 1472-1475.

205. P. Mariani, V. Luzzati and H. Delacroix, Cubic phases of lipid-containing systems. Structure analysis and biological implications, J. Mol. Biol., 1988, 204, 165-189.

206. K. Fontell, Cubic phases in surfactant and surfactant-like lipid systems, Colloid Polym. Sci., 1990, 268, 264-285.

207. P. Ström and D. M. Anderson, The cubic phase region in the system didodecyldimethylammonium bromide-water-styrene, Langmuir, 1992, 8, 691-709.

208. G. C. Shearman, O. Ces and R. H. Templer, Towards an understanding of phase transitions between inverse bicontinuous cubic lyotropic liquid crystalline phases, Soft Matter, 2010, 6, 256-262.

209. H. I. Lee, C. Pak, S. H. Yi, J. K. Shon, S. S. Kim, B. G. So, H. Chang, J. E. Yie, Y.U. Kwon and J. M. Kim, Systematic phase control of periodic mesoporous organosilicas using Gemini surfactants, J. Mater. Chem., 2005, 15, 4711-4717.

210. R. Koynova and B. Tenchov, Interactions of surfactants and fatty acids with lipids, Curr. Opin. Colloid Interface Sci., 2001, 6, 277-286.

211. D. W. Smith, Ionic hydration enthalpies, J. Chem. Educ., 1977, 54, 540.

212. P. Brown, C. Butts, R. Dyer, J. Eastoe, I. Grillo, F. Guittard, S. Rogers and R. Heenan, Anionic surfactants and surfactant ionic liquids with quaternary ammonium counterions, Langmuir, 2011, 27, 4563-4571.

213. R. S. Boethling, E. Sommer and D. DiFiore, Designing small molecules for biodegradability, Chem. Rev., 2007, 107, 2207-2227.

214. M. M. Adeva, G. Souto, N. Blanco and C. Donapetry, Ammonium metabolism in humans, Metab., Clin. Exp., 2012, 61, 1495-1511.

215. M. F. Chaplin, in Water and Life: The Unique Properties of $\mathrm{H}_{2} \mathrm{O}$, ed. R. M. Lynden-Bell, S. Conway Morris, J. D. Barrow, J. L. Finney and C. L. Harper, Jr, CRC Press, USA, 1st edn, 2010, ch. 5, pp. 1-20.

216. J. M. Seddon, A. Squires, O. Ces, R. H. Templer, J. Woenckhaus and R. Winter, in SelfAssembly, ed. B. H. Robinson, IOS Press, The Netherlands, 1st edn, 2003, ch. 24, pp. 212-221.

217. M. Ge and J. H. Freed, Hydration, structure, and molecular interactions in the headgroup region of dioleoylphosphatidylcholine bilayers: an electron spin resonance study, Biophys. J., 2003, 85, 4023-4040. 
218. P. Mariani, L. Q. Amaral and L. Saturni, Hexagonal-cubic phase transitions in lipid containing systems: Epitaxial relationships and cylinder growth, J. Phys. II, 1994, 4, 13931416.

219. J. Kherb, S. C. Flores and P. S. Cremer, Role of carboxylate side chains in the cation Hofmeister series, J. Phys. Chem. B, 2012, 116, 7389-7397.

220. A. Salis and B. W. Ninham, Models and mechanisms of Hofmeister effects in electrolyte solutions, and colloid and protein systems revisited, Chem. Soc. Rev., 2014, 43, 73587377.

221. D. F. Parsons and B. W. Ninham, Ab initio molar volumes and Gaussian radii, J. Phys. Chem. A, 2009, 113, 1141-1150.

222. C. I. Rich, in The Role of Potassium in Agriculture, ed. V. J. Kilmer, S. E. Younts and N. C. Brady, American Society of Agronomy, Crop Science Society of America, Soil Science Society of America, USA, 1st edn, 1968, ch. 5, pp. 79-108.

223. N. Vlachy, B. Jagoda-Cwiklik, R. Vácha, D. Touraud, P. Jungwirth and W. Kunz, Hofmeister series and specific interactions of charged headgroups with aqueous ions, Adv. Colloid Interface Sci., 2009, 146, 42-47.

224. P. Intharathep, A. Tongraar and K. Sagarik, Structure and dynamics of hydrated $\mathrm{NH}_{4}{ }^{+}$: An ab initio QM/MM molecular dynamics simulation, J. Comput. Chem., 2005, 26, 13291338.

225. W. I. Babiaczyk, S. Bonella, L. Guidoni and G. Ciccotti, Hydration structure of the quaternary ammonium cations, J. Phys. Chem. B, 2010, 114, 15018-15028.

226. Y. X. Wang, M. Lu and D. E. Draper, Specific ammonium ion requirement for functional ribosomal RNA tertiary structure, Biochemistry, 1993, 32, 12279-12282.

227. H. I. Petrache, T. Zemb, L. Belloni and V. A. Parsegian, Salt screening and specific ion adsorption determine neutral-lipid membrane interactions, Proc. Natl. Acad. Sci. USA, 2006, 103, 7982-7987.

228. K. D. Collins, Ion hydration: Implications for cellular function, polyelectrolytes, and protein crystallization, Biophys. Chem., 2006, 119, 271-281.

229. J. K. Blusztajn, Choline, a vital amine, Science, 1998, 281, 794-795.

230. S. H. Zeisel, K. A. Da Costa, P. D. Franklin, E. A. Alexander, J. T. Lamont, N. F. Sheard and A. Beiser, Choline, an essential nutrient for humans, FASEB J., 1991, 5, 2093-2098.

231. S. H. Zeisel and K.-A. da Costa, Choline: An essential nutrient for public health, Nutr. Rev., 2009, 67, 615-623.

232. A. A. Yates, S. A. Schlicker and C. W. Suitor, Dietary reference intakes: The new basis for recommendations for calcium and related nutrients, B vitamins, and choline, J. Am. Diet. Assoc., 1998, 98, 699-706. 
233. Institute of Medicine (US) Standing Committee on the Scientific Evaluation of Dietary Reference Intakes and its Panel on Folate, Other B Vitamins, and Choline, Dietary Reference Intakes for Thiamin, Riboflavin, Niacin, Vitamin $B_{6}$, Folate, Vitamin $B_{12}$, Pantothenic Acid, Biotin, and Choline, National Academy Press, USA, 1998.

234. A. Bidulescu, L. E. Chambless, A. M. Siega-Riz, S. H. Zeisel and G. Heiss, Usual choline and betaine dietary intake and incident coronary heart disease: the Atherosclerosis Risk in Communities (ARIC) study, BMC Cardiovasc. Disord., 2007, 7, 20.

235. Z. Li and D. E. Vance, Phosphatidylcholine and choline homeostasis, J. Lipid Res., 2008, 49, 1187-1194.

236. S. H. Zeisel, Nutritional importance of choline for brain development, J. Am. Coll. Nutr., 2004, 23, 621S-626S.

237. S. H. Zeisel, Choline: Critical role during fetal development and dietary requirements in adults, Annu. Rev. Nutr., 2006, 26, 229-250.

238. G. M. Shaw, R. H. Finnell, H. J. Blom and S. L. Carmichael, Choline and risk of neural tube defects in a folate-fortified population, Epidemiology, 2009, 20, 714-719.

239. G. M. Shaw, S. L. Carmichael, W. Yang, S. Selvin and D. M. Schaffer, Periconceptional dietary intake of choline and betaine and neural tube defects in offspring, Am. J. Epidemiol., 2004, 160, 102-109.

240. A. J. Copp, N. S. Adzick, L. S. Chitty, J. M. Fletcher, G. N. Holmbeck and G. M. Shaw, Spina bifida, Nat. Rev. Dis. Primers, 2015, 1, 15007.

241. P. M. Ueland, Choline and betaine in health and disease, J. Inherited Metab. Dis., 2011, 34, 3-15.

242. E. L. Cohen and R. J. Wurtman, Brain acetylcholine: Control by dietary choline, Science, 1976, 191, 561-562.

243. B. A. Trommer, D. E. Schmidt and L. Wecker, Exogenous choline enhances the synthesis of acetylcholine only under conditions of increased cholinergic neuronal activity, $J$. Neurochem., 1982, 39, 1704-1709.

244. J. W. Goodby, Liquid crystals and life, Liq. Cryst., 1998, 24, 25-38.

245. E. T. M. Leermakers, E. M. Moreira, J. C. K.-d. Jong, S. K. L. Darweesh, T. Visser, T. Voortman, P. K. Bautista, R. Chowdhury, D. Gorman, W. M. Bramer, J. F. Felix and O. H. Franco, Effects of choline on health across the life course: A systematic review, Nutr. Rev., 2015, 73, 500-522.

246. S. H. Zeisel and D. J. Canty, Choline phospholipids: Molecular mechanisms for human diseases: A meeting report, J. Nutr. Biochem., 1993, 4, 258-263.

247. B. Ramstedt and J. P. Slotte, Membrane properties of sphingomyelins, FEBS Lett., 2002, 531, 33-37. 
248. B. Engelmann, S. Streich, U. M. Schönthier, W. O. Richter and J. Duhm, Changes of membrane phospholipid composition of human erythrocytes in hyperlipidemias. I. Increased phosphatidylcholine and reduced sphingomyelin in patients with elevated levels of triacylglycerol-rich lipoproteins., Biochim. Biophys. Acta, 1992, 1165, 32-37.

249. P. Niemelä, M. T. Hyvönen and I. Vattulainen, Structure and dynamics of sphingomyelin bilayer: Insight gained through systematic comparison to phosphatidylcholine, Biophys. J., 2004, 87, 2976-2989.

250. S. H. Zeisel, Choline. A nutrient that is involved in the regulation of cell proliferation, cell death, and cell transformation., Adv. Exp. Med. Biol., 1996, 399, 131-141.

251. J. C. Lacal, Choline kinase as a precision medicine target for therapy in cancer, autoimmune diseases and malaria, Precis. Med., 2015, 2, e980.

252. N. D. Ridgway, The role of phosphatidylcholine and choline metabolites to cell proliferation and survival, Crit. Rev. Biochem. Mol. Biol., 2013, 48, 20-38.

253. S. Janardhan, P. Srivani and G. N. Sastry, Choline kinase: an important target for cancer, Curr. Med. Chem., 2006, 13, 1169-1186.

254. H. M. Awwad, J. Geisel and R. Obeid, The role of choline in prostate cancer, Clin. Biochem., 2012, 45, 1548-1553.

255. T. Skripuletz, A. Manzel, K. Gropengießer, N. Schäfer, V. Gudi, V. Singh, L. S. Tejedor, S. Jörg, A. Hammer, E. Voss, F. Vulinovic, D. Degen, R. Wolf, D.-H. Lee, R. Pul, D. Moharregh-Khiabani, W. Baumgärtner, R. Gold, R. A. Linker and M. Stangel, Pivotal role of choline metabolites in remyelination, Brain, 2015, 138, 398-413.

256. M. E. O'Leary and R. Horn, Internal block of human heart sodium channels by symmetrical tetra-alkylammoniums., J. Gen. Physiol., 1994, 104, 507-522.

257. M. E. O'Leary, R. G. Kallen and R. Horn, Evidence for a direct interaction between internal tetra-alkylammonium cations and the inactivation gate of cardiac sodium channels, $J$. Gen. Physiol., 1994, 104, 523-539.

258. M. R. Picciotto, M. J. Higley and Y. S. Mineur, Acetylcholine as a neuromodulator: Cholinergic signaling shapes nervous system function and behavior, Neuron, 2012, 76, 116-129.

259. R. Schliebs and T. Arendt, The significance of the cholinergic system in the brain during aging and in Alzheimer's disease, J. Neural Transm., 2006, 113, 1625-1644.

260. T. H. Ferreira-Vieira, I. M. Guimaraes, F. R. Silva and F. M. Ribeiro, Alzheimer's disease: Targeting the cholinergic system, Curr. Neuropharmacol., 2016, 14, 101-115.

261. M. Homma, A. Uzawa, H. Tanaka, N. Kawaguchi, T. Kanai, K. Nakajima, M. Narita, Y. Hara, H. Maruyama, Y. Ogawa, K. Himuro and S. Kuwabara, A novel fusion protein, AChR-Fc, ameliorates myasthenia gravis by neutralizing antiacetylcholine receptor antibodies and suppressing acetylcholine receptor-reactive B cells, Neurotherapeutics, 2016, 14, 191-198. 
262. M. Keijzers, G. Nogales-Gadea and M. de Baets, Clinical and scientific aspects of acetylcholine receptor myasthenia gravis, Curr. Opin. Neurol., 2014, 27, 552-557.

263. J. K. Blusztajn, M. Liscovitch and U. I. Richardson, Synthesis of acetylcholine from choline derived from phosphatidylcholine in a human neuronal cell line, Proc. Natl. Acad. Sci. USA, 1987, 84, 5474-5477.

264. P. J. Whitehouse, D. L. Price, A. W. Clark, J. T. Coyle and M. R. DeLong, Alzheimer disease: Evidence for selective loss of cholinergic neurons in the nucleus basalis, Ann. Neurol., 1981, 10, 122-126.

265. P. Whitehouse, D. Price, R. Struble, A. Clark, J. Coyle and M. Delon, Alzheimer's disease and senile dementia: Loss of neurons in the basal forebrain, Science, 1982, 215, 12371239.

266. H. Tohgi, T. Abe, K. Hashiguchi, M. Saheki and S. Takahashi, Remarkable reduction in acetylcholine concentration in the cerebrospinal fluid from patients with Alzheimer type dementia, Neurosci. Lett., 1994, 177, 139-142.

267. G. Ehrenstein, Z. Galdzicki and G. D. Lange, The choline-leakage hypothesis for the loss of acetylcholine in Alzheimer's disease, Biophys. J., 1997, 73, 1276-1280.

268. R. Schliebs and T. Arendt, The cholinergic system in aging and neuronal degeneration, Behav. Brain Res., 2011, 221, 555-563.

269. Z.-M. Wang, P. Cai, Q.-H. Liu, D.-Q. Xu, X.-L. Yang, J.-J. Wu, L.-Y. Kong and X.-B. Wang, Rational modification of donepezil as multifunctional acetylcholinesterase inhibitors for the treatment of Alzheimer's disease, Eur. J. Med. Chem., 2016, 123, 282-297.

270. J. K. Blusztajn and R. J. Wurtman, Choline and cholinergic neurons, Science, 1983, 221, 614-620.

271. R. J. Wurtman, M. Cansev and I. H. Ulus, in Handbook of Neurochemistry and Molecular Neurobiology: Neural Lipids, ed. A. Lajtha, G. Tettamanti and G. Goracci, Springer Science+Business Media, USA, 3rd edn, 2009, ch. 18, pp. 443-501.

272. R. Hayes, G. G. Warr and R. Atkin, Structure and nanostructure in ionic liquids, Chem. Rev., 2015, 115, 6357-6426.

273. M. J. Earle and K. R. Seddon, Ionic liquids. Green solvents for the future, Pure Appl. Chem., 2000, 72, 10-25.

274. N. V. Plechkova and K. R. Seddon, in Methods and Reagents for Green Chemistry: An Introduction, ed. P. Tundo, A. Perosa and F. Zecchini, John Wiley \& Sons, Inc., USA, 1st edn, 2007, ch. 5, pp. 105-130.

275. M. K. Potdar, G. F. Kelso, L. Schwarz, C. Zhang and M. T. W. Hearn, Recent developments in chemical synthesis with biocatalysts in ionic liquids, Molecules, 2015, 20, 1678816816. 
276. P. Wasserscheid and W. Keim, lonic liquids-New "solutions" for transition metal catalysis, Angew. Chem., Int. Ed., 2000, 39, 3772-3789.

277. K. S. Rao, P. S. Gehlot, T. J. Trivedi and A. Kumar, Self-assembly of new surface active ionic liquids based on Aerosol-OT in aqueous media, J. Colloid Interface Sci., 2014, 428, 267-275.

278. A. Sein and J. B. F. N. Engberts, Micelle to lamellar aggregate transition of an anionic surfactant in dilute aqueous solution induced by alkali metal chloride and tetraalkylammonium chloride salts, Langmuir, 1995, 11, 455-465.

279. G. C. Kalur and S. R. Raghavan, Anionic wormlike micellar fluids that display cloud points: Rheology and phase behavior, J. Phys. Chem. B, 2005, 109, 8599-8604.

280. M. Jansson, L. Eriksson and P. Skagerlind, The effect of tetraalkylammonium ions on the stability of dilute o/w emulsions, Colloids Surf., 1991, 53, 157-167.

281. M. Rappolt, A. Hickel, F. Bringezu and K. Lohner, Mechanism of the lamellar/inverse hexagonal phase transition examined by high resolution x-ray diffraction, Biophys. J., 2003, 84, 3111-3122.

282. D. P. Siegel, W. J. Green and Y. TALMON, The mechanism of lamellar-to-inverted hexagonal phase transitions: A study using temperature-jump cryo-electron microscopy, Biophys. J., 1994, 66, 402-414.

283. E. Shyamsunder, S. M. Gruner, M. W. Tate, D. C. Turner, P. T. So and C. P. Tilcock, Observation of inverted cubic phase in hydrated dioleoylphosphatidylethanolamine membranes., Biochemistry, 1988, 27, 2332-2336.

284. J. Erbes, C. Czeslik, W. Hahn, R. Winter, M. Rappolt and G. Rapp, On the existence of bicontinuous cubic phases in dioleoylphosphatidylethanolamine, Ber. Bunsenges. Phys. Chem., 1994, 98, 1287-1293.

285. J. M. Seddon, G. Cevc, R. D. Kaye and D. Marsh, X-ray diffraction study of the polymorphism of hydrated diacyl and dialkylphosphatidylethanolamines, Biochemistry, 1984, 23, 2634-2644.

286. M. F. Schneider, R. Zantl, C. Gege, R. R. Schmidt, M. Rappolt and M. Tanaka, Hydrophilic/hydrophobic balance determines morphology of glycolipids with oligolactose headgroups, Biophys. J., 2003, 84, 306-313.

287. O. G. Mouritsen and L. A. Bagatolli, Life - As a Matter of Fat: Lipids in a Membrane Biophysics Perspective, Springer International Publishing, Switzerland, 2016.

288. H. Hauser, M. C. Phillips and R. M. Marchbanks, Physical studies of the interactions of acetylcholine chloride with membrane constituents, Biochem. J., 1970, 120, 329-335.

289. R. H. Templer, J. M. Seddon, P. M. Duesing, R. Winter and J. Erbes, Modeling the phase behavior of the inverse hexagonal and inverse bicontinuous cubic phases in 2:1 fatty acid/phosphatidylcholine mixtures, J. Phys. Chem. B, 1998, 102, 7262-7271. 
290. J. M. Seddon, A. M. Squires, C. E. Conn, O. Ces, A. J. Heron, X. Mulet, G. C. Shearman and R. H. Templer, Pressure-jump X-ray studies of liquid crystal transitions in lipids, Philos. Trans. R. Soc., A, 2006, 364, 2635-2655.

291. D. S. Khvostichenko, J. J. D. Ng, S. L. Perry, M. Menon and P. J. A. Kenis, Effects of detergent $\beta$-octylglucoside and phosphate salt solutions on phase behavior of monoolein mesophases, Biophys. J., 2013, 105, 1848-1859.

292. S. S. Funari, C. di Vitta and G. Rapp, X-ray diffraction and NMR studies on mixtures of non-ionic surfactant $\left(\mathrm{C}_{12} \mathrm{EO}_{2}\right)$ and phospholipids (POPC), Acta Phys. Pol., A, 1997, 91, 953-960.

293. H. Qiu and M. Caffrey, The phase diagram of the monoolein/water system: metastability and equilibrium aspects, Biomaterials, 2000, 21, 223-234.

294. R. Beck and H. Hoffmann, in Bicontinuous Liquid Crystals, ed. M. L. Lynch and P. T. Spicer, CRC Press, USA, 1st edn, 2005, vol. 127, ch. 6, pp. 131-168.

295. A. Pal, P. Bharath, S. G. Dastidar and V. A. Raghunathan, Collapse and coacervation of a lamellar phase by inter-headgroup bridging, Soft Matter, 2012, 8, 927-930.

296. H. Hoffmann, C. Thunig, U. Munkert, H. W. Meyer and W. Richter, From vesicles to the $L_{3}$ (sponge) phase in alkyldimethylamine oxide/heptanol systems, Langmuir, 1992, 8, 26292638.

297. O. D. Lavrentovich, C. Quilliet and M. Kléman, Orientational dependence of the surface free energy of the $L_{\alpha}-L_{3}$ interface, J. Phys. Chem. B, 1997, 101, 420-428.

298. C. Blanc, O. Sanseau and V. Cabuil, The interface between the lamellar and the sponge phases, Mol. Cryst. Liq. Cryst. Sci. Technol., Sect. A, 1999, 332, 523-530.

299. S. B. Rizwan, Y. D. Dong, B. J. Boyd, T. Rades and S. Hook, Characterisation of bicontinuous cubic liquid crystalline systems of phytantriol and water using cryo field emission scanning electron microscopy (cryo FESEM), Micron, 2007, 38, 478-485.

300. G. C. Sutton, N. J. Russell and P. J. Quinn, The effect of salinity on the phase behaviour of purified phosphatidylethanolamine and phosphatidylglycerol isolated from a moderately halophilic eubacterium, Chem. Phys. Lipids, 1990, 56, 135-147.

301. H. N. Patrick and G. G. Warr, Counterion binding and regulation of interactions between charged bilayers, J. Phys. Chem., 1996, 100, 16268-16274.

302. A. A. Al-Badr and H. A. El-Obeid, Acetylcholine chloride: Physical profile, Profiles Drug Subst., Excipients, Relat. Methodol., 2005, 31, 1-19.

303. M. Dubois and T. Zemb, Swelling limits for bilayer microstructures: The implosion of lamellar structure versus disordered lamellae, Curr. Opin. Colloid Interface Sci., 2000, 5, 27-37.

304. M. Dubois and T. Zemb, Phase behavior and scattering of double-chain surfactants in diluted aqueous solutions, Langmuir, 1991, 7, 1352-1360. 
305. A. Sein, J. B. F. N. Engberts, E. van der Linden and J. C. van de Pas, Lyotropic phases of dodecylbenzenesulfonates with different counterions in water, Langmuir, 1996, 12, 29132923.

306. B. A. Coldren, H. Warriner, R. van Zanten, J. A. Zasadzinski and E. B. Sirota, Flexible bilayers with spontaneous curvature lead to lamellar gels and spontaneous vesicles, Proc. Natl. Acad. Sci. USA, 2006, 103, 2524-2529.

307. P. Bharmoria and A. Kumar, Unusually high thermal stability and peroxidase activity of cytochrome $c$ in ionic liquids colloidal formulation, Chem. Commun., 2015, 52, 497-500.

308. P. Bharmoria, T. J. Trivedi, A. Pabbathi, A. Samanta and A. Kumar, Ionic liquid-induced all- $\alpha$ to $\alpha+\beta$ conformational transition in cytochrome $c$ with improved peroxidase activity in aqueous medium, Phys. Chem. Chem. Phys., 2015, 17, 10189-10199.

309. S. Alexander, G. N. Smith, C. James, S. E. Rogers, F. Guittard, M. Sagisaka and J. Eastoe, Low-surface energy surfactants with branched hydrocarbon architectures, Langmuir, 2014, 30, 3413-3421.

310. W. Kunz and G. J. T. Tiddy, in Specific lon Effects, ed. W. Kunz, World Scientific Publishing Co. Pte. Ltd., Singapore, 1st edn, 2010, ch. 12, pp. 313-320. 